\title{
Proceeding
}

Supplementary Issue: Spring Conferences of Sports Science. International Seminar of Physical Education, Leisure and Health, 17-19 June 2019. Castelo Branco, Portugal

\section{Proceedings of the International Seminar of Physical Education, Leisure and Health, 17-19 June 2019. Castelo Branco, Portugal}

Cite this article as:

Proceedings of the International Seminar of Physical Education, Leisure and Health; Castelo Branco, Portugal. (2019). Journal of Human Sport and Exercise, 14(4proc), S1169-S1823. doi:https://doi.org/10.14198/jhse.2019.14.Proc4.82

Table of Contents

Muscle soreness and fatigue and their associations with internal and external load measures in professional soccer players. 1181

Internal and external training load associations in professional soccer players 1185 Comparison of motivational factors for the practice of exercise at gyms and nature and adventure sports 1189

Association of physical activity, self-concept and self-efficacy in high school students 1193 Effect of the Internet and online social media on awareness of ACSM physical activity recommendations 1197

Physical activity level and perceptions about exercise in patients with Osteoporosis 1201 Parental perceptions of physical activity benefits for children with autism spectrum disorders ...1205 Relationship of intrinsic motivation towards sport, with variables related to a healthy lifestyle....1209 Differences between gender and population groups, motivational variables and healthy lifestyles 1213

Short-term effects of myofascial release on isometric knee extensors strength 1217

Iron, phosphorus and magnesium erythrocyte concentrations in men with a high, moderate or low level of physical training 1221

Possible relationship between some trace metals and the hormone insulin in high-level athletes 1225

Erythrocyte concentrations of chromium, manganese and zinc in men with a high, moderate or low level of physical training. 1228 
Arsenic, cadmium and lead erythrocyte concentrations in men with a high, moderate or low level of physical training

Cyberbullying in school: A literature review 1235

The effects of a mind-body program on the cognomotor performance of high school children ....1239 Ethical issues on military physical conditioning

Relationships between psychological skills and European U19 rugby union tournament outcomes and performance indicators 1246

Physical performance tests and anthropometric data to predict selection in U19 rugby union players 1250

Physical exercise in higher education: Knowledge, attitudes and practices ..................................1254

Nutritional knowledge and eating attitudes and habits in higher education ...................................1258

Comparisons of external load variables between small-sided and real format games: An 8-week study in professional soccer training context. 1262

Volleyball selection process: How do coaches select? 1266

The importance of attitudes and values in sport and competition: The opinion of a group of coaches of Volleyball 1270

Short-term effects of myofascial release on isometric knee extensors strength 1273

Digital media in professional basketball: The case of supporters of the Hamburg Towers 1277 Planning and assessment by teachers of physical education classes in elementary school 1280 Pedagogical model for teaching combat sports in physical education class: Presentation of partial results 1283

Intra-week variations and associations between internal and external load measures in a elite volleyball team 1286

Frequency of sports participation, body image satisfaction and psychological well-being: Gender differences among vocational students 1290

Analysis of physical-sport motivations in adolescents according to gender, age and BMI 1294 Olympism: Level of knowledge between different University Degrees in the Faculty of Education. Differences by gender and University Degree 1298 Academic routine impact on physical activity level of university students: A longitudinal study .1302 Cooperative games vs competitive games in Primary School Education: What effects do they have on schoolchildren motivation? 1305

Physical activity index for Galician schoolchildren of primary school education according to age and gender 1308

Fitness professionals: Narrative review 1311

Competence towards Physical Education inclusion: Self-perception of Galician future teachers of Primary School Education

Principal complaints and reference to spine pain in young tennis players during a tournament: Data from massage therapy care 1319 
Terminal actions at Brazilian's Volleyball League 1322

Effects of a training program in post-menopausal women . 1326

"Destroying stereotypes, building on equality": Didactic intervention with Master in Teaching of Physical Education students 1330

Perception of primary school children about the playful and sport practice 1334

Socio-demographic characteristics of nursing students and the prevalence of physical activity .1338 Inequalities in female combat sports

Evaluation of coordinated motor ability in handball players 1346

How assessment the flexibility in handball players? Results of a systematic review 1349

Evaluation of urinary protein and creatinine concentration in athletes after high-performance physical exercise 1353

Lipid profile and associated factors among an academic community of Higher Education 1356 Sociological analysis of three dual combat practices in Portugal: The case study of aikido, judo and wrestling.... 1360

Comparison of propulsive forces between two head-out water exercises .....................................1364

Experiences of bullying in education and school paths. 1368

Insights on a sucessfull research-to-practice partnership with Matosinhos city hall: The case of surfing in schools 1372

Levels of insufficient health-related physical activity in Portuguese adolescents 1375

Gender differences in psychosocial benefits of physical activity and sports participation in youth 1379

Watching overweight: Monitoring in child health consultations 1383

An aesthetic reflection in school sports: Notes on the participation of the sportive delegation of Campus Santo Ângelo in the student games of IF Farroupilha (JEIF) .............................................1387

Self-perception of life quality and the practice of physical activity in elderly .................................1391

Anthropometric, somatotype and physical profile of young female roller skaters .........................1395

Functional physical fitness in elderly: Differences depending on the practice of physical activity 1399

Physical activity levels in adults with intellectual disabilities: The importance of physical education 1403

A comparison of physical fitness by competitive levels in youth basketball players ...................1407

Present and future of the soccer specialists perfomance ................................................................1411

Evaluation of physical activity status and cognitive function among breast cancer patients: A crosssectional study....

Physical conditioning of U16 national team players: Mediator effect of previous sport experiences and strength and conditioning practices 1419 
Evaluation of the efficacy of a sensorimotor program in the development of children in swimming lessons

Relationship between physical education, school satisfaction, psychological well-being and academic achievement in vocational students 1427

Water aerobics: The use of wearables. 1430

Influence of the full squat on short sprint performance in young adults 1433

Effect of previous ankle sprain in stride variables during basketball-specific drill: Insights about maturity offset 1437

Is low volume HIIT enough to induce changes in oxygen uptake kinetics? ...................................1441

Warm-up kinematics influence in 30m sprint performance............................................................1445

Bioelectrical impedance vector displacement and phase angle: Progonostic tools for swimmers? 1449

Jumping in the Brazilian Women's Volleyball "B" Super-league .....................................................1453

Smartphone fitness applications used by runners: For what reason? ...........................................1457

The educative role of judo for children in first-cycle primary school: Parents' opinion based on focus group

The development of emotional self-emotion in volleyball project "AVP SOCIAL": Under19 athletes perspective 1465

Self-determined motivation and subjective well-being in Portuguese veteran athletes in different sports. 1469

Self-determined motivation and subjective well-being of adapted sport athletes members of Special Olympics 1473

Monitoring workload in women's basketball based on player tracking device..... 1477

The athlete's perception of parents behaviors in sport context: A study in youth handball players of the Madeira Handball Association. 1481

Fair play : The perception of the young handball players on the parent's behavior......................1485

The importance of imagery in acquiring and improving motor skills and sports technique ..........1489 Comparing differences in motor proficiency of children with and without Autism spectrum disorders 1492

Pre-service physical education teachers tasks load vs. tactical game approach tasks load: A case study 1495

Comparative analysis of interlimb asymmetry in a RSA Test in basketball players .......................1499

Analysis of the action of penalty and double-penalty in football for blind people .........................1503

Loopboard: Device for acrobatics trainning in sliz sports .............................................................1507

Health education in patients with rheumatoid arthritis: A pilot education program ........................1510

Voluntary sports clubs and the participation of young refugees with uncertain perspective of staying: Access barriers and challenges 
In-season internal training load quantification of an under-17 European male soccer team: Starters versus Non-starters. 1518

Pre-season and in-season internal training load quantification of one-week schedules in under-17 European soccer team ..................................................................................................................1522

Bullying among medical students: Integrative literature review ....................................................1526

Perceived motivational climate and goal orientation in soccer athletes: A longitudinal perspective . .1529

Bullying victimization and family interactions of Brazilian students: A mixed study ....................1533

Habitual physical activity patterns of pre-school children from Bragança .....................................1537

The (In)discipline: Playtherapy as prevention ................................................................................1541

Students' opinion on Physical Education and School: An association with academic performance 1545

The curricular identity of Physical Education: New perspective .....................................................1548

The effect of combining general warm-up with specific warm-up in bench press performance ....1552

The importance of workplace health management in the context of skills shortage in small and medium-sized companies

Case study of the programs for soccer teaching of two teachers in training versus the Tactical Game Approach model. 1559

Social and personal skills in Physical Education: teachers and students' preception about an intervention program. 1563

Is $\mathrm{VO}_{2}$ kinetics influenced by swimming intensity in maximal and supramaximal velocities in young female swimmers? 1566

Training and leadership profile in adapted sport coaches and the implication in athletes with intellectual disabilities 1570

Adapted sports: An experience for initial skills development of sport professionals 1574

Perceived barriers and physical activity levels in older adults: The role of education 1578

Supervised vs. non-supervised physical activity: The impact on functional fitness in older adults 1582

Playfulness in education: A systematic review 1586

The 2019 Special Olympics World Games experience: Perspective of athletes who participated 1590 The importance of sports to the Inclusive Research Group of APPACDM Castelo Branco. 1593 Adapted sports: Curricular traineeship 1596

The observation: Adapted table tennis. 1600

Changing elderlies strength levels with a four months multicomponent training program ...........1604 Bone mineral density and muscle strenght in elderly: A cross-sectional study 1608 Effects of a multicomponent exercise program with duration of 12 weeks on the quality of life in breast cancer survivors 1612 
Orienteering sport and environmental education: A theoretical review 1620

Evaluation of the quality of life of schoolchildren of the EJA in the Municipality of Itacoatiara, Amazonas. 1624

Is performance in basketball referees affected by gender? 1628 Associations of physical activity with body composition and aerobic capacity in adults with Down syndrome 1632

Differences on body composition and biochemical parameters between practitioners and nonpractitioners of soccer 1636

Which factors are related with coaches' perception of young soccer players competence: Physical fitness, motor coordination or specific skill?

Sports attitudes of young people practicing orienteering: The influence of the additional practice of another sport. 1643

The thinking process of Football coaches: The training factors. 1647

Differences of Imagery ability between youth soccer and swimming practitioners. 1651 Comparison of physical fitness between young and middle-aged adults 1655

Adherence to physical activity guidelines and body composition in elderly people using objective measurements 1659

Correlation between pulmonary function and aerobic capacity in middle-aged adults 1663 Exploring relative age effect and maturity status on physical performance of school-age children 1667

Push-ups with hands or feet on unstable surface: Does it affects muscle activation and ground reaction forces? 1670

Correlation between vertical stiffness and agility performance in sport students 1674

Parental involvement in health promotion programs during pre-school aged children: A systematic review. 1678

Motor development in children from 12 to 46 months: Influence of the variable "type of breastfeeding" 1682

Physiological responses at maximal aerobic swimming pacing in different distance-trials ...........1686

What do students think in physical education?. 1690

Leisure-time physical activity and food consumption among Brazilian university students ..... 1693 Motor imagery and music: The influence of music on mental rotation of bodily-related pictures 1696 Study of differences in motor coordination, comparing individuals with eutrophic and overweight, with Intellectual Disability..... 1700 Validity and applicability of a web and mobile application to control the physical recovery of athletes 1704 Motor imagery and music: A function of arousal? 1707

Effects of a physical exercise program on body composition and functional physical fitness in the elderly 
Quality of life of Nursing students: Relationship with the level of Physical Activity .....................1715

Motor skills in childhood: From the family perceptions to the practices of the children 1719

Association between body composition and functional physical fitness in the elderly population

Correlation between sedentary behavior, physical activity and lung function in the elderly

Study of the relationship between global motor skills, fine motor skills and age

Functional exercise vs aquafit for seniors

Effects of a senior exercise program on functional capacity in institutionalized elderly in the municipality of Mação

Comparison of physical fitness tests and special judo fitness test performance and classificatory tables development for juvenile and cadet male athletes

Postural stability and handgrip strength in the older adults: Differences between fallers and nonfallers

Fine motor skills: An emergent competence in preschool age 1751

Physical education in primary school: From perceptions to practices 1755

Physical fitness level of a population with mild cognitive impairment 1758

The sports practice of karate in Portugal: Sociological analysis of the identities, ideologies, communities and cultures of the Portuguese karateka's (brown and black belts) .........................1762

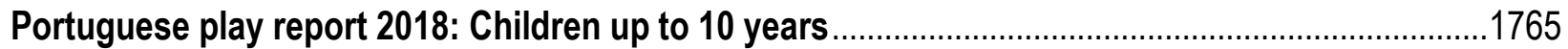

Prevalence of Methicillin - Resistant Staphylococcus Aureus in students of higher education .....1768

Risk factors and arterial hypertension

The importance of the electrocardiogram in the competitive pre-period.

Excess weight and obesity in a region of the interior of Portugal

Venous insufficiency and sedentary job activity

Peripheral arterial systolic-diastolic velocities in athletes and non-athletes by Doppler ultrasound

Pilates for elderly women: An improvement in functional mobility and balance 1792

Physical activity and subjective well-being in Health Sciences first-year students. 1796

Validation of the Intentionality Scale of being physically active in a Portuguese population .........1800

Motivations for the practice of adventure and nature physical activities on young people ............1804

The Cooperative Games with children: Communitarianism and citizenship ....................................1808

Assessent of Portuguese wheelchair basketball team motivation and anxiety levels ....................1812

Assessment of physical capacities of the Portuguese wheelchair basketball team ........................1816

Sociocultural animation in 1 st cycle for educational sucess

Is running kinematics of university trainned students changed by hipertrophy training? A pilot study 


\section{(c) (i) (3)}

This work is licensed under a Attribution-NonCommercial-NoDerivatives 4.0 International (CC BY-NC-ND 4.0). 


\title{
Leisure, education and work: Reflections on improving people's quality of life
}

\author{
AGUINALDO CESAR SURDI $\triangle$, GEORGE TAWLISTON SOARES GADELHA \\ DEF/UFRN, Brazil
}

\begin{abstract}
The article discusses leisure as a condition to improve human health and quality of life. Today, we can see in society the emphasis on the values of the world of work and the excessive pressure for results and income that go beyond real human possibilities. This process makes man a slave to work, so that his leisure becomes an extension of this work, affecting his health and, therefore, his quality of life. Hence the need to strengthen the discussion about the relationship between education, health and leisure and the role of physical education in this context. Keywords: Business; Education; Labour.
\end{abstract}

Corresponding author. Avenida Praia de Ponta Negra, 9119, Ponta Negra - Natal, RN cep 59094-100. Brazil.

E-mail: aguinaldosurdi@yahoo.com.br

Supplementary Issue: Spring Conferences of Sports Science. International Seminar of Physical Education, Leisure and Health, 17-19 June 2019. Castelo Branco, Portugal.

JOURNAL OF HUMAN SPORT \& EXERCISE ISSN 1988-5202

(C) Faculty of Education. University of Alicante.

doi:10.14198/jhse.2019.14.Proc4.82 


\section{INTRODUCTION}

With the process of globalization of the world of work, the conditions of the quality of workers' lives are aggravated, given that hard-won labour rights are being deregulated, and it can be seen that one can no longer speak in the world of homogeneous work, but in worlds of work. With this new configuration, the workforce is overexploited by extreme conditions such as meagre wages, long hours of work, which are "legalized" by overtime. The need for leisure is essential for every human being. It is possible that, in the scope of academic research and leisure sociology, this component of the human life routine has not yet received its proper valuation, given that in current reading there is still a negative view about this institution. For Elias and Dunning (1992), leisure is something that is configured with leisure activities. These are activities directly associated with the destruction of the routine, a characteristic of mimetic excitement that involves leisure activities, such as going to the theatre, or to a concert, to racing, cinema, hunting, fishing, playing bridge, doing mountaineering, betting, dancing or watching television. Marcelino (2004), people are ashamed to claim leisure, for the reason that it is still treated as a "vagabond" thing. This idea of leisure time must begin to change. It seems that we no longer have the right to play and we have to treat all everyday affairs as serious and productive. According to Requixa (1977), education is today understood as the great vehicle for development, and leisure, an excellent and gentle instrument to impel the individual to develop, to improve, to expand his interests and his sphere of responsibilities. The author suggests an education for leisure and leisure, which implies using the principles of leisure as an educational factor and using the sphere of the school as an impulse to make these elements part of the life of both the individual and the whole society. In this article, we intend to broaden the discussion that addresses leisure as an important cultural factor, by enabling an educational process that affects the health of the population and provides a substantial improvement in people's quality of life

\section{TIME OF WORK AND TIME OF LEISURE}

In the world of work, the reduction of the journey was always a subject insistently claimed by the workers, considering that such a measure would imply the increase of their free time with possibilities to take advantage of it in leisure time. We can see in history that this struggle has had an effect and that the working day has gradually diminished. Labour struggles, together with the trade unions which emerged in England in the nineteenth century from the industrial revolution, are instruments that have provided and still provide such gains and serve as resistance to the dictates of capitalist logic.

As a consequence of this historical reduction of the working day, Padilha (2006) points out that this would be a proposal to mitigate the alienation and exploitation of man by work, through the development and rational use of machines. In this sense, Lafargue (1999) comments that it could make the work again a source of pleasure for the worker. This reduction was seen by both Lafargue and Marx as an outlet to increase the "free time" of the worker.

We can understand, therefore, that the conquest of "free time" is a reduction of the working day. This reduction simply does not provide the worker, in the words of Antunes (1999), "life full of meaning," if the logic of capital continues to dictate the rules. Therefore, a human emancipation is necessary in the sense of confrontation and the desire for change. In the words of Pronovost apud Padilha (2006), if we want to conquer a leisure civilization, we will have to fight to overcome the capitalist logic. 


\section{THE IMPORTANCE OF EDUCATION FOR LEISURE}

The idea that leisure is something more than free time comes from the philosophers, especially from Aristotle and Plato. The Greek conception of leisure was based on a time for itself, which was a state or a condition, without preoccupations with any occupation. Over the years and with the Industrial Revolution, the reduction of working hours and the increase of leisure time, leisure has become the meaning of a period that can be understood as free time of work.

This time was available for any activity, which allowed the emergence of the leisure industry. This new, fastgrowing market was fast-moving, and the sectors that got the most out of it were alcoholic vendors, races, football, boxing, events, and all the activities that excited working people in their free time.

In countries such as Brazil, social reality prevents large sections of the population from accessing leisure activities in an integrative, desalinating and well-being perspective. Requixa (1977), seeks to rescue the different manifestations of leisure in the country, and defends the thesis that leisure is a product of the industrial development itself. Marcelino (2004: 53) believes in leisure as "being the favourable moment for cultural changes ..." The action of educators could assume a dual function: that of transforming leisure into an element of change or accommodation, on the one hand, stimulating its function as a factor of humanization or, on the other, exacerbating its alienating function of simple consumer good.

Socioeconomic conditions, as well as free time, influence the development of leisure. It can be seen, then, that the underprivileged classes have their reduced opportunities in relation to the use of leisure, unlike the elites, who manage to occupy and involve him, thanks to the fact that they hold the capital. The notion of leisure must be understood and defined by the quality of the leisure time experience, that is, the satisfaction of a free and pleasurable action and the creation of something like free expression of oneself.

Considering these aspects, the school cannot forget free time as a process of formation. It will be the school's task to provide students with knowledge and opportunities so that they can live, live and work, giving meaning to their lives. Nowadays, these objectives cannot be achieved, simply through an education for work, but parallel to a leisure education and, here, reinforces the role of physical education as a transformation agent.

Taking into consideration that the ideal conditions for leisure cannot be guaranteed only by the individual, it is necessary coordinated action by governments, non-governmental and voluntary organizations, industries, educational institutions and media, leisure education plays important role in reducing differences in conditions and ensuring equality of opportunity and resources.

\section{CONCLUSIONS}

Leisure, education, work and health are fundamental components of life and relate to every moment, day to day. They promote relationships with each other, like a football game on a Sunday, on a barbecue, in a reading and correlate to enable other functions, such as work, study, homework, among others. In the end, without good health one does not get the desired leisure and without a good leisure, in some aspect, our health will be compromised, that is, without them it will be very difficult to acquire a good level of quality of life. This union among education, leisure and work seems to be a fundamental point in improving the quality of life of the people. The Physical Education teacher has the appropriate knowledge to assist at this point. He acts in school and in the community and can strengthen the playful importance of the game, the physical, sports and recreational activities that people can do in their free time. Currently, there is no leisure 
professional, but a professional who understands leisure and believe that the Physical Education teacher can be a reference in this field.

\section{REFERENCES}

Antunes, R. (1995). Adeus ao trabalho? Campinas: Unicamp.

Elias, N.;Dunning, E. (1992). A busca da Excitação. Lisboa: Edições Difel.

Lafargue, P. (1999). O Direito à Preguiça. São Paulo: Hucitec/UNESP.

Marcelino, N. (2004). Lazer e Educação. São Paulo: Papirus.

Padilha, V. (2006). Shopping Center: A catedral das mercadorias. São Paulo: Boitempo editorial.

Requixa, R. (1977). O lazer no Brasil. São Paulo: Brasiliense.

\section{(c) $(\mathrm{B})(\mathrm{EY}$}

This work is licensed under a Attribution-NonCommercial-NoDerivatives 4.0 International (CC BY-NC-ND 4.0). 


\title{
Muscle soreness and fatigue and their associations with internal and external load measures in professional soccer players
}

\author{
FILIPE MANUEL CLEMENTE 1,2 \\ ${ }^{1}$ Polytechnic Institute of Viana do Castelo, School of Sport and Leisure, Melgaço, Portugal \\ 2Instituto de Telecomunicações, Delegação da Covilhã, Portugal
}

\begin{abstract}
The purpose of this study was to examine the correlations between perceived fatigue and muscle soreness (DOMS) and training load measures collected on the same day $(0 \mathrm{~d})$, as well as one day $(+1 \mathrm{~d})$, two days $(+2 \mathrm{~d})$ and three days $(+3 \mathrm{~d})$ before a well-being report. Twenty-three professional soccer players $(24.9 \pm 3.5$ years old; height: $168.8 \pm 41.4 \mathrm{~cm}$ ) from a team competing in the Portuguese first league were daily monitored for one month. Hooper questionnaire, CR10 Borg's scale, and 10-Hz GPS units were daily applied to track the perceived well-being of players and the load impact of the sessions. The correlations between fatigue and DOMS on the same day with the load measures revealed large inverse correlations between fatigue and total distance $(r=-0.53)$, running distance $(r=-0.56)$, and player load $(r=-0.66)$; a moderate inverse correlation between DOMS and player load $(r=-0.44)$. Interestingly, correlations between fatigue and DOMS and the training load measures of the training session two days before the well-being report revealed large positive correlations between fatigue and total distance $(r=0.52)$, as well as large and positive correlations between DOMS and total distance $(r=0.58)$ and DOMS player load $(r=0.60)$. The results suggest that smaller levels of fatigue and DOMS may potentiate a greater availability to support greater loads during training sessions and that the effect of load on fatigue and DOMS is delayed rather than immediate. Keywords: Training load monitoring; Wellness; Internal load; External load; Association football.
\end{abstract}

\footnotetext{
Corresponding author. Complexo Desportivo e Lazer Comendador Rui Solheiro - Monte de Prado, 4960-320, Melgaço. Portugal.

E-mail: filipe.clemente5@gmail.com

Supplementary Issue: Spring Conferences of Sports Science. International Seminar of Physical Education, Leisure and Health, 17-19 June 2019. Castelo Branco, Portugal.

JOURNAL OF HUMAN SPORT \& EXERCISE ISSN 1988-5202

(c) Faculty of Education. University of Alicante.

doi:10.14198/jhse.2019.14.Proc4.82
} 


\section{INTRODUCTION}

The majority of studies suggest that load (on the same day or one day after training) has an immediate effect on well-being parameters. However, information about the delayed effects of load on those well-being parameters is relatively scarce. Thus, the purpose of this study was to test the correlations between perceived fatigue and DOMS and various training load measures collected on the same day as $(0 \mathrm{~d})$, one day before $(+1 d)$, two days before $(+2 d)$, and three days before $(+3 d)$ a well-being report was administered.

\section{MATERIALS AND METHODS}

\section{Participants}

Twenty-three professional soccer players (age: $24.9 \pm 3.5$ years old; height: $168.8 \pm 41.4 \mathrm{~cm}$; body mass: $71.6 \pm 18.7 \mathrm{~kg}$; fat mass: $13.6 \pm 4.6 \%$ ) from a team competing in the first Portuguese league were daily monitored for one month (starting six months after the beginning of the season).

\section{Measures}

Fatigue and muscle soreness (DOMS) were rated based on responses on a scale of 0-7 on an adjusted Hooper questionnaire (Hooper \& Mackinnon, 1995). perceived exertion (RPE) was tested using the CR10 Borg's scale and session-RPE. Internal load (IL) was calculated by multiplying the score by the time of training in minutes (Foster et al., 2001). Total distance (TD), running distance (RD: 14.0-20.0 km.h-1), sprinting distance (SD: > $20.1 \mathrm{~km} / \mathrm{h}-1)$, and player load (PL) were monitored during all training sessions using a 10$\mathrm{Hz}$ GPS (JOHAN Sports, The Netherlands), which was properly validated and is reliable.

\section{Procedures}

Data from 19 training sessions and four matches were collected. The fatigue and DOMS reports were correlated with the training load measures taken on the same day as $(0 \mathrm{~d})$, one day before $(+1 \mathrm{~d})$, two days before $(+2 \mathrm{~d})$, and three days before $(+3 \mathrm{~d})$ he well-being scores. The well-being and RPE scores were reported immediately after players woke up in the morning and 30 minutes after the end of the training session using the JOHAN Sports smartphone app. The GPS units were used by the players during all sessions and matches.

\section{Analysis}

The Pearson correlation coefficient ( $r$ ) was calculated considering the DOMS, fatigue, and training load measured on the same day as and one, two, and three days before the well-being report. The confidence interval was set at $95 \%$. The following scale was used to classify the magnitude of the correlations: $0.0-0.1$ $=$ trivial; $0.1-0.3=$ small; $0.3-0.5=$ moderate $; 0.5-0.7=$ large $; 0.7-0.9=$ very large $>0.9=$ nearly perfect.

\section{RESULTS}

Figure 1 presents the correlations between fatigue and the training load measures reported on different days. Large inverse correlations were observed between fatigue and total distance $(r=-0.53,[-0.77 ;-0.15] ; p<0.05)$, running distance $(r=-0.56,[-0.79 ;-0.19] ; p<0.05)$, and player load $(r=-0.66,[-0.84 ;-0.34] ; p<0.05)$ on the same day as the well-being report (0d). Interestingly, a large positive correlation between fatigue and the total distance $(r=0.52$, [0.11;0.78]; $p<0.05)$ occurred two days before the well-being report $(+2 \mathrm{~d})$. Also, moderate correlations between fatigue and running distance $(r=0.45,[0.02 ; 0.74] ; p<0.05)$ and player load $(r=0.45$, $[0.02 ; 0.74] ; p<0.05)$ were recorded two days before the well-being report. 


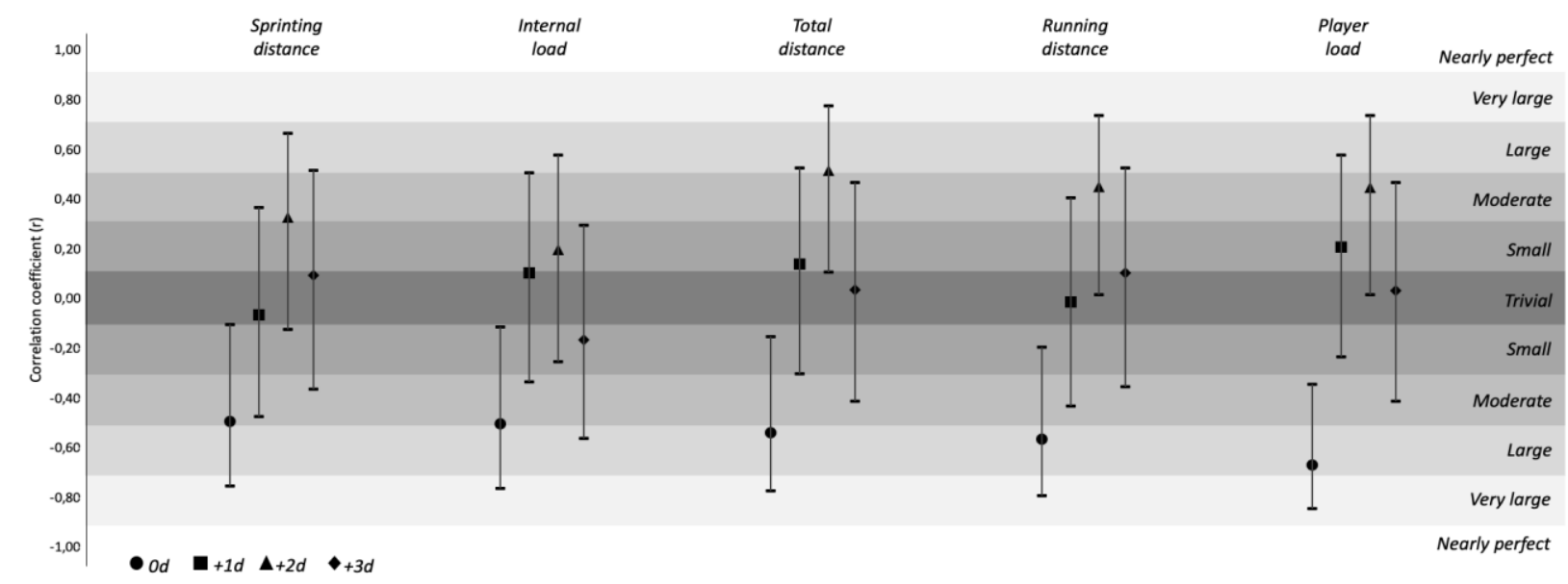

Figure 1. Correlation coefficients $(r)$ between fatigue and training load variables on the same day as $(0 d)$, one day before $(+1 d)$, two days before $(+2 d)$, and three days before $(+3 d)$ the well-being report.

Figure 2 shows the correlations between DOMS and training load measures reported on different days. A moderate inverse correlation between DOMS and player load ( $r=-0.44,[-0.72 ;-0.03] ; p<0.05)$ was recorded on the same day as the well-being report (0d). Large positive correlations were found between DOMS and total distance $(r=0.58,[0.20 ; 0.81] ; p<0.05)$ and between DOMS and player load $(r=0.60,[0.23 ; 0.82] ; p<0.05)$ two days before the well-being report.

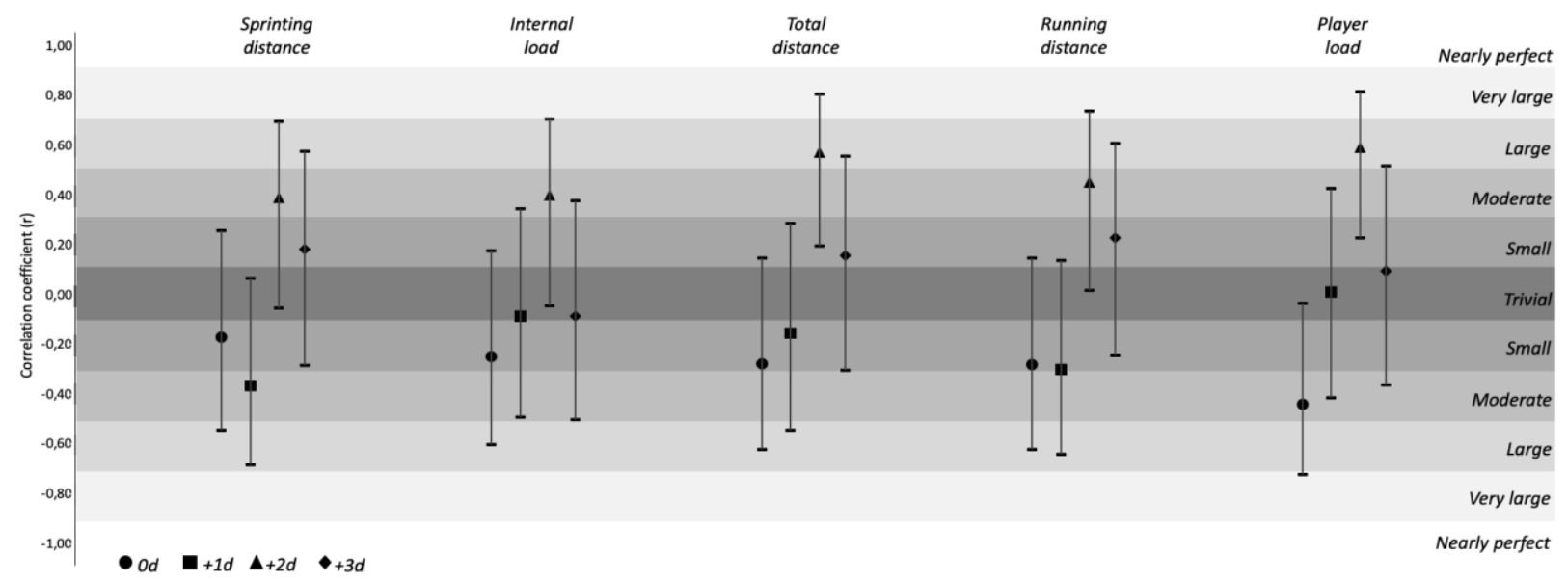

Figure 2. Correlation coefficients $(r)$ between muscle soreness and training load variables on the same day as $(0 \mathrm{~d})$, one day before $(+1 \mathrm{~d})$, two days before $(+2 \mathrm{~d})$, and three days before $(+3 \mathrm{~d})$ the well-being report.

\section{DISCUSSION}

The positive moderate-to-large correlations between training load measures reported two days before the well-being scores suggest that the effects of load on the fatigue and DOMS levels of players are delayed. This can be partially explained by the acute physiological responses to the load that may occur up to 72 hours after the load is imposed, depending on the magnitude of the load and the fitness status/recovery strategies of the players (Silva et al., 2014). Conversely, smaller levels of fatigue and DOMS were 
moderately-to-largely correlated with greater loads quantified in the training session completed on the same day as the well-being report, suggesting that a better recovery status may increase a player's chances of adequately supporting greater loads.

\section{CONCLUSIONS}

The results presented in this article suggest that lower levels of fatigue and DOMS may potentiate a greater availability for professional soccer players to support greater loads during training sessions. Moreover, it was found that the greatest correlations between well-being variables and training load measures occurred two days after the training session, suggesting that the effect of load on fatigue and DOMS is delayed rather than immediate.

\section{FUNDING}

This work is funded by FCT/MEC through national funds and when applicable co-funded by FEDER - PT2020 partnership agreement under the project UID/EEA/50008/2019.

\section{REFERENCES}

Foster, C., Florhaug, J. A., Franklin, J., Gottschall, L., Hrovatin, L. A., Parker, S., .. Dodge, C. (2001). A new approach to monitoring exercise training. Journal of Strength and Conditioning Research, 15(1), 109-115. https://doi.org/10.1519/00124278-200102000-00019

Hooper, S. L., \& Mackinnon, L. T. (1995). Monitoring Overtraining in Athletes. Sports Medicine, 20(5), 321-327. https://doi.org/10.2165/00007256-199520050-00003

Silva, J. R., Rebelo, A., Marques, F., Pereira, L., Seabra, A., Ascensão, A., \& Magalhães, J. (2014). Biochemical impact of soccer: an analysis of hormonal, muscle damage, and redox markers during the season. Applied Physiology, Nutrition, and Metabolism, 39(4), 432-438. https://doi.org/10.1139/apnm-2013-0180

\section{(@) $\odot \Theta \Theta$}

This work is licensed under a Attribution-NonCommercial-NoDerivatives 4.0 International (CC BY-NC-ND 4.0). 


\title{
Internal and external training load associations in professional soccer players
}

\author{
FILIPE MANUEL CLEMENTE ${ }^{1,2}$ \\ ${ }^{1}$ Polytechnic Institute of Viana do Castelo, School of Sport and Leisure, Melgaço, Portugal \\ 2Instituto de Telecomunicações, Delegação da Covilhã, Portugal
}

\begin{abstract}
The purpose of this study was to test the correlation levels between internal load (perceived exertion - RPE; session-RPE) and external load (total [TD], running [RD] and sprinting [SD] distances, and player load [PL]) measures. The study monitored 23 professional soccer players $(24.9 \pm 3.5$ years old; height: $168.8 \pm 41.4 \mathrm{~cm}$; body mass: $71.6 \pm 18.7 \mathrm{~kg}$ ) from a team competing in the first Portuguese league. The players were monitored daily for internal and external load measures over a one-month period. Very large positive correlations between RPE and the external load measures of TD ( $r=0.84), R D(r=0.79)$, SD ( $r=0.73)$. and PL ( $r=0.90)$ were found. Correlation levels between session-RPE and TD ( $r=0.74), R D(r=0.77)$, and PL ( $r=0.79)$ were positive and very large. The evidence of the present study suggests that RPE and session-RPE are valid methods for estimating the overall load exerted by players during matches and training sessions. This can be very important, especially for teams without the economic resources required to monitor RPE using objective measurement instruments. Keywords: Training load; Monitoring; Association football; RPE; Load quantification; Training control.
\end{abstract}

\footnotetext{
Corresponding author. Complexo Desportivo e Lazer Comendador Rui Solheiro - Monte de Prado, 4960-320, Melgaço. Portugal.

E-mail: filipe.clemente5@gmail.com

Supplementary Issue: Spring Conferences of Sports Science. International Seminar of Physical Education, Leisure and Health, 17-19 June 2019. Castelo Branco, Portugal.

JOURNAL OF HUMAN SPORT \& EXERCISE ISSN 1988-5202

(c) Faculty of Education. University of Alicante.

doi:10.14198/jhse.2019.14.Proc4.82
} 


\section{INTRODUCTION}

The use of perceived exertion scales is an easy-to-use and low-cost alternative to using objective measurement instruments (e.g., heart rate monitors) (Alexiou \& Coutts, 2008). The quantification of loads based on perceived scales has been progressively adopted at both professional and amateur levels as a way to provide information about load variations across training sessions (Haddad, Stylianides, Djaoui, Dellal, \& Chamari, 2017). RPE scales are a valid and reliable possible replacement for internal load instruments. However, the relationship of internal load with external load measures (derived from position data) is not wellestablished (Casamichana, Castellano, Calleja-Gonzalez, San Román, \& Castagna, 2013). Based on that, the purpose of this study was to test the correlation levels between perceived load scales and external load measures quantified by GPS (global positioning system).

\section{MATERIALS AND METHODS}

\section{Participants}

The study monitored 23 professional soccer players (age: $24.9 \pm 3.5$ years old; height: $168.8 \pm 41.4 \mathrm{~cm}$; body mass: $71.6 \pm 18.7 \mathrm{~kg}$; fat mass: $13.6 \pm 4.6 \%$ ) from a team competing in the first Portuguese league.

\section{Measures}

The CR10 Borg's scale was used to determine the perceived exertion (RPE) of the players after each training session and match. Each player's score on this scale was then multiplied by the time of the training session/match (in minutes) to provide the session-RPE value (Foster et al., 2001). The external load measures consisted of total distance (TD), running distance (RD: 14.0-20.0 km/h-1), sprinting distance (SD: $>20.1 \mathrm{~km} / \mathrm{h}-1$ ), and player load (PL). These variables were collected using a 10-Hz GPS (JOHAN Sports, The Netherlands) which was properly validated and is reliable.

\section{Procedures}

The players were monitored daily for internal and external load measures over a one-month period (starting six months after the beginning of the season). Nineteen training sessions and four matches were recorded in total. The RPE was scored 30 minutes after the end of each session by using the JOHAN smartphone app. The external load measures were quantified by a $10-\mathrm{Hz}$ GPS unit.

\section{Analysis}

The Pearson correlation coefficient ( $r$ ) was calculated to determine the magnitude of the associations between the training load measures. The confidence interval was set at $95 \%$. The following scale was used to interpret the magnitude of correlations (Hopkins, 2002): $0.0-0.1=$ trivial; $0.1-0.3=$ small; $0.3-0.5=$ moderate; $0.5-0.7=$ large; $0.7-0.9=$ very large; $>0.9=$ nearly perfect.

\section{RESULTS}

Table 1 presents the correlation coefficients between the training load measures. Very large positive correlations between RPE and external load measures of TD ( $r=0.84), R D(r=0.79), S D(r=0.73)$, and PL $(r=0.90)$ were found. Correlation levels between session-RPE and TD ( $r=0.74), R D(r=0.77)$ and PL ( $r=0.79)$ were also positive and very large. 
Table 1. Correlation coefficients ( $r$ ) and confidence intervals $(95 \% \mathrm{Cl})$ between training load measures

\begin{tabular}{|c|c|c|c|c|c|}
\hline & Session-RPE & TD & RD & SD & $\mathrm{PL}$ \\
\hline \multicolumn{6}{|l|}{ Internal load } \\
\hline \multirow{3}{*}{ RPE (A.U.) } & 0.86 & 0.84 & 0.79 & 0.73 & 0.90 \\
\hline & {$[0.69 ; 0.94]$} & {$[0.65 ; 0.93]$} & {$[0.56 ; 0.91]$} & {$[0.45 ; 0.88]$} & {$[0.78 ; 0.96]$} \\
\hline & Very large & Very large & Very large & Very large & Very large \\
\hline \multirow{3}{*}{$\begin{array}{r}\text { Session-RPE } \\
\text { (A.U.) }\end{array}$} & & 0.74 & 0.77 & 0.70 & 0.79 \\
\hline & & {$[0.47 ; 0.88]$} & {$[0.52 ; 0.90]$} & {$[0.40 ; 0.86]$} & {$[0.56 ; 0.91]$} \\
\hline & & Very large & Very large & Large & Very large \\
\hline \multicolumn{6}{|l|}{ External load } \\
\hline \multirow{3}{*}{$\mathrm{TD}(\mathrm{m})$} & & & 0.91 & 0.89 & 0.92 \\
\hline & & & {$[0.80 ; 0.96]$} & {$[0.75 ; 0.95]$} & {$[0.82 ; 0.97]$} \\
\hline & & & Nearly perfect & $\begin{array}{c}\text { Very large } \\
0.94\end{array}$ & $\begin{array}{c}\text { Nearly perfect } \\
0.85\end{array}$ \\
\hline \multirow[t]{2}{*}{$\mathrm{RD}(\mathrm{m})$} & & & & [0.86;0.97] & {$[0.67 ; 0.93]$} \\
\hline & & & & Nearly perfect & $\begin{array}{c}\text { Very large } \\
0.76\end{array}$ \\
\hline \multirow[t]{2}{*}{$\mathrm{SD}(\mathrm{m})$} & & & & & {$[0.51 ; 0.89]$} \\
\hline & & & & & Very large \\
\hline
\end{tabular}

RPE: rate of perceived exertion (10-point); TD: total distance; RD: running distance; SD: sprinting distance; PL: player load.

\section{DISCUSSION}

RPE was very largely and positively correlated with total distance, running distance, sprinting distance, and player load. Similar evidence was found for session-RPE. This suggests that a common perceived load measure, such as the CR10 Borg's scale, may represent a valid method for characterizing the load of the session. The results are in line with previous findings, suggesting the usefulness and validity of RPE methods to properly quantify load and represent the stimulus both internally and externally (Casamichana et al., 2013). Moreover, this confirms that derived calculations, such as acute, chronic, and acute: chronic workload ratios, may be computed based on RPE (Haddad et al., 2017) in different contexts without the need for expensive resources.

\section{CONCLUSIONS}

The results of the present study reveal that RPE is a valid and accurate perceived scale for quantifying the overall load performed during training sessions. Therefore, RPE may be more than an internal load measure and could be used in contexts for which the use of objective measurement instruments is not possible.

\section{FUNDING}

This work is funded by FCT/MEC through national funds and when applicable co-funded by FEDER - PT2020 partnership agreement under the project UID/EEA/50008/2019. 


\section{REFERENCES}

Alexiou, H., \& Coutts, A. J. (2008). A comparison of methods used for quantifying internal training load in women soccer players. International Journal of Sports Physiology and Performance, 3(3), 320330. https://doi.org/10.1123/iispp.3.3.320

Casamichana, D., Castellano, J., Calleja-Gonzalez, J., San Román, J., \& Castagna, C. (2013). Relationship between indicators of training load in soccer players. Journal of Strength and Conditioning Research, 27(2), 369-374. https://doi.org/10.1519/jsc.0b013e3182548af1

Foster, C., Florhaug, J. A., Franklin, J., Gottschall, L., Hrovatin, L. A., Parker, S., ... Dodge, C. (2001). A new approach to monitoring exercise training. Journal of Strength and Conditioning Research, 15(1), 109-115. https://doi.org/10.1519/00124278-200102000-00019

Haddad, M., Stylianides, G., Djaoui, L., Dellal, A., \& Chamari, K. (2017). Session-RPE Method for training load monitoring: Validity, ecological usefulness, and influencing factors. Frontiers in Neuroscience, 11. https://doi.org/10.3389/fnins.2017.00612

Hopkins, W. G. (2002). A New View of Statistics. Retrieved March 29, 2018, from http://www.sportsci.org/resource/stats/effectmag.html 


\title{
Comparison of motivational factors for the practice of exercise at gyms and nature and adventure sports
}

\author{
ROBERTA FRONTINI ${ }^{14}$, ANTÓNIO BRANDÃO², MARIA MONTEIRO², ROGÉRIO SALVADOR ${ }^{3}$, \\ RICARDO LIMA², DANIEL FERNANDES², FILIPE MANUEL CLEMENTE2,4 \\ ${ }^{1}$ CiTechCare, Centre for Innovative Care and Health Technology, Polytechnic Institute of Leiria, Portugal \\ 2 Instituto Politécnico de Viana do Castelo, Escola Superior de Desporto e Lazer, Melgaço, Portugal \\ ${ }^{3}$ School of Education and Social Sciences, Polytechnic Institute of Leiria, Portugal \\ ${ }^{4}$ Instituto de Telecomunicações, Delegação da Covilhã, Portugal
}

\begin{abstract}
Physical inactivity continues rising making it necessary to understand what motives the practice of physical exercise and sports. 901 Portuguese practitioners of exercise at gyms and nature and adventure sports modalities were recruited. Participants answered to the Portuguese version of the Exercise Motivations Inventory-2 (EMI-2) to assess the motives for the practice. Independent t-test were executed to compare the motives between the 2 groups and d Cohen was executed for effect size. We found that some motives were significantly higher in exercise at gyms compared to nature and adventure sports, namely Health Avoidance $(p=0.001 ; d=1.27)$, Health Pressures $(p=0.001 ; d=0.76)$, Positive Health $(p=0.001 ; d=1.09)$, Strength \& Endurance $(p=0.001 ; d=1.02)$ and Nimbleness $(p=0.001 ; d=0.80)$. These results are in line with previous studies suggesting that the pursuit of health benefits is usually most identified by physical exercise practitioners. These studies are important to create different motivational profiles which may have an impact in the practice (and in the avoidance of withdrawals) in physical activity practice. Keywords: Exercise at gyms; Nature and adventure sports; Motivation.
\end{abstract}

\footnotetext{
Corresponding author. IPL Campus 2, Morro do Lena - Alto do Vieiro, apartado 4163 | 2411-901 Leiria - Portugal.

E-mail: roberta frontini@hotmail.com

Supplementary Issue: Spring Conferences of Sports Science. International Seminar of Physical Education, Leisure and Health, 17-19 June 2019. Castelo Branco, Portugal.

JOURNAL OF HUMAN SPORT \& EXERCISE ISSN 1988-5202

(C) Faculty of Education. University of Alicante.

doi:10.14198/jhse.2019.14.Proc4.82
} 


\section{INTRODUCTION}

Physical exercise and sports are extremely important in an era where the pandemic of physical inactivity continues rising (Ding et al., 2016). Motivation is crucial for solving this problem. Identifying the main reasons that motivate people to engage in exercise and sports is extremely important. Physical exercise and sports have different specificities, but both contribute to an improvement in people's quality of life and wellbeing. Understanding what motivates people to practice exercise at gyms and/or nature and adventure sports may be related to different benefits: 1) will help improve the programs and classes presented; 2) may help people practice safer and structured physical exercise and sports; 3 ) will help find the most appropriate strategies to remove possible barriers for the practice and, thus, decrease dropouts.

\section{MATERIAL AND METHODS}

\section{Participants}

The sample consisted of 901 Portuguese practitioners of exercise at gyms (recruited in 2 gyms and social networks) and nature and adventure sports modalities (NAS): Canyoning, Mountain Biking, Paragliding, Climbing, Hiking and Canoeing (recruited in clubs, associations, federations and social networks). Participants had a mean age of $35.93 \pm 10.19$. 450 practised exercise at gyms (average age $33.79 \pm 9.91$ ) and 451 practised NAS (average age $38.06 \pm 10.03$ ). The majority of the sample $(71.37 \%)$ was male.

\section{Measures}

To assess the motives for the practice, the Exercise Motivations Inventory-2 (EMI-2) adapted to the Portuguese population (Alves \& Lourenço, 2003) was used. It consists of a 51 item questionnaire answered on a Likert scale ranging from 0 to $5\left(0=\right.$ "nothing true to $\mathrm{me}^{\prime}$ to $5=$ "completely true to me"). The items may be grouped into 5 dimensions (psychological motives, interpersonal motives, health motives, body-related motives, and fitness motives) and 14 factors (table 1). The questionnaire has good psychometric qualities.

\section{Procedures}

Participants filled an informed consent form and all data was collected and analysed anonymously, ensuring the principle of confidentiality.

\section{Analysis}

Dependent variables were tested for normality and homogeneity. After assumptions of normality and homogeneity being observed, independent t-test were executed to compare the motives between the 2 groups. The effect size was calculated using the $d$ Cohen. The following scale was used to classify the magnitude of the effect size (Batterham \& Hopkins, 2006): 0.00-0.20, trivial; 0.21-0.50, minimum; 0.51-0.80, moderate; $>0.80$, strong. The calculation of statistical procedures, with the exception of the magnitude of the effect, was performed using the software SPSS (v.24, IBM statistics, USA) for a level of significance of $5 \%$.

\section{RESULTS}

Table 1 presents the comparisons between the groups for the motivational factors. We found that the Health Avoidance motive was significantly higher in exercise at gyms (EG) compared to NAS with a strong effect size $(p=0.001 ; d=1.27)$. With a moderate dimension, it was also observed that the values of Health Pressures $(p=0.001 ; d=0.76)$, Positive Health $(p=0.001 ; d=1.09)$, Strength \& Endurance $(p=0.001 ; d=$ 1.02 ) and Nimbleness $(p=0.001 ; d=0.80)$ were higher in $E G$ practitioners. 
Table 1. Comparisons between activities for the motivational factors

\begin{tabular}{|c|c|c|c|c|c|}
\hline & $\begin{array}{c}E G(N=450) \\
M(S D)\end{array}$ & $\begin{array}{c}\text { NAS } \\
(\mathrm{N}=451) \\
\mathrm{M}(\mathrm{SD})\end{array}$ & $\begin{array}{l}\% \text { of difference } \\
\text { (EG-NAS) }\end{array}$ & $p$ & $\begin{array}{l}\mathrm{d} \text { (effect) } \\
\text { (EG-NAS) }\end{array}$ \\
\hline $\begin{array}{l}\text { Stress } \\
\text { Management }\end{array}$ & $3.18(1.16)$ & $3.15(1.22)$ & -0.94 & 0.763 & 0.03 , trivial \\
\hline Revitalisation & $4.08(0.98)$ & $3.97(0.86)$ & -2.70 & 0.060 & 0.12 , trivial \\
\hline Enjoyment & $3.93(1.10)$ & $3.56(1.24)$ & -9.41 & 0.001 & 0.32, minimum \\
\hline Challenge & $2.97(1.30)$ & $2.92(1.19)$ & -1.68 & 0.557 & 0.04, trivial \\
\hline Health Pressures & $1.47(1.29)$ & $0.61(0.94)$ & -58.50 & 0.001 & 0.76 , moderate \\
\hline Health Avoidance & $3.51(1.21)$ & $1.75(1.54)$ & -50.14 & 0.001 & 1.27, strong \\
\hline Positive Health & $4.35(0.88)$ & $2.99(1.52)$ & -31.26 & 0.001 & 1.09 , moderate \\
\hline $\begin{array}{l}\text { Strength \& } \\
\text { Endurance }\end{array}$ & $3.81(1.07)$ & $2.52(1.44)$ & -33.86 & 0.001 & 1.02 , moderate \\
\hline Nimbleness & $3.54(1.20)$ & $2.47(1.46)$ & -30.23 & 0.001 & 0.80, moderate \\
\hline Social Recognition & $0.81(1.03)$ & $0.97(1.16)$ & 19.75 & 0.031 & -0.15 , trivial \\
\hline Affiliation & $2.66(1.40)$ & $3.17(1.33)$ & 19.17 & 0.001 & -0.37 , minimum \\
\hline Competition & $1.61(1.40)$ & $1.84(1.47)$ & 14.29 & 0.017 & -0.16 , trivial \\
\hline $\begin{array}{l}\text { Weight } \\
\text { Management }\end{array}$ & $2.53(1.62)$ & $2.34(1.64)$ & -7.51 & 0.085 & 0.12 , trivial \\
\hline Appearance & $2.16(1.55)$ & $1.86(1.56)$ & -13.89 & 0.004 & 0.19 , trivial \\
\hline
\end{tabular}

\section{DISCUSSION}

Research has shown that the pursuit of health benefits is one of the reasons most identified by physical exercise practitioners which is in line with our results. It is possible that practitioners of exercise at gyms are more accustomed to hearing about the benefits of physical activity for health compared to NAS practitioners. The planning of NAS may also include aspects related to the health and fitness gains associated with these activities. In fact, studies have shown that the practice of these sports is related to physical and mental improvements (Calogiuri \& Elliott, 2017). Nonetheless, it is possible that this information is not well transmitted to NAS practitioners. An interesting finding was that Strength \& Endurance and Nimbleness were higher in EG practitioners than NAS. It is possible that NAS practitioners seek more convenience and experiencing nature (Calogiuri \& Elliott, 2017) and less physical and health improvements. These studies are important, especially since different fields of leisure-time physical activity have been shown to be associated with different motivational profiles (Calogiuri \& Elliott, 2017). Creating these profiles may be enlightening to help better tailor exercise plans that will meet the needs of the practitioners, helping them continue the practice and motivating them not to give up.

\section{CONCLUSIONS}

According to our results, gyms may use the importance of physical exercise for health in order to engage and recruit possible practitioners. They may also create partnerships with health institutions and professionals (e.g., nutritionists, physicians who prescribe exercise) as a way to enhance their gym. Future studies should continue exploring the motives usually more referred by people who exercise and practice sports. It would be important to create different motivational profiles to guide gyms, clubs, associations and federations. 


\section{REFERENCES}

Alves, J., \& Lourenço, A. (2003). Tradução e Adaptação do Questionário de Motivação para o Exercício. Desporto, Investigação e Ciência, 2, 3-23.

Batterham, A. M., \& Hopkins, W. G. (2006). Making Meaningful Inferences about Magnitudes. Int J Sport Physiol, 1(1), 50-57. https://doi.org/10.1123/ijspp.1.1.50

Calogiuri, G., \& Elliott, L. R. (2017). Why do people exercise in natural environments? Norwegian adults' motives for nature-, gym-, and sports-based exercise. Int J Env Res Pub He, 14(4). https://doi.org/10.3390/ijerph14040377

Ding, D., Lawson, K. D., Kolbe-Alexander, T. L., Finkelstein, E. A., Katzmarzyk, P. T., van Mechelen, W., \& Pratt, M. (2016). The economic burden of physical inactivity: a global analysis of major noncommunicable diseases. Lancet, 388(10051), 1311-1324. https://doi.org/10.1016/s0140$\underline{6736(16) 30383-x}$ 


\title{
Association of physical activity, self-concept and self-efficacy in high school students
}

\author{
GUILHERME DA S. GASPAROTTO1,2 , GISLAINE CRISTINA VAGETTI², BEATRIZ OLIVEIRA \\ PEREIRA ${ }^{3}$, VALDOMIRO DE OLIVEIRA ${ }^{2}$ \\ ${ }^{1}$ Federal Institute of Education, Science and Technology of Paraná, Brazil \\ ${ }^{2}$ Federal University do Paraná (UFPR), Brazil \\ 3University of Minho, Center for Research in Child Studies, Institute of Education, Portugal
}

\begin{abstract}
This study analyses the relationship between physical activity and the perception of self-concept and selfefficacy in high school students. The understanding of this relationship may lead to the development of pedagogical strategies, improving the perception of psychological constructs that might impact adolescents' academic success. Self-report instruments were employed to collect the following data from 330 high school students: self-concept and self-efficacy perception and weekly time spent on moderate-to-vigorous physical activity. Association was demonstrated through linear regression adjusted for age, sex and socioeconomic status. It was observed that physical activity partially explained the general perceived academic self-efficacy (Beta: 0.13; $p=0.02$ ), the mathematic academic self-efficacy (Beta: $0.24 ; p<0.01$ ), the general perceived self-efficacy, the academic self-concept (Beta: $0.14 ; p<0.01$ ), the social self-concept (Beta: $0.34 ; p<0.01$ ), the physical self-concept (Beta: 0.35; $p<0.01$ ), and the general self-concept (Beta: 0.29; $p<0.01$ ). It was found that moderate-to-vigorous physical activity contributed to the understanding of high school students' self-concept and self-efficacy perceptions. Keywords: Self-concept; Self-efficacy; Students; Physical activity; Adolescents.
\end{abstract}

Corresponding author. Federal Institute of Education, Science and Technology of Paraná, Brazil.

E-mail: guilhermegptt@gmail.com

Supplementary Issue: Spring Conferences of Sports Science. International Seminar of Physical Education, Leisure and Health, 17-19 June 2019. Castelo Branco, Portugal.

JOURNAL OF HUMAN SPORT \& EXERCISE ISSN 1988-5202

(c) Faculty of Education. University of Alicante.

doi:10.14198/jhse.2019.14.Proc4.82 


\section{INTRODUCTION}

The literature suggests that physical activity plays considerable role in the development of self-concept and self-efficacy, which in turn may be determinant of success in various life contexts of children and adolescents as well as in their school quotidian or sports activities (Babic et al., 2014). It is important to stress that many of the studies aiming at observing the relationship between self-concept and self-efficacy were carried out on children. The unravelling of a possible connection between those aspects among adolescents students is particularly important since adolescence is a crucial period to the settling of concepts and behaviours of individuals. Hence, this study sets out to verify the relationship between physical activity, self-concept and self-efficacy in a group of high school students.

\section{MATERIALS AND METHODS}

The study was conducted with 167 females and 163 males whose age averaged $16.4 \pm 0.3$ years. All subjects were students from the campuses belonging to the Instituto Federal de Educação, Ciência e Tecnologia do Paraná, Brazil.

Information on self-concept was collected in the Five-Factor Self-Concept Questionnaire AF5. The questionnaire scores from 01 to 99 and assesses the following five dimensions of the participants' selfconcept: academic, social, emotional, family, and physical. (Coelho et al. 2015).

The General Perceived Self-Efficacy Scale, a Likert-like instrument with ten items that score from 1 to 5, was used to assess the perception of individuals regarding their competence in various life contexts (Sbicigo et al. 2012). The youth's perception of his/her academic competence was measured with the help of a 26 -item Academic Self-Efficacy Scale. The answer options ranged from A to F (Neves e Faria, 2006).

The short version of the International Physical Activity Questionnaire was employed to evaluate the time (in minutes) spent a week on moderate-to-vigorous physical activity (MVPA) Guedes et al. (2005).

Concerning data analysis, the Pearson correlation coefficient was employed to measure the correlation between the variables adjusted for age, sex and socioeconomic status. Afterwards, the weekly time spent on MVPA was correlated with self-concept and self-efficacy via linear regression. The statistical significance was set at $p<0.05$.

Protocol 2.327.626 of the ethics committee of the Federal University of Paraná/Brazil.

\section{RESULTS}

The results of the correlation and the association between the weekly time spent in AFMV with self-concept and self-efficacy, adjusted for age, gender and socioeconomic status are shown in Table 1. 
Table 1. Correlation and the association between the weekly time spent in AFMV with self-concept and selfefficacy, adjusted for age, gender and socioeconomic level

\begin{tabular}{lccccc}
\hline & Correlation Coef. & $\mathbf{p}$ & $\mathbf{R}^{2}$ Adjusted & Beta & $\mathbf{p}$ \\
\hline General ASE & 0.11 & 0.06 & 0.09 & 0.13 & $0.02^{*}$ \\
Portuguese language ASE & 0.33 & 0.55 & 0.06 & 0.08 & 0.13 \\
Mathematics ASE & 0.28 & $<0.001^{*}$ & 0.11 & 0.24 & $<0.01^{*}$ \\
General Self-efficacy & 0.33 & $<0.001^{*}$ & 0.12 & 0.25 & $<0.01^{*}$ \\
Academics SC & 0.12 & $0.03^{*}$ & 0.07 & 0.14 & $0.01^{*}$ \\
Social SC & 0.36 & $<0.001^{*}$ & 0.17 & 0.34 & $<0.01^{*}$ \\
Emotional SC & 0.14 & $0.01^{*}$ & 0.12 & 0.05 & 0.28 \\
Family SC & 0.05 & 0.89 & 0.02 & 0.03 & 0.87 \\
Physical SC & 0.37 & $<0.001^{*}$ & 0.17 & 0.35 & $<0.01^{*}$ \\
General SC & 0.33 & $<0.001^{*}$ & 0.13 & 0.29 & $<0.01^{*}$ \\
\hline
\end{tabular}

ASE: Academic self-efficacy; SC: self-concept; ${ }^{*} p<0.05$.

\section{DISCUSSION}

Body-related activities have been regarded as important strategies to help academic improvement and enhance students' psychosocial competencies. In this perspective, studies indicate physical activity as a viable option to be employed in school contexts (Marques et al., 2016; Babic et al., 2014).

Herein, the linear regression adjusted for age, sex and socioeconomic status showed that the MVPA explained to a certain extent the variances of general academic and mathematics self-efficacy as well as the academic, social, physical and general self-concept. The variance of the general perceived self-efficacy was also explained by MVPA. These results support the idea that not only can regular physical activity improve well-known biological health indicators, it may also promote students' academic, human and psychosocial development (Marques et al., 2016). The results corroborate the study by Lobo et al. (2015) that showed a positive relationship between the time dedicated to physical activities and measures of self-concept and selfesteem.

According to Tamayo et al. (2001), physical activity may not only have physiological impact on psychological factors, but there seems to be a social dimension contained in that variable as well.

\section{CONCLUSION}

Moderate-to-vigorous physical activity has been found to correlate with psychological factors that are important to human and academic development.

\section{REFERENCES}

Babic, M. J., Morgan, P. J., Plotnikoff, R. C., Lonsdale, C., White, R. L., \& Lubans, D. R. (2014). Physical Activity and Physical Self-Concept in Youth: Systematic Review and Meta-Analysis. Sports Medicine, 44(11), 1589-1601. https://doi.org/10.1007/s40279-014-0229-z

Coelho, V. A., Sousa, V., Marchante, M., \& Romão, A. M. (2015). Validation of the Form 5 self-concept questionnaire in a sample of Portuguese children and adolescents. International Journal of Developmental and Educational Psychology. Revista INFAD de Psicología., 1(1), 67-78. https://doi.org/10.17060/ijodaep.2015.n1.v1.11 
Guedes, D.P., Lopes, C. C., \& Guedes, J. E. R. P. (2005). Reproducibility and validity of the International Physical Activity Questionnaire in adolescents. Revista Brasileira de Medicina do Esporte, 11(2), 151-158. https://doi.org/10.1590/s1517-86922005000200011

Lobo, R., Batista, M., \& Delgado, S. C. (2015). Physical activity practice as a potentiating factor of psychological variables and school performance of primary school students. Revista Iberoamericana de Psicología del Ejercicio y el Deporte, 10 (1), 83-93.

Marques, M. I., Pimenta, J., Reis, S., Ferreira, L. M., Peralta, L., Santos, M. I., Santos, S., \& Santos, E. (2016). (In)Satisfaction with body image in adolescence. Nascer e Crescer, 25(4), 217-221.

Neves, S. P., \& Faria, L. (2006). Construction, Adaptation and Validation of the Academic Self-Efficacy Scale (ASES). Psicologia, 20(2), 45-68.

Tamayo, A., Campos, A. P. M, Matos, D. R., Mendes, G. R., Santos, J. B., \& Carvalho, N. T. (2001). The influence of regular physical activity on the self-concept. Estudos de Psicologia (Natal), 6(2), 157165. https://doi.org/10.1590/s1413-294x2001000200004

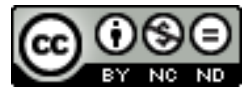

This work is licensed under a Attribution-NonCommercial-NoDerivatives 4.0 International (CC BY-NC-ND 4.0). 


\title{
Effect of the Internet and online social media on awareness of ACSM physical activity recommendations
}

\author{
DULCE ESTEVES ${ }^{1,2}$, PAULO PINHEIRO $0^{1,3}$, RUI BRÁS $^{1,2}$, RICARDO RODRIGUES $^{1,3}$, ANA \\ GOUVEIA ${ }^{1,4}$, KELLY O'HARA $^{1,2}$, PAULO DUARTE 1,3 \\ 1 Universidade da Beira Interior, Portugal \\ ${ }^{2}$ Research Center in Sports Sciences, Health Sciences and Human Development (CIDESD), Portugal \\ ${ }^{3}$ NECE Research Unit in Business Sciences, Portugal \\ ${ }^{4}$ Instituto de Biofísica e Engenharia Biomédica, Faculdade de Ciências, Universidade de Lisboa, Portugal
}

\begin{abstract}
Intervention strategies to increase physical activity (PA) that can reach many people in a cost-effective manner are desired. Web-based interventions have shown promising results, but little investigation focus the effect of use Internet Sites and online social media as information sources on PA knowledge. This investigation aims to evaluate the effect of seeking for PA information on Internet Sites and online social media on awareness of ACSM PA recommendations for health promotion, among college students. The study included a randomly recruited sample of 658 college students (32.1\% males; $67.9 \%$ females), aged 20.8, SD 2.9 years. A survey was designed to evaluate the use different PA information sources and the awareness of ACSM PA recommendations. $63.2 \%$ of respondents were not accurately knowledgeable of the ACSM PA recommendations. Interviewed use Internet Sites (55.8\%) and online social media (29.1\%) to seek for PA information. Looking for PA information online presents a negative influence on awareness of ACSM recommendations $(P=.003$ for Internet Sites and $P=.022$ for online social media).Despite efforts to promote PA and widespread knowledge on ACSM recommendations, about two thirds of interviewed students can not accurately identify adequate PA characteristics for health improvement. The use of new information technologies as PA information source seems to lead to lower level of knowledge, what could be related with poor quality of PA information on web. Keywords: Awareness of ACSM recommendations; Internet; Online Social Media; College Students.
\end{abstract}

Corresponding author. Departamento de Ciências do Desporto, Universidade da Beira Interior, 6200001 Covilhã. Portugal. E-mail: desteves@ubi.pt

Supplementary Issue: Spring Conferences of Sports Science. International Seminar of Physical Education, Leisure and Health, 17-19 June 2019. Castelo Branco, Portugal.

JOURNAL OF HUMAN SPORT \& EXERCISE ISSN 1988-5202

(C) Faculty of Education. University of Alicante.

doi:10.14198/jhse.2019.14.Proc4.82 


\section{INTRODUCTION}

Globally in 2016, $28 \%$ of adults aged $18+$ years were insufficiently physically active (World Health Organization, 2016) with severe consequences on development of several highly prevalent diseases (Rhodes et al., 2017).

Due to the widespread scope of this problem, prevention and intervention strategies are needed to reach large population segments at reasonable costs (Lewis et al., 2017) so, researchers have focused upon the Internet and online social media as a mean of engaging population in a more active lifestyle (Maher et al., 2014). Intervention strategies to increase physical activity (PA) that can reach many people in a cost-effective manner are desired (Esteves et al., 2016). Web-based interventions have shown promising results (Zhang et al., 2016), but little investigation focus the effect of use Internet Sites (IS) and online Social Media (SM) as information sources on PA knowledge (Knight et al., 2015).

This investigation aims to evaluate the effect of seeking for PA information on IS and SM on awareness of ACSM PA recommendations for health promotion, among college students.

\section{MATERIAL AND METHODS}

\section{Participants}

This investigation is a cross-sectional population-based study in Portugal. A total of 658 college students between 18 to 25 years old (mean=20.8; $S D=2.9$ ); $32.1 \%$ males; $67.9 \%$ females, were included in this study.

\section{Measures}

The study was based on a questionnaire specifically designed to meet investigation goals. The awareness of ACSM PA recommendations was assessed as indicated by Bennett et al. (2009). PA information sources were evaluated according to expressed by Pinheiro et al. (2012).

\section{Procedures}

Participants were completely free to participate in the study, after the presentation of researchers' affiliation, investigation goals and guaranty of total confidentiality. College students were asked to answer the questionnaire (time: 10 minutes). To ensure content and face validity, all items were reviewed by an expert panel of professors and researchers not involved in the study.

\section{RESULTS}

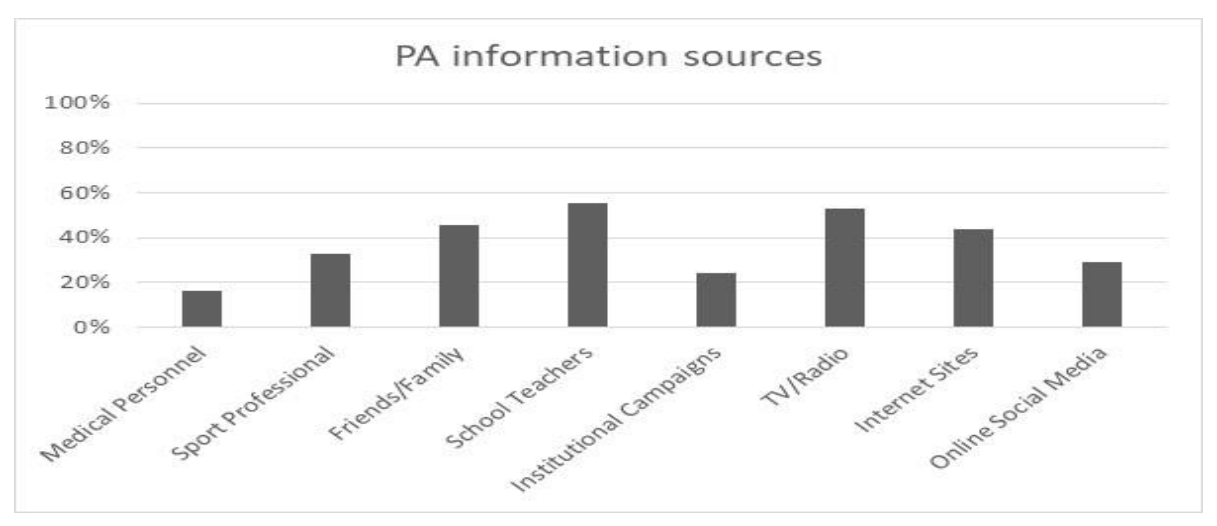

Figure 1. Self-reported use of different PA Information Sources by college students. 
Only 36.9 of the students correctly recognized ACSM PA recommendations. The information sources used to get PA information reported are expressed on Figure 1. Most participants receive PA information by schoolteachers, TV/Radio and Friends/family. Looking for PA information both in IS and in SM presents a negative influence on awareness of ACSM recommendations ( $P=.003$ for IS and $P=.022$ for $S M$ ).

\section{DISCUSSION}

Bennet et al. reported that only a third of general population were accurately knowledgeable of the ACSM PA recommendations. Our results are very similar, except our participants were college students. It is very peculiar that the use of IS and MS show a negative effect on knowing PA recommendation to a healthy lifestyle.

\section{CONCLUSIONS}

Despite efforts to promote PA and widespread knowledge on ACSM recommendations, almost two thirds of college students can not accurately identify adequate PA characteristics for health improvement. This lack of awareness may impair the adoption of an active lifestyle. These findings highlight the need for more effective campaigns to widespread ACSM recommendations for health promotion among universities.

\section{REFERENCES}

Bennett, G. G., Wolin, K. Y., Puleo, E. M., Mâsse, L. C., \& Atienza, A. A. (2009). Awareness of national physical activity recommendations for health promotion among US adults. Med Sci Sports Exerc., 41(10), 1849. https://doi.org/10.1249/mss.0b013e3181a52100

Esteves, D., Pinheiro, P., O'Hara, K., Brás, R. (2016). Internet and Social Network as Health/Physical Activity Information Sources, in The Encyclopaedia of E-Health and Telemedicine, Ed. IGI Global, 49, 634-645. https://doi.org/10.4018/978-1-4666-9978-6.ch049

Knight, E., Intzandt, B., MacDougall, A., \& Saunders, T. J. (2015). Information seeking in social media: A review of YouTube for sedentary behavior content. i-JMR, 4(1) e3. https://doi.org/10.2196/ijmr.3835

Lewis, B. A., Napolitano, M. A., Buman, M. P., Williams, D. M., \& Nigg, C. R. (2017). Future directions in physical activity intervention research: expanding our focus to sedentary behaviors, technology, and dissemination. J. Behav. Med., 40(1), 112-126. https://doi.org/10.1007/s10865-016-9797-8

Maher, C.A., Lewis, L.K., Ferrar, K., Marshall, S., De Bourdeaudhuij, I., Vandelanotte, C. (2014). Are health behavior change interventions that use online social networks effective? A systematic review. JMIR 16, e40. https://doi.org/10.2196/imir.2952

Pinheiro, P., Esteves, D., \& Brás, R. (2012). Evaluation of new information technologies exposure on knowledge retention regarding benefits of physical activity on health status. EJISE, 14(1), 122.

Rhodes, R. E., Janssen, I., Bredin, S. S., Warburton, D. E., \& Bauman, A. (2017). Physical activity: Health impact, prevalence, correlates and interventions. Psychol Health, 32(8), 942-975. https://doi.org/10.1080/08870446.2017.1325486

World Health Organization. (2016). Global Health Observatory (GHO) data: Prevalence of insufficient physical activity. Retrieved from http://www.who.int/gho/ncd/risk_factors/physical_activity/en/

Zhang, J., Brackbill, D., Yang, S., Becker, J., Herbert, N., \& Centola, D. (2016). Support or competition? How online social networks increase physical activity: A randomized controlled trialPrev Med Rep., 4, 453-458. https://doi.org/10.1016/j.pmedr.2016.08.008 


\section{(c) (i) (3)}

This work is licensed under a Attribution-NonCommercial-NoDerivatives 4.0 International (CC BY-NC-ND 4.0). 


\title{
Physical activity level and perceptions about exercise in patients with Osteoporosis
}

\author{
DULCE ESTEVES ${ }^{1,2}$, PAULO PINHEIRO ${ }^{1,3}$, RUI BRÁS $^{1,2}$, RICARDO RODRIGUES $^{1,3}$, ANA \\ GOUVEIA1,4, KELLY O'HARA ${ }^{1,2}$, PAULO DUARTE ${ }^{1,3}$ \\ 1 Universidade da Beira Interior, Portugal \\ ${ }^{2}$ Research Center in Sports Sciences, Health Sciences and Human Development (CIDESD), Portugal \\ ${ }^{3}$ NECE Research Unit in Business Sciences, Portugal \\ ${ }^{4}$ Instituto de Biofísica e Engenharia Biomédica, Faculdade de Ciências, Universidade de Lisboa, Portugal
}

\begin{abstract}
Osteoporosis is a major health concern around the world. Physical activity (PA) is an essential preventive and therapeutic approaches for osteoporosis. This investigation aims to evaluate PA level and of perceptions about exercise of osteoporosis patients, regarding (1) importance of exercise on osteoporosis condition (IE); (2) balance between exercise benefits and risks (B/R); (3) importance of participation in exercise programs (IP); (4) major risks (R); (5) major benefits (B). PA level of 73 postmenopausal Portuguese women (mean=74.3, SD=8.7 years) with diagnosed osteoporosis (T-score < - 2.5 by DXA) was assessed by IPAQ. A complementary questionnaire was designed to evaluate perceptions about exercise. Descriptive statistics were used to analyse data. IPAQ analysis reported $21.9 \%$ high; $35.6 \%$ moderate and $42.5 \%$ low PA. 35.6\% of patients consider that exercise has no impact in preventing osteoporosis evolution and $19.2 \%$ refers that it is only partially important. $63.0 \%$ considers that exercise has more disadvantages than benefits. About IP, $65.8 \%$ of women found daily activities more adequate then exercise programs. Main disadvantages reported (R) were risk of fall $(87.7 \%)$ risk of bone injury $(83.6 \%)$; risk of vertebral fractures $(63.0 \%)$ and risk of increase pain $(52.1 \%)$. Main benefits found were related to calcium fixation $(83.6 \%)$; lower body weight $(78.1 \%)$; increase bone density (69.9\%); and increase muscular tonus (61.6\%). Results suggest that the adherence to an active lifestyle is dependent on the capacity to change perceptions of osteoporosis patients about exercise. Exercise is medicine if patients acknowledge that exercise brings greater benefits than risks. Keywords: Osteoporosis; Physical activity level; Perceptions on exercise benefits and risks.
\end{abstract}

\footnotetext{
Corresponding author. Departamento de Ciências do Desporto, Universidade da Beira Interior, 6200001 Covilhã. Portugal. E-mail: desteves@ubi.pt Supplementary Issue: Spring Conferences of Sports Science. International Seminar of Physical Education, Leisure and Health, 17-19 June 2019. Castelo Branco, Portugal. JOURNAL OF HUMAN SPORT \& EXERCISE ISSN 1988-5202

(C) Faculty of Education. University of Alicante. doi:10.14198/jhse.2019.14.Proc4.82
} 


\section{INTRODUCTION}

Osteoporosis is an age-related disease, characterised by low bone mineral density and compromised bone geometry and microarchitecture, leading to reduced bone strength (McMillan et al., 2017) and increasing the risk of fractures (Lorbergs \& Holl, 2016). Worldwide, osteoporosis causes more than 8.9 million fractures annually, resulting in an osteoporotic fracture every $3 \mathrm{~s}$ (Bial et al., 2017).

Physical activity $(\mathrm{PA})$ is considered a valid instrument of prevention, since immobilization is a major risk factor for bone loss McMillan et al., 2017). In spite of general opinion about exercise benefits on osteoporosis, there is also the perception about injury risks aggregated to it. In fact, Osteoporosis is associated to bone becoming increasingly porous and weak, which leads to bone fragility and elevated fracture risk (Lorbergs et al., 2016). When acknowledging this risk, patients may avoid physical exercise, considering movement increases fracture possibilities (Baert et al., 2015). This may impair exercise adherence and lead to a "kinesiophobia" (Gunendi et al., 2018). Therefore, in order to promote exercise adherence among osteoporosis patients, it is a major concern to evaluate their perceptions about exercise (Baert, et al., 2015), since perceptions about exercise will determine patient behaviour towards an active lifestyle. Accordingly, our research objective is to evaluate PA level and of perceptions about exercise of osteoporosis patients, regarding (1) importance of exercise on osteoporosis condition (IE); (2) balance between exercise benefits and risks (B/R); (3) importance of participation in exercise programs (IP); (4) major risks (R); (5) major benefits (B).

\section{MATERIAL AND METHODS}

\section{Participants}

Lorbergs et al., (2016) refer that the majority of persons with or at risk of osteoporosis are women over 50 years of age. Therefore, our study was based only in women aged over 50 years: 73 postmenopausal Portuguese women (mean=74.3, SD=8.7 years) with diagnosed osteoporosis ( $\mathrm{T}$-score $<-2.5$ by DXA as inclusion criteria). Patients were recruited on Primary Care (medical appointments in Health centres, designed by a nurse).

\section{Measures}

PA level was evaluated using the International Physical Activity Questionnaire (IPAQ), validated to Portugal by Craig et al., 2003. Perceptions regarding exercise were evaluated by a specifically designed questionnaire, based on motivators and barriers referred by Baert et al., (2015); Lorbergs et al., (2016) Rodrigues et al. (2017) and Sale et al., (2019).

\section{Procedures}

Participants were completely free to participate in the study, after the presentation of researchers' affiliation, investigation goals and guaranty of total confidentiality. Osteoporosis patients in regular medical appointment were asked to answer the questionnaire, interview form. Descriptive statistics were used to analyse data.

\section{RESULTS}

IPAQ analysis reported $21.9 \%$ high; $35.6 \%$ moderate and $42.5 \%$ low PA, with $6.4 \pm 2.7$ hours sited/day.

Regarding the perceptions about IE, $35.6 \%$ consider that exercise has no impact in preventing osteoporosis evolution and $19.2 \%$ refers that it is only partially important. Considering $\mathrm{B} / \mathrm{R}, 63.0 \%$ considers that exercise has more disadvantages than benefits. About IP, $65.8 \%$ of interviewed women found daily activities more 
adequate to osteoporosis then exercise programs. Main disadvantages of exercise reported (R) were risk of fall $(87.7 \%)$ risk of bone injury $(83.6 \%)$; risk of vertebral fractures $(63.0 \%)$ and risk of increase pain $(52.1 \%)$. For $\mathrm{B}$, results found calcium fixation in bones (83.6\%); lower body weight $(78.1 \%)$; increase bone density $(69.9 \%)$; and increase muscular tonus $(61.6 \%)$ as major aspects valuated be the sample.

\section{DISCUSSION}

$42.5 \%$ of the sample is almost sedentary, with severe consequences on osteoporosis. Similar results are found by Rodrigues et al., (2017) reporting $50 \%$ of osteoporosis patients as inactive. Major barriers to exercise adherence pointed out by literature are lack of interest, time and fear of falling (Baert et al., 2015), lack of exercise-related knowledge, fear of fracturing, trust in exercise providers (Ziebart et al., 2018). These results are comparable to ours. Perceptions about exercise exposed by intervened patients reveal three main aspects: lack of information about exercise benefits, lack of trust on exercise providers and fear of injuries.

\section{CONCLUSIONS}

Most of interviewed women consider that exercise has little impact on osteoporosis, and have more disadvantages than benefits. Participation in exercise programs is not valuated, regarding benefits on the disease. Results suggest that adherence to an active lifestyle is dependent on the capacity to change perceptions of osteoporosis patients about exercise.

\section{REFERENCES}

Baert, V., Gorus, E., Mets, T., \& Bautmans, I. (2015). Motivators and barriers for physical activity in older adults with osteoporosis. J Geriatr Phys Ther., 38(3), 105-114. https://doi.org/10.1519/jpt.0000000000000035

Bilal, M., Haseeb, A., Merchant, A. Z., Rehman, A., Arshad, M. H., Malik, M., ... \& Shamsi, U. S. (2017). Knowledge, beliefs and practices regarding osteoporosis among female medical school entrants in Pakistan. Asia Pac Fam Med., 16(1), 6. https://doi.org/10.1186/s12930-017-0036-4

Craig, L., Marshall, L., Sjostrom, M., Bauman, A., Booth, M., Ainsworth, E., Pratt, M., Ekelund, U., Yngve, A., Sallis, J., \& Oja, P. (2003) International Physical Activity Questionnaire: 12- Country Reliability and Validity. Med Sci Sports Exerc., 35(8), 1381-1395. https://doi.org/10.1249/01.mss.0000078924.61453.fb

Gunendi, Z., Eker, D., Tecer, D., ... \& Ozyemisci-Taskiran, O. (2018). Is the word" osteoporosis" a reason for kinesiophobia?. Eur J Phys Rehabil Med. 54(5), 671-675. https://doi.org/10.23736/s19739087.18.04931-6

Lorbergs, L., \& Holl, A. (2016). Falling between the cracks: attitudes and perceptions toward osteoporosis prevention among postmenopausal women. J Osteoporos Phys Act., 1-6. https://doi.org/10.4172/2329-9509.1000169

McMillan, L., Zengin, A., Ebeling, P., \& Scott, D. (2017). Prescribing physical activity for the prevention and treatment of osteoporosis in older adults. Healthcare, 5(4), 85. https://doi.org/10.3390/healthcare5040085

Rodrigues, I. B., Armstrong, J. J., Adachi, J. D., \& MacDermid, J. C. (2017). Facilitators and barriers to exercise adherence in patients with osteopenia and osteoporosis: a systematic review. Osteoporosis Int., 28(3), 735-745. https://doi.org/10.1007/s00198-016-3793-2 
Sale, J.., Marwah, A. ... \& Meadows, L. (2019). Evidence of patient beliefs, values, and preferences is not provided in osteoporosis clinical practice guidelines. Osteoporosis Int.1-13. https://doi.org/10.1007/s00198-019-04913-y

\section{(c) $(\mathrm{B})(\mathrm{EY}$}

This work is licensed under a Attribution-NonCommercial-NoDerivatives 4.0 International (CC BY-NC-ND 4.0). 


\title{
Parental perceptions of physical activity benefits for children with autism spectrum disorders
}

\author{
CARLA LOURENÇO ${ }^{1,2,3}$, DULCE ESTEVES 1,2 \\ 1 Universidade da Beira Interior, Portugal \\ ${ }^{2}$ Research Center in Sports Sciences, Health Sciences and Human Development (CIDESD), Portugal \\ ${ }^{3}$ Center for Studies in Education, Technologies and Health (CI\&DETS), Portugal
}

\begin{abstract}
Physical activity (PA) programs are very valuable for children with autism spectrum disorders (ASD). Parental perceptions of physical activity benefits may influence children's behaviours. The purpose of this study was to investigate (1) parents' perceptions about their knowledge regarding the benefits of PA programs; (2) the perceived need of more PA programs addressed to ASD and (3) information sources used to obtain PA information. Participants were made up of nine parents from families with one child with ASD whose ages ranged from 4 to 10. Using a questionnaire, applied in interview form, we evaluate parental beliefs on the importance of PA. Results show that $77.8 \%$ of interviewed parents consider knowing the benefits that PA brings to their children. $44.4 \%$ of parents consider having enough information about PA, and $88.9 \%$ want to have more information about the adequate PA program to ASD children. Most parents receive PA information by ASD Associations and sport professionals. Results show that medical personnel do not emphasise the need of PA-based therapy to ASD children. In spite of the generalization of new communications tools, parents do not use the Internet nor Online social media to get information on PA programs to ASD children. In order to promote PA among ASD children, parents' involvement is crucial. Parents must acknowledge not only the effective benefits of PA programs to ASD children, but also to have proper information regarding the adequate program their specific child. Keywords: Autism spectrum disorders; Physical activity programs; Parents perceptions; Physical activity information sources.
\end{abstract}

\footnotetext{
Corresponding author. Universidade da Beira Interior, Portugal.

E-mail: ccvl@ubi.pt

Supplementary Issue: Spring Conferences of Sports Science. International Seminar of Physical Education, Leisure and Health, 17-19 June 2019. Castelo Branco, Portugal.

JOURNAL OF HUMAN SPORT \& EXERCISE ISSN 1988-5202

(C) Faculty of Education. University of Alicante.

doi:10.14198/jhse.2019.14.Proc4.82
} 


\section{INTRODUCTION}

PA offers an array of benefits for children with ASD on health domains (motor coordination, balance, flexibility) and reducing stereotypical behavioural patterns, improving self-stimulation behaviours, social and communication abilities, academic engagement, cognition, attention and sensory skills (Bremer et al., 2016). Healy et al. (2018) reported that made up of to be effective, it is necessary to involve parents in the intervention delivery, reporting that active parent involvement is an effective and ecological means of intervention delivery. Pitchford et al. (2016) refer that parental perceptions of physical activity benefits may influence children's behaviours towards PA and concluded that PA level was predicted by parental beliefs. This result induces the conclusion that PA promotion among ASD children must involve their parents. In order to involve parents on PA programs it is necessary to certify that they understand the potential benefits of those programs for their children. Accordingly, the purpose of this study was to investigate (1) parents' perceptions about their knowledge regarding benefits of PA programs; (2) the perceived need of more PA programs addressed to ASD and (3) information sources used to obtain PA information. Participants were made up of nine parents from families with one child with ASD whose ages ranged from 4 to 10 . Using a questionnaire, applied in interview form, we evaluate parental beliefs on the importance of PA.

\section{MATERIAL AND METHODS}

\section{Participants}

A purposeful sampling strategy was used to recruit parents of children with ASD for the Project JUMP FOR INCLUSION. Inclusion criteria for participants included: Parents with a child (age 4 to 10) who had a diagnosis of ASD, and participated in the program. Nine parents were recruited to collaborate in the current study and completed interviews.

\section{Measures}

A questionnaire was specifically designed to evaluate (1) parents' perceptions about their knowledge regarding benefits of PA programs; (2) the perceived need of more PA programs addressed to ASD and (3) information sources used to get PA information. Parent's perceptions and perceived needs of PA information were evaluated based on the work of Pitchford et al., (2016). PA information sources were evaluated according to what was stated by Pinheiro et al. (2012).

\section{Procedures}

Participants were completely free to participate in the study, after the presentation of the researchers' affiliation, investigation goals and guarantee of total confidentiality. Parents were asked to answer the questionnaire, interview form. To ensure content and face validity, all items were reviewed by an expert panel of professors and researchers who were not involved in the study. The board consisted of two sports scientists (with research experience), one expert researcher to market studies and survey development and one expert researcher on knowledge management. Fieldwork supervisors conducted a pre-test of the questionnaire with a sample of 15 individuals to ensure the clarity and completion time. The study protocol conformed to the Declaration of Helsinki and was conducted with the Scientific Committee of the Ph.D. in Sports Science approval, from the University of Beira Interior. Descriptive statistics were used to analyse data. 


\section{RESULTS}

Regarding parents' perceptions about their knowledge on benefits of PA programs, results report that $77.8 \%$ of respondents consider acknowledging those benefits, whereas $22.2 \%$ do not really understand the real effect of PA on ASD children.

Concerning the perceived need of more PA programs addressed to ASD, $44.4 \%$ of parents consider having enough information about PA, and $88.9 \%$ want to have more information about the adequate PA program to ASD children. The information sources used to obtain the PA information reported are expressed in figure 1. Most parents receive PA information by ASD Associations and sport professionals.

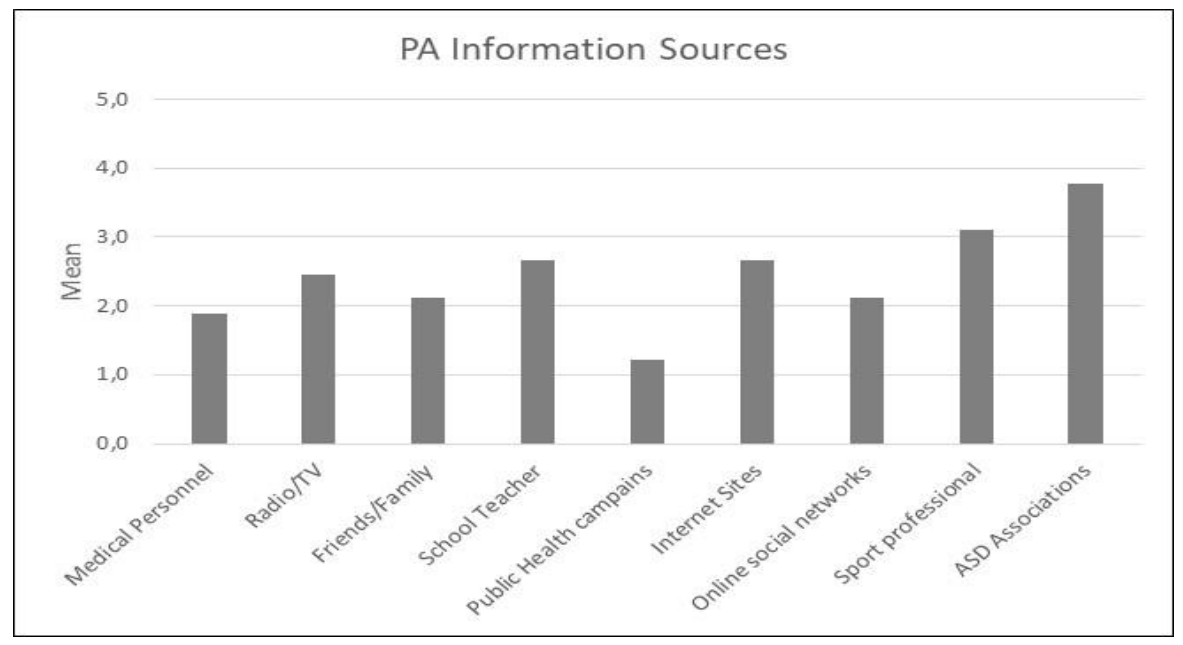

Figure 1. Use of different PA Information Sources by parents of children with ASD.

\section{DISCUSSION}

Results show that most parents already understand the potential benefits that PA brings to their life, but they show the need to have more information regarding PA. Similar results were described by Pitchford et al. (2016) and Healy et al. (2018). ASD Associations are the major PA information source reported. Results show that medical personnel do not emphasise the need of PA-based therapy to ASD children, what is a curious find. It is also important to emphasise that, in spite of the generalization of new communication tools, parents do not use the Internet nor Online social media to find get information on PA programs to ASD children.

\section{CONCLUSIONS}

In order to promote PA among ASD children parents' involvement is crucial. Thus, parents must acknowledge not only the effective benefits of PA programs to ASD children, but also to have proper information regarding the adequate program for their specific child. ASD Associations reveal to be the best mean to promote PA programs. 


\section{REFERENCES}

Bremer, E., Crozier, M., \& Lloyd, M. (2016). A systematic review of the behavioural outcomes following exercise interventions for children and youth with autism spectrum disorder. Autism, 20(8), 899-915. https://doi.org/10.1177/1362361315616002

Pitchford, E. A., Siebert, E., Hamm, J., \& Yun, J. (2016). Parental perceptions of physical activity benefits for youth with developmental disabilities. American journal on intellectual and developmental disabilities, 121(1), 25-32. https://doi.org/10.1352/1944-7558-121.1.25

Healy, S., Marchand, G., \& Williams, E. (2018). "I'm not in this alone" the perspective of parents mediating a physical activity intervention for their children with autism spectrum disorder. Research in developmental disabilities, 83, 160-167. https://doi.org/10.1016/j.ridd.2018.08.014

Pinheiro, P., Esteves, D., \& Brás, R. (2012). Evaluation of new information technologies exposure on knowledge retention regarding benefits of physical activity on health status. Electronic Journal of Information Systems Evaluation, 14(1), 122.

\section{(c)}

This work is licensed under a Attribution-NonCommercial-NoDerivatives 4.0 International (CC BY-NC-ND 4.0). 


\title{
Relationship of intrinsic motivation towards sport, with variables related to a healthy lifestyle
}

\author{
MARTA LEYTON 19 , MARCO BATISTA² ${ }^{1}$ RUTH JIMÉNEZ ${ }^{3}$ \\ ${ }^{1}$ Centre for Sport Studies, University of Rey Juan Carlos, Madrid, Spain \\ 2Sport, Health and Exercise Research Unit (SHERU), Polytechnic Institute of Castelo Branco, Portugal \\ ${ }^{3}$ Research Group on Didactic and Behavioural Analysis of Sport, University of Extremadura, Caceres, Spain
}

\begin{abstract}
This study's objective was to determine which lifestyle variables have a direct relationship with motivation towards the practice of physical activity. There was a sample of 858 subjects, aged between 18 and 77 years (41.62 \pm 13.30$)$, from different parts of Spain. The instruments used for data collection were the Behavioural Regulation Exercise Questionnaire, for Intrinsic Motivation measurement; the Intentionality to be Physically Active Measurement Scale, for the intention of future practice measurement; and the Healthy Lifestyles Questionnaire, for tobacco consumption, rest, balanced diet and respect for meal schedule variables measurement. The results determined that the Intention to be Physically Active variable was significant as a predictor of Intrinsic Motivation towards sport whereas the variables Rest Habits, Tobacco Consumption, Balanced Diet and Respect for Meals Schedule were not significant. The importance of promoting strategies for increasing intrinsic motivation is emphasized. Keywords: Motivation; Physical activity; Healthy lifestyles; Balanced diet; Tobacco; Rest habits.
\end{abstract}

Corresponding author. Centro de Estudios del Deporte. Universidad Rey Juan Carlos, Spain.

E-mail: marta.leyton@uric.es

Supplementary Issue: Spring Conferences of Sports Science. International Seminar of Physical Education, Leisure and Health, 17-19 June 2019. Castelo Branco, Portugal.

JOURNAL OF HUMAN SPORT \& EXERCISE ISSN 1988-5202

(c) Faculty of Education. University of Alicante.

doi:10.14198/jhse.2019.14.Proc4.82 


\section{INTRODUCTION}

It has become valid to study the importance of motivation towards the practice of physical activity throughout different population groups, from teenagers (López-Castedo et al., 2018) to adults and seniors (Leyton et al. , 2017). The goal of any sport professional is to achieve practice adherence. Intrinsic motivation, defined as being "related to the pleasure that is experienced when performing an activity" (Moreno and Martínez, 2006).), plays a very important role for participants, as demonstrated in many studies (Deci and Ryan, 2000; Deci and Ryan, 2012; Granero-Gallegos, et al., 2014). There is a universal consensus that everything related to proper nutrition, the practice of PA, good rest habits and personal hygiene encourages healthy behaviours. On the other hand, stress, lack of rest, smoking, a sedentary lifestyle and alcohol/or psychoactive substances abuse represent a risk to health (Casado-Pérez et al., 2015). Therefore, the objective of this study was to determine which lifestyle variables have a direct relationship with motivation towards the practice of physical activity.

\section{MATERIAL AND METHODS}

\section{Participants}

The study sample was formed by 858 subjects, aged between 18 and 77 years ( $41.62 \pm 13.30)$, coming from different areas of Spain. 455 subjects were female and 403 males. The whole sample performs physical activity of mild or moderate intensity.

\section{Measures}

- Intrinsic motivation. The Behavioural Regulation Exercise Questionnaire (BREQ-3) (Wilson, et al., 2006) in its Spanish version (González-Cutre, et al., 2010) was used. It is composed of 23 items, of which only the Intrinsic Motivation factor was used (e.g. "Because I find it enjoyable and satisfying to exercise"). In this study a Cronbach's alpha value of $=.93$ was obtained.

- Intention to be physically active: The Measurement Scale of Intentionality to be Physically Active (Hein, et al., 2004), validity to Spanish by Moreno, et al. (2007) was used. It consists of five items that are grouped in the same factor or variable, so they measure the same aspect (e.g., "I am interested in my physical form development"). "). In this study a Cronbach's alpha value of $=.87$ was obtained.

- Healthy lifestyles: For healthy lifestyles measurement, the Healthy Lifestyles Questionnaire (VHS) designed by Wold (1995), translated and validated by Leyton, et al. (2018) was used. It consists of 12 items divided into the following factors: Rest Habits (Cronbach's Alpha $=.85$ ) composed of 3 items (e.g. "I usually sleep 7 or 8 hours a day", Tobacco Consumption (Cronbach's Alpha $=.88$ ) composed of 3 items (e.g. "I feel good when I smoke"), Balanced Diet (Cronbach's alpha = .71) composed of 3 items (e.g. "Normally, I eat vegetables and fruits every day") and Respect for Meals Schedule (Cronbach's Alpha $=.70$ ) composed of 3 items (e.g. "Always have breakfast").

\section{Procedures}

Firstly, the online questionnaire was produced using the Google Form platform. Later we administered the questionnaires through different channels (WhatsApp, Facebook, Twitter).

\section{Analysis}

Factorial analysis and reliability analysis (Cronbach's alpha)were carried out. The Kolmogorov-Smirnov test and variances homogeneity using the Levene test were carried out. The results showed a normal data distribution. Next, the descriptive analyses were carried out, and finally, the Regression analysis by steps. 
The program used to analyse the data obtained in the different questionnaires is the statistical program IBM SPSS Statistics 19.0.

\section{RESULTS}

Table 1 shows the regression analysis by steps. It indicates that the Intention to be Physically Active variable was significant as a predictor of Intrinsic Motivation towards sport whereas the variables Rest Habits, Tobacco Consumption, Balanced Diet and Respect for meals schedule were not significant. Collinearity indices (VIF, Tolerance, Condition Index, Durbin-Watson) were observed, and do not exist. In relation to the variance explained, the intrinsic motivation explains a variance of $73 \%$.

Table 1. Coefficients of regression analysis by successive steps, considering as a dependent variable Intrinsic Motivation towards the practice of sports

\begin{tabular}{ccccc}
\hline VARIABLES & $\beta$ & $R^{2}$ & $t$ & $p$ \\
\hline Intention to be Physically Active & .73 & .53 & 31.64 & .00 \\
\hline EXCLUDED VARIABLES & & & & \\
\hline Rest Habits & .01 & - & .52 & .60 \\
Tobacco Consumption & .01 & - & .46 & .64 \\
Balanced Diet & .03 & - & 1.06 & .29 \\
Respect for Meals Schedule & .04 & - & 1.57 & .12 \\
\hline
\end{tabular}

\section{DISCUSSION}

The variable that correlates directly with intrinsic motivation towards practice is the intention to be physically active. Although it is demonstrated that the practice of physical activity involves habits related to a healthy lifestyle (Langille and Rodgers, 2010), no relationship has been found between variables of healthy lifestyles. However, there are several studies that support a direct relationship between intrinsic motivation and the intention of future practice (García, et al., 2012; Moreno, et al., 2012), as confirmed by our results.

\section{CONCLUSIONS}

There is a direct relationship between intrinsic motivation towards the practice of physical activity and the intention to be physically active. It is necessary to continue investigating the relationships between lifestyle variables and motivation towards the practice of physical activity.

\section{FUNDING}

This study was carried out thanks to the contribution of the Ministry of Economy and Infrastructure of the Council of Extremadura, through the European Regional Development Fund. A way to make Europe. (GR18129). We would like to specify that the financial assistance is only for the Professor Ruth Jiménez Castuera.

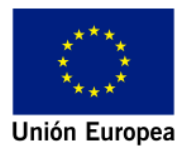

JUNTA DE EXTREMADURA

Consejeria de Economía e Infraestructuras 


\section{REFERENCES}

Deci, E. L., \& Ryan, R. M. (2012). Self-determination Theory. In A. W. Kruglanski, P. A. M. Van Lange \& E. T. Higgins (Eds.), Handbook of theories of social psychology (pp. 416-437). London: SAGE.

Leyton, M., Batista, M., Lobato, S., Aspano, M. I., \& Jiménez, R. (2017). Application of two intervention programs in order to optimize motivation and to improve eating habits in adult and elderly women. $J$ Hum Kinet, 59(1), 131-142. https://doi.org/10.1515/hukin-2017-0153

López-Castedo, A., Domínguez-Alonso, J., \& Portela-Pino, I. (2018). Predictive variables of motivation and barriers for the practice of physical exercise in adolescence. J Hum Sport Exerc, 13(4), 907-915. https://doi.org/10.14198/ihse.2018.134.17

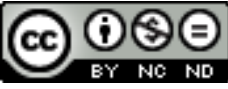

This work is licensed under a Attribution-NonCommercial-NoDerivatives 4.0 International (CC BY-NC-ND 4.0). 


\title{
Differences between gender and population groups, motivational variables and healthy lifestyles
}

MARTA LEYTON1 ${ }^{1}$, MARCO BATISTA², RUTH JIMÉNEZ3

${ }^{1}$ Centre for Sport Studies, University of Rey Juan Carlos, Madrid, Spain

${ }^{2}$ Sport, Health and Exercise Research Unit (SHERU), Polytechnic Institute of Castelo Branco, Portugal

${ }^{3}$ Research Group on Didactic and Behavioural Analysis of Sport, University of Extremadura, Caceres, Spain

\begin{abstract}
The objective of our study was to analyse the difference between genders in the population groups under and over 40 years old, in relation to healthy lifestyles variables such as the intention to be physically active, balanced diet, tobacco, rest, as well as motivational variables related to the practice of physical activity. The sample consisted of 858 subjects, aged between 18 and 77 years old (41.62 \pm 13.30$)$. The instruments used for collection data were the Behavioural Regulation Exercise Questionnaire, for the Intrinsic Motivation measurement, the Intentionality to be Physically Active Measurement Scale, for the intention of future practice measurement, and the Healthy Lifestyles Questionnaire, for the tobacco consumption, rest, balanced diet and respect to the meal schedule variables measurement. The results determined that men have more intention to be physically active, higher more self-determined motivation and greater balanced diet, than women, both in the group under 40 years, and in the group over 40 years It was concluded that men have higher means in variables related to lifestyle, in relation to women. Keywords: Healthy lifestyle; Gender; Physical activity; Motivation.
\end{abstract}

Corresponding author. Centro de Estudios del Deporte. Universidad Rey Juan Carlos, Spain.

E-mail: marta.leyton@uric.es

Supplementary Issue: Spring Conferences of Sports Science. International Seminar of Physical Education, Leisure and Health, 17-19 June 2019. Castelo Branco, Portugal.

JOURNAL OF HUMAN SPORT \& EXERCISE ISSN 1988-5202

(c) Faculty of Education. University of Alicante.

doi:10.14198/jhse.2019.14.Proc4.82 


\section{INTRODUCTION}

The importance of adopting a healthy lifestyle from an early age is essential, and then maintain these habits in the adult stage (Hallal, et al., 2006). Regarding gender, Marcos, Borges, Rodríguez, Huescar, \& Moreno (2011), stressed that the reasons for practice depend on the characteristics of the practitioners, adding that integrated motivation is key to achieving a greater commitment to practice. In their results they found that boys have a greater reason for affiliation and social recognition than girls, in addition to recognizing that the reasons at younger age are related to the image, and at older age the reason for health prevails. There are several studies (Lamoneda \& Huertas, 2017, Práxedes, Sevil, Moreno, del Villar, \& García, 2016) that establish that girls are still showing lower levels of participation in sports physical activities than boys. Therefore, the objective of our study was to analyse the difference between genders in relation to healthy lifestyles variable, in the population groups under and over 40 years old.

\section{MATERIAL AND METHODS}

\section{Participants}

The sample consisted of 858 subjects, with aged between 18 and 77 years $(41.62 \pm 13.30)$, from different areas of Spain. The following frequency groupings were made, between 18 and 40 years old (232 women, $192 \mathrm{men}$ ), and between 41 and 77 years old (223 women and $211 \mathrm{men}$ ).

\section{Measures}

It was used the Behavioural Regulation Exercise Questionnaire (BREQ-3) (Wilson, et al., 2006) in its Spanish version (González-Cutre, et al., 2010), composed of 23 items divided into the following factors: Intrinsic Regulation (Cronbach's Alpha $=.93$ ) composed of 4 items, Integrated Regulation (Cronbach's Alpha $=.92$ ) composed of 4 items, Identified Regulation (Cronbach's Alpha $=.83$ ) composed of 3 items, Regulation Introjected (Cronbach's alpha $=.70)$ composed of 4 items, External Regulation (Cronbach's alpha $=.82$ ) composed of 4 items, amotivation (Cronbach's alpha $=.72$ ) composed of 4 items.

It was used the Intentionality to be Physically Active Measurement Scale (Hein, et al., 2004), validity to Spanish by Moreno, et al. (2007), which consists of five items that are grouped into one factor. In this study it was obtained a Cronbach's alpha value of $=.87$.

For healthy lifestyles measurement, it was used the Healthy Lifestyles Questionnaire (VHS) designed by Wold (1995), translated and validated by Leyton, et al. (2018). It consists of 12 items divided into the following factors: rest habits (Cronbach's alpha $=.85$ ) composed of 3 items, Tobacco consumption (Cronbach's alpha $=.88$ ) composed of 3 items, balanced diet (Cronbach's alpha $=.71$ ) composed of 3 items and respect to the meal schedule (Cronbach's Alpha $=.70)$ composed of 3 items.

In all the questionnaires used, all the items are answered through a Likert scale of 5 points, whose range goes from the value 0 , totally in disagreement to 5 , totally according to the formulation of the question.

\section{Procedures}

First we elaborated the questionnaire in the Google Form platform, for the online questionnaire production. Later we administer the questionnaires through different channels (WhatsApp, Facebook, twitter). 


\section{Analysis}

It was carried out the reliability analysis (Cronbach's alpha). It was carried out the Kolmogorov-Smirnov test and variances homogeneity using the Levene test, the results show a normal data distribution. Next, the descriptive analyses were carried out, and the variance analysis was performed by an ANOVA analysis with Tukey's Post Hoc. The program used to analyse the data obtained in the different questionnaires is the statistical program IBM SPSS Statistics 19.0.

\section{RESULTS}

In Table 1, it is shown the Anova analysis' results, between gender, for the population groups under and over 40 years old.

Table 1. Variance Analysis between the different dependent variables, in the population groups under and over 40 years old, based on gender

\begin{tabular}{ccccccc}
\hline Variables & $\begin{array}{c}\text { Quadratic } \\
\text { mean }\end{array}$ & $\mathrm{F}$ & $\mathrm{P}$ & $\begin{array}{c}\text { Men } \\
\text { mean }\end{array}$ & $\begin{array}{c}\text { Women } \\
\text { mean }\end{array}$ \\
\hline \multirow{2}{*}{$<40$} & Intention to be physically active & 5.69 & 8.54 & .00 & 4.44 & 4.20 \\
years & Intrinsic Regulation & 5.30 & 5.86 & .00 & 4.35 & 4.12 \\
& Integrated regulation & 14.89 & 13.16 & .00 & 4.27 & 3.90 \\
\hline \multirow{2}{*}{$>40$} & Intention to be physically active & 5.69 & 8.54 & .01 & 4.30 & 4.11 \\
years & Balance diet & 5.30 & 5.86 & .03 & 4.86 & 4.04 \\
& Integrated regulation & 14.89 & 13.16 & .01 & 4.22 & 3.99 \\
\hline
\end{tabular}

P: significant between .00 and .05 .

\section{DISCUSSION}

According to Gutiérrez et al. (2018), the motivations differences according to gender exist, determining that in younger ages women tend more towards task orientation and greater intrinsic motivation towards practice (Berlanga, et al., 2018), which entails a greater intention to be physically active (Bermejo, et al., 2018). They also affirm that men tend more towards an orientation towards the ego, and therefore a more extrinsic motivation (Berlanga, et al., 2018). However, Ortuño et al. (2018), state that younger men have a greater intention to practice. In relation to a balanced diet, men obtained a higher average. This result agrees with Candía, et al. (2019). They concluded that women have a healthier diet than men, but as age advances, this diet improves quality, especially in men.

\section{CONCLUSIONS}

In both population groups, men have a greater intention to be physically active, more self-determined motivations and a more balanced diet.

\section{FUNDING}

This study was carried out thanks to the contribution of the Ministry of Economy and Infrastructure of the Council of Extremadura, through the European Regional Development Fund. A way to make Europe. (GR18129). We would like to specify that the financial assistance is only for the Professor Ruth Jiménez Castuera. 


\section{REFERENCES}

Bermejo, J. P., Torres, B. J. A., \& González, J. A. R. (2018). Factores motivacionales relacionados con la intención de seguir practicando ejercicio físico en mujeres adultas. Retos: Nuevas Tendencias en Educación Física, Deporte y Recreación, (34), 117-122.

Candía, S., Candia, P., Mena, R. P., \& Durán, S. A. (2019). Food quality in the elderly population in Santiago of Chile. Rev Esp Geriatr Gerontol (in press). https://doi.org/10.1016/.regg.2019.01.002

González-Cutre, D., Sicilia, A., y Fernández, A. (2010). Hacia una mayor comprensión de la motivación en el ejercicio físico: Medición de la regulación integrada en el contexto español. Psicoth, 22, 841847. https://doi.org/10.5232/ricyde2011.02506

Wilson, P. M., Rodgers, W. M., Loitz, C. C. y Scime, G. (2006). «lt's who I am ... really!». The importance of integrated regulation in exercise contexts. J Appl Biobehav Res, 11(2), 79-104. https://doi.org/10.1111/j.1751-9861.2006.tb00021.x

\section{(2) $(\Theta \Theta \Theta$}

This work is licensed under a Attribution-NonCommercial-NoDerivatives 4.0 International (CC BY-NC-ND 4.0). 


\title{
Short-term effects of myofascial release on isometric knee extensors strength
}

\author{
PEDRO CARVALHO ${ }^{1}$, MIGUEL MORAIS ${ }^{1}$, ALBERT DOS SANTOS², CAROLINA VILA-CHÃ $\tilde{A}^{1,3}$ \\ ${ }^{1}$ Polytechnic Institute of Guarda, Portugal \\ 2University Center Augusto Motta, Brazil \\ ${ }^{3}$ Research Center in Sports Sciences, Health Sciences and Human Development, CIDESD, Portugal
}

\begin{abstract}
Myofascial release (MFR) has been commonly applied by physiotherapists to enhance tissue recovery and to increase myofascial mobility. More recently, and the fitness and sports area myofascial release has been seen as pre-exercise technique restore movement patterns and to enhance motor performance. Nonetheless the results on short-term effects of MFR in muscle strength are controversial. Therefore the aim of this study was to investigate the acute effects of MFR in the maximal isometric strength of the knee extensors. Fourteen healthy men $(22.1 \pm 2.8$ years; mean $\pm S D$ ) were randomly assigned to control or to MFR protocol. Maximum voluntary isometric contraction (MVC) of the knee extensors were measured pre and post experimental protocol (either control or MFR protocol). MFR protocol consisted in a single bout of 90 s of slow stick rolling in the quadriceps muscle of the dominant leg, administered by a researcher, while control protocol consisted in a rest period with a time duration equivalent to MFR protocol duration. The results showed a significant decreased of the knee extensors MVC following MFR protocol (-10.14 $\pm 4.35 \% ; P<0.001)$, while no changes were observed in the control group. This suggests that the present MFR protocol causes transient decrease in strength, which might not be suitable for a plication prior to a workout with significant force demands. Keywords: Myofascial release; Muscle strength; Knee extensors.
\end{abstract}

Corresponding author. Polytechnic Institute of Guarda, Portugal.

E-mail: cvilacha@ipg.pt

Supplementary Issue: Spring Conferences of Sports Science. International Seminar of Physical Education, Leisure and Health, 17-19 June 2019. Castelo Branco, Portugal.

JOURNAL OF HUMAN SPORT \& EXERCISE ISSN 1988-5202

(c) Faculty of Education. University of Alicante.

doi:10.14198/jhse.2019.14.Proc4.82 


\section{INTRODUCTION}

Myofascial release (MFR) has been described as a set of manual therapy techniques through which mechanical pressure is applied to a muscle and fascia, aiming to reduce restrictive barriers or fibrous adhesions seen between layers of fascial tissue (McKnney et al. 2013). It has been postulated that in fascia can occur changes in the ground, such changes might cause fascia less flexible that might restrict muscular forces and movement patterns (Davies \& Davies, 2013). As a result, therapists and fitness professionals have implemented MFR as recovery and maintenance techniques to assist in the process of soft-tissue healing and to restore movement patterns efficiency (McKnney et al. 2013). The scientific literature is reporting a variety of acute effects induced by MFR, including increased flexibility, range of motion, arterial and vascular endothelial function (Davies \& Davies, 2013). However, the effects on muscle strength are inconsistent (Arroyo-Morales et al., 2008; Peacock et al., 2014) following MFR. Therefore the aim of this study was to investigate the acute effects of MFR in the maximal isometric strength of the knee extensors.

\section{MATERIAL AND METHODS}

\section{Participants}

Fourteen healthy young college-aged men $(22.1 \pm 2.8$ years, $177.8 \pm 6.7 \mathrm{~cm}$ of height, and $78.8 \pm 9.6 \mathrm{~kg}$ of body mass), volunteered for this study. Subjects were all university students and recreationally active (regular physical activity at least 3-4 times per week). Before participation, an explanation of the study was given, and the subjects were asked to read and sign an informed consent. The participants were then randomly assigned to control or experimental group (MFR protocol).

\section{Procedures}

Force measurements and experimental protocols were performed in separated lab rooms. In the room 1, each subject performed a light warm up of 5 minutes (cycle ergometer at $50 \mathrm{rpm}$ with a light load). Hereafter, the subject was seated on an isokinetic dynamometer (Humac Norm/ CSMi, USA), with the knee of the dominant leg flexed at $90^{\circ}$. The subject was asked to perform $2 \mathrm{MVC}$ of the knee extensors, over a period of $5 \mathrm{~s}$, with a 2 min of rest in between. The highest MVC value was considered. Then the subject moved to the room 2 and, after 2 minutes, was submitted to: a) control protocol or b) MFR protocol. The control protocol consisted in a rest interval equivalent to MRF protocol time duration (3 minutes). The subjects submitted to MFR were firstly familiarized with the target mechanical pressure by applying the stick in the quadriceps of the non-dominant leg. The subject was asked to classify the pain in a VAS-10 scale. The mechanical pressure that produced a pain of 5-6 in the VAS scale was then applied in the quadriceps of dominant leg by the researcher with a stick Aptonia, for 90 seconds. The, at room 1 and, after 2 min, MVC of the knee extensors were measured as previously described.

\section{Analysis}

A two-way ANOVA with repeated measures (time) was performed on all dependent variables recorded in the pre- and post-conditions test (SPSS). The two factors (2x2) included condition (control and MFR protocol) and time (pre- and post - conditions). Pairwise comparisons were performed with the Student-Newman-Keuls post hoc test when ANOVA was significant. The significance level was set to $P<0.05$. 


\section{RESULTS}

Figure 1 shows the results on MVC knee extensors strength, pre and post both protocols (control and MFR). An interaction condition $x$ time was observed $(P<0.001)$. After MFR protocol the MVC decreased significantly $(10.14 \pm 4.35 \%$; $P<0.001$; Fig. 1), while no changes were observed in the control group.

(A)

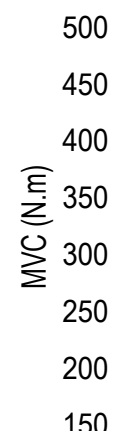

150

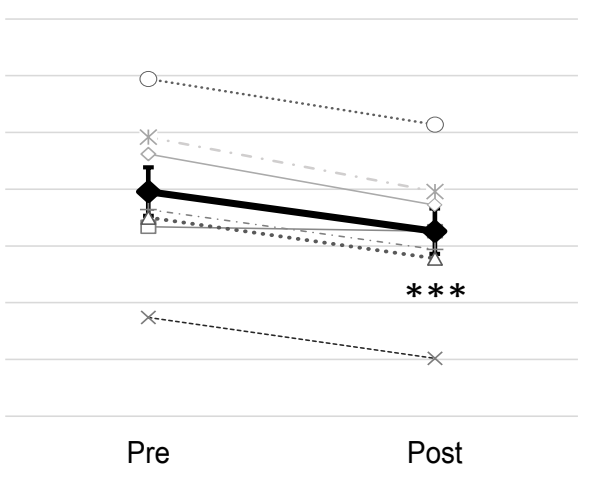

(B)

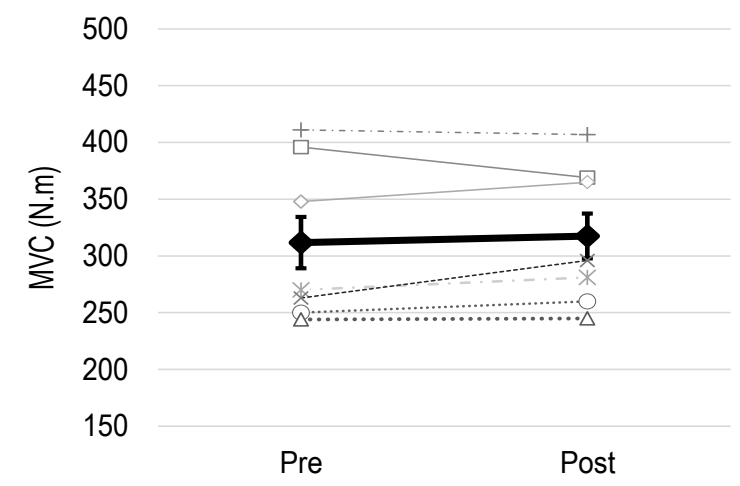

Each line represents 1 subject; thick black line represents the mean of the group. ${ }^{* * *} P<0.001$.

Figure 1. Maximal isometric torque of the knee extensors (MVC, pre and post myofascial release protocol (A) and control protocol (B).

\section{DISCUSSION}

This study investigated the effect of a single bout of 90 s of MFR on the MVC of the quadriceps. The results showed that this protocol negatively affected the isometric strength of the muscles submitted to this protocol. The number of studies on MFR are scarce. Wikktorson-Moller et al (1983), found that massage of 2 minutes decreased the knee extensors isometric strength. Arroyo-Morales et al. (2008), found a decrease in the EMG activity of the vastus medialis following a massage in the quadriceps, which might result in a transient loss of muscle strength, although this was not tested. Studies on self-myofascial release have reported no changes (Healey et al., 2014) or improvement in the muscle strength (Peacock et al., 2014). The controversial results might be explained by the specific characteristics of the MFR protocols.

\section{CONCLUSIONS}

In conclusion, the data from this study indicated that an acute bout of 90 s of slow stick rolling on the quadriceps significantly reduces knee extensors isometric strength. Although the results were driven form static conditions, they suggest that MFR prior to a workout that requires substantial force production are not desirable.

\section{REFERENCES}

Arroyo-Morales, M., Olea, N., Martinez, M., Hidalgo-Lozano, A., Ruiz-Rodriguez, C., \& Diaz-Rodriguez, L. (2008). Psychophysiological effects of massage-myofascial release after exercise: a randomized sham-control study. J Altern Completment, 14, 1223-1229. https://doi.org/10.1089/acm.2008.0253 
Davies, C., \& Davies, A. (2013). The trigger point therapy workbook: your self-treatment guide for pain relief. New Harbinger Publications.

Healey, C., Hatfield, L., Blanpied, P., Dorfman. R., \& Riebe, D. (2014). The effects of myofascial release with foam rolling on performance. J Strength Cond Res. 28. 61-68. https://doi.org/10.1097/01.jsc.0000395625.08079.28

Peacock, C., Krein, D., Silver, T., Sanders, G., \& Carlowitz, K. (2014). An Acute Bout of Self-Myofascial Release in the Form of Foam Rolling Improves Performance Testing. Int J Exerc Sci, 7, 202-211.

Wiktorsson-Moller, M., Oberg, B., Ekstrand, J., \& Gillquist, J. (1983). Effects of warming up, massage, and stretching on range of motion and muscle strength in the lower extremity. Am J Sports Med, 11, 249-252. https://doi.org/10.1177/036354658301100412

\section{(c) $(9)(\mathrm{F}$}

This work is licensed under a Attribution-NonCommercial-NoDerivatives 4.0 International (CC BY-NC-ND 4.0). 


\title{
Iron, phosphorus and magnesium erythrocyte concentrations in men with a high, moderate or low level of physical training
}

\author{
VÍCTOR TORO ROMÁN ${ }^{1}$, IGNACIO BARTOLOMÉ SÁNCHEZ ${ }^{1}$, JAVIER ALVES VAS², MARIO PÉREZ \\ QUINTERO1, JULIO MONTERO ARROYO'1, MARCOS MAYNAR MARIÑO1 \\ ${ }^{1}$ Sport Science Faculty, University of Extremadura, Spain \\ ${ }^{2}$ School of Sport Sciences, Pontifical University of Salamanca, Spain
}

\begin{abstract}
The present study aimed to determine changes occurring in the erythrocytes concentrations of Iron (Fe), Magnesium (Mg) and phosphorous $(\mathrm{P})$ of a high-level trained sportsman, moderate trained levels men and sedentary man's living in the same area of Extremadura (Spain). 30 sedentary subjects, with $24.34 \square 3.02$ years without sports practice and a less active lifestyle, formed the control group (CG); 24 moderately trained subjects with $23.53 \square 1.85$ years, who perform between 4 and 7 hours / week of moderate sports practice without any performance objective and without following any type of systematic training formed the group of subjects with moderate degree of training (MTG) and 22 professional cyclists at the beginning of their sports season, with an age of $23.29[2.73$, who performed more than 20 hours / week of training formed the highlevel training group (HTG). Serum samples were collected for subsequent analysis of the minerals by ICPMS. The results showed that there were statistically significant lower concentration of erythrocyte Fe, Mg and $P$ in the sportsman groups (MTG and HTG) than CG. The correlation study indicates that this change was correlated with training. All results are expressed in $\mu \mathrm{g} / \mathrm{g} \mathrm{Hb}$. We can conclude that physical training produces a decrease in erythrocyte concentration of $\mathrm{Fe}, \mathrm{Mg}$ and $\mathrm{P}$. This situation could produce alterations in the performance of athletes given the importance of the elements. For this reason, we recommend an erythrocyte control at the beginning of the training period and during the training period to avoid harmful deficits. Keywords: Iron; Magnesium; Phosphorous; Erythrocytes and training.
\end{abstract}

Corresponding author. Avenida de la Universidad s/n (Cáceres), Spain.

E-mail: tororomanvictor@gmail.com

Supplementary Issue: Spring Conferences of Sports Science. International Seminar of Physical Education, Leisure and Health, 17-19 June 2019. Castelo Branco, Portugal.

JOURNAL OF HUMAN SPORT \& EXERCISE ISSN 1988-5202

(c) Faculty of Education. University of Alicante.

doi:10.14198/jhse.2019.14.Proc4.82

VOLUME 14 | Proc4 | 2019 | S1221 


\section{INTRODUCTION}

Minerals comprise a large and diverse group of complex ion elements. Thus, trace elements become essential in the human organism. In recent years, interest in the nutritional significance of trace elements has increased considerably, both in the scientific community and in the food industry. (Buxaderas and FarréRovira., 1985). The present study aims to determine the changes that occur in the concentrations of Iron $(\mathrm{Fe})$, Magnesium $(\mathrm{Mg})$ and Phosphorus $(\mathrm{P})$ in erythrocytes of high-level trained athletes, moderately trained subjects and sedentary participants who live in the same area (Extremadura, Spain).

\section{MATERIAL AND METHODS}

\section{Participants}

The participants were divided into three groups: control (GC), moderately trained (MTG) and highly trained (HTG). GC was formed by 30 sedentary subjects (age (years) $=24.34 \pm 3.02$; height $(\mathrm{m})=1.75 \pm 0.18$; weight $(\mathrm{kg})=75.34 \pm 12.03 ; \mathrm{VO}_{2 \mathrm{Max}}(\mathrm{mL}$ min-1 $\left.\mathrm{kg}-1)=38.90 \pm 3.73\right)$ without sports practice and a less active lifestyle. MTG was constituted by 24 moderately trained subjects (age (years) $=23.53 \pm 1.85$; height $(\mathrm{m})=1.77 \pm 0.05$; weight $\left.(\mathrm{kg})=75.12 \pm 8.16 ; \mathrm{VO}_{2 \mathrm{Max}}(\mathrm{mL} \min -1 \mathrm{~kg}-1)=46.77 \pm 6.72\right)$, who perform between 4 and 7 hours / week of moderate sports practice without any performance objective and that imply an active lifestyle, without following any systematic training formed the group of subjects with a moderate degree of training. 22 high level athletes, professional cyclists at the beginning of their sport season (age (years) $=23.29 \pm 2.73$; height $(\mathrm{m})=1.73 \pm 0.07$; weight $\left.(\mathrm{kg})=65.61 \pm 5.22 ; \mathrm{VO}_{2 \mathrm{Max}}(\mathrm{mL} \min -1 \mathrm{~kg}-1)=61.23 \pm 3.00\right)$ who performed more than 20 hours/week of training formed the high-level training group.

\section{Measures}

The physical condition of the subjects was evaluated by performing an ergospirometric test. To perform the test, an ergospirometer system equipped with a gas analyser (Metamax, Biophysik, Gmbh, Germany), a treadmill (Powerjoc, United Kingdom) and a Polar heart rate monitor (Polar, Norway) were used.

\section{Procedures}

All subjects underwent an extraction of blood from the antecubital vein after an overnight fast, deposited in glass tubes containing EDTA as an anticoagulant and centrifuged immediately (2,500 rpm for $10 \mathrm{~min}$.) To separate the plasma of erythrocytes. The previously separated erythrocytes are washed with a $0.9 \%$ sodium chloride solution in ultra-pure water and stored at $-70^{\circ} \mathrm{C}$ until analysis. $\mathrm{Fe}, \mathrm{P}$ and $\mathrm{Mg}$ were determined by ICP-MS.

\section{Analysis}

Subsequently, a comparison of the variables between the three groups was made, using an ANOVA test, applying a Bonferroni test later on if there is significance. It was considered that there was a significant difference when $p<0.05$. 


\section{RESULTS}

Table 1. Characteristics of control group and sportsmen classified by metabolic modalities

\begin{tabular}{lccc} 
& CG & MTG & HTG \\
\hline $\mathrm{Fe}(\mu \mathrm{g} / \mathrm{gHb})$ & $917.78 \pm 113.00$ & $623.50 \pm 212.42^{+++}$ & $517.89 \pm 81.96 \mathrm{tt+}^{+}$ \\
$\mathrm{Mg}(\mu \mathrm{g} / \mathrm{gHb})$ & $41.65 \pm 5.98$ & $29.35 \pm 8.95^{+++}$ & $25.12 \pm 7.12 \mathrm{tt \dagger}^{+\dagger}$ \\
$\mathrm{P}(\mu \mathrm{g} / \mathrm{gHb})$ & $523.18 \pm 88.94$ & $440.78 \pm 111.35^{+++}$ & $398.42 \pm 47.26^{+t \dagger}$ \\
\hline
\end{tabular}

† Differences between HTG and CG ( $† p<0,05 ;+t p<0,01 ;+t+p<0,001) ;{ }^{*}$ Differences between MTG and HTG $\left({ }^{*} p<0,05\right.$; $\left.{ }^{* *} p<0,01 ;{ }^{* * *} p<0,001\right)+$ Differences between MTG and CG $(+p<0,05 ;++p<0,01 ;+++p<0,001)$.

\section{DISCUSSION}

When evaluating the results of the study, we found that the subjects who did physical exercise regularly had concentrations of erythrocytes of $\mathrm{Fe}, \mathrm{Mg}$ and $\mathrm{P}$ significantly lower than the subjects who did not exercise regularly and that these concentrations were correlated with the training. Fe deficiency is the most common nutritional deficiency in the world, even in the richest countries (Looker et al., 1997). Athletes, especially women and adolescents, have a higher risk of depleting their Fe deposits to a state of absolute or functional deficiency that, if not recognized or treated, can develop sideropenic anaemia (J. Beard and Tobin, 2000). The prevalence of this deficiency in adolescents and adult women competing in different sports has been described in a range of $25 \%$ to $36 \%$ (Di Santolo et al., 2008). When Fe deposits are inadequate, physical performance may decrease, presenting fatigue, intolerance to exercise and various cognitive disorders (Auersperger et al., 2013). The concentration of Mg erythrocytes is essential in the evaluation of the body state of Mg (Corica et al., 1997). Likewise, the subjects with the highest level of training have lower concentrations of $\mathrm{P}$. In the study by Maynar et al. found higher levels in the control group than in different groups of physical activity. Therefore, it would be necessary to increase the intake of foods rich in these minerals in athletes (Maynar et al., 2015).

\section{CONCLUSIONS}

The physical training produces a decrease in the concentration of erythrocytes of $\mathrm{Fe}, \mathrm{Mg}$ and $\mathrm{P}$. This situation could provide alterations in the performance of the athletes given the importance of the elements. For this reason, it would be advisable to control the erythrocyte levels of $\mathrm{Mg}, \mathrm{Fe}$ and $\mathrm{P}$ at the beginning of the training period and during it to avoid harmful deficits.

\section{REFERENCES}

Auersperger, I., Škof, B., Leskošek, B., Knap, B., Jerin, A., \& Lainscak, M. (2013). Exercise-induced changes in iron status and hepcidin response in female runners. PLoS One, 8(3), e58090. https://doi.org/10.1371/journal.pone.0058090

Beard, J., \& Tobin, B. (2000). Iron status and exercise. Am J Clin Nutr, 72(2), 594S-597S. https://doi.org/10.1093/ajcn/72.2.594s

Buxaderas, S. C., \& Farré-Rovira, R. (1985). Whole blood and serum zinc levels in relation to sex and age. Rev Esp fisiol, 41(4), 463-470.

Corica, F., Allegra, A., Lentile, R., \& Buemi, M. (1997). Magnesium concentrations in plasma, erythrocytes, and platelets in hypertensive and normotensive obese patients. Am J Hypertens, 10(11), 1311-1313. 
Di Santolo, M., Stel, G., Banfi, G., Gonano, F., \& Cauci, S. (2008). Anemia and iron status in young fertile non-professional female athletes. Eur J Appl Physiol, 102(6), 703-709. https://doi.org/10.1007/s00421-007-0647-9

Looker, A. C., Dallman, P. R., Carroll, M. D., Gunter, E. W., \& Johnson, C. L. (1997). Prevalence of iron deficiency in the United States. JAMA, 277(12), 973-976. https://doi.org/10.1001/jama.1997.03540360041028

Maynar-Marino, M., Crespo, C., Llerena, F., Grijota, F., Alves, J., Munoz, D., \& Caballero, M. J. (2015). Influence of Physical exercise on serum concentration of magnesium and phosphorus. Med Sport, 68(4), 577-584. 


\title{
Possible relationship between some trace metals and the hormone insulin in high-level athletes
}

\author{
VÍCTOR TORO ROMÁN 1 , IGNACIO BARTOLOMÉ SÁNCHEZ ${ }^{1}$, JAVIER ALVES VAS ${ }^{2}$, MARIO PÉREZ \\ QUINTERO1', JULIO MONTERO ARROYO1', MARCOS MAYNAR MARIÑO1 \\ ${ }^{1}$ Sport Science Faculty, University of Extremadura, Spain \\ ${ }^{2}$ School of Sport Sciences, Pontifical University of Salamanca, Spain
}

\begin{abstract}
Introduction: the relation between hormones and toxic metals is still incomplete. Insulin participated in anabolic process. Thus, the aim of this study was to determine the possible correlations between vanadium $(\mathrm{V})$, arsenic $(\mathrm{As})$, cadmium $(\mathrm{Cd})$ and lead $(\mathrm{Pb})$ in serum and the insulin in trained man athletes living in Caceres (Spain). Methods: 62 athletes with a national level that followed a systematic training plan for at least the last two years. Plasma insulin were analysed by ELISA and serum trace elements were analysed with inductively coupled plasma mass spectrometry (ICP-MS). Results: insulin concentrations were inversely related to $V(r=-0.411, p<0.05)$. No differences were found in the other metals. Conclusion: insulin correlated with vanadium. Keywords: Vanadium; Arsenic; Cadmium; Lead; Insulin; Athletes.
\end{abstract}

Corresponding author. Avenida de la Universidad s/n (Cáceres), Spain.

E-mail: tororomanvictor@gmail.com

Supplementary Issue: Spring Conferences of Sports Science. International Seminar of Physical Education, Leisure and Health, 17-19 June 2019. Castelo Branco, Portugal.

JOURNAL OF HUMAN SPORT \& EXERCISE ISSN 1988-5202

(C) Faculty of Education. University of Alicante.

doi:10.14198/jhse.2019.14.Proc4.82 


\section{INTRODUCTION}

Mineral trace elements are considered necessary for health although harmful in high concentrations, other metals to which we are exposed have been considered toxic for health (Kabata-Pendias, 2007). Changes in hormone concentrations could affect the metabolism and redistribution of trace metals due to the interactions between endocrine systems and trace metals (Soria et al., 2015). A high volume of exercise training can lead to a great physiological stress inducing changes in the mentioned systems. Toxic metals are considered as disruptors of hormonal metabolism (Hsieh et al., 2008). Insulin relation with toxic metals is has been studied in metabolic diseases such as diabetes. Thus, the aim of this study was to observe the relation between toxic metals $(\mathrm{Pb}, \mathrm{As}, \mathrm{Cd}$ and $\mathrm{V})$ with insulin.

\section{MATERIAL AND METHODS}

\section{Participants}

62 male professional athletes (age (years) $=22.87 \pm 4.04$; height $(\mathrm{m})=1.78 \pm 7.36$; weight $(\mathrm{kg})=64.97 \pm 7.36$; $\mathrm{VO}_{2 \max }(\mathrm{mL} / \mathrm{min} / \mathrm{kg})=67.57 \pm 8.51$ participated in the present survey. All of them had been living in the same region for, at least, two years. The athletes had trained regularly for the two previous years with a weekly training routine of 3-4 days/week of continued aerobic running or fartlek and 2-3 days/week of intense series and interval training depending on whether or not there was a competition at the weekend.

\section{Measures}

The participants completed a questionnaire consisted of a 3-day daily nutritional record, in two pre-assigned weekdays and one weekend day. In each day, participants indicated individually the type, frequency, and quantity (in grams) of every food consumed, then the nutritional composition of their diets was evaluated using different databases (Kabata-Pendias, 2007; Reilly, 2008).

\section{Procedures}

At nine o'clock in the morning, $10 \mathrm{~mL}$ of venous blood from the antecubital vein of each participant were drawn from all using a plastic syringe fitted with a stainless-steel needle by a physician. Later, the blood samples were centrifuged at $3000 \mathrm{rpm}$ for $10 \mathrm{~min}$ at room temperature to isolate the serum. Subsequently, the samples were frozen at $-80^{\circ} \mathrm{C}$ for further analysis in inductively coupled plasma mass spectrometry (ICPMS) for mineral trace elements and ELISA for insulin.

\section{Analysis}

Pearson's correlation coefficient ( $r$ ) was used to determine whether there were significant relationships between mineral concentrations in serum and hormones. A $p \leqq 0.05$ was considered statistically significant.

\section{RESULTS}

Table 1. Correlations between trace metals and hormones.

\begin{tabular}{cc}
\hline & Insulin \\
\hline \multirow{2}{*}{ As } & $r=-0.411$ \\
& $p=0.023$ \\
Cd & $r=-0.134$ \\
& $p=0.174$ \\
$r=0.129$ \\
$p=0.452$
\end{tabular}


$\mathrm{Pb}$

$r=0.217$

$p=0.098$

$p<0.05$ was considered statistically significant.

Insulin concentrations was inversely correlated to Vanadium $(r=-0.411, p<0.05)$. There was no correlation with the rest of metals.

\section{DISCUSSION}

An interesting correlation found was between the serum $V$ concentration and the serum insulin levels $(r=$ $0.411, p=0.023$ ) in the sense that lower serum V levels, higher serum insulin levels. Therefore, this could indicate the need for this element for the synthesis of insulin or its correct functioning. In this sense, the effects of $V$ as a mimetic substance of insulin (Jakusch et al., 2011) that could be used as an insulin supplement that would avoid its adverse effects that can cause its administration (insulin resistance) are known, however, more research is needed to establish the possible long-term effects of $\mathrm{V}$ treatment as well as to determine intervals and toxic doses in different groups of patients (Gruzewska et al., 2014).

\section{CONCLUSIONS}

Insulin correlated with the concentration of vanadium, in the sense that the higher the vanadium is in plasma/serum, the lower the insulin levels. Therefore, the relation already described between the said hormone and the element studied is clear.

\section{REFERENCES}

Gruzewska, K., Michno, A., Pawelczyk, T., \& Bielarczyk, H. (2014). Essentiality and toxicity of vanadium supplements in health and pathology. J Physiol Pharmacol, 65(5), 603-611.

Hsieh, F. I., Hwang, T. S., Hsieh, Y. C., Lo, H. C., Su, C. T., Hsu, H. S., Chen, C. J. (2008). Risk of erectile dysfunction induced by arsenic exposure through well water consumption in Taiwan. Environ Health Perspect, 116(4), 532-536. https://doi.org/10.1289/ehp.10930

Jakusch, T., Pessoa, J. C., \& Kiss, T. (2011). The speciation of vanadium in human serum. Coord Chem Rev, 255(19-20), 2218-2226. https://doi.org/10.1016/j.ccr.2011.02.022

Kabata-Pendias, A. (2007). Trace Elements from Soil to Human (Springer Ed.).

Reilly, C. (2008). The nutritional trace metals. John Wiley \& Sons.

Soria, M., Gonzalez-Haro, C., Anson, M., Lopez-Colon, J. L., \& Escanero, J. F. (2015). Plasma levels of trace elements and exercise induced stress hormones in well-trained athletes. J. Trace Elem. Med. Biol., 31, 113-119. https://doi.org/10.1016/j.jtemb.2015.04.004

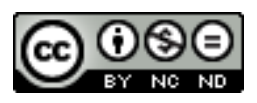

This work is licensed under a Attribution-NonCommercial-NoDerivatives 4.0 International (CC BY-NC-ND 4.0). 


\title{
Erythrocyte concentrations of chromium, manganese and zinc in men with a high, moderate or low level of physical training
}

\author{
JESÚS SIQUIER COLL ${ }^{1}$, JAVIER MÁRQUEZ DONCEL ${ }^{1}$, MARIA CONCEPCIÓN ROBLES GIL ${ }^{1}$, DIEGO \\ MUÑOZ MARIN ${ }^{1}$, GEMA BARRIENTOS VICHO², FRANCISCO JAVIER GRIJOTA PÉREZ1 \\ ${ }^{1}$ Sport Science Faculty, University of Extremadura, Spain \\ ${ }^{2}$ School of Sport Sciences, Pontifical University of Salamanca, Spain
}

\begin{abstract}
The aim of the present study was to determine changes occurring in the erythrocytes concentrations of chromium $(\mathrm{Cr})$, manganese $(\mathrm{Mn})$ and zinc $(\mathrm{Zn})$ of high-level trained, moderate-level trained and sedentary man living in the same area of Extremadura (Spain). 30 sedentary subjects, with $24.34 \pm 3.02$ years without sports practice and a less active lifestyle formed the control group (CG); 24 moderately trained subjects with $23.53 \pm 1.85$ years, who perform between 4 and 7 hours/week of moderate sports practice without any performance objective and without following any type of systematic training formed the group of subjects with moderate degree of training (MTG) and 22 professional cyclists at the beginning of their sports season, with an age of $23.29 \pm 2.73$, who performed more than 20 hours/week of training formed the high-level training group (HTG). The results showed a statistically significant lower concentration of erythrocyte $\mathrm{Mn}$ and $\mathrm{Zn}$ in the groups of athletes (MTG and HTG) than CG. We can conclude that physical training produces a decrease in erythrocyte concentration of $\mathrm{Mn}$ and $\mathrm{Zn}$. This situation could create a reduction in the performance of athletes given the importance of the elements. For this reason, we recommend an erythrocyte control at the beginning of the training period and during the training period to avoid negative effects. Keywords: Chromium; Manganese; Zinc and training.
\end{abstract}

Corresponding author. Avenida de la Universidad s/n (Cáceres), Spain.

E-mail: jsiquier@alumnos.unex.es

Supplementary Issue: Spring Conferences of Sports Science. International Seminar of Physical Education, Leisure and Health, 17-19 June 2019. Castelo Branco, Portugal.

JOURNAL OF HUMAN SPORT \& EXERCISE ISSN 1988-5202

(c) Faculty of Education. University of Alicante.

doi:10.14198/jhse.2019.14.Proc4.82 


\section{INTRODUCTION}

Micronutrients, trace minerals and vitamins are an integral component of many metabolic processes and are required daily in minimal amounts in the diet to maintain health. Usually, the body level of micronutrients is determined by measuring the trace elements in plasma. During the inflammation in response to damage, the plasma concentrations of $\mathrm{Zn}$ fail. The erythrocyte concentration of trace elements is not affected by the acute inflammatory response (Oakes et al., 2008) or by the short-term diet. Therefore, it is very important to know the intraerythrocytic concentrations of $\mathrm{Cr}, \mathrm{Mn}$ and $\mathrm{Zn}$ in sedentary men, with a moderate and high degree of physical training, given that a deficit may impair performance sports, and know if such concentrations can depend on the degree of training.

\section{MATERIAL AND METHODS}

\section{Participants}

Thirty sedentary subjects, with $24.34 \pm 3.02$ years without sports practice and a less active lifestyle formed the control group (CG). 24 moderately trained subjects with $23.53 \pm 1.85$ years, who perform between 4 and 7 hours/week of moderate sports practice without any performance objective and that imply an active lifestyle, without following any systematic training formed the group of subjects with a moderate degree of training (MTG). Twenty-two high-level athletes, professional cyclists at the beginning of their sports season, with $23.29 \pm 2.73$, who performed more than 20 hours/week of training formed the high-level training group (HTG).

\section{Measures}

The physical condition of the subjects was evaluated by performing an ergospirometric test. To perform the test, an ergospirometer system equipped with a gas analyser (Metamax, Biophysik, Gmbh, Germany), a treadmill (Powerjoc, United Kingdom) and a Polar heart rate monitor (Polar, Norway) were used.

\section{Procedures}

All subjects underwent an extraction of blood from the antecubital vein after an overnight fast, deposited in glass tubes containing EDTA as an anticoagulant and centrifuged immediately (2,500 rpm for $10 \mathrm{~min}$.) To separate the plasma of erythrocytes. The previously isolated erythrocytes are washed with a $0.9 \%$ sodium chloride solution in ultrapure water and stored at $-70^{\circ} \mathrm{C}$ until analysis. $\mathrm{Cr}, \mathrm{Mn}$ and $\mathrm{Zn}$ were determined by ICP-MS.

\section{Analysis}

Subsequently, a comparison of the variables between the three groups was made, using an ANOVA test, applying a Bonferroni test later on if there is significance. It was considered that there was a significant difference when $p<0.05$.

\section{RESULTS}

Table 1. Concentration of the trace elements in the control group and athletes classified by the level of training

\begin{tabular}{llll}
\hline & CG & MTG & HTG \\
\hline $\mathrm{Cr}(\mu \mathrm{g} / \mathrm{gHb})$ & $0.029 \pm 0.012$ & $0.021 \pm 0.011$ & $0.010 \pm 0.011$ \\
$\mathrm{Mn}(\mu \mathrm{g} / \mathrm{gHb})$ & $0.139 \pm 0.030$ & $0.120 \pm 0.013^{++}$ & $0.112 \pm 0.025^{\mathrm{t \dagger}}$ \\
$\mathrm{Zn}(\mathrm{mg} / \mathrm{gHb})$ & $0.061 \pm 0.009$ & $0.044 \pm 0.011^{++}$ & $0.041 \pm 0.004 \mathrm{tt}$ \\
\hline
\end{tabular}

† Differences between the HTG and CG ( $+p<0,05 ;+t p<0,01 ;+t+p<0,001) ;+$ Differences between the MTG and CG ( $+p<0,05$; $++p<0,01 ;+++p<0,001) ;{ }^{*}$ Differences between the HTG and MTG $\left({ }^{*} p<0,05 ;{ }^{* *} p<0,01 ;{ }^{* * *} p<0,001\right)$. 
When compared concentrations between CG and MTG, Mn $(p<0.01)$ and $Z n(p<0.01)$ concentrations were lower in MTG. When compared levels between CG and HTG, Mn $(p<0.01)$ and $\mathrm{Zn}(p<0.001)$ in HTG were lower.

\section{DISCUSSION}

We can observe low erythrocyte concentrations in trace minerals of great importance for cellular functions in the subjects who trained. It is possible that the mechanism by which exercise improves the response to insulin is related to an alteration in the metabolism of $\mathrm{Cr}$. Maynar et al., shows that serum concentrations in different sports modalities were significantly higher in athletes than in the control group (Maynar et al., 2018). Physical exercise increases the activity of manganese-dependent superoxide dismutase (Mn-SOD) at the level of the myocardium and in general of the mitochondria. This is significant because Mn-SOD is an antioxidant enzyme located at the level of the mitochondria that would neutralise the superoxide radicals that form inside it. Some studies report that $Z n$ increases in plasma and decreases in erythrocytes after an intense race on tape or an extraordinary effort on a cycle ergometer. These studies believe that these plasma increases are due to a $\mathrm{Zn}$ leakage from muscles damaged by physical activity (Mundie and Hare, 2001). It can be stated that functional displacements of $Z n$ can occur between tissues during exercise. For that reason, it seems quite difficult to determine the effects of exercise on the concentration of $\mathrm{Zn}$ and other elements (Buchmann et al.,1998).

\section{CONCLUSIONS}

In conclusion, our study shows a deficiency in $\mathrm{Cu}, \mathrm{Mn}$ and $\mathrm{Zn}$ in subjects who perform physical training, which does not exist in participants who do not complete training.

\section{REFERENCES}

Buchman, A. L., Keen, C., Commisso, J., Killip, D., Ou, C. N., Rognerud, C. L., ... \& Dunn, J. K. (1998). The effect of a marathon run on plasma and urine mineral and metal concentrations. J Am Coll Nutr, 17(2), 124-127. https://doi.org/10.1080/07315724.1998.10718737

Maynar, M., Llerena, F., Bartolomé, I., Alves, J., Grijota, F. J., Robles, M. C., \& Muñoz, D. (2018). Influence of an exercise until exhaustion in serum and urinary concentrations of toxic minerals among professional athletes, a preliminary approach. J. Trace Elem. Med. Biol.50, 312-319. https://doi.org/10.1016/j.jtemb.2018.07.022

Mundie, T. G., \& Hare, B. (2001). Effects of resistance exercise on plasma, erythrocyte, and urine Zn. Biol Trace Elem Res, 79(1), 23-28. https://doi.org/10.1385/bter:79:1:23

Oakes, E. J., Lyon, T. D., Duncan, A., Gray, A., Talwar, D., \& O’Reilly, D. S. J. (2008). Acute inflammatory response does not affect erythrocyte concentrations of copper, zinc and selenium. Clin Nutr, 27(1), 115-120. https://doi.org/10.1016/j.clnu.2007.10.003

\section{(9)}

This work is licensed under a Attribution-NonCommercial-NoDerivatives 4.0 International (CC BY-NC-ND 4.0). 


\title{
Arsenic, cadmium and lead erythrocyte concentrations in men with a high, moderate or low level of physical training
}

\author{
JESÚS SIQUIER COLL ${ }^{1}$, JAVIER MÁRQUEZ DONCEL ${ }^{1}$, MARIA CONCEPCIÓN ROBLES GIL ${ }^{1}$, DIEGO \\ MUÑOZ MARIN ${ }^{1}$, GEMA BARRIENTOS VICHO², FRANCISCO JAVIER GRIJOTA PÉREZ ${ }^{1}$ \\ ${ }^{1}$ Sport Science Faculty, University of Extremadura, Spain \\ ${ }^{2}$ School of Sport Sciences, Pontifical University of Salamanca, Spain
}

\begin{abstract}
Toxic metals can impair essential functions. Therefore, the objectives of the study are to know the intraerythrocytic concentrations of toxic elements arsenic $(\mathrm{As})$, cadmium $(\mathrm{Cd})$ and lead $(\mathrm{Pb})$ and check for different concentrations of these elements in erythrocytes in relation to physical activity. For it we had a total of 106 subjects separate into three groups: 32 to the group of highly trained, 34 to the trained group and 40 to the sedentary group, from Caceres. All subjects performed an assessment of cardiovascular fitness $\left(\mathrm{VO}_{2 \max }\right)$, blood sampling and determination of mineral elements by ICP-MS. As concentration, was lower in CG $(p<0.01)$ and MTG $(p<0.01)$ than HTG. $C d(p<0.001)$ and Pb $(p<0.05)$ concentrations were higher in CG than HTG. All results were expressed in $\mu \mathrm{g} / \mathrm{g} \mathrm{Hb}$. We can conclude that high physical training produces a decrease in erythrocyte concentration of $\mathrm{Cd}$ and $\mathrm{Pb}$ but a high level of As. For this reason, we recommend an erythrocyte control at the beginning of the training period and during the training period to avoid harmful deficits. Keywords: Arsenic; Cadmium; Lead; Erythrocyte and training.
\end{abstract}

Corresponding author. Avenida de la Universidad s/n (Cáceres), Spain.

E-mail: jsiquier@alumnos.unex.es

Supplementary Issue: Spring Conferences of Sports Science. International Seminar of Physical Education, Leisure and Health, 17-19 June 2019. Castelo Branco, Portugal.

JOURNAL OF HUMAN SPORT \& EXERCISE ISSN 1988-5202

(C) Faculty of Education. University of Alicante.

doi:10.14198/jhse.2019.14.Proc4.82 


\section{INTRODUCTION}

The effect of physical exercise above toxic metals toxic to the organism is unknown. Due to the negative effect of this metals above health, performance can be impaired. Thus, the objectives of the study are to know the intraerythrocytic concentrations of the essential trace elements of the toxic elements arsenic (As), cadmium $(\mathrm{Cd})$ and lead $(\mathrm{Pb})$ and check whether there are different concentrations of these elements in erythrocytes in relation to physical activity.

\section{MATERIAL AND METHODS}

\section{Participants}

The participants were divided into three groups: control (GC), moderately trained (MTG) and highly trained (HTG). GC was formed by 30 sedentary subjects (age (years) $=24.34 \pm 3.02$; height $(\mathrm{m})=1.75 \pm 0.18$; weight $(\mathrm{kg})=75.34 \pm 12.03 ; \mathrm{VO}_{2 \operatorname{Max}}(\mathrm{mL}$ min-1 $\left.\mathrm{kg}-1)=38.90 \pm 3.73\right)$ without sports practice and a less active lifestyle. MTG was constituted by 24 moderately trained subjects (age (years) $=23.53 \pm 1.85$; height $(\mathrm{m})=1.77 \pm 0.05$; weight $\left.(\mathrm{kg})=75.12 \pm 8.16 ; \mathrm{VO}_{2 \mathrm{Max}}(\mathrm{mL} \min -1 \mathrm{~kg}-1)=46.77 \pm 6.72\right)$, who perform between 4 and 7 hours / week of moderate sports practice without any performance objective and that imply an active lifestyle, without following any systematic training formed the group of subjects with a moderate degree of training. 22 high level athletes, professional cyclists at the beginning of their sport season (age (years) $=23.29 \pm 2.73$; height $(\mathrm{m})=1.73 \pm 0.07$; weight $\left.(\mathrm{kg})=65.61 \pm 5.22 ; \mathrm{VO}_{2 \mathrm{Max}}(\mathrm{mL} \min -1 \mathrm{~kg}-1)=61.23 \pm 3.00\right)$ who performed more than 20 hours/week of training formed the high-level training group.

\section{Measurements}

The physical condition of the subjects was evaluated by performing an ergospirometric test. To perform the test, an ergospirometer system equipped with a gas analyser (Metamax, Biophysik, Gmbh, Germany), a treadmill (Powerjoc, United Kingdom) and a Polar heart rate monitor (Polar, Norway) were used.

\section{Procedures}

All subjects underwent an extraction of blood from the antecubital vein after an overnight fast, deposited in glass tubes containing EDTA as an anticoagulant and centrifuged immediately (2,500 rpm for $10 \mathrm{~min}$.) To separate the plasma of erythrocytes. The previously separated erythrocytes are washed with a $0.9 \%$ sodium chloride solution in ultra-pure water and stored at $-70^{\circ} \mathrm{C}$ until analysis. $\mathrm{As}, \mathrm{Cd}$ and $\mathrm{Pb}$ were determined by ICP-MS.

\section{Analysis}

The statistical analyses were performed with the SPSS 16.0 software. Subsequently, a comparison of the variables between the three groups was made, using an ANOVA test, applying a Bonferroni test later on if there is significance. It was considered that there was a significant difference when $p<0.05$. 


\section{RESULTS}

Table 1.Concentrations of the trace elements $\mathrm{Cd}, \mathrm{Pb}$ and $\mathrm{As}$ in erythrocyte of the different groups of the study.

\begin{tabular}{cccc}
\hline & GC & MTG & HTG \\
\hline As $(\mu \mathrm{g} / \mathrm{gHb})$ & $1.51 \pm 1.23$ & $1.23 \pm 0.89$ & $3.42 \pm 2.98 \mathrm{t \dagger}^{* *}$ \\
$\mathrm{Cd}(\mu \mathrm{g} / \mathrm{gHb})$ & $0.0450 \pm 0.0013$ & $0.0051 \pm 0.009++$ & $0.0000 \pm 0.0000+\mathrm{t \dagger}^{* *}$ \\
$\mathrm{~Pb}(\mu \mathrm{g} / \mathrm{gHb})$ & $9.72 \pm 3.53$ & $11.09 \pm 6.02$ & $8.73 \pm 5.13 \dagger$ \\
\hline
\end{tabular}

ANOVA and Bonferroni tests

† Differences between the HTG and CG ( $+p<0,05 ;+t p<0,01 ;+t+p<0,001)$.

+ Differences between the MTG and $C G(+p<0,05 ;++p<0,01 ;+++p<0,001)$.

${ }^{*}$ Differences between the HTG and MTG $\left({ }^{*} p<0,05 ;{ }^{* *} p<0,01 ;{ }^{* * *} p<0,001\right)$.

It was found As concentration, was lower in CG $(p<0.01)$ and MTG $(p<0.01)$ than HTG. $C d(p<0.001)$ and Pb $(p<0.05)$ concentrations were higher in CG than HTG. MTG present a higher concentration of Cd than HTG $(p<0.05)$.

\section{DISCUSSION}

Arsenic was in higher concentrations in the more trained subjects. We cannot explain with the current knowledge about this situation. What is certain is that they are a low concentration that would not have to have negative repercussions on the subjects. Regarding cadmium, it is essential to check how this element, probably related to sports training, does not pass to erythrocytes in the subjects with the highest degree of training and if it does to subjects with less training. In a study conducted by Llerena et al., found a major urinary elimination of $\mathrm{Cd}$ in the more trained subjects (Llerena et al., 2012). The evaluation in the serum of these subjects Maynar et al., it was observed that the concentrations of $\mathrm{Cd}$ were higher in the subjects more trained compared to the control group (Maynar et al., 2018). As found in our previous study, the highest concentrations of $\mathrm{Cd}$ in athletes in serum would be due to the difficulty that the element would have in entering the interior of the cells, preventing their accumulation inside the body and that increase in serum concentration it would not be dangerous for the organism because it would eliminate it easily through the urine. We believe that what is found in this study may be of great interest in relation to avoiding the harmful effects of the element in the organism that are very negative for man.

\section{CONCLUSIONS}

We can conclude that high-level physical training produces a decrease in the concentration of $\mathrm{Cd}$ and $\mathrm{Pb}$ erythrocytes but a high level of As. For this reason, we recommend a control of erythrocytes at the beginning of the training period and during the training period to avoid deficits harmful.

\section{REFERENCES}

LLerena, F., Maynar, M., Barrientos, G., Palomo, R., Robles, M. C., \& Caballero, M. J. (2012). Comparison of urine toxic metals concentrations in athletes and in sedentary subjects living in the same area of Extremadura (Spain). Eur J Appl Physiol, 112(8), 3027-3031. https://doi.org/10.1007/s00421-011-2276-6

Maynar, M., Llerena, F., Bartolomé, I., Alves, J., Grijota, F. J., Robles, M. C., \& Muñoz, D. (2018). Influence of an exercise until exhaustion in serum and urinary concentrations of toxic minerals among 
professional athletes, a preliminary approach. J. Trace Elem. Med. Biol, 50, 312-319. https://doi.org/10.1016/j.jtemb.2018.07.022

\section{(c)}

This work is licensed under a Attribution-NonCommercial-NoDerivatives 4.0 International (CC BY-NC-ND 4.0). 


\title{
Cyberbullying in school: A literature review
}

\author{
PAULO OLIVEIRA , BEATRIZ PEREIRA \\ Research Centre on Child Studies, University of Minho, Portugal
}

\begin{abstract}
This work aims to perform a systematic review of literature on cyberbullying in the Scielo - Brazil database to verify the amount of studies on this theme in this database that is a reference in relation to Brazilian scientific production. For the accomplishment of this search the Scielo - Brazil database was used, from the descriptors cyberbullying and school in the Portuguese, English and Spanish languages. The sample consisted of four studies, among the twenty found in the search. Where are found eight articles in Portuguese, nine in English and three in Spanish related to the descriptors of the research. However, only four of these studies met the criteria of selection of this systematic literature review, two in Portuguese, one in English and one in Spanish. It was verified that all the investigations detected the presence of cyberbullying in the relations between the students, as well as there was a variation between $1.9 \%$ and $11.4 \%$ for the rate of victimization by cyberbullying and a variance between $3.9 \%$ and $12.5 \%$ for the rate of aggression by cyberbullying. With this investigation we conclude that cyberbullying is still little investigated in the Brazilian population and that new research is needed to deepen the knowledge about the subject, which is of great importance, since this type of violence can be seen as a public health problem, and should receive attention from public policy makers. Keywords: Cyberbullying; School; Peer relationship; Literature review.
\end{abstract}

Corresponding author. Largo do Paço, 4704-553, Braga, Portugal.

E-mail: paulofoliveira1982@hotmail.com

Supplementary Issue: Spring Conferences of Sports Science. International Seminar of Physical Education, Leisure and Health, 17-19 June 2019. Castelo Branco, Portugal.

JOURNAL OF HUMAN SPORT \& EXERCISE ISSN 1988-5202

(C) Faculty of Education. University of Alicante.

doi:10.14198/jhse.2019.14.Proc4.82 


\section{INTRODUCTION}

Violence is a phenomenon that is very present in modern society and presents different forms of manifestation. Among them we can highlight bullying, which occurs in school every day. With the advancement in the development of information and communication technologies, as well as the ease of acquiring electronic devices with Internet access, this phenomenon has started to manifest itself through the virtual medium known as cyberbullying (Slonje and Smith, 2008).

\section{MATERIAL AND METHODS}

A systematic review of the data search literature was made in the Scielo - Brazil database.

\section{Participants}

The sample consisted of four studies, among the twenty found in the search.

\section{Measures}

The criteria for inclusion of the articles in this literature review were the following: a) they are the result of empirical research; b) have been published in the last five years; and c) involving students.

\section{Procedures}

A research was carried out in the Scielo - Brazil database, with the following descriptors: cyberbullying and school, in Portuguese, English and Spanish. In the search where are found eight articles in Portuguese, nine articles in the English and three articles in the Spanish. The abstracts of these articles were read and duplicate articles were discarded, as were those that did not meet the criteria established for inclusion in this literature review. Remaining, two in Portuguese, one in English and one in Spanish.

\section{Analysis}

The analysis applied to the articles that were included in this literature review was of the quantitative and qualitative type in relation to the data found in these studies.

\section{RESULTS}

The articles included in this literature review present one quantitative and qualitative study, one exploratory study, one descriptive study and one quantitative study with a longitudinal design. In relation to the year of publication, the articles are distributed as follows: one in 2014, one in 2016, one in 2017 and one in 2019. For the collection, were used: Questionnaire on Digital Inclusion and Informational Education for Health, Diagnostic Questionnaire on Cyberbullying, Internet Experiences Questionnaire, Strength and Difficulties Questionnaire and Youth Self-Report.

In the first study, carried out in Brazil, the manifestation of homophobic and misogynist cyberbullying was observed through a virtual learning environment (Cavalcante et al., 2017). In the second study, conducted in Portugal, $7.6 \%$ of the students answered that they had been victims of cyberbullying and $3.9 \%$ of the students who responded were cyberbullying aggressors (Caetano et al., 2016). In the third study, conducted in Chile, $11.4 \%$ of the students who have access to a cell phones reported having been victims of some kind of cyberbullying, through text message, receiving photos or through the internet and $12.5 \%$ of the students reported having been perpetrators of cyberbullying. Regarding the means of victimization and aggression by cyberbullying, the study indicates that: $3.6 \%$ of students reported having been a victim of cyberbullying 
through text message, $6.6 \%$ by sending photographs and $7.9 \%$ by internet, while $4.4 \%$ of students reported having been cyberbullying abusers through text message, $7.8 \%$ through sending photographs and $5.9 \%$ through the internet (Varela et al., 2014). In the fourth study, conducted in Brazil, $1.9 \%$ of the students reported having been victims of cyberbullying (Vieira et al., 2019).

\section{DISCUSSION}

The studies were published between 2014 and 2019, which was expected due to cyberbullying being a relatively recent phenomenon. All the articles included in this literature review used questionnaires as instruments of data collection. However, there was a variation between $1.9 \%$ and $11.4 \%$ in relation to victimization by cyberbullying and a variation between $3.9 \%$ and $12.5 \%$ in relation to the aggression by cyberbullying. The means used to practice cyberbullying reported in the articles were: through text message, sending of photographs and through the internet.

The two studies performed in Brazil present very different results compared with the other two studies analysed in this literature review. This fact may be related to the type of research that was used in each of the studies, as well as the fact that each of these studies used a different questionnaire to collect the data, which have different methodologies for the investigation of cyberbullying.

\section{CONCLUSIONS}

All articles presented the manifestation of cyberbullying, either through a virtual learning environment or through the use of a cell phone. No classifications of the types of cyberbullying were found in any of the studies included in this literature review, which points to the need for a categorization of the types of manifestation of this phenomenon. One of the limitations of this study is to have used only the Scielo - Brazil database to perform this literature review. Although the database used for this study is Brazilian, few studies have been found related to cyberbullying in Brazil, which indicates that this phenomenon needs to be better investigated in the country.

\section{REFERENCES}

Caetano, A. P., Freire, I., Simão, A. M. V., Martins, M. J. D., \& Pessoa, M. T. (2016). Emoções no cyberbullying: um estudo com adolescentes portugueses. Educ. Pesqui., 42(1), 199-212. https://doi.org/10.1590/s1517-9702201603138125

Cavalcante, R. B., Silva, J. J., Martins, J. R. T., Passos, T. R., Magalhães, T. I., \& Esteves, C. J. S. (2017). Inclusão digital e uso de tecnologias da informação: a saúde do adolescente em foco. Perspect. Ciênc. Inf., 22(4), 3-21. https://doi.org/10.1590/1981-5344/2539

Slonge, R., \& Smith, P. K. (2008). Cyberbullying: Another main type of bullying? Scand. J. Psychol., 49(2), 147-154. https://doi.org/10.1111/j.1467-9450.2007.00611.x

Varela, J., Pérez, J. C., Schwaderer, H., Astudillo, J., \& Lecannelier, F. (2014). Caracterización de cyberbullying en el gran Santiago de Chile, en el año 2010. Psicol. Esc. Educ., 18(2), 347-354. https://doi.org/10.1590/2175-3539/2014/0182794

Vieira, M. A., Rønning, J. A., Mari, J. J., \& Bordin, I. A. (2019). Does cyberbullying occur simultaneously with other types of violence exposure? Braz. J. Psychiatr., 0(0), 00-00. https://doi.org/10.1590/15164446-2018-0047 


\section{(c) (i) (3)}

This work is licensed under a Attribution-NonCommercial-NoDerivatives 4.0 International (CC BY-NC-ND 4.0). 


\title{
The effects of a mind-body program on the cognomotor performance of high school children
}

\author{
ANDERSON AUGUSTO RIBEIRO ${ }^{1}$, CLEONICE TEREZINHA FERNANDES ${ }^{2}$, MARIA ISABEL MARTINS \\ MOURÃO CARVALHAL ${ }^{3}$, CILENE MARIA LIMA ANTUNES MACIEL ${ }^{4}$, LEONARDO GIROTTO DE \\ OLIVEIRA ${ }^{5}$ \\ ${ }^{1}$ Instituto Federal de Mato Grosso (IFMT), Campus Cuiabá, Bela Vista, Mato Grosso, Brazil \\ 2Universidade de Cuiabá (UNIC), Mato Grosso, Brazil \\ 3 Universidade de Trás-os-Montes and Alto Douro (UTAD), Portugal \\ ${ }^{4}$ Universidade de Cuiabá (UNIC), Mato Grosso, Brazil \\ 5 Universidade Estadual de Maringá (UEM), Paraná, Brazil
}

\begin{abstract}
The aim of this study is to explore the effects of ã mind-body program on the cognomotor performance of high school children. A qualitative-quantitative longitudinal design (pre-test intervention post-test) is being implemented during 12 weeks to 48 students $(17.83 \pm 0.87)$ from the Federal Institute of Mato Grosso, Campus, Cuiabá - Bela Vista, Brazil. Before and after the intervention period, were applied the following instruments: Physical Education, Nutrition and Psychology questionnaires and specific protocols, namely (sociodemographic questionnaire, anamnesis, perceptual questionnaire, well-being pentacle, DASS-21; silhouetted scales; flexibility of trunk and legs. Teaching strategies for mind-body exercises such as yoga, mindfulness meditation and circular dances included the activities of the intervention program. Preliminary data indicate that $60 \%$ of students do not practice three moderate I vigorous sessions of physical activity per week, nor time in daily relaxation or self-reflection activities. Psychological data reveal that $51 \%$ of the participants experience anxiety; $33 \%$ feel depressed or sadness; $24 \%$ report headaches; $42 \%$ admit disruptive daily behaviours, and $20 \%$ reported feelings that life no longer made sense. With the present study we hope to provide participants with a more contextualized practice about their body and their connection to the cognitive, which may include attitudinal changes regarding healthier habits, as well as greater sensitivity about the importance of recognition and development of the body. Keywords: Mind-body exercise; Embodied cognition; Cognomotor performance; High school.
\end{abstract}

\footnotetext{
Corresponding author. Rua Pedro Fernandes, №375; Apartamento 1004; Bairro: Boa Esperança. CEP: 78.068-710. Cuiabál MT. Brasil.

E-mail: anderson.ribeiro@blv.ifmt.edu.br

Supplementary Issue: Spring Conferences of Sports Science. International Seminar of Physical Education, Leisure and Health, 17-19 June 2019. Castelo Branco, Portugal.

JOURNAL OF HUMAN SPORT \& EXERCISE ISSN 1988-5202

(c) Faculty of Education. University of Alicante.

doi:10.14198/jhse.2019.14.Proc4.82
} 


\section{INTRODUCTION}

The combination of body movement, meditation and deep breathing is called mind-body exercise. A growing body of scientific research indicated that mind-body exercises (yoga, Tai chi and Qigong, Mindfulness) might generate substantial health benefits and improving brain function (Wang, 2019).

In cognitive neuroscience, the link between action, body, emotion and cognition has become known as the embodied approach (Gjelsvik et al., 2017). According to this approach, brain and body are indissociable integrated as a unity crucial for the cognition process because the biochemical neurotransmissors that make the connection between body and mind (Damásio, 2012; Herculano-Houzel, 2009). Programs that arouse body awareness lead the subject to rediscover essentially their emotional states and points of tension, and this is of fundamental importance for the reduction of the biopsychosocial and neuromuscular risks to which we are exposed (Lima, 2007).

However, we are currently immersed in a process where we assume a role of no reflection / analysis of our attitudes and of our own body consciousness. Sometimes we expose our biologic body to several negative situations involve us an opposite lifestyle, to which we can provide a healthy balance.

In this context, and in order to minimize this serious reality, this study seeks to make students more active and participative in the training environments.

Therefore, it will be important to develop research in order to explore the effects of body-mind exercise on cognomotor performance of high school children.

\section{MATERIAL AND METHODS}

A qualitative-quantitative longitudinal design (pre-test intervention post-test) is being implemented during 12 weeks in the first half of 2019 year, with students from the Federal Institute of Mato Grosso, Campus, Cuiabá - Bela Vista, Brazil.

\section{Participants}

The sample include 17 year-old students $(17.83 \pm 0.87)$ who attended the courses of environment and chemistry. After a large dissemination of the project to the school, 48 students ( $73 \%$ female and $27 \%$ male) integrated the project on a voluntary basis.

\section{Measures}

Before and after the intervention period, the following instruments will be applied: Physical Education, Nutrition and Psychology questionnaires and specific protocols, namely (sociodemographic questionnaire, anamnesis, perceptual questionnaire, well-being pentacle, DASS-21; silhouetted scales; flexibility of trunk and legs.

Teaching strategies for mind-body exercises such as yoga, mindfulness meditation and circular dances included the activities of the intervention program.

\section{Procedures}

This study was approved on 10/13/2018 by the Research Ethics Committee of the University of Cuiabá (UNIC), Brazil, under opinion № 2,892,382. 


\section{Analysis}

The repeated measure ANOVA will be applied to test the effects of the intervention program on cognomotor performance.

\section{RESULTS}

Preliminary data indicate that $60 \%$ of students do not practice three moderate I vigorous sessions of physical activity per week, nor time in daily relaxation or self-reflection activities. Psychological data reveal that $51 \%$ of the participants experience anxiety; $33 \%$ feel depressed or sadness; $24 \%$ report headaches; $42 \%$ admit disruptive daily behaviours, and $20 \%$ reported feelings that life no longer made sense.

\section{DISCUSSION}

The preliminary data are in consonance with epidemiological research that found that school-aged children suffer from psychological health problems and disruptive behaviours. These baseline data justify an intervention with alternative: yoga and mindfulness. Supported on the Wang's opinion (2019) we hope that these mind-body exercises (yoga, Circular dances and Mindfulness) might generate some mental health benefits and to improve cognomotor performance of high school children. The alternative intervention program is supported on the embodied perspective that exercises emotion, action, cognition and body all together and not in a dualistic perspective of Human being.

\section{CONCLUSIONS}

With the present study we hope to provide participants with a more contextualized practice about their body and their connection to the cognitive, which may include attitudinal changes regarding healthier habits, as well as greater sensitivity about the importance of recognition and development of the body. At the same time, we expect changes in participants 'lifestyles, minimizing the risk factors that affect participants' lives today.

\section{REFERENCES}

Damásio, A. R. (2012). O Erro de Descartes: emoção, razão e o cérebro humano. 1 ed. São Paulo: Companhia das Letras.

Herculano-Houzel, S. (2009). Neurociências na educação. Rio de Janeiro: CEDIC.

Gjelsvik, B., Lovric, D.; Williams, J.M. (2018). Embodied cognition and emotional disorders: Embodiment and abstraction in understanding depression. Journal of Experimental Psychopathology. pp: 1-41. https://doi.org/10.5127/pr.035714

Lima, V. (2007). Ginástica Laboral: atividade física no ambiente de trabalho. 3 ed. São Paulo: Phorte.

Wang, C., Li, K.;Gaylord, S. (2019). Prevalence, patterns, and predictors of meditation use among U.S. children: Results from the National Health Interview Survey Complementary Therapies in Medicine 43 (2019) 271-276. https://doi.org/10.1016/j.ctim.2019.02.004

\section{@(9)}

This work is licensed under a Attribution-NonCommercial-NoDerivatives 4.0 International (CC BY-NC-ND 4.0). 


\title{
Ethical issues on military physical conditioning
}

\author{
MANUEL COGE $1,2 \square$, DULCE ESTEVES ${ }^{1,3}$ \\ 1 Universidade da Beira Interior, Portugal \\ 2Instituto Superior Técnico Militar (ISTM), Angola \\ ${ }^{3}$ Research Center in Sports Sciences, Health Sciences and Human Development (CIDESD), Portugal
}

\begin{abstract}
Successful performance of military duties requires a high level of physical fitness. In addition to the physiological, biomechanical, biochemical and psychosocial dimensions of the military physical training, ethical questions should be considered. The aim of this study is to identify the main ethical issues related to military physical condition. A literature search was conducted in Web of Science and Scopus from January 2014 to October 2018, to include research studies that evaluate the ethical issues related to physical condition in military training. The reviewed studies revealed five main ethical issues related to military physical condition: (1) the overtraining / risk of injuries; (2) the safety paradox; (3) the use of drugs to enhance performance; (4) the use of wearable technology to get individual biological data and (5) how to train ethical behaviours wile training physical conditioning. We conclude that military training demands high ethical standards, and acknowledging ethical problems is the first step to promote ethical engagement during military physical condition. Keywords: Military; Physical Conditioning; Ethics.
\end{abstract}

Corresponding author. Universidade da Beira Interior, Portugal.

E-mail: mcoje@hotmail.com

Supplementary Issue: Spring Conferences of Sports Science. International Seminar of Physical Education, Leisure and Health, 17-19 June 2019. Castelo Branco, Portugal.

JOURNAL OF HUMAN SPORT \& EXERCISE ISSN 1988-5202

(c) Faculty of Education. University of Alicante.

doi:10.14198/jhse.2019.14.Proc4.82 


\section{INTRODUCTION}

Successful performance of military duties requires a high level of physical fitness, especially, aerobic fitness and muscular strength (Kyröläinen et al., 2018), since those duties include moving quickly and carrying heavy loads for long periods of time. In terms of special forces, fitness involves high endurance and strength performance, fatigue resistance, motivation and survivability (Dhahbi et al., 2018). Therefore, a specific training program has been developed for military in several countries, since physical condition is a corn stone of the military performance (Dhahbi et al., 2018). Beyond this "physical demands", the moral decisions and behaviours made in a military context can have profound effects on the military decision maker, their subordinates and peers, their adversaries, and civilians caught in a conflict as well as on mission effectiveness and support for the military (Thompson \& Jetly, 2014). As such, in addition to the physiological, biomechanical, biochemical and psychosocial dimensions of the military physical training, ethical questions should be considered (Van Baarle et al., 2015). From our literature review, we can conclude that ethical issues on military physical conditioning focus mainly in two complementary ways: (1) what are the anatomical and psychological limits that should be reached, in physical conditioning and (2) embracing ethical behaviours while training physical conditioning (Ojanen et al., 2018; Thompson \& Jetly, 2014; Van Baarle et al., 2018). From this initial systematization, the objective of this work is to highlight the main ethical issues in each of the ways described.

\section{MATERIAL AND METHODS}

A literature search was conducted in Web of Science and Scopus from January 2014 to October 2018, to include research studies that evaluate the effects of TAS, regarding ethical concerns. Search key words were military + physical conditioning or physical training + ethics. Exclusion criteria were: (1) articles that focus on medical ethics and (2) articles that aimed a philosophical redefinition of military ethics.

\section{RESULTS}

Of the 39 articles retrieved ( 16 from Web of Science; 23 from Scopus), 7 met the inclusion criteria. The reviewed studies revealed five major ethical problems concerning the military physical condition, presented in Figure 1.

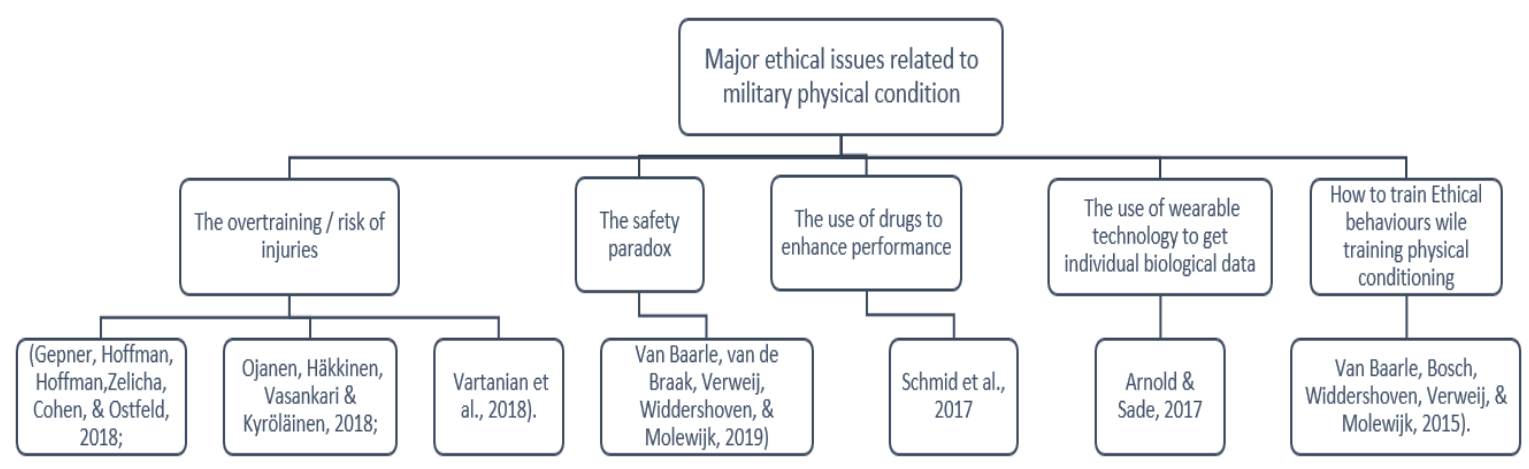

Figure 1. Major ethical issues related to military physical condition, according to 7 studies retrieved. 


\section{DISCUSSION}

The overtraining / risk of injuries - since military duties demand high "physical" performance, military training involve high loads, that may be harmful to health (Gepner et al., 2018; Ojanen et al., 2018; Vartanian et al., 2018). The safety paradox: how to introduce in training the stress lived in "real" battle situation? (Van Baarle et al., 2019). The use of drugs to enhance performance - Faced with such a high training requirement, many military personnel use drugs that allow them to enhance performance, to facilitate recovery or to withdraw pain, in order to continue training (Schmid et al., 2017). The use of wearable technology to get individual biological data, what raises issues like data ownership, informed consent (Arnold \& Sade, 2017).Finally, how to train ethical behaviours wile training physical conditioning - recent studies (Van Baarle et al., 2015) indicate that during the physical conditioning of the military, they are carried to physiological and psychological limits (extreme fatigue, sleep deprivation, deprivation of food, transportation of heavy loads). In this situation of near exhaustion, ethical behaviour, such as self-control, respect for the other, help the other, effort to complete the assignment successfully, among others, should be requested and trained. Thus, it should be a concern of the coaches' ethical training included in the military's "physical" training.

\section{CONCLUSIONS}

Military training demands high ethical standards, and acknowledging ethical problems is the first step to promote ethical engagement during military physical condition.

\section{REFERENCES}

Arnold, J. F., \& Sade, R. M. (2017). Wearable technologies in collegiate sports: the ethics of collecting biometric data from student-athletes. Am J Bioeth, 17(1), 67-70. https://doi.org/10.1080/15265161.2016.1251648

Dhahbi, W., Sellami, M., (...)., \& Chamari, K. (2018). Seasonal weather conditions affect training program efficiency and physical performance among special forces trainees: A long-term follow-up study. PloS one, 13(10), e0206088. https://doi.org/10.1371/journal.pone.0206088

Gepner, Y., Hoffman, J. R., Hoffman, M. W., Zelicha, H., Cohen, H., \& Ostfeld, I. (2018). Association between circulating inflammatory markers and marksmanship following intense military training. J R Army Med Corps, jramc-2018. https://doi.org/10.1136/jramc-2018-001084

Kyröläinen, H., Pihlainen, K., Vaara, J. P., Ojanen, T., \& Santtila, M. (2018). Optimising training adaptations and performance in military environment. J Sci Med Sport., 21(11), 1131-1138. https://doi.org/10.1016/j.jsams.2017.11.019

Ojanen, T., Jalanko, P., \& Kyröläinen, H. (2018). Physical fitness, hormonal, and immunological responses during prolonged military field training. Physiol Rep., 6(17), e13850. https://doi.org/10.14814/phy2.13850

Schmid, B., Tubman, D. S., Loomis, (...) \& Rigoglioso, J. (2017). Substance Use Disorders in the United States Military: Current Approaches and Future Directions. In Handbook of Military Psychology (115136). Springer, Cham. https://doi.org/10.1007/978-3-319-66192-6_9

Thompson, M. M., \& Jetly, R. (2014). Battlefield ethics training: integrating ethical scenarios in highintensity military field exercises. Eur J Psychotraumatol., 5(1), 23668. https://doi.org/10.3402/ejpt.v5.23668

Van Baarle, E., Bosch, J., Widdershoven, G., Verweij, D., \& Molewijk, B. (2015). Moral dilemmas in a military context. A case study of a train the trainer course on military ethics. J Moral Educ., 44(4), 457-478. https://doi.org/10.1080/03057240.2015.1087389 
Van Baarle, E., van de Braak, I., Verweij, D., Widdershoven, G., \& Molewijk, B. (2019). The safety paradox in ethics training: a case study on safety dynamics within a military ethics train-the-trainer course. Med Health Care Philos., 22(1), 107-117. https://doi.org/10.1007/s11019-018-9847-9

Vartanian, O., Fraser, B., (...)., \& Cheung, B. (2018). Changes in mood, fatigue, sleep, cognitive performance and stress hormones among instructors conducting stressful military captivity survival training. Physiol Behav., 194, 137-143. https://doi.org/10.1016/i.physbeh.2018.05.008

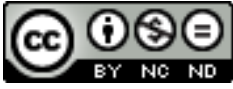

This work is licensed under a Attribution-NonCommercial-NoDerivatives 4.0 International (CC BY-NC-ND 4.0). 


\title{
Relationships between psychological skills and European U19 rugby union tournament outcomes and performance indicators
}

\author{
HELDER MIGUEL FERNANDES $1,2,3$, MARCO BATISTA ${ }^{3,4,5}$, LUIS VAZ ${ }^{1,2}$ \\ 1 Universidade de Trás-os-Montes e Alto Douro, Portugal \\ ${ }^{2}$ Research Centre in Sports, Health and Human Development (CIDESD-UTAD), Portugal \\ ${ }^{3}$ Research in Education and Community Intervention, RECI- Instituto Piaget, Portugal \\ ${ }^{4}$ Instituto Politécnico de Castelo Branco, Portugal \\ ${ }^{5}$ Sport, Health \& Exercise Research Unit (SHERU), Portugal
}

\begin{abstract}
The purpose of this study was to investigate and identify the psychological skills and strategies related to tournament outcomes and performance indicators in a European under-19 Rugby Union Championship. The sample included a total of 183 elite male $U 19$ rugby players, aged between 17 and 19 years $(M=18.67, S D=$ 0.51), who competed at the U19 European Championship 2014. These players represented seven national teams ranked by the teams' final classification in the championship, as follows: Georgia (1st place, $n=34$ ), Portugal (2nd place, $n=20$ ), Spain (3rd place, $n=26$ ), Russia (4th place, $n=27$ ), Netherlands (5th place, $n=$ 17), Belgium (6th place, $n=14$ ), and Romania (7th place, $n=45)$. Athletes responded to translated and adapted versions of the questionnaire Test of Performance Strategies (TOPS; Thomas et al., 1999) an hour before the first match of the tournament. Summary matches' statistics and the final ranking position (inverted) of the national teams were retrieved from an official website. Correlation results showed that a more frequent use of self-talk, emotional control and goal-setting strategies was positively related to points, tries and conversions scored, whereas imagery levels were positively associated to penalties scored. On the other hand, higher levels of negative thinking were positively correlated to points, tries and penalties conceded, whereas higher activation levels were negatively related to points, tries and conversions conceded. The teams' final ranking was significantly predicted by goal-setting $(\beta=0.21)$, imagery $(\beta=0.17)$, activation $(\beta=$ $0.32)$ and negative thinking $(\beta=-0.16)$ levels $\left(R^{2}=0.11, p=0.005\right)$. Keywords: Rugby Union; Under 19; Psychological skills and strategies; Tournament outcomes; Performance.
\end{abstract}

\footnotetext{
Corresponding author. Universidade de Trás-os-Montes e Alto Douro, Departamento de Ciências do Desporto, Exercício e Saúde, Quinta de Prados - Campus Desportivo, Apartado 1013, 5001-801 Vila Real - Portugal.

E-mail: hmfernandes@gmail.com

Supplementary Issue: Spring Conferences of Sports Science. International Seminar of Physical Education, Leisure and Health, 17-19 June 2019. Castelo Branco, Portugal.

JOURNAL OF HUMAN SPORT \& EXERCISE ISSN 1988-5202

(C) Faculty of Education. University of Alicante.

doi:10.14198/jhse.2019.14.Proc4.82
} 


\section{INTRODUCTION}

Much of the applied sport psychology literature has devoted a lot of attention to the relationships between psychological factors and individual or teams' performance. Regarding rugby union, some studies have investigated the association between certain psychological skills and competitive anxiety levels (e.g., Neil et al., 2006), but mainly in adult players. Consequently, knowledge is still lacking on the psychological skills and strategies used by youth rugby players before and when competing and, even more important, its influence on team's performance indicators and outcomes during an international tournament.

Therefore, the aim of the present study is to identify the psychological skills and strategies related to tournament outcomes and performance indicators in a European U19 Rugby Union Championship.

\section{MATERIAL AND METHODS}

\section{Participants}

A total of 183 elite male under-19 rugby players (backs, $n=91$; forwards, $n=92$ ), aged between 17 and 19 years $(M=18.67, S D=0.51)$, volunteered to participate in the current study. Participants competed at the U19 European Championship 2014, held in Lisbon - Portugal, representing seven national teams ranked by the teams' final classification in the championship, as follows: Georgia (1st place, $n=34)$, Portugal (2nd place, $n=20$ ), Spain (3rd place, $n=26$ ), Russia (4th place, $n=27$ ), Netherlands (5th place, $n=17$ ), Belgium (6th place, $n=14$ ), and Romania (7th place, $n=45$ ). Data from the Polish national team (8th place in the tournament; $n=17$ ) was not analysed because of low reliability issues (Cronbach's a values below 0.60 ).

\section{Measures}

Athletes completed the 32-item of the competition subscales of the Test of Performance Strategies (TOPS; Thomas et al., 1999), which assesses eight psychological skills and strategies (self-talk, emotional control, automaticity, goal-setting, imagery, activation, relaxation and negative thinking). A detailed description of the different language versions of the instrument that were used is available elsewhere (Vaz et al., 2017).

Summary matches' statistics and the final ranking position (inverted) of the national teams competing at the U19 European Championship 2014 were retrieved from an official website (old.rugbyeurope.tv/archivescompetition-71.htm, retrieved July, 8, 2015). The total tournament statistics collected for analysis were: i) points, tries, conversions and penalties scored, and ii) points, tries, conversions and penalties conceded.

\section{Procedures}

The institutional ethics committee of the first author provided ethical approval for this study (CE.UTAD 27/2015). After obtaining approval from the team managers or coaches, signed informed consents were obtained from the athletes or by the legal guardians of minors. Athletes completed the questionnaire one hour before the first match of the tournament. The instrument was completed, individually, in a quiet room.

\section{Analysis}

Statistical analysis included descriptive statistics (mean, standard deviation, skewness and kurtosis), internal consistency (Cronbach's alpha), Pearson correlations and multivariable linear regression. Significance was set at $p<0.05$. All analyses were performed using SPSS 17.0 (SPSS Inc., Chicago). 


\section{RESULTS}

TOPS' competition subscales presented acceptable values of skewness and kurtosis (between -0.67 and 0.98). All of the subscales demonstrated satisfactory reliability (i.e., Cronbach's alpha $>0.60$ ), with the exception of the automaticity subscale. Taking this into account, and also previous evidence (Hardy et al., 2010), the data from this subscale was omitted from the following analyses.

Table 1 shows the correlations between the study variables.

Table 1. Correlations between psychological skills/strategies and tournament performance indicators

\begin{tabular}{lccccccccc}
\hline \multirow{2}{*}{$\begin{array}{l}\text { Competition } \\
\text { subscales }\end{array}$} & \multicolumn{3}{c}{ Total tournament indicators scored } & \multicolumn{3}{c}{ Total tournament indicators conceded } & Final \\
\cline { 2 - 8 } & Points & Tries & Conver. & Penalt. & Points & Tries & Conver. & Penalt. & Ranking \\
\hline Self-talk & $0.29^{* *}$ & $0.28^{* *}$ & $0.28^{* *}$ & -0.10 & $-0.17^{*}$ & $-0.16^{*}$ & -0.13 & 0.01 & 0.02 \\
Emotional control & $0.16^{*}$ & $0.16^{*}$ & $0.24^{* *}$ & 0.10 & 0.00 & -0.07 & 0.03 & $-0.16^{*}$ & -0.09 \\
Goal-setting & $0.23^{* *}$ & $0.22^{* *}$ & $0.22^{* *}$ & 0.09 & -0.11 & -0.04 & -0.13 & $-0.15^{*}$ & 0.14 \\
Imagery & 0.10 & 0.08 & 0.06 & $0.25^{* *}$ & -0.06 & 0.00 & -0.14 & $-0.16^{*}$ & 0.14 \\
Activation & 0.09 & 0.09 & 0.08 & 0.00 & $-0.19^{*}$ & $-0.21^{* *}$ & $-0.26^{* *}$ & 0.13 & $0.15^{*}$ \\
Relaxation & -0.05 & -0.03 & -0.06 & -0.06 & -0.12 & -0.13 & -0.12 & 0.04 & -0.04 \\
Negative thinking & -0.14 & -0.13 & 0.02 & -0.11 & $0.25^{*}$ & $0.32^{* *}$ & 0.12 & $0.21^{* *}$ & -0.10 \\
\hline
\end{tabular}

Results showed small to moderate significant correlations between the study variables. A more frequent use of self-talk, emotional control and goal-setting strategies was positively related to points, tries and conversions scored, whereas imagery levels were positively associated to penalties scored. On the other hand, higher levels of negative thinking were positively correlated to points, tries and penalties conceded, whereas higher activation levels were negatively related to points, tries and conversions conceded.

Multivariable linear regression analysis using the teams' final ranking (inverted) as the dependent variable showed that goal-setting $(\beta=0.21, p<0.01)$, imagery $(\beta=0.17, p<0.05)$, activation $(\beta=0.32, p<0.001)$ and negative thinking $(\beta=-0.16, p<0.05)$ were the significant predictors in the model $\left(R^{2}=0.11, p=0.005\right)$.

\section{DISCUSSION}

Using a prospective design, the results of the current study indicate that pre-tournament levels of certain psychological skills and strategies predict the teams' final classification. More precisely, our findings expand previous evidence (Neil et al., 2006; Vaz et al., 2017) by suggesting that these effects are somehow explained by the specific influence of psychological dimensions on certain teams' performance indicators.

\section{CONCLUSIONS}

In sum, the findings of the present study suggest that certain competition psychological skills and strategies play an important role on U19 rugby union tournament outcomes and performance indicators. Taken as a whole, this evidence underscores the importance and relevance of planning and implementing appropriate psychological preparation before and during rugby union youth tournaments. 


\section{REFERENCES}

Hardy, L., Roberts, R., Thomas, P., \& Murphy, S. (2010). Test of Performance Strategies (TOPS): Instrument refinement using confirmatory factor analysis. Psychol Sport Exerc, 11(1), 27-35. https://doi.org/10.1016/i.psychsport.2009.04.007

Neil, R., Mellalieu, S. D., \& Hanton, S. (2006). Psychological skills usage and the competitive anxiety response as a function of skill level in rugby union. J Sci Med Sport, 5(3), 415-423.

Vaz, L., Martin, I., Batista, M., Almeida, L.M., \& Fernandes, H.M. (2017). Differences in the psychological skills and strategies used by elite male under-19 rugby union players in competition according to playing position. Ibero Am J Exerc Sport Psychol, 12(2), 225-230.

\section{(c) $(1)(9)$}

This work is licensed under a Attribution-NonCommercial-NoDerivatives 4.0 International (CC BY-NC-ND 4.0). 


\title{
Physical performance tests and anthropometric data to predict selection in U19 rugby union players
}

\author{
LUIS VAZ ${ }^{1,2}$, MARCO BATISTA ${ }^{3,4,5}$, SAMUEL HONÓRIO ${ }^{3,4,5}$, HELDER MIGUEL FERNANDES $1,2,3$ \\ 1 Universidade de Trás-os-Montes e Alto Douro, Portugal \\ ${ }^{2}$ Research Centre in Sports, Health and Human Development (CIDESD-UTAD), Portugal \\ ${ }^{3}$ Research in Education and Community Intervention, RECl- Instituto Piaget, Portugal \\ ${ }^{4}$ Instituto Politécnico de Castelo Branco, Portugal \\ ${ }^{5}$ Sport, Health \& Exercise Research Unit (SHERU), Portugal
}

\begin{abstract}
Anthropometric and performance data were collected from 184 rugby players (mean age $=17.9 \pm 0.5$ years, body mass $=84.2 \pm 13.5 \mathrm{~kg}$; height $=1.79 \pm 0.07 \mathrm{~m}$ ) to explore: (i) the correlation among indicators; and (ii) compare profiles from the selected $(n=39)$ and non-selected $(n=145)$ players to represent the Portuguese under-19 national team. Anthropometry characteristics included body height and body mass. Physical performance variables included push up and pull-up test, squat, Sargent test, flexed arm hang test, sit-andreach test, $20 \mathrm{~m}$ shuttle run test, handgrip strength test, $20-\mathrm{m}$ and $50-\mathrm{m}$ sprint and Illinois agility test. The results showed that the selected rugby athletes reported significantly higher levels of right $(t=2.77, p=0.01$, $E S=-0.5[-0.8$ to -0.2$])$ and left handgrip strength scores $(t=2.81, p=0.01, E S=-0.5[-0.8$ to -0.2$])$, and significantly better (i.e. lower) levels of agility scores $(t=-2.28, p=0.02$, $E S=0.4[0.1$ to 0.7$]$ ) than the nonselected rugby athletes. Accordingly, prospective players need to be constantly monitored and evaluated across age-categories in order to allow them to achieve the anthropometrical and fitness requirements needed to play at the international level. Keywords: Rugby Union; Performance; Field-based tests; Selective factors; Predict selection.
\end{abstract}

\footnotetext{
Corresponding author. Universidade de Trás-os-Montes e Alto Douro, Departamento de Ciências do Desporto, Exercício e Saúde, Quinta de Prados - Campus Desportivo, Apartado 1013, 5001-801 Vila Real - Portugal.

E-mail: hmfernandes@gmail.com

Supplementary Issue: Spring Conferences of Sports Science. International Seminar of Physical Education, Leisure and Health, 17-19 June 2019. Castelo Branco, Portugal.

JOURNAL OF HUMAN SPORT \& EXERCISE ISSN 1988-5202

(C) Faculty of Education. University of Alicante.

doi:10.14198/jhse.2019.14.Proc4.82
} 


\title{
INTRODUCTION
}

Traditionally, the recruitment, selection and development of talented rugby union players has focused on training and game perception aspects of skills performance, with less emphasis placed on the physical testing performance characteristics. However, the differences between specific positions in anthropometrical measures also illustrate the heterogeneous nature of rugby players (Vaz, Morais, Rocha \& James, 2014). Therefore, the purpose of this study was to explore how anthropometric and physical performance test indicators help to predict selection in U19 rugby union players.

\section{MATERIAL AND METHODS}

\section{Participants}

A total of 184 male U19 rugby union players from Portuguese national academies were part of this study (mean \pm SD; age $17.9 \pm 0.5$ years, body mass $84.2 \pm 13.5 \mathrm{~kg}$, height $1.79 \pm 0.07 \mathrm{~m}$ ). Players were divided into two groups based on selected $(n=39)$ and non-selected $(n=145)$ criteria to represent the Portugal national U19 rugby team. The study protocol followed the guidelines stated and conformed to the declaration of Helsinki and ethical approval was provided by the local University Ethics Committee.

\begin{abstract}
Measures
Anthropometric measurement included body mass $(\mathrm{kg})$ and body height $(\mathrm{m})$. Physiological and performance assessment tests included: Push Up test, Pull-up test, Free standing squat, 1RM, Sargent Test, Flexed arm hang test, Sit-and-reach test, 20 m shuttle-run test and $\mathrm{VO}_{2 m a x}$. Handgrip strength test (right and left hand). Speed across $20 \mathrm{~m}$ and $50 \mathrm{~m}$ using dual beam electronic timing gates and Illinois agility test with 10 meters long and 5 meters wide. The validity and reliability of all tests selected for this study had been previously confirmed in literature (Moore \& Murphy, 2003).
\end{abstract}

\section{Procedures}

The anthropometric and physical measurements were performed as follows: anthropometrics (morning), physical tests (afternoon), with an interval of $\approx 30$-min interspersing consecutive tests. Due to the specific routines of these athletes' training and to their frequent physical assessments performed in Portuguese national academies, all athletes were highly familiarized with the experimental procedures of this study.

\section{Analysis}

Mean (M) and standard deviation (SD) were calculated for all dependent variables. Correlation between variables was computed using Pearson ( $r$ ) correlation coefficient. Independent sample t-tests were used in order to identify significant differences between the two groups (selected vs. non-selected athletes). The effect sizes (ES) of obtained differences were expressed in standardized Cohen's differences and respecting intervals (Hopkins, Schabort, \& Hawley, 2001). All statistical analyses were performed using SPSS Version (IBM Corporation, USA) and statistical significance was set at $<0.05$.

\section{RESULTS}

The descriptive statistics and the correlation coefficients for the anthropometric and physical fitness variables in total sample were calculated. Moderate to high negative correlations were observed between the pull up and the Illinois agility scores, between the left handgrip strength and the Sargent test scores, and between the squat and the Sargent test scores. The anthropometric and physical performance levels for the selected and non-selected groups of athletes are presented in Table 1. 
Table 1. The anthropometric and physical performance levels for selected and non-selected groups of athletes

\begin{tabular}{|c|c|c|c|c|c|c|}
\hline Variables & $\begin{array}{l}\text { Total sample } \\
(\mathrm{n}=184) \\
M \pm S D\end{array}$ & $\begin{array}{l}\text { Selected } \\
(n=39) \\
M \pm S D\end{array}$ & $\begin{array}{c}\text { Non selected } \\
(n=145) \\
M \pm S D\end{array}$ & $t$ & $p$ & $\begin{array}{l}\text { Cohen'd } \\
{[90 \% \mathrm{Cl}]}\end{array}$ \\
\hline \multicolumn{7}{|c|}{ Anthropometric characteristics } \\
\hline Body mass $(\mathrm{kg})$ & $84.28 \pm 13.53$ & $86.72 \pm 12.76$ & $83.63 \pm 13.70$ & 1.27 & 0.20 & \\
\hline Body height (m) & $1.79 \pm 0.07$ & $1.80 \pm 0.09$ & $1.79 \pm 0.06$ & 0.72 & 0.46 & \\
\hline \multicolumn{7}{|l|}{ Physical fitness } \\
\hline Push Up test & $43.25 \pm 11.10$ & $42.64 \pm 12.48$ & $43.41 \pm 10.73$ & -0.38 & 0.70 & \\
\hline Pull up test & $11.83 \pm 4.29$ & $12.36 \pm 4.57$ & $11.68 \pm 4.21$ & 0.87 & 0.38 & \\
\hline Flexed arm hang test & $40.42 \pm 13.63$ & $38.82 \pm 10.34$ & $40.85 \pm 14.38$ & -0.82 & 0.41 & \\
\hline Handgrip strength (left) & $48.00 \pm 4.88$ & $49.89 \pm 6.54$ & $47.49 \pm 4.22$ & 2.81 & $0.01^{*}$ & $-0.5[-0.8$ to -0.2$]$ \\
\hline Handgrip strength (right) & $46.03 \pm 4.65$ & $47.86 \pm 5.98$ & $45.54 \pm 4.11$ & 2.77 & $0.01^{*}$ & $-0.5[-0.8$ to -0.2$]$ \\
\hline Free standing squat & $140.77 \pm 31.09$ & $142.31 \pm 31.22$ & $140.37 \pm 31.15$ & 0.34 & 0.73 & \\
\hline Sargent test & $44.95 \pm 5.06$ & $44.82 \pm 5.20$ & $44.99 \pm 5.04$ & -0.18 & 0.85 & \\
\hline $20 \mathrm{~m}$ sprint $(s)$ & $2.99 \pm 0.18$ & $2.97 \pm 0.16$ & $3.01 \pm 0.18$ & -1.16 & 0.24 & \\
\hline $50 \mathrm{~m}$ sprint $(s)$ & $6.85 \pm 0.27$ & $6.84 \pm 0.26$ & $6.86 \pm 0.18$ & -0.29 & 0.76 & \\
\hline Illinois agility test & $15.15 \pm 0.96$ & $14.84 \pm 1.09$ & $15.24 \pm 0.91$ & -2.28 & $0.02^{*}$ & $0.4[0.1$ to 0.7$]$ \\
\hline Sit-and-reach test & $32.93 \pm 4.88$ & $33.05 \pm 4.71$ & $32.89 \pm 4.95$ & 0.18 & 0.85 & \\
\hline $\mathrm{VO}_{2 \max }\left(\mathrm{ml} \cdot \mathrm{kg}^{-1} \cdot \mathrm{min}^{-1}\right)$ & $49.69 \pm 3.96$ & $49.62 \pm 4.31$ & $49.71 \pm 3.87$ & -0.12 & 0.90 & \\
\hline
\end{tabular}

Between-groups comparisons indicated that selected rugby athletes reported significantly higher levels of right (ES $=-0.5[-0.8$ to -0.2$]$ ) and left handgrip strength scores (ES $=-0.5[-0.8$ to -0.2$])$, and significantly better levels of agility scores (ES $=0.4[0.1$ to 0.7$]$ ) than the non-selected rugby athletes.

\section{DISCUSSION}

Findings highlight the importance and relationship between handgrip strength test and agility ability to team selection in Portuguese U19 rugby national team. Results regarding the left and right handgrip strength may provide additional insights. A combination of three variables, body mass, handgrip strength and agility, differentiated significantly between the selected and non-selected rugby players.

\section{CONCLUSIONS}

The main findings have several implications for the effective selection process design, particularly by helping to identify and to improve the accuracy of elite rugby talent identification programs.

\section{REFERENCES}

Hopkins, W. G., Schabort, E. J., \& Hawley, J. A. (2001). Reliability of power in physical performance tests. Sports Med, 31(3), 211-234. https://doi.org/10.2165/00007256-200131030-00005

Moore, A., \& Murphy, A. (2003). Development of an anaerobic capacity test for field sport athletes. J Sci Med Sport, 6(3), 275-284. https://doi.org/10.1016/s1440-2440(03)80021-x

Vaz, L., Morais, T., Rocha, H., \& James, N. (2014). Fitness profiles of elite Portuguese rugby union players. J Hum Kinet, 41, 235-244. https://doi.org/10.2478/hukin-2014-0051 


\section{(c) (i) (3)}

This work is licensed under a Attribution-NonCommercial-NoDerivatives 4.0 International (CC BY-NC-ND 4.0). 


\title{
Physical exercise in higher education: Knowledge, attitudes and practices
}

\author{
REGINA ALVES \\ CIEC, Center for Research in Child Studies, Institute of Education, University of Minho, Portugal
}

\begin{abstract}
Sedentary behaviours have been associated with the emergence of chronic diseases, such as diabetes or hypertension. Conversely, regular physical activity has a leading role in promoting a healthy lifestyle, as evidenced by the numerous physical, psychological and social benefits. This study includes a systematic review of studies on knowledge, attitudes and physical activity of college students, which included declared criteria of eligibility. In general, in order to measure physical exercise, students should be essentially asked about the frequency (in number of days per week) of moderate, vigorous and light exercise, and the number of hours spent sitting. Scales of attitudes should address items that include lack of energy; lack of motivation; lack of resources; lack of support and lack of time. The scales of knowledge about physical exercise should include items about the recommendations about the practice of physical exercise and the relation between physical exercise and diseases. The current review, 1) provides guidelines for the construction of standardized measurement scales related to knowledge, attitudes and practice of physical exercise. And, 2) the main results presented can help in the elaboration of programs of education and promotion of the physical exercise in the university. Keywords: Physical exercise; Higher education; Physical activity; Knowledge; Attitudes.
\end{abstract}

Corresponding author. CIEC, Center for Research in Child Studies, Institute of Education, University of Minho, Portugal.

E-mail: rgnalves@gmail.com

Supplementary Issue: Spring Conferences of Sports Science. International Seminar of Physical Education, Leisure and Health, 17-19 June 2019. Castelo Branco, Portugal.

JOURNAL OF HUMAN SPORT \& EXERCISE ISSN 1988-5202

(C) Faculty of Education. University of Alicante.

doi:10.14198/jhse.2019.14.Proc4.82 


\section{INTRODUCTION}

Regular physical exercise contributes to a healthy lifestyle, however, Portuguese university students have a high prevalence of inactivity (41\%) (Haase, Steptoe, Sallis, \& Wardle, 2004). The WHO argues that the increase in knowledge about the international guidelines on the exercise represents a positive motivation prerequisite for a physically active lifestyle (WHO, 2012). This systematic review study aims to identify, retrieve and analyse the measuring instruments of knowledge, attitudes and exercise practices of higher education students.

\section{MATERIAL AND METHODS}

To ensure transparency and integrity of literature proceeded in accordance with the guidelines of PRISMA (Moher, Liberati, Tetzlaff, \& Altman, 2010). From the research in five databases (PubMed, ERIC, CINAHL®, SciELO and ScienceDirect) of articles published in scientific journals, between 1980 and 2018, four terms are combined using Boolean operators. After carrying out literature searches, 16 articles were selected from a total of 169 studies found. It excluded a) studies on populations not only of higher education or samples of populations in specific situations; $b$ ) articles related to physical exercise and health problems and including the association with other risk behaviours; c) literature review articles or interventions; and, d) which did not present the psychometric measures of the instruments.

\section{RESULTS}

Analysis of assessment instruments of physical activity concluded that the Godin Leisure-Time Exercise Questionnaire was the most used instrument (Blanchard et al., 2007; Cardinal, Tuominen, \& Rintala, 2004; Holtz, Kokotilo, Fitzgerald, \& Frank, 2013; Keating et al., 2009; Yan, Cardinal, \& Acock, 2015). At the level of the attitudes towards the practice of physical exercise, categories were created according to the dimensions of analysis presented, highlighting those that present with more frequency: social and/or familiar support (Blanchard et al., 2007; King, Vidourek, English, \& Merianos, 2014; Nahar et al., 2016; Wallace et al., 2000; Yan et al., 2015); lack of time (Blanchard et al., 2007; King et al., 2014; Nahar et al., 2016; Wallace \& Buckworth, 2001) self-efficacy (Cardinal et al., 2004; Wallace et al., 2000; Yan et al., 2015); accessibility/resources (Nahar et al., 2016; Yan et al., 2015). In order to study the level of knowledge about physical exercise, we should mainly consider: the concept and components of physical exercise, the relation of physical exercise and diseases and health benefits (Haase et al., 2004; Keating et al., 2009).

\section{DISCUSSION}

In order to measure physical exercise practice, students should be, essentially asked about the frequency (in number of days per week) of moderate, vigorous and moderate exercise; and about the number of hours per day spent sitting. The scales of attitudes presented in the different instruments analysed are more comprehensive, so it is proposed to construct a scale that includes the following dimensions: lack of energy; lack of motivation; lack of resources; lack of support and lack of time. For the scale of knowledge about physical exercise, the following dimensions are proposed: recommendations about the practice of physical exercise and the relation between physical exercise and diseases. 


\section{CONCLUSIONS}

The current review provides guidelines for the construction of standardized and comparable measurement scales. Concomitantly, the verified low level of knowledge and the barriers to physical exercise reinforce the idea of the need to develop intervention programs in the promotion of physical exercise in Higher Education.

\section{REFERENCES}

Blanchard, C. M., Kupperman, J., Sparling, P., Nehl, E., Rhodes, R. E., Courneya, K. S., ... Hunt, T. (2007). Ethnicity as a moderator of the theory of planned behavior and physical activity in college students. Res Q Exercise Sport, 78(5), 531-541. https://doi.org/10.1080/02701367.2007.10599452

Cardinal, B. J., Tuominen, K. J., \& Rintala, P. (2004). Cross-Cultural Comparison of American and Finnish College Students' Exercise Behavior Using Transtheoretical Model Constructs. Res Q Exercise Sport, 75(1), 92-101. https://doi.org/10.1080/02701367.2004.10609137

Haase, A., Steptoe, A., Sallis, J. F., \& Wardle, J. (2004). Leisure-time physical activity in university students from 23 countries: Associations with health beliefs, risk awareness, and national economic development. Prev Med, 39(1), 182-190. https://doi.org/10.1016/j.ypmed.2004.01.028

Holtz, K. A., Kokotilo, K. J., Fitzgerald, B. E., \& Frank, E. (2013). Exercise behaviour and attitudes among fourth-year medical students at the University of British Columbia. Can Fam Physician, 59(5), 2632. https://doi.org/10.1714/1563.17025

Keating, X. D., Jose, C.-P., Erin, C., Louis, H., Tere, R., \& Li, C. (2009). Health-related fitness knowledge and its relation to student physical activity patterns at a large U.S. southern state university. Health Related Fitness, 5(2), 3-10.

King, K. A., Vidourek, R. A., English, L., \& Merianos, A. L. (2014). Vigorous physical activity among college students: Using the health belief model to assess involvement and social support. Archives of Exercise in Health and Disease, 4(2), 267-279. https://doi.org/10.5628/aehd.v4i2.153

Moher, D., Liberati, A., Tetzlaff, J., \& Altman, D. G. (2010). Preferred reporting items for systematic reviews and meta-analyses: The PRISMA statement. Int $J$ Surg, 8, 336-341. https://doi.org/10.1016/j.ijsu.2010.02.007

Nahar, V. K., Sharma, M., Catalano, H. P., Ickes, M. J., Johnson, P., \& Ford, M. A. (2016). Testing multitheory model (MTM) in predicting initiation and sustenance of physical activity behavior among college students. Health Promotion Perspectives, 6(2), 58-65. https://doi.org/10.15171/hpp.2016.11

Wallace, L. S., \& Buckworth, J. (2001). Application of the Transtheoretical Model to Exercise Behavior among Non-traditional College Students. American Journal of Health Education, 32(1), 39-47. https://doi.org/10.1080/19325037.2001.10609396

Wallace, L. S., Buckworth, J., Kirby, T. E., \& Sherman, W. M. (2000). Characteristics of Exercise Behavior among College Students: Application of Social Cognitive Theory to Predicting Stage of Change. Prev Med, 31(5), 494-505. https://doi.org/10.1006/pmed.2000.0736

WHO. (2012). Health education: theoretical concepts, effective strategies and core competencies: a foundation document to guide capacity development of health educators. Retrieved from http://www.who.int/iris/handle/10665/119953

Yan, Z., Cardinal, B. J., \& Acock, A. C. (2015). Understanding Chinese international college and university students' physical activity behavior. J Sport Health Sci, 4, 203-210. https://doi.org/10.1016/j.jshs.2013.07.002 


\section{(c) $\underset{\mathrm{EY}}{\mathrm{NO}} \mathrm{\Theta}$}

This work is licensed under a Attribution-NonCommercial-NoDerivatives 4.0 International (CC BY-NC-ND 4.0). 


\title{
Nutritional knowledge and eating attitudes and habits in higher education
}

\author{
REGINA ALVES \\ CIEC, Center for Research in Child Studies, Institute of Education, University of Minho, Portugal
}

\begin{abstract}
Eating habits involve behavioural predispositions related to intra and interpersonal factors, which include nutritional knowledge and attitudes towards the consumption of certain types of food. This study includes a systematic review of studies on knowledge, attitudes and eating habits of college students, which included declared criteria of eligibility. In general, to measure eating habits, it is proposed that questions be asked regarding the daily frequency of consumption of fruits, vegetables, fast foods and foods with sugar. Scales of attitudes towards food should include items about the ability to understand nutritional recommendations and food labels. The scales of knowledge about food, it is proposed the use of items related to the presence of nutrients in certain foods. The current review, 1) provides guidelines for the construction of standardized measurement scales related to knowledge, attitudes and eating habits. And, 2) the main results presented can help in the elaboration of programs of education and promotion of healthy eating in the university. Keywords: Eating habits; Higher education; Nutritional knowledge; Eating attitudes.
\end{abstract}

Corresponding author. CIEC, Center for Research in Child Studies, Institute of Education, University of Minho, Portugal. E-mail: rgnalves@gmail.com

Supplementary Issue: Spring Conferences of Sports Science. International Seminar of Physical Education, Leisure and Health, 17-19 June 2019. Castelo Branco, Portugal.

JOURNAL OF HUMAN SPORT \& EXERCISE ISSN 1988-5202

(c) Faculty of Education. University of Alicante.

doi:10.14198/jhse.2019.14.Proc4.82 


\section{INTRODUCTION}

Health and disease prevention is strongly related to food selection (WHO, 2017), and excessive consumption of saturated fat is associated with increased cholesterol and a higher risk of cardiovascular diseases. Eating habits involve behavioural predispositions related to intra and interpersonal factors, which include nutritional knowledge and attitudes towards the consumption of certain types of food. The present systematic review aims to identify, retrieve and analyse the instruments of knowledge, attitudes and eating habits of students of Higher Education.

\section{MATERIAL AND METHODS}

To ensure transparency and integrity of literature proceeded in accordance with the guidelines of PRISMA (Moher, Liberati, Tetzlaff, \& Altman, 2010). From the research in five databases (PubMed, ERIC, CINAHL®, SciELO and ScienceDirect) of articles published in scientific journals, between 1980 and 2018, four terms are combined using Boolean operators. After carrying out literature searches, 10 articles were selected from a total of 172 studies found. It excluded a) studies on populations not only of higher education or samples of populations in specific situations; b) articles related to eating habits or attitudes and health problems or including the association with other risk behaviours; c) literature review articles or interventions; and, d) which did not present the psychometric measures of the instruments.

\section{RESULTS}

The selected articles were published between 2007 (Kolodinsky, Harvey-Berino, Berlin, Johnson, \& Reynolds, 2007) and 2018 (Silva, Campos, \& Marôco, 2018). The analysis of the food habits assessment instruments allowed us to conclude that it is essential to evaluate the eating frequency of the following foods: fats or fast foods (Blotnicky, Mann, \& Joy, 2015; Kolodinsky et al., 2007; Kresić, Kendel Jovanović, Pavicić Zezelj, Cvijanović, \& Ivezić, 2009; Ozdoğan \& Ozcelik, 2011; White, Horwath, \& Conner, 2013); sugars or sweets (Blotnicky et al., 2015; Kolodinsky et al., 2007; Kresić et al., 2009; Ozdoğan \& Ozcelik, 2011; White et al., 2013); vegetables (Blotnicky et al., 2015; Kolodinsky et al., 2007; Kresić et al., 2009; White et al., 2013); and fruits (Kolodinsky et al., 2007; Kresić et al., 2009; White et al., 2013). The variables weight and height for the calculation of body mass index proved to be an important variable (Fyler, Schumacher, Banning, \& Gam, 2014; Hamilton et al., 2018; Ohara et al., 2014; Poínhos, Oliveira, \& Correia, 2015; Silva et al., 2018). The nutritional knowledge addressed by several studies is directed towards the understanding of nutrients in foods (Kolodinsky et al., 2007; Kresić et al., 2009) .(Kresić et al., 2009; Ozdoğan \& Ozcelik, 2011) and food recommendations (Kolodinsky et al., 2007; Kresić et al., 2009). Self-efficacy was a major factor in the analysis of positive attitudes toward healthy eating (Blotnicky et al., 2015; Fyler et al., 2014; Poínhos et al., 2015).

\section{DISCUSSION}

To measure eating habits, it is proposed that questions be asked regarding the daily frequency of consumption of fruits, vegetables, fast foods and food with sugar. Scales of attitudes towards food should include items about the ability to understand nutritional recommendations and food labels. For the food knowledge scale, the use of items related to the presence of nutrients in certain foods is proposed. 


\section{CONCLUSIONS}

The current review provides guidelines for the construction of standardized and comparable measurement scales. At the same time, it reinforces the idea of the need to develop intervention programs to promote healthy eating in higher education, so that university students have the opportunity to make informed decisions about the foods to be consumed, to develop more positive attitudes towards healthy eating and, therefore, to opt for a balanced diet.

\section{REFERENCES}

Blotnicky, K. A., Mann, L. L., \& Joy, P. R. (2015). An assessment of University Students' Healthy Eating Behaviors With the Expectancy Theory. ASBBS EJournal, 11(1), 31-44. Retrieved from http://www.asbbs.org/files/2015/eJournal_2015.pdf\#page=31

Fyler, M., Schumacher, J., Banning, J., \& Gam, H. J. (2014). Influence of Body Satisfaction, Body Mass Index, and Diet Quality on Healthy Eating Attitudes among College Students. Family and Consumer Sciences Research Journal, 42(4), 330-340. https://doi.org/10.1111/fcsr.12067

Hamilton, T., Hoffman, J., Arsiwalla, D., Volpe, R., Schmidt, E., \& Gropper, S. (2018). Gender comparisons of young adults' eating behavior regulation: Re-examination of the Regulation of Eating Behavior Scale (REBS). Appetite, 126, 80-89. https://doi.org/10.1016/j.appet.2018.03.014

Kolodinsky, J., Harvey-Berino, J. R., Berlin, L., Johnson, R. K., \& Reynolds, T. W. (2007). Knowledge of Current Dietary Guidelines and Food Choice by College Students: Better Eaters Have Higher Knowledge of Dietary Guidance. J Am Diet Assoc, 107(8), 1409-1413. https://doi.org/10.1016/j.jada.2007.05.016

Kresić, G., Kendel Jovanović, G., Pavicić Zezelj, S., Cvijanović, O., \& Ivezić, G. (2009). The effect of nutrition knowledge on dietary intake among Croatian university students. Collegium Antropol, 33(4), 1047-56. Retrieved from https://hrcak.srce.hr/51453

Moher, D., Liberati, A., Tetzlaff, J., \& Altman, D. G. (2010). Preferred reporting items for systematic reviews and meta-analyses: The PRISMA statement. Int $\mathrm{J}$ Surg, 8, 336-341. https://doi.org/10.1016/j.ijsu.2010.02.007

Ohara, K., Kato, Y., Mase, T., Kouda, K., Miyawaki, C., Fujita, Y., ... Nakamura, H. (2014). Eating behavior and perception of body shape in Japanese university students. Eat Weight Disord, 19(4), 461-468. https://doi.org/10.1007/s40519-014-0130-7

Ozdoğan, Y., \& Ozcelik, A. (2011). Evaluation of the nutrition knowledge of sports department students of universities. J Int Soc Sport Nutr, 8(1), 11. https://doi.org/10.1186/1550-2783-8-11

Poínhos, R., Oliveira, B. M. P. M., \& Correia, F. (2015). Eating behavior in Portuguese higher education students: The effect of social desirability. Nutrition, 31(2), 310-314. https://doi.org/10.1016/..nut.2014.07.008

Silva, W. R. da, Campos, J. A. D. B., \& Marôco, J. (2018). Impact of inherent aspects of body image, eating behavior and perceived health competence on quality of life of university students. PLOS ONE, 13(6), e0199480. https://doi.org/10.1371/journal.pone.0199480

White, B. A., Horwath, C. C., \& Conner, T. S. (2013). Many apples a day keep the blues away - Daily experiences of negative and positive affect and food consumption in young adults. Brit $\mathrm{J}$ Health Psych, 18(4), 782-798. https://doi.org/10.1111/bjhp.12021

WHO. (2017). Cardiovascular diseases (CVDs). Retrieved April 11, 2019, from https://www.who.int/en/news-room/fact-sheets/detail/cardiovascular-diseases-(cvds) 


\section{(c) (i) (3)}

This work is licensed under a Attribution-NonCommercial-NoDerivatives 4.0 International (CC BY-NC-ND 4.0). 


\title{
Comparisons of external load variables between small-sided and real format games: An 8-week study in professional soccer training context
}

\author{
LEANDRO SILVA ${ }^{1}$, BRUNO SOUSA ${ }^{1}$, RICARDO LIMA ${ }^{1}$, MIGUEL CAMÕES ${ }^{1}$, PEDRO BEZERRA ${ }^{1}$, FILIPE \\ MANUEL CLEMENTE 1,2 \\ ${ }^{1}$ Polytechnic Institute of Viana do Castelo, School of Sport and Leisure, Melgaço, Portugal \\ 2Instituto de Telecomunicações, Delegação da Covilhã, Portugal
}

\begin{abstract}
The purpose of this study was to compare the external load of professional soccer players between smallsided games (SSGs) and real format $(G R+10 \times 10+G R)$ during an 8-week training period. Thirty-two players $(25.1 \pm 2.9$ years, $181.9 \pm 6.3 \mathrm{~cm}, 73.1 \pm 6.3 \mathrm{~kg}$ ) from a team competing in the second professional Portuguese league were monitored. The external load was quantified using a 10-Hz GPS unit and all the training sessions of the first 8 weeks of the season were quantified. The variables compared were: duration of the drill (min), distance covered $(\mathrm{m})$, sprint distance $(\mathrm{m})$, player load $(\mathrm{g})$ and maximal velocity $(\mathrm{km} / \mathrm{h}-1)$. The results revealed significant and larger increases of sprinting distance $(>20 / \mathrm{km}-1)$ in real format than in SSGs $(p=0.006 ; d=1.321)$. Similar evidence was found in maximal velocity $(p=0.010 ; d=1.350)$. As conclusions, it is possible to analyse that the time dedicated to SSGs and real format is approximately the same during the training sessions, however there are significant and large differences in terms of the main important external load variables. The SSGs are not close enough to produce a similar stimulus of the sprinting load and maximal velocity during the training in comparison to real format. Keywords: External load; Small-sided; Real format games; Training load monitoring; Association football; Performance.
\end{abstract}

\footnotetext{
Corresponding author. Complexo Desportivo e Lazer Comendador Rui Solheiro - Monte de Prado, 4960-320, Melgaço, Portugal.

E-mail: filipe.clemente5@gmail.com

Supplementary Issue: Spring Conferences of Sports Science. International Seminar of Physical Education, Leisure and Health, 17-19 June 2019. Castelo Branco, Portugal.

JOURNAL OF HUMAN SPORT \& EXERCISE ISSN 1988-5202

(c) Faculty of Education. University of Alicante.

doi:10.14198/jhse.2019.14.Proc4.82
} 


\section{INTRODUCTION}

Small-sided games (SSGs) have been massively used in training context aiming to optimize the training time by stimulating both physical/physiological and technical/tactical capacities (Clemente, Martins, \& Mendes, 2014). SSGs can be considered smaller and adjusted versions of the real game format and usually are conditioned by concurrent task constraints (e.g., size of the pitch, players involved, rules modification) (Sarmento et al., 2018). The adaptations are often used to augment the perception of the players for a specific tactical content and this produces different acute responses to the exercise. Despite of the possible benefits of these games in training sessions, the fact is that the researches that have compared SSGs and real game format reported significant differences in external load variables related with high intensity demands (Casamichana, Castellano, \& Castagna, 2012; Clemente et al., 2019). This could seriously affect the proper stimulation of specific capacities in players, mainly considering that sprinting distances or maximal velocity are very hard to develop in small pitches. Despite the descriptive findings of the differences between SSGs and real format game, there is no evidence about the volume of time that both SSGs and real format games are occupying in the real training context. Only considering the volume will be possible to identify a potential risk of undertraining of specific capacities. Thus, the purpose of this study was to monitor the external load of professional soccer players during an 8-week training period and compare the average of time and load associated with SSGs and real format game during those weeks.

\section{MATERIAL AND METHODS}

\section{Participants}

Thirty-two professional soccer players $(25.1 \pm 2.9$ years, $181.9 \pm 6.3 \mathrm{~cm}, 73.1 \pm 6.3 \mathrm{~kg})$ who competed in the second professional Portuguese league in the 2017/2018 season were daily monitored during an 8-week training period (October-December). Only the players that participated in more than $80 \%$ of the sessions were included in the analysis. The study followed the ethical guidelines of Declaration of Helsinki.

\section{Measures}

The players daily used a 10-Hz GPS (JOHAN Sports, The Netherlands), which was properly validated and tested for his reliability. The following external load variables were collected: (i) total distance; (ii) sprinting distance (running > $20 \mathrm{~km} / \mathrm{h}-1$ ); (iii) player load (sum of instantaneous accelerations in the three axes); and (iv) maximal velocity.

\section{Procedures}

An observational study design was adopted. The duration of each task related with small-sided games (SSGs) and real format game was collected and the specific external load during those drills. Any conditioned format of the game was considered SSG and the range of these tasks varied between small (3 vs. 3) to large (9 vs. 9) sided games. The real format game was only considered when the specific format of play with any task constraints was implemented.

\section{Analysis}

The averages of external load variables during each training per type of exercise (SSGs or real format) were compared using a repeated measures ANOVA after confirmation of the normality and homogeneity of the sample. The calculus of the effects size using the Cohen $d$ was also executed. The magnitude thresholds of effect size were (Batterham \& Hopkins, 2006): 0.0-0.2, trivial; 0.2-0.6, small; 0.6-1.2, moderate; 1.2-2.0, large; $>2.0$, very large. The statistical procedures were made using the SPSS (version 24.0, IBM) for a $p<0.05$. 


\section{RESULTS}

The descriptive statistics comparing the external load values between SSGs and real format games per training session (average of the 8-week period of analysis) can be observed in the following table 1.

Table 1. Descriptive statistics of the mean per session dedicated to SSGs and RGs

\begin{tabular}{cccccc}
\hline & RFG & SSGs & $\begin{array}{c}\text { Dif. RFG- } \\
\text { SSGs }\end{array}$ & p-value & $\mathrm{d}$ \\
\hline Duration $(\mathrm{min})$ & $20.14 \pm 7.91$ & $20.82 \pm 7.83$ & -0.68 & 0.872 & 0.086 , trivial effect \\
Total distance $(\mathrm{m})$ & $1786.97 \pm 725.27$ & $1455.26 \pm 748.07$ & 331.71 & 0.355 & 0.450 , small effect \\
Sprint distance $(\mathrm{m})$ & $55.59 \pm 35.86$ & $18.47 \pm 17.10$ & 37.12 & 0.006 & 1.321 , large effect \\
Player load (g/min) & $102.03 \pm 36.68$ & $85.52 \pm 41.15$ & 16.51 & 0.388 & 0.424 , small effect \\
Maximal velocity (km/h $\left.\mathrm{h}^{-1}\right)$ & $24.92 \pm 2.26$ & $21.56 \pm 2.70$ & 3.37 & 0.010 & 1.350 , large effect \\
\hline RFG: real format game; SSGs: small-sided games; Dif: differences of the means (RFG-SSGs); d: value of the effect size and the \\
magnitude of the Cohen d.
\end{tabular}

The results comparing the both drills (RFG and SSGs) revealed no significant differences in the volume of time during the training sessions $(p=0.872 ; d=0.086)$, total distance $(p=0.355 ; d=0.450)$, and player load $(p=0.388 ; d=0.424)$. However, the sprinting distance was significantly greater in real format game $(+37.12$ $m$, average) than in SSGs $(p=0.006 ; d=1.321)$. Similarly, the maximal velocity was significantly higher in real format game $(+3.37 \mathrm{~km} / \mathrm{h}-1$, average $)$ than in SSGs $(p=0.010 ; d=1.350)$.

\section{DISCUSSION}

JRCs presented significantly lower values of sprinting sprint and maximal velocity than the real format of game during the average of all training session. Despite the fact that a greater number of high intensity repeated efforts can performed in SSGs (i.e., accelerations inferred by the player load) there are no evidence that these smaller formats provides the necessary conditions to stimulate high levels of high-intensity running (namely sprint) and maximal velocity (Clemente et al., 2019). Therefore, it seems that the argument of specificity to use the SSGs for physical development is, at the very least, rebuttable. For the specific case of sprinting or maximal velocity stimulus, it is necessary longitudinal space in which, by default, is scarce in SSGs. On the other hand, smaller spaces increase the frequency of accelerations and decelerations (Clemente et al., 2019) thus, resulting in a clear difference in the profile of physical requirement.

\section{CONCLUSIONS}

This study conducted in professional soccer players revealed that despite the time spent on SSGs and RFG being approximately the same during training sessions, there are significant differences in the result of the important external load variables (sprint distance, maximal velocity). Coaches should be aware of that to adopt compensatory or supplementary training drills to properly stimulate such variables.

\section{REFERENCES}

Batterham, A. M., \& Hopkins, W. G. (2006). Making Meaningful Inferences about Magnitudes. International Journal of Sports Physiology and Performance, 1(1), 50-57. https://doi.org/10.1123/ijspp.1.1.50 
Casamichana, D., Castellano, J., \& Castagna, C. (2012). Comparing the Physical Demands of Friendly Matches and Small-Sided Games in Semi-professional Soccer Players. Journal of Strength and Conditioning Research, 26(3), 837-843. https://doi.org/10.1519/jsc.0b013e31822a61cf

Clemente, F. M., Martins, F. M., \& Mendes, R. S. (2014). Developing Aerobic and Anaerobic Fitness Using Small-Sided Soccer Games : Methodological Proposals. Strength and Conditioning Journal, 36(3), 76-87. https://doi.org/10.1519/ssc.0000000000000063

Clemente, F. M., Sarmento, H., Rabbani, A., Van Der Linden, C. M. I. (Niels), Kargarfard, M., \& Costa, I. T. (2019). Variations of external load variables between medium- and large-sided soccer games in professional players. Research in Sports Medicine, 27(1), 50-59. https://doi.org/10.1080/15438627.2018.1511560

Sarmento, H., Clemente, F. M., Harper, L. D., Costa, I. T. da, Owen, A., \& Figueiredo, A. J. (2018). Small sided games in soccer - a systematic review. International Journal of Performance Analysis in Sport, 18(5), 693-749. https://doi.org/10.1080/24748668.2018.1517288 


\title{
Volleyball selection process: How do coaches select?
}

\author{
MARCELA DANTAS XIMENES ${ }^{1} \triangle$, ANA JOSÉ AGUIAR RODRIGUES², JEFFERSON JUREMA SILVA ${ }^{1}$, \\ JOÃO FILIPE PEREIRA NUNES PRUDENTE2 \\ ${ }^{1}$ University of the State of Amazonas, Brazil \\ 2University of Madeira, Portugal
}

\begin{abstract}
When it comes to children's sport, it is important to select these athletes in competitions, so that everyone has the opportunity to compete according to their level of development and abilities. The objective of this study was to analyse the selection process used by the coaches. The sample used was 30 volleyball coaches (21 men and 9 women), aged between 21 and 62 years (39.23 \pm 10.57$)$. In order to collect information on the selection criteria used, the study was adapted from Cardoso, (2007). The study project was approved by the scientific committee of the Department of Physical Education and Sport and ethics committee of the State University of Amazonas. The Kolmogorov-Smirnov test was used to analyse the data and the mean and standard deviation statistics were used. The Kolmogorov-Smirnov test was used and the Chi-square test for qualitative variables and Spearman correlations were used in quantitative variables. T-Student was used to determine differences between groups, the Anova of repeated measures to analyse differences in the relevance attributed by the coaches to the criteria between different levels of training. The results showed relevant data such as the predominance of observation of physical education classes in children and youngsters ( $76.7 \%$ and $63.3 \%$, respectively) and the application of tests in the levels of initiates and juveniles ( $40 \%$ and $50 \% \%$ respectively). There were no divergences between the selection methods considering the characteristics of the trainer ( $p>0.05$ ). Keywords: Selection; Competition; Coaches; Volleyball; Formation.
\end{abstract}

Corresponding author. University of the State of Amazonas, Brazil.

E-mail: mdximenes@hotmail.com

Supplementary Issue: Spring Conferences of Sports Science. International Seminar of Physical Education, Leisure and Health, 17-19 June 2019. Castelo Branco, Portugal.

JOURNAL OF HUMAN SPORT \& EXERCISE ISSN 1988-5202

(c) Faculty of Education. University of Alicante.

doi:10.14198/jhse.2019.14.Proc4.82 


\section{INTRODUCTION}

The participation of children and young people in sport is greatly influenced by the behaviour of adults, including coaches, whose intervention is decisive, especially when it comes to opportunities and participation in the competition, because it is the coaches who decide who participates when and how much you participate. The aim is to: i) Identify the criteria used by the coaches to summon the athletes to the competition; and ii) Determine the relationship between the coaches' biographical characteristics (gender, age, training and sports experience) criteria.

\section{MATERIAL AND METHODS}

\section{Participants}

Participants in the study were 30 volleyball coaches ( 21 men and 9 women), aged between 21 and 62 years $(39.23 \pm 10.57)$. Most coaches have a sporting past as an athlete $(83.3 \%, n=25)$. In relation to training, $20 \%$ $(n=6)$ had no training, 43.3\% ( $n=13)$ Volleyball coach level $1,10 \%(n=3)$ level 2 and $16.7 \%$ level 3.

\section{Measures}

To determine the relevance of the selection criteria by coaches, a questionnaire adapted from Cardoso (2007) was used. The adaptation was performed through the Delphin method by auscultation, analysis and consensual review of 3 doctoral researchers, specialized in the field of training. The questionnaire has a structure consisting of: section I (personal and biographical data) and section II (selection process), the coaches were asked to express their opinion independently for the levels of children, children, initiates and juveniles.

\section{Procedures}

The research project was approved by the scientific committee of the Department of Physical Education and Sport and by the Ethics Committee of the State University of Amazonas. The coaches were invited to participate, authorizing their participation through signing of informed consent. The questionnaire was applied by a member of the team properly trained and the filling had a duration of approximately 15 minutes.

\section{Analysis}

We used the mean descriptive statistics and standard deviation to characterize the sample in the variables under study. The Kolmogorov-Smirnov test was used to analyse the normality of distributions in continuous variables. The association between variables was determined through the Chi-square independence test for qualitative variables and Spearman correlations in quantitative variables. The T-Student test was used to determine the differences between groups in variables with normal distribution and the Anova of repeated measures to analyse the differences in the relevance attributed by the coaches to the criteria between the different training levels (children, children, initiated and juveniles ). The statistical software used was SPSS version 25.0 and the level of significance was $5 \%$.

\section{RESULTS}

The selection process of the athletes is based predominantly on the observation of physical education classes in children and children $(76.7 \%$ and $63.3 \%$, respectively) and the application of tests in the levels of initiates and juveniles (40\% and $50 \%$ respectively). There were no divergences between the selection methods reported considering the characteristics of the trainer (gender, sports past and training) $(p>0.05)$. 
From the criteria of convocation analysed, it is verified that the trainers assign in average greater importance to the attendance to the trainings and to the scholastic performance, independent of the step (table 1). Considering the same coach and the relevance that he assigns to the selection criteria in the different levels of competition, there is an appreciation of the importance of biological maturation, age, somatic measures, attendance at training and sports performance by coaches of the rank of little for juveniles $(p<0.05)$. However, it is also in these criteria that there is a greater discrepancy between the coaches' opinions (Table 1).

Table 1. Criteria for convening competitions and their importance coaches' opinion $(n=30)$

\begin{tabular}{|c|c|c|c|c|}
\hline Criteria for convening athletes & $\begin{array}{c}\text { Little } \\
(\mathrm{M} \pm \mathrm{SD})\end{array}$ & $\begin{array}{l}\text { Infantile } \\
(\mathrm{M} \pm \mathrm{SD})\end{array}$ & $\begin{array}{c}\text { Started } \\
(\mathrm{M} \pm \mathrm{SD})\end{array}$ & $\begin{array}{l}\text { Juvenile } \\
(\mathrm{M} \pm S D)\end{array}$ \\
\hline Biological Maturation (1 - 5) & $3.33 \pm 1.37$ & $3.57 \pm 1.25$ & $3.77 \pm 1.19$ & $4.07 \pm 1.36$ \\
\hline Assiduity to trainings $(1-5)$ & $4.30 \pm 0.84$ & $4.30 \pm 0.92$ & $4.53 \pm 0.97$ & $4.60 \pm 0.93$ \\
\hline ভ Sporting performance $(1-5)$ & $3.63 \pm 1.03$ & $3.93 \pm 0.89$ & $4.23 \pm 0.89$ & $4.60 \pm 0.86$ \\
\hline 䒺 Age $(1-5)$ & $3.33 \pm 1.21$ & $3.60 \pm 0.97$ & $3.97 \pm 0.96$ & $4.30 \pm 0.88$ \\
\hline 르 School Income (1 - 5) & $4.23 \pm 0.97$ & $4.22 \pm 0.95$ & $4.23 \pm 0.97$ & $4.27 \pm 0.91$ \\
\hline Somatic Measures $(1-5)$ & $3.27 \pm 1.05$ & $3.67 \pm 0.96$ & $3.90 \pm 0.96$ & $4.27 \pm 0.94$ \\
\hline Physical Education (1 - 5) & $3.97 \pm 0.89$ & $3.93 \pm 0.91$ & $3.97 \pm 0.93$ & $3.9 \pm 1.06$ \\
\hline
\end{tabular}

Characteristics such as age, sex and training of coaches are not shown to be associated with the importance attributed to the interview criteria analysed $(p>0.05)$. However, the number of years of practice as an athlete is associated with the importance attributed by coaches to biology maturation $(0.416<r<0.508 ; p<0.05)$, training attendance $(0.425<r<0.586 ; p<0.05)$, school performance $(0.532<r<0.627, p<0.05)$, somatic measures $(0.463<r<0.651, p<0.05)$ and sports performance $(0.499<r<0.508 ; p<0.05)$, as criteria for convening competitions.

\section{DISCUSSION}

From the results obtained in the present study, there was no difference with the studies of Cardoso (2012) regarding the handball coaches of Portugal, related to the selection criterion of the athletes. The assiduity factor $(0.425<r<0.586 ; p<0.05)$ was considered extremely relevant in both studies.

\section{CONCLUSIONS}

According to the objectives of the study, the Amazonian trainers consider as very important criteria such as attendance, school performance, biological maturation, sports performance, in the process of selection of athletes.

\section{REFERENCES}

Cardoso, A. R. (2012). Competition and Sport Participation of children and youth in Handball - The opinion of the coaches, the continent and the Autonomous Region of Madeira, on the long-term preparation of the young Handball players. Master's Degree in Physical Activity and Sport, University of Madeira. 
Silva Cardoso, M.F. (2007). For a theory of sport competition for children and young people: a study on the contents, structures and framework of sport competitions for the youngest in Portugal. Porto: Doctoral Dissertation submitted to the Faculty of Sport of the University of Porto.

\section{(c) $(9)\left(\Theta_{\mathrm{EY}}\right.$}

This work is licensed under a Attribution-NonCommercial-NoDerivatives 4.0 International (CC BY-NC-ND 4.0). 


\title{
The importance of attitudes and values in sport and competition: The opinion of a group of coaches of Volleyball
}

\author{
MARCELA DANTAS XIMENES ${ }^{1} \triangle$, ANA JOSÉ AGUIAR RODRIGUES², JEFFERSON JUREMA SILVA ${ }^{1}$, \\ JOÃO FILIPE PEREIRA NUNES PRUDENTE2 \\ ${ }^{1}$ University of the State of Amazonas, Brazil \\ 2University of Madeira, Portugal
}

\begin{abstract}
Behaviours of children and young people in sport are influenced by the attitudes and behaviours of adults. Behaviours related to fair play, respect for others and rules, conveyed through coaches' example are important, at a time when young people are his own identity, tending to identify with figures such as coaches and where the maxim "does what I say and not what I do" does not work. The objective of the study was to identify the importance that coaches attribute to values and attitudes in sport, and to study the association between the trainer's biographical characteristics and the relevance of these values and attitudes in sport and competition. A total of 30 volleyball coaches (21 men and 9 women) were interviewed, with a mean age of $39.23 \pm 10.57$ years. To collect information on the selection criteria, we used the survey adapted from Cardoso, (2007). The study project was approved by the scientific committee of the Department of Physical Education and Sport and ethics committee of the State University of Amazonas. For the analysis of the data, mean and standard deviation statistics were used. In the normality of the variables, the Kolmogorov-Smirnov test was used and the association between variables was the Chi-square test for qualitative variables and Spearman correlations in quantitative variables. T-Student was used to determine differences between groups in variables with normal distribution. Most of the Amazon coaches attach significant importance to all factors related to values and attitudes in sport and competition among young children. Keywords: Attitudes; Values; Formation; Coaches; Competition.
\end{abstract}

Corresponding author. University of the State of Amazonas, Brazil.

E-mail: mdximenes@hotmail.com

Supplementary Issue: Spring Conferences of Sports Science. International Seminar of Physical Education, Leisure and Health, 17-19 June 2019. Castelo Branco, Portugal.

JOURNAL OF HUMAN SPORT \& EXERCISE ISSN 1988-5202

(C) Faculty of Education. University of Alicante.

doi:10.14198/jhse.2019.14.Proc4.82

S1270 | 2019| Proc4| VOLUME 14

(C) 2019 University of Alicante 


\section{INTRODUCTION}

The behaviours of children and young people in sport are influenced by the attitudes and behaviours of adults, especially coaches. Attitudes and behaviours related to fair play, respect for others and rules, passed through the coaches' example are important, at a time when young people are building their own identity, tending to identify with such figures as coaches and where the maxim "does what I say and not what I do" does not work. In this context, we intend to identify the importance that coaches attach to values and attitudes in sport and to study the association between the coach's biographical characteristics and the relevance they attribute to values and attitudes in sport and competition.

\section{MATERIAL AND METHODS}

\section{Participants}

A total of 30 volleyball coaches (21 men and 9 women) were interviewed, with a mean age of $39.23 \pm 10.57$ years. Most coaches have a sports past as an athlete $(83.3 \%, n=25), 20 \%(n=6)$ have no specific training in the modality, $43.3 \%(n=13)$ volleyball coach level course $1,10 \%(n=3)$ level 2 and $16.7 \%(n=8)$ level 3.

\section{Measures}

In order to quantify the importance attributed by coaches to values and attitudes in sport, we used the appropriately adapted Cardoso (2007) questionnaire through the Delphin method for auscultation, analysis and consensus review of $3 \mathrm{PhD}$ researchers. The questionnaire consists of 12 statements evaluated on a Linkert scale from 1 (completely disagree) to 5 (agree completely). Coaches were also asked about biographical data (gender, age, professional and sports experience).

\section{Procedures}

The research project was approved by the scientific committee of the Department of Physical Education and Sport and by the Ethics Committee of the State University of Amazonas. The coaches were invited to participate, authorizing their participation by signing on informed consent. The questionnaire was applied by a member of the team properly trained and the filling had a duration of approximately 15 minutes.

\section{Analysis}

The mean and standard deviation descriptive statistics were used to describe the sample, and the Kolmogorov-Smirnov test to analyse the normality of the distributions of the continuous variables. The association between quantitative variables was determined through Spearman correlations. The T-Student test was used to determine the differences between groups in variables with normal distribution. The statistical software used was SPSS version 25.0 and the level of significance was $5 \%$.

\section{RESULTS}

The coaches attribute great importance to values and attitudes in sport and competition, but they are more relevant in having fun (4.63 \pm 0.81 ), integration in a group ( $4.60 \pm 0.81)$, commitment and to persistence $(4.57 \pm 0.82)$, to fairy-play and adequate behaviour $(4.53 \pm 0.86)$. Aspects such as "Overcome and win others", "Have a good public image, look good, people who like me" form those that the coaches report less relevance, however elevated from $3.77 \pm 1.04$ and $3.57 \pm 1.33$ respectively. There were no differences between male and female coaches regarding their relevance to values and attitudes ( $p>0.05)$, nor association between the age of coaches and their relevance to values and attitudes $(p>0,05)$. Untrained or 
lower specific trainers (level 1 trainers course), value more overcoming and winning others than coaches with higher specific training (level 2 or 3 coaching course), in the remaining non-training there were differences with statistical significance. Of the 12 statements of the questionnaire only in the statements "Show sportsmanship, have appropriate behaviour, do not be a bad loser" and "Have a good public image, look good, people who like me", there was no statistically significant difference between coaches with $(P<.05)$. In the other groups, coaches with a sports background attribute greater importance to attitudes and values compared to colleagues with no sports past $(p<0.05)$.

\section{DISCUSSION}

In the current study, most of the Amazonian coaches interviewed attribute great importance to values and attitudes in sport and competition. However, the main relevance is to have fun (4.63 \pm 0.81$)$, integration into a group $(4,60 \pm 0.81)$, commitment and persistence $(4.57 \pm 0.82)$ and fair play and adequate behaviour (4.53 \pm 0.86$)$. In agreement with the studies of Cardoso (2012), the coaches of the two groups (national and Autonomous Region of Madeira) attribute significant importance to the values and attitudes of the competition, presenting concern on factors such as: performance motivation, self-esteem development and self-concept, creating social capital, making friends and socializing with peers and creating values. The Amazon coaches, as well as the coaches of the Autonomous Region of Madeira (RAM), are less relevant in aspects such as "Overcome and win over others", "Have a good public image, look good, people who like me." There were no differences between the male and female coaches regarding the importance they attribute to values and attitudes ( $p>0.05$ ), nor the association between the age of the coaches and their relevance to values and attitudes $(p>0.05)$.

\section{CONCLUSIONS}

With regard to the results, we can conclude from the research that most of the Amazon coaches attribute significant importance to all factors related to values and attitudes in sport and competition among children and young people: the greatest relevance is in having fun $(4,63 \pm 0.81)$, integration into a group (4.60 \pm $0.81)$, commitment and persistence $(4.57 \pm 0.82)$ and fair play and adequate behaviour $(4.53 \pm 0.86)$. With regard to training, Amazonian level 1 coaches value more to overcome and win than coaches level 2 or 3 .

\section{REFERENCES}

Cardoso, A. R. (2012). Competition and Sport Participation of children and youth in Handball - The opinion of the coaches, the continent and the Autonomous Region of Madeira, on the long-term preparation of the young Handball players. Master's Degree in Physical Activity and Sport, University of Madeira.

Silva Cardoso, M.F. (2007). For a theory of sport competition for children and young people: a study on the contents, structures and framework of sport competitions for the youngest in Portugal. Porto: Doctoral Dissertation submitted to the Faculty of Sport of the University of Porto.

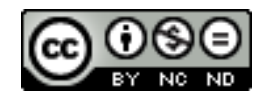

This work is licensed under a Attribution-NonCommercial-NoDerivatives 4.0 International (CC BY-NC-ND 4.0). 


\title{
Short-term effects of myofascial release on isometric knee extensors strength
}

\author{
PEDRO CARVALHO ${ }^{1}$, MIGUEL MORAIS ${ }^{1}$, ALBERT DOS SANTOS ${ }^{2}$, CAROLINA VILA-CHÃ ${ }^{1,3}$ \\ ${ }^{1}$ Polytechnic Institute of Guarda, Portugal \\ 2University Center Augusto Motta, Brazil \\ ${ }^{3}$ Research Center in Sports Sciences, Health Sciences and Human Development, CIDESD, Portugal
}

\begin{abstract}
Myofascial release (MFR) has been commonly applied by physiotherapists to enhance tissue recovery and to increase myofascial mobility. More recently, and the fitness and sports area myofascial release has been seen as pre-exercise technique restore movement patterns and to enhance motor performance. Nonetheless the results on short-term effects of MFR in muscle strength are controversial. Therefore the aim of this study was to investigate the acute effects of MFR in the maximal isometric strength of the knee extensors. Fourteen healthy men $(22.1 \pm 2.8$ years; mean $\pm S D$ ) were randomly assigned to control or to MFR protocol. Maximum voluntary isometric contraction (MVC) of the knee extensors were measured pre and post experimental protocol (either control or MFR protocol). MFR protocol consisted in a single bout of 90 s of slow stick rolling in the quadriceps muscle of the dominant leg, administered by a researcher, while control protocol consisted in a rest period with a time duration equivalent to MFR protocol duration. The results showed a significant decreased of the knee extensors MVC following MFR protocol (-10.14 $\pm 4.35 \% ; P<0.001)$, while no changes were observed in the control group. This suggests that the present MFR protocol causes transient decrease in strength, which might not be suitable for a plication prior to a workout with significant force demands. Keywords: Myofascial release; Muscle strength; Knee extensors.
\end{abstract}

Corresponding author. Instituto Politécnico da Guarda. Av. Francisco Sá Carneiro, n. 50; 6300- Guarda, Portugal.

E-mail: cvilacha@ipg.pt

Supplementary Issue: Spring Conferences of Sports Science. International Seminar of Physical Education, Leisure and Health, 17-19 June 2019. Castelo Branco, Portugal.

JOURNAL OF HUMAN SPORT \& EXERCISE ISSN 1988-5202

(c) Faculty of Education. University of Alicante.

doi:10.14198/jhse.2019.14.Proc4.82 


\section{INTRODUCTION}

Myofascial release (MFR) has been described as a set of manual therapy techniques through which mechanical pressure is applied to a muscle and fascia, aiming to reduce restrictive barriers or fibrous adhesions seen between layers of fascial tissue (McKnney et al. 2013). The scientific literature is reporting a variety of acute effects induced by MFR, including increased flexibility, range of motion, arterial and vascular endothelial function (Davies \& Davies, 2013). However, the effects on muscle strength are inconsistent (Arroyo-Morales et al., 2008; Peacock et al., 2014) following MFR. Therefore the aim of this study was to investigate the acute effects of MFR in the maximal isometric strength of the knee extensors.

\section{MATERIAL AND METHODS}

\section{Participants}

Fourteen healthy young college-aged men $(22.1 \pm 2.8$ years, $177.8 \pm 6.7 \mathrm{~cm}$ of height, and $78.8 \pm 9.6 \mathrm{~kg}$ of body mass), volunteered for this study. Subjects were all university students and recreationally active (regular physical activity at least 3-4 times per week). Before participation, an explanation of the study was given, and the subjects were asked to read and sign an informed consent. The participants were then randomly assigned to control or experimental group (MFR protocol).

\section{Measures}

To test the effects of MRF, the maximum isometric knee extensors strength of the subjects were measured pre and post intervention (MRF or control) in an isokinetic dynamometer (Humac Norml CSMi, USA).

\section{Procedures}

Force measurements and experimental protocols were performed in separated lab rooms. In the room 1, each subject performed a light warm up of 5 minutes (cycle ergometer at $50 \mathrm{rpm}$ with a light load). Hereafter, the subject was seated on the isokinetic dynamometer, with the knee of the dominant leg flexed at $90^{\circ}$. The subject was asked to perform 2 MVC of the knee extensors, over a period of $5 \mathrm{~s}$, with a 2 min of rest in between. The highest MVC value was considered. Then the subject moved to the room 2 and, after 2 minutes, was submitted to: a) control protocol or b) MFR protocol. The control protocol consisted in a rest interval equivalent to MRF protocol time duration (3 minutes). The subjects submitted to MFR were firstly familiarized with the target mechanical pressure by applying the stick in the quadriceps of the non-dominant leg. The subject was asked to classify the pain in a VAS-10 scale. The mechanical pressure that produced a pain of 5-6 in the VAS scale was then applied in the quadriceps of dominant leg by the researcher with a stick Aptonia, for 90 seconds. The, at room 1 and, after 2 min, MVC of the knee extensors were measured as previously described.

\section{Analysis}

A two-way ANOVA with repeated measures (time) was performed on all dependent variables recorded in the pre- and post-conditions test (SPSS). The two factors (2x2) included condition (control and MFR protocol) and time (pre- and post - conditions). Pairwise comparisons were performed with the Student-Newman-Keuls post hoc test when ANOVA was significant. The significance level was set to $P<0.05$. 


\section{RESULTS}

Figure 1 shows the results on MVC knee extensors strength, pre and post both protocols (control and MFR). An interaction condition $x$ time was observed $(P<0.001)$. After MFR protocol the MVC decreased significantly (10.14 $\pm 4.35 \%$; $P<0.001$; Fig. 1), while no changes were observed in the control group.

\section{(A)}

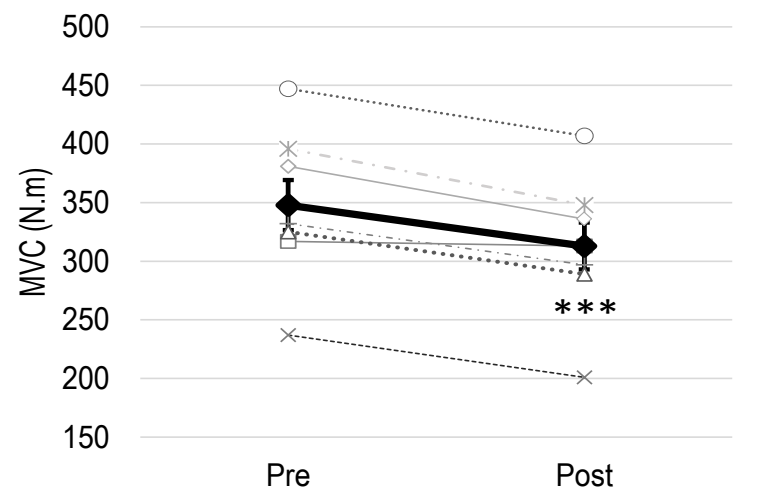

(B)

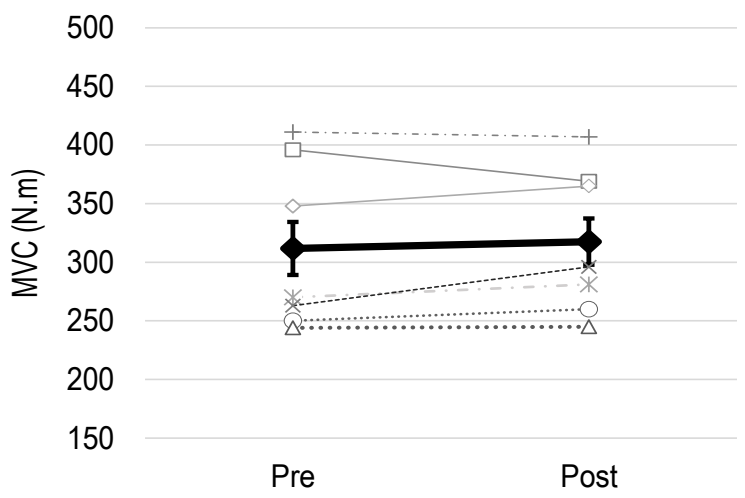

Each line represents 1 subject; thick black line represents the mean of the group. ${ }^{* * *} P<0.001$.

Figure 1. Maximal isometric torque of the knee extensors (MVC, pre and post myofascial release protocol (A) and control protocol (B).

\section{DISCUSSION}

This study investigated the effect of a single bout of 90s of MFR on the MVC of the quadriceps. The results showed that this protocol negatively affected the isometric strength of the muscles submitted to this protocol. The number of studies on MFR are scarce. Wikktorson-Moller et al (1983), found that massage of 2 minutes decreased the knee extensors isometric strength. Arroyo-Morales et al. (2008), found a decrease in the EMG activity of the vastus medialis following a massage in the quadriceps, which might result in a transient loss of muscle strength, although this was not tested. Studies on self-myofascial release have reported no changes (Healey et al., 2014) or improvement in the muscle strength (Peacock et al., 2014). The controversial results might be explained by the specific characteristics of the MFR protocols.

\section{CONCLUSIONS}

In conclusion, the data from this study indicated that an acute bout of 90s of slow stick rolling on the quadriceps significantly reduces knee extensors isometric strength. Although the results were driven form static conditions, they suggest that MFR prior to a workout that requires substantial force production are not desirable.

\section{REFERENCES}

Arroyo-Morales, M., Olea, N., Martinez, M., Hidalgo-Lozano, A., Ruiz-Rodriguez, C., \& Diaz-Rodriguez, L. (2008). Psychophysiological effects of massage-myofascial release after exercise: a randomized sham-control study. J Altern Completment, 14, 1223-1229. https://doi.org/10.1089/acm.2008.0253 
Davies, C., \& Davies, A. (2013). The trigger point therapy workbook: your self-treatment guide for pain relief. New Harbinger Publications.

Healey, C., Hatfield, L., Blanpied, P., Dorfman. R., \& Riebe, D. (2014). The effects of myofascial release with foam rolling on performance. J Strength Cond Res. 28. 61-68. https://doi.org/10.1097/01.jsc.0000395625.08079.28

Peacock, C., Krein, D., Silver, T., Sanders, G., \& Carlowitz, K. (2014). An Acute Bout of Self-Myofascial Release in the Form of Foam Rolling Improves Performance Testing. Int J Exerc Sci, 7, 202-211.

Wiktorsson-Moller, M., Oberg, B., Ekstrand, J., \& Gillquist, J. (1983). Effects of warming up, massage, and stretching on range of motion and muscle strength in the lower extremity. Am J Sports Med, 11, 249-252. https://doi.org/10.1177/036354658301100412

\section{(c) $(9)(\mathrm{F}$}

This work is licensed under a Attribution-NonCommercial-NoDerivatives 4.0 International (CC BY-NC-ND 4.0). 


\title{
Digital media in professional basketball: The case of supporters of the Hamburg Towers
}

\author{
ALEXANDER HODECK ${ }^{1}$, MARCIO JOSE KERKOSKI² \\ ${ }^{1}$ EBC Hamburg \& Chemnitz University of Technology, Germany \\ 2Universidade Tecnologica Federal do Parana, Brazil
}

\begin{abstract}
Digitalization is one of the global trends. It is relevant to sports and especially to professional sport clubs. As expectations and behaviour of supporters transform, clubs are forced to change their communication strategy to satisfy their customers. This study analyses the media usage and the expectations of supporters of a German professional basketball club. Younger people than in other sports are attracted by basketball, therefore it can be seen as interesting target group. Data $(n=285)$ was collected online and with paper and pencil by using a standardized questionnaire in the season 2018/19. SPSS was used for statistical analysis. Although basketball fans are open minded for new media and use social media more often than others, they still expect traditional communication instruments (as the printed match day programme) from their preferred club. As a consequence, professional sport clubs have to use both traditional and new instruments within their communication strategy. Keywords: Digital media; Basketball; Media usage; Supporters.
\end{abstract}

Corresponding author. EBC Hochschule Hamburg, Esplanade 6, 20354 Hamburg, Germany.

E-mail: hodeck.alexander@ebc-hochschule.de

Supplementary Issue: Spring Conferences of Sports Science. International Seminar of Physical Education, Leisure and Health, 17-19 June 2019. Castelo Branco, Portugal.

JOURNAL OF HUMAN SPORT \& EXERCISE ISSN 1988-5202

(C) Faculty of Education. University of Alicante.

doi:10.14198/jhse.2019.14.Proc4.82 


\section{INTRODUCTION}

Digitalization is a global megatrend influencing sports as well as other social areas. Using social media or other internet based communications tools are used to fulfil marketing communication goals (Eaglemen, 2013) has been increased during the last years. Co-creation processes in sports, especially in team sports, offer great potentials (e.g. Uhrich, 2014) regarding satisfaction and loyalty. Social media can help developing co-creation by driving digital participation (Hutchins, 2016). This paper investigates the expectations of supporters of a professional German basketball club (Hamburg Towers) in order to understand whether it is possible in professional sports nowadays to communicate only digitally to supporters or not.

\section{MATERIAL AND METHODS}

\section{Participants}

285 supporters of the Hamburg Towers (second league of German professional basketball) were 31.2 years old and 70.5 percent of them were male. Most of them visit games of the team together with friends (42.8 percent), their family (21.1. per cent) or partners (18.6 per cent). The sample is highly involved in basketball as the average of attended games per season is 8.4 per person.

\section{Measures}

A standardized questionnaire, based on previous studies among sport fans (PWC, 2016) and validated by a pre-test done with 15 undergraduate students from several disciplines, was used in this study. It consisted of four parts: involvement of the participants with Basketball and the Hamburg Towers, socio-demographic aspects, usage of social-media as well as smartphones and finally the importance of different information sources about the club.

\section{Procedures}

Participants were asked before and during three home games at the beginning of the regular season 2018/19 by trained undergraduate students of sports management. In addition, the questionnaire could be filled 0 during 24.11.18 and 01.12.18 online via the platform soscisurvey.

\section{Analysis}

Data were analysed by using SPSS and Excel descriptively. In addition, mean values were compared on a level of significance of 5 percent.

\section{RESULTS}

Basketball supporters use mobile devices and smart phones more than others. 96.5 percent of the sample own at least one smart phone and use it daily. Getting information about sports in general on a digital basis is quite common among them. Almost three of four of the basketball fans use sport specific apps for that - 40 percent even daily. 70 percent gain information about their favourite basketball team via apps. On the other side the fans of the Hamburg Towers are interested in traditional media like the match day programme. Reading the printed match day programme is very probable among the basketball fans (3.22; with 1: not likely at all and 4: very likely). A quite big number just uses this printed programme once before and during the game, but some (14 per cent) spectators even collect the programme at home. Asked for future communication instruments of the club, a mixture of traditional and modern media was rated most positively. A third of the population is even willing to pay money for a traditional match day programme (mean value of 2.00 Euro). Finally, it can be stated that supporters of the towers generally without regard to preferred media 
are most interested in current information about the club and the team itself (3.42; with 1: not important and 4: very important) followed by information about the league (3.36). Less interest is shown in youth teams (2.48), tickets (2.24) and sponsors (1.81).

\section{DISCUSSION}

Supporters of German professional basketball clubs are younger than those in other sports as football and much more involved in modern media. Only 32 per cent of German football fans use club apps or sport apps (24 per cent) in general (PWC, 2016). So development among this group of sport supporters can be a raw model for other sports. Regarding this, it is very important to take in consideration that despite the high involvement of basketball fans in modern media a traditional instrument of communication of a professional sport club as the printed match day programme is still important to the fans. A carefully adjusted mixture of traditional and modern instruments should be used within the communication strategy of a sport club (see also Hutchins, 2016). Basketball fans are even willing to pay for information, but only if the information fit their interests. Basketball fans are focussed on information about their favourite sport or club. Information about sponsors is less important.

\section{CONCLUSIONS}

Basketball fans are young and digital, but they are still interested in traditional sport communications instruments, if they meet their interests. At the moment, communication instruments of professional sport clubs are often financed by sponsors. That can lead to difficulties regarding the relationship of supporters and clubs, if clubs do not considerate this issue. Because of the limitations of this study, results can only be seen as representative for fans of the Hamburg Towers. Therefore, generalizations should be done carefully. But interesting outcomes regarding the media usage of the sample can be taken as first hint to sport supporters in general. In order to verify the results of this study further research among supporters of all professional German basketball clubs and also among other kind of sports should be done in future.

\section{REFERENCES}

Eagleman, A.N. (2013). Acceptance, motivations, and usage of social media as a marketing communications tool amongst employees of sport national governing bodies. Sport Management Review, 16, 488-497. https://doi.org/10.1016/j.smr.2013.03.004

Hutchins, B. (2016). Tales of the digital sublime: Tracing the relationship between big data and professional sport. Convergence, 22 (5), 494-509. https://doi.org/10.1177/1354856515587163

PWC (2016). Fußballfan-Befragung 2016 [Footballfan survey 2016]. Frankfurt: PwC.

Uhrich, S. (2014). Exploring customer-to-customer value co-creation platforms and practices in team sports. European Sport Management Quarterly, $14 \quad$ (1), 25-49. https://doi.org/10.1080/16184742.2013.865248

\section{(9) $\odot \Theta \Theta$}

This work is licensed under a Attribution-NonCommercial-NoDerivatives 4.0 International (CC BY-NC-ND 4.0). 


\title{
Planning and assessment by teachers of physical education classes in elementary school
}

\author{
DANIELLA MEDEIROS ${ }^{1,2} \triangleleft$, THIAGO ROGEL ${ }^{3}$, CATARINA MUNHOZ², BEATRIZ MUNHOZ², BRUNA \\ MERIGHI ${ }^{2}$ \\ ${ }^{1}$ University of São Paulo, Brazil \\ ${ }^{2}$ Santa Cecilia University, Brazil \\ 3University of Minho, Portugal
}

\begin{abstract}
Objective: to identify types of planning and assessment methods employed by teachers in Physical Education (PE) classes. Methods: transversal study involving the participation of $32 \mathrm{PE}$ teachers from elementary schools of the public school system, with at least 5 years of experience, answered a questionnaire with twelve opened questions about planning, assessment and experience practice, adapted from Luguetti (2010). The responses were verified through the method of content analysis and categorized to present the data considering the frequency of them. Results: teachers are concerned about the importance of planning and assessment in PE classes to improve cognitive, motor and social development aspects in their students. However, the majority of teachers' planning are based on the social aspect. Moreover the most used assessment method is observational without register. Conclusion: most of the participating teachers do not apply evaluations with registration and only consider the observation as students evolution during classes, which makes it difficult to follow the learning process and didactic-pedagogical planning. Keywords: Planning; Assessment; Physical education; Teachers.
\end{abstract}

Corresponding author. Santa Cecilia University. Rua Osvaldo Cruz, 277. 11045-907. Santos/SP, Brazil.

E-mail: danimedeiros@usp.br

Supplementary Issue: Spring Conferences of Sports Science. International Seminar of Physical Education, Leisure and Health, 17-19 June 2019. Castelo Branco, Portugal.

JOURNAL OF HUMAN SPORT \& EXERCISE ISSN 1988-5202

(c) Faculty of Education. University of Alicante.

doi:10.14198/jhse.2019.14.Proc4.82 


\section{INTRODUCTION}

There are many contents that can contemplate a PE class. However, any activity need to enhance cognitive, affective, social and motor skills (Betti and Zuliani, 2002). Planning and assessment are important to keep up with the students' learning process. It involves data collect to verify the necessity to maintain or change the practice that are using for the teacher in the classes (Pereira and Moreira, 2001). Because of this, assessment is considered a strategy to control the planning quality. Assessment can be formative, with a regulation of the control of the teaching learning process. Besides, it can be continuous, with feedbacks that improve the relation between teachers and students (Carvalho, 1994). Teachers can choose different kinds to track learning process and this is very important for the classes to become better. Drawing, written pedagogical activities and physical education diaries can be an evaluation instrument (Santos et al, 2015). Thus, the aim of this study was to identify types of planning and assessment methods that teachers employ in PE classes.

\section{MATERIAL AND METHODS}

\section{Participants}

Thirty-two PE teachers of elementary public schools in Santos city - Brazil, with at least five years of experience, and that were participating in a training about activities to improve classes.

\section{Measures}

This transversal study used a questionnaire with twelve opened questions about planning, assessment and experience practice, adapted of Luguetti (2010).

\section{Procedures}

After teachers' consent to participate in the research through a consent form. The questionnaires were given in the beginning of the training and returning at the end. Then the answers were categorized for data organization.

\section{Analysis}

The questionnaire answers went through a content analysis, where common elements were categorized in planning, objectives of classes, content and assessment. So the data were tabulated from the frequency distribution and displayed as a percentage in each category.

\section{RESULTS}

The results show that all teachers elaborate planning, which $31.40 \%$ make it daily. In the classes objectives, $25.3 \%$ of the teachers emphasize the social aspect and $2.5 \%$ or less the leisure (fun). Regarding activities developed in classes, the games are the most used (33\%). Most of the teachers make qualitative quarterly assessment through observation in developed activities without registration (26\%).

\section{DISCUSSION}

The observational method is widely used in PE classes. The study by Carvalho (1994) found that most teachers make use of observational assessment with registration and students self-assessment, which can be determinant for planning and consequently teaching learning process. The Brazilian curriculum (MEC, 1997) emphasizes the importance of valuing cultural productions in PE classes, but without restricting 
activities that foster students' motor development and the conceptual and procedural learning of different sports. The present study showed that teachers do not seek to plan the classes concerned with maintaining a certain balance between the contents and objectives of the classes.

\section{CONCLUSIONS}

In the present study there is an opposition in relation to the planning and its application in the classes, since most of the teachers perform an assessment through observation without records, making it difficult to follow the evolution of teaching learning process.

\section{REFERENCES}

Betti, M. \& Zuliani, R. L. (2002). Educação Física Escolar: uma proposta de diretrizes pedagógicas. Revista Mackenzie de Educação Física e Esporte, 1(1), 73-81. https://doi.org/10.1590/s180755092011000500011

Carvalho, L. (1994). Avaliação das aprendizagens em Educação Física. Boletim da Sociedade Portuguesa de Educação Física, 10(11), 135-151.

Luguetti, C. N. (2010). Práticas esportivas escolares no ensino fundamental. Município de santos-SP. Dissertação de Mestrado em educação física. Universidade de São Paulo. USP-SP. https://doi.org/10.11606/d.39.2010.tde-18082010-172722

Ministério da Educação. (MEC, 1997). Parâmetros curriculares nacionais: Educação física. Secretaria de Educação Fundamental. - Brasília.

Pereira, R. S. \& Moreira, E. C. (2011). Educação Física Escolar: desafios e propostas. Várzea Paulista: Fontoura.

Santos, W., Mathias, B., Matos, J. \& Vieira, A. (2015). Avaliação na educação física escolar: reconhecimento a especificidade de um componente curricular. Movimento, 21(1), 205-2018. https://doi.org/10.22456/1982-8918.46895

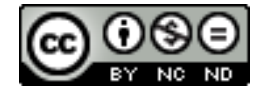

This work is licensed under a Attribution-NonCommercial-NoDerivatives 4.0 International (CC BY-NC-ND 4.0). 


\title{
Pedagogical model for teaching combat sports in physical education class: Presentation of partial results
}

\author{
DANIELLA MEDEIROS $1,2 \triangle$, THIAGO ROGEL ${ }^{3}$, LUIZ DANTAS $^{1}$ \\ 1 University of São Paulo, Brazil \\ ${ }^{2}$ Santa Cecilia University, Brazil \\ 3University of Minho, Portugal
}

\begin{abstract}
Objective: to present preliminary results of a qualitative study carried out by approaching to combat sports in Physical Education (PE) class and the necessity to develop a pedagogical model to such practice. Method: a select group of Brazilian experienced teachers in combat sports and physical education gathered together in ten meetings to discuss concepts about combat sports issues. All meetings sessions were voice recorded and transcribed, in order to be analysed leaving room for future content debate with "critical friend" from outside the group, aiming at organizing ideas and concepts with researchers, so, future plans could be outlined in the upcoming meeting sessions. Results: the group of physical educators dealt with arduous conceit when adapting combat sport to the reality of PE classes, despite of being qualified and experienced with the subject. They expressed preoccupation regarding infrastructure, fit, aggressive behaviour and limited learning skills which unfavourably impacts their teaching. Conclusion: The approach to combat sport in PE classes is accompanied by various difficulties, even for teachers with wide expertise and vast teaching experience. A construction of a pedagogical model is a broad and bold type pathway that could provide educators with combat sport and enhance their professional abilities in PE classes, hence it has crucial elements as central idea, critical elements and learning outcomes. Keywords: Pedagogical model; Combat sports; Physical education class.
\end{abstract}

Corresponding author. Santa Cecilia University. Rua Osvaldo Cruz, 277. 11045-907. Santos/SP, Brazil.

E-mail: danimedeiros@usp.br

Supplementary Issue: Spring Conferences of Sports Science. International Seminar of Physical Education, Leisure and Health, 17-19 June 2019. Castelo Branco, Portugal.

JOURNAL OF HUMAN SPORT \& EXERCISE ISSN 1988-5202

(c) Faculty of Education. University of Alicante.

doi:10.14198/jhse.2019.14.Proc4.82 


\section{INTRODUCTION}

PE classes can improve body practices based on aspects of content of results from the cultural movement approach. Considering the social aspect, the Brazilian national educational program remains steadily neglecting combat sport as a substantial content for PE classes (MEC, 2017). Sports can be a cultural asset to benefit wide range of physical, affective, cognitive and social learning outcomes (Banks and Reed, 2003). This content can be invigorating regarding the increasing value of confidence and self-esteem that can promote aggressive behaviours control (Olivier, 2000). In addition, combat sports require a wide range of motor skills movements (Vergara Marin, 2001). Unfortunately, PE teachers avoid teaching combat sports in their classes, due to the fact that many of them do not feel comfortable with the content and the poor literature about how to improve combat sports in classes (Correia and Francini, 2010). Some teachers believe that it is necessary to have an appropriate space that suits the improvement of the activities. Furthermore, they are concerned about the possible improvement towards aggression (Olivier, 2000). There are some possibilities to adequate combat sports in PE classes, as self-defence (Banks and Reed, 2003), opposition games and combat sports classification involving the distance between students during the combat activities (short, medium, and long distance) (Avelar and Figueiredo, 2009). Thus, it is necessary to improve combat sports controversy. A pedagogical model is co-constructed to achieve particular learning, and can provide teachers with alternatives to improve pedagogical knowledge about combat sport in their classes (Kirk et al, 2018).

\section{MATERIAL AND METHODS}

\section{Participants}

This research was conducted in Santos, Sao Paulo, Brazil, by a group of six physical educators specialized in combat sports, such as Taekwondo, Capoeira, Karate, Muay-Thai and Judo. This content counts also on "critical friend" and bears point of view from an outsider, who is specialized in Kendo.

\section{Measures}

The group of educators held together ten formal meeting sessions for one hour in alternate weeks. A conceptual map had been designed during the first and the last formal meeting session, besides, all approached topic were voice recorded to be used in further debate with "critical friend" in the future sessions.

\section{Procedures}

Firstly, the project was approved by the ethics committee at the University of Sao Paulo. Then, the group designed a conceptual map about individual previous knowledge regarding combat sports in PE class. Finally, the meetings involved deliberation of contents to understand and work on difficulties by the educators.

\section{Analysis}

The designed conceptual maps, audio content and their transcriptions had been analysed by researcher's members and "critical friend" considering the classification of combat sport and the components of a pedagogical model, such as key theme, critical elements and learning outcomes.

\section{RESULTS}

The participants of the research demonstrated that even specialized educators may have difficulties applying combat sports in their PE classes. Furthermore, they have presented limited knowledge regarding the importance of combat sports and its possible improvement in PE classes. Consequently, the participants would compose a group to provide support by designing an educational approach to combat sport in PE, 
however they demonstrate exceedingly limited potential for the reason that they don't understand the elements that form a pedagogical model.

\section{DISCUSSION}

The misunderstanding that educators face regarding combat sports and the elements involved in classes are present in the literature of the same content (Olivier, 2000; Correia e Franchini, 2010). A pedagogical model contributes helping educators to enhance their classes, hence this is a didactic depiction which conducts, clarifies, and details procedures to be followed, in order to reach an objective. (Kirk et al., 2018).

\section{CONCLUSIONS}

Considering all benefits of applying combat sports, the difficulties presented by the group and the disconcerted literature published, a pedagogical model is an alternative to develop and improve PE classes.

\section{REFERENCES}

Avelar, B., \& Figueiredo, A. (2009). La iniciación a los deportes de combate: interpretación de la estructura del fenómeno lúdico luctatorio. Revista de Artes Marciales Asiáticas, 4(3), 44-57. https://doi.org/10.18002/rama.v4i3.177

Banks, A., \& Reed, J. (2003). Applying mass media to self-defense instruction in Physical Education. Joperd, 74(2). 41-52.

Correia, W., \& Franchini, E. (2010). Produção acadêmica em lutas, artes marciais e esportes de combate. Revista Motriz, 16(1), 01-09. https://doi.org/10.5016/1980-6574.2010v16n1p01

Kirk, D., Lamb, C., Oliver, K., Ewing-Day, R., Fleming, C., Loch, A. \& Smedley, V. (2018). Balancing prescription with teacher and pupil agency: spaces for manoeuvre within a pedagogical model for working with adolescent girls. The curriculum journal. https://doi.org/10.1080/09585176.2018.1449424

Ministério da Educação. (MEC, 2017). Base nacional comum curricular. Retrieved from: http://basenacionalcomum.mec.gov.br/abase/

Olivier, J. (2000). Das brigas aos jogos com regras: enfrentando a indisciplina na escola. Porto Alegre, Brasil: Artmed. https://doi.org/10.5007/2175-8042.2008n31p251

Vergara Marin, E. (2001). Las atividades luctatorias y la formación en valores. Revista Educación Fisica y Deporte, . 21(2), 99-104.

\section{@ $\odot \Theta \Theta$}

This work is licensed under a Attribution-NonCommercial-NoDerivatives 4.0 International (CC BY-NC-ND 4.0). 


\title{
Intra-week variations and associations between internal and external load measures in a elite volleyball team
}

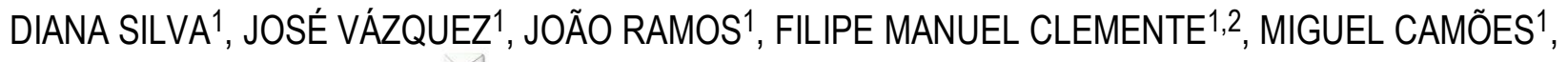
RICARDO FRANCO LIMA ${ }^{1,3}$

1 Instituto Politécnico de Viana do Castelo, Escola Superior de Desporto e Lazer de Melgaço, Portugal

2Instituto de Telecomunicações, Departamento da Covilhã, Portugal

${ }^{3}$ Research Center of in Sports Sciences, Health Sciences and Human Development (CIDESD), Portugal

\begin{abstract}
The purpose of the present study was: (i) to analyse the variation of the internal and external load during the training days; and (ii) to analyse the levels of association between internal and external load in professional volleyball players. Eight high-level volleyball athletes participated in the study. Players used an inertial measurement unit to quantify the number and the height of the jumps made during the session. The Borg CR-10 and the session-RPE were used as internal load measures. The results indicated statistically significant differences between training days in terms of training duration, number of jumps and RPE. There were no statistically significant differences in s-RPE. There were small magnitudes correlations between internal and external loads. The present study demonstrates that the training load is variable throughout the week, with the most voluminous and intense training occurring on the second and third days before the match. Keywords: Monitoring; Athletes; Volleyball.
\end{abstract}

Corresponding author. Instituto Politécnico de Viana do Castelo, Escola Superior de Desporto e Lazer de Melgaço, Portugal. E-mail: ricardo.lima@esdl.ipvc.pt

Supplementary Issue: Spring Conferences of Sports Science. International Seminar of Physical Education, Leisure and Health, 17-19 June 2019. Castelo Branco, Portugal.

JOURNAL OF HUMAN SPORT \& EXERCISE ISSN 1988-5202

(c) Faculty of Education. University of Alicante.

doi:10.14198/jhse.2019.14.Proc4.82 


\section{INTRODUCTION}

Training load monitoring has been seen by many as a key factor in assessing whether the training program is appropriate for athletes (Halson, 2014). Thus, there are two types of load: external and internal. The external load characterizes the physical impact of the training stimulus on the players. On the other hand, the internal load quantifies the impact of physical stimulus on the biological responses (Bourdon et al., 2017). The subjective perception of effort (RPE) is one of the most common methods to control the internal workload, since it allows to obtain valid information about the impact of the training stimulus on the players (Clemente et al., 2018). These internal differences can help determine if an athlete is fit for the type of training or fatigued (Bourdon et al., 2017). Considering the fewer studies analysing the weekly load profile of elite volleyball teams and the associations levels between internal and external load measures in this sport, the purpose of this study was twofold: (i) analyse the intra-week variations of internal and external load; and (ii) test the associations between internal and external load.

\section{MATERIAL AND METHODS}

\section{Participants}

Eight male elite volleyball athletes (age: 23.05 .22 yo; body mass: $84.57 .58 \mathrm{~kg}$; height: $193.09 .71 \mathrm{~cm}$; BMl: $22.00 .02 \mathrm{~kg} / \mathrm{m} 2$ ). The study procedures complied with international standards for human experiments (Declaration of Helsinki, 1975).

\section{Measures}

To determine the number of jumps and their height an inertial measurement device with three gyroscopes and three accelerometers (VERT Classic, Florida, USA) was used. The CR-10, from Borg - subjective effort scale was used to quantify the perceived load of the athletes during the training sessions evaluated ( 1 to 10 , where 1 would be considered the very light activity and 10 of a maximum effort). The RPE (s-RPE) session was calculated from the multiplication of CR-10 score by the training time (min).

\section{Procedures}

To measure vertical jumps, during the 1st phase of the senior national championship (October 2018 to March 2019 - monitoring 42 training sessions), athletes used the "VERT" inertial sensor that was placed on a belt that athletes held in the hip area from the start of the training to the end and it was possible to monitor all athletes in real time. Concerning the recording of the RPE, this was done immediately after the end of the training session.

\section{Data analysis}

The one-way ANOVA tested the variations of external and internal load between training sessions, after confirmation of the normality and homogeneity assumptions. The Tukey HSD post hoc test and the Cohen d (d) to test the effect size were also performed to perform the pairwise comparisons. The associations between external and internal loads were executed with Pearson $r$ test. The statistical procedures were executed in the SPSS (version 24.0, IBM) for a $p<0.05$.

\section{RESULTS}

The one-way ANOVA revealed statistically significant differences between training days in terms of training duration ( $p=0.001)$, number of jumps $(p=0.004)$, RPE $(p=0.025)$. Comparisons between pairs (Tukey HSD) showed that the most prolonged training occurred on day $-2(95.21 \pm 37.54 \mathrm{~min})$, differing statistically 
from days -1 ( $p=0.007 ; d=0.570$, small effect); $-3(p=0.001, d=0.801$, moderate effect) and $-4(p=0.014$, $d=0.563$, small effect). Regarding the number of jumps, it was observed that the largest amount occurred on day $-2(143.10 \pm 60.11 \mathrm{n})$, differing statistically from day $-1(p=0.003 ; d=0.695$, moderate effect). Finally, day $-3(7.07 \pm 2.0$ U.A.) presented the highest RPE intensity values, differing statistically from day $-1(p=$ $0.041, d=0.561$, small effect). On the other hand, the lower intensity of RPE was recorded on day -1 (6.02 \pm 1.79 U.A.) presenting significantly lower values of day $-2(p=0.031 ; d=0.598$, small effect).

Table 1. Descriptive statistics $(M \pm S D)$ of internal and external load values between days of the week

\begin{tabular}{ccccc}
\hline & RPE (U.A.) & Duration (min.) & s-RPE (U.A.) & Number of jumps (n) \\
\hline Day -1 & $6.02 \pm 1.79$ & $75.70 \pm 29.94$ & $462.04 \pm 330.05$ & $106.40 \pm 42.77$ \\
Day -2 & $7.06 \pm 1.71$ & $95.21 \pm 37.54$ & $586.68 \pm 365.66$ & $143.10 \pm 60.11$ \\
Day -3 & $7.07 \pm 1.99$ & $69.83 \pm 21.81$ & $477.44 \pm 267.34$ & $120.31 \pm 46.58$ \\
Day -4 & $6.56 \pm 1.74$ & $75.05 \pm 32.74$ & $466.68 \pm 295.71$ & $118.87 \pm 68.61$ \\
Day -5 & $6.57 \pm 1.70$ & $83.06 \pm 21.49$ & $430.21 \pm 215.77$ & $106.56 \pm 35.65$ \\
\hline
\end{tabular}

NOTES: s-RPE: RPE session derived from the multiplication of the RPE for the duration of training in minutes; U.A.: arbitrary units; n: number.

Comparing the internal and external load between training days, correlations were found with a large magnitude between the number of jumps and the duration of training $(r=0.596, p=0.001)$, with an R2 of 0.355. The association between the number of jumps and the RPE presented values of small magnitude $(r=$ $0.147 ; p=0.041)$. Regarding the association between the number of jumps and s-RPE, we observed correlations with moderate magnitude $(r=0.486 ; p=0.001)$.
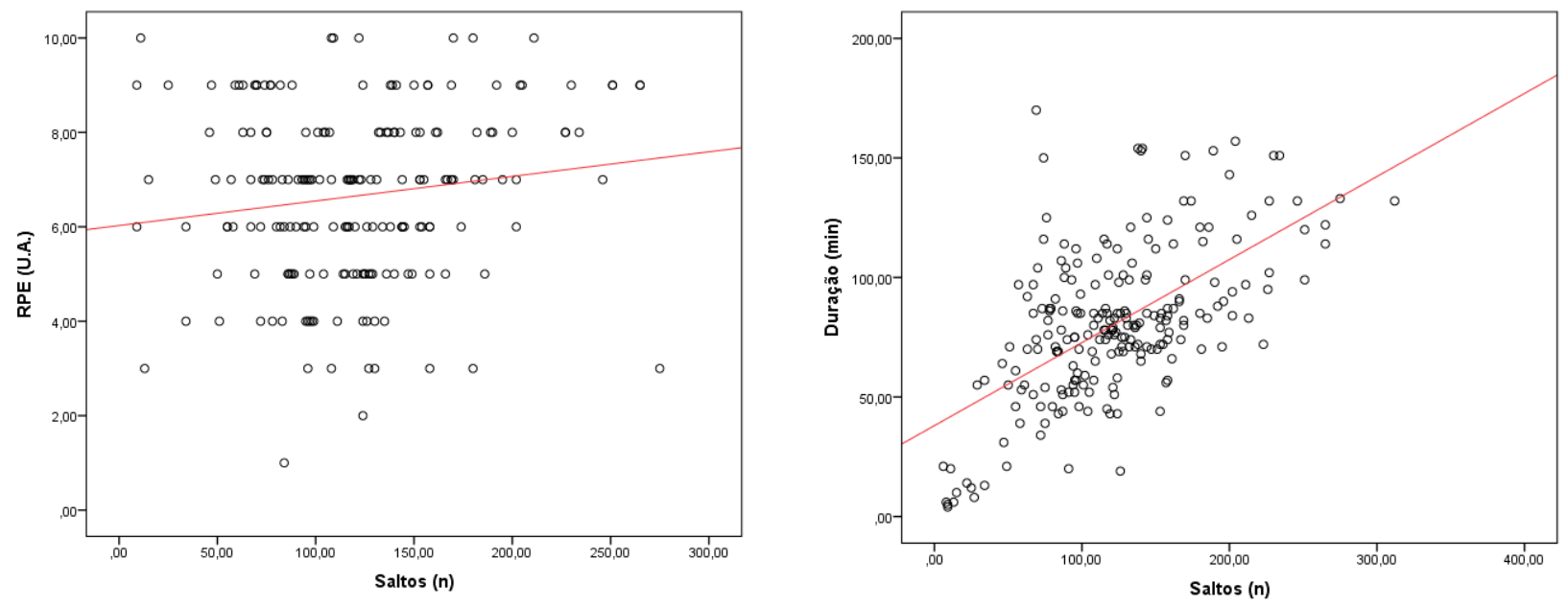

Figure 1. Scatterplot of the relationship between the number of jumps and the RPE variables and duration.

\section{DISCUSSION}

The days with the greatest volume and intensity occurred two and three days before the game, possibly trying to grant a tapering period on the day before the game and a recovery period with a lower load on days (48 hours) following the game. These results are in line with the literature (Mendes et al., 2018). The data of the correlations between loads showed small to moderate magnitudes and, therefore, both loads should be measured in the trainings in order to infer different acute responses and necessarily different implications. 


\section{CONCLUSIONS}

The study revealed that external and internal load monitoring are instruments that support the trainer in the process of constructing the training unit, as well as in the prevention of injuries of the athletes. Thus, it is fundamental for the coach to control different variables in the training process.

\section{REFERENCES}

Bourdon, P. C., Cardinale, M., Murray, A., Gastin, P., Kellmann, M., Varley, M. C., ... Cable, N. T. (2017). Monitoring Athlete Training Loads: Consensus Statement. International Journal of Sports Physiology and Performance, 12(Suppl 2), S2-161-S2-170. https://doi.org/10.1123/ijspp.2017-0208

Clemente, F. M., Mendes, B., Palao, J. M., Silvério, A., Calvete, F., \& Nakamura, F. Y. (2018). Seasonal player wellness and its longitudinal association with internal training load: study in elite volleyball load: study in elite volleyball. The Journal of Sports Medicine and Physical Fitness, (April). https://doi.org/10.23736/s0022-4707.18.08312-3

Halson, S. L. (2014). Monitoring Training Load to Understand Fatigue in Athletes. Sports Medicine, 44(2), 139-147. https://doi.org/10.1007/s40279-014-0253-z

Mendes, B., Palao, J. M., Silvério, A., Owen, A., Carriço, S., Calvete, F., \& Clemente, F. M. (2018). Daily and weekly training load and wellness status in preparatory, regular and congested weeks: a seasonlong study in elite volleyball players. Research in Sports Medicine, 26(4), 462-473. https://doi.org/10.1080/15438627.2018.1492393

\section{(@) $\odot \Theta$}

This work is licensed under a Attribution-NonCommercial-NoDerivatives 4.0 International (CC BY-NC-ND 4.0). 


\title{
Frequency of sports participation, body image satisfaction and psychological well-being: Gender differences among vocational students
}

\author{
HELDER MIGUEL FERNANDES $1,2 \checkmark$, LUÍS ALMEIDA3,4 \\ ${ }^{1}$ Research Centre in Sports Sciences, Health Sciences and Human Development, CIDESD-UTAD, Portugal \\ ${ }^{2}$ Research in Education and Community Intervention, RECI- Instituto Piaget, Portugal \\ ${ }^{3}$ Research Centre of Training Innovation and Intervention in Sport, CIFI2D-FADEUP, Portugal \\ ${ }^{4}$ Centre for Research and Intervention in Education, CIIE-FPCEUP, Portugal
}

\begin{abstract}
The main aim of the current study was to investigate gender-related variations in the scores and relationships between frequency of sports participation, body image satisfaction and psychological well-being among vocational students. The total sample was composed of 387 students ( 200 girls and 187 boys), aged between 15 and 24 years $(M=17.66, S D=1.68)$, and attending four different vocational high schools. This study involved two types of variables: single-items for the assessment of sports participation frequency and body image satisfaction, and composite scores obtained from the Portuguese adapted and validated version (Fernandes, Vasconcelos-Raposo, \& Teixeira, 2010) of Ryff's psychological well-being scales. The results indicated that boys reported higher levels of sports participation frequency, body image satisfaction and selfacceptance than girls, whereas the latter showed higher levels of personal growth than boys. Correlations separated by gender showed that girls' frequency of sports participation was positively associated with positive relationships with others, purpose in life and the total well-being score. On the other hand, higher frequency levels of sports participation among boys was related to better scores of body image satisfaction, environmental mastery, purpose in life, self-acceptance and total well-being. In sum, the current study shows that the effects of sports participation frequency on body image satisfaction and psychological well-being scores are gender-related, with male vocational students getting more psychosocial benefits from higher sports involvement in comparison to their female peers. Keywords: Sports participation; Body image; Psychological well-being; Vocational students.
\end{abstract}

\footnotetext{
Corresponding author. Universidade de Trás-os-Montes e Alto Douro, Departamento de Ciências do Desporto, Exercício e Saúde, Quinta de Prados - Campus Desportivo, Apartado 1013, 5001-801 Vila Real - Portugal.

E-mail: hmfernandes@gmail.com

Supplementary Issue: Spring Conferences of Sports Science. International Seminar of Physical Education, Leisure and Health, 17-19 June 2019. Castelo Branco, Portugal.

JOURNAL OF HUMAN SPORT \& EXERCISE ISSN 1988-5202

(C) Faculty of Education. University of Alicante.

doi:10.14198/jhse.2019.14.Proc4.82
} 


\section{INTRODUCTION}

Vocational education high school students comprise an increasing and significant percentage of the Portuguese school-based population. Available evidence has demonstrated that these students tend to engage more frequently in health-compromising and disruptive behaviours, as well as to report poorer mental health (e.g., Holmberg \& Hellberg, 2007). Not surprisingly, some studies have examined the associations between physical activity/sports participation and certain psychological dimensions (Almeida et al., 2018; Gerber et al., 2015), since a considerable proportion of the vocational students population fails to achieve the recommended health-related physical activity guidelines. As such, the main aim of the present study was to investigate gender-related differences in the scores and relationships between frequency of sports participation, body image satisfaction and psychological well-being among vocational students.

\section{MATERIAL AND METHODS}

\section{Participants}

The total sample was composed of 387 students (200 girls and 187 boys) attending four different vocational high schools located in two Portuguese districts (Aveiro and Oporto). Participants' age ranged from 15 to 24 years $(M=17.66, S D=1.68)$, with no differences between genders $(p=0.94)$.

\section{Measures}

Frequency of participation in organized sports was obtained through responses to a single item: "How many times a week do you practice (train or compete) organized sports?". Body image satisfaction was evaluated by one question ("How satisfied are you with your body/appearance?") using a 10-point response scale (1: very dissatisfied to 10: very satisfied). Psychological well-being was measured using an adapted and validated Portuguese version (Fernandes, Vasconcelos-Raposo, \& Teixeira, 2010) of Carol Ryff's Scales. This instrument consists of 30 items answered on a five-point Likert scale (1: strongly disagree to 5: strongly agree), which allow the measurement of six different facets of well-being, namely: autonomy, environmental mastery, personal growth, positive relationships with others, purpose in life, and self-acceptance. In the present study, the Cronbach's alpha for the total scale was 0.89 .

\section{Procedures}

Prior to the data collection process, the directors of the purposively sampled schools were contacted and authorization to administer the questionnaires was obtained. After obtaining this approval, written informed consent to participate in this study was obtained from all adult students or their parents/legal guardians. The instruments were completed individually at the beginning or end of a lesson, in quiet classroom conditions. In all phases of the study, the anonymity and confidentiality of the respondents were assured.

\section{Analysis}

Statistical analysis included descriptive statistics (mean, standard deviation, percentage, skewness and kurtosis), internal consistency (Cronbach's alpha), and Pearson correlations. Significance was set at $p<0.05$. All analyses were performed using SPSS 17.0 (SPSS Inc., Chicago).

\section{RESULTS}

All study variables presented acceptable values of skewness and kurtosis (between -1.53 and 2.85). 
The results of gender comparison showed that boys reported significantly $(p<0.01)$ higher levels of sports participation frequency $(2.15 \pm 2.29)$, body image satisfaction $(7.54 \pm 2.28)$ and self-acceptance $(4.07 \pm$ $0.68)$ than girls $(1.10 \pm 1.75,6.68 \pm 2.31$ and $3.90 \pm 0.74$, respectively). On the other hand, girls showed significantly $(p<0.01)$ higher levels of personal growth $(4.43 \pm 0.48)$ than boys $(4.28 \pm 0.65)$.

Correlation results showed small to moderate significant associations between the study variables. Among girls, frequency of sports participation was positively associated with positive relationships with others $(r=$ $0.15, p<0.05)$, purpose in life $(r=0.22, p<0.001)$, and the total well-being score $(r=0.19, p<0.01)$. On the other hand, higher levels of boys' sports participation frequency was related to higher scores of body image satisfaction $(r=0.20, p<0.01)$, environmental mastery $(r=0.15, p<0.05)$, purpose in life $(r=0.17, p<0.05)$, self-acceptance $(r=0.15, p<0.05)$, and total well-being $(r=0.16, p<0.05)$.

\section{DISCUSSION}

The main goal of the present study was to explore gender-related variations in the scores and associations between sports participation frequency, body image satisfaction and psychological well-being among vocational students. Taken together, our results indicate that higher levels of sports participation play a significant and protective role on vocational students' mental health, contributing to better intra- and interindividual outcomes. Nevertheless, the current evidence also suggests that male vocational students get more benefits from higher sports involvement in comparison to their female peers, which is consistent with some previous findings among the overall youth population (Ahn \& Fedewa, 2011). These variations in the relationships may be explained by the distinct patterns of sports participation among boys and girls, which in turn lead to the psychosocial differences observed. With this in mind, vocational schools should encourage and promote a more physically active lifestyle of their students, by providing (more) physical education and school sport participation through a wider offer of activities for both genders.

\section{CONCLUSIONS}

In sum, the current study shows that the effects of sports participation frequency on body image satisfaction and psychological well-being scores are gender-related, with male vocational students getting more psychosocial benefits from higher sports involvement in comparison to their female peers.

\section{REFERENCES}

Ahn, S., \& Fedewa, A. (2011). A meta-analysis of the relationship between children's physical activity and mental health. J Pediatr Psychol, 36(4), 385-397. https://doi.org/10.1093/jpepsy/jsq107

Almeida, L., Pereira, H., \& Fernandes, H.M. (2018). Efeitos de diferentes tipos de prática desportiva no bem-estar psicológico de jovens estudantes do ensino profissional. Ibero Am J Exerc Sport Psychol, 13(1), 15-21.

Fernandes, H.M., Vasconcelos-Raposo, J., \& Teixeira, C. (2010). Preliminary analysis of the psychometric properties of Ryff's Scales of Psychological Well-Being in Portuguese adolescents. Span J Psychol, 13(2), 1032-1043. https://doi.org/10.1017/s1138741600002675

Gerber, M., Lang, C., Feldmeth, A., Elliot, C., Brand, S., Holsboer-Trachsler, E., \& Pühse, U. (2015). Burnout and mental health in Swiss vocational students: The moderating role of physical activity. J Res Adolesc, 25(1), 63-74. https://doi.org/10.1111/jora.12097 
Holmberg, L., \& Hellberg, D. (2007). Health, health-compromising behavior, risk-taking behavior and sexuality in female and male high school students in vocational compared with theoretical programs in Sweden. Int J Adolesc Med Health, 19(4), 459-472. https://doi.org/10.1515/ijamh.2007.19.4.459

\section{(c) (i) $\ominus$}

This work is licensed under a Attribution-NonCommercial-NoDerivatives 4.0 International (CC BY-NC-ND 4.0). 


\title{
Analysis of physical-sport motivations in adolescents according to gender, age and BMI
}

\author{
A.D. AGRASO-LÓPEZ ${ }^{1}$, J.E. MORAL-GARCÍA² $\triangle$, S. LÓPEZ GARCÍA², J.M. DE MENA-RAMOS² \\ ${ }^{1}$ Master's Degree in Teacher Training, Universidad Pontificia de Salamanca, Spain \\ ${ }^{2}$ Department of Physical Activity and Sport Sciences, Faculty of Education, Pontifical University of \\ Salamanca, Spain
}

\begin{abstract}
A study has been carried out to verify the main motivations for the practice of physical-sport activity in adolescents, both at school and outside it. To this end, a sample of 345 students from 1 st to 4 th year of ESO, aged between 12 and 16 years old, has been taken. The questionnaire was used by those who formulated the self-perception test (CSAPPA) whose independent variables to be treated were the following: Adequacy, Predilection and Enjoyment. While the dependent variables to be related with the previous ones are: sex, age and BMI. With regard to the results obtained, with respect to the adaptation and predilection section, women and subjects aged 12-13 years present higher values with respect to men and the other age groups compared; on the other hand, the enjoyment section also shows relative differences in favour of women. The 16-year age group offers the lowest results. In conclusion, the students and the calculation of students whose age range is between 12 and 13 years of age, offers more motivational favourability towards the practice of physical-sports activity, relating the variables dependent on sex, age and BMI with the independent variables of Adequacy, Predilection and Enjoyment. Keywords: Physical activity; Fitness; Predilection; Enjoyment; Gender \& BMI.
\end{abstract}

Corresponding author. C/ Henry Collet, 52-70, CP: 37007, Salamanca, Spain.

E-mail: jemoralga@upsa.es

Supplementary Issue: Spring Conferences of Sports Science. International Seminar of Physical Education, Leisure and Health, 17-19 June 2019. Castelo Branco, Portugal.

JOURNAL OF HUMAN SPORT \& EXERCISE ISSN 1988-5202

(C) Faculty of Education. University of Alicante.

doi:10.14198/jhse.2019.14.Proc4.82 


\section{INTRODUCTION}

It is known that the practice of physical activity (AP) has physical and psychological benefits for people of all ages, besides helping (Ruiz-Ariza, de la Torre-Cruz, Latorre-Román, and Martínez-López, 2016). Therefore, the school environment is an ideal context to study the motivations for practicing physical activity (Kelder, Karp, Scruggs and Brown, 2014). This practice can affect academic performance (López de los MozosHuertas, 2018), also affecting self-esteem (Revuelta, Esnaloa and Goñi, 2016).

\section{MATERIAL AND METHODS}

A cross-sectional descriptive study was carried out, using as a measuring instrument the self-perception questionnaire on the appropriateness and predilection of physical activity in children (CSAPPA) (MorenoMurcia et al., 2011).

\section{Participants}

The total sample was 345 students of Compulsory Secondary Education between the ages of 12 and 16 $(50.7 \%$ female).

\section{Measures}

Adjustment, predilection and enjoyment factors (CSAPPA) were analysed as dependent variables, relating them to the independent variables gender, age and BMI.

\section{Procedures}

The questionnaires were supervised by the principal investigator and completed by the students in a class session. The ethical guidelines of the Declaration of Helsinki were followed.

\section{Analysis}

A descriptive analysis was carried out, the reliability coefficient and the internal consistency of the scale (Cronbach's Alpha) were obtained. Finally, an analysis of variance (ANOVA) and correlations was made.

\section{RESULTS}

The present study yielded a Cronbach's alpha of 0,817 . By factors, Cronbach's alpha was 0,70 in adequacy, 0,72 in predilection and 0.8 in enjoyment. There are significant differences $(p<0,00)$ according to gender in the factors adequacy, predilection and enjoyment favourable to women, in the age being younger adolescents (12-13 years) those who feel greater motivation in adequacy and predilection, and according to the BMI where those who experience more motivation related to adequacy are normopeso (see Table 1).

\section{DISCUSSION}

The present study yielded a Cronbach's alpha of 0,817 . Therefore, this is a good consistency index (Streiner, 2003). The level of motor commitment in Physical Education classes is higher in boys, in addition girls perform less extracurricular physical-sports activity (Carrillo, Rodriguez, Loaisa, Coll, Galindo, \& Gimeno (2017). Our results show that the predilection, enjoyment and suitability of the female sex is greater than the male sex. Motivation is higher among younger and normopeso students. For its part, a recent study (Moral-García et al., 2019) the predilection factor is more related to males and younger subjects. 
Table 1. Descriptive and variance analysis (ANOVA)

\begin{tabular}{|c|c|c|c|c|c|c|}
\hline \multirow[b]{2}{*}{ Factors } & & \multicolumn{3}{|c|}{ Descriptive } & \multicolumn{2}{|c|}{ ANOVA } \\
\hline & & $S$ & Average & SD & $F$ & Sig. \\
\hline \multirow{8}{*}{ Adequacy } & Feminine & 175 & 2.5257 & .53563 & \multirow{2}{*}{36.945} & \multirow{2}{*}{.000} \\
\hline & Masculine & 170 & 2.1655 & .56492 & & \\
\hline & $12-13$ years old & 147 & 2.4257 & .54243 & \multirow{3}{*}{3.198} & \multirow{3}{*}{.042} \\
\hline & $14-15$ years old & 84 & 2.2279 & .59343 & & \\
\hline & 16 years old & 114 & 2.3371 & .60044 & & \\
\hline & Normal weight & 271 & 2.3869 & .57996 & \multirow{3}{*}{7.853} & \multirow{3}{*}{.000} \\
\hline & Overweight & 33 & 2.0866 & .48437 & & \\
\hline & Obesity & 40 & 1.5714 & .26082 & & \\
\hline \multirow{8}{*}{ Predilection } & Feminine & 175 & 2.5989 & .39870 & \multirow{2}{*}{35.105} & \multirow{2}{*}{.000} \\
\hline & Masculine & 170 & 2.3327 & .43627 & & \\
\hline & $12-13$ years old & 147 & 2.5385 & .39254 & \multirow{3}{*}{4.980} & \multirow{3}{*}{.007} \\
\hline & $14-15$ years old & 84 & 2.3524 & .48308 & & \\
\hline & 16 years old & 114 & 2.4596 & .44378 & & \\
\hline & Normal weight & 272 & 2.4857 & .43945 & \multirow{3}{*}{2.278} & \multirow{3}{*}{.104} \\
\hline & Overweight & 33 & 2.3242 & .45417 & & \\
\hline & Obesity & 40 & 2.3000 & .16330 & & \\
\hline \multirow{8}{*}{ Enjoy } & Feminine & 175 & 3.1534 & .82644 & \multirow{2}{*}{12.387} & \multirow{2}{*}{.000} \\
\hline & Masculine & 170 & 2.8255 & .90597 & & \\
\hline & $12-13$ years old & 147 & 2.4257 & .88210 & \multirow{3}{*}{1.128} & \multirow{3}{*}{.325} \\
\hline & $14-15$ years old & 84 & 2.2279 & .95400 & & \\
\hline & 16 years old & 114 & 2.3371 & .82066 & & \\
\hline & Normal weight & 272 & 2.9877 & .89315 & \multirow{3}{*}{.676} & \multirow{3}{*}{.509} \\
\hline & Overweight & 33 & 2.9192 & .72183 & & \\
\hline & Obesity & 40 & 2.5000 & 1.23228 & & \\
\hline
\end{tabular}

\section{CONCLUSIONS}

Both girls and younger subjects show a higher level of motivation in the three selfless factors; normal-weight subjects feel more motivation in the predilection and enjoyment factor, however obese subjects have higher scores in appropriateness. In general, as age increases and BMI decreases, motivation towards sport.

\section{REFERENCES}

Carrillo, V. J. B., Rodríguez, A. C. S., Loaisa, A. J., Coll, D. G. C., Galindo, M. C. M., \& Gimeno, E. M. C. (2017). Diferencias según género en el tiempo empleado por adolescentes en actividad sedentaria y actividad física en diferentes segmentos horarios del día. Retos: nuevas tendencias en educación física, deporte y recreación, (31), 3-7. https://doi.org/10.24054/16927427.v2.n2.2016.2409

Kelder, S. H., Karp, G. G., Scruggs, P. W., \& Brown, H. (2014). Chapter 1 setting the stage: Coordinated approaches to school health and physical education. Journal of Teaching in Physical Education, 33(4), 440-448. https://doi.org/10.1123/itpe.2014-0087

López de los Mozos-Huertas, J. (2018). Condición física y rendimiento académico. Journal of Sport and Health Research, 10(3), 349-360.

Moral-García, J.E., Agraso-López, A., Pérez Soto, J.J., Rosa Guillamón, A., Tárraga Marcos, M.L., García-Canto, E. y Tárraga López, P.J. (2019). Práctica de actividad física según adherencia a la 
dieta mediterránea, consumo de alcohol y motivación en adolescentes. Nutrición Hospitalaria, 36(2):420-427. https://doi.org/10.20960/nh.2181

Moreno-Murcia, J.A., Martínez-Galindo, M.C., Ruíz, L.M., García, V. y Martín-Albo, J. (2011). Validation of the Spanish version of the children's self-perceptions of adequacy in and predilection for physical activity (CSAPPA) questionnaire. Psychology, Society \& Education, 3(2), 113-132. https://doi.org/10.25115/psye.v3i2.474

Revuelta, L., Esnaola, I., \& Goñi, A. (2016). Relaciones entre el autoconcepto físico y la actividad físicodeportiva adolescente. Revista internacional de Medicina y Ciencias de la Actividad Física del Deporte, 16(12), 561-581. https://doi.org/10.15366/rimcafd2016.63.010

Ruiz-Ariza, A., Ruiz, J. R., de la Torre-Cruz, M., Latorre-Román, P., \& Martínez-López, E. J. (2016). Influencia del nivel de atracción hacia la actividad física en el rendimiento académico de los adolescentes. Revista latinoamericana de psicología, 48(1), 42-50. https://doi.org/10.1016/j.rlp.2015.09.005

Streiner, D. L. (2003). Being inconsistent about consistency: When coefficient alpha does and doesn't $\begin{array}{llll}\text { matter. Journal of personality assessment, } & \text { 217-222. }\end{array}$ https://doi.org/10.1207/s15327752jpa8003 01 


\title{
Olympism: Level of knowledge between different University Degrees in the Faculty of Education. Differences by gender and University Degree
}

\author{
J.M. DE MENA-RAMOS ${ }^{1} \triangle$, S. LÓPEZ-GARCI'A ${ }^{1}$, A.D. AGRASO-LÓPEZ² , J.E. MORAL-GARCÍA ${ }^{1}$ \\ ${ }^{1}$ Department of Physical Activity and Sport Sciences, Faculty of Education, Pontifical University of \\ Salamanca, Spain \\ ${ }^{2}$ Master's Degree in Teacher Training, Pontifical University of Salamanca, Spain
}

\begin{abstract}
The objective of this research is to know the degree of knowledge of the university students of the Faculty of Education of the Pontifical University of Salamanca of the degrees of Teaching and Sciences of Physical Activity and Sport. An analysis was made on the variables gender and degree of studies that is being studied. To this end, the Molina questionnaire (2011) was administered with some update to a total of 426 university students. We can conclude that the level of knowledge is high except in the 5th and 9th items. There is greater knowledge in university men than in university women. In the grades whose curriculum includes more than one specific subject of physical education, the level of knowledge is also higher. Keywords: Olympic education; University students; Olympism; Gender; University Degree.
\end{abstract}

Corresponding author. Calle Trascastillo $n^{0}$ 13. 49026. Zamora, Spain.

E-mail: imenara@upsa.es

Supplementary Issue: Spring Conferences of Sports Science. International Seminar of Physical Education, Leisure and Health, 17-19 June 2019. Castelo Branco, Portugal.

JOURNAL OF HUMAN SPORT \& EXERCISE ISSN 1988-5202

(C) Faculty of Education. University of Alicante.

doi:10.14198/jhse.2019.14.Proc4.82 


\section{INTRODUCTION}

We can consider the Olympic Games as the supreme manifestation of sport worldwide, being that a phenomenon, in parallel, transcends a multitude of areas of our life. The strength of the Olympic Movement exceeds the mere sporting relevance, achieving a great repercussion in fundamental aspects of each country (Goig \& Ferrando, 2016) and, among them, education (Gutiérrez Sánchez \& Martínez Gorroño, 2015). Therefore, we believe it is necessary to implement Olympic education within the scope of general education and, in particular, in those university studies with a clear teaching vocation. Since the birth of the modern Olympic movement, the initial pedagogical action proposed by Coubertin in his Olympic ideology (Cortes, 2013) has been changing, gradually diluting the objective that sports practice should form the basis of a new educational method (Fernández Truan, 2015). Nowadays, the educational concept of Olympism has been almost forgotten to the detriment of the sports and media spectacle that constitute the Olympic Games to the point that many of the educational, humanistic and philosophical principles that support them have fallen into oblivion (Todt, 2007 ). For all the above, this research seeks to know not only aspects related to the celebration of the Olympic Games but also, the degree of knowledge of university students about the modern Olympic movement analysing such knowledge in terms of gender and the University Degree the are studying.

\section{MATERIAL AND METHODS}

A descriptive cross-sectional study was carried out, using as a measuring instrument the Molina questionnaire (2011) which consists of 10 multiple-choice questions with 4 answer options in each question, with only one correct answer. Two questions have been updated to the date of 2019.

\section{Participants}

The total sample was 426 students of the Teaching Degrees (Infant and Primary) and CAFYD, of which 305 are men $(71.6 \%)$ and 121 women (28.4\%). Dividing the sample by Degrees we find that in Teaching (infant and primary) there are 125 students of which 75 are women (60.0\%) and 50 are men (40.0\%). In the CAFYD Degree, the sample consists of 301 students of which there are 255 men (71.6\%) and 46 women (28.4\%). The ages ranged between 18 and 36 years.

\section{Measures}

The SPSS for Windows v.20 program was used, first analysing the different variables and then the descriptive statistics on successes and errors in each question according to different segmentation variables of the sample. They performed association tests between these variables and the correct answers.

\section{Procedures}

The questionnaires were supervised by the principal investigator and completed by the students in a class session. The ethical guidelines of the Declaration of Helsinki were followed.

\section{Analysis}

A descriptive analysis was carried out, the reliability coefficient and the internal consistency of the scale were obtained (Cronbach's alpha), and addition an analysis of variance (ANOVA) and the different correlations were made. 


\section{RESULTS}

Regarding knowledge of Olympism and gender, it is observed that men have a higher percentage of success than women, this difference being statistically significant especially in the $2 \mathrm{nd}, 6$ th and 10 th items. There are questions where the success of both men and women exceeds $50 \%$ and one where the level of success is less than $15 \%$ in both men and women, but these are those that have a higher percentage of success $(11.4 \%$ against to $8.6 \%$ of men). The distribution according to the Degree and the presence in the program of studies of specific subjects of physical education and sports gives us a total of 336 students called "specialists" (Degree of CAFYD and Teaching Physical Education) of the total of 426 students surveyed (78.9\%). The results show that in the ten items of the questionnaire the "specialists" get more hits than the "non-specialists" even in those items in which the degree of success is lower. In some items, the statistical difference regarding the successes is overwhelming.

\section{DISCUSSION}

The results obtained in this research reflect that in the University the knowledge about Olympism is higher in men than in women and among students in whose studies there are specific subjects on physical education and sports, data that confirm Molina's research (2011), Martínez, Morote \& Mármol (2015), and García, Arcas, Morote \& Pato (2018). In addition we can affirm that, in general, the knowledge of Olympism among the surveyed university students is medium - low, since in many of the referenced items the level of success is less than $70 \%$. Therefore, we believe that a greater knowledge of the Olympic movement is needed in university students, especially in those subjects related to the history of sports.

\section{CONCLUSIONS}

The objective of this research was to assess the degree of knowledge of university students of the Faculty of Education of the Pontifical University of Salamanca regarding Olympism, analysing the results according to gender and the Degree of Study. The knowledge can be considered medium - low since in the degree of success in more than half of the items is below $80 \%$ and most of them from a level of difficulty accessible to a population that is supposed to have an academic background high as are university students, especially those who focus their studies towards physical activity and sports. We must take into account as a possible limitation of the research that some of the students surveyed have not yet studied subjects related to the history of sports, so it is possible that the results would have improved especially in the specialists.

\section{REFERENCES}

Cortés, D. F. G. (2013). Pierre de Coubertin y su idea pedagógica del deporte y el olimpismo. VIREF Revista de Educación Física, 1(1), 51-61.

Fernández-Truan, J. C. (2015). Antecedentes del valor educativo de la instauración de los Juegos Olímpicos Modernos.

García, J. L. J., Arcas, A. L., Morote, J. M., \& Pato, A. S. (2018). Proyecto educativo Olimpízate: objetivos, metodología y actividades para enseñar Olimpismo en los centros de educación secundaria. Retos: nuevas tendencias en educación física, deporte y recreación, (33), 286-292. https://doi.org/10.15332/tg.mae.2018.00385

Goig, R. L., \& Ferrando, M. G. (2016). Los Juegos Olímpicos como NOMS. El olimpismo en la sociedad deportivizada global. Revista Española de Sociología, 25(1). 
Gutiérrez Sánchez, C. B., \& Martínez Gorroño, M. E. (2015). Los Centros de Estudios Olímpicos como parte de la estructura de la Academia Olímpica Española: una iniciativa y alternativa para la renovación y actualización del Olimpismo dentro de las universidades españolas. Citius, Altius, Fortius: Humanismo, Sociedad y Deporte: Investigaciones y ensayos. https://doi.org/10.15366/citius2015.8.2.003

Martínez, B. J. S. A., Morote, J. M., \& Mármol, A. G. (2015). Los contenidos educativos del Olimpismo en el currículum escolar: percepción de los estudiantes. Acciónmotriz, (15), 25-32.

Molina, J.M. (2011). Visión del olimpismo y sus repersusiones educativas entre universitarios de Magisterio de Educación Física, Grado de Educación Primaria y Grado en Ciencias de la Actividad Física y el Deporte de la Región de Murcia. Tesis Doctoral no publicada. https://doi.org/10.20868/upm.thesis.49175

Todt, N. (2007). Jogos Olimpicos da antiguidade. En K. Rubio; A. Reppold Filho; N. Todt \& R. Mesquita (Orgs.), Ética e compromisso social nos estudos olímpicos (pp. 217-222). Porto Alegre: EDIPUCRS. Tubino, M. (2017). O que é olimpismo. Brasiliense. 


\title{
Academic routine impact on physical activity level of university students: A longitudinal study
}

\author{
THIAGO ROGEL ${ }^{1,4}$, , GIOVANNA HARDER $^{3}$, LARISSA PERONDI ${ }^{3}$, DANIELLA MEDEIROS ${ }^{2,3}$, DÉBORA \\ ROCCO $^{3}$, BEATRIZ PEREIRA ${ }^{1,4}$ \\ 1 University of Minho, IE, Portugal \\ 2University of Sao Paulo, Brazil \\ ${ }^{3}$ Santa Cecilia University, Brazil \\ ${ }^{4}$ Research Centre on Child Studies, University of Minho, CIEC-UM, Portugal
}

\begin{abstract}
Objective: The aim of this study was to investigate the impact of routine academic physical activity level (PAL) in university students. Method: Participants were 30 students (17 girls and 13 boys) of physical education course, aged between 18 and 32 years. The PAL was accessed by the short version of International of Physical Activity Questionnaire (IPAQ) validated for Brazilian population by Matsudo et al. (2001). The participants included in this longitudinal study answered the IPAQ during every academic path $(2015,2016$, 2017 and 2018). For the statistical analysis was utilized the Anova-One Way for repeated measurements test with Bonferroni post-hoc. Results: The results showed significant differences between the 1st year and other schooling years. Conclusion: It is possible to conclude that the academic routine negatively impacts the PAL, especially in the last year of course (4th year).Keywords: Academic routine; Health; Physical activity.
\end{abstract}

Corresponding author. University of Minho, 4710-057 Braga, Portugal.

E-mail: thiago rogel@hotmail.com

Supplementary Issue: Spring Conferences of Sports Science. International Seminar of Physical Education, Leisure and Health, 17-19 June 2019. Castelo Branco, Portugal.

JOURNAL OF HUMAN SPORT \& EXERCISE ISSN 1988-5202

(c) Faculty of Education. University of Alicante.

doi:10.14198/jhse.2019.14.Proc4.82 


\section{INTRODUCTION}

Communication and strengthening of friendship bonds are made during the organized sports practices and spontaneous physically active play, that is, the movement has strong social and cultural meanings (Miyabayashi and Pimentel, 2011). Therefore, the relationship between peers has been suggested as a strong predictor of the physical activity levels in children, as youth are more likely to be active when they are in the company of friends (Haye et al, 2011). These influences have been associated with factors such as motivation and social modelling and are based on the social facilitation theory and social learning theory (Salvy et al, 2012). Specifically, imitation is a behaviour which emerges from the relations of the individuals in a complex social system and which establishes collective norms and restrictions on individual behaviour. Thus, the objective of the study was to investigate the relative importance of children in school friendship groups and their associations with LPA in the early years of basic school.

\section{MATERIAL AND METHODS}

\section{Participants}

This research included Thirty-two children (girls=13), aged 8-9 years, enrolled in the 3rd year of elementary education, in the city of Santos, São Paulo, Brazil. The children Who participated in the experiment were children with chronological age compatible with the school year and that presented the free and informed consent form signed by a person responsible.

\section{Measures}

To identify the ties of friendship a questionnaire was used to composed by a closed question, where the children indicated how often they play with each student in their classroom (often, sometimes or never). The physical activity was evaluated through the self-reporting questionnaire proposed and validated by Godin and Shephard (1985). Children reported the number of times they spent more than 15 minutes in mild activities (3 METs), moderate (5 METs) or strenuous (9 METs) in a typical week. The measurement of the weekly physical activity level was calculated by multiplying the frequency indicated by the respective MET value for each intensity.

\section{Procedures}

Data collection was performed on a typical day during the school period. Both questionnaires were applied individually through an interviewer assisted by the researcher. Then a database was created for statistical treatment.

\section{Analysis}

The relative importance of the child in the group was evaluated through the number of reciprocal appointments (degree centrality) through the Yed Software for analysis of networks. After the verification of normality of the data through the Bronferroni test, the data were submitted to the Pearson correlation coefficient test with significance level accepted at $p \leq 0.05$. The degree of centrality and the level of physical activity were the variables of investigation and the body mass index and the sex were the control variables of the study.

\section{RESULTS}

The results of this study showed a positive correlation between the degree of relative importance of the child in the group of friends and the frequency of moderate and vigorous physical activities ( $r=363, p=.041 ; r=362$, 
$p=.042$. In contrast the mild activities, BMI and sex are not associated with interpersonal relationships of children.

\section{DISCUSSION}

The associations found between interpersonal relations and physical activity in children are consistent with corroborate with the literature. Relationships between peers are essential for a physically active lifestyle in children, specifically in increasing motivation (Salvy, Roemmich and Bowker, 2009), distance between friend's group residence, active transportation in groups for school (Jerina et al, 2018), companionship in organized sports practices and free play (Todisco et al, 2017).

\section{CONCLUSIONS}

The interpersonal relationships are directly associated with physical activity levels in childhood. However, cross-sectional studies do not allow the identification of cause and effect relationships between variables.

\section{REFERENCES}

Miyabayashi, L. A. \& Pimentel, G. G. A. (2011) Interações sociais e proficiência motora em escolares do ensino fundamental, Revista Brasileira de Educação Física e Esporte, 25 (4), 649-662. https://doi.org/10.1590/s1807-55092011000400009

Haye, K., Robbins, G., Mohr, P. \& Wilson, C. (2011). How physical activity shapes, and is shaped by, adolescent friendships, Social Science \& Medicine, 73, 719-728. https://doi.org/10.1016/i.socscimed.2011.06.023

Salvy, S., Roemmich, J., Bowker, J., Romero, N., Stadler, P. \& Epstein, L. (2009). Effect of Peers and Friends on Youth Physical Activity and Motivation to be Physically Active, Journal of pedriatric Psychology, 34 (2), 217-225. https://doi.org/10.1093/jpepsy/jsn071

Salvy, S., Haye, K, Bowker, J. C. \& Hermans, R. C. J. (2012). Influence of peers and friends on children`s and adolescent's eating and activities behaviors, Physiology \& Behavior, 106, 369-378. https://doi.org/10.1016/j.physbeh.2012.03.022

Jerina, T., Pisot, R. \& Volmut, T. (2018). Social and Demographic factors of physical activity in 9-11 year old Slovenian Children, Kinesiology, 50 (1), 68-78. https://doi.org/10.26582/k.50.1.13

Todisco, W. M. D., Guilherme, F. R., Molena-Fernandes, C. A., Santo, S. R., Ravagnani, R. J. F., Del Vecchio, F. B. \& Rinaldi, W. (2017). Nível de atividade física e apoio dos pais e amigos em escolares da rede pública, Revista Brasileira de Atividade Física e Saúde, 22 (5), 457-463. https://doi.org/10.12820/rbafs.v.22n5p457-463

\section{@) $(\Theta \Theta \Theta$}

This work is licensed under a Attribution-NonCommercial-NoDerivatives 4.0 International (CC BY-NC-ND 4.0). 


\title{
Cooperative games vs competitive games in Primary School Education: What effects do they have on schoolchildren motivation?
}

\author{
RUBÉN NAVARRO-PATÓN ${ }^{1}$, JOSÉ EUGENIO RODRIGGUEZ-FERNÁNDEZ1 ${ }^{1}$, BEATRIZ OLIVEIRA - \\ PEREIRA ${ }^{2}$ \\ 1 University of Santiago de Compostela, Spain \\ 2University of Minho, Research Centre on Child Studies, Portugal
}

\begin{abstract}
As a school subject, the ultimate goal of Physical Education (PE) is to help schoolchildren to learn different concepts, skills and values. For this reason, it is important to understand student's motivation in PE learning process, and how different teaching strategies and methodologies influence children motivation. Then, the aim of this study was to assess the impact of two units (one of them based on cooperative games and the other one based on competitive games) on primary school student's motivation. The program involved the participation of 227 (114 males and 113 females) students belonging to fifth and sixth grade ( $M=9.79$; $\mathrm{DT}=1.30$ ) from state school in Galicia, Spain. The design was a quasi-experimental pre and post-test. The control group (CG) and Experimental Groups (Competitive Group -EG1- and Cooperative Group -EG2-) consisted of 61 (CG), 82 (EG1) and 84 (EG2) students, respectively. The Motivation Questionnaire in Physical Education for Primary Education (MQPE-PE) was used to measure the schoolchildren motivation. The results show that there are statistically significant differences in EG1 in IM $p=.008)$; EM ( $p<.001)$ and in DEM ( $p=.020)$. In EG2 only these differences have been found in IM ( $p=.030)$ and in DEM $(p=.034)$. Cooperative games caused significant improvements in IM while the opposite effect is observed in the competitive games participants; IM decreases and EM and DEM increases. Keywords: Self-Determined motivation; Physical education; Primary school education; Innovative strategies.
\end{abstract}

\footnotetext{
Corresponding author. Facultad de Ciencias de la Educación-Campus Norte. Avda. Xoán XXII, s/n. 15872. Santiago de Compostela, Spain.

E-mail: geno.rodriguez@usc.es

Supplementary Issue: Spring Conferences of Sports Science. International Seminar of Physical Education, Leisure and Health, 17-19 June 2019. Castelo Branco, Portugal.

JOURNAL OF HUMAN SPORT \& EXERCISE ISSN 1988-5202

(c) Faculty of Education. University of Alicante.

doi:10.14198/jhse.2019.14.Proc4.82
} 


\section{INTRODUCTION}

The motivation in Physical Education (PE) classes is related to the commitment to practice physical activity and sports, with improvements in social relationships, cooperation, responsibility, or the learning of certain motor skills. Different studies focused on teaching-learning, such as the use of methodologies based on cooperation (Navarro-Patón, Basanta-Camiño, \& Abelairas-Gómez, 2017) or competition (Navarro, Cons, \& Eirín, 2018), show different influences on the motivation according to gender. The objective of this work was to analyse the effects of two Didactic Units (DU) of EF, one based on cooperative games and the other on competitive games, on intrinsic motivation (IM), external regulation (ER) and demotivation (DEM) in Primary School (PS) students.

\section{MATERIAL AND METHODS}

\section{Participants}

A longitudinal, descriptive, analytical and comparative relational study was carried out, involving 227 students (113 girls and 114 boys) of PS from 3 schools in Galicia (Spain), with an age range between 9 and 11 years old $(M=9.79$, $D T=1.30)$. It is a sample for convenience, divided into 3 groups: the control $(C G)$ and Experimental Groups (Competitive Group -EG1- and Cooperative Group -EG2-) consisted of 61 (CG), 82 (EG1) and 84 (EG2) students, respectively.

\section{Measures}

The Motivation Questionnaire in Physical Education for Primary Education (MQPE-PE) was applied (Leo, García-Fernández, Sánchez-Oliva, Pulido, \& García-Calvo, 2016). It consists of 18 items on a Likert scale of 5 points, where the variables of IM, ER and DEM were analysed.

\section{Procedures}

In compliance with the provisions of the Declaration of Helsinki and with the informed consent of students, they completed the MQPE-PE at first (pre-test). Afterwards, the control group continued with the normal development of their Physical Education teacher's program, while with the Experimental Group 1 (EG1) students (competitive games) an UD was developed for 3 weeks ( 6 sessions) and, with Experimental Group 2 (EG2) an UD of cooperative games in the same time interval was applied.

\section{Analysis}

The quantitative variables are summarized in their mean and standard deviation (SD). A comparison of means (ANOVA) was made to analyse the previous differences between groups in the measurements of each of the variables. Parametric tests (t-Student) of related samples were used to observe the pre- and post-intervention differences in each of the groups and according to gender. The IBM SPSS Statistics v.20.0 Program was used, establishing a 95\% confidence interval.

\section{RESULTS}

Pre-intervention analyses indicated that the groups were homogeneous with respect to IM $(P=.667)$ (Control Group ( $M=4.61 ; S D=.40)$; Experimental Group $1(M=4.58 ; S D=.65)$; Experimental Group $2(M=4.66$; $\mathrm{SD}=.62$ ), but not in terms of Extrinsic Motivation ( $p<.001$ ) (Control Group ( $M=3.20 ; S D=1.11)$; Experimental Group $1(\mathrm{M}=1.73 ; \mathrm{SD}=1.10)$; Experimental Group $2(\mathrm{M}=3.53 ; \mathrm{SD}=1.27)$, or in the Demotivation ( $\mathrm{p}=.001)$ (Control Group ( $M=1.18 ; S D=.73$ ); Experimental Group $1(M=1.10 ; S D=.22)$; Experimental Group $2(M=1.45$; $\mathrm{SD}=.81$ ). Regarding the analysis of related samples in each group globally, no statistically significant 
differences were observed in the control group in any of the dimensions studied, IM ( $p=.965)$; EM ( $p=.391)$ and DEM ( $p=.674$ ); have been found in Experimental Group 1 in IM (t 83=2.70; $p=.008$ ); EM (t $83=-3.73$; $p<.001)$ and in DEM ( $83=-2.36 ; p=.020)$, while in Experimental Group 2 these differences were found only in IM ( $\mathrm{t} 81=-2.21 ; \mathrm{p}=.030)$ and in DEM ( $81=2.15 ; \mathrm{p}=.034)$. Regarding the analysis of related samples in each group according to gender and analysing the Experimental Groups, statistically significant differences were observed in Experimental Group 1 in children in IM ( $47=2.39 ; p=.021)$ and EM (t $47=-2.80 ; p=.007$ ) and in girls in EM (t $35=-2.60 ; p=.013)$ and DEM ( $35=-2,52 ; p=.016)$, whereas in Experimental Group 2 only these differences were found in girls in IM ( $\mathrm{t} 33=-2.65 ; p=.012)$ and in EM ( $33=-2.09 ; p=.044)$.

\section{DISCUSSION}

The results show positive effects of cooperative methodology used on the EG2, increasing IM significantly and decreasing the DEM, as in studies of Navarro-Patón et al. (2017), while in EG1, where competitive games have been applied, EM and DEM have increased significantly and IM has decreased, as in studies of Navarro et al. (2018).

Depending on gender, after the application of cooperative games, IM and EM have increased significantly in girls, but not in boys (Navarro-Patón et al., 2017), which may be due to the fact that cooperative games need the participation of all for the achievement of the final group objective (Navarro-Patón \& Basanta-Camiño, 2015). With competitive games, in girls, the EM and the DEM increases, and in boys the IM and the EM decreases (Navarro et al., 2018).

\section{CONCLUSIONS}

Cooperative games provoke significant improvements in IM, slightly higher in girls (the DEM decreases, and is lower in girls.) With the competitive games the opposite effect is observed, decreasing the IM in a general way and increasing the EM and the DEM, with higher incidence in girls.

\section{REFERENCES}

Leo, F.M., García-Fernández, J.M., Sánchez-Oliva, D., Pulido, J.J., \& García-Calvo, T.M. (2016). Validación del cuestionario de motivación en Educación Física en educación primaria (CMEF-EP). Universitas Psychologica, 15 (1), 315-326. https://doi.org/10.11144/javeriana.upsy15-1.vmpe

Navarro-Patón, R. \& Basanta-Camiño, S. (2015). Juegos y actividades cooperativas para educación física, deporte escolar y campamentos juveniles. En V. Arufe (Ed.), Actas del V congreso mundial del deporte escolar, educación física y psicomotricidad. Sportis: A Coruña. https://doi.org/10.17979/sportis.2016.2.3.1783

Navarro-Patón, R., Basanta-Camiño, S., \& Abelairas-Gómez, C. (2017). Los juegos cooperativos: incidencia en la motivación, necesidades psicológicas básicas y disfrute en Educación Primaria. Sportis Scientific Technical Journal, 11 (3), 589-604. https://doi.org/10.17979/sportis.2017.3.3.2088

Navarro, R., Cons, M., \& Eirín, R. (2018). Efecto de una unidad didáctica basada en juegos competitivos en la motivación, necesidades psicológicas básicas y disfrute en alumnado de Educación Primaria. Sportis Scientific Technical Journal, 4 (1), 111-125. https://doi.org/10.17979/sportis.2018.4.1.2900

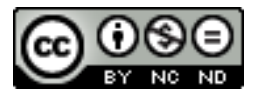

This work is licensed under a Attribution-NonCommercial-NoDerivatives 4.0 International (CC BY-NC-ND 4.0). 


\title{
Physical activity index for Galician schoolchildren of primary school education according to age and gender
}

\author{
RUBÉN NAVARRO-PATÓN ${ }^{1}$, JOSÉ EUGENIO RODRIGGUEZ-FERNÁNDEZ1 ${ }^{1}$, BEATRIZ OLIVEIRA - \\ PEREIRA ${ }^{2}$ \\ 1 University of Santiago de Compostela, Spain \\ 2University of Minho, Research Centre on Child Studies, Portugal
}

\begin{abstract}
The acquisition of an active lifestyle in childhood and adolescence help it to be maintained in adult life. For this reason, it is essential to know the current situation of the number of schoolchildren who are active in their free time, both at school and when they are going to school. Therefore, the objective of this study has been to know the rates of children school physical activity practice, voluntary and leisure time in schoolchildren aged between 10 to 12 years, by gender. Participants were primary school children $(n=1009)$, aged between 10 t0 12 years old, (boys=507; girls= 502); average age $=10.85, S D=0.79$ who were enrolled in different centres in Galicia (Spain). Statistically significant differences were found according to gender in the school physical activity index $(p<.001)$ and in voluntary physical activity practice index $(p<.001)$, being higher in boys than in girls. Statistically significant differences were also observed for age $(p<.001)$. Physical activity rates of Galician schoolchildren at school and leisure are higher in boys than girls. School physical activity index decreases as school age increases. Keywords: Physical activity index; Primary education; Schoolchildren; Galicia.
\end{abstract}

\footnotetext{
Corresponding author. Facultad de Ciencias de la Educación-Campus Norte. Avda. Xoán XXII, s/n. 15872. Santiago de Compostela, Spain.

E-mail: geno.rodriguez@usc.es

Supplementary Issue: Spring Conferences of Sports Science. International Seminar of Physical Education, Leisure and Health, 17-19 June 2019. Castelo Branco, Portugal.

JOURNAL OF HUMAN SPORT \& EXERCISE ISSN 1988-5202

(c) Faculty of Education. University of Alicante.

doi:10.14198/jhse.2019.14.Proc4.82
} 


\section{INTRODUCTION}

The World Health Organization (WHO) has been developing promotion and education programs for school health for more than three decades due to increase in sedentary lifestyles and unhealthy lifestyles (Muñoz, Fernández, \& Navarro, 2015), because the majority of schoolchildren aged 5-17 years do not reach the world recommendations on physical activity for health, which should mean 60 minutes a day of moderate to vigorous physical activity (WHO, 2010). Therefore, the objective of this study has been to know the rates of practice of school physical activity, voluntary and in their leisure time in schoolchildren aged between 10 to 12 years, as well as gender differences.

\section{MATERIAL AND METHODS}

\section{Participants}

1009 primary school students participated, 507 were boys and 502 girls; average age $=10.85, S D=0.79$ who were enrolled in different centres in provinces of Lugo, A Coruña, Ourense and Pontevedra (Galicia, Spain).

\section{Measures}

The Inventory of Physical Activity for Schoolchildren (IAFHE) validated by García-Cantó (2011) was used for the evaluation of physical activity indexes of students regarding their leisure physical-sport activity, their physical-sport practice in school context and physical-sport practice in leisure time. Possible range of values for the three indexes is $1-5$.

\section{Procedures}

To carry out the study, detailed information was provided and collaboration was requested from different educational centres. Likewise, permission was requested to parents, mothers and/or legal guardians of participating children. Students only participated in the study with written authorization from their legal representatives. After offering a brief initial explanation and resolving the existing doubts, a time of 25 minutes was provided to answer the questionnaire. The instrument was applied by researchers during a Physical Education (PE) session that the corresponding teacher authorized altruistically for this task. For this, it was requested that the teacher was not present, in order to avoid interference in the responses of the students. A time of 20 minutes was provided to answer the questionnaire: additionally time was given for explanation and resolution of doubts before starting.

\section{Analysis}

Descriptive statistical analyses (average and standard deviation) were performed for all dependent variables. Subsequently, after verifying that data complied with the assumptions of parametric statistical analyses, a multivariate analysis of variance (MANOVA), of two factors (age and gender), was carried out to examine differences between boys and girls in terms of their physical activity index. The size of the effect was estimated by eta squared $(\eta 2)$. All the statistical analyses were performed with the SPSS program version 18.0 for Windows. The level of statistical significance was established at $p=.05$.

\section{RESULTS}

A significant main effect of gender was observed in school physical activity practice index $[F(1,1003)=$ $19,392, p<.001, \eta 2=.019]$, and in the index of voluntary physical activity practice $[F(1,1003)=61,099, p$ $<.001, \eta 2=.057$ ], being higher in boys than girls. There was also a significant main effect on the age factor 
in the school physical activity index $[F(2,1003)=15,141, p<.001, \eta 2=.029]$, so that as age increases the physical activity index decreases.

\section{DISCUSSION}

If we focus on the levels of practice according to gender, as in our study, different investigations indicate a lower level of sports practice in girls with respect to boys (García-Cantó, 2011; Pantoja \& Montijano, 2012; Villagrán, Rodríguez-Martín, Novalbos, Martínez, \& Lechuga, 2010). In the case of differences according to age, these indexes decrease as the age of schoolchildren increases, confirming studies such as Hermoso, García \& Chinchilla (2010).

\section{CONCLUSIONS}

Galician schoolchildren physical activity rates are higher in boys than girls and decrease as age of schoolchildren increases.

\section{REFERENCES}

García-Cantó, E. (2011). Niveles de actividad física habitual en escolares de 10 a 12 años de la Región de Murcia. Tesis (Doctorado) - Departamento de Expresión Plástica, Musical y Dinámica. Universidad de Murcia, Murcia. https://doi.org/10.17533/udea.penh.v20n2a04

Hermoso, Y.M., García, A.V., \& Chinchilla, J.L. (2010). Estudio de la ocupación del tiempo libre de los escolares. Retos: Nuevas Tendencias en Educación Física, Deporte y Recreación, 18, 9-13.

Muñoz, A., Fernández, N. \& Navarro, R. (2015) Estudio descriptivo sobre los hábitos saludables en alumnado de Primaria desde la educación física escolar. Sportis. Scientific Technical Journal, 1 (1), 87-104. https://doi.org/10.17979/sportis.2015.1.1.1402

Organización Mundial de la Salud (OMS) (2010). Recomendaciones Mundiales sobre la actividad física para la salud. Ginebra, Suiza. Available at: http://whqlibdoc.who.int/publications/2010/9789243599977 spa.pdf

Pantoja, A., \& Montijano, J. (2012). Estudio sobre hábitos de actividad física saludable en niños de Educación Primaria de Jaén capital. Apunts. Educación Física y Deportes, 107, 13-23. https://doi.org/10.5672/apunts.2014-0983.es.(2012/1).107.01

Villagrán, S., Rodríguez-Martín, A., Novalbos, J. P., Martínez, J. M., \& Lechuga, J. L. (2010). Hábitos y estilos de vida modificables en niños con sobrepeso y obesidad. Nutrición Hospitalaria, 25(5), 823831.

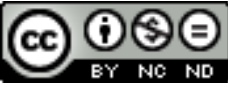

This work is licensed under a Attribution-NonCommercial-NoDerivatives 4.0 International (CC BY-NC-ND 4.0). 


\title{
Fitness professionals: Narrative review
}

\author{
LILIANA RAMOS ${ }^{1,2}$, DULCE ESTEVES ${ }^{3,4}$, SUSANA FRANCO ${ }^{1,2}$, ISABEL VIEIRA ${ }^{1,2}$, VERA SIMÕES ${ }^{1,2}$ \\ ${ }^{1}$ Sport Science School of Rio Maior, Polytechnic Institute of Santarém, Portugal \\ ${ }^{2}$ Centre of Research on Quality of Life, CIEQV, Portugal \\ ${ }^{3}$ University of Beira Interior: Department of Sports Sciences, UBI, Covilhã, Portugal \\ ${ }^{4}$ Research Centre in Sport, Health and Human Development, CIDESD, Portugal
}

\begin{abstract}
The increasing number of fitness professionals is one of the results associated with the growth of this industry. Understanding the sociodemographic characteristics and different variables related to the intervention of fitness professionals in Portugal will increase the knowledge about this work activity and can also contribute to the improvement of their working and living conditions. This study aims to carry out a narrative review in order to identify the main characteristics of fitness professionals. This identification will allow the building of the conceptual bases necessary for the construction of an instrument that will help to characterize these professionals. The research obtained seven results, three of them reports and four descriptive studies. Most of the characterization variables of fitness professionals refer to age, gender, place of residence and qualifications regarding sociodemographic aspects. Regarding aspects related to the profession, the professionals were characterized mainly in terms of hours of work, salary and the functions performed. Based on the results, the main characteristics were identified and allow us to propose a new evaluation instrument: a questionnaire that will characterize fitness professionals in Portugal - "VIDAPROFIT". Keywords: Fitness professionals; Narrative review; Sociodemographic data; Characteristics of the profession.
\end{abstract}

Corresponding author. Av. Dr. Mário Soares n 110, 2040-413 Rio Maior, Portugal.

E-mail: lilianaramos@esdrm.ipsantarem.pt

Supplementary Issue: Spring Conferences of Sports Science. International Seminar of Physical Education, Leisure and Health, 17-19 June 2019. Castelo Branco, Portugal.

JOURNAL OF HUMAN SPORT \& EXERCISE ISSN 1988-5202

(c) Faculty of Education. University of Alicante.

doi:10.14198/jhse.2019.14.Proc4.82 


\section{INTRODUCTION}

The number of places that provide fitness services and of participants of this type of activity has increased sharply (EHFA, 2012). Fitness related professions have grown in response to this increase (Papadimitriou \& Karteroliotis, 2000). The provision of a quality service that leads to the satisfaction of its participants is necessary, with industry professionals playing a very active role in this regard (Viallon et al., 2003). The objective of this study is to carry out a narrative review on the characteristics of fitness professionals (FPs), revealing the most used variables for this purpose. From this narrative review we intend, along with other methodological steps, to construct and validate the questionnaire "VIDAPROFIT" (questionnaire characterizing the FPs in Portugal).

\section{MATERIAL AND METHODS}

For this study, studies and reports were searched on the databases MEDLINE/PubMed, SCOPUS, DIALNET, Web of Science and Google Scholar from the year 2000 to the present day, as well as on the websites of relevant international organizations of the fitness industry. The keywords used were "fitness professionals", "fitness instructors", "exercise professionals", "exercise physiologists", "exercise experts" and "personal trainers (PTs)". The words "report", "profile", "characterization" and "characteristics" were also associated. The selection of the studies was based on the following inclusion criteria: $a$ ) reports and b) descriptive studies. The exclusion criteria established were articles written in other languages besides English, Spanish or Portuguese.

\section{RESULTS}

The research led to 7 results, of which 3 were reports and 4 were descriptive studies (Table 1).

Table 1. Reports \& descriptive studies

\begin{tabular}{|c|c|}
\hline $\begin{array}{l}\text { Reports } \\
\text { (name/year/country) }\end{array}$ & Sample | Results \\
\hline Profile of the Fitne & ample: $27500 \mathrm{FPs} \mid 56 \%$ women and $44 \%$ men $\mid$ Average age $=32$ years \\
\hline Industry in $A u$. & old | Most worked in Victoria | 32\% FPs full-time | Wage per week, gross \\
\hline 2016 & $\begin{array}{l}=\text { AUD } 692 \text { | Majority did not have a contract of employment | Majority had } \\
\text { certificate level III/IV, } 21 \% \text { bachelor degree and } 14 \% \text { with graduation in } \\
\text { the sport area | FPs with more qualifications had more working hours | } \\
\text { Majority worked in chains of gyms, own business or paid income. }\end{array}$ \\
\hline Salary Report for & Sample: More than $3000 \mathrm{FPs} \mid 75 \%$ of FPs had bachelor degree or \\
\hline & PT \\
\hline & wo) | Health \\
\hline & requent in more educated \\
\hline & $\begin{array}{l}\text { FPs | Since 2010, the hourly and annual value earned by FPs has } \\
\text { remained similar, with the exception of those who have achieved } \\
\text { advanced certifications. }\end{array}$ \\
\hline $\begin{array}{l}\text { Report about FPs } \\
2018 \text { | Portugal }\end{array}$ & $\begin{array}{l}\text { Sample: Approximately } 14000 \mathrm{FPs} \mid 62 \% \text { men and } 38 \% \text { women } \mid \text { Average } \\
\text { age }=35 \text { years old | } 50 \% \text { live in Lisbon and Porto | About } 65 \% \text { of the } \\
\text { foreianers are Brazilian and about } 25 \% \text { are from the European Union }\end{array}$ \\
\hline
\end{tabular}




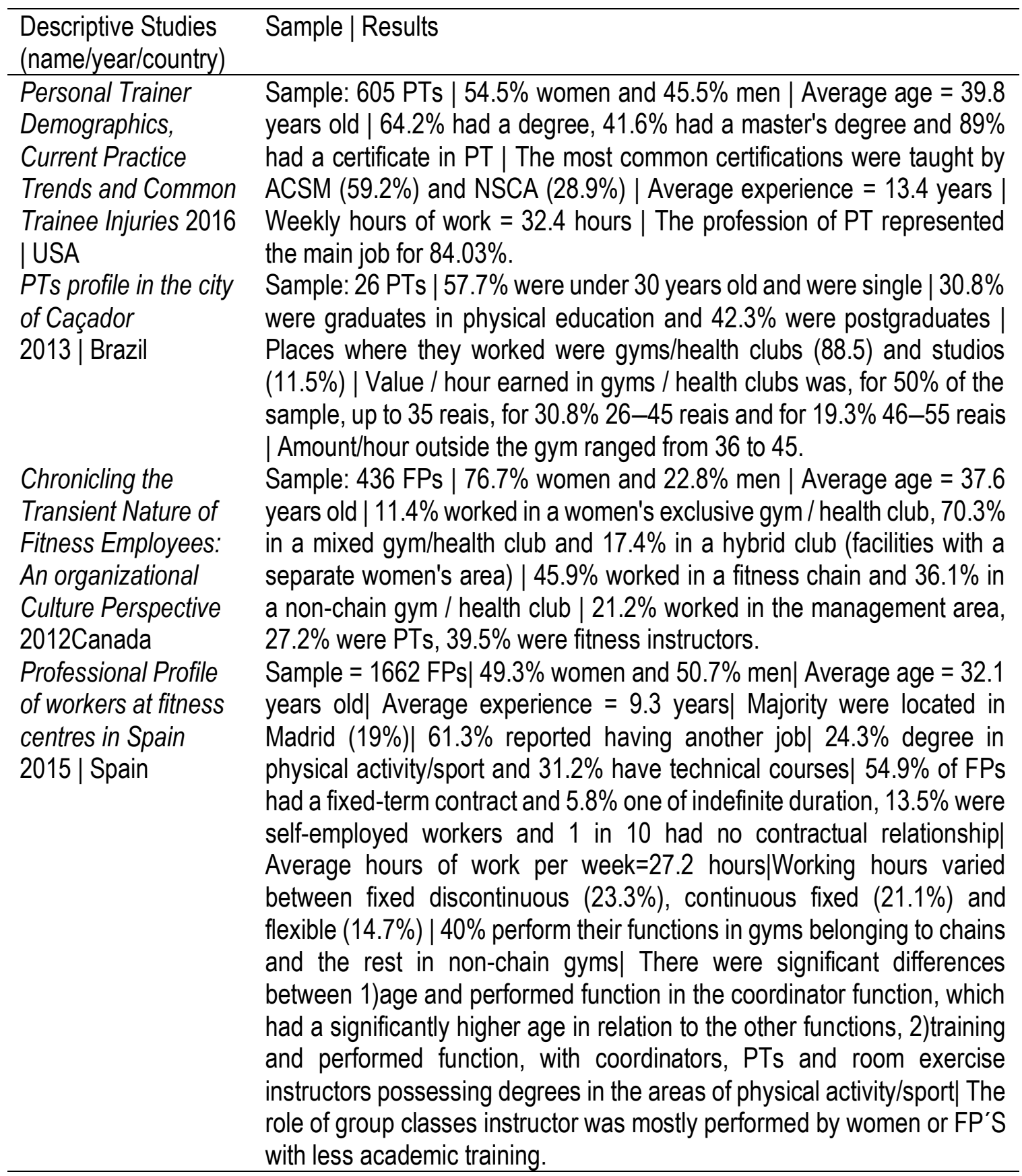

\section{DISCUSSION}

Most of the characterization variables of the FPs refer to sociodemographic aspects such as age, gender, place of residence and training. Regarding aspects related to their profession, the professionals were characterized mainly in terms of hours of work, salary and functions performed. This review allowed us to verify that there is a set of sociodemographic and other variables, related to specific aspects of FPs, that are presented in several studies/reports that can also be useful for making inferences about fitness professionals in different countries. 


\section{CONCLUSIONS}

This review intends to serve as a basis for the definition of variables that will constitute the questionnaire "VIDAPROFIT" (questionnaire characterizing FPs in Portugal), and thus contribute to an increasing knowledge about these professionals and allow the subsequent improvement of their working and living conditions.

\section{REFERENCES}

American Council on Exercise. (2013). Salary report for health \& fitness professionals. San Diego, CA.

Boned, C., Felipe, J., Barranco, D., Grimaldi-Puyana, M., \& Crovetto, M. (2015). Perfil profesional de los trabajadores de los centros de fitness en España. Rev Int Med Cienc Ac, 15(58), 195-210. https://doi.org/10.15366/rimcafd2015.58.001

EHFA. (2012). European health \& fitness association annual report 2012. Brussels: European Health \& Fitness Association.

Fitness Australia - The Health \& Fitness Association. (2016). Profile of the fitness industry in Australia Fitness industry workforce. Alexandria.

Infografia dos Técnicos de Desporto em Portugal. (2018). Instituto Português da Juventude e Desporto. Lisbon.

Melton, D., Katula, J. A., \& Mustian, K. (2008). The current state of personal training: An industry perspective of personal trainers in a small southeast community. J Strength Cond Res, 22, 883-889. https://doi.org/10.1519/jsc.0b013e3181660dab

Mineiro, L., Scolaro, A., De Assis Bardy, J., \& Da Rocha, R. (2013). Perfil dos personal trainers atuantes na cidade de Caçador/SC. RBPFE, 7, 41.

Papadimitriou, D. A., \& Karteroliotis, K. (2000). The service quality expectations in private sport and fitness centers: A reexamination of the factor structure. SMQ, 9(3), 157-164.

Viallon, R., Camy, J. F., \& Collins. (2003). The European integration of a new occupation, the training and education strategies of national professional organizations: The case of the fitness sector in France and the United Kingdom. Manag Leisure, 8, 85-96. https://doi.org/10.1080/1360671032000085693

Waryasz, G., Daniels, A., Gil, J. A., Surci, V., \& Eberson, C.P. (2016). Personal trainer demographics, current practice trends and common trainee injuries. Orthop Rev (Pavia), 8 (6600). https://doi.org/10.4081/or.2016.6600

\section{(2) $\odot \Theta \Theta$}

This work is licensed under a Attribution-NonCommercial-NoDerivatives 4.0 International (CC BY-NC-ND 4.0). 


\title{
Competence towards Physical Education inclusion: Self-perception of Galician future teachers of Primary School Education
}

\author{
JOSÉ EUGENIO RODRÍGUEZ-FERNÁNDEZ1 ${ }^{1}$ RUBÉN NAVARRO-PATÓN ${ }^{1}$, BEATRIZ OLIVEIRA - \\ PEREIRA 2 \\ 1 University of Santiago de Compostela, Spain \\ 2University of Minho, Research Centre on Child Studies, Portugal
}

\begin{abstract}
Quality Physical Education needs to adopt methodologies that allow the equal participation of all students, especially of those who have some type of disability. According to this it is necessary that teachers are trained regarding inclusion. The objective of this research was to study the perceived self-efficacy of the future teachers of Physical Education from three public Universities of Galicia (Spain) at the end of their studies. We have in this sample 112 students: 43 from University of Coruña, 36 from University of Santiago de Compostela and 33 from University of Vigo; 42,9\% were women and $57,1 \%$ men between 19 to 35 years old (M 21.43 SD=2.75). The Scale of Self-Efficacy in Teachers of Physical Education for Students with Disabilities (EA-PEF-AD) was used to collect data. We observed a significant main effect of gender on the perception of self-efficacy in Intellectual disability (ID) $[F(1,106)=4.060, p=.048, \eta 2=.033]$, in Physical Disability (FD) $[F(1,106)=6.796, p=.010, \eta 2=.060]$ and in Visual Disability $(V D)[F(1,106)=4.255, p=.042$, $\eta 2=.039]$. Disability training is an effective mean to improve perception of self-efficacy and disposition towards the inclusion of future Physical Education teachers, although this is lower in the female gender. Keywords: Self-efficacy; Inclusion; Teachers; Physical Education; Primary education.
\end{abstract}

Corresponding author. Facultad de Formación de Profesorado. Avda. Ramón Ferreiro, s/n. 27001. Lugo, Spain.

E-mail: ruben.navarro.paton@usc.es

Supplementary Issue: Spring Conferences of Sports Science. International Seminar of Physical Education, Leisure and Health, 17-19 June 2019. Castelo Branco, Portugal.

JOURNAL OF HUMAN SPORT \& EXERCISE ISSN 1988-5202

(c) Faculty of Education. University of Alicante.

doi:10.14198/jhse.2019.14.Proc4.82 


\section{INTRODUCTION}

The scientific evidence regarding perception of self-efficacy (SEP) towards inclusion has indicated a low perception on part of the teaching staff (Block \& Obrusnikova, 2007; Rust \& Sinelnikov, 2010), being this one of the main reasons for inadequate educational response. It has been shown that teachers who receive training on disability perceive themselves as more capable and have a better predisposition towards inclusion (Hwang \& Evans, 2011). The objective of this study was to study the perceived self-efficacy (SEP) of the future teachers of Physical Education (PE) from three Universities of Galicia (Spain).

\section{MATERIAL AND METHODS}

\section{Participants}

112 students from three public Universities of Galicia (Spain) took part in the study: 43 from University of Coruña, 36 from University of Santiago de Compostela and 33 from University of Vigo. 48 were women $(42.9 \%)$ and 64 men $(57.1 \%)$, with 19 to 35 years old $(M=21.43 \mathrm{SD}=2.75)$ of the Degree in Primary School Education (mention in Physical Education).

\section{Measures}

It was used the Scale of Self-Efficacy in Teachers of Physical Education for Students with Disabilities (EAPEF-AD) (Block, Hutzler, Barak, \& Klavina, 2013), whose Spanish version has been validated in previous studies (Reina, Hemmelmayr, \& Sierra-Marroquín, 2016), and three subscales are mainly collected: Intellectual Disability (ID), Physical Disability (FD) and Visual Disability (VD).

\section{Procedures}

In compliance with Declaration of Helsinki provisions and with informed consent of students, they completed the EA-PEF-AD after concluding specific training subject regarding inclusion in the specialty of Physical Education.

\section{Analysis}

Descriptive statistical analyses were performed for all dependent variables, proceeding with a multivariate analysis of variance (MANOVA) of two factors: University and gender. The statistical analysis was performed with SPSS v. 18.0 for Windows. The level of statistical significance was established at $p=.05$.

\section{RESULTS}

Table 1 shows the SEP at the end of the mention of PE studies for each group of disabilities according to University and gender. We observed a significant main effect of gender in the SEP in ID $[F(1,106)=4.060$, $\mathrm{p}=.048, \eta 2=.033]$, in $\mathrm{DF}[\mathrm{F}(1,106)=6.796, \mathrm{p}=.010, \eta 2=.060]$ and in $\operatorname{VD}[\mathrm{F}(1,106)=4.255, \mathrm{p}=.042, \eta 2=.039]$. No significant main effect was observed in the University factor or in the interaction of both factors in any of the variables studied. 
Table 1. Perceptions of global self-efficacy and according to the gender of the students of the three universities

\begin{tabular}{|c|c|c|c|c|c|c|c|}
\hline \multirow[b]{2}{*}{ University } & & \multicolumn{2}{|c|}{ Total } & \multicolumn{2}{|c|}{ Men } & \multicolumn{2}{|c|}{ Women } \\
\hline & & $M$ & $D E$ & $M$ & $D E$ & $M$ & $D E$ \\
\hline \multirow[t]{3}{*}{ Coruña (n=43) } & ID & 3.14 & .53 & 3.23 & 11 & 3.06 & .13 \\
\hline & FD & 3.11 & .59 & 3.33 & .12 & 3.02 & .14 \\
\hline & VD & 3.12 & .65 & 3.11 & .12 & 3.04 & .14 \\
\hline \multirow{3}{*}{$\begin{array}{l}\text { Santiago } \\
(\mathrm{n}=36)\end{array}$} & ID & 3.17 & .56 & 3.20 & 11 & 3.03 & .17 \\
\hline & FD & 3.13 & .52 & 3.23 & .12 & 3.02 & .18 \\
\hline & VD & 3.02 & .57 & 3.21 & .12 & 3.09 & 18 \\
\hline \multirow[t]{3}{*}{ Vigo $(n=33)$} & ID & 3.08 & .62 & 3.27 & .14 & 2.97 & .13 \\
\hline & FD & 3.17 & .76 & 3.23 & 16 & 2.81 & .14 \\
\hline & VD & 3.07 & .66 & 3.17 & .15 & 2.97 & .14 \\
\hline
\end{tabular}

Note. ID = Intellectual Disability; FD = Physical Disability; VD = Visual Disability. The ID, FD and VD scales have a range of 1-5.

\section{DISCUSSION}

The results obtained are in agreement with those observed in previous investigations as in studies of Rust \& Sinelnikov (2010) and Taliaferro, Hammond, \& Wyant (2015). Paying attention to the differences observed between men and women according to the types of disability, it was appreciated that the inclusion of a student with ID, FD and VD aroused the lowest value of perception of self-efficacy in the female gender, as in the Abellán, Sáez-Gallego, Férriz, Reina \& Navarro-Patón (2019) studies, but which differ from a Meegan \& Macphail (2006) studies, in which it is affirmed that women are the most predisposed.

\section{CONCLUSIONS}

Training on disability issues received at public universities of Galicia (Spain) are an effective mean to improve self-efficacy perception and therefore the willingness to include future Physical Education teachers, although this is less in the gender female.

\section{REFERENCES}

Abellán, J., Sáez-Gallego, N., Férriz, R., Reina, R., \& Navarro-Patón, R. (2019). Percepción de autoeficacia hacia la inclusión en futuros maestros de educación física. Revista de Psicología del Deporte, 28 (1), 143-156. https://doi.org/10.6018/sportk.343011

Block, M.E. \& Obrusnikova, I. (2007). Inclusion in physical education: a review of the literature from 19952005. Adapted Physical Activity Quarterly, 24, 103-124. https://doi.org/10.1123/apaq.24.2.103

Block, M.E., Hutzler, Y., Barak, S., \& Klavina, A. (2013). Creation and validation of the self- efficacy instrument for physical education teacher education majors toward inclusion. Adapted Physical Activity Quarterly, 29, 184-205. https://doi.org/10.1123/apaq.30.2.184

Hwang, Y. \& Evans, D. (2011). Attitudes towards inclusion: gaps between belief and practice. International Journal for Special Education, 26 (1), 136-146.

Meegan, S. \& Macphail, A. (2006). Irish physical educators' attitude toward teaching students with special educational needs. European Physical Education Review, 12 (1), 75-97. https://doi.org/10.1177/1356336x06060213 
Reina, R., Hemmelmayr, I., \& Sierra-Marroquín, B. (2016). Autoeficacia de profesores de educación física para la inclusión de alumnos con discapacidad y su relación con la formación y el contacto previo. Psychology, Society \& Education, 8 (2), 93-103. https://doi.org/10.25115/psye.v8i2.455

Rust, R. \& Sinelnikov, O. (2010). Practicum in a self-contained environment: pre-service teacher perceptions of teaching students with disabilities. Physical Educator, 67 (1), 33-46.

Taliaferro, A.R., Hammond, L., \& Wyant, K. (2015). Preservice physical educators' self-efficacy beliefs toward inclusion: the impact of coursework and practicum. Adapted Physical Activity Quarterly, 32, 49-57. https://doi.org/10.1123/apaq.2013-0112

\section{(c) $(\mathrm{B})(\mathrm{EY}$}

This work is licensed under a Attribution-NonCommercial-NoDerivatives 4.0 International (CC BY-NC-ND 4.0). 


\title{
Principal complaints and reference to spine pain in young tennis players during a tournament: Data from massage therapy care
}

\author{
LIIVIA PIMENTA RENÓ GASPAROTTO ${ }^{1}$, GUILHERME DA SILVA GASPAROTTO², MARTA SILVA DE \\ SOUZA ${ }^{1}$, CÉLIA REGINA ALVES DE ARAÚJO ${ }^{1}$, CLÁUDIA MONTEIRO DOS SANTOS BONTORIN ${ }^{1}$ \\ 1 Instituto Federal do Paraná, Campus Curitiba, Brazil \\ 2Instituto Federal do Paraná, Campus Pinhais, Brazil
}

\begin{abstract}
Tennis practice requires locomotor abilities from young players and causes different osteomuscular overcharges. Sports massage therapy is designed to prepare athletes to achieve the desired performance, relieve muscle tension, and prevent injuries. The aim of this study was to identify the main physical complaints in young tennis athletes who were treated with massage therapy during a tournament. Participants comprised 87 athletes of both genders aged between 08 and 21 years. Of the $77 \%$ who reported discomfort in some area of the body, $33.3 \%$ complained of upper limb pain, $25.3 \%$ of lower limb pain, $21.8 \%$ of spinal discomfort, and $8.0 \%$ reported pain in other areas of the body. Concerning the spinal area, pain prevalence was in the lumbar region for both genders $(55 \%)$. While thorax pain was more prevalent in females, aches in more than one spot of the spinal area were more preponderant in males. It can be concluded that young tennis players suffer from some physical discomfort in the spinal area and upper and lower limbs at a high rate during tournaments. Distinct spinal pain locations among the genders call for a personalized training plan along with massage therapy treatment based on biomechanical aspects according to the life stage (childhood or adolescence). Keywords: Massage therapy; Tennis; Pain; Young people.
\end{abstract}

Corresponding author. Instituto Federal do Paraná, Campus Curitiba, Brazil.

E-mail: livia.gasparotto@ifpr.edu.br

Supplementary Issue: Spring Conferences of Sports Science. International Seminar of Physical Education, Leisure and Health, 17-19 June 2019. Castelo Branco, Portugal.

JOURNAL OF HUMAN SPORT \& EXERCISE ISSN 1988-5202

(c) Faculty of Education. University of Alicante.

doi:10.14198/jhse.2019.14.Proc4.82

VOLUME 14 | Proc4 | 2019 | S1319 


\section{INTRODUCTION}

According to Kovacs, Ellenbecker, Kibler, Roetert \& Lubbers (2014), tennis practice requires good locomotor skills and demands specificities from the musculoskeletal system. When in-season, proper care is imperative to maintain the quality and efficiency of the movements whilst staying resilient to injury. As stated by Moraska (2005), sports massage therapy promotes blood circulation in the working tissues and minimizes muscle fatigue. The aim of this study is to identify the most common complaints by children and young athletes who received massage therapy care during a tennis competition.

\section{MATERIAL AND METHODS}

\section{Participants}

52 male $(59.8 \%)$ and 35 female $(40.2 \%)$ tennis athletes totalizing 87 participants aged from 08 to 21 years (13.92 \pm 2.37$)$. They participated in the Brazilian interclub championship, in November 2018, at the club curitibano, city of Curitiba, Parana, Brazil.

\section{Measures}

The research is descriptive and retrospective based on the data of the extension project "traveling hands: massage therapy in events" of IFPR Curitiba, approved under the number 23397.000277 / 2014-35. The questionnaire reports on pain and complaints during a competition, prepared by the authors.

\section{Procedures}

A questionnaire collected information on pain complaints for the massotherapists to treat for either muscle relaxation or stimulation. All the participants received massage therapy care during a tennis competition.

\section{Analysis}

Insertion of data into an Excel spreadsheet and statistical analysis with the SPSS 24.0 software. Spinal pain among genders was evaluated via frequency distribution and the chi-squared test.

\section{RESULTS}

$77 \%$ of the 87 athletes reported pain in some region of the body. Of those who had some sort of pain, $33.3 \%$ complained of upper limb pain, $25.3 \%$ of lower limb pain, $21.8 \%$ of spinal pain, and $8.0 \%$ reported pain in other areas of the body. Focusing on the spinal area, pain prevalence was in the lumbar region for both genders $(55 \%)$. While thorax pain was more prevalent in females, aches in more than one spot of the spinal area were more preponderant in males.

Table 1. Complaint of spinal pain in young tennis players grouped according to gender

\begin{tabular}{lccc}
\hline \multicolumn{1}{c}{ SPINE PAIN } & $\begin{array}{c}\text { TOTAL } \\
\%(\mathrm{n})\end{array}$ & $\begin{array}{c}\text { MALE } \\
\%(\mathrm{n})\end{array}$ & $\begin{array}{c}\text { FEMALES } \\
\%(\mathrm{n})\end{array}$ \\
\hline \multirow{3}{*}{ Cervical } & & \multicolumn{2}{c}{$X^{2}: 6,98 ; p<0,05$} \\
Thoracic & 0 & 0 & 0 \\
Lumbar & $15 \%(03)$ & 0 & $15 \%(03)$ \\
Two or more & $55 \%(11)$ & $25 \%(05)$ & $30 \%(06)$ \\
\hline \multicolumn{4}{c}{$X^{2}:$ Qui-square test } \\
\hline
\end{tabular}




\section{DISCUSSION}

A study by Pluin, Loeffen, Clarsen, Bahr \& Verhagen (2013) on young athletes revealed discomfort or musculoskeletal lesions at a rate of $67 \%$ in-season, which corroborates the $77 \%$ found in this work. Guedes, Barbieri \& Fiabane (2010) and Silva, Cohen, Matsumoto \& Gracitelli (2005) suggested that the incidence of pain and discomfort in young athletes increases with age, reaching a lesion rate of $36 \%$ in athletes older than 14 years. The high rate of discomfort reported in this study calls for preventive measures to avoid future injuries. The sports massage therapy may be an option since it reduces muscle overcharge via tissue nutrition and relieves tension in muscle fibres, as pointed out by Moraska (2005).

This study revealed a $21.8 \%$ prevalence of pain in the spinal region, a result that corroborates the findings of Guedes et al. (2010) who determined a value of $15 \%$. Pain prevalence was in the lumbar region for both genders, thorax pain was mainly reported by females, and aches in more than one spot of the spinal area were preponderant in males. Fu, Ellenbecker, Renstron, Windler \& Dines (2008) indicated the overuse of paravertebral muscles and that the number of lesions is higher during competitions than in trainings. Torso lesions are mostly related to chronic problems whereas extremities suffer from acute complications. Thus, it is imperative to investigate the origins of back pain since it may be associated with bone growth and body development. A higher prevalence of lesions has been observed in young athletes who participate in tournaments.

\section{CONCLUSIONS}

Tennis practice is considerably demanding for the locomotor system, in particular for muscle groups and ligaments. Among the main causes of lesions are repeated motion patterns, inadequate equipment, biomechanically incorrect sport movement, and poor physical preparation. In young athletes back lesions are more predominant while elbow and shoulder injuries are more common in adults.

\section{REFERENCES}

Guedes, J.M., Barbieri, D.F., \& Fiabane, F. (2010). Lesões em tenistas competitivos. Revista Brasileira de Ciencias do Esporte 31(3), 217-229. https://doi.org/10.1590/s0101-32892010000300015

Fu, M.C., Ellenbecker, T.S., Renstron, P.A., Windler, G.S., \& Dines, D.M. (2018) Epidemiology of injuries in tennis players. Curr Rev Musculoskelet Med. 11(1), 1-5. https://doi.org/10.1007/s12178-018$\underline{9452-9}$

Kovacs, M., Ellenbecker, T., Kibler, W., Roetert, E., \& Lubbers, P. (2014) Injury trends in American competitive junior tennis players. J Med Sci Tennis. 19(1), 19-23.

Moraska, A. (2005). Sports massage: a comprehensive review. Journal of Sports Medicine and Physical Fitness 45(3), 1-11.

Pluim, B.M., Loeffen, F.G., Clarsen, B., Bahr, R., \& Verhagen, E.A.(2016) A one-season prospective study of injuries and illness in elite junior tennis. Scand J Med Sci Sports 26(5), 564-571. https://doi.org/10.1111/sms.12471

Silva, R.T. Cohen, M., Matsumoto, M.H., Gracitelli, G.C (2005). Avaliação de lesões ortopédicas em tenistas amadores competitivos. Rev Bras Ortop. 40(5).

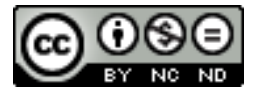

This work is licensed under a Attribution-NonCommercial-NoDerivatives 4.0 International (CC BY-NC-ND 4.0). 


\title{
Terminal actions at Brazilian's Volleyball League
}

\author{
LUIS HENRIQUE MERCANTE DA SILVA ${ }^{1}$, MÁRCIO JOSÉ KERKOSKI ${ }^{1}$, ANDERSON CAETANO \\ PAULO ${ }^{1}$, ALEXANDER HODECK ${ }^{2}$ \\ ${ }^{1}$ Federal Technological University of Brazil, Brazil \\ ${ }^{2} E B C$ Hamburg \& Chemnitz University of Technology, Germany
}

\begin{abstract}
Efficacy in volleyball is observed by success in "terminal" procedural actions of attack, block and serve, (Shondell \& Reynaud, 2005). Regarding the literature, it can be stated that studies involving observation of women's games in any category are few (Silva, Marcelino, Lacerda, \& João, 2016). The objective of this study is to associate the performance indicators (draw, block, attack) and their influence for the ranking score of the women and men's volleyball teams participating in the 2016/2017 Brazilian Super league. Twenty-four adult teams (12 men and 12 women) were investigated by using the Volleyball Information System (VIS). The linear regression in the male competition showed that aces predict $51 \%$ of the ranking score, while point attack predicts $87 \%$ and the combination of ace and serve ratio giving continuity to the game predict $92 \%$. Looking to female competitions, the point attack predicts $90 \%$, along with block / point blocking ratio returning to the opponent predict $95 \%$ and both added to the total attacks predict $97 \%$ of the ranking score. Keywords: Volleyball; Ranking; Performance; Match Analysis.
\end{abstract}

Corresponding author. Sports Science Research Group. Federal Technological University of Brazil, Brazil.

E-mail: Ihmercante@gmail.com

Supplementary Issue: Spring Conferences of Sports Science. International Seminar of Physical Education, Leisure and Health, 17-19 June 2019. Castelo Branco, Portugal.

JOURNAL OF HUMAN SPORT \& EXERCISE ISSN 1988-5202

(c) Faculty of Education. University of Alicante.

doi:10.14198/jhse.2019.14.Proc4.82 


\section{INTRODUCTION}

The most used indicator in volleyball for performance analysis is the effectiveness of the game's actions (Silva et al., 2016). Studies involving terminal actions (attack, block and serve) mostly observe games in adult male's categories (Marcelino, Mesquita, \& Afonso, 2008; Silva et al, 2016). The purpose of this study is to associate performance indicators (serve, block, attack) and reasons for the final ranking of the women's and men's teams participating in the 2016/2017 Brazilian Volleyball Super League.

\section{MATERIAL AND METHODS}

\section{Participants}

Twelve male and twelve female teams who participated in the 2016/2017 Brazilian Super League were investigated.

\section{Measures}

The numbers of points and errors points by serve, attack and blocks. The attack ratio was calculated by the attacks that generated the point divided by the wrong attacks. The serving is calculated directly by the serving point. The serve serves as the continuity of the game. The block ratio was generated by block points divided by the wrong blocks. The block continues the owner team ratio, was generated by block points added to the blocks that allowed the continuity of the actions by the team itself divided by the wrong blocks and the blocks continuity the opponent ratio was generated by block points divided by the sum of the wrong blocks and blocks that allowed the continuity of the game by the opponent.

\section{Procedures}

The data of the terminal actions of the Volleyball Information System, were extracted from the report of the Brazilian Volleyball Confederation (CBV, 2017). A total of 25,447 / 23,791 (male / female). service actions, 27,052 / 35,348 attack actions and 10,360 / 10,903 block actions were analysed. Points in the final ranking were defined as dependent variable in the model of the study. Used the procedures adopted by Drikos, Kountouris, Laios, \& Laios (2009).

\section{Analysis}

To verify the relationship between the variables a multivariate linear regression model was used. Differences between means genders were tested by the unpaired $t$ test.

\section{RESULTS}

The linear regression at male teams, showed that the variables that correlate (correlation coefficient) with the ranking's points are: ace serve (.748), points attack (.885), serve continuity ratio (.912), attack ratio (.922), block ratio (.781) and blocks continuity opponent ratio (.894). Ace serve predict $51 \%$ of the points in the ranking. The ace serve plus the points attack predict $87 \%$ of the points in the ranking and both plus serve continuity ratio (in the champion team for every 10 serves executed 01 generates direct point) predict $92 \%$ of the points ranking's. The linear regression at female teams, showed that the variables that correlate (correlation coefficient) with the ranking's points are: serve continuity ratio (.877), total attacks (.864), points attack (.954) attack ratio (.951), points block (.829), block ratio (.902) and blocks continuity the opponent ratio (.913). The points attack predicts $90 \%$ of the points ranking score, the points attack plus the blocks continuity the opponent ratio (in the champion team for every 08 blocks that return to the opponent 04 generate points for the owner team) predict $95 \%$ of the point ranking and both plus the total number of attacks predict $97 \%$ 
of the ranking score. It was observed that the mean values of male and female significantly differed $(p=0.05)$ regarding the following variables: errors serve (395.6 male / 186.5 female), the block ratio ( 0.49 male / 0.61 female) and the blocks continuity the opponent ratio ( 0.39 male / 0.44 female).

\section{DISCUSSION}

The study conducted by Castro, Souza, \& Mesquita, (2011) at the Volleyball World Cup / 2007 identified a strong association of the effectiveness of serve point (ace serve) and point attack in the final result. In the 2005 World Men's League, Marcelino et al (2008) observed that the attack and blocking determines the outcome. In the Brazilian's Super League of Men's Volleyball 2012/2013, Campos, Campos, Bezerra, \& Pellegrinotti (2015), found that the attack has the greatest impact, followed by the block and finally the serve. Marcelino et al (2010) found that among men's teams, that the serve is correlated to the final result, with the winning team averaging 1.32 points per set and that the block is a predictor of victory. This is not proved by our study. For female competitions the points blocked appeared as a predictor in the final ranking. Contrary to theory, maybe due to less aggressiveness in the female attack, the block is not a predictor of set victory (Afonso \& Mesquita, 2011).

\section{CONCLUSIONS}

The study concluded that in the men's competition ace serve provides $51 \%$ of the score in the ranking. The ace serve plus point attack's predict $87 \%$ of the score in the ranking and the two added serve continuity ratios, predict $92 \%$ of the points ranking score. In the women's competition, the point attack's predicts $90 \%$ of the points score in the ranking, the point attack's plus blocks continuity the opponent ratio predict $95 \%$ of the ranking and the two added to the total of attacks predicts $97 \%$ of the ranking score.

\section{REFERENCES}

Afonso, J., \& Mesquita, I. (2011). Determinants of block cohesiveness and attack efficacy in high-level women's volleyball. European Journal of Sport Science, 11(1), 69-75. https://doi.org/10.1080/17461391.2010.487114

Campos, F. A. D., Campos, L. C. B., Bezerra, T. A. R., \& Pellegrinotti, I. L. (2015). Eficácia do saque, ataque e bloqueio no voleibol masculino brasileiro. Cinergis, 16(4). https://doi.org/10.17058/cinergis.v16i5.6912

Castro, J., Souza, A., \& Mesquita, I. (2011). Attack efficacy in volleyball: elite male teams. Perceptual and Motor Skills, 113(2), 395-408. https://doi.org/10.2466/05.25.PMS.113.5.395-408

Drikos, S., Kountouris, P., Laios, A., \& Laios, Y. (2009). Correlates of Team Performance in Volleyball. International Journal of Performance Analysis in Sport, 9(2), 149-156. https://doi.org/10.1080/24748668.2009.11868472

Marcelino, R., Mesquita, I., \& Afonso, J. (2008). The weight of terminal actions in Volleyball. Contributions of the spike, serve and block for the teams' rankings in the World League 2005. International Journal of Performance Analysis in Sport, 8(2), 1-7. https://doi.org/10.1080/24748668.2008.11868430

Marcelino, R., Mesquita, I., Sampaio, J., \& Moraes, J. C. (2010). Estudo dos indicadores de rendimento em voleibol em função do resultado do set. Revista Brasileira de Educação Física e Esporte, 24(1), 69-78. https://doi.org/10.1590/\$1807-55092010000100007

Silva, M., Marcelino, R., Lacerda, D., \& João, P. V. (2016). Match Analysis in Volleyball: a systematic review. Montenegrin Journal of Sports Science and Medicine, 5(1), 35-46. 


\section{(c) (i) (3)}

This work is licensed under a Attribution-NonCommercial-NoDerivatives 4.0 International (CC BY-NC-ND 4.0). 


\title{
Effects of a training program in post-menopausal women
}

\author{
ALVARO DE PANO-RODÍGUEZ ${ }^{1} \triangle$, VICENÇ HERNÁNDEZ-GONZÁLEZ ${ }^{1}$, TERESA FONSECA², \\ JOAQUÍN REVERTER-MASIA ${ }^{1}$
}

${ }^{1}$ Grupo de Investigación Movimient Humà, Universidad de Lleida, Spain

${ }^{2}$ Escola Superior de Educação, Comunicação e Desporto, Instituto Politécnico da Guarda, IPG, Guarda, Portugal

\begin{abstract}
Sleep is a function that has a direct influence on health. The current investigation examined the acute relationship between exercise behaviour and self-reported sleep in older adults. For this purpose, a quantitative approach to the influence of a 10-week physical activity program (PAP) on the quality and quantity of sleep was attempted. Thirty-four community-dwelling, initially sedentary, post-menopausal women (mean age $=67.4$ years, SD $=10,8$ years) completed two days per week training program. "Time to fall asleep", "duration of sleep" and "altered sleep frequency" were estimated from the Pittsburgh Sleep Quality Index (PSQI) before and after the PAP. We found no significant differences in time to fall asleep and sleep duration after the PAP. We found a significant reduction in the altered sleep frequency after the PAP. Conclusions: The PAP had no influence on the time to fall asleep or on the duration of sleep. The PAP exerted a positive influence on the quality of sleep by reducing the frequency of its alterations. Keywords: Sleep; Physical activity program; Pittsburgh Sleep Quality Index; Post-menopausal.
\end{abstract}

Corresponding author. Campus de Cappont Av. de l'Estudi General, 425001 Lleida. Spain.

E-mail: alvarodepano@gmail.com

Supplementary Issue: Spring Conferences of Sports Science. International Seminar of Physical Education, Leisure and Health, 17-19 June 2019. Castelo Branco, Portugal.

JOURNAL OF HUMAN SPORT \& EXERCISE ISSN 1988-5202

(C) Faculty of Education. University of Alicante.

doi:10.14198/jhse.2019.14.Proc4.82 


\section{INTRODUCTION}

Sleep has a significant impact on the health and quality of life and its scarcity or low quality is associated with obesity, type 2 diabetes, hypertension and cardiovascular problems (Tobaldini et al., 2017).In the case of adults, a minimum of $7 \mathrm{~h}$ per night is recommended ("2020 Topics and Objectives - Objectives A-Z | Healthy People 2020," n.d.; Watson, Badr, Belenk, \& Bliwise, 2015). Although sleep patterns are related to genetics (Gutierrez-Amavizca, Gutierrez-Amavizca, \& Perez-Leon, 2019) and certain factors are related to the environment and to lifestyle (Wang et al., 2017), older women show a greater propensity to manifest low quality of sleep and more nocturnal sleep interruptions than any other population subgroup. A recent study suggests that sleep duration and quality sleep are associated with physical activity in elderly people (Rup", 2018). Because of this, in the present study, a quantitative approach to the influence of a 10-week physical activity program on the quality and quantity of sleep of post-menopausal women was attempted. The following hypotheses were generated: 1) continued physical exercise has a positive influence on the time it takes to get to sleep 2) continued physical exercise has a positive influence on sleep time 3) the quality of sleep is improved by continued physical exercise.

\section{MATERIAL AND METHODS}

\section{Participants}

A total of 34 post-menopausal untrained women were the object of the study, with an age range between 55 and 69 years and an average age of 67.4 (SD 10.8) years. None of the subjects presented cardiac diseases, metabolic disorders, tumour processes, or neurological disturbances. All participants signed an informed consent in which they were informed about the benefits and risks.

\section{Measures}

The Pittsburgh Sleep Quality Index (PSQI) is a validated instrument for the analysis of the quantity and quality of sleep that allows the possibility of establishing in a quantitative manner the discrimination between people with adequate sleep and people with poor sleep (Buysse, Reynolds, Monk, Berman, \& Kupfer, 1989). In the present study, the variables "time in conciliating sleep", "duration of sleep" and "altered sleep frequency" have been analysed.

\section{Procedures}

The sessions of the proposed training program lasted $40 \mathrm{~min}$. In the initial 10 minutes, a walking warm-up at low intensity was carried out on the treadmill. Later on we proceeded to exercise strength (squat, deadlift and bench press). This push and pull movements are appropriate for improving strength in older people (AragãoSantos et al., 2018).

\section{Analysis}

The normality of the distributions of the variables was tested by means of the Kolmogorov-Smirnov test for the total sample. The Student T-Test was used to evaluate the differences in the variable "sleep time" between pre-test and post-test data. The nonparametric Wilcoxon test for related samples was used in the variable "time to fall asleep" to evaluate the differences between pre-test and post-test. For the analysis of the pre/post-test differences for the categorical variables, the Pearson Chi-square test was used. The level of significance for all the analyses was set at $P<0.05$. The statistical analysis was performed using the SPSS Statistics 24 software. 


\section{RESULTS}

In the variable "time to fall asleep" there is a non-significant tendency to be reduced which is positive $(p=$ .131). In the variable "sleep hours" there are no variations between both moments (zeta $=-2.62, p=.79$ ). The quality of sleep is positively affected because the participants had a lower number of sleep disturbances $(P=.040)$ throughout the night, once the proposed training program was finished.

\section{DISCUSSION}

The first hypothesis of this study is not fulfilled because there are no significant differences in the variable "time to fall asleep". This may be due to the fact that the sample performed their training late in the afternoon and the physical exercise can have, as an immediate effect, the alteration of the circadian rhythms creating certain problems in adults when it comes to falling asleep (Weinert \& Waterhouse, 2007). This stagnation in the time necessary to fall asleep, when doing physical activity in the last hours of the day, could lead to the recommendation of physical exercise at earlier hours. The morning exercise could favour the achievement of consistent daily routines, which have been related to fewer problems in sleep conciliation (Monk, Reynolds, Buysse, DeGrazia, \& Kupfer, 2003). The second hypothesis is not fulfilled because the variable "sleep time" does not show significant differences. However, there is a slight improvement that suggests that there may be some relationship between continuous physical exercise and the number of hours of sleep. This finding would be in agreement with Murray et al (2017) who found an improvement in sleep time when the participants performed moderate-vigorous physical activity in the open air. It is possible that in this study the differences are not as remarkable as the aforementioned, due to the characteristics of the exercises proposed in our program. In our program the exercises were oriented towards the improvement of strength instead of being entirely focused on aerobic work. The third hypothesis of this study is proven to be true. A considerable improvement in sleep quality was observed taking into account that the participants showed a significant reduction of sleep disturbances throughout the night. The results of this study in terms of sleep quality coincide with Dzierzewski et al. (2014) who also found that the immediate effect of physical exercise benefited most palpably in people with sleep difficulties. This information should be taken into account in the recommendations of physical exercise when dealing with people with altered sleep.

\section{CONCLUSIONS}

- The PAP had no influence on the time to fall asleep and the duration of sleep.

- The PAP exerted a positive influence on the quality of sleep by reducing the frequency of its alterations.

\section{REFERENCES}

Besedovsky, L., Schmidt, E.-M., Linz, B., Diekelmann, S., Lange, T., \& Born, J. (2017). Signs of enhanced sleep and sleep-associated memory processing following the anti-inflammatory antibiotic minocycline in men. Journal of Psychopharmacology (Oxford, England), 31(2), 204-210. https://doi.org/10.1177/0269881116658991

Giulio Tononi, \& Chiara Cirelli. (2014). Sleep and the Price of Plasticity: From Synaptic and Cellular Homeostasis to Memory Consolidation and Integration. Neuron, 81(1), 12-34. https://doi.org/10.1016/j.neuron.2013.12.025 
Gutierrez-Amavizca, B. E., Gutierrez-Amavizca, J. P., \& Perez-Leon, J. A. (2019, April). Genetic contribution in primary sleep disorders. Revista medica del Instituto Mexicano del Seguro Social. Mexico.

Rico-Rosillo, M. G., \& Vega-Robledo, G. B. (2018). [Sleep and immune system]. Revista alergia Mexico (Tecamachalco, Puebla, Mexico : 1993), 65(2), 160-170. https://doi.org/10.29262/ram.v65i2.359

Rup", T. (2018). Sleep Duration and Sleep Quality Are Associated with Physical Activity in Elderly People Living in Nursing Homes.

Tobaldini, E., Costantino, G., Solbiati, M., Cogliati, C., Kara, T., Nobili, L., \& Montano, N. (2017). Sleep, sleep deprivation, autonomic nervous system and cardiovascular diseases. Neuroscience and Biobehavioral Reviews, 74(Pt B), 321-329. https://doi.org/10.1016/j.neubiorev.2016.07.004

Wang, S., Wu, Y., Ungvari, G. S., Ng, C. H., Forester, B. P., Gatchel, J. R., .. Xiang, Y.-T. (2017). Sleep duration and its association with demographics, lifestyle factors, poor mental health and chronic diseases in older Chinese adults. Psychiatry Research, 257, 212-218. https://doi.org/10.1016/j.psychres.2017.07.036 


\title{
"Destroying stereotypes, building on equality": Didactic intervention with Master in Teaching of Physical Education students
}

\author{
ALBA GONZÁLEZ-PALOMARES, MARÍA PERRINO-PEÑA \\ Faculty of Education, Universidad Pontificia de Salamanca, Spain
}

\begin{abstract}
This experience is focused on fighting the gender stereotypes in Physical Education (PE) teaching. This is important because future teachers are responsible of changing these non-sense and unfair practices. The population who received the intervention were 70 students of a master's in teaching in Physical Education, in the Sports Faculty of the University of Porto. The experience consisted in three different parts: first, a theoretical seminar of visual alphabetization, working with the didactic tool "Gender in the eyes: Strategies to educate with (and despite of) the photographs of the curricular materials". Secondly, we developed a practical workshop, focusing in two traditionally stereotyped contents: combat sports and a postmodern dance called Contact Improvisation. Lastly we leaded a global reflexion in order to conclude that the gender is not determinant for dealing with these contents. The students found that both -combat and rhythmic-expressive activities- can be perfectly suitable for male and female, without real or scientific reasons for considering them as stereotyped physical activities because of the gender. Keywords: Didactic intervention; Stereotypes; Sports and physical activities; Combat sports; Rhythmic-expressive activities.
\end{abstract}

Corresponding author. C/Henry Collet, 52-70. Facultad de Educación. UPSA. CP: 37007, Salamanca. Spain.

E-mail: mperrinope@upsa.es

Supplementary Issue: Spring Conferences of Sports Science. International Seminar of Physical Education, Leisure and Health, 17-19 June 2019. Castelo Branco, Portugal.

JOURNAL OF HUMAN SPORT \& EXERCISE ISSN 1988-5202

(c) Faculty of Education. University of Alicante.

doi:10.14198/jhse.2019.14.Proc4.82 


\section{INTRODUCTION}

Equality between Women and Men is one of the main principles of de European Union, what is reflected in different programmes and political actions, promoted and performed in the member countries. The approach of this teaching/didactic intervention, "Destroying stereotypes, building on equality", which has been developed under the frame of European Teaching Mobility Erasmus +KA103, started from the relevance of a gender equality in the developed societies. It should be reflected in the physical and sports practices, especially in those loaded with stereotypes.

From a sociological and pedagogical perspective, it is essential to help future PE teachers to adopt gender equality philosophy, reducing stereotypes. We consider that it is possible to remove gender stereotypes working with awareness in both formal and informal perspectives of the educational field from the earliest age. Furthermore, sport is a social sphere where inequalities are usually reflected; and we defend that it should be a way to fight, precisely, these gender inequalities.

\section{MATERIAL AND METHODS}

According to Touriñan (2011), a teaching intervention is defined as "the international action to perform some actions leading to the achievement of the pupil's integral development" (p. 283). The aim of this intervention is to the future Portuguese professionals in physical activity and sport -and possible PE teachers-, reflect on the current body culture and the gender stereotypes existing.

\section{Participants}

The population object of study consisted of the students registered in the Master in PE Teaching at basic and secondary level in the Sport Faculty of the University of Porto. In the academic period 2018/2019 it rises to 70 students, with ages between 21 and 33 years old. The sample selected was intentional and non-aleatory.

\section{Measures}

We used a didactic tool: "Gender in the eyes: Strategies to educate with (and despite of) the photographs of the curricular materials" (Rey-Cao and González-Palomares, 2017), to develop the seminar of visual literacy. It consists in 9 activities related with 8 stereotypes existing in the body and physical culture. The practical motor activities were planned and leaded by us, as well as the subsequent reflection and final dialogue through different questions asked to the students.

\section{Procedures}

The Erasmus experience was implemented between the 4th and the 8th of March of 2019. The teaching intervention last eight hours total, divided in three different seminars: visual literacy, physical and sports stereotyped activities (including combat sports and rhythmic-expressive activities) and the final reflection, which are detailed in the following section.

\section{RESULTS}

The intervention: "Destroying stereotypes, building on equality": was divided in these were the three different modules:

1. Visual Literacy Seminar (2 hours, in the classroom). Firstly we exposed to the student the social importance of photographs with images about the physical and sports culture. They also viewed the 
need of analyse to face up their professional future as teachers, being aware of the existing stereotypes in sports and physical activity field and trying to avoid transmitting them. In a second part, we worked with the didactic tool "Gender in the eyes" and the students did the 9 activities through the analysis of the photographs included in schoolbooks/manuals of PE.

2. Workshop of physical and sports stereotyped practises (4 hours, in the tatami and gymnastics hall). We leaded a common warm up to begin, adequate for both different contents. This part was divided in 2 practises, 2 hours long each one. The first part consisted in a workshop of combat sports (such as boxing, kickboxing, judo and taekwondo) doing a circuit in small groups; the second was an initial workshop of contact improvisation dance, learning in pairs the main techniques an basic exercise of this content. In both parts they worked out physical and coordinative capacities as balance, flexibility, coordination, strength and change of weight, mainly.

3. Final Reflection Seminar (2 hours, in the classroom). All the participants thought about the activities performed through several questions proposed by the teachers leading the intervention, with the aim of inquiring into their conceptions, feelings and opinions about the experience lived, in a common reflection where they could speak and analyse the gender stereotyped contents in PE.

\section{DISCUSSION}

In the first part, the Visual Literacy Seminar, it was found that the students observe and recognize different stereotypes in the teaching of PE in the schools. After the first reflection, they identified the most common ones, finding in the literature (Calvo-Ortega \& Perrino-Peña, 2017), and the huge importance that de main socialization agents play on its transmission (family, scholar education and media). They detailed some practises and behaviours of their past experiences with the PE as prejudiced and stereotyped. The result was satisfactory, contributing to the theory by first, recognizing and second, to fighting these preconceived ideas.

During the second part, the Workshop of physical and sports stereotyped practises, they could live that theory in a practical sphere, making it closer to their future as teachers. The objective was to realise that the gender stereotypes associated to these contents come from the traditional body and physical culture, and not from a scientific foundation, so without a valid justification. The students discovered that the gender is not a determining factor for its practise, and they found very similar physical and coordinative capacities or even exercises needed and required for both contents combat sports and contact dance, as we saw before.

Last, we exposed these similarities in the Final Reflection Seminar, through several questions that the teachers threw to the participants and they took the word in order to affirm that the gender is not a conditioning to choose and work a content or another, but the physical and coordinative capacities are the important thing. In this case both contents shared technical and physical aspects that are not ownership of men or women. In other words: the gender does not justify the content.

\section{CONCLUSIONS}

Throughout this didactic intervention, the participants were shown that they can change the traditional and stereotyped body and physical culture, as future PE teachers. They could also experience it, what is determinant. The fact of choosing contents based on the capacities that we want to work and not for the social believes or conventions, and not segregate them by the gender when practicing a content or other, 
promote a coeducative and more egalitarian physical sports practice in the scholar PE. We should teach from a critical and reflective perspective, and to serve to the society for a better physical activity and sports practice.

\section{REFERENCES}

Calvo-Ortega \& Perrino-Peña (2017). Physical-sports habits in adolescents from Castile and Leon. Movimento, 23(4), 1341-1352. https://doi.org/10.22456/1982-8918.71852

Rey-Cao, A., \& González-Palomares, A. (2017). Gender in the eyes: Strategies to educate with (and despite of) the photographs of the curricular materials. A Coruña, España: Discotrompo S. L. Retrieved from http://xeneronosollos.webs.uvigo.gal/

Touriñan, J. M. (2011). Educational Intervention, Pedagogical Intervention and Education: The Pedagogical Look. Revista Portuguesa de Pedagogía, Extra-Série, 283-307. 


\title{
Perception of primary school children about the playful and sport practice
}

\author{
JOSÉ EUGENIO RODRÍGUEZ-FERNÁNDEZ1 ${ }^{1}$ RUBÉN NAVARRO-PATÓN ${ }^{1}$, BEATRIZ OLIVEIRA - \\ PEREIRA 2 \\ 1 University of Santiago de Compostela, Spain \\ 2University of Minho, Research Centre on Child Studies, Portugal
}

\begin{abstract}
The study of the perceptions of physical activity is a topic with great interest at present, and can help to implement a change to an active lifestyle of schoolchildren. The objective of this study was to establish the perception of physical activity (play and sport) by students of primary school education by their classmates at school and at leisure active play and sport practice. The sample consists of 1009 children of elementary school (boys $=50.3 \% 507$; girls $=49.7 \%$ ); with the age average $=10.85, S D=0.79$. They were studying in schools in the provinces of Lugo, A Coruña, Ourense and Pontevedra (Galicia, Spain). We found a directly proportional association between gender and the perception that schoolchildren have about the leisure play and sports of their peers $(p<0.001)$, as well as a directly proportional relationship between gender and the perception of practice of active play involvement at school, and there is a significant association with gender $(p<0.001)$. Girls perceive that their classmates of the opposite sex practice more physical activity than they do both in their leisure time and at school. Regarding age ranges, there are no differences in the perception of physical activity practice. Keywords: Subjective perception; Physical activity; Children; Primary school education.
\end{abstract}

Corresponding author. Facultad de Formación de Profesorado. Avda. Ramón Ferreiro, s/n. 27001. Lugo, Spain.

E-mail: ruben.navarro.paton@usc.es

Supplementary Issue: Spring Conferences of Sports Science. International Seminar of Physical Education, Leisure and Health, 17-19 June 2019. Castelo Branco, Portugal.

JOURNAL OF HUMAN SPORT \& EXERCISE ISSN 1988-5202

(c) Faculty of Education. University of Alicante.

doi:10.14198/jhse.2019.14.Proc4.82 


\section{INTRODUCTION}

Physical activity practice is related to motivations and perceptions of schoolchildren about it. The study of these perceptions about physical activity is a subject of great interest at present, since the behaviours related to health are influenced by personal, environmental and sociocultural factors; this knowledge can contribute to the modification of lifestyles in school population (Keating, Guan, Castro \& Bridges, 2005). Therefore, the objective of this study was to know the perception of physical activity by elementary students in relation to their classmates at school and voluntary sports practice.

\section{MATERIAL AND METHODS}

\section{Participants}

1009 children of elementary school participated, 507 were boys and 502 girls; with 10, 11 and 12 years old (average $=10.85, S D=0.79$ ) from different educational centres in the provinces of Lugo, A Coruña, Ourense and Pontevedra (Galicia, Spain).

\section{Measures}

The Inventory of Physical Activity for Schoolchildren (IAFHE) validated by García-Cantó (2011) was used to evaluate the perception of physical activity of children with respect to their peers in relation to their leisure play and sports and also in school context.

\section{Procedures}

For the realization of this study, permission was requested to school management as well as to legal tutors of the students. Only the children who had signed informed consent participated. Subsequently they completed the physical activity inventory in Physical Education class without the presence of the teacher.

\section{Analysis}

Descriptive statistical analyses were performed. For the study of the association between categorical variables [2 of Pearson statistic test was used with the contingency coefficient for the comparisons related to regular practice of leisure physical activity outside school and at school context according to Age (10,11 and 12 years) and gender (boys vs. girl).

The statistical analysis was performed with SPSS v.20.0 for Windows. The level of statistical significance was established at $p=.05$.

\section{RESULTS}

From the results obtained, we can say that there is a statistically significant association between gender and the perception that schoolchildren have about the sports practice by their peers $(X 2(4)=27.327, p<0.001)$, as well as a statistically significant relationship and directly proportional (contingency coefficient $=162, p$ $<0.001$ ), but not in age. Regarding the perception of practice and sports involvement in school, there is a significant association with gender $(X 2(4)=39.867, p<0.001)$ with a statistically significant and directly proportional relationship (contingency coefficient $=199, p<0.001$ ), this does not happen with age. 
Table 1. Perception of physical activity practice with respect to their classmates according to age and gender

\begin{tabular}{|c|c|c|c|c|c|c|c|}
\hline & \multicolumn{3}{|c|}{ Age } & \multicolumn{2}{|c|}{ Gender } & \multirow[b]{2}{*}{ Total } \\
\hline & & 10 & 11 & 12 & Boys & Girls & \\
\hline \multirow{5}{*}{$\begin{array}{l}\text { Boys and } \\
\text { girls of my } \\
\text { age practice } \\
\text { sports ... }\end{array}$} & $\begin{array}{c}\text { A lot more than } \\
\text { me }\end{array}$ & $9(0.9 \%)$ & $22(2.2 \%)$ & $16(1.6 \%)$ & $15(1.5 \%)$ & $32(3.2 \%)$ & $47(4.7 \%)$ \\
\hline & More than me & $68(6.7 \%)$ & 109 10.8\%) & $29(2.9 \%)$ & $89(8.9 \%)$ & $117(11.6 \%)$ & $206(20.4 \%)$ \\
\hline & Like me & $207(20.5 \%)$ & $317(31.4 \%)$ & $98(9.7 \%)$ & $315(31.2 \%)$ & $307(30.4 \%)$ & $622(61.6 \%)$ \\
\hline & Less than me & $29(2.9 \%)$ & $54(5.4 \%)$ & $26(2.6 \%)$ & $67(6.7 \%)$ & $42(4.2 \%)$ & $109(10.8 \%)$ \\
\hline & $\begin{array}{c}\text { Much less than } \\
\text { me }\end{array}$ & $11(1.1 \%)$ & $9(0.9 \%)$ & $5(0.5 \%)$ & $21(2.1 \%)$ & $4(0.4 \%)$ & $25(2.5 \%)$ \\
\hline \multirow{5}{*}{$\begin{array}{c}\text { Boys and } \\
\text { girls of my } \\
\text { age practice } \\
\text { sports at } \\
\text { school ... }\end{array}$} & $\begin{array}{c}\text { A lot more than } \\
\text { me }\end{array}$ & $8(0.8 \%)$ & $19(1.9 \%)$ & $14(1.4 \%)$ & $18(1.8 \%)$ & $23(2.3 \%)$ & $41(4.1 \%)$ \\
\hline & More than me & $53(5.3 \%)$ & $71(7.0 \%)$ & 31 (3.1\%) & $50(5.0 \%)$ & $105(10.4 \%)$ & $155(15.4 \%)$ \\
\hline & Like me & $235(23.3 \%)$ & $361(35.8 \%)$ & $107(10.6 \%)$ & $361(35.8 \%)$ & $342(33.9 \%)$ & $703(69.7 \%)$ \\
\hline & Less than me & $21(2.1 \%)$ & $49(4.9 \%)$ & $15(1.5 \%)$ & $60(5.9 \%)$ & $25(2.5 \%)$ & $85(8.4 \%)$ \\
\hline & $\begin{array}{c}\text { Much less than } \\
\text { me }\end{array}$ & $7(0.7 \%)$ & $11(1.1 \%)$ & $1(0.7 \%)$ & $18(1.8 \%)$ & $7(0.7 \%)$ & $25(2.5 \%)$ \\
\hline
\end{tabular}

\section{DISCUSSION}

The great majority of schoolchildren perceive that peers perform the same physical and sport activity as them. On the other hand, it is observed that girls perceive boys practice more physical activity or sport than they do. The boys perceive that girls perform less sport activity than they outside the school environment as occurs in studies such as those of Garcia-Soler (2015). As can be seen, most students consider that students of their age perform the same physical activity and sport as they do in school, with very small percentage of students who consider that older children do more physical-sport activities than their peers. These findings aren't in agreement with those of Higher Sports Council (CSD, 2011) which indicate that as age increases, physical activity increases to. Maybe this happens because these ages are very similar.

\section{CONCLUSIONS}

Girls in this study generally perceive that their classmates of the opposite sex engage in more physical activity than they do, both on a regular basis and at school. Regarding age, there is no difference in the perception of physical activity practice.

\section{REFERENCES}

Consejo Superior de Deportes (CSD) (2011). Estudio Los hábitos deportivos de la población escolar en España. Madrid: CSD.

García-Soler, S. (2016). Percepción de Utilidad, Diversión y Motivación en la Asignatura de Educación Física y su Relación con el Nivel de Actividad Física Habitual en Escolares de 10-12 Años de la Región de Murcia. (Tesis Doctoral). Universidad de Murcia. https://doi.org/10.18294/sc.2015.797

Keating XD, Guan J, Castro J, Bridges DM. (2005). A meta-analysis of college students' physical activity behaviors. J. Am. Coll. Health, 54(2),116-125. https://doi.org/10.3200/jach.54.2.116-126 


\section{(c) $\underset{\mathrm{EY}}{\mathrm{NO}} \mathrm{\Theta}$}

This work is licensed under a Attribution-NonCommercial-NoDerivatives 4.0 International (CC BY-NC-ND 4.0). 


\title{
Socio-demographic characteristics of nursing students and the prevalence of physical activity
}

\author{
CARLA NUNES 11 , CLÁUDIA CHAVES ${ }^{2}$, JOÃO DUARTE ${ }^{2}$ \\ 1 USF Cidade Jardim, ACeS Dão Lafões, Viseu, Portugal \\ 2Instituto Politécnico de Viseu, CI\&DETS, Escola Superior de Saúde, Portugal
}

\begin{abstract}
The purpose of this quantitative, descriptive and correlational research study is to identify whether nursing students 'sociodemographic variables interfere in the practice of physical activity. It is a non-probabilistic sample, for convenience, composed of 372 nursing and health students, from mainland Portugal. It was conducted a social-demographic characterization questionnaire as well as the International Physical Activity Questionnaires (IPAQ). The feminine gender $(86,3 \%)$ prevails, with a mean age of $23.33 \pm 6.41$,excelling the students in the age group $\leq 20$ years $(41,7 \%)$. Masculine gender students present an higher average ordination in relation to the total physical activity index $(M=227,67)$, with statistically significant differences $(p=0,003) ; 2 n d$ and 3 rd grade students have identical results $(M=15,24 \pm 2,548 ; M=15,24 \pm 2,936$, respectively), suggesting that they are the students with the higher physical activity rate. The Sociodemographic predictor variables of physical activity are age and male gender, highlighting that the older the students are the lower physical activity they practice; there is a larger physical activity among male gender students. Keywords: Nursing students; Physical activity; Sociodemographic determinants.
\end{abstract}

Corresponding author. Avenida da Bélgica, 52 A 1 DT 3510 - 159 Viseu, Portugal.

E-mail: carlacgnunes@hotmail.com

Supplementary Issue: Spring Conferences of Sports Science. International Seminar of Physical Education, Leisure and Health, 17-19 June 2019. Castelo Branco, Portugal.

JOURNAL OF HUMAN SPORT \& EXERCISE ISSN 1988-5202

(C) Faculty of Education. University of Alicante.

doi:10.14198/jhse.2019.14.Proc4.82 


\section{INTRODUCTION}

Despite the numerous health benefits physical activity has, there is a decline of physical activity also in leisure activities of the higher education students. In this sense, the aim of the present study is to identify whether nursing students 'sociodemographic variables interfere in the practice of physical activity.

\section{MATERIAL AND METHODS}

\section{Participants}

Non-probabilistic sample, for convenience, composed of 372 nursing and health students, from continental Portugal higher education schools, mainly feminine (86.3\%), with an average age of $23.33 \pm 6.41$, predominating the students in the age group $\leq 20$ years $(41,7 \%) .86,8 \%$ are single, mostly living in a village $(57,7 \%)$, cohabiting with relatives $(53.8 \%)$, with prevalence of students whose father $(64.0 \%)$ and mother $(54.8 \%)$ have academic qualifications until the 3rd grade of the elementary education, professionally actives (86,3\% father VS. $73.1 \%$ mother), the majority with 1 brother $(63.7 \%)$, without remunerated activity $(75.3 \%)$, with prevalence of those who reveal a normal weight $(73.4 \%)$.

\section{Measures}

It was conducted a social-demographic characterization questionnaire and the International Physical Activity Questionnaires (IPAQ), developed by investigators elected by the Word Health Organisation (WHO), with acceptable psychometric characteristics to use in studies about the prevalence of the participation in physical activities. The results of that study, that include data from Portugal, have been published by Craig, Marshall, Sjöström, Bauman, et al. (2003), with a Spearman`s correlation coefficient, $p=0.76$ (95\%, IC 0.73-0.77) and the validity of criteria, revealing a value of 0.30 (95\%, IC $0.23-0.36)$.

\section{Procedures}

Data were obtained through an online evaluation protocol, with favourable opinion of the Ethics Committee of the ESSV ( $\left.\mathrm{N}^{0} 1 / 2014\right)$. It was included an introductory text with goals and explanations for completing the form as well as to thank for the cooperation.

\section{Analysis}

Descriptive statistics and analytical or inferential parameters were applied, using SPSS software (Statistical Package for the Social Sciences) version 21.0 (2013) for Windows. As for the descriptive statistics, as statistical measures were used absolute and percentage frequencies, central tendency measures (mean), measures of dispersion (standard deviation) as well as Skewess asymmetry measures (SK) and kurtosis. Pearson's correlation coefficient test, the U-Mann-Whitney e Kruskal Wallis test, the variance test (ANOVA), the $t$ test and the chi-squared test were also used.

\section{RESULTS}

The prevalence of inactive students is $42.7 \%, 13.4 \%$ are intermediate and $43.8 \%$ are active. The higher percentage regards the inactive students with 20 years old or less (45.3\%); 40\% of the students, aged $21-22$ reveal intermediate physical activity, $39.9 \%$ of the active students are 20 years old or less. Masculine gender students present an higher average ordination in relation to the total physical activity index $(M=227,67)$, with statistically significant differences $(p=0,003)$; The year the students attend is shown to be a variable that interfere in the total physical activity index, with a $f=3.096$ and a value of $p=0.016$. In terms of means, $2 n d$ and 3 rd grade students have identical results $(M=15,24 \pm 2,548 ; M=15,24 \pm 2,936$, respectively), suggesting 
that they are the students with the higher physical activity rate. The Sociodemographic predictor variables of physical activity are age and male gender, highlighting that the older the students are the lower physical activity they practice; there is a larger physical activity among male gender students. The regression coefficient or the age trajectory ( $\beta$ age $=-0.18$ ) of the masculine gender ( $\beta$ masculine gender $=0.17$ ), there is a larger physical activity among students of the masculine gender.

\section{DISCUSSION}

It has been found that students of the masculine gender have a higher mean regarding the total physical activity index, with statistically significant differences. In Saraiva, Júnior, Petrolini et al. (2018) study, 61.8\% of the participants were more physically active, showing that women prefer moderate activities while men more vigorous activities. In Melo, Carvalho, Sá et al. (2016) study, the feminine gender students have been classified as insufficiently active when compared to the masculine gender $(p=0,008)$. The comparison between Courses for the feminine gender reveals that women of the degree Course have higher results classified as insufficiently active when compared to the bachelor's degree students ( $p=0,027)$, but without statistically significant differences in what the levels of activity for the masculine gender is related, between Courses. In the study of Oliveira, Gordiab, Quadros and Campos (2014), the younger students are those who practise more physical activity, which is not in accordance with this study, once were the 2nd and 3rd grade students, with higher age bracket, who revealed higher levels of physical activity.

\section{CONCLUSIONS}

This study indicates that further initiatives are indispensable by higher education institutions which envisages a more a comprehensive and coordinated approach, that promotes the health and well-being of students through the practise of physical activity. The adoption of strategies and health educational programmes at the University It is very important as a precautionary measure for the adoption of healthy lifestyles, for the improvement of quality of life, in the short and the long term. Higher Education Institutions should disseminate a set of knowledge, culture and values, among which physical activity cannot be excluded. Despite the promising results, new scientific studies will be necessary with more representative samples as well as an improved methodological quality.

\section{REFERENCES}

Craig, C.L., Marshall, A.L., Sjöström, M., Bauman, A.E., Booth, M.L., Ainsworth, B.E., Pratt, M., Ekelund, U., Yngve, A., Sallis, J.F., \& Oja, P. (2003). International physical activity questionnaire: 12-country reliability and validity. Med Sci Sports Exerc; 35: 1381-1395. https://doi.org/10.1249/01.mss.0000078924.61453.fb

Melo, A.B., Carvalho, E.M., De Sá F,G.S., Cordeiro, J.P., Leopoldo, A.S., \& Leopoldo, A.P.L. (2016). Nível de atividade física dos estudantes de graduação em educação física da Universidade Federal do Espírito Santo. J Phys Educ; 27, 2723. https://doi.org/10.4025/jphyseduc.v27i1.2723

Oliveira, C.S., Gordiab, A.P., Quadros, T.M.B., \& Campos, W. (2014). Atividade física de universitários brasileiros: uma revisão da literatura. Revista de Atenção à saúde; 12(42), 78-89. https://doi.org/10.13037/rbcs.vol12n42.2457

Saraiva, L.C., Júnior, J.R.A.N., Petrolini, A.L. et al. (2018). Frequency and duration of physical activity practice of university teachers and servants. J. Phys. Educ. v. 29, e2958. https://doi.org/10.4025/jphyseduc.v29i1.2958 


\section{(c) (i) (3)}

This work is licensed under a Attribution-NonCommercial-NoDerivatives 4.0 International (CC BY-NC-ND 4.0). 


\title{
Inequalities in female combat sports
}

\author{
CRISTINA RODRÍGUEZ-RODRÍGUEZ, MARÍA PERRINO-PEÑA \\ Universidad Pontificia de Salamanca, Spain
}

\begin{abstract}
Sport is a sociocultural phenomenon with capacity to mobilize a huge mass of people. It has been considered traditionally as a male leisure activity in which female participation was disapproved. In combat sports the situation is more complicated because of the gender stereotypes. The incursion of women is still hard and induces non-acceptance and exclusion situations and differences in opportunities. This study aims to investigate inequalities of sportswomen, especially in combat modalities. An analysis of the historic evolution of woman in sport and her progressive incorporation has been developed, through a qualitative methodology; se used a bibliographic review and the discourse analysis have been performed. Continuous prohibitions and barriers that they have overcome have been highlighted, as well as inequalities and established stereotypes for women among combat sport modalities. We observed some behaviours and opinions from different authors and their discourses. After having reflecting about their meanings we conclude that sports women have always existed and have achieved successes, also in combat modalities. Furthermore, their presence increases each year, as statistics show. Keywords: Combat sports; Sportswomen; Inequalities; Stereotyped sports; Gender.
\end{abstract}

Corresponding author. C/Henry Collet, 52-70. Facultad de Educación. UPSA. CP: 37007, Salamanca, Spain.

E-mail: mperrinope@upsa.es

Supplementary Issue: Spring Conferences of Sports Science. International Seminar of Physical Education, Leisure and Health, 17-19 June 2019. Castelo Branco, Portugal.

JOURNAL OF HUMAN SPORT \& EXERCISE ISSN 1988-5202

(C) Faculty of Education. University of Alicante.

doi:10.14198/jhse.2019.14.Proc4.82 


\section{INTRODUCTION}

Sport is a sociocultural phenomenon nowadays, in which the sociological constructions and the gender roles are greatly relevant. It has been traditionally considered as a men's leisure activity (Sagarzazu \& Lallana, 2012; Calvo-Ortega \& Perrino-Peña, 2017), where the female participation has been even prohibited and disapproved (Hardin \& Greer, 2009; Jones, 2011). In some stereotyped modalities, for example in combat sports (boxing, Muay Thai, Brazilian jujitsu, etc.), the possibilities for a woman are more complicated than for a man. Physical and psychological capacities required for having success in these modalities include a high level of strength, aggressiveness, competitiveness, no fear of contact or pain, socially considered as appropriate of men (Lucimí, 2012). Men who did not like sports or did not practise it, could be seen as little masculine or effeminate (COSUDE, 2005). And otherwise for a woman who practise hard and tough sports, they are seen very masculine, even many people question their femininity. In these contexts this essay has the objective of standing out the inequalities of sportswomen -mainly in combat sports-, fighting for the abolition of unjustifiable stereotypes, and recognizing their value. This study shows some opinions and relevant ideas of different authors in order to contribute to identify and delegitimize the existing inequalities.

\section{MATERIAL AND METHODS}

\section{Participants}

An analysis of the historic evolution of woman in sport and her progressive incorporation has been developed, through a qualitative methodology. The total quantity of references consulted rises up to 43 scientific documents, (articles and books) and besides that, news in press or web sites (blogs and others).

\section{Measures}

The documentation was registered with the Access program, including different aspects such as type of document; year of publication; authorship and gender; title; topic, key words; discriminatory expressions or ideas; difficulties or barriers. In brief, eight categories were defined.

\section{Procedures}

We started with a bibliographic review of the topic, searching in online sites (Sportdiscus, Dialnet and Google Scholar), using the descriptors: 'woman'; 'combat sports'; 'inequality'; 'stereotypes'; 'discrimination', and different combinations. Next step was a scientific reading of the most remarkable ones, related to the topic. Then, we applied the technique of discourse analysis (Wodak y Meyer, 2003; Rodríguez, 2011). Printed and online sports press (news and images) were also investigated, those we considered stereotyped or discriminatory to women.

\section{Analysis}

According to these materials, we compiled and described the situation of inequality of women in sports, especially in combat sports, detailing the information found in the categories exposed.

\section{RESULTS}

According to Vega et al., (2014) we found an important amount of very rooted stereotypes related to women and sport. It has been conforming some ideas and social configurations that, without being true, have conditioned the equal dealing of sportswomen. 
In the same way, sports and physical activities performed in public spaces, as well as the relationship of women with the private spaces, have provoked huge barriers for all the women who was interested in practising sport. For instance, they considered impossible to combine their private and home tasks as well as their wives and mothers roles with their leisure, such as sport (Vázquez, 2002). Other barrier was the fact that women have to prove or reinforce their femininity, by wearing sexy clothes and having a typically considered feminine behaviour.

\section{DISCUSSION}

The stereotypes and believes that we found, affect the social view, to the point that we identify some sports and physical activities like 'male' ones, and others like 'female' ones (Moreno et al., 2006). Combat sports are defined are identified with strength, aggressiveness, competition and physical contact.

We can add the social invisibility and the wrong dealing of sportswomen in the media, even more in combat sports, strongly stereotyped, accomplishing some established canons (Brown, 2012). However, there have been always women that stand out in combat sports, breaking the stereotypes, fighting against the abuses, strong language or disqualifiers adjectives (Ezzel, 2009, in Moreno, 2011). Current MMA (Mixed Martial Arts) have great famous women fighters, thanks to the confidence and trust of promoters, sponsors and media. Only in this way, they could have the same recognition as athletes than their male homologous.

Different researches consulted affirm that women in sport have evolved since the beginnings, thanks to their effort and persistence for overcoming the barriers. Nevertheless, we still attend to several inequalities in combat sports, such as the very late incorporation of women boxing to de Olympic Games.

\section{CONCLUSIONS}

After having analysing the discourses and a later reflection, we conclude that despite the barriers and difficulties, such as continuous criticism, abuses, lack of economic support, invisibility in media and others negative aspects, women have been always existed in sport and also in combat modalities, although in a minor proportion. We have noticed some progress, however, we should continue with the abolition of stereotypes and recognizing the value and bravery of sportswomen in these modalities. It is necessary to continue fighting for the same opportunities and rewards, and not only for the material ones.

\section{REFERENCES}

Brown, S. (2012). De Coubertin's Olympism and the Laugh of Michel Foucault: Crisis Discourse and the Olympic Games. Quest, 64(3), 150-163. https://doi.org/10.1080/00336297.2012.693750

Calvo-Ortega \& Perrino-Peña (2017). Calvo-Ortega, E., \& Perrino-Peña, M. (2017). Hábitos físicodeportivos en adolescentes de Castilla y León. Movimento, 23(4), 1341-1352. https://doi.org/10.22456/1982-8918.71852

COSUDE (Agencia Suiza para el desarrollo y la cooperación) (2005). Integrar la equidad de género en los proyectos deportivos. Agencia Suiza para el Desarrollo y la Cooperación. Berna. https://doi.org/10.5944/reppp.5.2015.14237

Hardin, M., y Greer, J. D. (2009). The influence of gender-role socialization, media use and sports participation on perceptions of gender-appropriate sports. Journal of Sport Behavior, 32(2), 207. 
Jones, A. H. (2011). Visual and verbal gender cues in the televised coverage of the 2010 winter Olympics. International Journal of Interdisciplinary Social Sciences, 6(2), 199-216. https://doi.org/10.18848/1833-1882/cgp/v06i02/52015

Latorre, P. A., González, M. A., Moreno, A. J., Sierra, M. D., Carmona, F. J., Molina, D., y Vergillos, L. (2007). Mujer, deporte y medios de comunicación. Revista Digital de Buenos Aires, (11), 106.

Lucumí, Y. (2012). Women contributions to the transformation of the sociocultural stereotypes of Colombian. Revista UDCA Actualidad \& Divulgación Científica, (15), 27-35.

Moreno, H. (2011). El boxeo como tecnología de la masculinidad. La ventana. Revista de estudios de género, 4(33), 152-196.

Sagarzazu, I., y Lallana, I. (2012). La influencia de los estereotipos de género tradicionales en la participación deportiva de las mujeres y su reproducción en los medios de comunicación. En I Congreso Internacional de Comunicación y Género. Libro de Actas: 5, 6 y 7 de marzo de 2012. (pp. 2032-2047). Facultad de Comunicación. Universidad de Sevilla. https://doi.org/10.15304/ricd.1.2.2657

Vega, L., Ávalos, M. A., Merma, G., y Gavilán, D. (2014). Percepciones del alumnado sobre los estereotipos en la práctica de la Gimnasia Rítmica. En XII Jornadas de redes de investigación en docencia universitaria. El reconocimiento docente: Innovar e investigar con criterios de calidad (pp. 2783-2793). Instituto de Ciencias de la Educación. https://doi.org/10.4067/s0718$\underline{45652011000200011}$ 


\title{
Evaluation of coordinated motor ability in handball players
}

\author{
MARTA CAMACHO-CARDENOSA ${ }^{1}$, ALBA CAMACHO-CARDENOSA ${ }^{1}$, JAVIER BRAZO-SAYAVERA ${ }^{2,3}$ \\ ${ }^{1}$ Faculty of Sport Science, University of Extremadura, Caceres, Spain \\ 2Instituto Superior de Educación Física, Universidad de la República, Rivera, Uruguay \\ ${ }^{3}$ Polo de Desarrollo Universitario EFISAL, Rivera, Uruguay
}

\begin{abstract}
Modern handball is quicker and more explosive and is made up of varying physically intense movements, which require high-level coordinated motor skills. Although these abilities are considered highly interrelated in team handball players, test involving balls and handball specific movements were used without clear logic and there are no standard test batteries for adults or young players. So, the aim of this review was to summarise current scientific knowledge about how are being assessed the coordinated motor abilities in handball players. Articles published before 10 April 2019 were retrieved by using searches of PubMed, Web of Science and SPORT Discuss. Inclusion criteria were as follows 1) Type of study. All articles published between January 2002 and July 2019 of the English or Spanish languages literature were considered. 2) Type of participant. Studies that evaluated handball players of any age were included and 3) Type of outcome measures: coordinated motor ability outcomes were measured. Ninety-nine original studies were identified in our systematic search from which data were extracted. Accuracy and precision and speed and reaction time are the coordinated motor abilities more evaluated. Only T-test and Illinois agility test show validity and reliability in male and female handball athletes to assess agility. Due to the fact that the included intervention studies were predominantly low in methodological quality and use non-validity test, further high quality studies are needed to develop standard test batteries for adult or young handball players. Keywords: Outcome assessment; Athletic performance; Motor skills and sports.
\end{abstract}

Corresponding author. Avenida da Bélgica, 52 A 1 DT 3510 - 159 Viseu, Portugal.

E-mail: albacc@unex.es

Supplementary Issue: Spring Conferences of Sports Science. International Seminar of Physical Education, Leisure and Health, 17-19 June 2019. Castelo Branco, Portugal.

JOURNAL OF HUMAN SPORT \& EXERCISE ISSN 1988-5202

(C) Faculty of Education. University of Alicante.

doi:10.14198/jhse.2019.14.Proc4.82 


\section{INTRODUCTION}

Between all coordinated motor abilities, the ability to run and sprint quickly and the accuracy may be the most determinants for success (Mohamed et al., 2009). Although these abilities are considered highly interrelated in team handball players only a skill test was a good indicator (Lidor et al., 2005). Test involving balls and handball specific movements were used in assessing coordinated motor abilities, however, these are used without clear logic and there are no standard test batteries for adults or young players (Visnapuu \& Jurimae, 2009). So, the aim of this review was to summarise current scientific knowledge about how are being assessed the coordinated motor abilities in handball players.

\section{MATERIAL AND METHODS}

In accordance with the Preferred Reporting Items for Systematic Reviews and Meta-Analyses (PRISMA) statement, articles published between January 2002 and July 2019 of the English or Spanish languages literature were considered were retrieved by using searches of PubMed, Web of Science and SPORT Discuss. Studies that evaluated coordinated motor abilities outcomes in handball players of any age were included. For experimental studies, the Physiotherapy Evidence Database (PEDro) scale was used to quantify the quality of the included studies.

\section{RESULTS}

Accuracy $(n=6)$, precision movements $(n=29)$ and visual-motor coordination $(n=7)$ and speed and reaction time were the most popular coordinated motor ability measured in 5,417 handball players. Median methodological quality score for intervention studies was 5.04 point (standard deviation 0.53 ), which can be interpreted as low methodological quality. T- Test $(n=12)$ the most popular test in the precision assessment. Sprint 5- 50m test were used to measure speed (m.s-1) or time involve going over these distances (s). Sprinting time (s) were also evaluated in a specific handball test: Gamed-Based Performance test $(n=2)$. Reaction time (mms) were specify evaluated in visual reaction time test $(n=1)$ and in the first step in some above sprint test $(n=3)$.

\section{DISCUSSION}

Even though laboratory tests are considered reliable and useful, previous studies noted the importance of sport-specific test in testing reactive-agility in handball (Sekulic et al., 2014). Some specific field-tests such as Slalom Dribbling Test or Eight Bending Test also includes movements or actions with the ball (determinant mainly in offense), which increases the validity of this (Kutlu et al., 2017). By other hand, defensive players have to quickly respond to opponents' actions cannot be pre-planned. Of this way, T-test and Illinois agility test were satisfactory reliability in male and female athletes to assess reactive-agility (Negra et al., 2016). On the other hand, some studies that have investigated the movement patterns of field-based team sports showed the mean distance of sprints between 10-20m; so, the distances performed in 10-m, 15-m and 20-m sprint test could be suitable. However, further research is required to document the time-motion analysis of maximal speed during handball activity (Spencer et al., 2005).

As have already been mentioned, most of used tests are general or specific tests, which are performed stepwise under standardized conditions. However, Wagner et al. (2019) developed and validated a teamhandball game-based performance (GBPT) that including similar movements and intensities as in competition match. Of this way, it is possible to measure specific aerobic performance, throwing performance, 
speed and agility under conditions that are similar to competition. Although, GBPT test showed valid and reliable test to analyse team-handball performance under conditions similar to competition, only one study administered this test to compare specific physiological and biomechanical performance in elite, sub-elite and non-elite male team handball players (Wagner et al., 2018). Thus, more investigations more investigations could be necessary to confirm and determinate the valid and reliable test in different categories and levels.

\section{CONCLUSIONS}

An analysis of validity and reliability of coordinated motor abilities tests is necessary in the handball field. Currently, only T-test and Illinois agility test show validity and reliability in male and female handball athletes to assess agility.

\section{REFERENCES}

Kutlu, M., Yapici, H., \& Yilmaz, A. (2017). Reliability and Validity of a New Test of Agility and Skill for Female Amateur Soccer Players. J Hum Kinet, 56, 219-227. https://doi.org/10.1515/hukin-2017$\underline{0039}$

Mohamed, H., Vaeyens, R., Matthys, S., Multael, M., Lefevre, J., Lenoir, M., \& Philppaerts, R. (2009). Anthropometric and performance measures for the development of a talent detection and identification model in youth handball. J Sports Sci, 27(3), 257-266. https://doi.org/10.1080/02640410802482417

Negra, Y., Chaabene, H., Hammami, M., Amara, S., Sammoud, S., Mkaouer, B., \& Hachana, Y. (2017). Agility in Young Athletes: Is It a Different Ability From Speed and Power? J Strength Cond Res, 31(3), 727-735. https://doi.org/10.1519/jsc.0000000000001543

Sekulic, D., Krolo, A., Spasic, M., Uljevic, O., \& Peric, M. (2014). The development of a new stop'n'go reactive-agility test. J Strength Cond Res, 28(11), 3306-3312. https://doi.org/10.1519/jsc.0000000000000515

Spencer, M., Bishop, D., Dawson, B., \& Goodman, C. (2005). Physiological and metabolic responses of repeated-sprint activities: specific to field-based team sports. Sports Med, 35(12), 1025-1044. https://doi.org/10.2165/00007256-200535120-00003

Visnapuu, M., \& Jurimae, T. (2009). Relations of anthropometric parameters with scores on basic and specific motor tasks in young handball players. Percept Mot Skills, 108(3), 670-676. https://doi.org/10.2466/pms.108.3.670-676

Wagner, H., Fuchs, P. X., \& von Duvillard, S. P. (2018). Specific physiological and biomechanical performance in elite, sub-elite and in non-elite male team handball players. Journal of Sports Medicine and Physical Fitness, 58(1-2), 73-81. https://doi.org/10.1123/ijspp.2018-0014

Wagner, H., Sperl, B., Bell, J. W., \& von Duvillard, S. P. (2019). Testing Specific Physical Performance in Male Team Handball Players and the Relationship to General Tests in Team Sports. Journal of Strength and Conditioning Research, 33(4), 1056-1064. https://doi.org/10.1519/jsc.0000000000003026

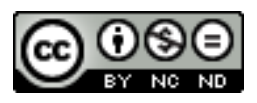

This work is licensed under a Attribution-NonCommercial-NoDerivatives 4.0 International (CC BY-NC-ND 4.0). 


\title{
How assessment the flexibility in handball players? Results of a systematic review
}

\author{
ALBA CAMACHO-CARDENOSA ${ }^{1}$, MARTA CAMACHO-CARDENOSA ${ }^{1}$, JAVIER BRAZO-SAYAVERA ${ }^{2,3}$ \\ ${ }^{1}$ Faculty of Sport Science, University of Extremadura, Cáceres, Spain \\ 2Instituto Superior de Educación Física, Universidad de la República, Rivera, Uruguay \\ ${ }^{3}$ Polo de Desarrollo Universitario EFISAL, Rivera, Uruguay
}

\begin{abstract}
In handball players, the application of flexibility measurement test prior to and during the competitive season may be useful to determine specific changes, identify players at a high risk for injury, initiate preventive therapeutic strategies or for early detection of possible consequent structural damage. In this way, different tests could be used to evaluate flexibility and, generally these are used without clear logic, independently of age, level or gender. Thus, the aim of this article was to summarise current scientific knowledge about flexibility assessment in handball players of different ages and categories. Articles published before 10 April 2019 were retrieved by using searches of PubMed, Web of Science and SPORT Discuss. Inclusion criteria were as follows 1) Type of study: all articles published between January 2002 and July 2019 of the English or Spanish languages literature were considered; 2) Type of participant: studies that evaluated handball players of any age were included; and 3) Type of outcome measures: flexibility outcomes were measured. Seventeen original studies were identified in our systematic search from which data were extracted. Eleven different tests were used to measure flexibility in lower and upper limbs of 1,565 handball players of different age categories. Sit-and-Reach and hamstring extensibility could be the best choice in the handball sport to assess the flexibility. Keywords: Hamstring muscles; Sport and athletic injuries.
\end{abstract}

Corresponding author. Faculty of Sport Science, University of Extremadura. Avd. Universidad, s/n, 10003, Cáceres, Spain. E-mail: mcamachocardenosa@unex.es

Supplementary Issue: Spring Conferences of Sports Science. International Seminar of Physical Education, Leisure and Health, 17-19 June 2019. Castelo Branco, Portugal.

JOURNAL OF HUMAN SPORT \& EXERCISE ISSN 1988-5202

(c) Faculty of Education. University of Alicante.

doi:10.14198/jhse.2019.14.Proc4.82 


\section{INTRODUCTION}

Lack of muscle flexibility is one of the most commonly postulated risk factors for the development of muscle injuries. In handball players, the application of flexibility measurement test prior to and during the competitive season may be useful to identify players at a high risk for injury and initiate preventive therapeutic strategies (Cejudo et al., 2015). In this way, different tests could be used to evaluate flexibility and, generally these are used without clear logic, independently of age, level or gender. Thus, the aim of this article was to summarise current scientific knowledge about flexibility assessment in handball players.

\section{MATERIAL AND METHODS}

The study was undertaken in accordance with the Preferred Reporting Items for Systematic Reviews and Meta-Analyses (PRISMA) statement. Articles published before 10 April 2019 were retrieved by using searches of PubMed, Web of Science and SPORT Discuss. Inclusion criteria were all articles published between January 2002 and July 2019 of the English or Spanish languages literature that evaluated flexibility outcomes of handball players of any age. Information was extracted from each including original article on: characteristics of handball players (age category and size sample) and information about the outcome measure (test name and outcomes).

\section{RESULTS}

Nine different tests were used to measure flexibility in lower and upper limbs of 1,565 handball players of different age categories. Distance $(\mathrm{cm})$ or time $(\mathrm{s})$ were measured with different test to evaluate lower limbs flexibility. A flexibility test was used to evaluate upper limb flexibility in 3 studies: flexibility with bat test.

\section{DISCUSSION}

Although the angular tests have the advantage of being the criterion measure to assess flexibility, have the disadvantage of having a limited used in several settings (Castro-Pinero et al., 2009). Contrary, lineal tests, such as sit-and-reach test are easy to administer, require-minimal skills training for application and need affordable equipment (Castro-Pinero et al., 2009). Like in this systematic review, Sit-and-reach is probably the most widely used lineal measures of flexibility in different population and seems to be a useful and reliable tool to estimate hamstring extensibility (Matthys et al., 2013).

\section{CONCLUSIONS}

Sit-and-Reach and hamstring extensibility could be the best choice in the handball sport to assess the flexibility.

\section{REFERENCES}

Bolanča, M., Čavala, M., \& Rogulj, N. (2010). Differences between the of Handball Players and Players from Younger Motor Abilites Non-Hanball Age Groups. Proceedings of the Faculty of Physical Education, University of Banja Luka(2), 170-174.

Castro-Pinero, J., Chillon, P., Ortega, F. B., Montesinos, J. L., Sjostrom, M., \& Ruiz, J. R. (2009). Criterion-Related Validity of Sit-and-Reach and Modified Sit-and-Reach Test for Estimating 
Hamstring Flexibility in Children and Adolescents Aged 6-17 Years. Int J Sports Med, 30(9), 658662. https://doi.org/10.1055/s-0029-1224175

Cejudo, A., Sainz de Baranda, P., Ayala, F., \& Santonja, F. (2015). Test-retest reliability of seven common clinical tests for assessing lower extremity muscle flexibility in futsal and handball players. Physical Therapy in Sport, 16(2), 107-113. https://doi.org/10.1016/i.ptsp.2014.05.004

Emeish, M. K. (2015). Effect of S.A.Q Exercises on Certain Physical VAriables and Jump Shotin. Ovidius University Annals, Series Physical Education \& Sport/Science, Movement \& Health, 15(2), 462-467.

Fernandez-Romero, J. J., Vila-Suarez, H., \& Cancela-Carral, J. M. (2017). Selection of talents in handball: anthropometric and performance analysis. Rev Bras Med Esporte, 23(5), 361-365. https://doi.org/10.1590/1517-869220172305141727

Galal El-Din, H., Zapartidis, I., \& Ibrahim, H. (2011). A Comparative Study between Talented Young Greek and German Handball Players in Some Physical and Antropometric Characteristics. Biol Sport, 28(4), 245-248. https://doi.org/10.5604/965488

Marković, S., Vučković, I., Sekulić, Ž., \& Gadžić, A. (2015). Physical Performance Modeling Among Young Basketball and Handball. Facta Univ Phys Educ Sport, 13(2), 263-273.

Matthys, S. P., Vaeyens, R., Fransen, J., Deprez, D., Pion, J., Vandendriessche, J., . . . Philippaerts, R. (2013). A longitudinal study of multidimensional performance characteristics related to physical capacities in youth handball. $\mathrm{J}$ Sports Sci, 31(3), 325-334. https://doi.org/10.1080/02640414.2012.733819

Mohamed, H., Vaeyens, R., Matthys, S., Multael, M., Lefevre, J., Lenoir, M., \& Philippaerts, R. (2009). Anthropometric and Performance Measures for the Development of a Talent Detection and Identification Model in Youth Handball. J Sport Sci, 27(3), 257-266. https://doi.org/10.1080/02640410802482417

Muratović, A., Petković, J., Bojanić, D., \& Vasiljević, I. (2015). Comparative Analysis of Motor and Specific Motor Abilities between Handball Players and Non-Athletes in the Cadet Age from Montenegro. International Scientific Journal of Kinesiology, 9(1), 70-74.

Nikolaidis, P. T., \& Ingebrigtsen, J. (2013a). Physical and physiological characteristics of elite male handball players from teams with a different ranking. J Hum Kinet, 38, 115-124. https://doi.org/10.2478/hukin-2013-0051

Nikolaidis, P. T., \& Ingebrigtsen, J. (2013b). The relationship between body mass index and physical fitness in adolescent and adult male team handball players. Indian J Physiol Pharmacol, 57(4), 361371.

Nikolaidis, P. T., Ingebrigtsen, J., Povoas, S. C., Moss, S., \& Torres -Luque, G. (2015). Physical and physiological characteristics in male team handball players by playing position - Does age matter? Journal of Sports Medicine and Physical Fitness, 55(4), 297-304.

Pavlovič, S., Talovič, M., Kazazovič, B., Kazazovič, E., \& Lakota, R. (2013). Differences in Basic Motor and Situational Motor Abilities of Female Handball Players at Varying Levels of Competition. Homo Sporticus, 15(1), 20-25. https://doi.org/10.5550/sgia.130902.en.008p

Pavlović, S., Talović, M., Kazazović, E., \& Lakota, R. (2013). Analysis and Differences in the Basic Motor and Situational Motor Abilities Between Female Handball Players in the Bih First Federal League and the First League of the Republika Srpska. SportLogia, 9(2), 217-224. https://doi.org/10.5550/sgia.130902.en.008p

Pori, P., Šibila, M., Justin, I., Kajtna, T., \& Pori, M. (2012). Correlation between the Motor Abilities and Competitive Performance os Slovenian Hadball Goalkeepers. KinSi, 18(2), 19-26.

Rousanoglou, E. N., Noutsos, K. S., \& Bayios, I. A. (2014). Playing Level and Playing Position differences of Anthropometric and Physical Fitness Characteristics in Elite Junior Handball Players. J Sport Med Phys Fit, 54(5), 611-621. 
Sebastia-Amat, S., Espina-Agullo, J. J., \& Chinchilla-Mira, J. J. (2017). Jump height, velocity, flexibility and anthropometric profile of handball goalkeepers in young categories. Retos, 32, 248-251.

Zapartidis, I., Kororos, P., Christodoulidis, T., Skoufas, D., \& Bayios, I. (2011). Profile of Young Handball Players by Playing Position and Determinants of Ball Throwing Velocity. J Hum Kinet, 27, 17-30. https://doi.org/10.2478/v10078-011-0002-4

Zapartidis I., N. M.-E., Vareltzis I., Kororos P. (2011). Sex differences in the motor abilities of young male and female handball players. Biology of Sport, 28, 171-176. https://doi.org/10.5604/959283

\section{(c) $(9)\left(\Theta_{\mathrm{EY}}\right.$}

This work is licensed under a Attribution-NonCommercial-NoDerivatives 4.0 International (CC BY-NC-ND 4.0). 


\title{
Evaluation of urinary protein and creatinine concentration in athletes after high-performance physical exercise
}

\author{
ANA SOFIA TAVARES ${ }^{1,2}$, CARINA LADEIRA ${ }^{1,2,3}$, JOÃO FILIPE QUINTÃO ${ }^{1}$, MARIA CAROLINA LUÍS $^{1}$, \\ MIGUEL OLIVEIRA PEREIRA ${ }^{1}$, RENATO DANTON ABREU ${ }^{1}$ \\ ${ }^{1}$ IPL - ESTeSL, Instituto Politécnico de Lisboa, Escola Superior de Tecnologia da Saúde de Lisboa, Portugal \\ ${ }^{2}$ H\&TRC, Health \& Technology Research Center, IPL-ESTeSL, Portugal \\ ${ }^{3}$ Centro de Investigação em Saúde Pública, Escola Nacional de Saúde Pública, Universidade Nova de \\ Lisboa, Portugal
}

\begin{abstract}
Intense physical exercise without a planned or structured physical activity can induce potentials risks in the human body. The objective of this study is to verify the existence of a modulation of the values of creatinine and urinary proteins after performing a training session of high intensity. Furthermore, we intend to verify the existence of significant alterations of these analytes in sportsmen who regularly use protein supplementation, compared to a group of athletes who do not use supplementation after performing the same highperformance training. A convenience sample of 18 sportsmen attending gyms, both genders and between the ages of 18 and 35 was divided in two groups: users of protein supplementation $(n=9)$ and individual's non-users of protein supplementation $(n=9)$. The two groups were subjects to a physical activity training session of high intensity, performed by a qualified professional. Urine samples were collected before and

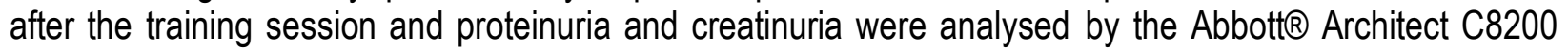
system. Statistical treatment of the results was performed using the SPSS (version 22), as well as descriptive and inferential statistics. Results showed that in both groups, physical exercise induced a significant increase in all these parameters. Both levels of protein and creatinine increased in urine approximately twice more after the physical training with estimated time of 45 minutes. However, no statistically significant changes were observed from one group to another, although there were slight changes in the user group of protein supplementation. Keywords: Exercise; Protein; Creatinine; Urine; RPC; Protein Supplementation.
\end{abstract}

\footnotetext{
Corresponding author. Escola Superior de Tecnologia da Saúde, Instituto Politécnico de Lisboa, Av. D. João II, Lote 4.69.01 | 1990-096 Lisboa, Portugal. https://orcid.org/0000-0002-5335-7498

E-mail:ana.tavares@estesl.jpl.pt

Supplementary Issue: Spring Conferences of Sports Science. International Seminar of Physical Education, Leisure and Health, 17-19 June 2019. Castelo Branco, Portugal.

JOURNAL OF HUMAN SPORT \& EXERCISE ISSN 1988-5202

(C) Faculty of Education. University of Alicante.

doi:10.14198/jhse.2019.14.Proc4.82
} 


\section{INTRODUCTION}

Nowadays, we assist to a growing discussion about the potential risks that the exacerbated physical exercise can induce in the human body, namely at the renal system level. Physical exercise requires a metabolic adaptation, depending on the type of intensity and its duration, and after its completion some organs such as the kidneys, heart and spleen may undergo changes (Neto, Carvalho, Nicolau \& Costa, 2017). Considering that in Portugal, about $6.6 \%$ of the population currently attend at gymnasia and fitness centres (AGAP, 2016), it is relevant to evaluate proteinuria and creatininuria (and its ratio - since this is an excellent marker of daily proteinuria) in a sample of regularly gym users, using or not protein supplementation, before and after performing a training session of high intensity. The findings obtained in this study pretend to contribute to the state of art about this issue.

\section{MATERIAL AND METHODS}

\section{Participants}

A convenience sample of 18 sportsmen attending gyms, of both sexes ( 9 female and 9 male), aged between 19-31 years old, that trained regularly for more than 6 months, carried out a diet considered normal and using or not protein supplementation.

\section{Measures}

Questionnaire about frequency of training, type of diet and presence of renal, hepatic or metabolic diseases, in order to avoid bias in the values of urinary protein and creatinine caused by pathological alterations and / or by physiological states that are prone to alteration of values. The values of creatinine and proteins in the urine were quantitatively measured (modified Jaffe's kinetic method and pyrogallol red method, respectively) in automatic system - Abbott@ Architect C8200.

\section{Procedure}

Participants were previously divided into 2 groups - users of protein supplementation and not users of protein supplementation. Prior to the training and after explaining the objectives of the training, the participants gave their informed consent to participate and answered a questionnaire. After receiving the first urine sample, all participants performed the planned high-performance training, with an average duration of 45 minutes, performed by a qualified professional. At the end of the training the participants were given an extra 15 minutes to collect the second urinary sample, thus making a 60 -minute time period from the first urine collection.

\section{Analysis}

The statistical analysis was performed using the SPSS (version 22) and was based on descriptive statistic (e.g. mean, median, standard deviation) and statistical inference (e.g. Wilcoxon-test; Mann-Whitney Test and t-Student paired test-test).

\section{RESULTS}

Our sample includes individuals of both sexes, being $50 \%(n=9)$ of male participants and $50 \%(n=9)$, of female participants, aged between 19-31 years old $(M=23.11 ; S D=3.67)$. Analysing the results obtained by assaying the urinary protein and creatinine concentrations, as well as the respective ratio, in the participants who were supplemented with protein, we found that an average of $10.42 \pm 7.36 \mathrm{mg} / \mathrm{dL}$ of urinary protein before high-performance training, rising to $22.38 \pm 23.93 \mathrm{mg} / \mathrm{dL}$ after the same training. On the other 
hand, urinary creatinine in the same group had mean values of $96.07 \pm 64.11 \mathrm{mg} / \mathrm{dL}$ and $123.05 \pm 71.85 \mathrm{mg}$ $/ \mathrm{dL}$, before and after training (respectively). The mean values of the proteinuria/creatinuria ratios also changed, with a mean value of $0.12 \pm 0.13 \mathrm{mg} / \mathrm{mg}$ before training and $0.19 \pm 0.11 \mathrm{mg} / \mathrm{mg}$ thereafter obtained. In the non-protein supplementation group, mean values of urinary proteinuria before training of $8,06 \pm 2,00$ $\mathrm{mg} / \mathrm{dL}$ were recorded and this value increased to $16.28 \pm 8.70 \mathrm{mg} / \mathrm{dL}$ after training. Urinary creatinine also increased after high-performance training, with a mean of $113.42 \pm 84.49 \mathrm{mg} / \mathrm{dL}$ and $168.50 \pm 117.49 \mathrm{mg} / \mathrm{dL}$. The mean proteinuria /creatinuria ratio observed before and after training was, respectively, $0.08 \pm 0.06$ $\mathrm{mg} / \mathrm{mg}$ and $0.10 \pm 0.04 \mathrm{mg} / \mathrm{mg}$.

\section{DISCUSSION}

It was verified statistically significant differences in urinary protein (Wilcoxon-test, $p=0.009$ and $p=0.006$ ) and creatinine concentrations (t-Student paired test-test, $p=0.0005$ and $p=0.004$ ), both in users and non-users of protein supplementation, respectively, before and after performing high intensity physical exercise. In both groups the concentrations of these two analytes had risen about twice as much after training indicating that high-throughput physical exercise in a small fraction of time may induce a substantial increase in urinary protein and creatinine excretion. These results are in line with the findings of Neto et al. (2017), which could indicate that intense physical exercise may aggravate this condition in individuals predisposed to renal pathologies. The proteinuria/creatinuria ratio was not significantly increased in the user group of protein supplementation before performing physical training. However, we can admit that RPC after exercise was significantly superior (Mann-Whitney test, $p=0,035$ ) in the user group of protein supplementation, leading us to think that athletes who are supplemented with protein may be more likely to develop some type of kidney disease.

\section{CONCLUSIONS}

It was verified that intense physical exercise, even in a short time, significantly influences the urinary excretion of proteins and creatinine. Although is not possible to say that protein supplementation has harmful effects on the renal system, it seems that athletes predisposed to kidney disease should not take protein supplements. This research is of great importance due to the increase of people that unduly engage in intensive physical exercise and uncontrolled intake of protein supplementation and education programmes regarding protein supplementation are recommended.

\section{REFERENCES}

AGAP. (2016). Sumário executivo - Barómetro 2016 [Executive summary - Barometer 2016匹. Retrieved from http://www.agap.pt/images/userfiles/files/BAROMETRO 2016_AF_SM.pdf

Neto, O., Carvalho, A., Nicolau, L., \& Costa, A. (2017). Analysis of proteinuria after intense physical exercise. Revista Brasileira de Análises Clínicas, 256-262.

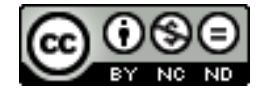

This work is licensed under a Attribution-NonCommercial-NoDerivatives 4.0 International (CC BY-NC-ND 4.0). 


\title{
Lipid profile and associated factors among an academic community of Higher Education
}

\author{
ANA SOFIA TAVARES ${ }^{1,2} \triangle$, AMADEU BORGES-FERRO ${ }^{1}$, ALEXANDRA VENTURA ${ }^{1}$, CATARINA \\ SANTOS ${ }^{1}$, MAGDA CAETANO ${ }^{1}$ \\ ${ }^{1}$ IPL - ESTeSL, Instituto Politécnico de Lisboa, Escola Superior de Tecnologia da Saúde de Lisboa, Portugal \\ ${ }^{2}$ H\&TRC, Health \& Technology Research Center, IPL-ESTeSL, Portugal
}

\begin{abstract}
Lipid profile, glycaemia and body mass index (BMI) present a direct relationship with lifestyles, eating habits and practice of physical activity. The aim of this study was to evaluate the lipid profile, glycaemia and relate these variables to each other and to the level of physical activity and BMI in an academic community of Higher Education. Furthermore, it was analysed whether gender and age group influence these parameters. A convenience sample of sixty-six participants complete an anonymous questionnaire and were submitted to capillary blood sample collection. Lipid profile and glucose levels were determined by the enzymatic assay. BMI was determined from weight and height measurements, and the level of physical activity was calculated through the application of a questionnaire (IPAQ). Findings showed a positive correlation between glucose values with triglycerides and $\mathrm{BMI}$ and the same for Total cholesterol with the remaining values of the lipid profile. A negative correlation was found between HDL cholesterol and BMI. Concerning age group and gender it was verified that there were higher values of Total cholesterol and HDL in female compared to male counterparts and increased glucose levels, Total cholesterol, LDL, triglycerides and BMI, with increasing age. Concerning lipid profile, there were alterations in $42 \%$ of the individuals, and in relation to the glycaemia there were alterations in $2 \%$ of the individuals. $21 \%$ of individuals showed risk of pre-obesity and $3 \%$ of obesity. Findings could support educational strategies in academic context in favour of cardiovascular risk reduction. Keywords: Lipid profile; Glycaemia; Body Mass Index; Physical activity; Academic community.
\end{abstract}

\footnotetext{
Corresponding author. Escola Superior de Tecnologia da Saúde, Instituto Politécnico de Lisboa, Av. D. João II, Lote 4.69.01 | 1990-096 Lisboa, Portugal. https://orcid.org/0000-0002-5335-7498

E-mail: ana.tavares@estesl.jpl.pt

Supplementary Issue: Spring Conferences of Sports Science. International Seminar of Physical Education, Leisure and Health, 17-19 June 2019. Castelo Branco, Portugal.

JOURNAL OF HUMAN SPORT \& EXERCISE ISSN 1988-5202

(C) Faculty of Education. University of Alicante.

doi:10.14198/jhse.2019.14.Proc4.82
} 


\section{INTRODUCTION}

Although there are several factors which contribute to a higher risk of developing cardiovascular diseases, it is well-known that some of them are modifiable, such as physical inactivity, eating patterns and smoking habits (Robbins, Dietz, Cox, \& Kuklina 2013). An increase in physical activity is associated with a significant reduction in the risk of cardiovascular-related death and also can attenuate or reverse the disease process in patients with cardiovascular disease, which can be mainly explained by a reduction in the plasma triglyceride concentration (Bellou et al., 2013; Warburton, Nico \& Bredin, 2006). The aim of this study was to evaluate the lipid profile, glycaemia and relate these variables to each other and to the level of physical activity and Body Mass Index (BMI) in an academic community of Higher Education. Furthermore, it was analysed whether gender and age group are also related with these parameters.

\section{MATERIAL AND METHODS}

\section{Participants}

Participants were recruited from 3 high schools of the Polytechnic Institute of Lisbon (students, teachers and other staff) originating a convenience sample of 66 participants (52 women; 14 men), aged between 18-61 years old.

\section{Measures and Procedure}

Prior to data collection, subjects signed an informed consent. Data collection was performed in two stages. Firstly, participants completed the International Physical Activity Questionnaire (IPAQ), concerning duration and level of physical activity. Secondly, capillary blood sample collection was performed to determine lipid and glycaemia profile, using Cholestech LDX equipment, using enzymatic methods. LDL value was calculated using Friedwald's formula. Height and weight were measured for BMI calculation. All procedures performed in this study were in accordance with the ethical standards of the institutional and national research committee and with the 1964 Helsinki declaration and its later amendments or comparable ethical standards.

\section{Analysis}

The statistical analysis was based on a descriptive statistic (e.g. mean, median, standard deviation) and nonparametric statistic inference (e.g. Spearman's Ordinal Correlation Coefficient and Mann-Whitney Test).

\section{RESULTS}

The results for the different parameters were as follows: glycaemia $-M=90.20, S D=17.05$; Total cholesterol $-M=161.23, S D=31.02 ; \mathrm{HDL}-M=53.53, S D=13.82 ; L D L-M=84.77, S D=25.86$; triglycerides $-M=115.61$, $S D=67.30 ; B M I-M=23.52, S D=3.60$. Concerning lipid profile and glycaemia, $42 \%$ and $2 \%$ of the individuals, respectively, showed abnormal results. Regarding BMI values, $21 \%$ of the individuals are in pre-obesity and $3 \%$ are already obese.

There was a significant correlation between the parameters: glycaemia and triglycerides ( $\mathrm{rs}[66]=.251$, $p<.050)$; glycaemia and BMI (rs[66]=.269, $p<.050)$, Total cholesterol and triglycerides (rs[66]=.457, $p<.001$ ) and HDL cholesterol and BMI (rs[66] $=-.440, p<.001)$.

The Mann-Whitney test showed that Total and HDL cholesterol was statistically significantly higher in women $(p<.050 ; p<.001$, respectively). The same test gave statistically significantly higher glycaemia, Total 
cholesterol, LDL, triglycerides and BMI in the over 34 years old group $(p<.050 ; p<.001 ; p<.001 ; p<.050$; $p<.001$, respectively).

\section{DISCUSSION}

The findings confirm the presence of $42 \%$ of individuals with one of the lipid profile parameters altered, which is higher than what was reported in similar studies (Assis et al., 2011). As was expected, there was an association between the increase in Total Cholesterol levels and the increase in the levels of LDL Cholesterol and Triglycerides in the study population. On the contrary, a positive association between Total Cholesterol and HDL Cholesterol was found. A significant negative correlation was confirmed, between HDL levels and $\mathrm{BMI}$ values, which is in line with the expected, since a decrease in weight and, consequently, a decrease in BMI, leads to an increase in HDL Cholesterol (Coelho et al., 2005). Regarding physical activity, there was no correlation between the remaining variables. These results are not according to that reported in some studies, since there is an association of a decrease of triglycerides; as well as a substantial increase in HDL levels, decreased levels of LDL, BMI, Glucose and Total Cholesterol, when increasing physical activity (Bellou et al., 2013; Wang \& Xu, 2017). Females showed higher values of Total cholesterol and HDL cholesterol compared to male counterparts, which is aligned with other studies (Coelho et al., 2005). Concerning age, it was found that individuals with more advanced ages presented higher values for glucose, Total cholesterol, LDL, triglycerides parameters and higher BMI.

\section{CONCLUSIONS}

The information presented in this study highlights that academic context, could provide an additional setting for intervention and prevention efforts targeting cardiovascular risk disease and could guide prevention strategies based on educational campaigns.

\section{REFERENCES}

Assis C, Duarte G, Silva I, Tostão I, Abreu R, Caria E. (2011). Perfil lipídico em estudantes do ensino superior: caracterização numa população de jovens [Lipid profile in higher education students: characterization of a young population]. Saúde \& Tecnologia, 6, 32-7.

Bellou, E., Siopi, A., Galani, M., Maraki, M., Tsekouras, Y. E., Panagiotakos, D. B., ... Sidossis, L. S. (2013). Acute effects of exercise and calorie restriction on triglyceride metabolism in women. Medicine and Science in Sports and Exercise, 45(3), 455-61. https://doi.org/10.1249/mss.0b013e318278183e

Coelho, V. G., Caetano, L. F., Roio, R. Del, Júnior, L., Cordeiro, J. A., Rossi, D., \& Souza, S. (2005). Perfil Lipídico e Fatores de Risco para Doenças Cardiovasculares em Estudantes de Medicina [Lipid Profile and Risk Factors for Cardiovascular Diseases in Medicine Students]. Arquivos Brasileiros de Cardiologia, 85(1). https://doi.org/10.1590/s0066-782x2005001400011

Wang, Y., \& Xu, D. (2017). Effects of aerobic exercise on lipids and lipoproteins. Lipids in Health and Disease, 16(1), 132. https://doi.org/10.1186/s12944-017-0515-5

Robbins, C. L., Dietz, P. M., Cox, S., \& Kuklina, E. V. (2013). Cholesterol screening for women: who is "at- risk" ? Journal of Women's Health, 22(5), 404-11. https://doi.org/10.1089/jwh.2012.4074

Warburton, D. E. R., Nicol, C. W., \& Bredin, S. S. D. (2006). Health benefits of physical activity: the evidence. CMAJ: Canadian Medical Association, 174(6), 801-9. https://doi.org/10.1503/cmaj.051351 


\section{(c) (i) (3)}

This work is licensed under a Attribution-NonCommercial-NoDerivatives 4.0 International (CC BY-NC-ND 4.0). 


\title{
Sociological analysis of three dual combat practices in Portugal: The case study of aikido, judo and wrestling
}

\author{
VÍTOR ROSA \\ CPES, Centro de Pesquisa e Estudos Sociais, Portugal
}

\begin{abstract}
The aim of the present paper it's to show the results of a sociological study on three sports of combat (aikido, judo and wrestling) in Portugal. There is no comparative study on these sports practices. In our initial question, we wonder if the sociocultural characteristics of individuals determine the affinities that are generated in the practice of dual combat sports of prehension, in particular of aikido, judo and amateur fights. Based on the problematization, we assume that the sociocultural characteristics of the practitioners of three dual combat sports determine the relations and affinities in the practice of the modalities in appreciation, although family tradition, career opportunities, visibility and social recognition demarcate the choice between them and the type of involvement in sports practice. We have delimited the empirical object to the coaches of aikido, judo and amateur fights in Portugal and Autonomous Regions (Madeira and Azores), in the sport season 2018/2019. Three theoretical hypotheses were defined to be analysed and discussed in order to verify their truthfulness. The questionnaire and the interviews are ongoing and we have currently 31 questionnaires and 5 interviews validated. The data is being processed in SPSS Statistic. The provisional results show that sociocultural characteristics of the practitioners determine the relations and affinities in the practice. Keywords: Sociology of sport; Martial arts; Sport of combat.
\end{abstract}

Corresponding author. Universidade Lusófona, Campo Grande, 376, Edifício U, 1749-024 Lisboa.

E-mail: vitor.rosa@ulusofona.pt

Supplementary Issue: Spring Conferences of Sports Science. International Seminar of Physical Education, Leisure and Health, 17-19 June 2019. Castelo Branco, Portugal.

JOURNAL OF HUMAN SPORT \& EXERCISE ISSN 1988-5202

(c) Faculty of Education. University of Alicante.

doi:10.14198/jhse.2019.14.Proc4.82 


\section{INTRODUCTION}

The objective of this paper it's to present the results of a comparative study on three sports of combat (aikido, judo and wrestling) in Portugal. There is no comparative study on these sports practices. There are some doctoral studies, but with a focus on karate (Figueiredo, 2006; Rosa, 2017; Tomás, 2018). In France, the only doctoral thesis known, which compares the three sports practices in question, is Clément (1995). We ask ourselves if the sociocultural characteristics of individuals determine the affinities that are generated in the practice of dual combat sports of prehension, of aikido, judo and amateur fights. We assume that the socio-cultural characteristics of the practitioners of three dual combat sports in Portugal determine the relations and affinities in the practice of the present modalities, even though the family tradition, career opportunities, visibility and social recognition mark the choice between them and the type of involvement in sports practice. In the deepening of our object of study, we will delimit the empirical object to the coaches of aikido, judo and amateur fights in Continental Portugal and Autonomous Regions (Madeira and Azores), in the sport season 2018/2019. A questionnaire survey of coaches and semi-directive interviews are planned for practitioners, who are coaches. The interviews with the privileged interlocutors will help in the discussion of the hypotheses. Three theoretical hypotheses have been defined to be analysed and discussed in order to verify their truthfulness. We define our Disaggregated Analysis Model (MAD), based on the relation of a broad set of variables and indicators capable of making them observable. With this research, and in the wake of our previous work, we hope to deepen a topic that has not been approached in Portugal.

\section{MATERIAL AND METHODS}

In the deepening of our object of study, we will delimit the empirical object to the coaches of aikido, judo and wrestling in Continental Portugal and Autonomous Regions (Madeira and Azores), in the sport season 2018/2019. Three theoretical hypotheses were defined to be analysed and discussed in order to verify their truthfulness.

\section{Participants}

In the present we have 31 participants of the three sports of combat (aikido, judo and amateur fights): 15 (judo), 14 (aikido) and 2 (wrestling), of the both sexes. The survey and the interviews it's in progress, using non-probabilistic "snowball" sampling. We hope to have a good sample at the end of this sociological research.

\section{Measures}

We assume that there is a homogeneity between the social profiles of the practitioners of the three combat sports and that there are differences regarding the level of habits and values (H1). We also consider that there is a differentiation in terms of social provisions, visibility, career opportunities and social reproduction (H2). We have also hypothesized to investigate, that there is a differentiation in terms of involvement, type of practice/conception, access conditions, family influence, practice intensity and associative participation (H3). For processing the quantitative data, we use SPSS and the convenient tests and MAXQDA for the qualitative information. In this paper only provisional data are presented.

\section{Procedures}

In the operationalization of the hypotheses we define our Disaggregated Analysis Model (MAD), based on the relation of a broad set of variables and indicators capable of making them observable. As elucidated to Quivy \& Campenhoudt (2008), the model of analysis, which operationalizes the hypotheses, is nothing more than the constitution of a gap between the problem set by us and the work of elucidation on the precise field 
of analysis. Four dimensions were defined in MAD: D1. Social Arrangements for AM\&DC; D2. Involvement in AM\&DC; D3. Values, Habits and Tastes; D4. Sociodemographic Characteristics of Practitioners.

\section{Analysis}

We will make some analysis with the software SPSS and MAXQDA. This two software are used by researchers for complex statistical data analysis.

\section{RESULTS}

The data we have available suggests that the sociocultural characteristics of the practitioners of three dual combat sports in Portugal determine the relations and affinities in the practice of the modalities in appreciation, although family tradition, career opportunities, visibility and social recognition demarcate the choice between them and the type of involvement in sports practice.

\section{DISCUSSION}

All combat sports and martial arts constitute a relatively autonomous field within the space of sporting practices. In relation to the majority sports (collective sports, athletics, swimming, etc.), of Anglo-Saxon inspiration, the practices of combat are distinguished by their cultural origins. In this sense, the deployment and development of combat sports and martial arts cannot be reduced to the appropriation (or the discovery) of different body models successively imported. The logic of appropriation implies a process of transformation and a creation and innovation of bodily models.

\section{CONCLUSIONS}

Characterized by traditional and modern elements, repetitive and innovative, the adaptation of the oriental arts and combat sports has been gradually happening, even though there is visible a sporting turnaround in the last decades, marked by the predominance of competition. After its introduction in the West, the Asian combat practices, popularized and later mediated, did not stop questioning the reasons of the success of its implantation and diffusion.

\section{REFERENCES}

Clément, J.-P. (1995). Étude comparative de trois disciplines de combat (lutte, judo, aikido) et de leurs usages sociaux, Thèse de doctorat en Sociologie, Paris : Université Paris 7 : UFR Sciences Sociales.

Figueiredo, A. (2006). A Institucionalização do Karaté - Os Modelos Organizacionais do Karaté em Portugal. Tese de doutoramento (não publicada) em Ciências do Desporto. Cruz Quebrada: Faculdade de Motricidade Humana.

Quivy, R. \& Campenhoudt, L. ([1992] 2008). Manuel de investigação em ciências sociais. Lisboa: Gradiva.

Rosa, V. (2017). A prática desportiva do karaté em Portugal. Análise sociológica sobre as identidades, ideologias, comunidades e culturas dos karatecas (cintos castanho e negro) portugueses. Tese de doutoramento (não publicada) em Educação Física e Desporto, Didática. Lisboa: ULHT.

Tomás, J. (2018). Socialização, desporto e qualidade de vida: exemplo dos praticantes de karaté-do. Tese de doutoramento (não publicada) em Sociologia. Évora: Universidade de Évora. https://doi.org/10.3895/rbqv.v7n3.2810 


\section{(c) (i) (3)}

This work is licensed under a Attribution-NonCommercial-NoDerivatives 4.0 International (CC BY-NC-ND 4.0). 


\title{
Comparison of propulsive forces between two head-out water exercises
}

\author{
CATARINA C. SANTOS ${ }^{1}$, LUÍS M. RAMA ${ }^{1,2}$, RAUL F. BARTOLOMEU 3,4 , TIAGO M. BARBOSA ${ }^{4,5,6}$, \\ MÁRIO J. COSTA 4,7 \\ ${ }^{1}$ Faculty of Sports Science and Physical Education, University of Coimbra, Portugal \\ ${ }^{2}$ Research Unit for Sport and Physical Activity (CIDAF), Coimbra, Portugal \\ 3 University of Trás-os-Montes and Alto Douro, Vila Real, Portugal \\ ${ }^{4}$ Research Centre in Sport, Health and Human Development (CIDESD), Vila Real, Portugal \\ ${ }^{5}$ Department of Physical Education and Sports Sciences, Nanyang Technological University, Singapore \\ ${ }^{6}$ Department of Sports Sciences, Polytechnic Institute of Bragança, Portugal \\ ${ }^{7}$ Department of Sports Sciences, Polytechnic Institute of Guarda, Portugal
}

\begin{abstract}
The aim of this study was to analyse and compare the propulsive force between two basic head-out water exercises. Twenty-nine young healthy participants (age: $21.7 \pm 1.9$ years-old, body mass: $68.5 \pm 10.8 \mathrm{~kg}$, height: $168.2 \pm 9.6 \mathrm{~cm}$ ) performed an incremental protocol for each exercise (horizontal adduction and rocking horse) from 105 beats per minute (b-min-1) until $150 \mathrm{~b} \cdot \mathrm{min}-1$ with increments of $15 \mathrm{~b} \cdot \mathrm{min}-1$ every 30 seconds. Data acquisition required a differential pressure system to obtain propulsive forces in upper limbs', especially the peak force for the dominant member (DPeakF) and non-dominant member (NDPeakF). Force values from both exercises were higher in DPeakF and NDPeakF even when increasing the music cadence and higher forces were found in HAAdd. Differences $(p \leq 0.05)$ were found when comparing two exercises at lower music cadences. The main conclusion is that there are significantly differences between two basic head-out water exercises at lower cadences. Keywords: Water exercises; Music cadence; Propulsive forces.
\end{abstract}

\footnotetext{
Corresponding author. Estádio Universitário de Coimbra, Pavilhão 3 / 3040-256 Coimbra, Portugal.

E-mail: katasantos6@sapo.pt

Supplementary Issue: Spring Conferences of Sports Science. International Seminar of Physical Education, Leisure and Health, 17-19 June 2019. Castelo Branco, Portugal.

JOURNAL OF HUMAN SPORT \& EXERCISE ISSN 1988-5202

(C) Faculty of Education. University of Alicante.

doi:10.14198/jhse.2019.14.Proc4.82
} 


\section{INTRODUCTION}

Previous research in the aquatic fitness has been conducted to understand the acute and chronic physiological adaptations to head-out water exercises or programmes (e.g. Costa et al., 2018). However, there is little evidence on the kinetics of water based exercises. The existing studies just reported propulsive force values in swimming and rehabilitation during vertical head-out water exercises at maximum velocity (Prins et al., 1994; Becker and Havriluk, 2006). As such, there is no insight about the forces produced in different head-out exercises. The aim of the study was to compare the propulsive forces between two basic head-out water exercises at different music cadences.

\section{MATERIAL AND METHODS}

\section{Participants}

Twenty-nine young healthy subjects, eleven women and eighteen man (age: $21.7 \pm 1.9$ years-old, body mass: $68.5 \pm 10.8 \mathrm{~kg}$, height: $168.2 \pm 9.6 \mathrm{~cm}$ ), volunteered to participate in this study. The following inclusion criteria were considered: (i) at least one year of experience in basic head-out water exercises; (ii) clinically healthy and physically active; (iii) non-pregnant women, and; (iv) not having a muscle-skeletal or neurologic injury in the past six months. The failure to meet any of the criteria excludes the subject from the study.

\section{Measures}

Height and body mass were measured using a stadiometer (Harpenden 98.603, Holtain Ltd., Crosswell, UK) and a scale (SECA 770, Hanover, USA), respectively. A differential pressure sensor system (Aquanex, 4.1, Swimming Technology Research, USA), previous validated (Havriluk, 1988) and reported in swimming analysis (e.g. Becker and Havriluk, 2006) was used to collect propulsive forces. Two independent sensors were positioned in both subjects' hands (between the 3rd and 4th fingers) to obtain the following variables: (i) peak force of dominant member (DPeakF, N); (ii) peak force of non-dominant member (NDPeakF, N).

\section{Procedures}

The study was held in a $25-\mathrm{m}$ indoor pool with a mean water temperature of $29^{\circ} \mathrm{C}$. After a 3 minutes warmup, each participant performed at the "water tempo" two basic head-out water exercises: (i) horizontal arms adduction (HAAdd), and; (ii) rocking horse with horizontal arms adduction (RHAdd). The RHAdd is characterized as perform horizontal arms' adduction, fully extended and the hands at $90^{\circ}$ angle of attack, with the simultaneous lower limbs' action (Barbosa et al. 2010). In every cycle it was assumed same knee flexion and opposite leg hyperextension between leaps. The level of water surface was set at above jugular notch point.

Each exercise was performed over an incremental protocol, with 4 music cadences or stages, starting at 105 beats per minute (b-min-1) and increasing every 30 seconds by $15 \mathrm{~b} \cdot \min -1$, up to $150 \mathrm{~b} \cdot \mathrm{min}-1$. The music cadence was controlled by a metronome (Korg, MA-30, Tokyo, Japan) connected to a sound system. All participants gave their written informed consent for the participation and all procedures were in accordance with the Helsinki Declaration in respect to human research.

\footnotetext{
Analysis

Descriptive statistics (mean and standard deviation) were calculate for pooled sample. A non-parametric Wilcoxon Signed-Rank Test was used to compare differences between exercises in the selected variables. The level of statistical significance was set at $p \leq 0.05$.
} 


\section{RESULTS}

Table 1 presents the comparison of dominant and non-dominant upper-limb between HAAdd and RHAdd according to the music cadence.

Table 1. Comparison of dominant and non-dominant upper-limb between two exercises at the incremental protocol

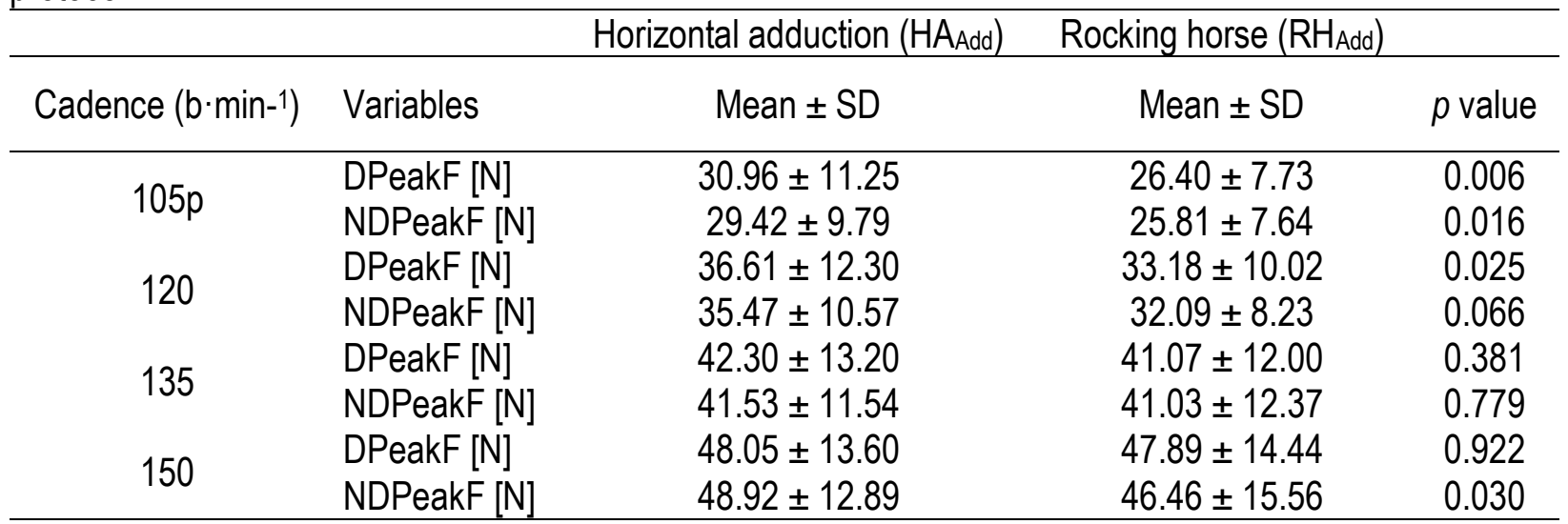

Note: $b \cdot$ min-1, beats per minute; SD, standard deviation; DPeakF, dominant peak force; NDPeakF, non-dominant peak force; $N$, Newton.

\section{DISCUSSION}

As expected, the DPeakF was higher than the NDPeak force in both exercises at majority of the cadences. When comparing exercises, there were higher forces for the HAAdd which were more pronounced at lower cadences. This means that at slower cadences the RHAdd promote lower force values compared to HAAdd probably due to imbalances, caused by multiple hops and the range of motion of the knee. For instance, we can have similar exertion pattern when adopted a static position or an imbalance position at higher music cadences, because the short time contact with the ground can lead to similar force values and even less hypothetic asymmetries.

\section{CONCLUSIONS}

To conclude, there are significantly differences between two basic head-out water exercises at lower cadences. So, head-out water fitness professionals should pay major attention and prescribe the exercises properly, when attempting to develop strength in water.

\section{REFERENCES}

Barbosa T.M., Sousa, V.F., Silva, A.J., Reis, V.M., Marinho, D.A., Bragada, J.A. (2010) Effects of musical cadence in the acute physiologic adaptations to head-out aquatic exercises. J Strength Cond Res, 24(1): 244-250. https://doi.org/10.1519/jsc.0b013e3181b296fd

Becker, T., \& Havriluk, R. (2006). Bilateral and anterior-posterior muscular imbalances in swimmers. In J. P. Vilas-Boas, F. Alves, A. Marques (Eds.), X Biomechanics and Medicine in Swimming. PJSS, 6(2): 327-328.

Costa, M.J., Cruz L., Simão, A., Barbosa T.M. (in press) Cardiovascular and perceived effort in head-out water exercises: effect of limbs' action and resistance equipment. J Hum Kinet. 
Havriluk, R. (1988). Validation of a criterion measure for swimming technique. J Swimming Res, 4(4): 11-16.

Prins, J.H., Hartung, G.H., Merritt, D.J., Blancq, R.J., Goobert, D.A. (1994) Effect of aquatic exercise training in persons with poliomyelitis disability. Sports Medicine, Training and Rehabilitation, 5(1): 29-39. https://doi.org/10.1080/15438629409511999

\section{(c) $(\mathrm{B})(\mathrm{EY}$}

This work is licensed under a Attribution-NonCommercial-NoDerivatives 4.0 International (CC BY-NC-ND 4.0). 


\title{
Experiences of bullying in education and school paths
}

\author{
AMÁLIA REBOLO ${ }^{1}$, BEATRIZ PEREIRA ${ }^{2}$ \\ 1 Instituto Piaget, Campus de Almada, RECl, Portugal \\ 2Universidade do Minho, CIEC, IE, Portugal
}

\begin{abstract}
According to Saraiva (2014) and Nery (2016) the memories of bullying experiences perpetuate themselves throughout life and produce long-term effects. In the scope of longitudinal research on school bullying, 17 young adults (8 males and 9 females) were interviewed in order to identify their memories of bullying experiences during compulsory schooling. The participants had been observed and interviewed during the first cycle of schooling considering the categories in which they were nominated by peers through questionnaires of peer nomination: aggressors, victims, aggressive victims, conflict managers, victim support. Each participant was interviewed individually, and interviews were recorded in digital audio format and transcribed retrospectively. We verified that regardless of their status all refer to have lived or observed situations of bullying and it is noteworthy that the strongest memories are related to the frequency of secondary education. We found that some experiences were well marked in the memory considering the details that were mentioned to us. We found that the school paths are different between groups considering the appointment as involved or not in conflicts. We can highlight the school course marked by the retention and abandonment of those who were named as aggressors while the students considered as conflict managers or supporters of the victims by their colleagues were successful at school during compulsory schooling and at the moment the interviews were held were attend degrees and master's degrees in their areas of preference. Keywords: School bullying; Adult memories; School routes.
\end{abstract}

Corresponding author. Instituto Piaget, Avenida Jorge Peixinho, $n .{ }^{\circ}$ 30, Quinta da Arreinela, 2805-059 Almada, Portugal. E-mail: amalia.rebolo@almada.ipiaget.pt Supplementary Issue: Spring Conferences of Sports Science. International Seminar of Physical Education, Leisure and Health, 17-19 June 2019. Castelo Branco, Portugal.

JOURNAL OF HUMAN SPORT \& EXERCISE ISSN 1988-5202

(c) Faculty of Education. University of Alicante.

doi:10.14198/jhse.2019.14.Proc4.82 


\section{INTRODUCTION}

The study "Playfight and Bullying in school playground" (Rebolo Marques, 2002-2006) showed that the children presented different characteristics according to the groups in which they were considered by their classmates in relation to: participation and type of participation in Game of Fight and Fight Seriously; school integration; family context; concerns and desires; networks of friends (Rebolo Marques, 2006). Five years later we found that children involved in conflict and aggression were less successful at school, and $40 \%$ of pupils nominated as aggressors had already repeated one or more years of schooling (Rebolo Marques, 2016). Fifteen years later we interviewed some of the participants to understand their school paths.

\section{MATERIAL AND METHODS}

\section{Participants}

16 young adults (ages between 21 and 25) who were part of the initial sample in the 2002-2003: 2 Aggressors (male), 2 Victim (1 male and 1 female), 3 Aggressor-Victim or provocative victim (males), 4 conflicts management (female) and 5 Victim Support (4 females and 1 male).

\section{Measures}

Semi-structured interview with thirteen questions - based on interviews of Rebolo Marques (2006), Saraiva (2011) and Nery (2016). We wanted to identify their school memories with questions like: How was your behaviour in school? What do you like most about school? What do you like least about school? Was there violence and / or bullying in school? Were they threatening or asking for money or other forms of aggression?

Do you remember any episode that was more serious? Do you remember the recreational games? What were the favourites? How was your behaviour at home? Were there conflicts at home?

We also try to understand their life's today with questions like: Who do you live with today? What are the prospects for the future? What were the most important moments in life?

\section{Procedures}

For each participant the starting point was the childhood interview that everyone had access (on paper). The interview was recorded using a VIMX digital recorder and later transcribed for content analysis and identification of analysis categories. They all consented the interview recording, and they were pleased to remember some episodes that happened during childhood.

\section{Analysis}

The data were subject to content analysis considering the interviews. The information was divided into eight points: as described in the pass and today; behaviour at school and at home during the first interview; the games and games in the playground, referred to in the first interview and remembered in the second; aspects that they like and did not like at school; involvement in conflict, aggression, bullying; family relationships in the past and present; prospects for the future taking into account what they wanted and what actually happened and foresees in the near future; important moments in life in the past and the present.

\section{RESULTS AND DISCUSSION}

The two considered as aggressors only finished primary school and are the ones with the highest rate of retention and drop out. In the group that included aggressors, victims and provoking victims we found that 
$71 \%$ of the students suffered retentions along the school course while in the remaining students of the sample only $20 \%$ were subject to withholdings. Other studies report the risk of aggression and/or victimization in the students that suffer retentions in their school course (Saraiva et al., 2011, Saraiva, 2014, Melin, 2016 and Pereira, 2002).

Only two of the interviewees are still full-time students, all the others are working part-time or full-time depending on whether they are still studying or not. The military career was the choice of the two aggressors and an aggressor victim, but one of the aggressors did not follow up and is in a phase of looking for solutions of employment and study. Those who are studying intend to pursue master's and doctoral studies having some already defined their areas of future interest.

All respondents reported having observed and / or participated in conflicts and struggles. Some have even used the term bullying to refer to some situations. They pointed out that serious situations happened especially after the 6th grade and with greater seriousness in secondary school.

One of the aggressor's recalls being provocative involving many schoolmates, but also defending his friends and sometimes engaging in fights for them.

One of the young women considered as "victim support" and two of the young women considered "conflict managers" by their colleagues, after the second cycle were victims of colleagues in their classes. They say they did not realize why, but they associate envy with the fact that they usually have above-average grades and are well liked by teachers.

\section{CONCLUSIONS}

Aggressors have a higher incidence of retention and dropout than all others (in general and per group).

When we bring together those directly involved - aggressors, victims and aggressive victims - in the same group, we find that levels of retention and dropout are higher than in other groups. Involvement in bullying seems to be related to school failure. All interviewees observed and / or participated in bullying situations.

Respondents are generally satisfied with their life decisions and intend to pursue studies.

\section{REFERENCES}

Melin, F. (2011). Na escola és feliz? Dissertação de Doutoramento. Braga: Universidade do Minho.

Nery, M. (2016). Bullying no contexto da Formação desportiva em Portugal. Estudo Exploratório a nível nacional de modalidades individuais, coletivas e de combate. Dissertação de doutoramento. Cruz Quebrada: FMH-UL.

Pereira, B.O. (2002). Para uma escola sem violência. Estudo e prevenção das práticas agressivas entre crianças, Porto: FCG-FCT.

Rebolo Marques, A. (2006). Jogo de Luta e bullying no recreio escolar. Dissertação de Doutoramento. Cruz Quebrada: FMH-UTL.

Rebolo Marques, A. (2016). Vivências de bullying e risco de insucesso escolar. In Beatriz Pereira, Altemir José Gonçalves Barbosa, Lélio Moura Lourenço (orgs.) Estudos sobre bullying: família, escola e atores, Curitiba-Brasil: Editora CRV, pp. 101-120. https://doi.org/10.24824/978854440904.6 
Saraiva, A. B. (2014). Memórias de infância e violência escolar: Estudo em contexto prisional. Dissertação de Doutoramento. Braga: IE- Universidade do Minho.

Saraiva, A. B.; Pereira, B, \& Zamith-Cruz, J. M. (2011). Trajetórias, vidas e bullying escolar, in Altemir Gonçalves Barbosa, Lélio Moura Lourenço \& Beatriz Pereira (organizadores) Bullying, conhecer e intervir, Juiz de Fora: Editora UFJF.

\section{(c) $(\mathrm{B})(\mathrm{EY}$}

This work is licensed under a Attribution-NonCommercial-NoDerivatives 4.0 International (CC BY-NC-ND 4.0). 


\title{
Insights on a sucessfull research-to-practice partnership with Matosinhos city hall: The case of surfing in schools
}

\author{
FERNANDO SANTOS ${ }^{1} \triangle$, CORLISS BEAN² ${ }^{2}$ NUNO AZEVEDO ${ }^{1}$, ANTÓNIO CARDOSO ${ }^{1}$, PAULO \\ PEREIRA ${ }^{1}$, HUGO CRUZ $^{3}$ \\ ${ }^{1}$ Polytechnic Institute of Porto, School of Higher Education, Portugal \\ ${ }^{2}$ School of Health and Exercise Sciences, University of British Columbia, Okanagan, Canada \\ ${ }^{3}$ Matosinhos City Hall, Portugal
}

\begin{abstract}
Throughout the last decades, researchers and practitioners have highlighted the need for quality physical education programs that include a life skills focus. However, few studies have provided insight on how organizations may develop an explicit approach toward life skills development and transfer. The purpose of this article was to provide insight on a partnership between Matosinhos City Hall and the School of Higher Education of Porto. For the past two years, the institutions have worked together to develop a local curriculum for physical education teachers to use to foster life skills development and transfer in their students. In this article, we provide an overview about (a) how an implicit approach to life skills development and transfer was embedded within the local curriculum, (b) the facilitators and barriers that emerged from the curriculum pilot, and (c) plans to integrate an explicit approach to life skills development and transfer. Awareness about the need for a life skill focus, time and positive influence from program participation were needed to foster buyin for the development of an explicit curriculum that included all stakeholders. This step-by-step approach that involved program creation and implementation with an implicit to an explicit focus helped the research team avoid substantial changes in short-periods of time that may create barriers for a life skill mandate. This is the first attempt to use the implicit/explicit continuum to understand and guide a research-to-practice partnership. Keywords: Life skills; Sport; Physical education teacher; Curriculum; Positive youth development.
\end{abstract}

Corresponding author. Polytechnic Institute of Porto, School of Higher Education, Portugal.

E-mail: fernando.sfsantos@hotmail.com

Supplementary Issue: Spring Conferences of Sports Science. International Seminar of Physical Education, Leisure and Health, 17-19 June 2019. Castelo Branco, Portugal.

JOURNAL OF HUMAN SPORT \& EXERCISE ISSN 1988-5202

(c) Faculty of Education. University of Alicante.

doi:10.14198/jhse.2019.14.Proc4.82 


\section{INTRODUCTION}

There has been a call for high quality youth development programs that use physical education to include a life skills focus (Chinkov \& Holt, 2016). Life skills have been defined in the literature (Gould \& Carson, 2008) as positive skills that are taught and applied by youth in physical education and transferred to other life domains. Different approaches have been taken to foster life skills. On one hand, an explicit approach that involves deliberate efforts towards life skills development and transfer (i.e., consider the need to use concrete strategies, objectives and activities) has been considered effective in generating positive developmental outcomes (Weiss, Stuntz, Bhalla, Bolter, \& Price, 2013). On the other hand, an implicit approach toward life skills development and transfer is less time-consuming and more manageable, and implies that teachers set rules, create a physically and psychologically safe environment, foster positive relationships and model positive behaviours. Based on these notions, few researches has attempted to understand the challenges related to embedding an implicitexplicit approach within physical education settings. In this article, we provide an overview about (a) how an implicit approach to life skills development and transfer was embedded within a local physical education curriculum, (b) the facilitators and barriers that emerged from the curriculum pilot in Matosinhos City Hall, and (c) plans to integrate an explicit approach to life skills development and transfer.

\section{CREATING AND IMPLEMENTING SURFING IN SCHOOLS IN PRIMARY SCHOOLS}

\section{First wave: Using an implicit approach to life skills development and transfer}

The first wave of Surfing in Schools was conducted between 2017 and 2018. The first step was to understand stakeholders' expectations and explore their initial understanding and perspectives toward life skills development and transfer. From conversations with stakeholders, an implicit approach was favoured because: (a) learning to teach surf was perceived as time-consuming and demanding task on its own; (b) developing life skills was seen as a complex endeavour; and (c) teaching surf and fostering a safe climate were crucial for youth participants' safety and adherence to the program.

\section{Second wave: Moving toward an explicit approach to life skills development}

In 2019, the research team attempted to integrate an explicit approach towards life skills development and transfer. Based on several researcher-stakeholder discussions, several life skills were deemed relevant: (a) emotional control, (b) focus, (c) perseverance, (d) teamwork, (e) decision-making, and (f) leadership. Through discussions with stakeholders, it was deemed crucial to engage other key social agents with the program curricula such as school managers and board of the Matosinhos City Hall. Short-duration modules will be created for the board of the Matosinhos City Hall and school managers.

\section{Lessons learned}

Throughout this two-year process of developing curriculum and delivering Surfing in Schools, the research team was able to gain valuable insight on how to implement a life skill focus within surf-based programs, and connected research to practice by utilizing the implicit/explicit continuum (Bean et al., 2018). This step-bystep approach that involved program creation and implementation with an implicit to an explicit focus helped the research team avoid substantial changes in short-periods of time that may create barriers for a life skill mandate. This is the first attempt to use the implicit/explicit continuum to understand and guide a researchto-practice partnership. 


\section{REFERENCES}

Bean, C., Kramers, S., Forneris, T., \& Camiré, M. (2018). The implicit/explicit continuum of life skills development and transfer. Quest, 70, 456-470. https://doi.org/10.1080/00336297.2018.1451348

Chinkov, A., \& Holt, N. (2016). Implicit transfer of life skills through participation in Brazilian jiu-jitsu. J. Appl. Sport Psychol., 28, 139-153. https://doi.org/10.1080/10413200.2015.1086447

Gould, \& Carson, S. (2008). Life skills development through sport: current status and future directions. Int Rev Sport Exerc Psychol., 1(1), 21-58. https://doi.org/10.1080/17509840701834573

Weiss, M., Stuntz, C. P., Bhalla, J. A., Bolter, N. D., \& Price, M. S. (2013). 'More than a game': Impact of the first tee life skills programme on positive youth development: Project introduction and Year 1 findings. Qual Res in Sport, Exerc Health, 5, 214-244. https://doi.org/10.1080/2159676x.2012.712997 


\title{
Levels of insufficient health-related physical activity in Portuguese adolescents
}

\author{
HENRIQUE COSTA ${ }^{1}$, HELDER MIGUEL FERNANDES ${ }^{2}$ \\ 1 Universidade de Trás-os-Montes e Alto Douro, Portugal \\ ${ }^{2}$ Research Centre in Sports Sciences, Health Sciences and Human Development, CIDESD-UTAD, Research \\ in Education and Community Intervention, RECl- Instituto Piaget, Portugal
}

\begin{abstract}
The negative consequences of physical inactivity and insufficient health-related physical activity during adolescence represent a major public health concern. Therefore, the aims of the present study are twofold: i) to investigate the inadequate levels of moderate to vigorous physical activity (MVPA) in a sample of Portuguese youth, and ii) to examine sex-, age category- and sports participation-related effects on the MVPA levels. A total of 1054 adolescents (612 girls and 442 boys), aged between 12 and 18 years $(M=14.40$, SD = 1.55), completed a sociodemographic survey and the PACE+ measure (Prochaska et al., 2001). The total score of this last measure was used to categorize the total sample into three groups (physically inactive: $\geq$ $60 \mathrm{~min}$ of MVPA in < 1 day/week; insufficiently active: $\geq 60 \mathrm{~min}$ in $<5$ days/week; and, physically active: $\geq$ $60 \mathrm{~min}$ in $\geq 5$ days/week). Descriptive statistics included means, standard deviations, and percentages. The chi-square test was employed in order to compare proportions between variables $(p<0.05)$. Analysis of the total sample showed relatively moderate to high percentages of physically inactive $(22.7 \%)$ and insufficiently active adolescents (69.6\%), whereas the physically active group only represented $7.7 \%$ of the total sample. Comparisons between subgroups showed that the percentage of physically inactive adolescents was significantly higher among girls ( $+17.2 \%$ than boys), late adolescents $(+11.2 \%$ than early adolescents) and non-sports youth $(+28.6 \%$ than young athletes). In sum, this cross-sectional study shows high and worrying levels of insufficient health-related physical activity in a sample of Portuguese youth. Keywords: Physical inactivity; Adolescence; Health-related physical activity; Sports participation.
\end{abstract}

Corresponding author. Tapada da Mareca s/n, Quinta da Pocariça, 6300-777 Guarda. Portugal.

E-mail: henriquefcosta@gmail.com

Supplementary Issue: Spring Conferences of Sports Science. International Seminar of Physical Education, Leisure and Health, 17-19 June 2019. Castelo Branco, Portugal.

JOURNAL OF HUMAN SPORT \& EXERCISE ISSN 1988-5202

(c) Faculty of Education. University of Alicante.

doi:10.14198/jhse.2019.14.Proc4.82

VOLUME 14 | Proc4 | 2019 | S1375 


\section{INTRODUCTION}

Insufficient physical activity (i.e., not achieving the recommended physical activity guidelines) and/or the adoption of sedentary behaviours in youth have been associated with several adverse health outcomes, such as unfavourable body composition, higher clustered metabolic risk, decreased fitness, and lower self-esteem, pro-social behaviour and health-related quality of life (Carson et al., 2016; Kumar et al., 2015).

Since both physical inactivity and insufficient health-related physical activity represent major public health concerns, the aims of the present study are twofold: i) to investigate the inadequate levels of MVPA in a sample of Portuguese youth, and ii) to examine sex-, age category- and sports participation-related effects.

\section{MATERIAL AND METHODS}

\section{Participants}

The total sample comprised 1054 adolescents ( 612 girls and 442 boys), aged between 12 and 18 years (M $=14.40, \mathrm{SD}=1.55)$, and attending the 7 th to 12 th grades at public schools located in four of the country's central region districts. Participants were grouped into three age categories: early (12-13 yrs.: $32.3 \%)$, middle (14-15 yrs.: $41.5 \%)$ and late adolescence (16-18 yrs.: 26.2\%).

\section{Measures}

The PACE+ measure, developed for adolescents by Prochaska et al. (2001), was used to assess levels of physical activity. This 2-item measure asks the number of days, on a typical week and previous week, of at least 60 minutes of MVPA, not including physical education or gym class. A composite score is computed from the average of the two items. The authors of this instrument suggested that a score of less than 5 would indicate that the adolescent did not meet the guideline of accumulating MVPA (i.e., 60 minutes) at least 5 days per week, which was also adopted in our study. As such, the total sample was categorized into three groups as follows (physically inactive: $<1$ day/week; insufficiently active: $<5$ days/week; and, physically active: $\geq 5$ days/week).

\section{Procedures}

All study's phases were carried out in accordance with the recommendations and approval of the ethics committee of the Portuguese Directorate-General for Education (Study Registration No 0395700001/MIME).

After obtaining the schools board's approval and a written informed consent of the participants' parents/guardians, the adolescents completed the questionnaire individually in quiet classroom conditions. The confidentiality and anonymity of the participants were guaranteed and participation was voluntary.

\section{Analysis}

Descriptive statistics included means, standard deviations, and percentages. The chi-square test was employed in order to compare proportions between variables. Significance was set at $p<0.05$. All analyses were performed using SPSS 17.0 (SPSS Inc., Chicago).

\section{RESULTS}

Analysis of the total sample showed relatively moderate to high percentages of physically inactive (22.7\%) and insufficiently active adolescents (69.6\%), whereas the physically active group only represented $7.7 \%$ of the total sample. Figure 1 presents the percentages of MVPA levels by sex, age and sports participation. 
All chi-square tests were significant at $p<0.01$, as follows: sex: $x^{2}(2)=72.31, p<0.001$; age groups: $x^{2}(4)=$ 13.62, $p<0.01$; and, sports club participation: $X^{2}(2)=148.84, p<0.001$. Comparisons between subgroups showed that the percentage of physically inactive adolescents was higher among girls ( $+17.2 \%$ than boys), late adolescents $(+11.2 \%$ than early adolescents) and non-sports youth $(+28.6 \%$ than young athletes).

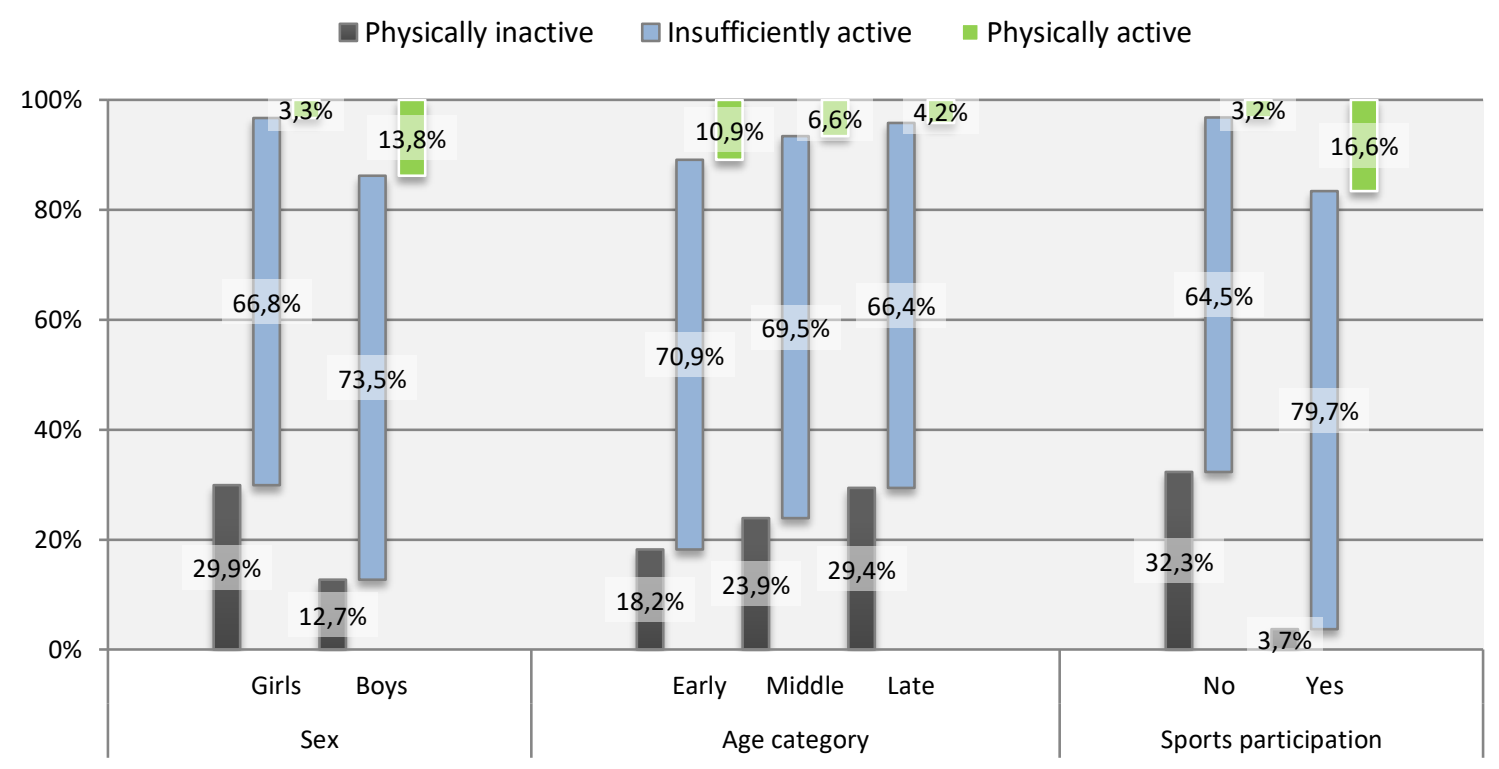

Figure 1. Percentages of the MVPA levels by sex, age categories and sports participation.

\section{DISCUSSION}

The present findings suggest that a considerable percentage of the adolescents in our sample do not meet the MVPA guidelines, with girls, older adolescents and non-sports youth representing the most at-risk subgroups. The results both confirm and extend previous findings in the epidemiology of insufficient healthrelated physical activity in young people (Fernandes, 2018; Kumar et al., 2015), reinforcing the need for more/better interventions and policies aimed at promoting a more active lifestyle throughout this life period.

\section{CONCLUSIONS}

In sum, this cross-sectional study shows high and worrying levels of insufficient health-related physical activity in a sample of Portuguese youth. However, these findings should be interpreted with caution because of the reliance on a self-report measure, the non-assessment of physical education time and the geographical scope of the sample.

\section{REFERENCES}

Carson, V., Hunter, S., Kuzik, N., Gray, C. E., Poitras, V. J., Chaput, J., ... Tremblay, M. S. (2016). Systematic review of sedentary behaviour and health indicators in school-aged children and youth: An update. Appl Physiol Nutr Metab, 41(6), S240-S265. https://doi.org/10.1139/apnm-2015-0630

Fernandes, H.M. (2018). Physical activity levels in Portuguese adolescents: A 10-year trend analysis (2006-2016). J Sci Med Sport, 21(2), 185-189. https://doi.org/10.1016/j.jsams.2017.05.015 
Kumar, B., Robinson, R., \& Till, S. (2015). Physical activity and health in adolescence. Clin Med (Lond), 15(3), 267-272. https://doi.org/10.7861/clinmedicine.15-3-267

Prochaska, J., Sallis, J., \& Long, B. (2001). A physical activity screening measure for use with adolescents in primary care. Arch Pediatr Adolesc Med, 155, 554-559. https://doi.org/10.1001/archpedi.155.5.554

\section{(c) $(\mathrm{B})(\mathrm{EY}$}

This work is licensed under a Attribution-NonCommercial-NoDerivatives 4.0 International (CC BY-NC-ND 4.0). 


\title{
Gender differences in psychosocial benefits of physical activity and sports participation in youth
}

\author{
HENRIQUE COSTA ${ }^{1}$, HELDER MIGUEL FERNANDES ${ }^{2}$ \\ 1 Universidade de Trás-os-Montes e Alto Douro, Portugal \\ ${ }^{2}$ Research Centre in Sports Sciences, Health Sciences and Human Development, CIDESD-UTAD, Research \\ in Education and Community Intervention, RECl- Instituto Piaget, Portugal
}

\begin{abstract}
The aim of the current study is to examine gender differences in the association of moderate to vigorous physical activity (MVPA) and sports participation with selected psychosocial dimensions, namely self-esteem, emotional intelligence (EI), social anxiety and life satisfaction. A total of 1054 adolescents (612 girls and 442 boys), aged between 12 and 18 years $(M=14.40, S D=1.55)$, volunteered to participate in this study. Participants completed a set of self-report measures: the PACE+ (Prochaska et al., 2001), a single item (yes/no) sports participation, and Portuguese adapted and validated versions of the Rosenberg Self-Esteem Scale (Raposo \& Freitas, 1999), the Wong and Law Emotional Intelligence Scale (Rodrigues et al., 2011), the Social Anxiety Scale for Adolescents (Cunha et al., 2004) and the Satisfaction with Life Scale (Neto, 1993). Results showed that boys reported higher levels of MVPA and sports participation than girls. Among girls, MVPA levels were positively associated with the El use of emotions dimension and life satisfaction, whereas boys' MVPA levels were positively correlated with self-esteem, others' emotional appraisal (EI), use of emotions (EI) and life satisfaction. Girls participating in organized sports showed higher levels of use of emotions (EI) and lower levels of social avoidance and distress in new situations than non-athlete females. On the other hand, boys who participated in organized sports reported better levels of self-esteem and El use of emotions, than their counterparts. The present results indicate that the psychosocial outcomes of physical activity and sports participation differ by gender in a sample of Portuguese youth, with boys receiving more psychological benefits from an active lifestyle than their female peers. Keywords: Physical inactivity; Adolescence; Health-related physical activity; Sports participation.
\end{abstract}

Corresponding author. Tapada da Mareca s/n, Quinta da Pocariça, 6300-777 Guarda. Portugal.

E-mail: henriquefcosta@gmail.com

Supplementary Issue: Spring Conferences of Sports Science. International Seminar of Physical Education, Leisure and Health, 17-19 June 2019. Castelo Branco, Portugal.

JOURNAL OF HUMAN SPORT \& EXERCISE ISSN 1988-5202

(C) Faculty of Education. University of Alicante.

doi:10.14198/jhse.2019.14.Proc4.82

VOLUME 14 | Proc4 | 2019 | \$1379 


\section{INTRODUCTION}

Accumulated empirical evidence has clearly demonstrated different levels of physical activity and sports participation by gender throughout adolescence (e.g., Fernandes, 2018a). At a psychological level, these distinct patterns of involvement levels are expected to lead to different effects and benefits (Ahn \& Fedewa, 2011; Fernandes, 2018b). However, few studies have explored gender variations in multiple psychosocial benefits of physical activity and sports participation in youth. Therefore, the aim of the current study is to examine gender differences in the association of moderate to vigorous physical activity (MVPA) and sports participation with selected psychosocial dimensions [self-esteem, emotional intelligence (EI), social anxiety and life satisfaction].

\section{MATERIAL AND METHODS}

\section{Participants}

A total of 1054 adolescents ( 612 girls and 442 boys), aged between 12 and 18 years $(M=14.40, S D=1.55$ ), volunteered to participate in this study. With respect to sports participation, 156 girls $(25.5 \%$ of the female sample) and 198 boys ( $44.9 \%$ of the male sample) reported to regularly participate and compete in organized sports events.

\section{Measures}

The PACE+ self-report measure, developed for adolescents by Prochaska et al. (2001), was used to assess levels of MVPA. Sports participation was assessed with a yes/no question. Participants also completed Portuguese adapted and validated versions of the Rosenberg Self-Esteem Scale (Raposo \& Freitas, 1999), the Wong and Law Emotional Intelligence Scale (Rodrigues et al., 2011), the Social Anxiety Scale for Adolescents (Cunha et al., 2004) and the Satisfaction with Life Scale (Neto, 1993). All scales and subscales showed acceptable to good levels of internal consistency (Cronbach's alphas >0.70).

\section{Procedures}

All study's phases were carried out in accordance with the recommendations and approval of the ethics committee of the Portuguese Directorate-General for Education (Study Registration N N 0395700001/MIME).

Firstly, we obtained the schools board's approval and a written informed consent of the participants' parents/guardians. Secondly, adolescents completed the questionnaire individually in quiet classroom conditions. The confidentiality and anonymity of the participants were guaranteed.

\section{Analysis}

Statistical analysis included descriptive statistics (mean, standard deviation and percentage), internal consistency (Cronbach's alpha), Pearson correlations and one-way ANOVAs. The chi-square test was employed in order to compare proportions between variables. Significance was set at $p<0.05$. All analyses were performed using SPSS 17.0 (SPSS Inc., Chicago).

\section{RESULTS}

Gender comparison of physical activity and sports levels indicated that boys reported significantly higher ( $p<$ $0.001)$ levels of MVPA (3.39 \pm 1.44$)$ and sports participation (44.9\%) than girls $(2.36 \pm 1.41$ and $25.5 \%$, respectively). 
Among girls, MVPA levels were positively associated with the El use of emotions dimension $(r=0.11, p<$ $0.05)$ and life satisfaction $(r=0.08, p<0.05)$. On the other hand, boys' MVPA levels were positively correlated with self-esteem $(r=0.13, p<0.01)$, El others' emotional appraisal $(r=0.15, p<0.01)$, El use of emotions $(r=$ $0.22, p<0.01)$ and life satisfaction $(r=0.11, p<0.05)$.

Girls participating in organized sports showed higher levels of El use of emotions ( $F=7.13, p<0.01)$ and lower levels of social avoidance and distress in new situations $(F=4.88, p=0.03)$ than non-athlete females. Conversely, boys who participated in organized sports reported better levels of self-esteem $(F=10.97, p=$ $0.001)$ and El use of emotions ( $F=28.31, p<0.001)$, than their counterparts.

\section{DISCUSSION}

The main aim of the current study was to explore gender variations in multiple psychosocial benefits of physical activity and sports participation in youth. The analysis of different levels of MVPA and sports participation observed in girls and boys resulted in a distinct pattern of psychosocial benefits, with boys showing more positive effects on certain psychological dimensions. Taken together, this evidence suggests that the psychosocial outcomes of physical activity and sports participation vary according to the genderspecific pattern of MVPA and sports undertaken. Furthermore, our results also confirm and extend previous findings (Ahn \& Fedewa, 2011) which demonstrate that, when analysed separately, boys receive more psychological benefits from an active lifestyle than their female peers do.

Although they are of different magnitudes, our results also suggest that increased levels of MVPA contribute to a better psychological functioning and well-being in adolescents of both genders. Future research should aim to better understand the psychological and sociocultural processes and mechanisms that may help explain the differentiated effects observed in adolescent girls and boys.

\section{CONCLUSIONS}

The present results indicate that the psychosocial outcomes of physical activity and sports participation differ by gender in a sample of Portuguese youth, with boys receiving more psychological benefits from an active lifestyle than their female peers.

\section{REFERENCES}

Ahn, S., \& Fedewa, A. (2011). A meta-analysis of the relationship between children's physical activity and mental health. J Pediatr Psychol, 36(4), 385-397. https://doi.org/10.1093/jpepsy/isq107

Cunha, M., Pinto-Gouveia, J., Alegre, S., \& Salvador, M. (2004). Avaliação da ansiedade na adolescência: A versão portuguesa da SAS-A. Psychologica, 35, 249-263.

Fernandes, H.M. (2018a). Physical activity levels in Portuguese adolescents: A 10-year trend analysis (2006-2016). J Sci Med Sport, 21(2), 185-189. https://doi.org/10.1016/j.jsams.2017.05.015

Fernandes, H.M. (2018b). Physical activity and mental health in adolescents: The mediating effect of self-esteem and body satisfaction. Rev Psicol Deporte, 27(1), 67-76.

Neto, F. (1993). Satisfaction with life scale: Psychometric properties in an adolescent sample. J Youth Adolesc, 22(2) 125-134.

Prochaska, J., Sallis, J., \& Long, B. (2001). A physical activity screening measure for use with adolescents in primary care. Arch Pediatr Adolesc Med, 155, 554-559. https://doi.org/10.1001/archpedi.155.5.554 
Raposo, J., \& Freitas, C. (1999). Avaliação da auto-estima em jovens transmontanos. Estud Psicol (Campinas), 16(3), 32-46. https://doi.org/10.1590/s0103-166x1999000300004

Rodrigues, N., Rebelo, T., \& Coelho, J.V. (2011). Adaptação da Escala de Inteligência Emocional de Wong e Law (WLEIS) e análise da sua estrutura factorial e fiabilidade numa amostra portuguesa. Psychologica, 55, 189-207. https://doi.org/10.14195/1647-8606 $55 \quad 10$

\section{(c) $(\mathrm{B})(\mathrm{EY}$}

This work is licensed under a Attribution-NonCommercial-NoDerivatives 4.0 International (CC BY-NC-ND 4.0). 


\title{
Watching overweight: Monitoring in child health consultations
}

\author{
PAULO FERNANDES ${ }^{1}$, INÊS SILVA ${ }^{2}$, ANA SILVA ${ }^{2}$, BEATRIZ PEREIRA ${ }^{2}$ \\ 1 USF Águeda + Saúde, Portugal \\ ${ }^{2}$ Centro de Investigação em Estudos da Criança, Universidade do Minho, Portugal
}

\begin{abstract}
Overweight and obesity in children is considered an epidemic but it can be prevented with one of the strategies, such as the monitoring of children in the child health consultations provided in the National Program for Children and Youth. Objectives: To identify the prevalence of overweight and obesity in children; Identify the number of child health consultations attended up to 18 months old; Verify if greater monitoring by the family health team up to 18 months is associated with the nutritional status of children. Methodology: Participants - 285 children aged 0 to 17 years. Instruments / Procedures: The percentile of body mass index (BMI) was used to determine nutritional status and the SClínico program was used to collect data of child health consultations. The BMI value used was related to the age of 5-6 years or in the case of children under this age was used the last value registered. Results: It was verified that $14.2 \%$ of the children were overweight and $12.3 \%$ were obese and that only $1.8 \%$ participated in all the consultations. There was a negative correlation between the number of consultations participated up to 18 months and the BMl of the children ($0.123, p=0.04)$, that is, as the number of consultations observed increases, BMl decreases. Conclusion: Since the birth of children, close and regular monitoring of the family health team is essential so that problems such as overweight and obesity can be prevented. Keywords: Overweight; Obesity; Children; Medical Attention; Prevention.
\end{abstract}

Corresponding author. Centro de Investigação em Estudos da Criança, Universidade do Minho, Portugal.

E-mail: paulo brites 18@hotmail.com

Supplementary Issue: Spring Conferences of Sports Science. International Seminar of Physical Education, Leisure and Health, 17-19 June 2019. Castelo Branco, Portugal.

JOURNAL OF HUMAN SPORT \& EXERCISE ISSN 1988-5202

(c) Faculty of Education. University of Alicante.

doi:10.14198/jhse.2019.14.Proc4.82 


\section{INTRODUCTION}

Overweight and obesity in children is currently presented as an epidemic, whose deleterious consequences have increasingly recognized impact. Physical inactivity as well as inappropriate dietary patterns are cited as the leading causes of increased overweight in the world population (World Health Organization (WHO), 2002). Despite the alarming results, overweight and obesity can be prevented, and this is one of the goals of the child health consultations that are part of the National Program for Child and Youth Health (DGS, 2013). Therefore, the objectives of the present study are to identify the prevalence of overweight and obesity in children, as well as the number of children's health consultations that they attended until the age of 18 months, and finally, to verify if a greater monitoring by the team of family health up to 18 months is associated with the nutritional status of the children.

\section{MATERIAL AND METHODS}

\section{Participants}

A total of 285 children participated (149 males (52.3\%) and 136 females (47.7\%)). The children were aged between 0 and 17 years old $(8.47 \pm 5.08)$. The study was conducted at a Family Health Unit $(F H U)$ in the central region of Portugal.

\section{Measures}

To determine the nutritional status of the children it was used BMl's percentile which was obtained by a calculation based on the child's height and weight. This percentile was categorized according to WHO guidelines (2006). The weight and height were measured, until approximately the 2 years of age, by digital scale and infant stadiometer and for older ages a digital scale with stadiometer.

Data on participation in child health consultations were obtained through SClínico program and subsequently exported to an Excel database and SPSS statistical program for analysis.

\section{Procedures}

This study is an integral part of the FHU internal health monitoring plan. This follow-up plan aims to evaluate the children seen by each family doctor regarding their nutritional status.

Data on child weight and height were collected at planned child health consultations by the family nurse. The $\mathrm{BMI}$ used refers to the age of 5-6 years or in the case of children under this age, the last value registered.

All data processing ensured the anonymity of the children.

\section{Analysis}

Descriptive analyses were used to verify the nutritional status and the number of child health consultations participated up to 18 months and Spearman correlation to verify the association between the variables. A significance level of $5 \%$ was adopted for all tests.

\section{RESULTS}

Regarding BMI, it was found that $14.2 \%$ of the children were overweight and $12.3 \%$ were obese. 
A total of 131 children (46.3\%) never attended the family health team until 18 months, $16.6 \%$ participated in 1 to 6 consultations, $35.3 \%$ in 7 to 8 consultations and only $1.8 \%$ participated in the total number of consultations planned up to 18 months. There was a negative correlation, however weak, between the number of consultations by the age of 18 months and the BMI of the children $(-0.123, p=0.04)$, in other words, as the number of consultations taken place increases, the BMI decreases.

\section{DISCUSSION}

The main results are the high prevalence of overweight children $(24.1 \%)$, corroborated by several investigations (COSI, 2016, PNPAF, 2016) but also the lack of participation of children in child health consultations. Since childhood is the main period for the adoption of life habits affecting the health of the child and its family, monitoring by the family health team during childhood, mainly in the first months of life, is fundamental for the control of their nutritional status. Sedentary lifestyles, namely the increasingly evident physical inactivity associated with changes in dietary patterns in new generations, as a result of the declining adherence to the Mediterranean diet and the higher consumption of foods with low nutritional value, correspond to two of the main causes of these dietary imbalances (Khan et al., 2017).Despite the limitations of the study as the lack of knowledge about other monitoring that children had, the study shows that the se consultations, where food plans are elaborated and revised as well as explained which motor and cognitive stimuli parents should performed in children according to their developmental stage, are essential for a harmonious growth. Thus, since birth, a closer and regular monitoring of the family health team is essential to physical, psychomotor and social development and the adoption of healthy lifestyles are evaluated (DGS, 2013) leading towards a fit approach to the child's growth that can be defined to prevent situations of overweight and obesity with consequences in short, medium and long term.

\section{CONCLUSIONS}

As a disease that affects more and more children in Portugal and the rest of the world, overweight and obesity should be a concern of the whole society, and its combat a priority. Awareness about all of its consequences in short, medium and long term is vital not only to children's physical health but also mental. Therefore, the active participation in child health consultations, essentially in the first months of life seems to correspond to one of the strategies of prevention of overweight and obesity and for that reason all parents must be alerted to the vital importance of actively participating in these.

\section{REFERENCES}

Childhood Obesity Surveillance Initiative: COSI Portugal 2016. (2016). Lisboa: Instituto Nacional de Saúde Doutor Ricardo Jorge.

DGS (2013). Saúde Infantil e Juvenil: Programa Nacional. Retrieved from https://nocs.pt/programanacional-saude-infantil-juvenil/

Khan, A., Khan, S., Marwat, M., Zia-Ul-Islam, S., Khan, M., \& Shah, A. J. (2017). Causes and Complication of Obesity among the Children. IJNHS, 02-05.

Programa Nacional para a Promoção da Atividade Física (PNPAF). (2016). Direção Geral de Saúde.

WHO. (2002). Reducing Risks, Promoting Healthy Life. Geneva: WHO.

WHO Multicentre Growth Reference Study Group. (2006). WHO Child Growth Standards: length/heightfor-age, weight-for-age, weight-for-length, weight-for-height and body mass index-for-age: methods and development. Geneva: WHO. https://doi.org/10.1111/j.1651-2227.2006.tb02378.x 


\section{(c) (i) (3)}

This work is licensed under a Attribution-NonCommercial-NoDerivatives 4.0 International (CC BY-NC-ND 4.0). 


\title{
An aesthetic reflection in school sports: Notes on the participation of the sportive delegation of Campus Santo Ângelo in the student games of IF Farroupilha (JEIF)
}

\author{
LARISSA ZANETTI THEIL ${ }^{1} \triangle$, MARIANNE S. FAULSTICH FERNANDES ${ }^{2}$, ALINE SALIHA OLIVEIRA ${ }^{3}$ \\ ${ }^{1}$ University of Coimbra, Portugal \\ ${ }^{2}$ Federal Institute Farroupilha, Brazil \\ 3University of Coimbra, Portugal
}

\begin{abstract}
The relevance and promotion of sports in educational environments is especially noteworthy, especially in the Brazilian Federal Institutes. The Farroupilha Federal Institute (JEIF) Student Games are examples of this premise. This study aims to interpret and analyse the participation of the sports delegation of the Santo Ângelo Campus in JEIF (2018) from the imagery interpretation methodology - Iconography and Iconology. It was used a collection of more than 2800 photographs, which were catalogued, indexed according to sports modality and suits. As results, it was possible to carefully capture the richness of singularities of the educational sport, such as: the athletes' physical disposition, how they deal with victories and defeats, the vibration when they climb on the podium, aspects of sociability and friendships on and off the court, that is, everything that makes up the world of sport, its ethical and aesthetic productions. Finally, the reflection on the sports image reveals the construction of text by the body language, in addition, allows to unveil new knowledge, bringing to the fore an aesthetic reflection about the sport in the educational space, more specifically of the sports delegation of the Federal Institute Farroupilha Campus Santo Ângelo. Keywords: Educational sport; Photographs; School; Teens.
\end{abstract}

Corresponding author. University of Coimbra, Portugal.

E-mail: larissatheil@gmail.com

Supplementary Issue: Spring Conferences of Sports Science. International Seminar of Physical Education, Leisure and Health, 17-19 June 2019. Castelo Branco, Portugal.

JOURNAL OF HUMAN SPORT \& EXERCISE ISSN 1988-5202

(c) Faculty of Education. University of Alicante.

doi:10.14198/jhse.2019.14.Proc4.82 


\section{INTRODUCTION}

Sport actions in the school environment constitute "privileged spaces for the development of culture, adoption of an active lifestyle for the full exercise of citizenship" (Brasil, 2009, p.14). The Federal Institute Farroupilha (IFFar) seeks to promote spaces that encourage and promote sports, the IFFar Student Games (JEIF) is an example of this premise. The JEIF is an annual sporting event that brings together the ten Campuses of IFFar: Campus Alegrete, São Augusto, São Vicente do Sul, Santa Rosa, Júlio de Castilho, Frederico Westphalen, São Borja, Jaguari, Panambi, Uruguaiana and Santo Ângelo, in which it aims to promote school integration through different sports modalities. The 9th JEIF had the participation of more than 600 students/athletes, from May 24 to 26, 2018, in the city of Panambi - Rio Grande do Sul/Brazil. Given this context, this study aims to interpret and analyse the participation of the sports delegation of the Santo Ângelo Campus in JEIF (2018), based on the methodology of imaging interpretation.

\section{MATERIAL AND METHODS}

We rely on the support derived from the methodology of imagery interpretation, based on the methods of Iconography and Iconology, attributed by Panofsky (2011). Iconography is based on the description and reconstitution of the visible elements of the same, as Iconology, on the detailed retrieval of the codified information that compose the image, that is, on the symbolic dimension embodied in the visual identity, actions, postures, feelings, voices, clothing of the athletes in the different modalities. (Panofsky, 2011). In this way, giving meaning not only to the content of the image, but the way this content is expressed, so as to seek more accurately the iconic and indicial vestiges of photography. According to Camargo (2011), the universe of images permeates meanings and representations, since they represent both visual information and meaning. In this way the imagery sources have brought us closer to the subtleties and many peculiar explicit and implicit details of the sports universe of the IFFar delegation. It used a collection of more than 2800 photographs, images taken by the servant Marianne Faulstich. First, a photographic document was screened, later a cataloguing of images, creating terms of indexing according to sports modality and suits. All images are stored in the Google Photos virtual repository, accommodated next to the IFFar physical imagery collection. The use of the images of the subjects involved in the research was made possible by the signature of the Image Use Authorization Term.

\section{RESULTS AND DISCUSSION}

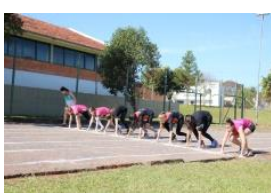

Figure 1

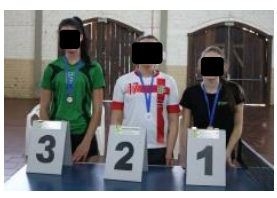

Figure 6

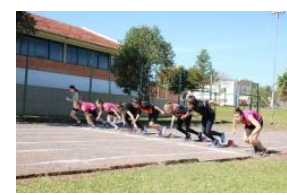

Figure 2

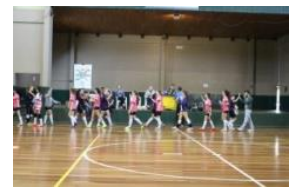

Figure 7

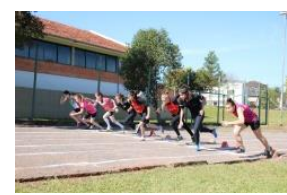

Figure 3

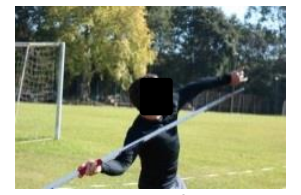

Figure 8

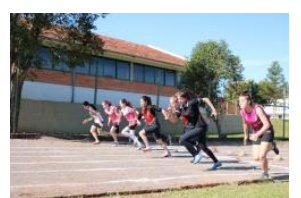

Figure 4

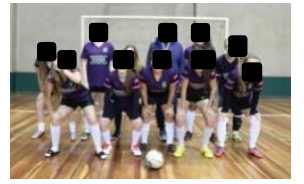

Figure 9

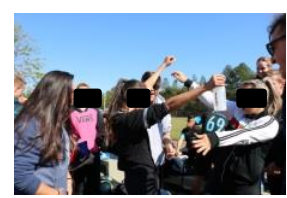

Figure 5

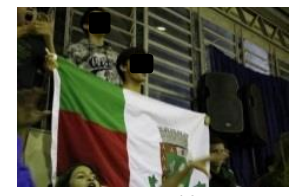

Figure 10

Source: Physical Education Collection, JEIF 2018. 
As a result, it is allowed to say that the participation of the sports delegation, acquisitions brought physical, cognitive and social learners. It was verified the involvement of more than 50 athletes, divided into modalities: indoor soccer, soccer field, volleyball, sand volleyball, athletics, chess and table tennis, the male and female suits. On the featured images, reading and meaningful understanding of some of the aesthetic peculiarities of the delegation participation is possible. According to Cruz \& Antunes (1996), sport involves the constant presence of social interactions, that the level of competition play a character intergroup and interpersonal. The coach-athlete relationship can be seen in figure 5. This relationship is relevant in sporting success, since the links in the group affect all actions they are involved. Respect and recognition of the athlete to the teacher image are explicit in the image. Already implicitly, you can see the joy of achievement, the euphoria of victory, the joy of long duty of good performance.

The sporting performance is considered as an integral component of the sport, both as a process, technical development, and as a result of sports stock (Schuler, 1987). Such concepts can be seen in the images 1 to 4 and 8 . Following 1-4, we can observe the first round of the athletics $100 \mathrm{~m}$ event, to observe the advantage that the athlete's Campus Santo Ângelo, second right athlete left, takes over the others already in the first seconds of race, which culminated in his victory. Specifically concerning figure 8 , the motor gesture technique allows us to affirm the high level of technical performance of the athlete, checked employed in the instrument handle and breath view through facial expression. The performance as a result is seen in picture 6 .

Sport is also an important tool to influence the behaviour and way of acting of adolescents. As shown in picture 7, when the women's indoor soccer team recognizes the opponent's merit after the defeat in court, maintaining harmonious relations of the sport at the expense of winning or losing. (Florentino \& Saldanha, 2007) in their study argue that the sport is pedagogical and educational for the possibility of providing obstacles and challenges, making teens experience the rules and learn to deal with others.

The uniform is one of the main symbols of a sports team is the visual identity that sets a team. In the photograph number 9 , this identity is highlighted when you realize that athletes and teacher make use of the same shirt. The uniform colours reinforce the thinking about identity, the colour purple represents the planning of actions and movements, and the colour pink the femininity of athletes who compete for the invasion of sport, indoor soccer, without leaving aside their vanity and lightness, represented in the picture by concern for the appearance and the presence of some loose hair. In Figure 10, you can see the involvement of athletes and fans. For Machado (1997), the sport is a means where you experience emotions very strongly. Competitions arouse feelings not only in athletes as the spectators, cheering. This feeling is described in the picture, the expression of the player who is in possession of the flag, in which denotes belonging pride for your institution. The force committed to hold the flag and the expression on his face show the energy used to encourage and support their peers, who are representing it in court. This interaction has generated a social link between the group of athletes and the educational institution. According to Thomas (1983), the contagion of feelings between fans and athlete expresses positive and negative responses, But the gestures apparently expressing the successful team of joy infects the audience delirious with excitement. This strengthened bond is positively distinguishing feature to the learning process.

\section{CONCLUSIONS}

The use of iconographic and iconological analysis is an effective possibility of educational research. Reflection on the sports imagery reveals the construction of text body language, allows uncover new knowledge, bringing out another possibility of contact with the history of sports culture, more specifically on the sporting culture of the IFFar Campus Santo Ângelo athletes. Finally, the photographs include not only 
moments of learning, knowledge production, the gesture engine significance, but also an aesthetic reflection on the sport in the educational space.

\section{REFERENCES}

Brasil (2009). Câmara dos Deputados Federais / Comissão de Turismo e Esporte. Diretrizes para Ações e Políticas para Educação Física e Esporte Escolar.

Cruz, J. \& Antunes, J. (1996). Dinâmica de grupos e coesão nas equipas desportivas. In: Cruz, J. (Ed), Manual de psicologia do desporto Braga S.H.O.

Florentino, J. \& Saldanha, R. P. (2007) Esporte, educação e inclusão social: reflexões sobre a prática pedagógica em educação física. E.F. Deportes.com, Revista Digital. Buenos Aires, nº 112.

Machado, A.A. (1997). Piscologia do esporte: Temas emergentes. Jundiaí. Àpice.

Panafsky, E. (2011) Iconografia e Iconologia: Uma introdução ao estudo da arte da Renascença. $2^{a}$ ed. SP: Perspectiva.

Schüler, D.(1987) Der Sport. Mannheim. Meyers Lexikonverlag.

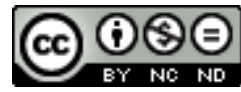

This work is licensed under a Attribution-NonCommercial-NoDerivatives 4.0 International (CC BY-NC-ND 4.0). 


\title{
Self-perception of life quality and the practice of physical activity in elderly
}

\author{
JORGE SANTOS ${ }^{1} \triangle$, JOÃO PETRICA ${ }^{1}$, RUI CARDOSO², JOÃO SERRANO ${ }^{1}$, MARCO BATISTA $^{1}$, \\ SAMUEL HONÓRIO ${ }^{1}$, LUIS MAIA ${ }^{3}$ \\ ${ }^{1}$ SHERU (Sport, Health and Exercise Research Unit), Instituto Politécnico de Castelo Branco, Portugal \\ 2Instituto Politécnico de Castelo Branco, Portugal \\ 3Universidade da Beira Interior, Departamento de Psicologia e Educação, Covilhã, Portugal
}

\begin{abstract}
The aging of population is an increasingly observable fact in several developed countries. We know that this life period can mean loss of life quality due to biological losses, psychological problems and social relations. The present study intends to verify if elderly people practicing a regular program of formal physical activity have a better perception of their quality of life compared to non-practicing elderly. The number of participants consisted of 64 elderly people $(n=64)$, living in Proença-a-Nova, being divided into two groups of 32 elements, in which one group had formal physical activity and the one was non-practitioners. The perceived quality of life was measured using the MOS SF-36 questionnaire. The results revealed that the perception of the quality of life presents only statistically significant differences in the physical and social functions and the health change item, in which that the group of practitioners presents higher values. Keywords: Aging; Active aging; Quality of life; Physical activity.
\end{abstract}

\footnotetext{
Corresponding author. Escola Superior de Educação de Castelo Branco - Rua Prof Dr. Faria de Vasconcelos 6000-266 Castelo Branco, Portugal.

E-mail: jorgesantos@ipcb.pt

Supplementary Issue: Spring Conferences of Sports Science. International Seminar of Physical Education, Leisure and Health, 17-19 June 2019. Castelo Branco, Portugal.

JOURNAL OF HUMAN SPORT \& EXERCISE ISSN 1988-5202

(c) Faculty of Education. University of Alicante.

doi:10.14198/jhse.2019.14.Proc4.82
} 


\section{INTRODUCTION}

Recently the increase in the elderly population has grown, and in Portugal, as a result of several changes in society, such as an increase in life expectancy and a decrease in the birth rate. Aging and old age has assumed an undeniable importance in our day (Oliveira, 2010). In Portugal, according to the NSI (2017), the population aged 65 or over may increase from 2.1 to 2, 8 million people between 2015 and 2080. We know that aging is a process characterized by functional weakness, so health is an essential condition for the wellbeing of this population. But not only, the WHO reports (2015) that "old age often involves significant changes beyond biological losses. These changes include changes in social roles and positions, as well as the need to deal with the loss of close relationships "(p.12). As a result, there has been a growing concern about the well-being and quality of life of this population. Our society, which, according to the WHO (2002), aims to optimize the possibilities of good health, participation and safety in order to increase the quality of life during old age. Quality of life is then the main purpose of active aging. According to Antão (2017), is related to selfesteem and personal well-being, which includes several concepts such as satisfaction with daily activities or with the environment, lifestyle and functional level. This functionality of the elderly, according to the literature, depends very much on the avoidance of sedentary behaviour. Therefore, a good perception of quality of life can be influenced by the regular practice of physical activity. Although the existing literature indicates that formal physical activity brings with it many benefits. Therefore, it is important that they reveal to us what they think and feel differently in their quality of life with physical activity. In addition, it is also important to realize the extent to which this perception may be different from that of elderly people who do not practice physical activity.

\section{MATERIAL AND METHODS}

\section{Participants}

A quantitative and descriptive study, in which 64 elderly individuals aged 65 years and over, all of them living in Proença-a-Nova, were evaluated. The participants were selected according to subjective criteria of the researcher, and individuals were invited to participate voluntarily in the research. After the acceptance, two groups of 32 elements were defined, one being the group of practitioners, with 32 individuals practicing formal physical activity in two programs (gymnastics and hydrogymnastics). The other group was referred to as a group of non-practitioners and had also 32 non-physically active elderly individuals.

\section{Measures}

In consideration with ethical aspects, all individuals who participated in this research signed the informed consent form, agreeing to participate in all activities established in the study. In order to evaluate the quality of life we used the MOS SF - 36v2 questionnaire, as an interview and duly validated for application for the Portuguese population (Camões et al, 2016). This questionnaire contains 36 enclosed responses items that allow us to evaluate 8 dimensions: physical function, physical performance, body pain, general health, vitality, social function, emotional performance and mental health. There is also an item related to the transition or change in health status. The eight dimensions mentioned above are grouped into two components: physical and mental.

\section{Procedures}

This study was intended to be a "photographic picture" of the study population. For this reason, the application of the data collection instrument was carried out in a single moment and on the same day. For this, we used the Proença-a-Nova sports facilities, in which tables and chairs were prepared to complete the questionnaire. After completing the informed consent form, a copy of the MOS SF - 36v2 questionnaire was sent to 
everyone. Later, we read it aloud, in order to confirm that everyone understood each item well, and this step was done individually. The elderly have responded to the questionnaire as they were being asked.

\section{Analysis}

For the results analysis, the computer program "IBM SPSS Statistics version 24 was used. Descriptive statistics were performed and the normality was verified by the Shapiro-Wilk test. According to the data obtained, the T-test was used. To measure the internal consistency of the questionnaires, we used Cronbach's alpha. Regarding acceptable reliability values, in this study, we considered values above 0.6, according to Morocco and Garcia-Marques (2006), and almost all variables revealed higher values, except for the social function that was of 0.463 . The level of significance was $p<0.05$.

\section{RESULTS}

From the analysis of the results we found that almost in all domains, the group of practitioners obtained higher values than the group of non-practitioners. The only exception was the item of health change, in which the values were higher in the group of non-practitioners. In the variables of physical function, social function and health change, there are statistically significant differences.

\section{DISCUSSION}

The results of this study revealed that the elderly practicing formal physical activity have higher quality of life values when compared to the elderly who do not practice. This trend is only contradicted in the health change item. Also in other similar studies with this questionnaire (MOS SF - 36v2), the results are similar as well. Camões, et al., (2016), sought to describe the perception of quality of life in individuals over 70 years of age, considering the participation in physical exercise programs, having verified higher values in physical exercise practitioners and statistically significant differences in physical function, general health, mental health, vitality and social function. In the study by Gomes (2010), who compared two groups of elderly people, one of them practicing physical activity and one of non-practitioners, it was concluded that, except for the body pain variable, in which the groups had very similar mean values, in all other dimensions had statistically significant differences, and the group of practitioners had higher values. In the present study, there were only statistically significant differences in the variables of physical function, social function and health change.

\section{CONCLUSIONS}

We found that perceived quality of life was not significantly different between the two groups. This means that the quality of life of practicing and non-practicing seniors is similar and tends to be higher for those who practice.

\section{REFERENCES}

Antão, L. (2017). Capacidade Funcional e Qualidade de Vida dos Seniores Praticantes e não Praticantes de Hidroginástica. Dissertação de mestrado, Escola Superior de Saúde - Instituto Politécnico de Bragança. https://doi.org/10.3895/rbqv.v7n3.2810

Gomes, T. (2010). Qualidade de vida, atividade e aptidão física em idosos participantes e não participantes em programas regulares de atividade física. Dissertação de mestrado, Universidade de Évora. https://doi.org/10.1590/s1517-86922013000600006 
Maroco, J. \& Garcia-Marques, T. (2006). Qual a fiabilidade do alfa de Cronbach? Questões antigas e soluções modernas? Laboratório de Psicologia, 4(1), 65-90. https://doi.org/10.14417//p.763

Oliveira, M. (2010). Autopercepção do corpo: diferenças entre idosos do sexo masculino e feminino. Estudo com idosos inscritos num programa de exercício físico. Dissertação de mestrado, Faculdade de Ciências do Desporto e Educação Física - Universidade do Porto. https://doi.org/10.5628/rpcd.01.01.44

Organização Mundial de Saúde. (2002). Active Ageing: a policy framework. Geneva: World Health Organization. Rocha, S. (2012). Efeitos do aumento da atividade física na funcionalidade e qualidade das pessoas idosas do centro social de Ermesinde. Lisboa: Faculdade de Motricidade Humana Universidade Técnica de Lisboa. [Dissertação de mestrado]. https://doi.org/10.32385/rpmgf.v29i2.11056

Camões, M.; Fernandes, F.; Silva, B.; Rodrigues, T.; Costa N. \& Bezerra, P. (2016). Exercício Físico e qualidade de vida em idosos: diferentes contextos sociocomportamentais. Motricidade, 12(1), 96 105. https://doi.org/10.6063/motricidade.6301

\section{(9) $(\Theta \Theta \Theta$}

This work is licensed under a Attribution-NonCommercial-NoDerivatives 4.0 International (CC BY-NC-ND 4.0). 


\title{
Anthropometric, somatotype and physical profile of young female roller skaters
}

\author{
CÉSAR LEÃO 14 , ANA SOFIA MEIRELES ${ }^{2}$, SILVIA ROCHA-RODRIGUES ${ }^{1,3}$, MIGUEL CAMÕES ${ }^{1,4}$ \\ ${ }^{1}$ Polytechnic Institute of Viana do Castelo, Higher School of Sport and Leisure, Viana do Castelo, Portugal \\ 2Juventude Pacense, Patinagem Artística, Paços de Ferreira, Portugal \\ ${ }^{3}$ Laboratory of Metabolism and Exercise, Research Centre for Physical Activity, health and Leisure (CIAFEL), \\ Faculty of Sport, University of Porto, Portugal \\ ${ }^{4}$ Research Center in Sports Sciences, Health Sciences and Human Development (CIDESD), Vila Real, \\ Portugal
}

\begin{abstract}
Aim: Although the anthropometric and somatotype profiles of elite athletes in some sports are well known, there is still a need for specific reference data for each sport, such as artistic roller skating, given its own characteristics. Therefore, we aimed to evaluate anthropometric and somatotype profile of female artistic roller skaters and then associate with physical performance variables. Methods: Nineteen Portuguese artistic roller skaters aged between 11 and 18 years old with more than 8 years of skating experience were submitted to anthropometric measurements based on the ISAK protocol for athletes (Height /weight2 - determine: body mass index - BMI). The somatotype was calculated using the Heath-Carter methods. Physical performance tests were evaluated based on lower and upper limbs, and trunk strength, including vertical and horizontal jumps, squats, sit-ups and push-ups. Data were expressed as mean and standard deviation (SD), by age group. Results: The mean BMl of female artistic roller skaters was $20.65 \pm 3.29 \mathrm{~kg} / \mathrm{m} 2$, the \%BF was $19.23 \pm 5.11$ and the skinfold sum was $86.75 \pm 31.70 \mathrm{~mm}$. The mean somatotype was 3.7-3.6-2.9 showing a predominant endo-mesomorphic somatotype. When we categorized by age group [10-13y], [14-16y] and [1718y], we observed that the mean somatotype was 2.7-3.2-3.7, 4.9-4.1-2.2 and 4.2-3.8-2.2, respectively. Squat jump (SJ) and squats negatively correlated with endomorphism $(r=-0.48, p=0.03 ; r=-0.51, p=0.27$, respectively) and \%BF ( $r=-0.46, p=0.046 ; r=-0.57, p=0.01$, respectively). Moreover, BMI was negatively associated with squats $(r=-0.488, p=0.034)$. Conclusions: Data suggest that young female roller artistic roller skaters exhibit a endo-mesomorphic somatotype. Both, anthropometric and somatotype profiles were associated with physical performance. Keywords: Roller skating; Body composition; Power; Strength; Endurance.

\footnotetext{
Corresponding author. Polytechnic Institute of Viana do Castelo, Higher School of Sport and Leisure, Viana do Castelo, Portugal.

E-mail: ces.leao@gmail.com

Supplementary Issue: Spring Conferences of Sports Science. International Seminar of Physical Education, Leisure and Health, 17-19 June 2019. Castelo Branco, Portugal.

JOURNAL OF HUMAN SPORT \& EXERCISE ISSN 1988-5202

(c) Faculty of Education. University of Alicante.

doi:10.14198/jhse.2019.14.Proc4.82
}

VOLUME 14 | Proc4 | 2019 | S1395 


\section{INTRODUCTION}

Anthropometry has been shown a key measure for athlete selection and/or performance criteria in sports while somatotyping is more specific for sports in which the body may impact the biomechanics of movement and the resulting performance (Carter \& Heath, 1990). Moreover, recent studies described somatotype profile in elite players as essential for the study of the development of a specific body shape under the influence of various intensive purposeful training processes and competitive periods (Sánchez-Muñoz et al, 2012). Indeed, somatotype is a convenient shorthand descriptor of overall physique in terms of body shape and composition independent of body size, and thus important in identifying talented young athletes for particular sports, such as artistic roller skating (Carter \& Heath, 1990). Artistic roller skating is a sport with two strands, recreational and competitive, which combines technique, balance, aesthetic, flexibility, precision and demands skater's ability to perform difficult content items like jumps, spins and footwork (Vila et al, 2015). At a competitive level, artistic roller skating demands both physical strength and endurance of the athletes that, at the same time, have to look like effortless graceful and beautiful (Ziegler et al, 2001). It is well known that the stereotype of artistic skaters is mainly characterized by shortness, lightness and leanness than their sedentary counterparts (Ziegler et al., 2001). Despite of its long history, there is very little research on anthropometric and somatotype profile of artistic roller skaters. We aimed to evaluate anthropometric and somatotype profile of female artistic roller skaters and then associate with physical performance variables.

\section{MATERIAL AND METHODS}

\section{Participants}

Nineteen Portuguese artistic roller skaters aged ranging between 11 and 18 years old voluntarily participated in the present study. The study group was with $8.3 \pm 2.7$ years of skating experience and have been competing for more than 4 years. All athletes train an average of $10.3 \pm 1.3$ hours per week. Then, the athletes were grouped by age as follows, $10-13$ years, $14-16$ years and $17-18$ years.

\section{Measures and Procedures}

Anthropometry

All anthropometric (height, weight, skinfolds, girths and diameter) variables were measured according to The International Society for the Advancement of Kinanthropometry (ISAK) protocol by a single professional (ISAK Level 2). Height and weight of the skaters were measured and body mass index (BMI) was calculated as follows weight/height2. Eight skinfolds (triceps, subscapular, biceps, suprailiac, supraspinal, abdominal, front thigh and medial calf), three limb girths (relaxed arm, flexed arm and calf) and 2 skeletal breadths (biepicondylar humerus and bi-epicondylar femur) were measured. The percentage of body fat (\%BF) was calculate using Slaughter prediction equation. The 3 somatotype components, endomorphy, mesomorphy and ectomorphy, were calculated according to Heath-Carter somatotype equations (Carter \& Heath, 1990).

\section{Physical Performance}

The physical performance was tested by squat jump (SJ), countermovement jump (CJ), Abalakov jump (ABK), horizontal jump (HJ). For the jumps, the best of the 3 trials was recorded to the nearest $0.1 \mathrm{~cm}$.. Additionally, participants performed the maximum number of squats, sit-ups and push-ups in 60 seconds each. Prior to each performance, test procedures were demonstrated for the participants.

\section{Analysis}

Statistical analyses were performed using IBM SPSS version 25.0 (SPSS, Chicago, USA). Data were expressed as mean and standard deviation (SD). The normality of all variables was tested using the 
Kolmogorov-Smirnov test. Associations between somatotype, body composition and physical variables were assessed by calculating the Pearson's linear correlation coefficients. Significance level was maintained at $\mathrm{p} \leq 0.05$ for all analyses.

\section{RESULTS}

The mean BMI of female artistic roller skaters was $20.65 \pm 3.29 \mathrm{~kg} / \mathrm{m} 2$, the $\%$ of Body Fat (\%BF) was $19.23 \pm 5.11$ and the skinfold sum was $86.75 \pm 31.70 \mathrm{~mm}$. Of all sample, the mean somatotype was 3.7-3.62.9 showing a predominant endo-mesomorphic somatotype. When we categorized by age group [10-13y], [14-16y] and [17-18y], we observed that a mean somatotype was 2.7-3.2-3.7, 4.9-4.1-2.2 and 4.2-3.8-2.2, respectively. As expected, BMI strongly correlated with endomorphism ( $r=0.86 ; p<0.001)$, mesomorphism $(r=0.74 ; p<0.001)$ and negatively associated with ectomorphism $(r=-0.89 ; p<0.001)$ in female roller skaters. The \%BF was associated to endomorphism ( $r=0.99 ; p<0.001)$ and mesomorphism $(r=0.69 ; p=0.001)$ and negatively associated to ectomorphism $(r=-0.82 ; p<0.001)$.

Regarding physical tests, we found a positive correlation between ectomorphism and $S J(r=0.458 ; p=0.048)$, and a negative correlation between endomorphism and SJ and squats number $(r=-0.479, p=0.038, r=-0.506$, $p=0.27$, respectively). Also, we found negative correlations between $\% B F$ and SJ and squats $(r=-0.46$, $p=0.046 ; r=-0.57, p=0.01$, respectively), as well between $B M I$ and squats $(r=-0.49, p=0.03)$.

\section{DISCUSSION}

To our best knowledge, this is the first study evaluating anthropometric and somatotype profiles and their association with physical characteristics and capabilities in young female artistic roller skaters. Based on anthropometric data obtained of young artistic roller skaters in the present study, they are lean, which are in line with previous findings conducted in ice skaters (Ziegler et al. 2002). However, some studies (Vila et al. 2015) reported higher stature and low values of $\mathrm{BF} \%$. This happen possibly due to the fact that the participants of the present study are younger.

Somatotype profile characterizes body physique, using measures relating to body shape and composition, assessing adiposity (fatness), musculo-skeletal robustness, and linearity or slenderness which likely associated with better performance (Carter \& Heath, 1990), especially for physically demanding sports as artistic roller skating. In this line, we found that young roller skaters were predominately endo-mesomorphism at [14-18y], which is mostly associated with a muscular-skeletal robustness. However, youngest skaters showed a ectomesomorphism profile. Previous reports (Vila et al. 2015) suggested that mesomorphism is associated with skeletal muscle strength and motor performance in general. We found that the endomorphic profile was negatively associated with squat jump and squats, suggesting that endomorphic skaters may have some limitations regarding the strength in lower limbs. These findings are in line with \%BF and BMI data obtained from the participants of the present study suggesting that both BMI and \% BF should be considered, in part, as indicators for physical performance in this sport.

\section{CONCLUSIONS}

Data suggest that young female roller artistic roller skaters exhibit a endo-mesomorphic somatotype. Both, anthropometric and somatotype profiles were associated with physical performance. 


\section{REFERENCES}

Carter, J. E. L., \& Heath, B. H. (1990). Somatotyping: Development and Applications.

Sánchez-Muñoz, C., Zabala, M., \& Williams, K. (2012). Anthropometric variables and its usage to characterise elite youth athletes. In Handbook of Anthropometry - Physical Measures of Human Form in Health and Disease. https://doi.org/10.1007/978-1-4419-1788-1

Vila, H., Abraldes, J. A., Rodriguez, N., \& Ferragut, C. (2015). Anthropometric and somatotype characteristics of world class male roller skaters by discipline. J. Sports Med. Phys. Fitness, 55(78), 742-748.

Ziegler, P. J., Jonnalagadda, S. S., \& Lawrence, C. (2001). Dietary intake of elite figure skating dancers. Nutrition Research, 21(7), 983-992. https://doi.org/10.1016/S0271-5317(01)00320-7

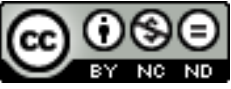

This work is licensed under a Attribution-NonCommercial-NoDerivatives 4.0 International (CC BY-NC-ND 4.0). 


\title{
Functional physical fitness in elderly: Differences depending on the practice of physical activity
}

\author{
PAULO SILVEIRA ${ }^{1,2}$, RUI PAULO ${ }^{1,2}$, SAMUEL HONÓRI01,2, MARCO BATISTA ${ }^{1,2}$, PEDRO DUARTE- \\ MENDES ${ }^{1,2}$, JOÃO ROCHA ${ }^{1,2}$, JOÃO PETRICA ${ }^{1,2}$, JORGE SANTOS ${ }^{1,2}$, ANTÓNIO FAUSTINO $^{1,2}$, HELENA \\ MESQUITA ${ }^{3}$, JOÃO SERRANO ${ }^{1,2}$ \\ ${ }^{1}$ Instituto Politécnico de Castelo Branco, Portugal \\ 2SHERU - Sport, Health and Exercise Research Unit, Portugal \\ 3 Instituto Politécnico de Castelo Branco, CICS.NOVA SHERU, Portugal
}

\begin{abstract}
This study aims to verify the impact of physical activity on the physical fitness of elderly. The sample consisted of 348 elderly individuals aged between 61 and 97 years, divided into 2 groups ( 254 practitioners of Physical Activity and 94 non-practitioners). From the first group 48 practice hydrogymnastics and 206 practice gymnastics.. Physical fitness was assessed following the test protocol of the Senior Fitness Test battery (Rikli \& Jones, 2001). In addition to descriptive statistics, the Kolmogorov Smirnov, Mann-Whitney and Kruskal Wallis statistical tests were used with comparisons by pairs. The results allowed to identify that in each and every one of the tests of functional physical fitness there are statistically differences between practitioners and non-practitioners of PA, with mean averages significantly higher by the practitioners. The practice of any of these activities (Hydrogymnastics and Gymnastics) reveals significant differences with the group of nonpractitioners, and the means obtained in any of the physical fitness tests of the two groups of practice much superiors compared to the non-practitioners. There are also significant differences between Hydrogymnastic and Gymnastics practitioners in the test sitting, walking $2.44 \mathrm{~m}$ and sitting again, and walking for 6 minutes, where the gymnastic practitioners obtained better results in both tests. Keywords: Aging; Physical activity and health; Functional physical fitness.
\end{abstract}

Corresponding author. Rua Prof. Dr Faria de Vasconcelos. 6000-266 Castelo Branco, Portugal.

E-mail: paulo.silveira@ipcb.pt

Supplementary Issue: Spring Conferences of Sports Science. International Seminar of Physical Education, Leisure and Health, 17-19 June 2019. Castelo Branco, Portugal.

JOURNAL OF HUMAN SPORT \& EXERCISE ISSN 1988-5202

(c) Faculty of Education. University of Alicante.

doi:10.14198/jhse.2019.14.Proc4.82 


\section{INTRODUCTION}

Physical activity (PA) is determinant for the health and autonomy of the elderly . It has been established as a preventive strategy to mitigate the effects of aging (Strath, Pfeiffer, \& Whitt-Glover, 2012). This study aims to verify the impact of physical activity on the physical fitness of the elderly. Its intended to analyse if there are differences between practitioners and non-practitioners of supervised PA and if there are differences between gymnastics and hydrogymnastics practitioners.

\section{MATERIAL AND METHODS}

\section{Participants}

A number of 348 elderly individuals aged between 61 and 97 years (mean $74.48 \pm 7.36$ ) participated in the study. They were divided into 2 groups (254 practitioners and 94 non-practitioners). The group of PA practitioners was divided into 2 subgroups (48 practiced hydrogymnastics and 206 gymnastic). For inclusion criteria the PA practice group had to perform at least 2 weekly sessions.

\section{Measures}

For elderly evaluation, the test "Senior Fitness Test" developed by Rikli and Jones (2001) and validated for the Portuguese population by Baptista and Sardinha (2005) was applied.

\section{Procedures}

All participants filled an individual informed consent form, and were evaluated under the same conditions, complying with the protocol established by the researchers. All ethical and legal research principles with Human Beings have been considered (Declaration of Helsinki, 2013).

\section{Analysis}

Descriptive statistics and the Kolmogorov Smirnov normality test $(n>30)$ was applied for data analysis. The comparison between the practice and non-practice groups of was assessed by the Mann-Whitney test. For each of the functional physical fitness tests, the non-parametric Kruskal-Wallis test for comparison of the three groups (Hydrogymnastics, Gymnastics and non-practitioners) was used for normality verification.

\section{RESULTS}

The functional physical fitness assessment tests used present a psychometric sensitivity because they present a distribution of values between the minimum and the maximum of the scale, with absolute values of asymmetry less than 3 and of kurtosis lower than 7 . The analysis of the $p$-values of table 1 allows to identify, statistical differences between practitioners and non-practitioners. PA practitioners present mean values significantly higher than the non-practitioners. The practice of Hydrogymnastics and Gymnastics reveals significant differences in the comparison between groups, with higher means in the practice group. 
Table 1. Comparison between groups and activities

\begin{tabular}{|c|c|c|c|c|c|c|c|c|c|c|c|c|c|c|}
\hline & & & & & & & & & & & & & $\begin{array}{r}\mathrm{P} \\
\text { comr }\end{array}$ & $\begin{array}{l}\text { airs } \\
\text { arisons }\end{array}$ \\
\hline & Group & $n$ & Mean & $\begin{array}{l}\text { Standard } \\
\text { deviation }\end{array}$ & $\begin{array}{c}\mathrm{p}- \\
\text { value } \\
\mathrm{K}-\mathrm{S}\end{array}$ & $\begin{array}{c}p \text {-value } \\
M-W\end{array}$ & Group & $\mathrm{n}$ & Mean & $\begin{array}{l}\text { Standard } \\
\text { deviation }\end{array}$ & $\begin{array}{c}\mathrm{p}- \\
\text { value } \\
\mathrm{K}-\mathrm{S}\end{array}$ & $\begin{array}{c}\text { p-value } \\
\text { K-W }\end{array}$ & Pair & p-value \\
\hline \multirow{3}{*}{$\begin{array}{l}\text { Get up and } \\
\text { sit on the } \\
\text { chair (30s) }\end{array}$} & \multirow{2}{*}{ Practice } & \multirow{2}{*}{254} & \multirow{2}{*}{15.08} & \multirow{2}{*}{3.481} & \multirow{2}{*}{.000} & \multirow{3}{*}{$<0.001$} & Hydrogymnastics(1) & 48 & 13.75 & 2.016 & .034 & \multirow{3}{*}{$<0.001$} & $(1)-(3)$ & $<0.001$ \\
\hline & & & & & & & Gymnastics (2) & 206 & 15.39 & 3.677 & .000 & & $(2)-(3)$ & $<0.001$ \\
\hline & $\begin{array}{l}\text { Non- } \\
\text { practice }\end{array}$ & 94 & 8.10 & 3.534 & .008 & & Non-practice (3) & 94 & 8.10 & 3.534 & .008 & & (1)-(2) & 0.098 \\
\hline \multirow{3}{*}{$\begin{array}{l}\text { Forearm } \\
\text { flexion } \\
\text { (30s) }\end{array}$} & \multirow{2}{*}{ Practice } & \multirow{2}{*}{254} & \multirow{2}{*}{20.40} & \multirow{2}{*}{6.359} & \multirow{2}{*}{.000} & \multirow{3}{*}{$<0.001$} & Hydrogymnastics(1) & 48 & 20.33 & 5.684 & .018 & \multirow{3}{*}{$<0.001$} & (1)-(3) & $<0.001$ \\
\hline & & & & & & & Gymnastics (2) & 206 & 20.42 & 6.519 & .000 & & (2)-(3) & $<0.001$ \\
\hline & $\begin{array}{l}\text { Non- } \\
\text { practice }\end{array}$ & 94 & 11.45 & 5.398 & .009 & & Non-practice (3) & 94 & 11.45 & 5.398 & .009 & & (1)-(2) & 1 \\
\hline \multirow{3}{*}{$\begin{array}{l}\text { Seat and } \\
\text { reach }(\mathrm{cm})\end{array}$} & \multirow{2}{*}{ Practice } & \multirow{2}{*}{254} & \multirow{2}{*}{4.31} & \multirow{2}{*}{11.087} & \multirow{2}{*}{.000} & \multirow{3}{*}{$<0.001$} & Hydrogymnastics(1) & 48 & .56 & 11.054 & .002 & \multirow{3}{*}{$<0.001$} & $(1)-(3)$ & $<0.001$ \\
\hline & & & & & & & \begin{tabular}{|l|} 
Gymnastics (2) \\
\end{tabular} & 206 & 5.18 & 10.938 & .000 & & (2)-(3) & $<0.001$ \\
\hline & $\begin{array}{l}\text { Non- } \\
\text { practice }\end{array}$ & 94 & -8.80 & 12.956 & .002 & & Non-practice (3) & 94 & -8.80 & 12.956 & .002 & & (1)-(2) & 0.1 \\
\hline \multirow{3}{*}{$\begin{array}{l}\text { Sit down. } \\
\text { walk } 2.44 \\
m \text { and sit } \\
\text { again }\end{array}$} & \multirow{2}{*}{ Practice } & \multirow{2}{*}{254} & \multirow{2}{*}{6.5562} & \multirow{2}{*}{2.02338} & \multirow{2}{*}{.000} & \multirow{3}{*}{$<0.001$} & \begin{tabular}{|l|l} 
Hydrogymnastics(1) \\
\end{tabular} & 48 & 8.3427 & 1.97471 & .003 & \multirow{3}{*}{$<0.001$} & $(1)-(3)$ & $<0.001$ \\
\hline & & & & & & & Gymnastics (2) & 206 & 6.1400 & 1.79924 & .000 & & (2)-(3) & $<0.001$ \\
\hline & $\begin{array}{l}\text { Non- } \\
\text { practice }\end{array}$ & 94 & 18.6148 & 14.28547 & .000 & & Non-practice (3) & 94 & 18.6148 & 14.28547 & .000 & & (1)-(2) & 0.004 \\
\hline & Practice & 254 & -1126 & 10671 & 016 & & Hydrogymnastics(1) & 48 & -14.37 & 11.094 & .177 & & (1)-(3) & $<0.001$ \\
\hline $\begin{array}{l}\text { Reach } \\
\text { behind }\end{array}$ & Practice & 254 & -11.26 & $10.6 / T$ & .070 & & \begin{tabular}{|l|} 
Gymnastics (2) \\
\end{tabular} & 206 & -10.53 & 10.466 & .013 & & $(2)-(3)$ & $<0.001$ \\
\hline $\begin{array}{l}\text { benind } \\
\text { back }\end{array}$ & $\begin{array}{l}\text { Non- } \\
\text { practice }\end{array}$ & 94 & -29.61 & 14.039 & .200 & $<0.001$ & Non-practice (3) & 94 & -29.61 & 14.039 & $.200^{*}$ & $<0.001$ & $(1)-(2)$ & 0.188 \\
\hline & & & & & & & Hydrogymnastics(1) & 48 & 482.85 & 62.053 & .082 & & $(1)-(3)$ & $<0.001$ \\
\hline Walk 6 min. & Practice & 254 & 543.05 & 151.448 & .000 & $<0 \cap 001$ & Gymnastics (2) & 206 & 557.07 & 162.413 & .000 & $<0001$ & (2)-(3) & $<0.001$ \\
\hline & $\begin{array}{l}\text { Non- } \\
\text { practice }\end{array}$ & 94 & 237.44 & 176.564 & .008 & $<0.001$ & Non-practice (3) & 94 & 237.44 & 176.564 & .008 & $<0.001$ & (1)-(2) & 0.007 \\
\hline
\end{tabular}

\section{DISCUSSION}

With aging there is a decline in physical fitness, strength, endurance, agility and flexibility (Milanović et al., 2013). Being sufficiently active is important to maintain or improve functional physical fitness on elderly (Santos et al., 2012). These results have important implications for public health, since the decline in physical fitness has negative consequences on health (Rejeski et al., 2011). Our results are in accordance with the results of Ofei-Dodoo et al. (2016), where PA is associated with greater aerobic endurance, strength and agility / dynamic balance capacity. Similar results were also found in Santos et al. (2012), where PA was shown to be associated with better physical fitness, Nawrocka et al. (2017). Even in advanced ages, the human body is sensitive to stimuli, so maintain a PA programme becomes important in order to improve physical fitness.

\section{CONCLUSIONS}

The elderly group with supervised PA practice presented better results in all components of functional physical fitness, statistically acceptable differences, regardless the type of PA practiced. By the comparison between the two PA activities, there are differences in agility and cardiorespiratory fitness, with gymnastics better performances obtained by the gymnastic practitioners.

\section{REFERENCES}

Baptista, F., \& Sardinha, L. (2005). Avaliação da aptidão física e do equilíbrio de pessoas idosas. Baterias de Fullerton. Cruz Quebrada: Faculdade de Motricidade Humana. 
Milanovic, Z., Pantelic, S., Trajkovic, N., Sporis, G., Kostic, R., \& James, N. (2013). Age-related decrease in physical activity and functional fitness among elderly men and women. Clinical Interventions in Aging, 8, 549-556. https://doi.org/10.2147/cia.s44112

Nawrocka, A., Mynarski, W., \& Cholewa, J. (2017). Adherence to physical activity guidelines and functional fitness of elderly women, using objective measurement. Annals of Agricultural and Environmental Medicine, 4, 632-635. https://doi.org/10.5604/12321966.1231388

Ofei-Dodoo, S., Rogers, N., Morgan, A., Amini, S., Takeshima, N., \& Rogers, M. (2016). The Impact of an Active Lifestyle on the Functional Fitness Level of Older Women. Journal of Applied Gerontology, 37, 119. https://doi.org/10.1177/0733464816641390

Rejeski, W. K., Brubaker, P. H., Goff Jr, D. C., Bearon, L. B., McClelland, J. W., Perri, M. G., \& Ambrosius, W. (2011). Traslating Weight Loss and Physical Activity Programs Into the Community to Preserve Mobility in Older, Obese Adults in Poor Cardiovascular Health. Arch Inter Med, 171, 880-886. https://doi.org/10.1001/archinternmed.2010.522

Rikli, E. \& Jones, J. (2001). Senior Fitness Test Manual. Champaign, IL: Human Kinetics.

Santos, D., Silva, A., Baptista, F., Santos, R., Vale, S., Mota, J., \& Sardinha, L. (2012). Sedentary behavior and physical activity are independently related to functional fitness in older adults. Experimental Gerontology, 47, 908-912. https://doi.org/10.1016/j.exger.2012.07.011

Strath, S., Pfeiffer, K., \& Whitt-Glover, M. (2012). Accelerometer use with children, older adults, and adults with functional limitations. Med Sci Sports Exerc, 41, S77-S85. https://doi.org/10.1249/mss.0b013e3182399eb1

\section{(9) $(\mathbb{Q} \Theta \Theta$}

This work is licensed under a Attribution-NonCommercial-NoDerivatives 4.0 International (CC BY-NC-ND 4.0). 


\title{
Physical activity levels in adults with intellectual disabilities: The importance of physical education
}

\author{
ANA JOSÉ AGUIAR RODRIGUES $\triangleleft$, DUARTE SOUSA, HELDER LOPES, JOÃO PRUDENTE \\ Departamento de Educação Física e Desporto, Faculdade de Ciências Sociais, Universidade da Madeira, \\ Portugal
}

\begin{abstract}
The relationship between physical activity levels and health indicators is widely reported in the literature. However, the number of studies that characterize the physical activity profile in intellectually disabled adults is still small. The aims of this study are: (i) to determine the levels of physical activity in adults with intellectual disability, (ii) to study differences in physical activity levels between gender and between days with or without physical education. A total of 78 subjects (41 males and 37 females) with intellectual disability (mild and moderate), with a mean age of $30.18 \pm 8.55$ years, participated in the study. The physical activity levels were evaluated by pedometer (Yamax Digiwalkers SW-700), placed in two working days (one with Physical Education and another without Physical Education classes). There were reduced levels of physical activity in days with Physical Education (4865.21 \pm 1611.04$)$ and without (3739.22 \pm 1243.34$)$, clearly below the recommended values. Men were more active compared to women, but only on days with Physical Education $(p<.05)$. In the days with Physical Education classes, there is a $24 \%$ increase in physical activity levels $(27 \%$ in men and $20 \%$ in women). The performance of physical education classes seems to have benefic effects on physical activity levels, however, it is necessary to develop a study that helps to understand more deeply the factors that influence the levels of physical activity in this population. Keywords: Intellectual disability; Physical activity; Physical education; Adults.
\end{abstract}

Corresponding author. Campus da Penteada, 9020-105 Funchal, Portugal.

E-mail: anajar@staff.uma.pt

Supplementary Issue: Spring Conferences of Sports Science. International Seminar of Physical Education, Leisure and Health, 17-19 June 2019. Castelo Branco, Portugal.

JOURNAL OF HUMAN SPORT \& EXERCISE ISSN 1988-5202

(c) Faculty of Education. University of Alicante.

doi:10.14198/jhse.2019.14.Proc4.82 


\section{INTRODUCTION}

The relationship between physical activity (PA) and health is widely reported in the scientific community (Bouchard, Blair, \& Haskell, 2018). Among adults with intellectual disabilities (ID), this relationship is also reported, with indicators as obesity, lipid profile (Gawlik, Zwierzchowska, \& Celebanska, 2018) and physical fitness (Jo, Rossow-Kimball, \& Lee, 2018). However, studies on people with intellectual disabilities are still limited (Dairo, Collett, Dawes, \& Oskrochi, 2016). Thus, the aims of this study are: (i) characterize the profile of PA in adults with ID; (ii) To study the differences in the PA profile between men and females, and between days with and without Physical Education (PE) classes.

\section{MATERIAL AND METHODS}

\section{Participants}

A total of 78 subjects (41 males and 37 females) with ID (mild and moderate), aged between 18 and 53 years $(30.18 \pm 8.55)$ participated in the study. All participants have PE classes, 120 to 180 minutes/week.

\section{Measures}

All participants were assessed in PA through the pedometer (Yamax Digiwalkers SW-700). Participants were asked to use it for 2 working days, one with PE classes and another without.

\section{Procedures}

Participants were invited to participate, through informed consent signed by the tutor, as well as by the organizations attended by the participants. The pedometers were placed for 2 working days contemplating a day with and two without PE classes.

\section{Analysis}

The mean and standard deviation were used to describe the sample at the levels of PA. The KolmogorovSmirnov test to analyse the normality of distributions in quantitative variables. The gender difference in PA levels was determined using the Student $T$ test. Pearson's correlations were used to determine the association between age and PA levels. The Student T-test for paired samples was used to determine differences in PA levels in the same subject between days with and without PE. The statistical software used was SPSS version 25.0 and the level of significance was $5 \%$.

\section{RESULTS}

Reduced levels of PA were observed on working days (3739.22 \pm 1243.34 and $4865.21 \pm 1611.04)$. The boys are more active than girls, but only with statistical significance on days with PE classes $(t(76)=2.674, p=.009)$. There was no association between age and levels of $P A(p>.05)$. There is a $24 \%$ increase in PA levels on weekdays with PE classes, compared to days without PE classes. Statistically significant differences were observed when the same subject was analysed on two days (male $t(40)=5.957, p<.001$; female $t(36)=4.664$, $p<.001)$. The difference between the two days is of greater magnitude in the men's comparatively the woman's (increase of $27 \%$ Vs $20 \%$ in the levels of PA, in the days with PE), without statistical significance. 
Table 1. Description of daily physical activity levels by gender (mean \pm standard deviation)

\begin{tabular}{|c|c|c|c|c|c|}
\hline & Total $(n=78)$ & Men $(n=41)$ & Woman ( $n=37)$ & $t$ & $p$ \\
\hline $\begin{array}{l}\text { Physical activity day whit } \\
\text { Physical Education (steps. }\end{array}$ & $4865.21 \pm 1611.04$ & $5302.71 \pm 1818.8$ & $4380.41 \pm 1189.99$ & 2.674 & .009 \\
\hline $\begin{array}{l}\text { Day) } \\
\text { Physical activity day without }\end{array}$ & & & & & \\
\hline $\begin{array}{l}\text { Physical Education (steps. } \\
\text { Day) }\end{array}$ & $3739.22 \pm 1243.34$ & $3912.12 \pm 1494.76$ & $3547.62 \pm 972.03$ & 1.324 & 190 \\
\hline $\begin{array}{l}\text { Difference between days with } \\
\text { and without Physical education } \\
\text { (steps. Day) }\end{array}$ & $1125.99 \pm 1338.19$ & $1390.58 \pm 1494.76$ & $832.78 \pm 1086.07$ & 1.868 & .066 \\
\hline
\end{tabular}

\section{DISCUSSION}

Similar to other studies, there were low levels of PA among participants with ID (Dairo et al., 2016; Hsieh, Hilgenkamp, Murthy, Heller and Rimmer, 2017), values well below those recommended. The supply of PA organized as PE classes represents an increase in PA levels. Even on days with PE classes, participants have PA levels clearly below those recommended. The small differences between days with and without PE deserve, in our view, the same emphasis, and the PE should be the object of future studies. In the same way, we are aware of the limitations of our instrument, particularly in relation to its ability to deal with our analysis. However, there is inconsistent evidence of the effects of interventions to improve levels of physical activity in individuals with ID (Hassan, Landorf, Shields, \& Munteanu, 2019). This aspect seems to indicate that behaviour is complex and sustained by multiple personal factors and involvement (Bossink, van der Putten, \& Vlaskamp, 2017). Thus, it is essential to develop studies that deepen the conception, dynamization and evaluation of intervention programs (Bossink et al., 2017; Brooker, van Dooren, McPherson, Lennox, \& Ware, 2015). Due to the reduced values of PA and the lower effect of the classes of PE in the levels of PA, women should be a priority group of study and intervention.

\section{CONCLUSIONS}

There is a reduced level of physical activity among adults with intellectual impairment. Physical Education classes contribute to increases in physical activity levels. However, it is crucial to deepen the knowledge about the physical activity profile of this population and to promote the conception, dynamization and evaluation of a physical activity promotion program.

\section{REFERENCES}

Bossink, L. W. M., van der Putten, A. A., \& Vlaskamp, C. (2017). Understanding low levels of physical activity in people with intellectual disabilities: A systematic review to identify barriers and facilitators. Research in Developmental Disabilities, 68, 95-110. https://doi.org/10.1016/.j.ridd.2017.06.008

Bouchard, C., Blair, S. N., \& Haskell, W. (2018). Physical Activity and Health (2th Eds). Champaign: Human Kinetics.

Brooker, K., van Dooren, K., McPherson, L., Lennox, N., \& Ware, R. (2015). A systematic review of interventions aiming to improve involvement in physical activity among adults with intellectual disability. Journal of Physical Activity \& Health, 12(3), 434-444. https://doi.org/10.1123/ipah.2013$\underline{0014}$ 
Dairo, Y. M., Collett, J., Dawes, H., \& Oskrochi, G. R. (2016). Physical activity levels in adults with intellectual disabilities: A systematic review. Preventive Medicine Reports, 4, 209-219. https://doi.org/10.1016/i.pmedr.2016.06.008

Gawlik, K., Zwierzchowska, A., \& Celebanska, D. (2018). Impact of physical activity on obesity and lipid profile of adults with intellectual disability. Journal of Applied Research in Intellectual Disabilities: JARID, 31(2), 308-311. https://doi.org/10.1111/jar.12406

Hassan, N. M., Landorf, K. B., Shields, N., \& Munteanu, S. E. (2019). Effectiveness of interventions to increase physical activity in individuals with intellectual disabilities: a systematic review of randomised controlled trials. Journal of Intellectual Disability Research : JIDR, 63(2), 168-191. https://doi.org/10.1111//ir.12562

Hsieh, K., Hilgenkamp, T. I. M., Murthy, S., Heller, T., \& Rimmer, J. H. (2017). Low Levels of Physical Activity and Sedentary Behavior in Adults with Intellectual Disabilities. International Journal of Environmental Research and Public Health, 14(12). https://doi.org/10.3390/ijerph14121503

Jo, G., Rossow-Kimball, B., \& Lee, Y. (2018). Effects of 12-week combined exercise program on selfefficacy, physical activity level, and health related physical fitness of adults with intellectual disability. Journal of Exercise Rehabilitation, 14(2), 175-182. https://doi.org/10.12965/jer.1835194.597

\section{@९@}

This work is licensed under a Attribution-NonCommercial-NoDerivatives 4.0 International (CC BY-NC-ND 4.0). 


\title{
A comparison of physical fitness by competitive levels in youth basketball players
}

\author{
PEDRO FORTE $1,2,3$, LUÍS FERREIRA ${ }^{1,2}$, PEDRO FLORES $^{1,2}$, CARLOS SOARES $^{1,2}$, CARLOS \\ MOREIRA ${ }^{1,2}$, ANTÓNIO M. MONTEIRO $^{4}$ \\ ${ }^{1}$ Instituto Superior de Ciências Educativas do Douro (ISCE Douro), Penafiel, Portugal \\ ${ }^{2}$ Núcleo de Investigação em Desporto e Exercício Físico do ISCE Douro, Penafiel, Portugal \\ ${ }^{3}$ Research Center in Sports Sciences, Health Sciences and Human Development, Vila Real, Portugal \\ ${ }^{4}$ Instituto Politécnico de Bragança, Bragança, Portugal
}

\begin{abstract}
Some basketball teams use train two teams at the same time. Even more, some younger players train and compete in a higher competitive level. The aim of this study was to compare the physical fitness (PF) between under sixteen (U16) and under eighteen (U18) basketball players. Body mass and height were assessed with a bioimpedance balance and a stadiometer. The strength levels were assessed for upper limbs by the number of push-ups (per 30s) and lower limb's with a horizontal jump without preparatory race (in centimetres). Coordination was measured by dribbling 5 barriers in the diagonal with a distance of $1 \mathrm{~m}$ (The first barrier at a distance of $8 \mathrm{~m}$ from the start and the last at $1 \mathrm{~m}$ of the basket). The speed was measured with a 20 meters sprint test (seconds). The seat and reach flexibility test and the up the back test in centimetres measured the flexibility. One way ANOVA assessed the statistical differences between groups. The significance level was $5 \%$. Only significant different were founded between U16 and U18 players in weight. No significant differences were founded between groups in anthropometrics, strength, speed, coordination and flexibility. In this team, no significant differences in physical fitness between U16 and U18 competitive levels were founded. Thus, youngers basketballers should be encouraged to train and compete in a higher competitive level. Keywords: Physical fitness; Performance; Youth; Basketball.
\end{abstract}

Corresponding author. Rua Vitorino da Costa,.$^{\circ}$ 96, 4560-708 Penafiel, Portugal.

E-mail: pedromiguel.forte@iscedouro.pt

Supplementary Issue: Spring Conferences of Sports Science. International Seminar of Physical Education, Leisure and Health, 17-19 June 2019. Castelo Branco, Portugal.

JOURNAL OF HUMAN SPORT \& EXERCISE ISSN 1988-5202

(c) Faculty of Education. University of Alicante.

doi:10.14198/jhse.2019.14.Proc4.82 


\section{INTRODUCTION}

Some team sports include two competitive levels in one team. However, sometimes there are physical differences that may compromise the younger players' performance. In basketball, speed, strength and motor coordination are three elemental motor abilities and the individual technique, strength and resistance are also associated with the team performance (Forte et al., 2016). The competitive level are determined by the birth age in junior basketball. That may result in a difference of two years between players (Wattie, et al., 2008). Intending to improve player's performance, several teams encourage the young players to practice in a higher competitive level or older team in the same club (Figueira, et al., 2018). However, younger's performance may be affected by the physical fitness differences. Considering the importance of train and compete in a higher competitive level, the aim of this study was to compare the physical fitness (PF) between under sixteen (U16) and under eighteen basketball (U18) players.

\section{MATERIAL AND METHODS}

\section{Participants}

This was a convenience sample, composed by nine U16 with $14.67( \pm 0.50)$ years old and seven U-18 players with 16.71 ( \pm 0.48 ) years old. All the players competed in Nacional Cup for U18 and U16 competitive levels. All the procedures were in accord to the Helsinki's declaration regarding human research. A written consent by the parents or tutors was obtained beforehand.

\section{Measures}

Body mass and height were assessed with a bioimpedance balance (Tanita, BC-601, USA) and a stadiometer. The PF assessment were made considering Forte et al., (2016) evaluations. Strength levels, coordination, speed and flexibility were evaluated.

\section{Procedures}

The upper limbs strength was measured by the number of push-ups (per 30s) and lower limb's with a horizontal jump without preparatory race (in centimetres). Coordination was measured by dribbling 5 barriers in the diagonal with a distance of $1 \mathrm{~m}$ (The first barrier at a distance of $8 \mathrm{~m}$ from the start and the last at $1 \mathrm{~m}$ of the basket). The speed was measured with a 20 meters sprint test (seconds). The seat and reach flexibility test and the up the back test in centimetres measured the flexibility.

\section{Statistical Analysis}

The Levene's and Kolmogorov-Smirnov tests assessed the equality of variances and normality respectively. The One way ANOVA evaluated the statistical differences between groups. The significance level was $5 \%$.

\section{RESULTS}

The mean values of anthropometrics, PF and statistical significance are presented in Table 1. 
Table 1. Means and standard deviation values of anthropometrics and physical fitness between U16 and U18 players

\begin{tabular}{lcccc}
\hline Variables & U16 & U18 & Mean $( \pm$ SD) & $p$ \\
\hline Age (years) & $14.67( \pm 0.50)$ & $16.71( \pm 0.49)$ & 67.411 & $<0.001^{*}$ \\
Weight $(\mathrm{kg})$ & $71.44( \pm 3.28)$ & $78.43( \pm 4.79)$ & 12.007 & $0.004^{*}$ \\
Height $(\mathrm{cm})$ & $175.11( \pm 8.02)$ & $179.43( \pm 4.47)$ & 1.619 & 0.224 \\
Wingspan (cm) & $174.00( \pm 9.72)$ & $177.29( \pm 3.99)$ & 0.699 & 0.417 \\
Upper Limbs Strength (reps) & $23.5556( \pm 8.37)$ & $22.71( \pm 6.87)$ & 0.046 & 0.833 \\
Lower Limbs Strength (cm) & $198.33( \pm 24.70)$ & $207.71( \pm 11.54)$ & 0.854 & 0.371 \\
Coordination (sg) & $7.29( \pm 0.40)$ & $8.0486( \pm 0.65)$ & 3.139 & 0.098 \\
Speed $(\mathrm{sg})$ & $3.77( \pm 0.39)$ & $3.4386( \pm 0.19)$ & 3.058 & 0.102 \\
Sit and reach $(\mathrm{cm})$ & $-1.44( \pm 7.52)$ & $-1.7( \pm 8.83)$ & 0.004 & 0.948 \\
Up the back $(\mathrm{cm})$ & $10.4444( \pm 7.02)$ & $5.57( \pm 3.789)$ & 2.727 & 0.121 \\
\hline \multicolumn{2}{c}{${ }^{*} p<0.05$} & & &
\end{tabular}

\section{DISCUSSION}

The aim of this study was to assess the differences in PF between U16 and U18 team. No significant differences in PF were observed between the two teams. Forte et al. (2016), applied a strength program on a U18 team. The authors made the same evaluations in two distinctive moments, the beginning of the season and four months later. Only age, upper limbs strength, coordination and up the back flexibility presented significant differences. In our study, no strength program was applied. However, the evaluations were at the beginning of the season and trainee was not assessed.

Different studies presented differences in strength levels (Fort-Vanmeerhaeghe et al., 2016; Forte et al., 2016). The mainly causes for physical fitness differences are strength levels. The main limitations of this study were: (i) the differences between U16 and U18 players were assessed in one single moment, at the beginning of the season; (ii) only sixteen players made part of this research. Thus, these findings cannot be extrapolated for all basketball players. However, it should be considered to predict significant differences in young player's physical fitness.

\section{CONCLUSIONS}

In this study that, no significant differences in physical fitness between U16 and U18 competitive levels were found. Strategies such as train and compete in a higher competitive level should be adopted.

\section{REFERENCES}

Fort-Vanmeerhaeghe, A., Montalvo, A., Latinjak, A., \& Unnithan, V. (2016). Physical characteristics of elite adolescent female basketball players and their relationship to match performance. Journal of human kinetics, 53(1), 167-178. https://doi.org/10.1515/hukin-2016-0020

Wattie N, Cobley S, Baker J. Towards a unified understanding of relative age effects. Journal of Sports Sciences. 2008;26(13):1403-9. https://doi.org/10.1080/02640410802233034

Figueira, B., Gonçalves, B., Masiulis, N., \& Sampaio, J. (2018). Exploring how playing football with different age groups affects tactical behaviour and physical performance. Biology of sport, 35(2), 145. https://doi.org/10.5114/biolsport.2018.71603 
Forte, P, Bartolomeu, R. F., Monteiro, A. M., \& Barbosa, T. M. (2016). Physical fitness in youth basketball players in pre and post season under a strength program effect. In Proceedings of the International Congress of the Research Center in Sports Sciences, Health Sciences \& Human Development (pp. 104-105). Universidade de Évora.

\section{(c) $(\mathrm{B})(\mathrm{EY}$}

This work is licensed under a Attribution-NonCommercial-NoDerivatives 4.0 International (CC BY-NC-ND 4.0). 


\title{
Present and future of the soccer specialists perfomance
}

\author{
JOAQUÍN REVERTER-MASIA , ALVARO DE PANO-RODÍGUEZ, VICENÇ HERNÁNDEZ-GONZÁLEZ, \\ JOSE GREGORIO BOLLADO-ESTEBA
}

Grupo de Investigación Movimiento Humano, Universidad de Lleida, Spain

\begin{abstract}
Professional soccer has become an important global business in which players have become substantial assets that must be selected, developed and carefully protected. Human resources teams have increasingly become involved with professional sports organizations, providing a wide range of services, such as scientific support or complex data analysis. With an increasing number of sports scientists entered into soccer clubs, it should be clarified and articulated the specific roles and responsibilities of their practice. In the current work we analyse the professional profiles of the staff of the Premier League. Keywords: Sport science; Performance specialist; Work; Soccer.
\end{abstract}

Corresponding author. Av. de l'Estudi General, n.4 E-25001 Lleida, Spain.

E-mail: reverter@didesp.udl.cat

Supplementary Issue: Spring Conferences of Sports Science. International Seminar of Physical Education, Leisure and Health, 17-19 June 2019. Castelo Branco, Portugal.

JOURNAL OF HUMAN SPORT \& EXERCISE ISSN 1988-5202

(c) Faculty of Education. University of Alicante.

doi:10.14198/jhse.2019.14.Proc4.82 


\section{INTRODUCTION}

Conditioning services in the sports sciences and more specifically in professional soccer clubs have changed the sports organization. There is a team of experts leading the players that must be equally organized and efficient (Onag y Tepeci, 2014). Therefore, in a high-level organization there can be two distinct teams: the players and the experts that are responsible for all athlete's health and performance. These teams are collectively called high-performance teams. These staff are now frequently destined to occupy specialist roles as opposed to the generic job descriptions they were once associated with (Juki et al., 2018). These roles commonly include strength and conditioning specialists (who may be predominantly gym based), fitness coaches (who may be field based), sport scientists, performance analysts and rehabilitation coaches who focus on the return to fitness of recently injured player. This increase in staff and the diversification of responsibilities is a significant departure from the strategies of the past that used to rely on a single individua. These staff are now also regularly supplemented by an expert input from consultants and other specialists often from the world of academia (Reverter-Masia et al., 2009; Drust, 2019). As the need for an even better support of elite athletes grows, elite sports organizations seek to employ knowledgeable and skilled professionals with high specialization at their respective fields (Tod et al., 2012). Currently, there is a lack of scientific articles regarding the structure and organization of high performance systems (Juki et al., 2018). The purpose of this paper is to describe the organization of high performance systems in English elite soccer clubs.

\section{MATERIAL AND METHODS}

\section{Participants}

For the present investigation we analysed the clubs of the Premier League 2018-2019 including: Arsenal (A), Bournemouth (B), Brighton \& Hove Albion (BI), Burnley (BU), Cardiff City (C), Chelsea (CH), Crystal Palace (CR), Everton (E), Fulham (F), Huddersfield Town (H), Leicester City (L), Liverpool (LI), Manchester City (MC), Manchester United(MU), Newcastle United (N), Southampton (S), Tottenham Hotspur(T), Watford (W), West Ham United (WE), Wolverhampton Wanderers (WO).

\section{Measures}

This study examined the web content of English football top-flight clubs. To carry out the evaluation, the web pages were selected: principal and technical staff.

\section{Procedures}

The methodology followed in this study is descriptive and comparative through document analysis.

\section{Analysis}

A descriptive statistic was used.

\section{RESULTS}

For this work an overview of professional specialties major sports team systems has been made (Table1). As most teams observe, they have hired a coach, assistants, first-team coach, goalkeeping, head performance, head medical, head physiotherapy, strength-conditioning, sports scientist and analyst. Likewise, we also found in some teams, professional profiles that we have not included in the table because they are not common, such as podiatrist, academy manager, psychologist masseurs, research of development or technical director. 


\section{DISCUSSION}

The high performance sport industry is a global, multi-billion dollar entity that provides employment opportunities for a vast range of professions (Juki et al., 2018). Given the growing need for personnel to manage sports organizations, it is logical to assume that the recruitment of professionals. According to these profiles of work in high performance systems, there is a need for specialists in a system that is multidimensional and interdisciplinary (Reverter-Masia et al., 2009, Drust, 2019). It is very important that the head coach and general manager bet on professionals with a high level of scientific and practical competence.

Table 1. Profiles of the technical staff in the football teams of the Premier League

\begin{tabular}{|c|c|c|c|c|c|c|c|c|c|c|c|c|c|c|c|c|c|c|c|c|c|c|c|}
\hline$\sum_{\infty}^{1}$ & 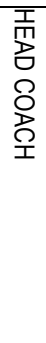 & 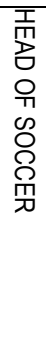 & 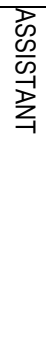 & 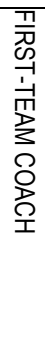 & 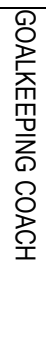 & 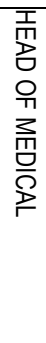 & 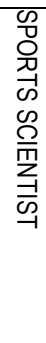 & 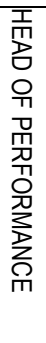 & 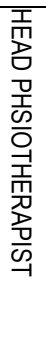 & 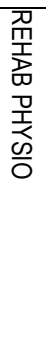 & $\begin{array}{l}\text { T) } \\
\text { ত } \\
\text { oD } \\
\text { O }\end{array}$ & 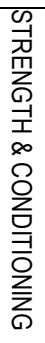 & 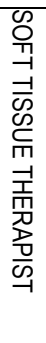 & 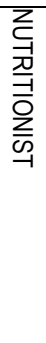 & 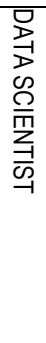 & 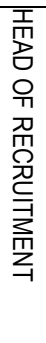 & 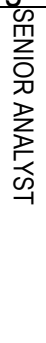 & 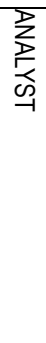 & 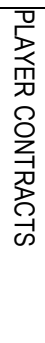 & $\begin{array}{l}\text { 紊 } \\
\text { 号 } \\
\text { 典 }\end{array}$ & 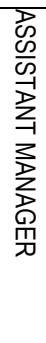 & 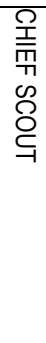 & 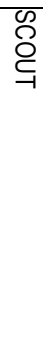 \\
\hline A & $x$ & $x$ & $x$ & $x$ & $x$ & $x$ & $x$ & $x$ & $x$ & $x$ & $x$ & $x$ & $\mathrm{x}$ & $\mathrm{x}$ & $x$ & $\mathrm{x}$ & $x$ & $x$ & $x$ & $x$ & $x$ & & \\
\hline$B$ & $x$ & & $x$ & $x$ & $x$ & $x$ & $x$ & & & $x$ & $x$ & $x$ & & & & $x$ & & $x$ & & $x$ & & & \\
\hline $\mathrm{BI}$ & & & $x$ & $x$ & $x$ & $x$ & & $x$ & & & $x$ & $x$ & & & & & & $x$ & & $x$ & $x$ & & \\
\hline $\mathrm{BU}$ & & & $x$ & $x$ & $x$ & & $x$ & $x$ & $x$ & & $x$ & $x$ & & & & & & $x$ & & $x$ & $x$ & & \\
\hline$c$ & & & $x$ & $x$ & & $x$ & $x$ & & $x$ & & $x$ & $x$ & $x$ & & & & & $x$ & $x$ & $x$ & & $x$ & $X$ \\
\hline $\begin{array}{l}\mathrm{C} \\
\mathrm{H}\end{array}$ & $x$ & & $x$ & $x$ & $x$ & $x$ & $x$ & $x$ & $x$ & & $x$ & $x$ & & & & & & $x$ & & $x$ & $x$ & $x$ & $x$ \\
\hline $\begin{array}{l}\mathrm{C} \\
\mathrm{R}\end{array}$ & & $x$ & $x$ & $x$ & $x$ & $x$ & $x$ & $x$ & $x$ & $x$ & $x$ & $x$ & $x$ & & & $x$ & & $x$ & $x$ & $x$ & $x$ & $x$ & \\
\hline$E$ & & $x$ & $x$ & $x$ & $x$ & $x$ & $x$ & $x$ & $x$ & $x$ & $x$ & $x$ & $x$ & $x$ & $x$ & & & $x$ & & $x$ & $x$ & & $x$ \\
\hline$F$ & & & $x$ & $x$ & $x$ & $x$ & $x$ & $x$ & & & $x$ & $x$ & & & & & & $x$ & & $x$ & $x$ & & \\
\hline $\mathrm{H}$ & $x$ & & $x$ & $x$ & $x$ & $x$ & & $x$ & $x$ & & & $x$ & & & & $x$ & & $x$ & & & & & \\
\hline $\mathrm{L}$ & & & $\mathrm{X}$ & $x$ & $x$ & $x$ & $x$ & $x$ & $x$ & & $x$ & $x$ & & $x$ & & $x$ & & $x$ & & $x$ & $x$ & $x$ & $x$ \\
\hline $\mathrm{LI}$ & & & & & $\mathrm{x}$ & $\mathrm{x}$ & $x$ & $x$ & $x$ & & $x$ & $x$ & & $\mathrm{x}$ & $\mathrm{x}$ & & & & $x$ & $X$ & $X$ & & $\mathrm{x}$ \\
\hline $\begin{array}{l}M \\
C\end{array}$ & & & & & $x$ & $x$ & & $x$ & $x$ & & $x$ & $x$ & $x$ & $x$ & & & & $x$ & $x$ & $x$ & $x$ & $x$ & $x$ \\
\hline $\begin{array}{l}M \\
U\end{array}$ & & & $x$ & $x$ & $x$ & & $x$ & $x$ & $x$ & $x$ & $x$ & $x$ & $x$ & & & & & $x$ & & $x$ & $x$ & $x$ & $x$ \\
\hline $\mathrm{N}$ & & & $x$ & $x$ & $x$ & $x$ & $x$ & $\mathrm{X}$ & & $\mathrm{X}$ & $x$ & $\mathrm{X}$ & & & & $x$ & & & & $x$ & $x$ & & \\
\hline$S$ & & & $x$ & $x$ & $x$ & & $x$ & $x$ & $x$ & $x$ & & & & $\mathrm{x}$ & & $x$ & & & $\mathrm{x}$ & $x$ & $x$ & $\mathrm{x}$ & $\mathrm{X}$ \\
\hline $\mathrm{T}$ & & & & $x$ & $x$ & & $x$ & $x$ & $x$ & $x$ & $x$ & & & $x$ & $x$ & $x$ & & $x$ & $x$ & & $x$ & $x$ & $x$ \\
\hline W & & & $\mathrm{x}$ & & $x$ & $\mathrm{X}$ & & & & $\mathrm{X}$ & $\mathrm{x}$ & & & $\mathrm{x}$ & & & & $\mathrm{x}$ & & & $\mathrm{x}$ & & \\
\hline $\begin{array}{l}\text { W } \\
E\end{array}$ & & & $x$ & & $x$ & $X$ & $x$ & $x$ & $x$ & & & $x$ & $x$ & & & & & $x$ & & & $x$ & & \\
\hline
\end{tabular}

The staff of a team is a complex social network in which people interact to achieve common goals (for example, improve the position in the classification or the development of players), and in combination with personal interests (for example, personally and / or professional growth, personal gratification or job security) (Juki et al., 2018, Yann et al., 2019 Drust, 2019). The role of performance specialists in soccer organizations is increasingly important in the creation of the best players, the best teams and the best sporting achievements.

\section{CONCLUSIONS}

These results provide managers, coaches and managers of football clubs with useful information to help staffs with better and more effective services. 


\section{REFERENCES}

Drust B. (2019). Applied science and soccer: a personal perspective on the past, present and future of a discipline. Sport Perform Sci Rep, 56.

Jukic, I.,et al.. (2018): Performance specialist: a new job of renaissance experts in team sports. Conference: 16th International Conference "Physical Conditioning of Athletes 2018", At Zagreb. Croatia.

\section{(c)}

This work is licensed under a Attribution-NonCommercial-NoDerivatives 4.0 International (CC BY-NC-ND 4.0). 


\title{
Evaluation of physical activity status and cognitive function among breast cancer patients: A cross-sectional study
}

\author{
SÍLVIA ROCHA-RODRIGUES1,2,3 $\triangleleft$, ANDREIA CARVALHO², ANTÓNIO ASCENSÃO2, MARIA JOÃO \\ CARDOSO ${ }^{3,4}$, JOSÉ MAGALHÃES 2 \\ ${ }^{1}$ Instituto Politécnico de Viana do Castelo, Escola Superior Desporto e Lazer de Melgaço, Viana do Castelo, \\ Portugal \\ 2Laboratory of Metabolism and Exercise, Research Centre for Physical Activity, health and Leisure (CIAFEL), \\ Faculty of Sport, University of Porto, Portugal \\ ${ }^{3}$ Mama Help Association, Support Centre for Breast Cancer Patients, Porto, Portugal \\ ${ }^{4}$ Breast Unit, Champalimaud Foundation and Nova Medical School, Lisbon, Portugal
}

\begin{abstract}
Background: Cancer treatment-related cognitive impairment $(\mathrm{CRCl})$ is frequently reported in breast cancer patients and regular physical exercise has been described as an effective way to decrease this negative effect of the disease. Methods: Women with a breast cancer diagnosis ( $n=29$, aged between [34 -80y]) were recruited from a Breast Cancer Support Association, Mama Help. The international Physical Activity Questionnaire (IPAQ) was used in all included volunteers to register the three intensity levels of physical activity - vigorous and moderate-intensity activity and /or walking. Data were then converted to Metabolic Equivalent minutes per week (MET, min/week). The $\mathrm{CRCl}$ was evaluated using the Functional Assessment of Cancer Therapy-Cognitive Function (FACT-Cog questionnaire). Results: A moderate-sized effect for total score FACT-Cog was observed in women during chemotherapy ( $d=0.4 ; 37.0$ [8.3-65.7]) and in postchemotherapy $(d=0.5 ; 36.2$ [19.9-52.6] groups compared to no-chemotherapy group (46.6 [-10.5-103.9]). No associations between age, total score FACT-Cog or its domains $(p>0.05)$, but a positive association between perceived cognitive abilities and months of diagnosis was observed $(r=0.507 ; p=0.05)$. A large-sized effect $(\mathrm{d}=0.93 ; 1315$ [279.9-2350] vs.733.4 [462.2-1002.6] was found in METs between current chemotherapy vs.no-chemotherapy groups. A moderate-sized effect ( $d=0.5 ; 30.541 .6$ [28-54.6] vs. 30.5 [3.4-57.5]) in total score and in quality of life (QoL) (d=0.4; 14.5 [7.2-18.9] vs. 10.3 [-1.9-22.7]) were observed in active group vs. inactive group. Conclusions: Data from this study provide evidence for the presence of cognitive complaints and poor physical activity status in women during chemotherapy which likely reflect on a negative impact on QoL. Keywords: Cognitive complaints; Exercise; Chemotherapy.

Corresponding author. Escola Superior Desporto e Lazer; Complexo Desportivo e de Lazer Comendador Rui Solheiro, 4960320 Melgaço, Viana do Castelo, Portugal.

E-mail: silviadarocharodrigues@gmail.com

Supplementary Issue: Spring Conferences of Sports Science. International Seminar of Physical Education, Leisure and Health, 17-19 June 2019. Castelo Branco, Portugal.

JOURNAL OF HUMAN SPORT \& EXERCISE ISSN 1988-5202

(C) Faculty of Education. University of Alicante.

doi:10.14198/jhse.2019.14.Proc4.82
\end{abstract}




\section{INTRODUCTION}

Cancer treatment-related cognitive impairment ( $\mathrm{CRCl})$ is a common deleterious consequence among breast cancer patients with estimates suggesting that more than $75 \%$ patients experience deficits during and after treatment. Evidence has shown that $\mathrm{CRCl}$ is not only associated with treatments, but also results from common risk factors for both the development of cancer and age-related cognitive decline as well as an accelerated aging caused by cancer treatments (Myers, Wick, \& Klemp, 2015). Evidence showed the negative effects of breast cancer treatments, such as chemotherapy, on cognitive function deficits in one or more domains and overall quality of life (QoL), compared to pre-treatment cancer and/or population controls. These cognitive deficits have been observed to persist for variable periods of time from one to as many as 10-20 years post-treatment (Myers et al., 2015). Physical exercise has been emerged as a promising treatment for age-related cognitive decline (Northey, Cherbuin, Pumpa, Smee, \& Rattray, 2018); however few studies have investigated the $\mathrm{CRCl}$ in breast cancer patients. Recent research reports that cognitive functioning is preserved with higher levels of physical activity in breast cancer patients (Northey et al., 2018). Therefore, we aimed to analyse the physical activity status and the $\mathrm{CRCl}$ among breast cancer patients, as well as their association to health-related quality of life.

\section{MATERIAL AND METHODS}

\section{Participants}

Twenty-nine women diagnosed with breast cancer were recruited from a Breast Cancer Support Association, Mama Help, after signing the informed consent. The study was approved by Mama Help's Ethics Committee. Participants aged between $34-80$ years, $52.7 \%$ were currently on treatments, chemotherapy $(25.1 \%)$ or radiation therapy $(27.6 \%)$. Exclusion criteria were confirmed psychiatric disorders and metastatic breast cancer.

\section{Measures}

Physical Activity Status

Data of The International Physical Activity Questionnaire (IPAQ) were converted to Metabolic Equivalent minutes per week (MET, min/week) based on an equation. Women were classified as "inactive" if they had achieved less than 600 METs or "active" if they had reached at least 600 METs as recommended for breast cancer patients /survivors.

Cancer treatment-related cognitive impairment

The CRCl was tested using the Functional Assessment of Cancer Therapy-Cognitive Function (FACT-Cog) V3 (Van Dyk et al., 2017). The FACT-Cog contains four subscales that assess perceived cognitive impairments (PCI); perceived cognitive abilities (PCA); impact of perceived cognitive impairment on QoL; and comments from others on cognitive function (Oth). Negatively worded items are reverse scored to create subscale scores, with higher scores reflecting fewer cognitive problems and better quality of life. Participants were classified into: 1-No chemotherapy ( $n=5,17.1 \%), 2$-currently receiving chemotherapy $(n=10,34.6 \%)$ and 3-post-chemotherapy $(n=14,48.3 \%)$. This classification was based on previous studies indicating that a subset of survivors continue to experience cognitive complaints past 1-2 years of completing chemotherapy (Myers et al., 2015).

\section{Analysis}

The effect sizes for the pairwise changes in FACT-Cog total score and each domain of its domains. Effect sizes were calculated as Cohen's $d$ using the pooled standard deviation of the random effects. 


\section{RESULTS}

When patients were categorized by chemotherapy treatment, no statistically significant effect was observed for FACT-Cog total score, PCl, or Oth or QoL domains. However, a moderate-sized effect for total score FACT-Cog was observed in women during chemotherapy $(\mathrm{d}=0.4 ; 37.0[8.3,65.7])$ and post-chemotherapy $(d=0.5 ; 36.2$ [19.9-52.6]) groups compared to no-chemotherapy group (46.6 [-10.5-103.9]). A moderate-sized effect was observed in post-chemotherapy group compared to current chemotherapy group for $\mathrm{PCl}(\mathrm{d}=0.3$; $20.3[7.8,32.7]$ vs15 [-1.6,31.6]) and for $\mathrm{QoL}(\mathrm{d}=0.55 ; 8.6[-1.4,21.4]$ vs.8.1 [3.8,17.5]). No associations between age, total score FACT-Cog or its domains ( $p>0.05)$, but a positive association between PCA and months of diagnosis was observed $(r=0.507 ; p=0.05)$. Of all, $10(35.5 \%)$ were classified as inactive and 19 $(65.5 \%)$ as active patients. When we analysed the physical activity status between receiving chemotherapy or not, no changes we observed but a large-sized effect ( $d=0.93 ; 733.4$ [462.2,1002.6] vs. 1315 [279.9,2350]) was found in current chemotherapy group vs. no-chemotherapy group. This finding suggests that in our group, women that are currently receiving chemotherapy do less physical activity $(\sim 45 \%)$ than the nochemotherapy group. Although there was no statistical significance between inactive and active groups regarding FACT-Cog total score or domains, a moderate-sized effect $(d=0.5 ; 30.541 .6[28,54.6]$ vs. 30.5 $[3.4,57.5])$ in total score and in QoL ( $d=0.4 ; 14.5[7.2,18.9]$ vs. $10.3[-1.9,22.7])$ was observed in the active group vs. the inactive group. A moderate association between months to diagnosis and METs $(p=0.05$, $r=0.370$ ) was also found.

\section{DISCUSSION}

The present study provides evidence of cognitive deleterious complaints in women under chemotherapy treatment for breast cancer and an increase in $\mathrm{PCl}$ after chemotherapy treatment with a negative impact on QoL. Moreover, women currently receiving chemotherapy with more cognitive complaints performed less than $45 \%$ of (METs/wk) than the no-chemotherapy group. Considering previous studies conducted in breast cancer patients (Northey et al., 2018) suggesting that physical exercise is an important strategy to attenuate $\mathrm{CRCl}$, data from the present study demonstrate that women who exercised more (METs/wk) have less cognitive complaints when compared to those of the with the inactive group. Although this is a very small study, results support the potential benefit of regular physical exercise as an intervention to mitigate $\mathrm{CRCl}$ associated with chemotherapy and improve QoL in breast cancer patients. In addition, $\mathrm{CRCI}$ seems to be not only dependent on treatments, but also with age-related cognitive decline. In the present study, we did not observe any effect of age on $\mathrm{CRCl}$ which is in line with previous studies showing that cognitive complaints are more frequent in younger women (Myers et al., 2015). A similar finding was observed by Cimprich et al. (2005) who hypothesized that alterations associated with treatment for breast cancer are more noticeable in younger women who are still working and/or face with balancing multiple tasks as compared to older women who may be retired and facing less cognitively challenging activities or less demoing life roles, or better able to cope with stressors due to life experience. In fact, $70 \%$ of the participants in our study were retired or on extended medical leave at the moment of the interview.

\section{CONCLUSIONS}

In spite of the small sample data provided evidence for the presence of cognitive complaints and poor physical activity status in women during chemotherapy which likely reflect on a negative impact on QoL. 


\section{REFERENCES}

Cimprich, B., So, H., Ronis, D. L., \& Trask, C. (2005). Pre-treatment factors related to cognitive functioning in women newly diagnosed with breast cancer. Psychooncology, 14(1), 70-78. https://doi.org/10.1002/pon.821

Myers, J. S., Wick, J. A., \& Klemp, J. (2015). Potential factors associated with perceived cognitive impairment in breast cancer survivors. Support Care Cancer, 23(11), 3219-3228. https://doi.org/10.1007/s00520-015-2708-7

Northey, J. M., Cherbuin, N., Pumpa, K. L., Smee, D. J., \& Rattray, B. (2018). Exercise interventions for cognitive function in adults older than 50: a systematic review with meta-analysis. $\mathrm{Br} \mathrm{J}$ Sports Med, 52(3), 154-160. https://doi.org/10.1136/bjsports-2016-096587

Van Dyk, K., Hunter, A. M., Ercoli, L., Petersen, L., Leuchter, A. F., \& Ganz, P. A. (2017). Evaluating cognitive complaints in breast cancer survivors with the FACT-Cog and quantitative electroencephalography. Breast Cancer Res Treat, 166(1), 157-166. https://doi.org/10.1007/s10549$\underline{017-4390-6}$ 


\title{
Physical conditioning of U16 national team players: Mediator effect of previous sport experiences and strength and conditioning practices
}

\author{
JORGE AREDE ${ }^{1} \triangle$, PEDRO ESTEVES ${ }^{12}$, ANTÓNIO PAULO FERREIRA ${ }^{3}$, NUNO LEITE \\ ${ }^{1}$ Research Center in Sports Sciences, Health Sciences and Human Development, CIDESD, University of \\ Trás-os-Montes and Alto Douro, Portugal \\ 2School of Education, Communication and Sport, Polytechnic Institute of Guarda, Guarda, Portugal \\ ${ }^{3}$ CIPER, Faculty of Human Kinetics, University of Lisbon, Cruz-Quebrada, Portugal
}

\begin{abstract}
The aims of this study were (a) to investigate the effects of previous strength and conditioning in physical performance; (b) to examine the mediator effect of previous sport experiences in physical performance, according to the training group (c) to examine the relationship between training frequency and physical performance. Twenty-four young under-16 (U-16) male basketball players performed several physical (jumping, sprinting, and change-of-direction speed) and functional (ankle dorsiflexion and lower limb asymmetry index [ASI]) tests during the national team training camp. Previous sport experiences, and strength and conditioning practices in club settings were collected using customized questionnaires. Cluster analysis was used to analyse the between strength and conditioning practices status differences (Group 1 and 2) in physical and functional parameters. No significant differences were found between groups, in physical and functional parameters. However, we found a significant main effect of non-specific deliberate practice in T-test, right ankle dorsiflexion and ankle dorsiflexion symmetry. Also, the ASI and strength/power training frequency were significantly related. Early non-specific deliberate practice and strength/power sessions involvement seem to play a key role on the development of the neuromuscular system and injury prevention. Keywords: Talent development; Long-term athlete development; Adolescence.
\end{abstract}

\footnotetext{
Corresponding author. Research Center in Sports Sciences, Health Sciences and Human Development, CIDESD, University of Trás-os-Montes and Alto Douro, Quinta de Prados, Apartado 202, 5001-911 Vila Real, Portugal.

E-mail: jorge arede@hotmail.com

Supplementary Issue: Spring Conferences of Sports Science. International Seminar of Physical Education, Leisure and Health, 17-19 June 2019. Castelo Branco, Portugal.

JOURNAL OF HUMAN SPORT \& EXERCISE ISSN 1988-5202

(c) Faculty of Education. University of Alicante.

doi:10.14198/jhse.2019.14.Proc4.82
} 


\section{INTRODUCTION}

Basketball is a fast-paced sport that requires the athletes to perform according to a solid physical conditioning (Schelling \& Torres-Ronda, 2016). The ability to repeat high-intensity efforts such as short-distance sprints, vertical jumps and physical contacts with teammates and opponents is dependent on extensive strength and conditioning practices (Schelling \& Torres-Ronda, 2016). Prior research has alluded to the importance of previous sport experiences on the player development in basketball, especially in physical abilities (Santos, Mateus, Sampaio, \& Leite, 2016). However, more research is needed to understand the relations between previous involvement in sporting activities, strength and conditioning practices in club settings and physical conditioning of youth athletes playing for national teams. The aims of this study were (a) to investigate the effects of previous strength and conditioning in physical performance; (b) to examine the mediator effect of previous sport experiences in physical performance, according to the training group (c) to examine the relationship between training frequency and physical performance.

\section{MATERIAL AND METHODS}

\section{Participants}

Twenty-four young U-16 male basketball players were selected by the Portuguese national coaching staff to participate in a training camp.

\section{Measures}

The strength and conditioning practices in last season (physical testing, flexibility, speed, agility, plyometrics, and strength/power development) in team club setting was obtained using standardized survey (Ebben \& Blackard, 2001). The detailed longitudinal profile of each player's involvement in specific and non-specific sporting activities was collected through a validated questionnaire (Santos et al., 2016). The countermovement (CMJ), and Abalakov Jumps (ABA), 25-meters sprint time and agility (T-test) were assessed. The lower limb asymmetry index (ASI) was determined based in single-leg vertical jumps. Ankle dorsiflexion was evaluated through the Weight-Bearing Lunge Test. The bilateral WBLT symmetry were calculated by subtracting the mean of the right limb from the mean of the left limb for each subject.

\section{Procedures}

Physical performance tests were performed during training session time with verbal encouragement. Institutional research ethics committee approved the present study, and it conformed to the recommendations of the Declaration of Helsinki.

\section{Analysis}

Ward's method was chosen for cluster analysis, taking strength and conditioning practices into consideration and using the squared Euclidian distance as the distance measure (Group 1, $n=13$; Group 2, $n=11$ ). Independent sample T-tests were conducted for differences in different variables within all groups. Univariate analysis of covariance (ANCOVA) was used to test the effect of previous sport experiences (i.e. sport starting age, basketball starting age, specialization age, number of sports practiced, basketball and non-specific deliberate and free practice) as covariate in physical parameters. The Pearson product-moment correlation was employed to determine the relationship between training frequency and physical parameters. 


\section{RESULTS}

The group 2 performed more flexibility $(p=0.000)$ and speed development $(p=0.021)$ than group 1 . No significant differences were found between groups, in physical and functional parameters, but also in other strength and conditioning practices. Results showed a significant main effect of non-specific deliberate practice in T-test $(p=0.048)$, right ankle dorsiflexion $(p=0.012)$ and ankle dorsiflexion symmetry $(p=0.012)$. The ANCOVA results shown that athletes pertaining to group 1 were more agile, less flexible in right ankle and presented higher symmetry between ankles. Significant relationship was found between the ASI and strength/power training frequency $(r=-0.405 ; p \leq 0.05)$.

\section{DISCUSSION}

The physical and functional parameters in national team players appear to be influenced by previous sport experiences. We found that non-specific deliberate practice affects positively the agility along with ankle dorsiflexion and ankle symmetry. Thus, the neuromuscular system seems to take advantage from performing non-specific stretch-shortening cycles in different conditions and directions (e.g. horizontal displacements in football and jumps in swimming), movement (e.g. kicking in swimming) or manipulative skills which involve controlling objects with the foot (e.g. dribbling, passing and shooting in football) during early ages in connection with involving in strength and conditioning practices in later stages of development. Current findings also revealed that higher strength/power training frequency is associated with lower ASI, which is a strong injury risk factor that somehow features national team players (Arede, Ferreira, Gonzalo-Skok, \& Leite, 2018). In fact, the frequent involvement in strength/power-based sessions elicits improvements at ASI level (Arede, Vaz, \& Leite, 2018). These practices are in line with previous recommendations which suggests to focus on injury prevention and general strength before the 16 years old (Schelling \& Torres-Ronda, 2016).

\section{CONCLUSIONS}

Non-specific deliberate practice sports during childhood might be beneficial in generating adaptations in neuromuscular system which underpin enhanced agility and functional performance. Also, strength and power programmes could decrease the risk of injury. Finally, multiple sport experiences at an early phase of the training programs should be strongly encouraged and disseminated.

\section{FUNDING}

This work was supported by the Foundation for Science and Technology (FCT, Portugal) and European Social Fund (ESF), through a Doctoral grant endorsed to the first author [SFRH/BD/122259/2016] and under project UID/DTP/04045/2019.

\section{REFERENCES}

Arede, J., Ferreira, A. P., Gonzalo-Skok, O., \& Leite, N. (2018). Maturational development as key aspect in physiological performance and national team selection in elite male basketball players. International Journal of Sports Physiology and Performance, 20, 1-24. https://doi.org/10.1123/ijspp.2018-0681

Arede, J., Vaz, R., \& Leite, N. (2018). The effect of playing status on adaptations to strength and conditioning intervention and detraining in youth basketball. In 6th NSCA International Conference : Oral abstract presentations (Vol. 32, p. e35). 
Ebben, W. P., \& Blackard, D. O. (2001). Strength and Conditioning Practices of National Football League Strength and Conditioning Coaches. Journal of Strength and Conditioning Research. https://doi.org/10.1519/1533-4287(2001)015<0048:SACPON>2.0.C0;2

Santos, S., Mateus, N., Sampaio, J., \& Leite, N. (2016). Do previous sports experiences influence the effect of an enrichment programme in basketball skills? Journal of Sports Sciences, 35(17), 17591767. https://doi.org/10.1080/02640414.2016.1236206

Schelling, X., \& Torres-Ronda, L. (2016). An Integrative Approach to Strength and Neuromuscular Power Training for Basketball. Strength and Conditioning Journal, 38(3), 72-80. https://doi.org/10.1519/SSC.0000000000000219 


\title{
Evaluation of the efficacy of a sensorimotor program in the development of children in swimming lessons
}

\author{
CLEONICE TEREZINHA FERNANDES ${ }^{1} \triangle$, JEFFERSON CARVALHO NEVES ${ }^{2}$, MARIA ISABEL \\ MARTINS MOURÃO CARVALHAL ${ }^{3}$, CILENE MARIA LIMA ANTUNES MACIEL ${ }^{1}$, CARLOS BARRIGAS ${ }^{5}$, \\ ALEXANDRE KONIG GARCIA PRADO 6 \\ ${ }^{1}$ Universidade de Cuiabá (UNIC), Cuiabá, Mato Grosso, Brazil \\ 2PPGEn, UNICVIFMT, Cuiabá, Mato Grosso, Brazil \\ ${ }^{3}$ Universidade de Trás-os-Montes and Alto Douro (UTAD), Vila Real, Portugal \\ ${ }^{4}$ Universidade de Lisboa (UTL), Lisboa, Portugal \\ ${ }^{5}$ Universidade Federal do Mato Grosso (UFMT), Cuiabá, Mato Grosso, Brazil
}

\begin{abstract}
We have achieved in recent years many advances in neuroscience studies, with new proposals being applied in several areas of human knowledge. Also in teaching we have made a lot of progress, but in some sports, as swimming has not taken advantage of all this potential, because in the present day we still have in most of the environments where classes of this modality are taught, these being taught as in the last century, not taking into account, for example, the indissociable body-to-person relationship. With a quasi-experimental type of qualitative-quantitative approach, this study will be carried out for 12 weeks, with two weekly meetings for each of the participating groups, where they will perform swimming lessons of 50 minutes, being a group with traditional classes of swimming, and the second performing the same classes but associated with a sensorimotor program developed for the aquatic environment. The research seeks to verify if sensorimotor development exercises influence the results in children's learning and performance in swimming practice, and if so, an alternative to the traditional teaching method of the modality that is currently based on mechanistic replications and reproductions of movements, which often discourage their practitioners, causing withdrawal and failures in their respective learning processes. Keywords: Cognitive-motor development; Teaching, Physical education, Palmateios.
\end{abstract}

Corresponding author. Av. Pres. Marques, No 833; Apartamento 22; Bairro: Quilombo. CEP: 78.045-175. Cuiabál MT. Brazil. E-mail: cleo terezinha@hotmail.com

Supplementary Issue: Spring Conferences of Sports Science. International Seminar of Physical Education, Leisure and Health, 17-19 June 2019. Castelo Branco, Portugal.

JOURNAL OF HUMAN SPORT \& EXERCISE ISSN 1988-5202

(c) Faculty of Education. University of Alicante.

doi:10.14198/jhse.2019.14.Proc4.82

VOLUME 14 | Proc4 | 2019 | S1423 


\section{INTRODUCTION}

Historically the teaching of swimming has its structuring consolidated in the mechanization of movements, often not taking into account the sensorial form of the gestural development, its efficiency and mainly the neural stimulation. Studies have shown that neural stimulation associated with improvement in physical characteristics has the capacity to greatly increase the subject's development (Thomas and Knowland, 2009). A good technique associated with sensory stimulation and communication opportunities are supports for cognitive gains, language development, motor skills, adaptive and socio-emotional aspects.

In the area of swimming sensory work should be the starting point for the construction of a solid and successful learning in the respective teaching, which would provide the training of a swimmer more prepared to face the challenges of the modality. In this sense, we believe that sensory development should be performed in a way associated with physico-technical work, especially for children.

While having great potential in the emotional and intellectual development of the subject, swimming is little explored in its practice in this area, thus neglecting its power to opportunize the positive confrontation of the emotions and sensations for example linked inherent to frustrations and achievements.

In this sense, the present study aims to verify the impact of a sensorimotor program when introduced in conventional swimming lessons.

\section{MATERIAL AND METHODS}

A quasi-experimental study with a total sample $(\mathrm{N}=40)$, equitable between control group $(C G)$ and experimental group (SG), with students between 8 and 12 years old, of both sexes, swimming practitioners, from a private college in Cuiabá, Mato Grosso, Brazil.

\section{Participants}

Inclusion criteria:

- Expected decimal age (difference not exceeding 1 month -8 and 12 years);

- Subjects with Biological Age verification;

- Enrolled in 2019 at the swimming school, with more than 1 year of practice in the modality.

Exclusion criteria:

- Absenteeism in more than 3 classes in the sessions of the GC and GE swimming program;

- Students with traits of ID - Intellectual Disability.

\section{Measurements}

The design will be with two independent groups, where GC and GE will initially be performed with the normal distribution test (Shapiro-Wilk) and the homogeneity of variance test (Levene). If the assumptions are accepted the $T$ test will be used to compare the results between the groups.

\section{Procedures}

The study is in the implementation phase and already has the opinion of the Committee of Ethics in Research with Human Beings (CEP) and respective opinion substantiated; distributed in three phases: 
1. Sample selection (and recruitment): swimming students being 10 females and 10 males in each group, randomly distributed;

2. Aquatic performance assessments will be performed on the subjects by means of taking time at distances of 25 and 50 meters freestyle (with a time interval of 20 minutes between tests), the specific force in the water will also be tested, using a traction dynamometer of the brand Cefise;

3. Pedagogical intervention performed for three months (to avoid maturity bias) composed of 2450 minute sessions; twice a week, the 2 groups being:

a. GC- 20 children who will perform traditional 50-minute swimming lessons (10 minutes of warm-up, 35 minutes of traditional specific exercises and 5 minutes of calm back);

b. GE - 20 children who will perform swimming lessons associated with sensory-motor development stimuli (10 minutes of warm-up, 10 minutes of Sensory-Motors exercises described, 25 minutes of traditional specific exercises and 5 minutes of calm).

\section{Analysis}

The study is ongoing.

\section{RESULTS}

We hope to see if there are developmental differences between groups; if sensory-motor development exercises contribute to better swimming results. With this work we intend to suggest an alternative to the traditional method of teaching swimming that is currently based on repetitions and reproductions of movements, without concern for the interaction \ integration of the body (sensation/ perception) of the subject with the aquatic environment.

\section{DISCUSSION}

Taking into account that a swimmer's success equals his ability to generate propulsion while reducing the resistance to the advance of his body (Toussaint and Beek, 1992), and with the current understanding of the propulsive mechanisms, we understand that in confirming the expected result of the present study, we may have the possibility of improving performance in swimming practice, not only with repetitive exercises and endless volumes of exercises; 'we may seek sensory stimulation so that once associated with cognitive gains, this evolution is more stimulating and creative, enjoyable, and without the usual excessive demands.

Thus, we could have a proposal where sensorial development becomes the starting point for the construction of a solid and successful learning in swimming teaching, which would provide the training of a swimmer more prepared to deal with the challenges of the modality, an understanding that goes against literature when it states that the domain of movement in water also develops by improving the feel and exerted on it, making swimmers who can improve these skills are considered more " talented "(Colwin, 2002).

\section{CONCLUSIONS}

Study in progress.

\section{REFERENCES}

Colwin, C.M. (2002). Nadando para o século XXI. São Paulo: Manole. 
Thomas, M, S.C.; Knowland, V.C.P. (2009). Sensitive periods in brain development: Implications for education policy. Eur. Psych. Rev., 2 (1), 17-20.

Toussaint, Huub M; Beek, Peter J. (1992).Biomechanics of Competitive Front Crawl Swimming. Sports Medicine 13(1); 8-24. https://doi.org/10.2165/00007256-199213010-00002

\section{(c) $(\mathrm{B})(\mathrm{EY}$}

This work is licensed under a Attribution-NonCommercial-NoDerivatives 4.0 International (CC BY-NC-ND 4.0). 


\title{
Relationship between physical education, school satisfaction, psychological well-being and academic achievement in vocational students
}

\author{
LUÍS ALMEIDA ${ }^{1,2}-$, HELDER M. FERNANDES 3,4 \\ ${ }^{1}$ Research Centre of Training Innovation and Intervention in Sport, CIFI2D-FADEP, Portugal \\ ${ }^{2}$ Centre for Research and Intervention in Education, CIIE-FPCEUP, Portugal \\ ${ }^{3}$ Research Centre in Sports Sciences, Health Sciences and Human Development, CIDESD-UTAD, Portugal \\ ${ }^{4}$ Research in Education and Community Intervention, RECl- Instituto Piaget, Portugal
}

\begin{abstract}
The objective was to study the relationships between attendance at physical education (PE) classes, satisfaction with school, psychological well-being and academic achievement. A case study was carried out at a vocational school known for the academic failure of its students. A total of 139 students (54.7\% boys), aged between 15 and 24 years $(M=17.95, S D=1.79)$, completed a survey that included sociodemographic, academic, and satisfaction-with-school variables, as well as the Ryff's Psychological Well-Being Scales adapted for youth (Fernandes et al., 2010). The correlational study indicated the existence of significant positive associations between PE attendance, satisfaction with school and psychological well-being, as well as a negative association between PE attendance and the total number of retentions. In short, the assiduous participation in PE classes seems to be related to better academic (school achievement and satisfaction) and psychosocial outcomes (psychological global well-being) among vocational students. Keywords: Physical education; Psychological well-being; Academic achievement; Vocational schools.
\end{abstract}

\footnotetext{
Corresponding author. Faculdade de Desporto da Universidade do Porto, Laboratório de Psicologia do Desporto, R. Dr. Plácido da Costa 91, 4200-450 Porto, Portugal.

E-mail: luismanuelalmeida@gmail.com

Supplementary Issue: Spring Conferences of Sports Science. International Seminar of Physical Education, Leisure and Health, 17-19 June 2019. Castelo Branco, Portugal.

JOURNAL OF HUMAN SPORT \& EXERCISE ISSN 1988-5202

(c) Faculty of Education. University of Alicante.

doi:10.14198/jhse.2019.14.Proc4.82
} 


\section{INTRODUCTION}

It is unanimously recognized that the causes and factors associated with school (un)success are several and diverse. Vocational education (VE) is an alternative school pathway, with increasing implementation in Portugal, predominantly at Vocational Schools (VS), which are mostly attended by students with school histories of dropout and failure (Almeida et al., 2018).

Scientific literature suggests that the practice of physical activity, besides benefiting the physical, social and mental health of individuals (Almeida et al., 2018; Fernandes et al., 2010), seems to contribute to the reduction of psychosocial problems (internalization and externalization) and also to improve academic achievement of adolescents (Bastos et al., 2015; Spruit et al., 2016). Moreover, a study by Fernandes et al. (2011) evidenced a positive correlation between school satisfaction and all dimensions of psychological wellbeing, with a moderate positive effect on global well-being.

Considering the increasing implementation of $\mathrm{PE}$, its objectives, student profile and the lack of research about the role of PE in improving academic achievement in this population, the objective was to study the relationships between attendance at PE classes, satisfaction with school, well-being and academic achievement among vocational students.

\section{MATERIAL AND METHODS}

\section{Participants}

A case study was carried out in a VS located in Aveiro (Portuguese district), which is known for the academic histories of dropout and failure of its students. The sample consisted of the all students of the school, a total of 139 students ( $54.7 \%$ boys), aged between 15 and 24 years $(M=17.95, S D=1.79)$.

\section{Measures}

PE attendance was converted into a percentage by calculating the relation between the total number of classes taught and the total number of classes in which each student participated in a physically active way. The satisfaction index with the school was obtained through a question "How satisfied are you with your current school?" answered in a scale from 1 to 10 (1: very dissatisfied to 10: very satisfied). Global well-being was measured by using the Psychological Well-Being Scales (Fernandes et al., 2010), a Portuguese version adapted from the Carol Ryff's scales, which is an instrument developed to evaluate autonomy, mastery of the environment, personal growth, positive relationships with others, goals in life and acceptance of self. A set of 30 items was answered on a 5-point Likert scale (from 1: strongly disagree to 5: strongly agree). Global well-being encompasses the sum of all items. The Cronbach's alpha for global well-being was 0.88 .

\section{Procedures}

After obtaining the school director's approval, written informed consent to participate in this study was obtained from all adult students or their parents/legal guardians. The instruments were applied individually at the beginning or end of a lesson, under quiet classroom conditions. In all phases of the study, the anonymity and confidentiality of the respondents were guaranteed.

\section{Analysis}

Statistical analysis included mean, standard deviation, percentage, skewness and kurtosis, internal consistency (Cronbach's alpha) and Pearson correlations. Significance was set at $p<0.05$. All analyses were performed using SPSS 25.0 . 


\section{RESULTS}

The majority of skewness and kurtosis coefficients of the variables were between -1 and +1 . The descriptive analysis showed that only 46 students $(33.1 \%)$ had no retention during their school history and that 93 $(66.9 \%)$ had one or more retentions. Correlation results showed small to moderate significant associations between the study variables. PE attendance correlated positively with school satisfaction $(r=0.18, p<0.05)$ and negatively with the total number of retentions $(r=-0.28, p<0.01)$. Satisfaction with school was positively associated with the total well-being score $(r=0.35, p<0.001)$.

\section{DISCUSSION}

The high percentage of students with retentions during their schooling years seems to confirm the school failure profile that characterizes the students of VE (Almeida et al., 2018). In addition to reinforcing the association between physical activity and better school performance (Bastos et al., 2015; Spruit et al., 2016), the results suggest that PE participation, in a formal school setting, seems to be related to better indices of satisfaction with school, thus highlighting the potential of PE as a mechanism to promote school satisfaction, and expectantly, an improvement in academic achievement. The evidence also confirms and extends Fernandes et al.'s (2011) findings among students enrolled in regular programs, by showing an association between school satisfaction and eudaimonic well-being, thus emphasizing the importance of promoting psychological well-being for the integration and identification of VE students with their schools.

\section{CONCLUSIONS}

The present study reinforces the importance of $\mathrm{PE}$ in promoting more effective teaching-learning processes among VE students and the need to address these processes in an ecological way, so as to reduce the school dropout and failure that characterizes students who opt for VE.

\section{REFERENCES}

Almeida. L., Pereira, H., \& Fernandes, H. M. (2018). Efeitos de diferentes tipos de prática desportiva no bem-estar psicológico de jovens estudantes do ensino profissional. Ibero Am J Exerc Sport Psychol, 13(1), 15-21.

Bastos, F., Reis, V. M., Aranha, A. C., \& Garrido, N. D. (2015). Relação entre atividade física e desportiva, níveis de IMC, perceções de sucesso e rendimento escolar. Motricidade, 11(3), 41-58. https://doi.org/10.6063/motricidade.3771

Fernandes, H. M., Vasconcelos-Raposo, J., Bertelli, R., \& Almeida, L. (2011). Satisfação escolar e bemestar psicológico em adolescentes portugueses. Rev Lusofona Educ, 18, 155-172.

Fernandes, H. M., Vasconcelos-Raposo, J., \& Teixeira, C. (2010). Preliminary analysis of the psychometric properties of Ryff's Scales of Psychological Well-Being in Portuguese adolescents. Span J Psychol, 13(2), 1032-1043. https://doi.org/10.1017/s1138741600002675

Spruit, A., Assink, M., van Vugt, E., van der Put, C., \& Stams, G. J. (2016). The effects of physical activity interventions on psychosocial outcomes in adolescents: A meta-analytic review. Clin Psychol Rev, 45, 56-71. https://doi.org/10.1016/..cpr.2016.03.006

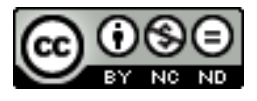

This work is licensed under a Attribution-NonCommercial-NoDerivatives 4.0 International (CC BY-NC-ND 4.0). 


\title{
Water aerobics: The use of wearables
}

\author{
LUÍS BRANDÃO FAÍL ${ }^{1,2}$, HENRIQUE PEREIRA NEIVA ${ }^{1,2}$, MARIA HELENA GIL $^{1,2}$, ANTÓNIO CARLOS \\ SOUSA ${ }^{1,2}$, PEDRO POMBO NEVES ${ }^{1,2}$, DANIEL ALMEIDA MARINHO ${ }^{1,2}$ \\ ${ }^{1}$ Department of Sports Science, University of Beira Interior, Covilhã, Portugal \\ ${ }^{2}$ Research Center in Sports Sciences, Health Sciences \& Human Development, CIDESD, Vila Real, Portugal
}

\begin{abstract}
Nowadays within the popularity of aquatic exercise programs, water-aerobics is one of the most recognized programs by the scientific community. Due to the importance of water-aerobics, it is essential to monitor and evaluate the exercise effects. However, little is known about exercise control in water-aerobics. Thus, the aim of this study was to characterize the use of wearable technology by the Portuguese who practice wateraerobics. For this, a questionnaire was applied to 188 subjects of both genders, with aged between 19 and 88 years, who regularly practice water-aerobics. The results show that most subjects have heard of wearable technology $(60 \%)$, but few have ever experienced it during exercise (12\%), and only $29 \%$ of these subjects used wearable technology during performing water-aerobics. Main reasons are: due to the lack of opportunity $(62 \%)$, for considering the technology useless (15\%), and due to the difficulty to perceive its functioning (9\%). These people who have never experienced, considered using it (79\%), especially if the technology allows to monitor heart rate $(34 \%)$ and calories spent $(31 \%)$. Overall, people have knowledge about the existence of wearable technology, but are few subjects who have ever experienced to perform physical exercise with the aid of these devices, especially when speaking in the water-aerobics. However, there is some interest in starting to use the technology, especially for monitoring heart rate and calories spent. Therefore, it is necessary to make known and to improve the technology according to the mode of exercise and to the preferences of its users. Keywords: Water-aerobics; Wearables; Questionnaire; In-water activities; Elderly.
\end{abstract}

\footnotetext{
Corresponding author. Department of Sports Sciences, University of Beira Interior, Convento de Santo António 6201-001 (Covilhã), Portugal.

E-mail: luisfail 93@hotmail.com

Supplementary Issue: Spring Conferences of Sports Science. International Seminar of Physical Education, Leisure and Health, 17-19 June 2019. Castelo Branco, Portugal.

JOURNAL OF HUMAN SPORT \& EXERCISE ISSN 1988-5202

(c) Faculty of Education. University of Alicante.

doi:10.14198/jhse.2019.14.Proc4.82
} 


\section{INTRODUCTION}

Nowadays within the popularity of aquatic exercise programs, water-aerobics is one of the most recognized exercises by the scientific community (Antunes et al., 2015; Benelli, Ditroilo and De Vito, 2004). This type of exercise provides a variety of movements performed using the properties of water to create resistance to movement, in a situation with decreasing force of gravity on the body, and with favourable results in regard to the physical capacities of practitioners (Green, 1989). This significant increase in the practice of physical exercise in the aquatic environment in recent years has led to an increase in the concern of health and sports professionals regarding their monitoring in water through the use of wearable technology (Raffaelli, Galvani, Lanza, and Zamparo, 2011). However, little is known about the use of wearables in water-aerobics. Thus, the purpose of this study was to characterize the use of this technology by the Portuguese who practice water-aerobics.

\section{MATERIAL AND METHODS}

\section{Participants}

188 subjects of both genders ( $65 \%$ female and $35 \%$ male), with aged between 19 and 88 years (mean \pm SD: $60 \pm 16.20$ years of age; $72.70 \pm 12.44 \mathrm{~kg}$ body mass; $1.63 \pm 0.08 \mathrm{~m}$ height) were recruited to participate voluntarily in this study. The study included individuals who practice water-aerobics, with 18 years-old or more, and who were present on the days determined for the questionnaire.

\section{Measures}

It was applied a questionnaire composed by 20 questions, divided in two parts. In the first part, data were collected about personal characterization, namely the year of birth, gender, weight, height, occupation, area of residence and academic qualifications. In the second part were placed questions about the knowledge and use of wearable technology during exercise, specifically in water-aerobics.

\section{Procedures}

An anonymous questionnaire was applied at each place for a period of several days ( 5 days) in order to include as many individuals practicing water-aerobics as long as they met the inclusion criteria. The questionnaires were presented to all individuals entering the swimming pools and that agreed to participate in the study, together with another sheet describing the study, also containing the declaration of confidentiality of personal data.

\section{Analysis}

We used the Microsoft Office Excel 16 program and the Statistical Package of Social Science (SPSS) 22.0, both for Windows. The calculation of means, standard deviations were performed by standardized statistical methods, as well as graphic representations for better interpretation of the obtained results.

\section{RESULTS}

When subjects were asked about the knowledge of wearables, approximately $60 \%$ have already heard about this technology, with only $12 \%$ that have experimented this technology during physical exercise, and of those, only $29 \%$ used it while performing water-aerobics. Of the subjects that never used wearable technology, the main reasons were the lack of opportunity $(62 \%)$, the uselessness of the equipment $(15 \%)$ and the difficulty to perceive its functioning (9\%). Despite this, the large majority of the subjects showed interested in using wearables during the performance of water-aerobics, if they had this possibility $(79 \%)$, provided the 
technology allowed to measure parameters such as heart rate $(34 \%)$, calories spent $(31 \%)$, oxygen consumption $(10 \%)$, exercise time $(9 \%)$, travelled distance $(7 \%)$, displacement speed $(5 \%)$ and technical corrections (4\%). Finally, to complement the results, it was observed that more than half of the respondents $(52 \%)$ are retired.

\section{DISCUSSION}

The results show that most individuals know of the existence of wearable technology, however, are few those who have experienced when performing water-aerobics. This may be due to the fact that water-aerobics practitioners are mostly retired, thus having a more advanced age (Lopes, Streit, Medeiros and Mazo, 2014), which can lead to a lack of knowledge about the functioning of the technology, as well as its usefulness for exercise monitoring. Despite the lack of knowledge, individuals put the hypothesis to start using this type of equipment, fundamentally to measure heart rate and calories spent.

\section{CONCLUSIONS}

In general, the Portuguese have little knowledge and little use of wearable technology in water-aerobics. However, there is some interest in starting to use the technology, especially for monitoring heart rate and calories spent. Therefore, it is necessary to make known and to improve the technology according to the modality and to the preferences of its users.

\section{ACKNOWLEDGEMENT}

This work is supported by national funding through the Portuguese Foundation for Science and Technology, I.P., under project UID/DTP/04045/2019.

\section{REFERENCES}

Antunes, A.H., Alberton, C.L., Finatto, P., Pinto, S.S., Cadore, E.L., Zaffari, P. \& Kruel, L.F. (2015). Active female maximal and anaerobic threshold cardiorespiratory responses to six different water aerobics exercises. Res Q Exerc Sport, 86(3), 267-273. https://doi.org/10.1080/02701367.2015.1012577

Benelli, P., Ditroilo, M. \& De Vito, G. (2004). Physiological responses to fitness activities: a comparison between land-based and water aerobics exercise. J Strength Cond Res, 18(4), 719-722. https://doi.org/10.1519/00124278-200411000-00006

Green, S. (1989). Effects of a Water Aerobics Program on the Blood Pressure, Percentage of Body Fat, Weight, and Resting Pulse Rate of Senior Citizens. J Appl Gerontol, 8(1), 132-138. https://doi.org/10.1177/073346488900800110

Lopes, A.L., Streit, I.A., Medeiros, P.A., \& Mazo, G.Z. (2014). Motivos de ingresso e permanência de idosos em um programa de atividades aquáticas: um estudo longitudinal. J Phys Educ, 25(1), 2332. https://doi.org/10.4025/reveducfis.v25i1.20371.

Raffaelli, C., Galvani, C., Lanza, M., \& Zamparo, P. (2011). Different methods for monitoring intensity during water-based aerobic exercises. Eur J Appl Physiol, 112(1), 125-134. https://doi.org/10.1007/s00421-011-1963-7

\section{@) $(\Theta \Theta \Theta$}

This work is licensed under a Attribution-NonCommercial-NoDerivatives 4.0 International (CC BY-NC-ND 4.0). 


\title{
Influence of the full squat on short sprint performance in young adults
}

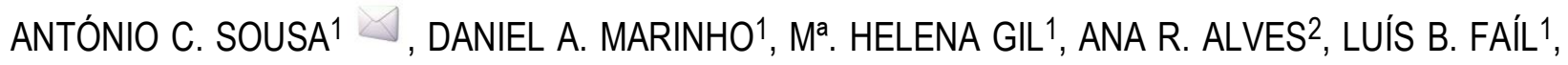 \\ PEDRO P. NEVES ${ }^{1}$, HENRIQUE P. NEIVA ${ }^{1}$, MÁRIO C. MARQUES ${ }^{1}$ \\ ${ }^{1}$ CIDESD, University of Beira Interior, Covilhã, Portugal \\ ${ }^{2}$ CIDESD, Polytechnic Institute of Beja, Beja, Portugal
}

\begin{abstract}
The aim of this study was to investigate the influence of the full squat exercise on sprint performance. Two experimental groups were implemented: one group performed a combined sprint and strength (squat) training protocol, and the other group performed sprint training protocol only. The sample compromised 24 subjects, with ages between $20 \pm 1$ years, $170 \pm 8 \mathrm{~cm}$ body mass, $63 \pm 7 \mathrm{~kg}$ weight. To collect data, a full squat position linear meter (T-Force System) and a Browner instrument (Sprint Wireless System) were used. The results of this study indicated that a 6 -week training period appears to be sufficient to cause significant strength gains. Moreover, a sprint program which include a faster full squat exercise provides higher gains in short sprint performance than when this ability is trained in isolation. In short, these results suggest that full squat exercise can be effective in order improve sprint performance. Keywords: Strength; High velocity; Sprint Capacity.
\end{abstract}

\footnotetext{
Corresponding author. Department of Sports Sciences, University of Beira Interior (Covilhã), Portugal; Convento de Santo António, 6201 - 001 (Covilhã) Portugal.

E-mail: antonio carlossousa@hotmail.com

Supplementary Issue: Spring Conferences of Sports Science. International Seminar of Physical Education, Leisure and Health, 17-19 June 2019. Castelo Branco, Portugal.

JOURNAL OF HUMAN SPORT \& EXERCISE ISSN 1988-5202

(c) Faculty of Education. University of Alicante.

doi:10.14198/jhse.2019.14.Proc4.82
} 


\section{INTRODUCTION}

In order to development lower body muscle strength and power, the squat exercise is one of the most implemented exercises. In addition, it is an exercise that has been related to the sprint performance enhancement (González-Badillo, 2000). In most studies a half or a parallel squat movement for training intervention have been implemented, and few studies also examine the training outcomes using a full squat (FS) movement (Marques et al., 2015). Some authors have observed a significant relationship between squat and short sprints (Wisloff et al., 2004). However, there is a lack of studies related to both exercises using a FS exercise. Therefore, aim of this study was to determine the influence of the high velocity FS exercise on sprint performance.

\section{MATERIAL AND METHODS}

\section{Participants}

Twenty-four male active subjects ( $20 \pm 1$ years; body mass: $63 \pm 7 \mathrm{~kg}$; height: $170 \pm 8 \mathrm{~cm}$ ) were divided into 2 groups: a combined sprint and strength (squat) training protocol $(n=12)$; and a sprint training protocol only $(n=12)$. At baseline, no differences between groups were observed in anthropometric characteristics, strength, power and sprint performances.

\section{Procedures}

Neuromuscular performance was assessed before and after a 6-week training period using a battery of tests performed in 2 sessions separated by a 48 -hour rest interval.

\section{Sprint performance}

Each participant performed three 30-m sprints separated by a 3-minute rest. Photocell timing gates (Brower Timing System, Fairlee, VT, USA) were placed at 0, 10, and 20-m, and the times needed to cover 0-10 m (T10), 0-20 m (T20) and 0-30 m (T30) could be determined. A standing start with the lead-off foot placed 1 $\mathrm{m}$ behind the first timing gate was used. The average of the best 3 sprints for the analysis was used. Warmup consisted of 5 minutes of running at a self-selected intensity, 5 minutes of joint mobilization exercises, followed by several sets of faster progressively $30-\mathrm{m}$ running accelerations.

Isoinertial Squat Loading Test

A smith machine (Multipower Fitness Line; Peroga, Murcia, Spain) was used. The initial load was set at 17 $\mathrm{kg}$ and progressively increased in $10 \mathrm{~kg}$ increments until the attained propulsive mean velocity (PMV) was $\sim 1.00$ m.s-1 The participants performed 3 repetitions with each load, with 3-min recovery. A linear velocity transducer (T-Force System; Ergotech, Murcia, Spain) to register bar velocity was used.

\section{Analysis}

The normality and homogeneity of variance were assessed by the Shapiro-Wilk. The descriptive statistics (mean \pm SD) for the different variables was calculated. To evaluate the differences between each evaluation moments, the independent measures $t$-test was used. To evaluate the performance changes within training groups, a t-test with repeated measures for each exercise was performed. The level of significance was set at $p \leq 0.05$. 


\section{RESULTS}

Table 1 shows the mean, standard deviation and significance values of the sprint times obtained in the 10 , 20 and $30-\mathrm{m}$, and also the PMV, propulsive mean power (PMP) and 1RM values in FS groups 1 and 2, during the pre- and post-test.

Table 1. Mean, standard deviation and significance values of the sprint times obtained in the 10, 20 and $30 \mathrm{~m}$ also the PMV, PMP and 1RM values in FS groups 1 and 2, during the pre- and post-test

\begin{tabular}{clccc} 
& & Group 1 (Sprint Only) & Group 2 (Combined) & p (inter-group) \\
\hline \multirow{3}{*}{ Sprint 10m (s) } & Pré-Test & $1.93 \pm 0.18$ & $1.93 \pm 0.16$ & $0.944 \mathrm{~ns}$ \\
& Post-Test & $1.89 \pm 0.18$ & $1.86 \pm 0.16$ & $0.625 \mathrm{~ns}$ \\
& p (intra-group) & $0.300 \mathrm{~ns}$ & $0.002^{* *}$ & \\
& Pré-Test & $3.34 \pm 0.32$ & $3.33 \pm 0.32$ & $0.940 \mathrm{~ns}$ \\
Sprint 20m (s) & Post-Test & $3.27 \pm 0.36$ & $3.22 \pm 0.29$ & $0.747 \mathrm{~ns}$ \\
& p (intra-group) & $0.175 \mathrm{~ns}$ & $0.000^{* *}$ & \\
& Pré-Test & $4.66 \pm 0.46$ & $4.67 \pm 0.48$ & $0.928 \mathrm{~ns}$ \\
Sprint 30m (s) & Post-Test & $4.60 \pm 0.54$ & $4.54 \pm 0.43$ & $0.751 \mathrm{~ns}$ \\
& p (intra-group) & $0.171 \mathrm{~ns}$ & $0.000^{* *}$ & \\
& Pré-Test & $1.14 \pm 0.30$ & $1.15 \pm 0.25$ & $9.953 \mathrm{~ns}$ \\
MPV (m/s) & Post-Test & $1.27 \pm 0.22$ & $1.32 \pm 0.22$ & $0.571 \mathrm{~ns}$ \\
& p (intra-group) & $0.062 \mathrm{~ns}$ & $0.002^{* *}$ & \\
& Pré-Test & $247.65 \pm 78.32$ & $248.26 \pm 69.17$ & $0.984 \mathrm{~ns}$ \\
PMP (W) & Post-Test & $286.20 \pm 69.39$ & $297.17 \pm 70.74$ & $0.706 \mathrm{~ns}$ \\
& p (intra-group) & $0,023^{*}$ & $0.002^{* *}$ & \\
& Pré-Test & $60.42 \pm 27.65$ & $63.33 \pm 24.37$ & $0.787 \mathrm{~ns}$ \\
1RM (Kg) & Post-Test & $63.17 \pm 26.68$ & $74.42 \pm 28.13$ & $0.326 \mathrm{~ns}$ \\
& p (intra-group) & $0.235 \mathrm{~ns}$ & $0.003^{* *}$ & \\
\hline
\end{tabular}

PMV: propulsive mean velocity; PMP: propulsive mean power; significance: * $p \llbracket 0,05$ and ** $\square \square 0,01$; ns: not significant.

\section{DISCUSSION}

The present data suggest that the inclusion of the FS exercise can be an important strategy in order to improve short sprint performance. In the present study, the results further indicated that a reduced strength training period (i.e. 6-weeks), appears to be sufficient to cause significant gains in sprint capacity.

Moreover, it was also found that only the combined group presented significant improvements from the preto the post-test for distances of 10,20 and 30-m sprint. The results may be associated with the potentiation caused by the FS exercise. According to McBride et al. (2005), performing muscle contractions under near maximal load conditions can produce subsequent improvements in performance, namely in movements that require large muscle power of the stimulated muscle groups. Still, through the obtained results, it can easily verify that both groups improved their pre-test results in the FS exercise. However, the sprint group only achieved significant improvements in the PMP ( $p=0.023)$, while the combined group obtained significant improvements in all measured force parameters. Such differences are probably related to the complexity of the motor task required by the squat. Exercises involving a larger number of muscle groups require a longer period of time to establish a balance in neural adjustments and in learning the motor task of movement. 


\section{CONCLUSIONS}

The inclusion of the FS exercise in a sprint program provides higher gains in short sprint ability than when it is trained in isolation. In short, based on the current results, it is suggested that strength training (based on exercises such as FS) can be determinant for developing short sprint ability.

\section{REFERENCES}

González-Badillo, J. (2000). Concepto y medida de la fuerza explosiva en el deporte. Posibles aplicaciones al entrenamiento. Rev Entren Deport, 14(1), 5-15.

Marques, M.C., Gabbett, T.J., Marinho, D.A., Blazevich, A.J., Sousa, A., van den Tillaar, R., and Izquierdo, M. (2015). Influence of Strength, Sprint Running, and Combined Strength and Sprint Running Training on Short Sprint Performance in Young Adults. Int J Sports Med, 36(10), 789-795. https://doi.org/10.1055/s-0035-1547284

McBride, J.M., Nimphius, S., and Erickson, T.M. (2005). The acute effects of heavy-load squats and loaded countermovement jumps on sprint performance. J Strength Cond Res, 19(4), 893-897. https://doi.org/10.1519/r-16304.1

Wisloff, U., Castagna, C., Helgerud, J., Jones, R., and Hoff, J. (2004). Strong correlation of maximal squat strength with sprint performance and vertical jump height in elite soccer players. Br J Sports Med, 38(3), 285-288. https://doi.org/10.1136/bjsm.2002.002071

\section{@) $\Theta \Theta \Theta$}

This work is licensed under a Attribution-NonCommercial-NoDerivatives 4.0 International (CC BY-NC-ND 4.0). 


\title{
Effect of previous ankle sprain in stride variables during basketball-specific drill: Insights about maturity offset
}

\author{
JORGE AREDE ${ }^{1} \triangle$, PEDRO FORTE ${ }^{1,2}$, KATHRIN REHFELD $^{3}$, DANGUOLE SATKUNSKIENE ${ }^{4}$, NUNO \\ LEITE1 \\ ${ }^{1}$ Research Center in Sports Sciences, Health Sciences and Human Development, CIDESD, University of \\ Trás-os-Montes and Alto Douro, Portugal \\ 2Higher Institute of Educational Sciences of the Douro, ISCE Douro, Penafiel, Portugal \\ 3 Institute for Sport Science, Otto-von-Guericke University, Magdeburg, Saxony-Anhalt, Germany \\ ${ }^{4}$ Lithuanian Sports University, Kaunas, Lithuania
}

\begin{abstract}
The aim of this study was to examine the effect of previous ankle sprain in stride variables during basketballspecific drills and analyse of mediator effect of maturity offset in these analyses. Ten young under-16 (U-16) male basketball players performed $5 \times 5$ half court situations ( 3 sets of 8 minutes, with 5 minutes of rest between sets) using the WIMU PRO Local Positioning System to assess steps kinetics and kinematics. No significant differences were founded between injured and non-injured young basketball players. The injured athletes shown higher vertical stiffness (Kvert) in strong leg than weaker leg $(p<0.05)$. A significant effect of the $\mathrm{MO}$ in the average acceleration (Acc) in both legs and in $\mathrm{K}_{\text {vert }}$ of weak leg was found. The previous ankle injury is associated with load attenuation strategy. Between-limbs differences in Kvert and biological maturation should be considered in rehabilitation process. Keywords: Ankle instability; Sports injury; Biomechanics; Ankle injuries; Gait; Biological maturation.
\end{abstract}

\footnotetext{
Corresponding author. Research Center in Sports Sciences, Health Sciences and Human Development, CIDESD, University of Trás-os-Montes and Alto Douro, Quinta de Prados, Apartado 202, 5001-911 Vila Real, Portugal.

E-mail: jorge arede@hotmail.com

Supplementary Issue: Spring Conferences of Sports Science. International Seminar of Physical Education, Leisure and Health, 17-19 June 2019. Castelo Branco, Portugal.

JOURNAL OF HUMAN SPORT \& EXERCISE ISSN 1988-5202

(c) Faculty of Education. University of Alicante.

doi:10.14198/jhse.2019.14.Proc4.82
} 


\section{INTRODUCTION}

The ankle sprain is a common injury in basketball and its effects include training and competition absence at short-term, incomplete recovery at medium-term, and ultimately, the development of osteoarthritis in the long term (Maffulli, Longo, \& Gougoulias, 2011). Both incomplete sport injuries rehabilitation and maturation are associated with body dysfunctions (Read, Oliver, De Ste Croix, Myer, \& Lloyd, 2016). These aspects may prevent that youth athletes can foster their talent. Microsensors has been widely used in team sports, their use in sports rehabilitation and return to play filed has have been scarcely explored (Buchheit, Gray, \& Morin, 2015). The aim of this study was to examine the effect of previous ankle sprain in stride variables during basketball-specific drill and analyse of mediator effect of maturity offset in these analyses.

\section{MATERIAL AND METHODS}

\section{Participants}

Ten young U-16 male basketball players were selected to participate in this study.

\section{Measures}

Previous ankle injuries were self-reported by the basketball players. The Cumberland Ankle Instability Tool (CAIT) was used to assess the to assess feelings of instability of both lower limbs (Noronha, Refshauge, Kilbreath, \& Figueiredo, 2008). Maturity offset (MO) was estimated according to non-invasive method (Mirwald, Baxter-Jones, Bailey, \& Beunen, 2002). The steps kinetics and kinematics during basketball-specific drill were assessed using the WIMU PRO Local Positioning System (Realtrack Systems, Almeria, Spain).

\section{Procedures}

The subjects performed $5 \times 5$ half court situations ( 3 sets of 8 minutes, with 5 minutes of rest between sets). The players were allocated in which team according previous ankle sprain status (injured, $n=5$; non-injured, $\mathrm{n}=5$ ). The contact time (CT) in milliseconds (ms), maximal (MaxAcc) and average acceleration (Acc) in $\mathrm{G}$ force, and vertical stiffness ( $\mathrm{K}_{\text {vert; }} \mathrm{N} / \mathrm{m}$ ) were collected for each leg (weak and strong) using the WIMU PRO. Each leg was categorized according to the score obtained in CAIT. In all cases, the weak leg was the previous injury leg. All data was computed using SPRO Software v1.0.0.

\section{Analysis}

The Wilcoxon signed rank test and non-parametric paired samples t-test was performed to assess the differences between strong and weak ankle for previous injured and non-injured players. Analysis of covariance (ANCOVA) with MO as the covariate was to evaluate differences between groups. The level of statistical significance was set at 5\%. All analyses were performed using SPSS (SPSS, Inc., Version 24.0, Chicago, IL).

\section{RESULTS}

No significant differences were founded between injured and non-injured young basketball players in step kinetics and kinematics in both legs (Table 1). The injured athletes shown higher $\mathrm{K}_{\text {vert }}$ in strong leg than weaker leg $(p<0.05)$. The ANCOVA revealed a significant effect of the MO in the Acc in both legs and in Kvert of weak leg (Table 1). The estimated marginal means shown that previous injured players presented higher Acc than non-injured in strong leg $(0.210>0.198 \mathrm{G})$ and in weak leg $(0.213>0.204 \mathrm{G})$, but also higher $\mathrm{K}_{\text {vert }}$ in weak leg $(36.17>34.51 \mathrm{~N} / \mathrm{m})$. 
Table 1. Between-group comparisons in the stride variables in both legs. Analysis of Covariance (ANCOVA) to examine the effect of MO in the steps kinetics and kinematics

\begin{tabular}{|c|c|c|c|c|c|c|}
\hline \multirow{2}{*}{\multicolumn{2}{|c|}{ Variables Comparisons }} & \multirow{2}{*}{ Mean ( \pm SD) Injured } & \multirow{2}{*}{$\begin{array}{l}\text { Mean ( } \pm \text { SD) Non- } \\
\text { Injured }\end{array}$} & \multicolumn{2}{|c|}{ Wilcoxon } & \multirow{2}{*}{$\frac{\text { ANCOVA }}{\text { MO }}$} \\
\hline & & & & U & $p$ & \\
\hline \multirow{4}{*}{ 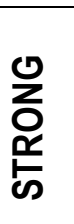 } & $\mathrm{CT}(\mathrm{ms})$ & $270.15( \pm 7.94)$ & $276.40( \pm 5.86)$ & 19.000 & 0.222 & 0.209 \\
\hline & $\operatorname{Max} \operatorname{Acc}(G)$ & $8.80( \pm 1.58)$ & $8.70( \pm 0.76)$ & 14.000 & 0.841 & 0.169 \\
\hline & $\operatorname{Acc}(G)$ & $0.21( \pm 0.03)$ & $0.19( \pm 0.03)$ & 8.000 & 0.421 & 0.000 \\
\hline & $K_{\text {vert }}(\mathrm{N} / \mathrm{m})$ & $38.43( \pm 4.83)^{*}$ & $34.40( \pm 4.82)$ & 7.000 & 0.309 & 0.182 \\
\hline \multirow{4}{*}{ 㭊 } & $\mathrm{CT}(\mathrm{ms})$ & $271.62( \pm 3.67)$ & $274.30( \pm 3.88)$ & 18.000 & 0.309 & 0.123 \\
\hline & $\operatorname{Max} \operatorname{Acc}(G)$ & $8.71( \pm 1.66)$ & $9.31( \pm 1.36)$ & 15.000 & 0.691 & 0.560 \\
\hline & $\operatorname{Acc}(G)$ & $0.22( \pm 0.04)$ & $0.19( \pm 0.03)$ & 9.500 & 0.548 & 0.000 \\
\hline & $K_{\text {vert }}(\mathrm{N} / \mathrm{m})$ & $36.62( \pm 4.95)$ & $34.06( \pm 4.29)$ & 11.000 & 0.841 & 0.027 \\
\hline
\end{tabular}

${ }^{*}$ Significant difference $(p<0.05)$ between Strong and Weak Leg. Note: The MO considered to perform estimated marginal means was 1.3.

\section{DISCUSSION}

We found that movement techniques are associated with previous injury status, but also that biological maturation can underpin between-groups differences. The injured athletes adopted the movement style with higher step frequency (expressed by lower CT), but also higher Kvert comparing their counterparts. Thus, the injured athletes may performed a load attenuation strategy, which includes higher dynamic stability, lower vertical oscillations of the centre of mass and greater muscle pre-activation, and consequently less stress in passive structures and lower likelihood of injury (Girard, Brocherie, Morin, \& Millet, 2017). Considering the ANCOVA results, this strategy may be underpin by more efficient neuromuscular feed-forward strategy prior to foot contact associated to greater biological maturation (Read et al., 2016). However, the Kvert was significantly lower in weak leg in injured athletes. This could be indicative of decreased muscle pre-activation generated by the inability to control the muscles around ankle joint, due to previous injury.

\section{CONCLUSIONS}

The previous ankle injury is associated with altered lower extremity biomechanics, in weaker leg, comparing with stronger leg. The practitioners should consider this information to develop training program to increase

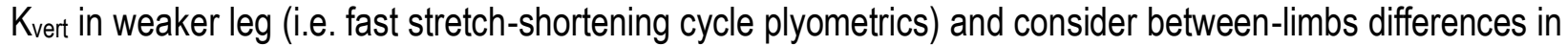
return to train or play protocols. Also, biological maturation should be taken in account as confound variable in both processes.

\section{ACKKNOWLEGEMENTS}

This work was supported by the Foundation for Science and Technology (FCT, Portugal), through a Doctoral grant endorsed to the first author [SFRH/BD/122259/2016] and under project UID/DTP/04045/2019.

\section{REFERENCES}

Buchheit, M., Gray, A., \& Morin, J. (2015). Assessing Stride Variables and Vertical Stiffness with GPSEmbedded Accelerometers : Preliminary Insights for the Monitoring of Neuromuscular Fatigue on the Field. Journal of Sports Science and Medicine, (14), 698-701. 
Girard, O., Brocherie, F., Morin, J., \& Millet, G. P. (2017). Lower limb mechanical asymmetry during repeated treadmill sprints. Human Movement Science, 52(February), 203-214. https://doi.org/10.1016/i.humov.2017.02.008

Maffulli, N., Longo, U. G., \& Gougoulias, N. (2011). Sport injuries : a review of outcomes. British Medical Bulletin, 97, 47-80. https://doi.org/10.1093/bmb/ldq026

Mirwald, R. L., Baxter-Jones, A. D. G., Bailey, D. A., \& Beunen, G. P. (2002). An assessment of maturity from anthropometric measurements. Medicine and Science in Sports and Exercise, 34(4), 689-694. https://doi.org/10.1097/00005768-200204000-00020

Noronha, M. D. E., Refshauge, K. M., Kilbreath, S. L., \& Figueiredo, V. G. (2008). Cross-cultural adaptation of the Brazilian-Portuguese version of the Cumberland Ankle Instability Tool ( CAIT ). Disability and Rehabilitation, 30(26), 1959-1965. https://doi.org/10.1080/09638280701809872

Read, P. J., Oliver, J. L., De Ste Croix, M. B. A., Myer, G. D., \& Lloyd, R. S. (2016). Neuromuscular Risk Factors for Knee and Ankle Ligament Injuries in Male Youth Soccer Players. Sports Medicine. https://doi.org/10.1007/s40279-016-0479-z 


\title{
Is low volume HIIT enough to induce changes in oxygen uptake kinetics?
}

\author{
DALIA CURTO ${ }^{1}$, RITA PINTO $^{1,2}$, MÁRIO ESPADA ${ }^{3}$, JORGE INFANTE ${ }^{1}$, FRANCISCO B. ALVES ${ }^{1,2}$, JOANA \\ F. REIS $1,2,4$
}

${ }^{1}$ Faculdade de Motricidade Humana, Universidade de Lisboa, Cruz Quebrada, Portugal

2 Interdisciplinary Center for the Study of Human Performance (CIPER), Faculdade de Motricidade Humana, Universidade de Lisboa, Cruz Quebrada, Portugal

${ }^{3}$ Escola Superior de Educação, Instituto Superior Politécnico de Setúbal, Setúbal, Portugal

${ }^{4}$ Universidade Europeia, Lisbon, Portugal

\begin{abstract}
The literature shows that endurance training induces a speeding of the initial oxygen uptake kinetics $\left(\mathrm{VO}_{2 \mathrm{k}}\right)$ and maximal oxygen consumption $\left(\mathrm{VO}_{2 \mathrm{max}}\right)$. However, the specific characteristics of training that optimizes said parameters is not yet clearly established. We aimed to investigate the effects of low volume high intensity interval training (HIIT) on $\mathrm{VO}_{2 \max }$ and $\mathrm{VO}_{2 \mathrm{k}}$ during intensity transitions within the moderate domain. Twenty healthy active subjects were randomly assigned to either complete 12 sessions of low volume HIIT over a 4wk period (HIIT group) or a control group (CON) that did not change their regular physical activity. Before (PRE) and after (POST) the intervention period, subjects completed on the treadmill an incremental test and square wave transitions to moderate intensity exercise. In all tests, pulmonary $\mathrm{VO}_{2}$ was collected breath-bybreath (Metamax3b, Cortex, Germany). The parameters of the $\mathrm{VO}_{2 k}$ for the moderate exercise were determined using a monoexponential model. All the physiological variables did not change from PRE to POST in the $\mathrm{CON}$ group. However, HIIT training improved the $\mathrm{VO}_{2 \max }$ and maximal aerobic velocity (MAV), whereas there were no significant changes in the $\mathrm{VO}_{2 k}$. Four weeks of low volume HIIT was not sufficient to induce changes on $\mathrm{VO}_{2 \mathrm{k}}$ in moderate running. Nevertheless, this training provoked improvements in $\mathrm{VO}_{2 \text { peak }}$ and MAV, consequently low volume HIIT seems to be a time-efficient approach to increase these parameters. The dissociation between changes in $\mathrm{VO}_{2 \mathrm{k}}$ and $\mathrm{VO}_{2 \max }$ seems to reflect the different underlying mechanisms regulating these adaptations. Keywords: $\mathrm{VO}_{2}$ kinetics; Interval training; Endurance, $\mathrm{VO}_{2 \max }$.
\end{abstract}

Corresponding author. Universidade Europeia 1500-210, Lisbon, Portugal.

E-mail: joana.reis@universidadeeuropeia.pt

Supplementary Issue: Spring Conferences of Sports Science. International Seminar of Physical Education, Leisure and Health, 17-19 June 2019. Castelo Branco, Portugal.

JOURNAL OF HUMAN SPORT \& EXERCISE ISSN 1988-5202

(c) Faculty of Education. University of Alicante.

doi:10.14198/jhse.2019.14.Proc4.82 


\section{INTRODUCTION}

For the same increment in work rate, individuals with slow overall oxygen uptake kinetics $\left(\mathrm{VO}_{2 k}\right)$ will rely on a greater extent on non-oxidative metabolic pathways to meet the energy demands of the exercise task, reaching early fatigue (Poole \& Jones, 2012). The literature shows that endurance training induces a speeding of the initial $\mathrm{VO}_{2 \mathrm{k}}$ and is also commonly associated with increased maximal oxygen uptake $\left(\mathrm{VO}_{2 \max }\right)$ in healthy subjects (Daussin et al., 2008; Montero, Diaz-Canestro, \& Lundby, 2015). However, there is evidence that adaptations to these two variables can be dissociated, since in healthy individuals, the $\mathrm{VO}_{2 \max }$ seems to be mainly determined by the cardiovascular capacity of $\mathrm{O} 2$ delivery, whereas the $\mathrm{VO}_{2 \mathrm{k}}$ is more dependent on processes at the site of the muscle (Poole \& Jones, 2012). On the other hand, the specific characteristics of training that optimizes said parameters is not yet clearly established, but low volume high intensity interval training (HIIT) has been recently pointed as an effective strategy to induce improvements in these parameters. Thus, the purpose of the present study was to investigate the effects of HIIT low volume on $\mathrm{VO}_{2 \max }$ and $\mathrm{VO}_{2 \mathrm{k}}$ during transitions to the moderate intensity domain.

\section{MATERIAL AND METHODS}

All tests were performed in a motorized treadmill (HP Cosmos Pulsar, Germany) with pulmonary oxygen uptake collected breath-by-breath (Metamax3b, Cortex, Germany). The participants were instructed to report to the laboratory with no alcohol or stimulant beverage consumption, or exercise participation taking place within the last 24 hours.

\section{Participants}

Twenty healthy active subjects, with no endurance training background were randomly assigned to a HIIT $(n=13 ; 25.1 \pm 5.8 \mathrm{yrs} ; 1.70 \pm 0.08 \mathrm{~m} ; 65.1 \pm 7.9 \mathrm{~kg})$ or a control group (CON) $(n=7 ; 27.8 \pm 6.6 \mathrm{yrs} . ; 1.68 \pm 0.07 \mathrm{~m}$; $60.5 \pm 8.8 \mathrm{~kg}$ ). The study was approved by the local ethic committee and all participants provided their written informed consent to participate.

\section{Measures}

Incremental test

The participants performed an incremental test until exhaustion to determine the $\mathrm{VO}_{2 \max }$ and the ventilatory threshold (VT). The test started at $6 \mathrm{kmh}-1$ and an increase of $1 \mathrm{kmh}-1$ per minute was imposed until exhaustion. $\mathrm{VO}_{2 \max }$ was recorded as the highest 30 s average of $\mathrm{VO}_{2}$ values and the maximal aerobic velocity (MAV) as the minimal velocity that elicit $\mathrm{VO}_{2 \text { max. }}$.

$\mathrm{VO}_{2 k}$

All subjects performed four square wave transitions from rest to $80 \%$ of $\mathrm{VT}$ in two separate days. The $\mathrm{VO}_{2}$ data was then time aligned and ensembled averaged to obtain a representative time series for each moment and the $\mathrm{VO}_{2 \mathrm{k}}$ parameters were determined using a monoexponential model, with an amplitude, a time delay and a time constant (Reis, Millet, Bruno, Vleck, \& Alves, 2017).

\section{Procedures}

Study Design

The HIIT group performed 12 sessions of $6-8 \times 30$ s running at $120-2 \%$ of MAV, with interval rest of 30 s over a 4-wk period. The CON group was instructed to maintain their regular physical activity. Before (PRE) and after (POST) the intervention period, subjects completed all the evaluations described in the previous section. 


\section{Statistical Analysis}

Paired t-tests were used for exploring possible differences in the physiological variables from PRE- to POST, within each group. Significance was set at $p<0.05$.

\section{RESULTS}

In the CON there were no significant differences between the PRE and POST moments in any of the variables. The HIIT training resulted in significant increases $(p<0.05)$ in $\mathrm{VO}_{2 \max }$ and MAV. Regarding $\mathrm{VO}_{2 k}$ there were no significant changes in any of its parameters in both the CON and HIIT training groups.

Table 1. Mean \pm Standard deviation of the physiological parameters obtained in the incremental and square wave transitions for the HIIT and CON groups for PRE and POST

\begin{tabular}{lcccc}
\hline & HIIT Pre & HIIT Post & CON Pre & CON Post \\
\hline $\mathrm{VO}_{2} \mathrm{max}(\mathrm{ml} / \mathrm{kg} / \mathrm{min})$ & $49.8 \pm 6.8$ & $54,7 \pm 5.5^{*}$ & $50.0 \pm 8.3$ & $50.14 \pm 7.90$ \\
$\mathrm{MAV}(\mathrm{km} / \mathrm{h})$ & $14.3 \pm 1.2$ & $15.0 \pm 1.3^{*}$ & $14.3 \pm 2.6$ & $14.29 \pm 2.56$ \\
$\mathrm{Amp}(\mathrm{ml} / \mathrm{kg} / \mathrm{min})$ & $25.6 \pm 3.2$ & $28.0 \pm 3.8$ & $26.6 \pm 4.00$ & $25.74 \pm 4.66$ \\
td $(\mathrm{s})$ & $6.3 \pm 5.0$ & $5.6 \pm 5.3$ & $3.3 \pm 4.5$ & $5.29 \pm 5.21$ \\
$\tau(\mathrm{s})$ & $29.3 \pm 7.2$ & $26.8 \pm 7.3$ & $29.2 \pm 10.3$ & $30.2 \pm 9.5$ \\
$\mathrm{VO}_{2}$ end $(\mathrm{ml} / \mathrm{kg} / \mathrm{min})$ & $31.5 \pm 3.4$ & $33.0 \pm 4.4$ & $31.9 \pm 5.0$ & $31.1 \pm 4.7$ \\
\hline
\end{tabular}

$\mathrm{VO}_{2 \text { max: }}$ maximal oxygen consumption; MAV: maximal aerobic velocity; amp, $t d$ and $r$ : amplitude, time delay and time constant for the primary phase $\mathrm{VO}_{2}$ response; $\mathrm{VO}_{2 \text { end: }}$ steady state oxygen uptake for the moderate intensity exercise. ${ }^{*}$ significantly different from $P R E, p<0.05$.

\section{DISCUSSION}

The main finding of the present investigation was that HIIT training induced improvements in $\mathrm{VO}_{2 m a x}$, whilst the $\mathrm{VO}_{2 \mathrm{k}}$ in moderate exercise remained unaltered. This is not in agreement with the literature, where improvements in $\mathrm{VO}_{2 \mathrm{k}}$ are reported after interventions of this type of training (Daussin et al., 2008). However, most of the training protocols comprised higher training volumes. Thus, it is hypothesized that the training volume performed in the present investigation was not enough to induce peripheral adaptations that could affect $\mathrm{VO}_{2 k}$, namely mitochondrial biogenesis, or the activity of mitochondrial oxidative enzymes (Holloszy \& Coyle, 1984). Conversely, HIIT training was able to induce significant increases in $\mathrm{VO}_{2 \max }$ and MAV, the latter being extensively considered as good performance predictor (Kilding, Winter, \& Fysh, 2006). Moreover, our results seem to confirm that $\mathrm{VO}_{2 \mathrm{~K}}$ and $\mathrm{VO}_{2 \max }$ are determined by different physiological mechanisms. Though, considering these results, low volume HIIT training seems to be an effective low time-consuming strategy to improve $\mathrm{VO}_{2 m a x}$ in untrained young participants.

\section{CONCLUSIONS}

The results of the present investigation demonstrated that four weeks of low volume HIIT training were not enough to change the velocity of $\mathrm{VO}_{2 \mathrm{k}}$. However, this training was able to induce significant improvements in

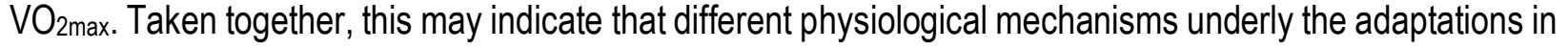
these two physiological parameters. 


\section{REFERENCES}

Daussin, F. N., Zoll, J., Dufour, S. P., Ponsot, E., Lonsdorfer-Wolf, E., Doutreleau, S., ... Richard, R. (2008). Effect of interval versus continuous training on cardiorespiratory and mitochondrial functions: relationship to aerobic performance improvements in sedentary subjects. Am J Physiol. Regul, Integr Comp Physiol, 295(1), R264-72. https://doi.org/10.1152/aipregu.00875.2007

Holloszy, J. O., \& Coyle, E. F. (1984). Adaptations of skeletal muscle to endurance exercise and their metabolic consequences. J Appl Physiol: Resp, Environ Exerc Physiol, 56(4), 831-838. https://doi.org/10.1152/jappl.1984.56.4.831

Kilding, A, E., Winter, E. M., \& Fysh, M. (2006). Moderate-domain pulmonary oxygen uptake kinetics and endurance running performance. J Sports Sci, 24(9), 1013-22. https://doi.org/10.1080/02640410500457208

Montero, D., Diaz-Canestro, C., \& Lundby, C. (2015). Endurance Training and VO2max: Role of Maximal Cardiac Output and Oxygen Extraction. Med Sci Sports Exerc, 47(10), 2024-2033. https://doi.org/10.1249/mss.0000000000000640

Poole, D. C., \& Jones, A. M. (2012). Oxygen uptake kinetics. Comprehensive Physiology, 2(2), 933-996.

Reis, J. F., Millet, G. P., Bruno, P. M., Vleck, V., \& Alves, F. B. (2017). Sex and exercise intensity do not influence oxygen uptake kinetics in submaximal swimming. Front Physiol, 8:72. https://doi.org/10.3389/fphys.2017.00072

\section{(9) $(\mathbb{0} \Theta \Theta$}

This work is licensed under a Attribution-NonCommercial-NoDerivatives 4.0 International (CC BY-NC-ND 4.0). 


\title{
Warm-up kinematics influence in $30 \mathrm{~m}$ sprint performance
}

\author{
MåHELENA GIL , HENRIQUE P. NEIVA, ANTÓNIO C.SOUSA, LUÍS B. FAÍL, PEDRO P. NEVES, MÁRIO \\ C. MARQUES, DANIEL A. MARINHO
}

CIDESD, University of Beira Interior, Covilhã, Portugal

\begin{abstract}
The scientific community has been looking for warm-up strategies to optimize sprint ability. However, most of the studies focused on performance and physiological variables without the full understanding of the effects of warm-up on the biomechanical variables of running. Therefore, this study aimed to analyse the effect of the manipulation of the running technique during warm-up on $30 \mathrm{~m}$ sprint performance. Thirty-one men physically active participated in this study. The subjects underwent into two warm-up protocols in a randomized order. One of warm-up protocols consisted of exercises with focus on the stride frequency (WUF) and the other with focus on the stride length (WUL). The variables tested were the sprint time of $0-15 \mathrm{~m}$ (TO15), of 15-30 m (T15-30) and 0-30m (T30), stride frequency (SF) and stride length (SL). Results revelled that WUF produced improvements of performance in first $15 \mathrm{~m}$ (WUF: $2.59 \pm 0.11 \mathrm{~s}$ vs. WUL: $2.63 \pm 0.15 \mathrm{~s} ; p=$ $0.03, d=0.30$ ) and the WUL produced improvements of performance in second $15 \mathrm{~m}$ (WUF: $1.94 \pm 0.19 \mathrm{~s}$ vs. WUL: $1.88 \pm 0.09 \mathrm{~s} ; p=0.05, d=0.39$ ). We can conclude that manipulating the sprinting technique may cause positive changes in the motor pattern of this movement. Keywords: Warm-up; Kinematics; Performance; Sprint.
\end{abstract}

Corresponding author. CIDESD, University of Beira Interior, Covilhã, Portugal.

E-mail: maria.helena.gil@hotmail.com

Supplementary Issue: Spring Conferences of Sports Science. International Seminar of Physical Education, Leisure and Health, 17-19 June 2019. Castelo Branco, Portugal.

JOURNAL OF HUMAN SPORT \& EXERCISE ISSN 1988-5202

(c) Faculty of Education. University of Alicante.

doi:10.14198/jhse.2019.14.Proc4.82 


\section{INTRODUCTION}

In recent years, warm-up has emerged as one of the main topics for researches, evidenced by the increase of multiple publications (Mackala et al., 2015; Morin et al., 2012; Nagahara et al., 2014a,b). A considerable number of sports depends significantly on sprinting performance (Byrne et al., 2014). Consequently, the scientific community has been searching for warm-up specific strategies to optimize this ability, however, most of the studies focused on performance and physiological variables without the full understanding of the effects of warm-up. In fact, there is a lack of knowledge about the effect of warm-up on the biomechanical variables of running and that could be critical to training and performance. Thus, the main goal of the current study was to analyse the effect of manipulating running technique during warm-up on sprint performance, comparing two warm-ups strategies focusing on different running techniques (higher stride frequency vs. higher stride length).

\section{MATERIAL AND METHODS}

\section{Participants}

Thirty-one male physically active aged 18-23 years (mean \pm SD: $19.35 \pm 1.08$ years of age; $1.77 \pm 0.07 \mathrm{~m}$ height; $71.90 \pm 10.37 \mathrm{~kg}$ body mass) volunteered to participate in the current study.

\section{Measures}

Each participant completed 2 sessions of maximal $30-\mathrm{m}$ sprint, in randomized order, separated by $48 \mathrm{~h}$. Each session comprised different warm-ups conditions, one stimulating maximal stride length (SL) and the other stimulating maximal stride frequency (SF). The dependent variables were time, kinematics during the sprint trial. This design was able to test whether the warm-ups using different technical running stimulation set affected running performance.

\section{Procedures}

Subjects was randomly assigned to a warm-up protocol, waiting posteriorly 5 minutes until the sprint. Both warm-ups consisted on $5 \mathrm{~min}$ of easy run (lower than $65 \%$ of estimated maximal heart rate), four exercise drills (2x20m): i) rhythmic jumps from foot to foot; ii) ankle drill; iii) skipping drills; iv) high-knee running; run demarcated by flags between steps. Then, these technical exercises were followed by $2 \times 40-\mathrm{m}$ running at gradually increasing intensity, performed with maximal $(69 \pm 3)$ or minimal number of steps (16 \pm 1$)$, depending on SF (WUF) or SL (WUL) focus of warm-up. During sprint the variables monitored have been the sprint time of 0-15m (T0-15), of 15-30 m (T15-30) and 0-30m (T30); SF and SL.

\section{Analysis}

The normality of all distributions was verified using Kolmogorov-Smirnov tests, and parametric statistical analysis was adopted. To compare the 2 trials, Student's paired t-tests and Cohen's $d$ effect sizes were calculated $(p \leq 0.05)$. An effect size of 0.2 was deemed small, 0.5 medium, and 0.8 large.

\section{RESULTS}

No difference was found between the WUF and WUL conditions assessed (WUF: $4.53 \pm 0.20 \mathrm{~s}$ vs. WUL: $4.52 \pm 0.22 \mathrm{~s} ; p=0.72, d=0.05$ ) in the $30 \mathrm{~m}$ sprint. However, these authors could found that subjects were faster in the first $15 \mathrm{~m}$ sprint when performed the WUF (WUF: $2.59 \pm 0.11 \mathrm{~s}$ vs. WUL: $2.63 \pm 0.15 \mathrm{~s} ; p=0.03$, $d=0.30$ ) and they were also faster in the second $15 \mathrm{~m}$ sprint when performed the WUL (WUF: $1.94 \pm 0.19 \mathrm{~s}$ vs. WUL: $1.88 \pm 0.09 \mathrm{~s} ; p=0.05, d=0.39$ ). 
Warm-ups assessed resulted not only in larger SF (WUF: $2.13 \pm 0.17 \mathrm{~Hz}$ vs. WUL: $2.10 \pm 0.16 \mathrm{~Hz} ; p=0.47$, $d=0.18$ ) but also larger SL (WUF: $2.75 \pm 0.18 \mathrm{~m}$ vs. WUL: $2.73 \pm 0.16 \mathrm{~m} ; p=0.74, d=0.11$ ) when the subjects performed WUF during the first $15 \mathrm{~m}$. Also during the second $15 \mathrm{~m}$, the subjects performed not only larger SL (WUF: $3.69 \pm 0.30 \mathrm{~m}$ vs. WUL: $3.73 \pm 0.34 \mathrm{~m} ; p=0.62, d=0.12$ ) but also larger SF (WUF: $2.12 \pm$ $0.27 \mathrm{~Hz}$ vs. WUL: $2.16 \pm 0.22 \mathrm{~Hz} ; p=0.46, d=0.16$ ) when the subjects performed WUL.

\section{DISCUSSION}

Our results showed that WUF could produce significant improvements of performance in first $15 \mathrm{~m}$ (the initial or starting acceleration), and the WUL produced improvements of performance in second $15 \mathrm{~m}$ (the main acceleration). According to Hunter et al. (2004), the SF may be the most important factor in the short term, whereas longer steps may require the development of strength and power. Murphy et al. (2003) confirmed that athletes who are able to generate higher sprint speed over short distances do so because of better SF. On the other hand, application of vertical and horizontal types of jumping exercises could contribute to longer SL (Coh and Mackala, 2013; Mackala et al., 2012). So, improvements in second 15m sprint may be resulted by stimulus of warm-up with focus on stride length because this are mostly composted by horizontal jumping exercises.

\section{CONCLUSIONS}

We can conclude that manipulation running technique through the stimulation of stride frequency (WUF) permitted a better performance during the acceleration phase $(\mathrm{T} 0-\mathrm{T} 15 \mathrm{~m})$ because of a bigger SF, however, the stimulation of stride length (WUL) permitted a better performance in second phase (T15-T30) because of a bigger SL without interfering with SF.

\section{FUNDING}

This project was supported by FCT (UID/DTP/04045/2019; POCI-01-0145-FEDER-006969). It was also supported by research fellowship provided by the University of Beira Interior/Faculty of Social Sciences and Humanities and Santander Universities (BI/Santander/UBI/FCSH/CD/2015).

\section{REFERENCES}

Byrne, P.J., Kenny, J., and O'Rourke, B. (2014). Acute potentiating effect of depth jumps on sprint performance. J Strength Cond Res, 28(3), 610-615. https://doi.org/10.1519/jsc.0b013e3182a0d8c1

Coh, M., and Mackala, K. (2013). Differences between elite and sub-elite sprinters in kinematic and dynamic determinations of countermovement jump and drop jump. J Strength Cond Res, 27(11), 3021-3027. https://doi.org/10.1519/jsc.0b013e31828c14d8

Hunter, J.P., Marshall, R.N., and McNair, P.J. (2004). Interaction of step length and step rate during sprint running. Med Sci Sports Exerc, 36(2), 261-271. https://doi.org/10.1249/01.mss.0000113664.15777.53

Mackala, K., Fostiak, M., and Kowalski, K. (2015). Selected Determinants of Acceleration in the $100 \mathrm{~m}$ Sprint. J Hum Kinet, 45(1), 135-148. https://doi.org/10.1515/hukin-2015-0014

Mackala, K., Stodolka, J., Siemienski, A., and Coh, M. (2012). Biomechanical analysis of standing long jump from varying starting position. J Strength Cond Res, 27(10), 2674-2684. https://doi.org/10.1519/jsc.0b013e31825fce65 
Morin, J.B., Bourdin, M., Edouard, P., Peyrot, N., Samozino, P., and Lacour, J.R. (2012). Mechanical determinants of 100-m sprint running performance. Eur J Appl Physiol, 112(11), 3921-3930. https://doi.org/10.1007/s00421-012-2379-8

Murphy, A.J., Lockie, R.G., and Coutts, A.J. (2003). Kinematic determinants of early acceleration in field sport athletes. J Sports Sci Med, 2(4), 144-150.

Nagahara, R., Matsubayashi, T., Matsuo, A., and Zushi, K. (2014a). Kinematics of transition during human accelerated sprinting. Open Biol, 3(8), 689-699. https://doi.org/10.1242/bio.20148284

Nagahara, R., Naito, H., Morin, J.-B, and Zushi, K. (2014b). Association of acceleration with spatiotemporal variables in maximal sprinting. Int $J$ Sports Med 35(9), 755-761. https://doi.org/10.1055/s-0033-1363252

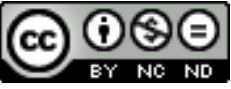

This work is licensed under a Attribution-NonCommercial-NoDerivatives 4.0 International (CC BY-NC-ND 4.0). 


\title{
Bioelectrical impedance vector displacement and phase angle: Progonostic tools for swimmers?
}

\author{
CATARINA N. MATIAS ${ }^{1,2}$, JOSÉ P. MORGAD01,3, PAULO FRANCO ${ }^{4}$, PEDRO QUARESMA ${ }^{1}$, DALIA \\ CURTO ${ }^{1}$, CRISTINA P. MONTEIRO ${ }^{1,2}$, JOANA F. REIS 1,2,5 \\ ${ }^{1}$ Faculdade de Motricidade Humana, Laboratory of Physiology and Biochemistry of Exercise, Universidade \\ de Lisboa, Portugal \\ 2Interdisciplinary Center for the Study of Human Performance (CIPER), Faculdade de Motricidade Humana, \\ Universidade de Lisboa, Portugal \\ 3 Instituto Superior de Ciências Educativas, Lisbon, Portugal \\ ${ }^{4}$ Federação Portuguesa de Natação, Lisbon, Portugal \\ 5 Universidade Europeia, Lisbon, Portugal
}

\begin{abstract}
The present investigation aimed to evaluate the response of body composition, hydration and cellular integrity to a training macrocycle in elite swimmers. 12 national and international level swimmers (4 females, 8 males) were evaluated for phase angle (PhA), fat-free mass (FFM), fat mass (FM), total body water (TBW), intracellular water (ICW) and extracellular water (ECW) at the beginning of the macrocycle (M1) and immediately before the main competition of the macrocycle (M2) with multi-frequency bioelectrical Impedance Analysis. At each moment of evaluation the swimmers also performed a $50 \mathrm{~m}$ all-out first stroke sprint with track start $(\mathrm{T} 50 \mathrm{~m})$ and time was recorded. Comparison of group means between both periods was performed using paired sample t-Test (or Wilcoxon test when normality was not observed). A 95\% confidence ellipse was plotted to understand the R/Xc vector displacement, standardized for height. FM, FFM, R and PhA changed between M1 and M2 in males and females. Additionally, TBW, ECW, and ICW increased in males, while $750 \mathrm{~m}$ decreased. The swimming training cycle induced a performance enhancement concomitantly with a R-Xc vector displacement to the left, indicating an increase in TBW and FFM, and therefore an improved cellular health. BIA data should be evaluated as routine assessment, specifically PhA and vector displacement, as their integrated evaluation might contribute to identify alterations in body composition and hydration as well as gauge about performance in athletes. Keywords: Swimming; Training; Bioimpedance; Hydration.
\end{abstract}

Corresponding author. Universidade Europeia 1500-210, Lisbon, Portugal.

E-mail: joana.reis@universidadeeuropeia.pt

Supplementary Issue: Spring Conferences of Sports Science. International Seminar of Physical Education, Leisure and Health, 17-19 June 2019. Castelo Branco, Portugal.

JOURNAL OF HUMAN SPORT \& EXERCISE ISSN 1988-5202

(C) Faculty of Education. University of Alicante.

doi:10.14198/jhse.2019.14.Proc4.82 


\section{INTRODUCTION}

Monitoring body composition is fundamental in sport, because of its relevance to athletes' health and performance, with lean mass considered as a predictor of muscular fitness. Bioelectrical impedance analysis (BIA) can be a practical solution from a field point of view as it is a rapid, safe and non-invasive method for estimating hydration and fat free mass (FFM). It is based on the different ability of tissues to conduct an electric current, with FFM being one of the major conductors of the body and fat mass showing relatively poor conductance properties. BIA analysis can be performed using raw data, namely phase angles ( $\mathrm{PhA}$ ) and/or bioelectrical impedance vectors and ellipses. According to classic BIVA (Piccoli et al., 1994), variations of the vectors along the $y$ axis of tolerance ellipses indicate changes in total body water (dehydration towards the upper pole, fluid overload towards the lower pole), while the $x$ axis refers to variations of absolute amount of body cell mass (left side: more FFM; right side: less FFM). Within this classic approach, the left upper side would correspond to athletic individuals, whereas the left lower side to obese ones (Piccoli et al., 1994). Also PhA is a measurement of interest as it has been reported to be positively associated with most of the nutritional markers and as an indicator of cell membrane integrity and water distribution between intra- and extracellular spaces (Selberg \& Selberg, 2002). The present investigation aimed to evaluate the response of body composition, hydration and cellular integrity to a training macrocycle in elite swimmers. Bearing in mind the co-dependency between hydration, FFM and performance, we hypothesize that a training cycle will increase PhA and displace the vector to the left side.

\section{MATERIAL AND METHODS}

All measurements were performed at rest and after an overnight fast, with no alcohol or stimulant beverage consumption, or exercise participation taking place within the last 12 hours.

\section{Participants}

12 national and international level swimmers (4 females, 8 males), undertaking $18-22 \mathrm{~h}$ of pool training and $5 \mathrm{~h}$ of dry-land training per week, were evaluated. All procedures were approved by the Scientific Committee of the Faculty of Human Kinetics and were conducted in accordance with the Declaration of Helsinki for human studies and all subjects provided their written informed consent to participate.

\section{Measures}

Body composition

Multi-frequency bioelectrical Impedance Analysis (BodyStat QuadScan 4000, Isle of White, United Kingdom) was used to assess resistance $(R)$ and reactance $(X c)$, according to the standard procedures, from which impedance, PhA, FFM, FM, total body water (TBW), intracellular water (ICW) and extracellular water (ECW) were obtained.

\section{Performance}

After standardized warm up, the swimmers performed a $50 \mathrm{~m}$ all-out first stroke sprint with track start (T50m) and time was recorded.

\section{Procedures}

Study Design

This study followed a swimming training macrocycle lasting 14 weeks. Swimmers were evaluated at the beginning of the macrocycle (M1) and immediately before the main competition of the macrocycle (M2). 


\section{Statistical Analysis}

Comparison of group means between both periods was performed using paired sample t-Test (or Wilcoxon test when normality was not observed). A 95\% confidence ellipse was plotted to understand the R/Xc vector displacement, standardized for height.

\section{RESULTS}

FM, FFM, R and PhA changed between baseline and the end of the macrocycle period in males and females. Additionally, TBW, ECW, and ICW increased in males, while T50m decreased.

The vector displacements plotted on the R-Xc graph, from M1 to M2, are shown in Figure 1.
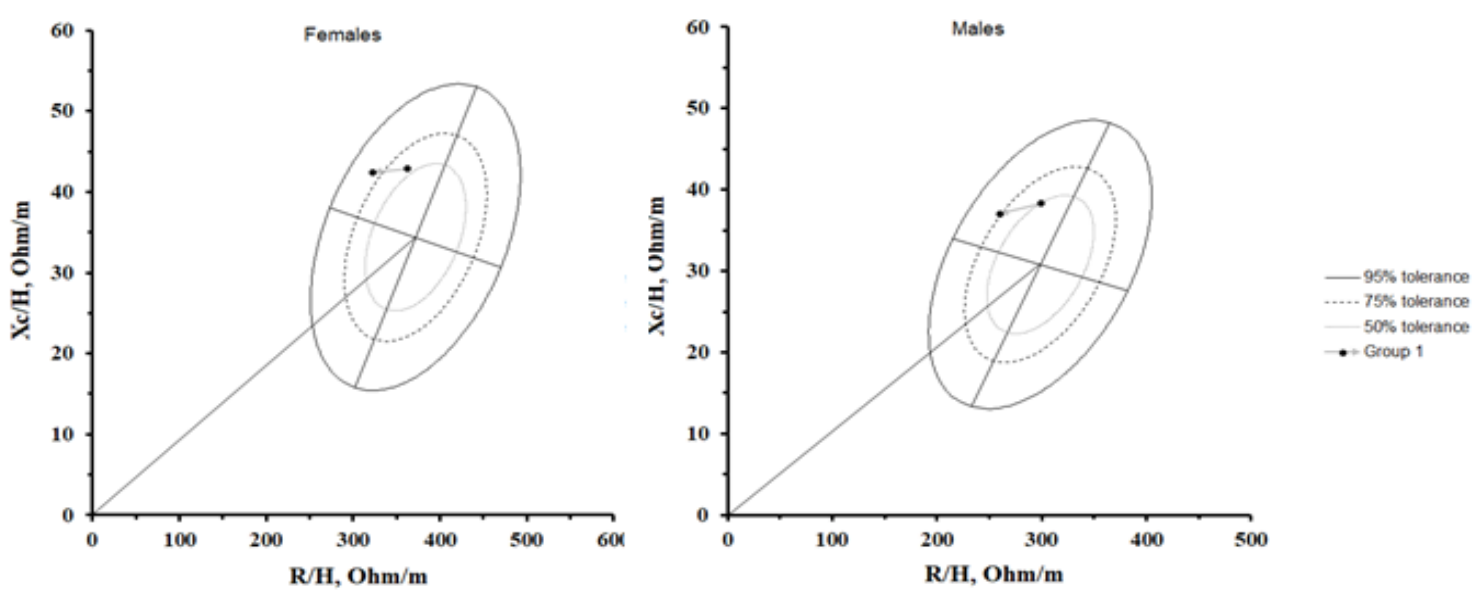

Figure 1. Paired graph and vector displacement with $95 \%$ confidence ellipses, with $\mathrm{R}$ representing resistance and $\mathrm{Xc}$ representing reactance, both corrected for $\mathrm{H}$ (height). The arrow represents the change from $\mathrm{M} 1$ to M2.

\section{DISCUSSION}

The main findings of the present investigation are an impedance vector displacement to the left and an increase of PhA from M1 to M2 in both groups. This resulted from increases in TBW and ICW, and correspondingly FFM, in athletes. Concomitantly an increase in performance was observed. This is consistent with the results observed in soccer, showing increases in body fluids occurring at the end of both the pre-season and the season, and fluid leaks occurring during the competitive period (Mascherini et al., 2015). It has been suggested that an increase in PhA is associated with improved power output in elite road cyclists (Pollastri et al., 2016). Contrarily, low PhA values emerged as a useful predictor for impaired muscle function (Beberashvili et al., 2014). From a practical point of view, a higher PhA is associated with large quantities of intact cells membranes and body cell mass (Selberg \& Selberg, 2002) whereas, lower PhA appears to be consistent with either cell death or an increase in the fragility of the cell membranes, therefore higher PhA values reflect higher cellularity, cell membrane integrity and better cell function. PhA monitoring throughout the competitive season may be an important non-invasive indicator of cellular health adaptation to training. 


\section{CONCLUSIONS}

The swimming training cycle induced a performance enhancement concomitantly with a R-Xc vector displacement to the left, indicating an increase in TBW and FFM, and therefore an improved cellular health. BIA data should be evaluated as routine assessment, specifically PhA and vector displacement, as their integrated evaluation might contribute to identify alterations in body composition and hydration as well as gauge about performance in athletes.

\section{REFERENCES}

Beberashvili, I., Azar, A., Sinuani, I., Shapiro, G., Feldman, L., Stav, K., Sandbank, J., \& Averbukh, Z. (2014). Bioimpedance phase angle predicts muscle function, quality of life and clinical outcome in maintenance hemodialysis patients. Eur J Clin Nutr, 68(6), 683-689. https://doi.org/10.1038/ejcn.2014.67

Mascherini, G., Gatterer, H., Lukaski, H., Burtscher, M., \& Galanti, G. (2015). Changes in hydration, body-cell mass and endurance performance of professional soccer players through a competitive season. J Sports Med Phys Fitness, 55(7-8), 749-755.

Piccoli, A., Rossi, B., Pillon, L., \& Bucciante, G. (1994). A new method for monitoring body fluid variation by bioimpedance analysis: the RXc graph. Kidney Int, 46(2), 534-539. https://doi.org/10.1038/ki.1994.305

Pollastri, L., Lanfranconi, F., Tredici, G., Burtscher, M., \& Gatterer, H. (2016). Body Water Status and Short-term Maximal Power Output during a Multistage Road Bicycle Race (Giro d'Italia 2014). Int J Sports Med, 37(4), 329-333. https://doi.org/10.1055/s-0035-1565105

Selberg, O., \& Selberg, D. (2002). Norms and correlates of bioimpedance phase angle in healthy human subjects, hospitalized patients, and patients with liver cirrhosis. Eur J Appl Physiol, 86(6), 509-516. https://doi.org/10.1007/s00421-001-0570-4

\section{(9) $(\mathbb{Q} \Theta \Theta$}

This work is licensed under a Attribution-NonCommercial-NoDerivatives 4.0 International (CC BY-NC-ND 4.0). 


\title{
Jumping in the Brazilian Women's Volleyball "B" Super-league
}

\author{
MÁRCIO JOSÉ KERKOSKI ${ }^{1}$, LUIS HENRIQUE MERCANTE DA SILVA ${ }^{1}$, ALEXANDER HODECK², \\ JAQUELINE TUCHEL ${ }^{2}$, LUISA HENTE 2 \\ 1 University Technological Federal of Paraná, Research Group on Sport Sciences, GPCE, Brazil \\ ${ }^{2}$ Chemnitz University of Technology, Germany
}

\begin{abstract}
The number of jumps during training and Volleyball games is directly related to knee injuries (Farina and Navarro 2008). Quantitative data with the jump magnitude serve to guide the training load (Berriel A, Foppa, G. 2004, Horta et al., 2007, Rocha and Barbanti 2007). There are no data on quantities and magnitudes of jumps in female athletes. The objective of this study was to verify the amount and magnitudes of jumps of setter, outside hitter and middle-blocker of a female team that played in the Brazilian B league of volleyball in training and competition. Participants of the case study were one setter, one middle-blocker and one outside hitter of the team. The accompaniment of each athlete was carried out during 5 training sessions and 2 games in the competition, including the final game. The jumps of the athletes were monitored with the equipment VERT Powered by G-VERT Technology. The results observed in training and competition, respectively, were: average number of jumps (147.2 / 134), average jump height (45.3 / 45.1), highest jump height ( 51.7 / 53) on the setter. The average jumps (71.8/95), average jump height (46.8 / 48), highest jump height (62 / 69.5) of the middle-blocker. The average number of jumps (64/116), average jump height (49.2 /55.2), highest jump height (53.5 / 64.5) of the outside hitter. The jump means of the present study are higher than in previous studies, perhaps because of monitoring jumps of all athletes. The athlete who jumps most within the competition was the setter, followed by the outside hitter and finally the middle-blocker. Keywords: Volleyball; Jump; Women.
\end{abstract}

Corresponding author. University Technological Federal of Paraná, Research Group on Sport Sciences, GPCE, Brazil.

E-mail: marciok@utfpr.edu.br

Supplementary Issue: Spring Conferences of Sports Science. International Seminar of Physical Education, Leisure and Health, 17-19 June 2019. Castelo Branco, Portugal.

JOURNAL OF HUMAN SPORT \& EXERCISE ISSN 1988-5202

(c) Faculty of Education. University of Alicante.

doi:10.14198/jhse.2019.14.Proc4.82

VOLUME 14 | Proc4 | 2019 | S1453 


\section{INTRODUCTION}

When analysing the men's volleyball superliga, Moraes and Bassedone (2007) observed that the area of most injuries is the knee (24.6\%). Data on quantity and magnitude of jumps are used to guide and quantify the training load (Berriel A., Foppa, G. 2004, Horta et al., 2007; Rocha and Barbanti 2007). However, there are no data on quantities and magnitude of jumps in female athletes who compete for the Brazilian Volleyball B-league in both training and competition. The objective of this study was to verify the quantity and magnitude of jumps of setter, outside hitter and middle-blocker of a female team that played the Brazilian Volleyball Bleague in training and competition.

\section{MATERIAL AND METHODS}

\section{Participants}

Three female athletes participated in the case study: one setter, one outside hitter and one middle-blocker who played in the Women's Brazilian Volleyball B-League.

\section{Measures}

The movements and jumps of the athletes were monitored with the equipment VERT Powered by G-VERT Technology. Data were collected through APP myVERT.

\section{Procedures}

The investigation of each athlete was performed during 5 training sessions (average 160 min duration) and during 2 games, including the final. The following variables were observed: the total of jumps, the average of height of jump, highest of jump, lower height jump, average jump per minute.

\section{Analysis}

The analysis was of the jump frequencies. The average of jumps in training and competition.

\section{RESULTS}

Table 1. Jumping means, jump height, highest and lowest jump and jumps per minute in training and competition

\begin{tabular}{llccccc}
\hline & & Jumps & Hight & Highest & Lower & Jump/Min \\
\hline Setter & Training & 147.2 & 45.3 & 51.7 & 18.3 & 1.7 \\
& Competition & 134.0 & 45.1 & 53.0 & 16.0 & 1.0 \\
Middle-blocker & Training & 71.8 & 46.8 & 62.0 & 22.4 & 1.0 \\
& Competition & 95.0 & 48.0 & 69.5 & 16.5 & 1.0 \\
\multirow{3}{*}{ Outside Hitter } & Training & 64.2 & 49.2 & 53.5 & 19.6 & 0.6 \\
& Competition & 116.0 & 55.2 & 64.5 & 20.0 & 0.8 \\
\hline
\end{tabular}

\section{DISCUSSION}

When analysing professional athletes of the volleyball super-league, Horta (2017), observed an average of 47 jumps per session including jumps for serve and block. The attackers (outsiders, opposites and middleblocker) presented average $32.1 \pm 17.4$ attack jumps and the setters $80.1 \pm 44.5$ setters jumps per session. When evaluating the women's volleyball Superliga A (Rocha and Barbanti 2007), it observed that average 
values of 43.1 jumps for setter per game. In the same study he observed average values of 85.3 jumps per game for the outside hitters. The middle-blockers players showed the highest averages per game in the jump to block of 27.1. Analysing a men's team that played the volleyball superliga, Cardoso (2018) pointed out that the number of jumps in games and training do not have significant differences. In the same study, the setter is the second athlete in number of jumps performed in games and training. But this person presented lower heights of jumps in the two evaluated situations. The middle-blockers player showed the highest number of jumps in games and training compared to other positions. In the current study, the comparison between training jumps and jumps in the competition was not performed. The jump means of the present study are higher than previous studies, perhaps because of the methodology of the study which included not all players of a team. The athlete who jumps the most in the competition is the setter, followed by outside hitter and finally the middle-blocker.

\section{CONCLUSIONS}

The results observed in training and competition were average number of jumps (147.2 / 134), average jump height (45.3 / 45.1), highest jump height (51.7 / 53) and the number of jumps per minute (1.7 / 1.0) of the setter, the average number of jumps (71.8 / 95), average jump height (46.8 / 48), highest jump height (62 I $69.5)$ and the number of jumps per minute $(1.0 / 1.0)$ of the middle-blocker and finally the average number of jumps (49.2 / 55.2), average jump height (53.5 / 64.5) and the number of jumps per minute (0.6 / 0.8$)$ of the outside hitter. In the current study, the comparison between training jumps and jumps in the competition was not performed. The means of number of jumps of the present study are higher than previous studies, perhaps because of monitoring all athletes' jumps in these studies. The athlete who jumps the most in the competition is the setter, followed by the outside hitter and finally the middle-blocker.

\section{REFERENCES}

Berriel A.; Foppa, G., G.P.; Fontoura. (2004). Avaliação Quantitativa de Saltos Verticais Em Atletas de Voleibol Masculino Na Superliga 2002/2003. Revista Digital de Educación Física y Deportes 10(73):1-8. Retrieved from https://www.efdeportes.com/efd73/volei.htm

Cardoso, A. S. (2018). Comportamento Do Desempenho de Saltos, Fadiga e Recuperação de Atletas de Voleibol Durante Jogos e Treinos. (Dissertação de mestrado). Universidade Federal do Rio Grande do Sul, Porto Alegre, RS, Brasil. https://doi.org/10.29289/259453942018v28s1059

Farina, K. G., and Navarro A. C. (2008). Analise De Atletas De Voleibol Lesionados Referente À Posição Exercida Em Quadra. Revista Brasileira de Prescrição e Fisiologia do Exercício 2(7): 27-35. https://doi.org/10.33233/fb.v8i3.1771

Horta, T. A. G., Bara Filho, M. G., Miranda, R., Ciombra D. R. and Werneck, F. Z. (2017). Influência Dos Saltos Verticais Na Percepção Da Carga Interna de Treinamento No Voleibol. Revista Brasileira de Medicina do Esporte 23(5): 403-6. https://doi.org/10.1590/1517-869220172305172132

Moraes, J. C., and Bassedone, D. da R. (2007). Estudo Das Lesões Em Atletas de Voleibol Participantes $\mathrm{Da}$ Superliga Nacional. Revista Digital - Buenos Aires 111: 1-8. https://doi.org/10.11606/t.5.2005.tde-14102014-111109

Rocha, M. A., and Barbanti, V. J. (2007). Análise Das Ações de Saltos de Ataque, Bloqueio e Levantamento No Voleibol Feminino. Revista Brasileira de Cineantropometria e Desempenho Humano 9(3): 284-290. https://doi.org/10.5007/1980-0037.2012v14n1p41 


\section{(c) (i) (3)}

This work is licensed under a Attribution-NonCommercial-NoDerivatives 4.0 International (CC BY-NC-ND 4.0). 


\title{
Smartphone fitness applications used by runners: For what reason?
}

\author{
ANDRÉ LUIS ARONI ${ }^{4}$, MARCO BATISTA ${ }^{2,3,4}$, AFONSO ANTONIO MACHADO ${ }^{5}$, ANTONIO RUI \\ GOMES 6 \\ 1 UniMetrocamp Wyden, Brazil \\ 2Instituto Politécnico de Castelo Branco, Portugal \\ ${ }^{3}$ Sport, Health \& Exercise Research Unit (SHERU), Portugal \\ ${ }^{4}$ Research in Education and Community Intervention (RECI), Instituto Piaget, Portugal \\ 5 Universidade Estadual Paulista, Brazil \\ 6Universidade do Minho, Portugal
}

\begin{abstract}
The aim of this study was to explore which smartphones applications features have most been appreciated by runners. This descriptive and exploratory study consisted of 278 respondents from Brazil and Portugal. A survey composed of demographic and closed questions was developed and distributed via social media. Findings revealed that $40.8 \%$ of the runners in Brazil prefer the "calories spent" as main feature, while also $40.8 \%$ of the participants in Portugal prefer the "heart rate control". The difference between the preferred features in Brazil and Portugal indicates that more cross-cultural research is needed to unravel the biopsycho-social mechanisms that might explain why members of some groups have different opinions than other ones. Keywords: Smartphones; Applications; Physical activity; Runners.
\end{abstract}

Corresponding author. Av. Dom Joaquim Mamede da Silva Leite 40, apto 102 bloco E, Jardim do Lago, CEP 13050-006, Campinas/SP, Brazil.

E-mail: andre-aroni@hotmail.com

Supplementary Issue: Spring Conferences of Sports Science. International Seminar of Physical Education, Leisure and Health, 17-19 June 2019. Castelo Branco, Portugal.

JOURNAL OF HUMAN SPORT \& EXERCISE ISSN 1988-5202

(C) Faculty of Education. University of Alicante.

doi:10.14198/jhse.2019.14.Proc4.82 


\section{INTRODUCTION}

Physical activity is defined as bodily movement that requires energy expenditure and includes physical activities done as part of daily living, occupation, leisure, exercise, and sports (Tenenbaum, Eklund, \& Kamata, 2011). Physical inactivity has been identified as the fourth leading risk factor for premature death around the world, accounting for slightly over three million deaths annually (Middelweerd et al., 2014; World Health Organization, 2016), and running appeared as one of the favourite physical activities practiced around the world (Aroni et al., 2017). About the technologies, nearly two-thirds of Americans reported owning a smartphone (Pew Research Internet Project, 2015). A smartphone is defined as any cellular device that has additional functions including a camera, global positioning system (GPS), and Wi-Fi capabilities, and is running in one of the following mobile devices: iPhone, Android, BlackBerry, Windows Mobile and others (Bert et al., 2014; Ozdalga, Ozdalga, \& Ahuja, 2012). Applications (apps) are downloaded from the platform to a mobile device and can be applied in various fields (e.g., social, entertainment, educational and fitness), and are often free and easy to use (Bert et al., 2014). Information technology companies and researchers have devoted attention to sports and physical activities to support people with new apps for indoor and outdoor use (Buttussi \& Chittaro, 2008). In the present study, we were interested in exploring which smartphones apps features have most been appreciated by runners, understanding the reasons to download.

\section{MATERIAL AND METHODS}

\section{Participants}

A total of 278 participants responded to the survey via the use of social networks Facebook® and Linkedln®. Participants were from Brazil (161) and Portugal (117), the inclusion criterion to participate in the study required participants to own a smartphone and have the running as main physical activity. The gender split was even across the two countries being sampled: Brazil ( 96 men - 59.6\%, 65 women - 40.4\%) and Portugal (76 men - 64.9\%, 41 women - 35.1\%). Although, five age categories were sampled (under 20; $21-30 ; 31$ 40; 41-50; above 51), the 21-30 age range comprised the vast majority of responses across the both countries: Brazil (118 participants $-73.2 \%$ ) and Portugal (76 participants $-64.9 \%$ ). Of the total participants, 203 respondents $(73 \%)$ reported downloading at least one fitness app on their smartphone.

\section{Measures}

An informed consent section, presented immediately after the survey link was opened, informed to the participants of the purpose of the study, confidentiality terms and explained what would be required of them during the data collection. The survey was composed of four demographic questions (age, gender, country of residence and city of residence), four closed questions (type of physical activity, physical activity app ever downloaded onto smartphone, which physical activity app and which app features considered most beneficial in helping the physical activity). The survey was made available in the Portuguese language.

\section{Procedures}

The research was conducted according to the ethical research principles involving human subjects. Data collection commenced after obtaining approval from the Research Ethics Committee at UNESP

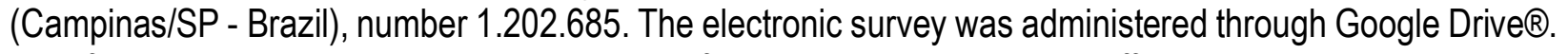
This free online service provides storage and file synchronization, which offers online solutions such as creating and editing documents, spreadsheets, presentations and surveys for research. As mentioned, the primary means of data collection was through social networks: Facebook $₫$ and Linkedln $\circledast$. Through the use of these engines the authors posted a message for runners to participate, and provided the link to the survey. 


\section{RESULTS AND DISCUSSION}

About the runners, 113 respondents in Brazil (70\%) reported downloading fitness app on their smartphones, in Portugal the total was 90 participants (77\%). Results from the online questionnaire suggest that people that are "into exercising" are more prone to follow technological trends (Agarwal et al., 1998; Lu, Yao,\& Yu, 2005).

Furthermore, runners in Brazil prefer the feature "calories spent" (40.8\%) while the Portuguese the "heart rate control" (40.8\%), related with the exercise goals (Troiano, Berrigan, \& Dodd, 2008).

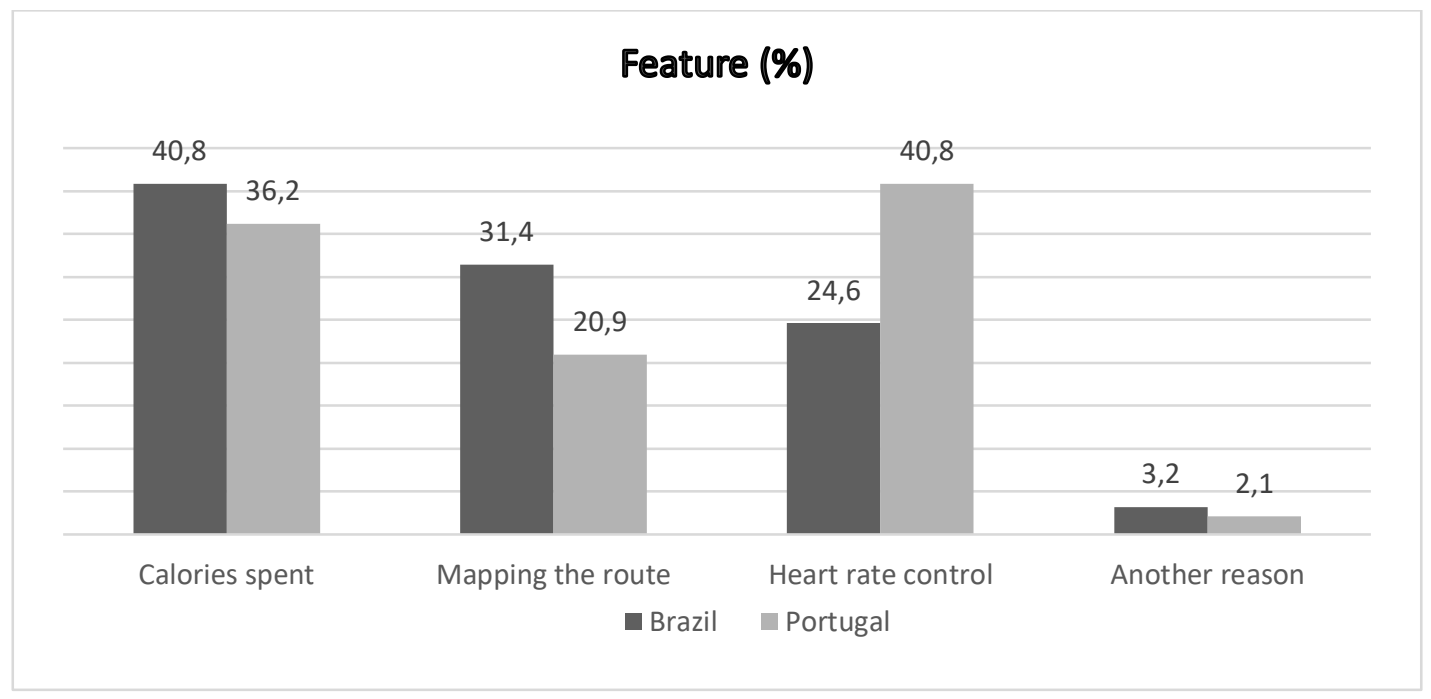

Figure 1. Preference about the applications feature.

\section{CONCLUSIONS}

The difference between the preferred features in Brazil and Portugal indicates that cross-cultural research is needed to unravel the bio-psycho-social mechanisms that might explain why members of some societies have different opinions than other societies.

\section{REFERENCES}

Aroni, A., Castillo, E., Sousa, C., Machado, A. A., Filho, E., Tenenbaum, G. (2017). Smartphone applications used for initiating and maintaining physical activity: an exploratory analysis. Revista de Psicologia del Deporte, 27, 100-112.

Agarwal, R., Ahuja, M., Carter, P. E., Gans, M. (1998). Early and late adopters of IT innovations: extensions to innovation diffusion theory. In Proceedings of the DIGIT Conference (pp. 1-18).

Bert, F., Giacometti, M., Gualano, M.R. et al. (2014). Smartphones and health promotion: A review of the evidence. Journal of Medical Systems, 38, 9995-9997.

Buttussi, F., Chittaro, L. (2008). MOPET: A context-aware and user-adaptive wearable system for fitness training. Artificial Intelligence in Medicine, 42, 153-163. https://doi.org/10.1016/.artmed.2007.11.004

Lu, J., Yao, J. E., Yu, C. S. (2005). Personal innovativeness, social influences and adoption of wireless Internet services via mobile technology. The Journal of Strategic Information Systems, 14(3), 245268. https://doi.org/10.1016/j.jsis.2005.07.003 
Middelweerd, A., Mollee, J.S., Van der Wal, N., Brug, J., te Velde, S. J. (2014) Apps to promote physical activity among adults: A review and content analysis. International Journal of Behavioral Nutrition \& Physical Activity, 11, 97. https://doi.org/10.1186/s12966-014-0097-9

Ozdalga, E., Ozdalga, A., Ahuja, N. (2012) The smartphone in medicine: A review of current and potential use among physicians and students. Journal of Medical Internet Research, 14, e128. https://doi.org/10.2196/jmir.1994

Tenenbaum, G., Eklund, R.C. and Kamata, A. (2011). Measurement in Sport and Exercise Psychology. Champaign, IL: Human Kinetics.

Troiano, R. P., Berrigan, D., Dodd, K. W. et al. (2008). Physical activity in the United States measured by accelerometer. Medicine and Science in Sports and Exercise, 40(1), 181. https://doi.org/10.1249/mss.0b013e31815a51b3

World Health Organization (2016) Health topics: physical activity. http://www.who.int/topics/physical_activity/en/. Accessed March 30, 2016. 


\title{
The educative role of judo for children in first- cycle primary school: Parents' opinion based on focus group
}

\author{
ANDRÉ LUIS ARONI ${ }^{1} \triangle$, AFONSO ANTONIO MACHADO ${ }^{2}$, ANTONIO RUI GOMES ${ }^{3}$, SAMUEL \\ HONÓRIO4,5,6, JORGE SANTOS ${ }^{4,5}$, MARCO BATISTA 4,5,6 \\ 1 UniMetrocamp Wyden, Brazil \\ 2Universidade Estadual Paulista, Brazil \\ 3 Universidade do Minho, Portugal \\ 4 Instituto Politécnico de Castelo Branco, Portugal \\ ${ }^{5}$ Sport, Health \& Exercise Research Unit (SHERU), Portugal \\ ${ }^{6}$ Research in Education and Community Intervention, RECI-Instituto Piaget, Portugal
}

\begin{abstract}
The recognition of pedagogy and method of Judo was made effective by UNESCO, as a world-wide sport utility in the development of the human being and, as an activity whose spectrum of approach in children's age ranges is quite broad, exerts a preponderant function in the global development of its practitioners, allowing them to transfer the sport context to their specific socio-cultural context. The objective of this study was to relate the practice of Judo in the development of self-concept, self-esteem and academic performance in children's first-cycle primary school. The study group included 8 parents in charge of education, aged 33$45(\mathrm{M}=39.75 \mathrm{SD}=4.33), 6$ female and 2 males, 4 had a bachelor's degree, 2 had an undergraduate degree and two had the 12th grade. According to these parents, the practice of Judo is very much linked to the development of self-concept, emphasizing through the promotion of social acceptance, compliance with rules and responsibility or balanced behaviour. It is also linked to the development of self-esteem, insofar as it is an activity that promotes self-confidence. It also exerts an important influence on school performance, since it promotes concentration. It is also emphasized that in the parents' opinion, the inclusion of Judo in the Physical Education curricular programme would bring several benefits for the integral conception of the child as a citizen. Keywords: Quantitive analyses; Focus group; Judo; Parents; Sports development.
\end{abstract}

\footnotetext{
Corresponding author. Av. Dom Joaquim Mamede da Silva Leite 40, apto 102 bloco E, Jardim do Lago, CEP 13050-006, Campinas/SP, Brazil.

E-mail: andre-aroni@hotmail.com

Supplementary Issue: Spring Conferences of Sports Science. International Seminar of Physical Education, Leisure and Health, 17-19 June 2019. Castelo Branco, Portugal.

JOURNAL OF HUMAN SPORT \& EXERCISE ISSN 1988-5202

(c) Faculty of Education. University of Alicante.

doi:10.14198/jhse.2019.14.Proc4.82
} 


\section{INTRODUCTION}

The recognition of pedagogy and method of Judo was made effective by UNESCO, as a world-wide sport utility in the development of the human being and, as an activity whose spectrum of approach in children's age ranges is quite broad, exerts a preponderant function in the global development of its practitioners, allowing them to transfer the sport context to their specific socio-cultural context.

Practicing physical activity, in particular Judo, is a component of a complex process involving the development, learning and assimilation of competences, values, norms, self-perceptions, identities and roles provided by variables other than the framework family and social relations, such as the sociodemographic group, the profession, education and place of residence, which may influence the physical activity of children and adolescents in their free time (Batista et al., 2016). The present study aims to relate the practice of Judo in the development of self-concept, self-esteem and academic performance in children at the first-cycle of basic education, using parent's opinion so they can choose this sport as a formative context for their students.

\section{MATERIAL AND METHODS}

\section{Participants}

The study group included 8 parents in charge of education, aged 33-45 ( $M=39.75 \mathrm{SD}=4.33), 6$ were female and 2 were male, 4 had a bachelor's degree, 2 had an undergraduate degree and the other two the 12th grade.

\section{Measures}

The qualitative focus group (Cubo-Delgado et al., 2011) focused on parents in charge of education, as it allows us to ascertain opinions and arguments about the practice of Judo and its importance and relation with self-concept, self- esteem and academic performance.

\section{Procedures}

We proceeded to establish contact with the individuals that would be part of the focused group, choosing the parents in a simple random choice. We were careful by including a father or mother that each one of them belonged to a club of the Judo Association of the Santarém District.

\section{Analysis}

According to the analysis technique proposed by Cubo-Delgado et al. (2011) of the ideas presented and discussed, an analysis was elaborated on key ideas or phrases that were grouped in sub-dimensions, which allowed us to perform statistical analyses by frequencies, which resulted in graphics percentages. The relationship of dimensions to be debated through the focus group was: a) Judo practice as a content of the national Physical Education programme; b) The practice of Judo as an influence of self-concept (school competence, social acceptance, athletic competence, physical appearance and behaviour); c) The practice of Judo as an influence on Self-esteem; d) The practice of Judo as an influence on academic performance.

\section{RESULTS}

From the results obtained, we present in graphics the interpretations or choices of the parents in charge of education for the practice of Judo by its students. The opinions of the parent's group about Judo being integrated in the practice of Physical Education national programme, the importance of Judo practice in the 
development of self-concept, in the development of self-esteem and the academic performance obtained by its students are evident.
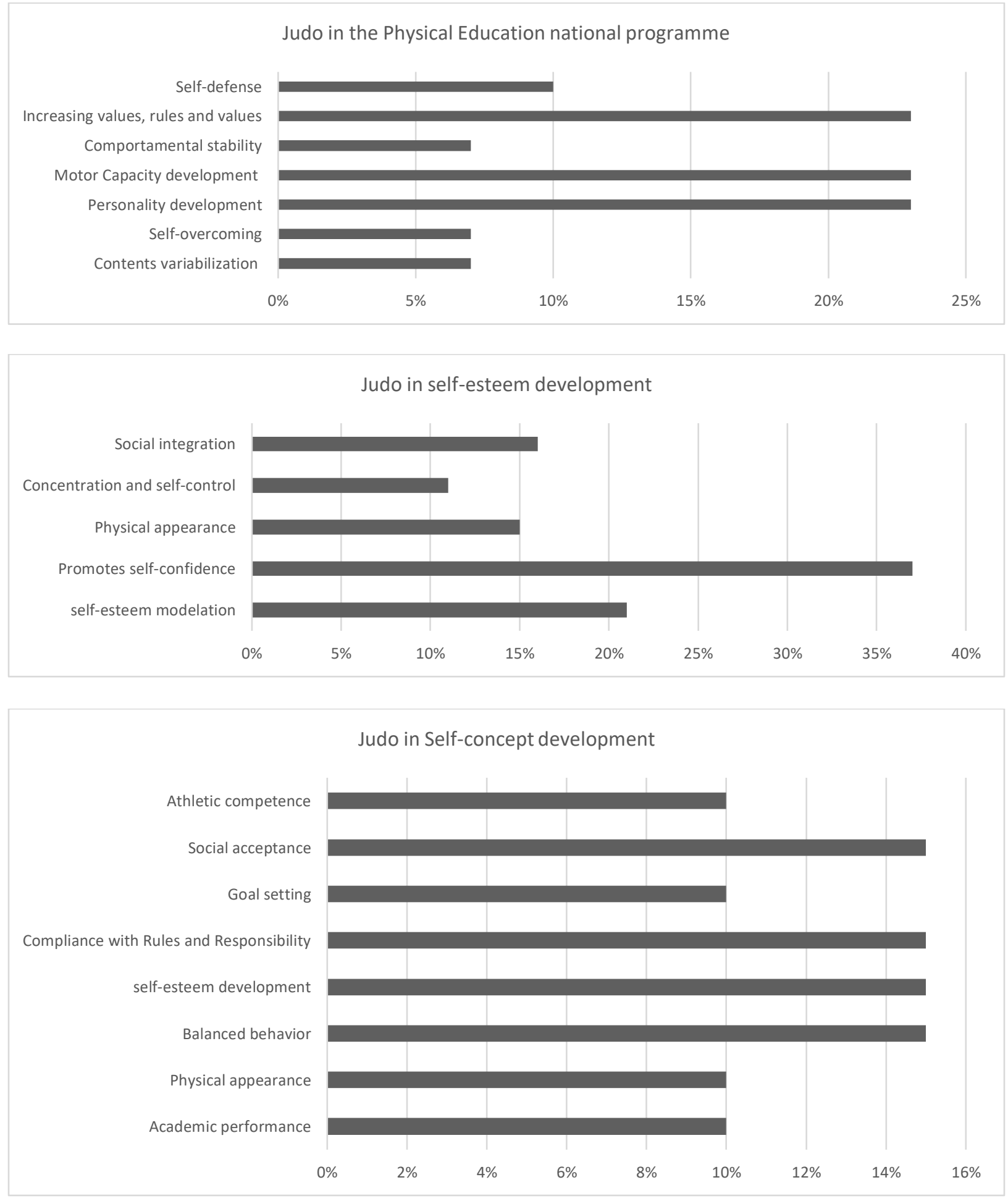


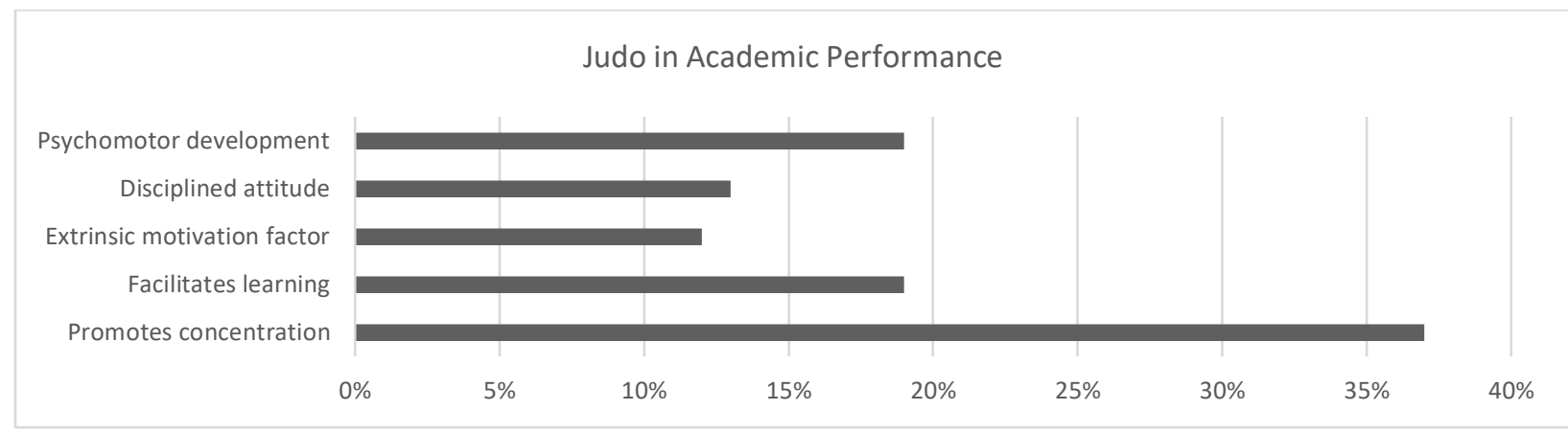

\section{DISCUSSION}

The results followed the opinion of parents; the practice of Judo by children presents multiple benefits for their self-concept and self-esteem development and also academic performance. These results corroborate the quantitative findings of Batista et al. (2016) who emphasize that Judo practice by students promotes a significant evolution in the development of self-concept and academic performance, as well as suggests a positive evolutionary tendency in the development of students' self-esteem in practicing this sport.

\section{CONCLUSIONS}

In the opinion of the parents in charge of education, the practice of Judo is very much linked to the development of self-concept, emphasizing these, through the promotion of social acceptance, compliance with rules and responsibility or balanced behaviour. It is also linked to the development of self-esteem, insofar as it is an activity that promotes self-confidence. It also exerts an important influence on academic performance, since it is seen as an activity that promotes concentration. It is also emphasized that in the parents' opinion, the inclusion of Judo in the Physical Education programme would bring several benefits for the integral formation of the child as a citizen.

\section{REFERENCES}

Batista, M.; Cubo, S.; Honório, S.; Martins, J. (2016). Judo: A Practice Enhancer of Psychological Variables and School Performance. Saarbrucken: LAP Lambert Academic Publishing: ISBN: 978-3659-85141-4.

Cubo-Delgado, S.; Martín-Marin, B. \& Ramos-Sanchez, J. L. (2011). Métodos de Investigación y Análisis de Datos en Ciências Sociales y de la Salud. Madrid: Pirâmide.

\section{(9) $\oplus \Theta \Theta$}

This work is licensed under a Attribution-NonCommercial-NoDerivatives 4.0 International (CC BY-NC-ND 4.0). 


\title{
The development of emotional self-emotion in volleyball project "AVP SOCIAL": Under19 athletes perspective
}

\author{
ALESSANDRA WEISS FERRAZ DE OLIVEIRA ${ }^{1}$, MARCIO JOSÉ KERKOSKI ${ }^{1}$, WANDERLEY MARCHI \\ JÚNIOR², ANA PAULA CABRAL BONIN MAOSKI ${ }^{1}$, GILMAR FRANCISCO AFONSO ${ }^{1}$ \\ 1 University Technological Federal do Paraná, Brazil \\ 2University Federal do Paraná, Brazil
}

\begin{abstract}
The sport has undergone transformations due to the modernization process, be it sport spectacle or educational sport. During its development, sport has been a means for the social and emotional balance of individuals. The objective of this study was to verify if the volleyball sports practice develops the emotional self-mastery of adolescent athletes of the U19 category of the "AVP Social" project. The methodology used has qualitative and quantitative characteristics. We interviewed 12 male athletes, all federated by FPV. In order to do this, we used a questionnaire with seven questions, four closed and three semi-open questions, elaborated from a research in the Portal of the Periodicals of Capes, which evidenced the teacher's pedagogical work from an emotional perspective, specifically on the skills of self-control and empathy. After data collection with the athletes, we transcribed the data using graphs and later analysed and discussed the responses using Norbert Elias's (1992) Theory of Civilization Process and Goleman's (2012) theory of Emotional Intelligence. The results demonstrated that the athletes' emotions (self-control and empathy) U19 volleyball project are present in sports practice and may be fundamental to the success of these young athletes in development. Keywords: Volleyball; Adolescence; Emotions.
\end{abstract}

Corresponding author. University Technological Federal do Paraná, Brazil.

E-mail:alewfo@hotmail.com

Supplementary Issue: Spring Conferences of Sports Science. International Seminar of Physical Education, Leisure and Health, 17-19 June 2019. Castelo Branco, Portugal.

JOURNAL OF HUMAN SPORT \& EXERCISE ISSN 1988-5202

(c) Faculty of Education. University of Alicante.

doi:10.14198/jhse.2019.14.Proc4.82 


\section{INTRODUCTION}

Despite the scarcity of specific studies about sports and the development of emotional self-mastery in adolescents, we understand that it may be useful to know the coach's work through the athlete's gaze, under the emotional bias, considering social sport. After all, sport is a "symbolic representation of the non-violent form," and a conducive place for creating tensions, releasing emotions in a controlled environment, creating an imaginary picture of human life (Elias and Dunning 1992: 45). Our field of study is limited to volleyball practiced in a social Project in the U19 category, which also includes children and adolescents. Among the objectives mentioned in the project are: formation of ethical, critical citizens, respectful of themselves and others; teamwork; effective enhancement of their sports "talent"; opportunity in the sport (Associação de Volleyball do Paraná, 2018). Using the understanding of Kerkoski (2009) during the trainings, the emotions are present and can be developed and improved to reach several goals. In order to verify if the coach seeks to include in his methodological procedures the emotional self-mastery, we elaborate the following problematic: does the volleyball practice develop emotional self-mastery in adolescent athletes of the U19 category of the "AVP Social" project?

\section{MATERIAL AND METHODS}

The nature of this research can be classified as qualitative and quantitative. We used as a tool a questionnaire, containing seven questions, four closed and three semi-open questions, elaborated from previous studies.

\section{Participants}

The questionnaire was applied to 12 male athletes federated by the Federação Paranaense de Voleibol (FPV), inserted in the project called "AVP Social", Under19 category, in the city of Curitiba, State of Paraná, Brazil.

\section{Measures}

The questionnaire "Self-mastery Emotional" was constructed based on a research carried out in the Portal of Periodicals of CAPES, which sought to identify studies on emotional self-control and empathy in the process of teaching learning (Goleman, 2012). In this way, we elaborate the following questions: 1) What do you feel during the training sessions? Containing 11 possible alternatives to be marked (joy, sadness, fear, anger, shame, pride, satisfaction, frustration, pleasure, gratitude and indignation). 2) During training do you usually express your ideas and feelings? If yes, give an example. 3) In addition to performing the activities proposed by the coach, do you usually perform other functions? Which are? 4) During the training, are there moments of discussion and dialogue? If so, how do you manifest yourself? 5) Does the coach have the habit of performing feedbacks (reporting mistakes and correctness) during the exercises? If yes, are they usually positive or negative? Containing 3 possible alternatives (yes, they are usually positive; he does not communicate errors and corrects; yes he does, but usually are negative). 6) Do you usually participate in the construction of rules or exercises during training? Yes or no? 7) Are there moments during training for you to choose a favourite game or exercise? Yes or no?

\section{Procedures}

The research was conducted in the first half of 2018, at the headquarters of the "AVP Social" project, with the athletes who were attending the training in the U19 category. The questionnaires were delivered before the training, the questions were read aloud and the doubts were healed, the 12 athletes answered all the questions in about 20 minutes. 


\section{Analysis}

For data analysis, we used the support of graphics, and soon afterwards, we conducted the discussion, crossing the information collected with Norbert Elias' (1992) theory of the Civilization Process and Daniel Goleman's (2012) theory of Emotional Intelligence.

\section{RESULTS}

In the first question, all athletes were able to identify: joy (18\%), pleasure (16\%) and anger (13\%). In the second question three athletes answered "yes" and the others "no". In the third question, 8 athletes answered "yes", but considered the activities practiced outside of training time. In the fourth question, we find 7 "yes" answers, and among the justifications we identified 3 athletes who affirmed the same proposition "I avoid talking what I am not sure". In the fifth question, it was possible to perceive that the coach usually does the feedback, but in a negative way. In the sixth question, most of the athletes presented a negative answer. Finally, in the last question all athletes mentioned that there are no moments for the choice of a game or exercise.

\section{DISCUSSION}

We understand that the didactic-pedagogical elaboration used by the Physical Education technician during the training with adolescent athletes needs to involve intentional practices aimed at the formation of the subject in its entirety, considering the demands and needs of this phase of life. For this, the support of the adult, besides fundamental, can be efficient if there is clarification and understanding of these changes that interfere in the behaviour of the subjects. However, we found little evidence mentioning methodological procedures dedicated to understanding and identifying ideas and feelings.

\section{CONCLUSIONS}

The emotions of the U19 athletes of the social project of volleyball are present during the training sessions and can be fundamental for the success of these young athletes in development. However, we noticed through the reports of the athletes that the coach does not offer moments of dialogue during the training for the development of emotional self-mastery. In this scenario, we understand that there is a long way to reach the transformation of teaching practice, whether in the sporting or social context. Emotion-oriented training needs to be discussed and dialogued in undergraduate and graduate courses, with new research that can clarify and elicit other possibilities for the practical reality of the Physical Education professional during sports training.

\section{REFERENCES}

Associação de Voleibol do Paraná (12 de outubro de 2018). Disponível em: https://www.avp.org.br/ Elias, N; Dunning, E. (1992). A busca da excitação. Lisboa: Difel.

Goleman, D. (2012). O cérebro e a inteligência emocional: novas perspectivas. Rio de Janeiro: Objetiva. Kerkoski, M. J. (2009). Prática Desportiva e Inteligência Emocional: estudo da influência do desporto na aquisição de aptidões e competências da inteligência emocional. Tese (doutorado). Instituto de Estudo da Criança, Universidade de Minho, Portugal. https://doi.org/10.21011/apn.2017.1502 


\section{(c) (i) (3)}

This work is licensed under a Attribution-NonCommercial-NoDerivatives 4.0 International (CC BY-NC-ND 4.0). 


\title{
Self-determined motivation and subjective well- being in Portuguese veteran athletes in different sports
}

\author{
MARCO BATISTA $1,2,3$, MARTA LEYTON4, JOÃO SERRANO1,2, JOÃO PETRICA ${ }^{1,2}$, SAMUEL \\ HONÓRIO1,2,3, JOÃO ROCHA ${ }^{1,2}$, JORGE SANTOS ${ }^{1,2}$, PAULO SILVEIRA ${ }^{1,2}$, RUTH JIMENEZ ${ }^{5}$ \\ ${ }^{1}$ Sport, Health \& Exercise Research Unit (SHERU / RECI), Castelo Branco, Portugal \\ 2 Instituto Politécnico de Castelo Branco, Castelo Branco, Portugal \\ ${ }^{3}$ Research in Education and Community Intervention, RECl- Instituto Piaget, Portugal \\ ${ }^{4}$ Center for Sport Studies, University of Rey Juan Carlos, Madrid, Spain \\ ${ }^{5}$ Research Group on Didactic and Behavioral Analysis of Sport, University of Extremadura, Cáceres. Spain
}

\begin{abstract}
With the increase of life expectancy associated to the need for a healthy lifestyle, there has been an increase in the number of persons involved in veterans' sports activities. The objective of this study was to evaluate the levels of self-determination for sports practice and subjective well-being in veteran athletes, depending on the different type of sports practiced. For data collection, the validated Portuguese versions of the Basic Psychological Needs Exercise Scale (BPNES), the Behaviour Regulation Sport Questionnaire (BRSQ), the Satisfaction with Life Scale (SWLS) and the Positive and Negative Affects Scale (PANAS) were used. The study sample consisted of 684 Portuguese veterans athletes of both genders, aged between 30 and 90 years $(\mathrm{M}=43.78 \mathrm{SD}=8.61$ ), of which $86.3 \%$ (590 individuals) of the male gender and $13,7 \%$ (94 individuals) of the female gender, mostly with 3 to 5 hours of training per week, with $19.5 \pm 12.2$ years of practice, competitors in several individual sports (43\%), team sports (37\%) and individual and teams sports (20\%). Veteran athletes are highly satisfied with basic psychological needs. When compared, athletes who practice individual sports and simultaneously individual and team sports differ significantly in the autonomous, controlled and amotivation compared to athletes who only practice team sports, as well as in positive and negative effects. Keywords: Self-determination; Satisfaction with life; Subjective well-being; Sport; Veterans.
\end{abstract}

\footnotetext{
Corresponding author. Instituto Politécnico de Castelo Branco, Av. Pedro Alvares Cabral 12, 6000-084 Castelo Branco, Portugal.

E-mail: marco.batista@ipcb.pt

Supplementary Issue: Spring Conferences of Sports Science. International Seminar of Physical Education, Leisure and Health, 17-19 June 2019. Castelo Branco, Portugal.

JOURNAL OF HUMAN SPORT \& EXERCISE ISSN 1988-5202

(C) Faculty of Education. University of Alicante.

doi:10.14198/jhse.2019.14.Proc4.82
} 


\section{INTRODUCTION}

Our society is constantly changing, and in the last decades an increase in life expectancy has been observed, accompanied by an increase in the percentage of individuals above 35 years of age to participate in physical activities of a competitive and non-competitive nature. In the case of veteran athletes, motivation must also be considered a psychological determinant that may interfere with the athlete's athletic performance (Batista et al., 2017). According to Sancho and Ruiz-Juan (2015), the motivations of these athletes are mainly intrinsic, although the extrinsic motivations, health reasons, the pleasure of practice, social relations and the competition itself are also very important. Diener et al. (1999) tells us that subjective well-being is a global judgment that the individual makes of himself in face of events and circumstances of life, which integrates a cognitive dimension, satisfaction with life and an affective dimension, regarding affections. The present study aims to evaluate the levels of self-determination for sports practice and subjective well-being in veteran athletes, depending on the type of sports practiced.

\section{MATERIAL AND METHODS}

\section{Participants}

The study sample consisted of 684 Portuguese veterans athletes of both genders, aged between 30 and 90 years ( $M=43.78 \mathrm{SD}=8.61$ ), of which $86.3 \%$ (590 individuals) of the male gender and $13,7 \%$ (94 individuals) of the female gender, mostly with 3 to 5 hours of training per week, with $19.5 \pm 12.2$ years of practice, competitors in several individual sports (43\%), team sports (37\%) and individual and teams sports $(20 \%)$.

\section{Measures}

The validated Portuguese versions of the Basic Psychological Needs Exercise Scale (BPNES) by Moutão, Cid, Alves, Leitão and Vlachopoulos (2012), the Behaviour Regulation Sport Questionnaire (BRSQ) by Monteiro, Moutão, Batista and Cid (2014) were used. Also, the Satisfaction with Life Scale (SWLS), Neto (1993) and the Positive and Negative Affects Scale (PANAS) by Galinha and Pais-Ribeiro (2005).

\section{Procedures}

Sports centres, such as clubs and associations, were selected, for a convenience sample (Cubo Delgado et al., 2011). The veteran athletes were contacted for the collaboration in the study, after their agreement, filled an informed consent. The described scales were administered in the presence of the investigator. The approximate time of was fifteen minutes.

\section{Analysis}

Statistical analysis included descriptive statistics (mean, standard deviation), internal consistency (Cronbach's alpha), One Way ANOVA with Sheffé post-hoc analysis and Cohen's d. Significance was set at $p<0.05$. All analyses were performed using SPSS 21.0 (SPSS Inc., Chicago).

\section{RESULTS}

All the subscales demonstrated satisfactory reliability (i.e., Cronbach's alpha $>0.70$ ). The descriptive values obtained were more favourable in all variables under analysis, in the athletes who practice individual and team sports, or only individual sports. Significant differences were observed between groups, particularly among athletes who only practice team sports compared to the other two groups, at the level of autonomous motivation, controlled motivation, amotivation, positive and negative effects, using Cohen's effect-size evaluated from small to moderates (Hattie, 2009). 
Table 1. Descriptive values of the variables under study and statistical significance according to the type of sports

\begin{tabular}{lcccccc}
\hline \multicolumn{1}{c}{ Variable } & $\alpha$ & \multicolumn{2}{c}{ Individual sports Team sports Individual and team sports } & Sig & Cohen's \\
\hline Autonomy perception & 0.78 & $4.04 \pm 0.63$ & $4.02 \pm 0.57$ & $4.14 \pm 0.53$ & 0.13 & \\
Competence perception & 0.71 & $4.04 \pm 0.53$ & $3.98 \pm 0.46$ & $4.07 \pm 0.50$ & 0.20 & \\
Social relations perception & 0.78 & $4.35 \pm 0.54$ & $4.27 \pm 0.55$ & $4.32 \pm 0.56$ & 0.27 & \\
Autonomous motivation & 0.88 & $5.72 \pm 0.94$ & $5.50 \pm 0.82$ & $5.75 \pm 0.75$ & $0.01^{* *}$ & 0.29 \\
Controlled motivation & 0.89 & $1.96 \pm 1.08$ & $2.11 \pm 1.15$ & $1.77 \pm 0.89$ & $0.01^{* *}$ & 0.32 \\
Amotivation & 0.85 & $2.16 \pm 1.27$ & $2.50 \pm 1.32$ & $2.03 \pm 1.00$ & $0.01^{* *}$ & 0.38 \\
Satisfaction with life & 0.87 & $5.17 \pm 1.05$ & $5.00 \pm 0.88$ & $5.12 \pm 0.95$ & 0.14 & \\
Positive affects & 0.85 & $3.66 \pm 0.60$ & $3.58 \pm 0.60$ & $3.73 \pm 0.46$ & $0.04^{*}$ & 0.26 \\
Negative affects & 0.86 & $1.70 \pm 0.54$ & $1.84 \pm 0.64$ & $1.73 \pm 0.56$ & $0.02^{*}$ & 0.24 \\
\hline
\end{tabular}

\section{DISCUSSION}

The results showed that the athletes who practice individual and team sports, or only individual sports, showed a higher satisfaction of the basic psychological needs, as well as a higher autonomous motivation. The values of controlled motivation and amotivation were shown to be lower, following the findings of Sancho and Ruiz-Juan (2015) or Batista et al. (2017). The levels of satisfaction with life and positive effects were higher, in disagreement with the production of negative effects, however in agreement with Diener et al. (1999) as well as the study by Batista et al. (2017).

\section{CONCLUSIONS}

Veteran athletes are highly satisfied with basic psychological needs. When compared, athletes who practice individual sports and simultaneously individual and team sports differ significantly in the autonomous, controlled and amotivation once compared with athletes who only practice team sports, as well as in the production of positive and negative effects.

\section{REFERENCES}

Batista, M.; Jimenez Castuera, R.; Leyton, M.; Aspano, M.; Lobato, S. (2017). Self-determined motivation and life satisfaction in Portuguese veterans athletes. Retos. 32, 124-129. ISSN: Edición impresa: 1579-1726. Edición Web: 1988-2041.

Cubo-Delgado, S.; Martín-Marin, B. \& Ramos-Sanchez, J. L. (2011). Métodos de Investigación y Análisis de Datos en Ciências Sociales y de la Salud. Madrid: Pirâmide.

Diener, E., Suh, E. M., Lucas, R. E., \& Smith, H. L. (1999). Subjective well-being: Three decades of progress. Psychological Bulletin, 125, 276-302. https://doi.org/10.1037/0033-2909.125.2.276

Hattie, J. (2009). Visible Learning. London: Routledge.

Sancho, A. Z., \& Ruiz-Juan, F. (2015). Factores determinantes de la motivación en atletas veteranos españoles. Revista Latinoamericana de Psicología, 47(1), 34-42. https://doi.org/10.1016/s0120$\underline{0534(15) 30004-2}$ 


\section{(c) (i) (3)}

This work is licensed under a Attribution-NonCommercial-NoDerivatives 4.0 International (CC BY-NC-ND 4.0). 


\title{
Self-determined motivation and subjective well- being of adapted sport athletes members of Special Olympics
}

\author{
MARCO BATISTA ${ }^{1,2,3}$, ANA DOMINGUES 2 , INÊS FERNANDES 2 , JOÃO DOMINGUES 2 , SANDRA \\ RIBEIRO2 ${ }^{2}$ PEDRO PIRES ${ }^{2,4}$, HELENA MESQUITA ${ }^{2,5}$, SERGIO IBAÑEZ 6 \\ ${ }^{1}$ Sport, Health \& Exercise Research Unit (SHERU / RECI), Castelo Branco, Portugal \\ 2 Instituto Politécnico de Castelo Branco, Castelo Branco, Portugal \\ ${ }^{3}$ Research in Education and Community Intervention, RECl- Instituto Piaget, Portugal \\ ${ }^{4}$ Associação Portuguesa de Pais e Amigos do Cidadão Deficiente Mental, Castelo Branco, Portugal \\ ${ }^{5}$ Centro Interdisciplinar de Ciências Sociais (CICS.NOVA), Portugal \\ 6 Universidad de Extremadura, Facultad de Deporte, Cáceres, Spain
}

\begin{abstract}
Adapted sport is an organized participation whose objective is to obtain improvement in physical and psychic conditions, that is, it represents one of the most important factors promoting educational success, inclusion and psychosocial development. The objective of this study was to evaluate the self-determined competences for the practice of sport and the levels of well-being, in athletes with Intellectual and Developmental Difficulties (DID) participating in the Special Olympics in Portugal, to be correlated as variables in study and which variables of self-determination are predictors of subjective well-being in this collective. Participated 54 Adapted Sport athletes, with DID, belonging to Portuguese Private Social Solidarity Institutions, participating in the Special Olympics competitive regime. Of the 54 athletes, 12 are female $(22.22 \%)$ and 42 males (77.78\%), aged between 11 and 53 years old ( $\dot{x}=29.57$ and $S D=10.59)$. For the data collection, the following validated scales were applied for the Portuguese population, The Basis Psychological Needs in Exercise Scale, The Behavioural Regulation in Sport Questionnaire, The Satisfaction with Life Scale, and The Positive and Negative Affect Scale. The athletes with intellectual and developmental difficulties (DID) participating in the Special Olympics revealed a high self-determined motivation for sports practice, and their subjective wellbeing was high enough and dependent on their levels of autonomous motivation. The regression models revealed that the best predictor of subjective well-being in this group was the autonomous motivation. Keywords: Adapted Sport; Intellectual and Developmental Difficulties (DID); Self-Determination theory; Subjective well-being; Special Olympics.

\footnotetext{
Corresponding author. Health \& Exercise Research Unit, SHERU, Instituto Politécnico de Castelo Branco, Castelo Branco, Portugal.

E-mail: marco.batista@ipcb.pt

Supplementary Issue: Spring Conferences of Sports Science. International Seminar of Physical Education, Leisure and Health, 17-19 June 2019. Castelo Branco, Portugal.

JOURNAL OF HUMAN SPORT \& EXERCISE ISSN 1988-5202

(c) Faculty of Education. University of Alicante.

doi:10.14198/jhse.2019.14.Proc4.82
} 


\section{INTRODUCTION}

The research developed in the sphere of Sport Sciences is based on the benefits of sport, being a psychological, physical and social level, and which have a positive impact on the gains of autonomy and selfconfidence (Celestino \& Pereira, 2015). The Self-Determination Teria (Deci \& Ryan, 2012) allows us to motivate the athletes to a sporting practice by providing an opportunity for better intervention in the training and competition process, guaranteeing the consequence, levels of subjective wellbeing in the highest practice (Batista, Lercas, Santos, Honório, Serrano and Petrica, 2018). In this sense, the objective of this study was to evaluate the self-determined competences for the practice of sport and the levels of well-being, in athletes with Intellectual and Developmental Difficulties (DID) participating in the Special Olympics in Portugal, to be correlated as variables in study and which variables of self-determination are predictors of subjective well-being in this collective.

\section{MATERIAL AND METHODS}

\section{Participants}

Participated 54 Adapted Sport athletes, with DID, belonging to Portuguese Private Social Solidarity Institutions, participating in the Special Olympics competitive regime. Of the 54 athletes, 12 are female $(22.22 \%)$ and 42 males $(77.78 \%)$, aged between 11 and 53 years old $(\dot{x}=29.57$ and $S D=10.59)$.

\section{Instruments}

For the data collection, the following validated scales were applied for the Portuguese population, The Basis Psychological Needs in Exercise Scale (Moutão, Cid, Alves, Leitão \& Vlachopoulos, 2012), The Behavioural Regulation in Sport Questionnaire (Monteiro, Moutão, Batista \& Cid, 2014), The Satisfaction with Life Scale (Albuquerque, Sousa \& Martins, 2010), and The Positive and Negative Affect Scale (Galinha \& Pais-Ribeiro, 2005).

\section{Procedures}

The institutional ethics committee of the first author provided ethical approval for this study. After obtaining approval from the team managers or coaches, signed informed consents were obtained from the athletes or by the legal guardians. Athletes completed the questionnaire one hour before the first match of the tournament. The instrument was completed, individually, with support the of researchers.

\section{Analysis}

Statistical analysis included descriptive statistics (mean, standard deviation), internal consistency (Cronbach's alpha), Spearman correlations and multivariable linear regression. Significance was set at $p<$ 0.05. All analyses were performed using SPSS 21.0 (SPSS Inc., Chicago).

\section{RESULTS}

All of the subscales demonstrated satisfactory reliability (i.e., Cronbach's alpha $>0.60)$ (Hardy et al., 2010).

We observed that athletes with DID presented high levels of satisfaction of basic psychological needs, as well as autonomous motivation for sports practice and, consequently, high satisfaction with life and positive effects. They also showed reduced values of controlled motivation, amotivation, and negative effects. The correlations between the studied variables were moderate, respecting the expected meaning. 
Multivariable linear regression analysis using the satisfaction with life as the dependent variable showed that autonomous motivation $(\beta=0.40, p<0.05)$, was the significant predictor in the model $\left(R^{2}=0.23, p=0.046\right)$, and the analysis using the positive effects as the dependent variable showed that autonomous motivation $(\beta=0.65, p<0.01)$, was the significant predictor in the model $\left(R^{2}=0.33, p=0.026\right)$.

Table 1. Descriptive data of the variables analysed in the sample, normality and Cronbach's

\begin{tabular}{ccccccc}
\hline & min. & Max. & Mean & SD & KS & $\alpha$ \\
\hline Autonomy & 2.00 & 5.00 & 4.44 & 0.71 & $0.01^{*}$ & 0.60 \\
Competence & 3.63 & 5.00 & 4.78 & 0.34 & $0.01^{*}$ & 0.60 \\
Relationship & 3.63 & 5.00 & 4.88 & 0.32 & $0.01^{*}$ & 0.60 \\
\hline Mot. Autonomous & 3.00 & 7.00 & 6.69 & 0.68 & $0.02^{*}$ & 0.81 \\
Mot. Controlled & 1.00 & 7.00 & 2.30 & 1.68 & $0.01^{*}$ & 0.75 \\
Amotivation & 1.00 & 6.00 & 2.19 & 1.48 & $0.01^{*}$ & 0.62 \\
\hline SWL & 1.75 & 7.00 & 6.46 & 1.06 & $0.00^{*}$ & 0.85 \\
Aff. Positives & 2.78 & 5.00 & 4.32 & 0.52 & $0.00^{*}$ & 0.60 \\
Aff. Negatives & 1.00 & 3.89 & 1.70 & 0.74 & $0.09^{*}$ & 0.85 \\
\hline
\end{tabular}

\section{DISCUSSION}

The descriptive results obtained in relation to self-determined motivation and subjective well-being of athletes with DID respect the findings of Batista et al. (2018) and Ferreira, Petrica \& Batista (2018) developed with adapted athletes without DID. The regression models revealed that the best predictor of subjective well-being in this group was the autonomous motivation, which is not in complete agreement with the studies cited, since the satisfaction of the basic psychological needs was equally good predictors.

\section{CONCLUSION}

The athletes with intellectual and developmental difficulties (DID) participating in the Special Olympics revealed a high self-determined motivation for sports practice, and their subjective well-being was high enough and dependent on their levels of autonomous motivation.

\section{REFERENCES}

Batista, M., Lercas, A., Santos, J., Honório, S., Serrano, J. \& Petrica, J. (2018). Practice motivation and life satisfaction of athletes of team sports: Comparative study between adapted and regular sport. Journal of Human Sport \& Exercise, 14(1), p. 20-29. https://doi.org/10.14198/jhse.2019.14.proc1.03

Celestino, T. \& Pereira, A. (2015). Orientação Adaptada: Caraterísticas e Potencialidade de um Desporto de Inclusão.

Deci, E, \& Ryan, R. (2012). Self-determination theory. In A. W. Kruglanski, P. A. M. Van Lange, \& E. T. Higgins (Eds.), Handbook of theories social psychology (pp. 416-437). London: Sage.

Ferreira, D., Petrica, J. \& Batista, M. (2018). Motivação Autodeterminada, Satisfação com a Vida e Integração Social de Atletas de Desporto Adaptado no Atletismo. Desporto e Atividade Física para Todos - Revista Científica da FPDD, 4(1), p. 14-23. https://doi.org/10.24197/aefd.2-3.2018.256-278 
Hardy, L., Roberts, R., Thomas, P., \& Murphy, S. (2010). Test of Performance Strategies (TOPS): Instrument refinement using confirmatory factor analysis. Psychol Sport Exerc, 11(1), 27-35. https://doi.org/10.1016/i.psychsport.2009.04.007

\section{(c)}

This work is licensed under a Attribution-NonCommercial-NoDerivatives 4.0 International (CC BY-NC-ND 4.0). 


\title{
Monitoring workload in women's basketball based on player tracking device
}

\author{
MARÍA REINA ${ }^{1}$, JAVIER GARCÍA-RUBIO¹, ANTONIO ANTÚNEZ², LUIS CÓRDOBA², SERGIO J. \\ IBÁÑEZ1
}

${ }^{1}$ Grupo de Optimización del Entrenamiento y el Rendimiento Deportivo, Facultad de Ciencias del Deporte, Universidad de Extremadura, Cáceres, Spain

${ }^{2}$ Grupo de Optimización del Entrenamiento y el Rendimiento Deportivo, Facultad de Educación, Universidad de Extremadura, Badajoz, Spain

\begin{abstract}
Knowing how the efforts are distributed during the sports competition is necessary to plan the training loads. However, in the field of women's basketball there is not enough information. So, the objective of this study was to characterize the competition load of women's basketball. The participants were forty-eight U18 women's basketball players belonged to four teams classified for the final four junior championship, a total of forty-eight U18 women's basketball players. Twelve matches were registered. The variables analysed were: Total distance, explosive distance, distance and percentage of time in each intensity group (walking, jogging, running and sprinting), number of accelerations, decelerations, impacts, jumps and player load. Accumulative measurement and intensity measurement were used. Each player was equipped with a WIMUPROTM inertial device Ultra-Wide Band (UWB) systems on the court. The main results were that the players can perform 5000 meters during a match but only $15 \%$ explosively. A greater number of accelerations than decelerations are performed. The jumping actions are superior to what was found in other studies. Finally, the load supported by the players differs greatly depending on the minutes played. In conclusion, the training load should be modulated in microcycles determined by the matches. In this way, it allows prescribing to specific training, preventing injuries and, therefore, increasing the performance of athletes. Keywords: Basketball; Women; External load; Tracking devices; Competition.
\end{abstract}

Corresponding author. Avda de la Universidad s/n, Facultad de Ciencias del deporte, 10003, Cáceres, Spain.

E-mail: mreinarom@unex.es

Supplementary Issue: Spring Conferences of Sports Science. International Seminar of Physical Education, Leisure and Health, 17-19 June 2019. Castelo Branco, Portugal.

JOURNAL OF HUMAN SPORT \& EXERCISE ISSN 1988-5202

(c) Faculty of Education. University of Alicante.

doi:10.14198/jhse.2019.14.Proc4.82 


\section{INTRODUCTION}

The monitoring of the training and competition load in collective sports is booming. There are many studies that support this topic and, as scientific knowledge advances, the measurement systems are more precise and the data they provide increases considerably (Fox et al., 2018). However, in the field of women's basketball, the most investigated object of study are injuries (Boles and Ferguson, 2010). Therefore, the analysis of the load caused by training and competition would have to assume a more important role, in order to comprehensively study women's basketball. For that reason, the objective of this study was to characterize the competition load of a final four of women's basketball U' 18 .

\section{MATERIAL AND METHODS}

\section{Participants}

The participants belonged to four teams, classified for the final four junior women's championship, a total of forty-eight U18 women's basketball players. Each team played three matches; therefore, twelve competition matches were analysed.

\section{Measures}

The variables used to describe the external load (EL) were:

- Distance (D): Volume of meters made by the player while on the field. It also includes the distance travelled explosively (ED), $>15 \mathrm{~km} / \mathrm{h}$ and percentage of time in each intensity group (walking, jogging, running and sprinting);

- Acceleration (ACC) and deceleration (DEC): Speed changes made during the match, total and per minute. It's are specific movements of basketball, including starts, braking and changes of direction;

- Jumps (J): Movement that consists in elevating oneself from the court with an impulse that implies more than $400 \mathrm{~ms}$ of flight time before landing again, in the same or another place. The manufacturer's software (SPROTM) reference measurements have been used;

- Player Load (PL): Neuromuscular load that the player receives during the activity per minute. It is an objective load measurement, validated and calculated from accelerometer signal in the 3 axes.

\section{Procedures}

Data related to EL were recorded using a position-tracking and Ultra-Wide Band (UWB) systems on the court. Each player was equipped with a WIMUPROTM inertial device that was placed into a specific custom-made vest fitted tightly onto the back of the upper torso. The UWB system was adjusted to the reference field before the start of the investigation, by going around the perimeter of the field so that it would be recognized as the reference system. This system is composed of six antennas placed in a hexagon around the playing field.

\section{Analysis}

Data were normalised in two different ways: Accumulative measurement (sum of all actions performed in the total game time) and intensity measurement (actions per minute of play). A descriptive analysis was performed using means and standard deviations. The statistical analysis was performed with SPSS (IBM Corp. Released 2017. IBM SPSS Statistics for Windows, Version 25.0. Armonk, NY:IBM Corp.).

\section{RESULTS}

These data allow to have a first view of the demand generated by the competition in women's basketball (Table 1). It is observed how the players travel a maximum distance of around 5000 meters per game, of 
which not even $15 \%$ is covered at the maximum intensity. The players spend most of the game in speeds between 5 and $7 \mathrm{~km} / \mathrm{h}$ (jogging), exceeding in very few occasions the $14 \mathrm{~km} / \mathrm{h}$ (Figure 1). They perform around 10-20 accelerations per minute, and can perform up to 6 jumps per minute. A match can assume a load measured in Player Load of 84 arbitrary units. By establishing this value as the maximum load, fatigue caused by competition in the other players can be established.

Table 1. Load of competition in women's basketball U'18

\begin{tabular}{cccccccccccc}
\hline & $\mathrm{D}$ & $\mathrm{D} / \mathrm{min}$ & $\mathrm{ED}$ & $\mathrm{ACC}$ & $\mathrm{ACC} / \mathrm{min}$ & $\mathrm{DEC}$ & $\mathrm{DEC} / \mathrm{min}$ & $\mathrm{J}$ & $\mathrm{J} / \mathrm{min}$ & $\mathrm{PL}$ & $\mathrm{PL} / \mathrm{min}$ \\
\hline MAX & 5097 & 104 & 712 & 1144 & 20 & 475 & 10 & 237 & 6 & 84 & 2 \\
AVG & 2305 & 72 & 296 & 343 & 11 & 205 & 6 & 62 & 2 & 36 & 1 \\
SD & 1412 & 19 & 176 & 290 & 6 & 123 & 2 & 50 & 1 & 23 & 0 \\
\hline
\end{tabular}

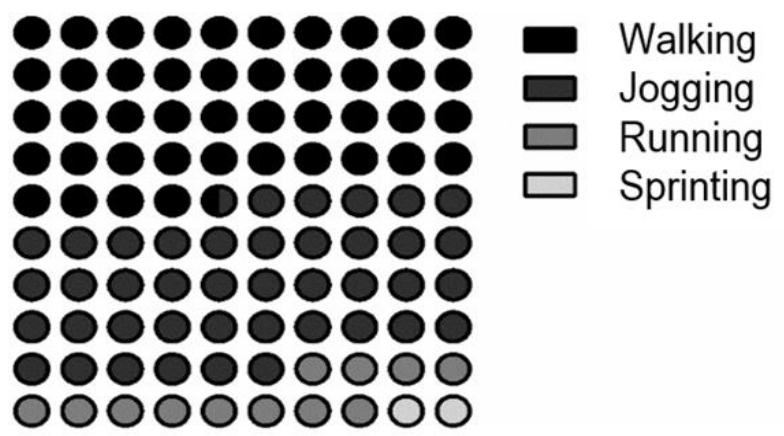

Figure 1. Meters performed by speed zones

\section{DISCUSSION}

This information is of vital importance because it allows prescribing a specific training, preventing injuries and, therefore, increasing the performance of athletes (Fox et al., 2017). According to Vargas et al., (2015), the training load should be modulated in microcycles determined by the matches, reducing fatigue and preparing the necessary effects for facing the competition in the best possible condition.

\section{CONCLUSIONS}

According to the results obtained, it can be considered important: work in a high-intensity race without forgetting the aerobic work done during most of the game; the competition gives rise to a greater number of accelerations, so it would be convenient to pay more attention; abdominal and lumbar work is considered necessary to combat the load and impact caused by the jumping action; it would be important to regulate the training load depending on the minutes played since the fatigue value differs.

\section{REFERENCES}

Boles, C. A., \& Ferguson, C. (2010). The female athlete. Radiologic Clinics, 48(6), 1249-1266. https://doi.org/10.1016/j.rcl.2010.07.015

Fox, J., Scanlan, A., \& Stanton, R. (2017). A review of player monitoring approaches in basketball: Current trends and future directions. J Strength Cond Res, 31(7), 2021-2029. https://doi.org/10.1519/jsc.0000000000001964 
Fox, J., Stanton, R., \& Scanlan, A. (2018). A Comparison of Training and Competition Demands in Semiprofessional Male Basketball Players. Res Q Exerc Sport, 89(1), 103-111. https://doi.org/10.1080/02701367.2017.1410693

Vargas, A., Urkiza, I., \& Orozko, S. (2015). Efecto de los partidos de pretemporada en la planificación deportiva: Variabilidad en las sesiones de entrenamiento. Retos, (27), 45-51.

\section{(c) $(\mathrm{B})(\mathrm{EY}$}

This work is licensed under a Attribution-NonCommercial-NoDerivatives 4.0 International (CC BY-NC-ND 4.0). 


\title{
The athlete's perception of parents behaviors in sport context: A study in youth handball players of the Madeira Handball Association
}

\author{
DUARTE SOUSA ${ }^{1} \triangle$, AMÉRICO CARDOSO ${ }^{1}$, ANA RODRIGUES ${ }^{1}$, JOÃO NOITE${ }^{1}$, HELDER LOPES ${ }^{1,2}$, \\ CATARINA FERNANDO ${ }^{1,2}$, JOÃO PRUDENTE ${ }^{1,2}$ \\ ${ }^{1}$ Department of Physical Education and Sport, Faculty of Social Sciences, University of Madeira, Portugal \\ ${ }^{2}$ C.I.D.E.S.D., Portugal
}

\begin{abstract}
The participation of parents in their children's sports is very important. However, many parents end up having negative and deviant behaviours in sports and social context, generating this participation some controversy and misunderstandings. With this study, we intend to assess the perception of the athletes about the behaviour of the parents in the sport context, considering the demographic characteristics of the athletes (gender and age). The sample is composed by young handball players from the Madeira Handball Association, aged $9-16$ years of both sexes $(n=284$, male $n=122$, female $n=162)$ registered by ten clubs and with a minimum of one year of organized sports practice. For the collection of data, a questionnaire previously constructed and duly validated was used. The results confirm the perception of the role of parents in supporting their children 's sports practices, as well as motivating them for the sport. There is also a perception of the young players of a pressure exerted by the parents in obtaining immediate results and little appreciation of the progress of learning. Keywords: Parents; Handball; Perception; Behaviour; Training.
\end{abstract}

Corresponding author. Campus da Penteada, 9020-105 Funchal, Portugal.

E-mail: duartesousaa@gmail.com

Supplementary Issue: Spring Conferences of Sports Science. International Seminar of Physical Education, Leisure and Health, 17-19 June 2019. Castelo Branco, Portugal.

JOURNAL OF HUMAN SPORT \& EXERCISE ISSN 1988-5202

(C) Faculty of Education. University of Alicante.

doi:10.14198/jhse.2019.14.Proc4.82 


\section{INTRODUCTION}

With this study we intend to gauge how the young athletes perceive the behaviour and attitudes of their parents in the sporting context. Some authors point out the involvement of the family as an important factor in the physical, social and psychological development of young athletes (Buceta, 2015; Byrne, 1993; Sobral, Coelho-e-Silva, 2002). In recent years there have been reports of problems in competitions involving the parents of young handball players with increasingly frequent deviant behaviours, which will help us find answers about how young people perceive and characterize these behaviours and attitudes, that previous studies refer to the discomfort and embarrassment of young people about their parents' wrong attitudes and behaviours (Buceta, 2015).

\section{MATERIAL AND METHODS}

\section{Participants}

A total of 284 children and adolescents ( 122 boys and 162 girls), aged between 9 and 16 years old from u10 to $u-16(12.82+1.67)$, enrolled at least one year in 10 clubs of the Handball Association of Madeira.

\section{Measures}

For this study, a questionnaire was constructed and validated through the Delphi method (Severino, 2014), by discussion and consensus among six handball experts. Subsequently, reliability was determined through the pilot study to quantify the consistency of the information collected and to identify possible ambiguous issues, high levels of intraclass correlation coefficient demonstrate the consistency of the information collected (ICC between .890 and .993). In this way, the final version of the questionnaire was composed of 38 affirmations according to a Likert scale from 1 (Never) to 5 (Always) and structured in the following dimensions: Characterization of the Practitioner, Participation of Parents in Children's Sports Practice, Fair play, Parent Types, and Athlete Perception of Parent Behaviour.

\section{Procedures}

The project was approved by the Scientific Committee of the Department of Physical Education and Sport of the University of Madeira. Participants were allowed to participate in the study through consent of the caregivers. Fulfilment of the questionnaires lasted for approximately 15 minutes. The questionnaires were applied by trained field team members who showed up for any requests for clarification that might arise.

\section{Analysis}

The mean and standard deviation were used to characterize the sample. Factor analysis was used to determine the dimensions, with 3 dimensions being extracted ("Extrinsic valorisation", "Support and Involvement of the Parents" "Individual Intrinsic Valorisation".

Pearson's correlations were used to analyse the association between scores of three dimensions and age. Student's t-test was used to study the differences between genders in the obtained scores. The statistical software used was SPSS v.25.0 and the level of significance was $5 \%$.

\section{RESULTS}

The results show that the parents are participative, with presence in competitions $(73.6 \%)$ and in training (73.1\%). However, only $43.5 \%$ realize that parents are actively participating in the monitoring of their participation in the sport. 
Two in three athletes $(73.5 \%)$ have the perception that parents want them to be champions, but only $17 \%$ realize that parents want them to be called to the regional team or that they are the best players in the team $(11.8 \%)$.

The perception that parents are angry when they lose was reported by $20 \%$ of the young respondents, although $92.9 \%$ of the athletes perceived that parents wanted them to be happy in sports and $81.4 \%$ said that parents value the acquisition of new techniques.

Through the extraction of the scores by analysis in main components, three scores were determined: (i) "Extrinsic Valorisation" (ii) "Support and Involvement of Parents" and (iii) "Intrinsic Individual Valorisation". It is verified that between the genders the boys have a perception of a greater pressure on the part of the parents in the dimension "Extrinsic Valorisation" ( $t(282)=2.731, p=.010)$, not being verified differences in the remaining dimensions. Considering age, we found only association with statistical significance with the dimension "Support and Involvement of Parents" ( $r=-0.334, p<.001)$ and only in boys.

\section{DISCUSSION}

Most of the young players live with the perception of a pressure on the immediate results as the fact that the parents want their children to be champions or that they reach the level of their team or of calls to the regional selections, results that go to meet the Hollins study (2016) which states that young people feel pressure rather than support when their parents are very involved in their sport. These results are more felt by the boys, probably because their parents have a greater desire of the children to reach prominent positions in the modality (Buceta, 2015). In this case, the Hollins study (2016) states that in many cases parents are more emotionally involved in children's sports participation than they are, supporting early specialization because they are too focused on immediate outcomes.

\section{CONCLUSIONS}

The presence and participation of the parents is unequivocal, starting from the perception of the athletes, which reveals some negative aspects. Although most young people are aware of the importance given by parents to the positive aspects of sports as fundamental to their personal and social development, there is a high number of young people who feel exaggerated pressure from their parents. It is important to highlight the perception of many athletes that their parents are angry when the results of the team are not what they want.

\section{REFERENCES}

Byrne, T. (1993). Sport: it's a family affair. In M. Lee (ed.), Coaching children in sport: principles and practice. London: E \& FN Spon.

Buceta, J.M.(2015). Mi hijo es el mejor, y además es mi hijo: manual de cabecera para padres de deportistas jóvenes. Madrid: Editorial Dykinson.

Hollins, D. K. (2016). When enough is enough: The impact os parental involvement in children's participation in organized sports. University Honors Theres. Paper 223. https://doi.org/10.15760/honors.222

Severino, A. (2014). Metodologia de um trabalho ciêntifico. Cortez Editora. S. Paulo. 


\section{(c) (i) (3)}

This work is licensed under a Attribution-NonCommercial-NoDerivatives 4.0 International (CC BY-NC-ND 4.0). 


\title{
Fair play : The perception of the young handball players on the parent's behavior
}

\author{
DUARTE SOUSA ${ }^{1} \triangle$, AMÉRICO CARDOSO ${ }^{1}$, ANA RODRIGUES ${ }^{1}$, JOÃO NOITE ${ }^{1}$, HELDER LOPES ${ }^{1,2}$, \\ CATARINA FERNANDO 1,2, JOÃO PRUDENTE ${ }^{1,2}$ \\ ${ }^{1}$ Department of Physical Education and Sport, Faculty of Social Sciences, University of Madeira, Portugal \\ ${ }^{2}$ C.I.D.E.S.D., Portugal
}

\begin{abstract}
In recent years, the deviant and negative behaviours of parents during follow-up in their children's sports practice have reached alarming proportions in various sports. This increase may be associated with greater participation of parents, both in the presence in games and in training. With this study, we intend to assess the perception of the athletes about the behaviour of the parents in the sports context, namely in relation to fair play. The sample consisted of young handball players from the Madeira Handball Association, aged 916 years of both genders $(n=284$, male $n=122$, female $n=162$ ) registered by ten clubs and with a minimum of one year of organized sports practice. For the data collection, a questionnaire previously constructed and duly validated was used. In this study were used descriptive statistics (mean and standard deviation), chisquare and Spearman correlations. The statistical program used was SPSS v.25.0 and the level of significance was $5 \%$. The results confirm that the athletes consider positive the presence of their parents, however, the results point to a perception of inadequate behaviours of the parents regarding the referees, mainly by the boys. It was found that the younger the athletes are the greater is the perception of the enthusiastic support of the parents, the greater the volume of instructions to the field, and the perception of a greater emotional discontent of the parents in relation to the problems and incidents of the game and training. Keywords: Parents; Handball; Perception; Fair play; Training.
\end{abstract}

Corresponding author. Campus da Penteada, 9020-105 Funchal, Portugal.

E-mail: duartesousaa@gmail.com

Supplementary Issue: Spring Conferences of Sports Science. International Seminar of Physical Education, Leisure and Health, 17-19 June 2019. Castelo Branco, Portugal.

JOURNAL OF HUMAN SPORT \& EXERCISE ISSN 1988-5202

(c) Faculty of Education. University of Alicante.

doi:10.14198/jhse.2019.14.Proc4.82 


\section{INTRODUCTION}

The development of young athletes requires family involvement (Byrne, 1993) and the presence of parents in the various areas of action and interest of the children. Sports context is one of these areas and parental intervention has been increasing. However, this greater participation has led to the occurrence of many negative and controversial situations arising from this presence, which leads us to understand how young people perceive and characterize their parents' behaviours in this context, regarding fair play.

\section{MATERIAL AND METHODS}

\section{Participants}

A total of 284 children and adolescents ( 122 boys and 162 girls), aged between 9 and 16 years old from u10 to $u-16(12.82+1.67)$, enrolled at least one year in 10 clubs of the Madeira Handball Association.

\section{Measures}

For this study, a questionnaire was constructed and validated through the Delphi method (Severino, 2014), by discussion and consensus among 6 Handball specialists. Subsequently reliability was determined through the pilot study to quantify the consistency of the information collected and identify possible ambiguous questions. High levels of intraclass correlation coefficients demonstrate the consistency of the information collected (.911 to .976). In this way, the final version of the questionnaire was composed of 38 affirmations according to a Likert scale from 1 (Never) to 5 (Always) and structured in the following dimensions: Characterization of the Practitioner, Participation of Parents in Children's Sports Practice, Fair play, Parent Types, and Athlete Perception of Parent Behaviour. In this study, only Fair play data was used.

\section{Procedures}

The project was approved by the Scientific Committee of the Department of Physical Education and Sport of the University of Madeira. Participants were allowed to participate in the study through consent of the tutors. Questionnaires making lasted for approximately 15 minutes. The questionnaires were applied by field team members, who appeared to be available for any clarification requests that might arise.

\section{Analysis}

Descriptive statistics (mean and standard deviation) were used to characterize the sample of the variables under study, the chi-square test was used to determine the association between ordinal and nominal variables. Spearman correlations were used to determine the association between ordinal and discrete variables. The statistical program used was SPSS v. 25.0 and the level of significance was $5 \%$.

\section{RESULTS}

The results show that the majority of respondents have a perception of parental support for good team practices (83.6\%), but one in five states that parents have difficulties in dealing with problems and incidents of the game in a controlled manner (20.3\%). $42.5 \%$ of the athletes report that parents give instructions to the field, sometimes using offensive language $(11.2 \%)$. About one in three say that parents criticize their performance during games / training because they have made mistakes.

Regarding the referees, the young people reported that they respected them (75.8\%), although only $55.2 \%$ accepted their decisions. As for the opponents, most participants perceive that the parents respect them $(89.7 \%)$, value their abilities $(70.1 \%)$, however, only 1 in 3 applauds the handsome moves of the opponents 
$(33,7 \%)$. Considering the coaches, $23.1 \%$ of the participants have the perception that the parents question them, as well as their options.

There were differences between the genders in the perception of the behaviour of the parents in accepting the decisions of the referees, in applauding the handsome moves of the opponents and in the use of offensive language. The boys have a more negative perception of their parents' behaviour regarding the acceptance of referee decisions ( $[2=11.711, p=0.020)$ and the use of offensive language $([2=13.173, p=0.010)$ compared to girls. girls say that they have more difficulty applauding their opponents' handsome moves ([2 $=16.590, p=0.002$ ) compared to boys.

It is verified that the younger the athletes are: (i) the greater is the enthusiastic support to the team $(r=$ $0.247 ; p<0.001$ ); (ii) greater perception of the difficulty in dealing with problems and incidents of the game and training in a controlled way $(r=-0.140 ; p=0.019)$; (iii) and higher the perception of constant instructions within the field $(r=-0.166, p=0.005)$.

\section{DISCUSSION}

The presence of the parents proves to be positive for the majority of the young players. However, values associated with negative aspects still present high levels of in-field instruction by parents, often with inadequate language. It is also verified that the athletes perceive a questioning of the parents to their coaches, a situation that, according to the bibliography, confirms the results of Ross, Mallet and Parkes (2015) that report more negative interactions than positive in this relationship. This same study points to a considerable amount of negative communication between parents and children. However, Byrne (1993) refers to the need to work with parents so that they focus on their children's performance rather than outcome, and Buceta (2015) refers to the need for parents to be helped to train their emotions.

\section{CONCLUSIONS}

The results of the study refer to a mostly positive perception of athletes regarding their parents fair play and sportive behaviour. However, younger athletes are faced with a different perception from older ones, namely the amount of information and instruction given by the parents during training or competition. These athletes still perceive a greater difficulty of the parents in controlling themselves through the problems and incidents of the games and instructions constant in the field. Differences between genders were also found in the perception of parents' behaviour regarding referees and in applauding opponents.

\section{REFERENCES}

Byrne, T. (1993). Sport: it's a family affair. In M. Lee (ed.), Coaching children in sport: principles and practice. London: $E$ \& FN Spon.

Buceta, J.M. (2015). Mi hijo es el mejor, y además es mi hijo: manual de cabecera para padres de deportistas jóvenes. Madrid: Editorial Dykinson.

Ross, A.J., Mallet, C.J. and Parkes, J.F. (2015). The Influence of Parent Sport Behaviours on Children's Development: Youth Coach and Administrator Perspectives. International Journal of Sports Science \& Coaching. Vol 10, 4. https://doi.org/10.1260/1747-9541.10.4.605

Severino, A. (2014). Metodologia de um trabalho ciêntifico. S. Paulo. Cortez Editora. 


\section{(c) (i) (3)}

This work is licensed under a Attribution-NonCommercial-NoDerivatives 4.0 International (CC BY-NC-ND 4.0). 


\title{
The importance of imagery in acquiring and improving motor skills and sports technique
}

\author{
JOÃO PETRICA ${ }^{1,2} \triangle$, PEDRO DUARTE-MENDES ${ }^{1,2}$, DANIEL MARINHO ${ }^{3,4}$ \\ ${ }^{1}$ Polytechnic Institute of Castelo Branco, Portugal \\ ${ }^{2}$ SHERU (Sport, Health \& Exercise Research Unit), Portugal \\ 3 University of Beira Interior, Portugal \\ ${ }^{4}$ Research Centre in Sports, Health and Human Development (CIDESD), Portugal
}

\begin{abstract}
Imagery, in a simplistic way, is the mental image we have of movement. In order to understand whether there is an association between the imagery capability, as the ability we have to see and mentally feel the movement, and motor activity, we have been developing some complementary works seeking to better understand its relation with dribbling, passing, ball handling and shooting in football, with the technical skills of Basketball, with different levels of sports performance in swimming, and even with global and fine praxis or balance in individuals with disabilities and also to see if the gender has any relation to it. Using MIQ-3 as the main working instrument and specific motor tests for the skills to study, some works with different samples were carried out in a complementary logic that would allow us to better understand the extent of the importance of the study relationship and for which several statistical techniques were used, such as the Kaiser-Meyer-Olkino sampling adequacy measure, the Bartlett sphericity test, the exploratory factorial analysis, the Kolmogorov-Smirnov test, the Shapiro-Wilk test, the T-Student, the T-Test and the MannWhitney and Wilcoxon tests, as appropriate. We conclude that in most studies there is a clear association between imagery capacity and performance, achievement, or motor improvement. Keywords: Imagery; Motor improvement; Improvement of sports technique.
\end{abstract}

Corresponding author. Rua Prof. Dr. Faria de Vasconcelos, 6000-266 Castelo Branco, Portugal.

E-mail: ..petrica@ipcb.pt

Supplementary Issue: Spring Conferences of Sports Science. International Seminar of Physical Education, Leisure and Health, 17-19 June 2019. Castelo Branco, Portugal.

JOURNAL OF HUMAN SPORT \& EXERCISE ISSN 1988-5202

(c) Faculty of Education. University of Alicante.

doi:10.14198/jhse.2019.14.Proc4.82 


\section{INTRODUCTION}

The concept of imagery is related to the mental image we make of movement, being also understood as the mental creation or recreation of sensory experiences in the mind, and according to Hall (2001), refers to the visual and kinaesthetic properties of movement that can be reproduced mentally in the absence of physical movement. In turn, the capacity of imagery, which varies from subject to subject, is the ability to control and generate mental images of movement, which can be modifiable through training and experimentation (Cumming \& Williams, 2012; Hall, 2001) and, for Morris, Spittle \& Watt (2005), is the strategy and technique of mental training most used by professional athletes to perfect motor skills in order to optimize the technique of sports. Imagery is therefore a cognitive process that may play an important role in the planning and execution of movements or motor actions to improve their learning and improvement. In this sense we decided to study the relation between the capacity of imagery measured through MIQ-3, which we translated and validated (work 1), and motor execution, trying to understand: if it is associated to the learning of basic soccer sports techniques, such as the execution of the pass, dribbling and shooting in children (work 2); if there are differences in the different capacities of imagery in young soccer players (work 3); if it is related to the performance of technical skills (defensive sliding, dribbling, passing, throwing and technical circuit) in basketball, in young practitioners (work 4); if there are differences in the ability of imagery depending on the level of performance in swimming (work 5); or in the context of working with individuals with disabilities: if it is related to global praxis and fine praxis (work6); and if it has an influence on balance (work 7); and finally, if there is difference in the capacity of imagery between genders (work 8).

\section{MATERIAL AND METHODS}

\begin{tabular}{cccccl}
\multicolumn{2}{l}{ Participants } \\
\hline Work & $\mathbf{N}$ & \multicolumn{1}{c}{ O } & 9 & Age & Modality (N) \\
\hline 1 & 176 & 134 & 42 & $20.1 \pm 5.9$ & Football (113); Basketball (43); \& Tennis (20) \\
2 & 26 & 26 & & $12.3 \pm 0.5$ & Futebol (26) \\
3 & 53 & 53 & & $15.0 \pm 1.2$ & Football (53) \\
4 & 62 & 31 & 31 & $15.0 \pm 1.2$ & Basketball (62) \\
5 & 79 & 37 & 42 & $17 \pm 3.1$ & Swimming (79): Elite (29). Sub-elite (27) \& not-elite (23) \\
6 & 42 & & & $37 \pm 12$ & Global and fine praxis - People with disabilities \\
7 & 39 & & & $48 \pm 12$ & Balance - People with disabilities \\
8 & 62 & 31 & 31 & $15.0 \pm 1.2$ & Basketball (62) \\
\hline
\end{tabular}

\section{Measures}

As an instrument to measure or train the imagery capacity, the MIQ-3 was used (Mendes, Marinho, Petrica, Silveira, Monteiro \& Cid, 2016), in its internal visual, external visual and kinaesthetic dimensions, and to measure the motor skills were used specific tests of the modalities in question.

\section{Procedures}

In some cases the imagery capacity and the motor performance in specific tasks were measured in order to be able to perform the correlations, in others, MIQ-3 was simply used to measure imagery and in others a pre- and post-test was performed on specific skills and the evolution was studied after a training program based on MIQ-3. 


\section{Analysis}

For the analysis and processing of data were used various statistical techniques as the Kaiser-Meyer-Olkino sampling adequacy measure, the Bartlett sphericity test, the exploratory factorial analysis, the KolmogorovSmirnov test, the Shapiro-Wilk test, the T-Student, the T-Test, and the Mann-Whitney and Wilcoxon tests, as it was intended to check, correlate or compare values, taking care to ensure normality.

The probability of .05 was used as level of significance.

\section{RESULTS}

Work 2 - individuals with higher imagery capacity obtained better results in all specific tests, presenting a weak positive correlation $(0.1 \leq r<0.5)$.

Work 3 - the results showed significant differences between the kinaesthetic capacity and any of the visual abilities (external and internal) and between these, differences were not significant.

Work 4 - in the female gender, athletes with higher imagery capacity obtained better values in all the specific tests and a statistically significant weak / moderate correlation $(0.1 \leq r<0.5 / 0.5 \leq r<0.8)$, whereas in boys, this relation was not so evident.

Work 5 - In all modalities of imagery, the averages obtained show significant differences. The Elite group scored the highest, followed by Sub-Elite and Non-Elite.

Work 6 - we verified a positive evolution even if the differences were not statistically significant.

Work 7 - a relation between imagery and balance was not clear, slight improvements were observed in the values obtained in both static and dynamic balance.

Work 8 - there are statistically significant differences between averages $(p \leq 0.05)$, both in the kinaesthetic modality and in the total MIQ -3 , where the male gender presents a higher average.

\section{CONCLUSIONS}

The ability of imagery measured through MIQ-3, as a generic instrument of assessment or training, often shows a positive relationship with motor performance, whether in terms of learning, development and improvement, suggesting the need to evolve and take the next step for specific instruments for each activity to enhance its application and the generalization of its use.

\section{REFERENCES}

Cumming, J. \& Williams, S. (2012). The role of imagery in performance. In S. Murphy (Ed),Handbook of Sport and Performance Psychology (p. 213-232). New York: Oxford University Press.

Hall, C. (2001). Imagery in sport and exercise. In R. N. Singer, H. Hausenblas, \& C. Janelle, Handbook of sport psychology ( $2^{\mathrm{a}}$ ed., pp. 529-549). New York: John Wiley \& Sons.

Mendes, P., Marinho, D., Petrica, J., Silveira, P., Monteiro, D., e Cid, L. (2016). Tradução e Validação do Movement Imagery Questionnaire - 3 (MIQ - 3) com Atletas Portugueses. Motricidade, 12(1), 149-158. https://doi.org/10.6063/motricidade.7006

Morris, T., Spittle, M. \& Watt, A. (2005). Technical Aids to Imagery. In Imagery in Sport (pp. 237-266). Champaign, Illinois: Human Kinetics.

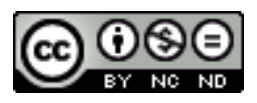

This work is licensed under a Attribution-NonCommercial-NoDerivatives 4.0 International (CC BY-NC-ND 4.0). 


\title{
Comparing differences in motor proficiency of children with and without Autism spectrum disorders
}

\author{
LUIZA ALBUQUERQUE ${ }^{1} \triangleleft$, CARLA LOURENÇO1,2, DULCE ESTEVES ${ }^{1,3}$, MÁRCIO SOARES $^{1}$ \\ 1 Universidade da Beira Interior, Portugal \\ ${ }^{2}$ Centers of Studies in Education, Technologies and Health (CI \& DETS), Portugal \\ ${ }^{3}$ Research Center in Sports Sciences, Health Sciences and Human Development (CIDESD), Portugal
}

\begin{abstract}
Individuals diagnosed with autism spectrum disorder (ASD) present a range of physical, cognitive, social, and behavioural deficits, including motor coordination impairments and deficits in social communication. Research suggests that exercise is beneficial for improving motor coordination, balance, and flexibility of children with ASD, however, relevant empirical research on comparing differences in motor proficiency of children with and without ASD is limited. With a case study method, we aim to examine differences presented in motor proficiency of a child with ASD, compared with others with a typical development in order to evaluate the need of a specific intervention in those competences. A group of 4 children (1 with ASD, 3 with a typical development), aged 4 years were evaluated. Motor proficiency was evaluated using Bruininks-Oseretsky test, short form. Results show that the child with ASD presents motor proficiency well below average, while other children present motor proficiency below average. Based on these findings, there is a need for an individualized intervention: a specific exercise program that meets the deficits in motor proficiency of the chid with ASD. Keywords: Autism spectrum disorders; Case study; Motor proficiency.
\end{abstract}

Corresponding author. Rua São João de Deus, 21- $1^{\circ}$ Esq., Covilhã, Portugal.

E-mail: mluiza.laurentino@gmail.com

Supplementary Issue: Spring Conferences of Sports Science. International Seminar of Physical Education, Leisure and Health, 17-19 June 2019. Castelo Branco, Portugal.

JOURNAL OF HUMAN SPORT \& EXERCISE ISSN 1988-5202

(c) Faculty of Education. University of Alicante.

doi:10.14198/jhse.2019.14.Proc4.82

S1492 | 2019| Proc4 | VOLUME 14

C 2019 University of Alicante 


\section{INTRODUCTION}

Autism spectrum disorder (ASD) is a neurological disorder characterized by impairments in communication and social skills, and associated with repetitive behaviour and stereotypical movements (Dillon et al., 2017). Research suggests that exercise is beneficial for improving children with ASD motor coordination, balance, and flexibility (Clare, Wong, Lo, So \& Chan, 2018). It also reduce their stereotypical behaviour and has positive effects on socialization, and communication (Bremer et al., 2016; Lang et al., 2018; Lourenço, et al., 2015; Sam et al., 2015). These investigations aims to examine differences presented in motor proficiency of a child with ASD, compared with others with a typical development. The secondary objective is designing an individualised exercise program that meets the major deficits identified, in order to improve motor proficiency.

\section{MATERIAL AND METHODS}

\section{Participants}

We sampled 4 boys, 1 diagnosed with ASD by specialized medical personnel and 3 with a typical development. These children were 4 years of age and were attending preschool.

\section{Measures}

To evaluate de motor proficiency of all participants we used the Bruininks-Oseretsky test (2nd ed., 2005), short form (Bot-2). This battery consists of the following 12 trials: fine motor precision (two tests: completion of a star and line drawing through a path); fine motor integration (two tests, drawing two overlapping circles and drawing a diamond as similar as possible to the images presented); manual dexterity (threading blocks); bilateral coordination (two tests: touching one's nose with the tip of the index finger, pivoting index and thumb while keeping the eyes closed); balance (move forward on a line - heel-to toe); speed and flexibility (one leg walk from one side of a line to the other); coordination of upper limbs (two tests: catching and throwing a ball with one hand and dribbling the ball alternating hands), and strength (performing kneeling push-ups). These tests were applied individually to each child, according to the published protocol (Bruinninks \& Bruininks, 2005), and lasted from 15 to 20 minutes.

\section{Procedures}

Parents were completely free to allow their child to participate in the study, after the presentation of researchers' affiliation, investigation goals and guaranty of total confidentiality. An informed consent was presented and signed. The study protocol is conformed to the Declaration of Helsinki.

\section{RESULTS}

Bot-2 results are presented in Figure 1. Considering the descriptive categories to standard scores of motor proficiency, child with ASD presents motor proficiency well below average, while other children present motor proficiency below average. Results show that the child with ASD presents several deficits in motor proficiency, such as balance, coordination (upper and lower limbs), bilateral coordination and fine motor skills.

\section{DISCUSSION}

Regarding motor proficiency, differences were found between a child with ASD and others with a typical development. Similar results were found by Liu et al. (2017) and Lourenço et al. (2015). The exercise should be seen as a complementary therapy for children with autism, given their potential to improve motor 
proficiency items such as coordination, balance, proprioceptive sense and laterality (Clare et al., 2018, Dillon et al, 2017). Therefore, results are the basis to design exercises to improve the deficits found.

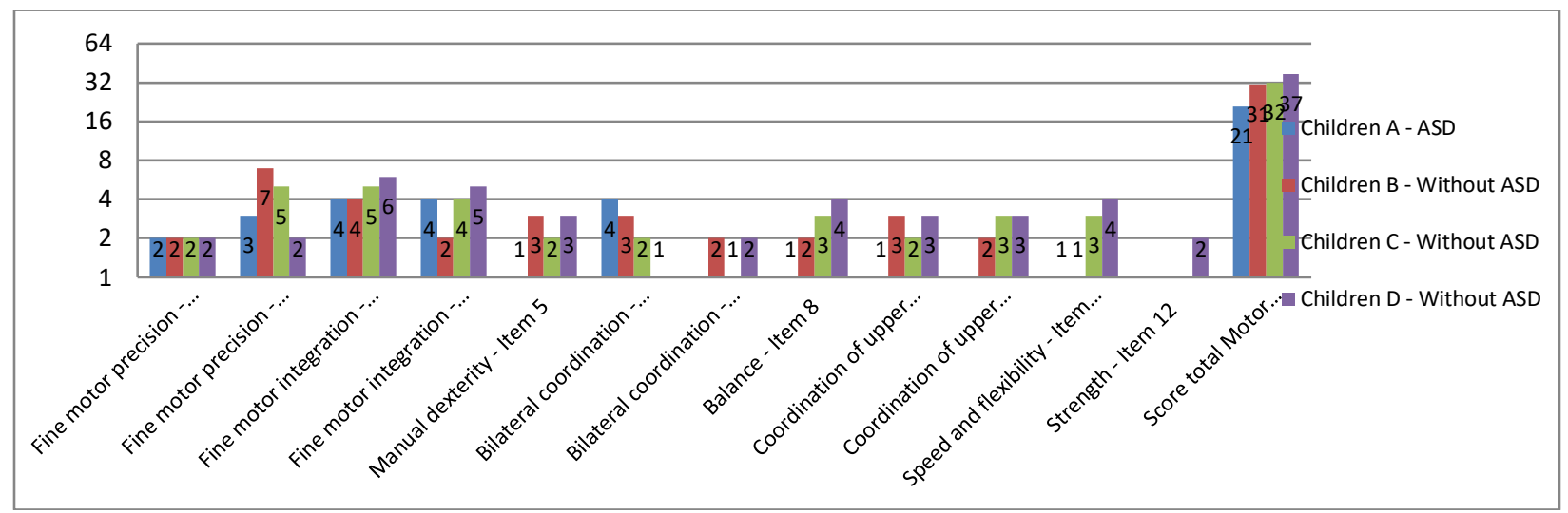

Figure 1. Motor proficiency of all children (Bot-2 results)

\section{CONCLUSIONS}

Results show a deficit in motor proficiency, for the child with ASD. Based on these findings, there is a need for an individualized intervention: a specific exercise program, hat meets the deficits in motor proficiency of the chid with ASD.

\section{REFERENCES}

Liu, T., Breslin, C. M., \& El Garhy, S. (2017). Motor Skill Assessment in Autism Spectrum Disorder: A Case Study. Physical Educator, 74(2), 239. https://doi.org/10.18666/tpe-2017-v74-i2-7148

Clare, C. W., Wong, S. W., Lo, F. S., So, R. C., \& Chan, D. F. (2018). Study protocol: a randomized controlled trial study on the effect of a game-based exercise training program on promoting physical fitness and mental health in children with autism spectrum disorder. BMC psychiatry, 18(1), 56. https://doi.org/10.1186/s12888-018-1635-9

Dillon, S. R., Adams, D., Goudy, L., Bittner, M., \& McNamara, S. (2017). Evaluating Exercise as evidence-based practice for individuals with autism spectrum disorder. Front Public Health., 4, 290. https://doi.org/10.3389/fpubh.2016.00290

Lang, R., Liu, T., \& Ledbetter-Cho, K. (2018). Exercise in Autism. In Encyclopedia of Autism Spectrum Disorders (pp. 1-6). Springer New York. https://doi.org/10.1007/978-1-4614-6435-8_102189-1

Lourenço, C., Esteves, D., Corredeira, R., \& Seabra, A. (2015). The effect of a trampoline-based training program on the muscle strength of the inferior limbs and motor proficiency in children with autism spectrum disorders. JPES, 15(3), 592. https://doi.org/10.7752/jpes.2015.03089

Sam, K. L., Chow, B. C., \& Tong, K. K. (2015). Effectiveness of exercise-based interventions for children with autism: a systematic review and meta-analysis. IJLT, 1, 98-103. https://doi.org/10.18178/ij|t.1.2.98-103.

\section{(9) $(\mathbb{0} \Theta \Theta$}

This work is licensed under a Attribution-NonCommercial-NoDerivatives 4.0 International (CC BY-NC-ND 4.0). 


\title{
Pre-service physical education teachers tasks load vs. tactical game approach tasks load: A case study
}

\author{
JUAN M. GARCÍA-CEBERINO $\checkmark$, MARÍA G. GAMERO, SERGIO GONZÁLEZ-ESPINOSA, ANTONIO \\ ANTÚNEZ, SEBASTIÁN FEU \\ Optimization of Training and Sports Performance Research Group (GOERD), Department of Didactics of \\ Music, Plastic and Body Expression, University of Extremadura, Spain
}

\begin{abstract}
Tasks analysis allows to know how the teachers plan lessons. The aim of this study was to quantify the external Training Load (eTL) of the tasks designed by physical education teachers in pre-service phase. Later, a comparative study was made of the eTL planned by the teachers with the eTL of tasks under the Tactical Game Approach (TGA) method. A total of 130 teaching tasks were categorized (pre-service teachers $n=101$; TGA $n=29$ ) through the Integral System for Training Tasks Analysis. The eTL of the tasks was calculated through six eTL variables: degree of opposition, density of the task, percentage of simultaneous performers, competitive load, game space and cognitive implication. A descriptive and inferential analysis were performed using Kruskal-Wallis $\mathrm{H}$ and Mann-Whitney $\mathrm{U}$ statistical tests. The effect size was calculated using Cohen's d. Tasks under TGA method cause higher levels of eTL than pre-service teachers tasks. Keywords: Planning; Didactic unit; External Training Load.
\end{abstract}

\footnotetext{
Corresponding author. Faculty of Education (office 0.1 ground-floor, main building), Avda. de Elvas S/N 06006, Badajoz, Spain.

E-mail: jgarciaxp@alumnos.unex.es

Supplementary Issue: Spring Conferences of Sports Science. International Seminar of Physical Education, Leisure and Health, 17-19 June 2019. Castelo Branco, Portugal. JOURNAL OF HUMAN SPORT \& EXERCISE ISSN 1988-5202

(c) Faculty of Education. University of Alicante. doi:10.14198/jhse.2019.14.Proc4.82
} 


\section{INTRODUCTION}

The study of teaching tasks designed by physical education teachers, PETEs, allows to analyse how they plan lessons. The tasks can be studied according to three criteria: i) pedagogical; ii) organizational; and iii) Training Load (Ibáñez, Feu, \& Cañadas, 2016). Specifically, this study focuses its interest on the analysis of the external Training Load (eTL), according with the requirements to which students will be exposed during soccer practise at school. The study about the teaching tasks will allow to know if the pre-service PETEs are well trained to plan lessons (Feu, García-Rubio, Gamero, \& Ibáñez, 2019). Therefore, the general aim of the study was to analyse the teaching tasks designed by pre-service PETEs, who were studying the area of physical education, for the teaching of soccer. Additionally, we also aim to analyse: i) quantify the eTL of the tasks from different eTL variables; and ii) compare it with the eTL level of the tasks under the Tactical Game Approach (TGA) method.

\section{MATERIAL AND METHODS}

The study used a comparative and transversal associative strategy (Ato, López, \& Benavente, 2013), in which the pre-service PETEs were asked to design tasks to be compared with tasks under the TGA method.

\section{Participants}

A total of 130 units of teaching tasks composed the study. 101 tasks were designed by two pre-service PETEs (teacher1 $n=53$; teacher2 $n=48$ ). They were free to design, during 12 sessions, the soccer tasks. The remaining 29 tasks, distributed in 12 sessions, constitute a soccer teaching programme (didactic unit) following the TGA method (García-Ceberino, Feu, \& Ibáñez, 2019).

The two teachers (age: $22.50 \pm 0.707$ years) were studying the speciality of physical education in a university in the southwest of Spain. Previously, they studied three years of primary education.

\section{Measures}

The quantification of eTL (in a subjective way) of the tasks was calculated through six eTL variables, which are registered in the Integral System for Training Tasks Analysis (SIATE, Spanish acronym): degree of opposition (DO), density of the task (DT), percentage of simultaneous performers (PSP), competitive load $(C L)$, game space (GS) and cognitive implication (Cl) (calculated as: eTL=DO+DT+PSP+CL+GS+Cl). Each eTL variable was structured as a categorical/ordinal system with a five-level definition. Four ranges were determined to categorize the value of the eTL : 6-12 (very-low), 13-18 (somewhat-low), 19-24 (somewhathigh) and 25-30 (very-high) (Ibáñez et al., 2016).

The Tactical Game Approach Soccer programme, TGAS (García-Ceberino et al., 2019), was used to establish comparisons. The tasks of this programme were validated by 13 expert judges and it shows excellent levels of validity $(\mathrm{V} \geq 0.69)$ and reliability $(\alpha=0.97)$.

\section{Procedures}

First, the pre-service PETEs were asked to plan a didactic unit for learning soccer. All the teaching tasks were categorized, using the SIATE, by an external coder with training and experience in the subject of study: i) PETE; and ii) formative soccer coach. Lastly, a TGAS programme was used to establish comparisons. 


\section{Analysis}

Non-parametric tests were used following the distribution of the sample. The eTL of the teaching tasks of each didactic unit and TGAS programme tasks were calculated independently. Subsequently, an inferential analysis was performed using Kruskal-Wallis $\mathrm{H}$ statistical test (x2). Finally, to establish pairwise comparisons between the didactic units and the TGAS programme was used the Mann-Whitney $U(U)$. The effect size was calculated through the Cohen's d (d).

\section{RESULTS}

Pre-service PETEs planned with somewhat-low eTL levels (teacher1 $M=18.09$; teacher2 $M=17.83$ ) and the TGAS programme tasks reached somewhat-high eTL levels (TGAS M=20.21). The results of the KruskalWallis $\mathrm{H}$ test indicate that there are statistically significant differences with a small effect size in the eTL between the didactic units and the TGAS programme $(x 2=8.273 ; g l=2 ; p=0.016 ; d=0.456)$. Likewise, pairwise comparisons are shown in Table 1.

Table 1. Results of the differences in the eTL between the didactic units and the TGAS programme

\begin{tabular}{|c|c|c|c|c|}
\hline & $U$ & $p$ & $d$ & Effect size \\
\hline DU T1*DU T2 & 1215.500 & 0.700 & 0.077 & No effect \\
\hline DU T1*TGAS & 507.000 & 0.011 & 0.584 & Intermediate effect \\
\hline DU T2*TGAS & 448.000 & 0.009 & 0.622 & Intermediate effect \\
\hline
\end{tabular}

Note: DT T1=didactic unit teacher1; DU T2= didactic unit teacher 2; TGAS: Tactical Game Approach Soccer programme;

${ }^{*} p<0.05$

\section{DISCUSSION}

Attendings to the loads, a better use of the DO, DT and GS cause that the level of eTL (somewhat-high) of the TGAS programme tasks is higher than the level of eTL (somewhat-low) of the tasks designed by preservice PETEs. Pre-service PETEs also planned lessons on handball with somewhat-low eTL levels (GarcíaCeberino, Gamero, González-Espinosa, García-Rubio, \& Feu, 2018).

\section{CONCLUSIONS}

The TGAS programme uses games with the presence of opponents, with modified rules and in small spaces; therefore, it causes higher levels of eTL. By contrast, pre-service PETEs designed tasks with assessment of technical gestures, which cause low levels of eTL.

\section{FUNDING}

This project has been partially subsidised by the Aid for Research Groups (GR18170) from the Regional Government of Extremadura (Department of Employment, Companies and Innovation); with a contribution from the European Union from the European Funds for Regional Development.

\section{REFERENCES}

Ato, M., López, J. J., \& Benavente, A. (2013). Un sistema de clasificación de los diseños de investigación en psicología. Anales de Psicología, 29(3), 1038-1059. https://doi.org/10.6018/analesps.29.3.178511 
Feu, S., García-Rubio, J., Gamero, M. G., \& Ibáñez, S. J. (2019). Task planning for sports learning by physical education teachers in the pre-service phase. Plos One, 14(3). https://doi.org/10.1371/journal.pone.0212833

García-Ceberino, J. M., Feu, S., \& Ibáñez, S. J. (2019). Comparative Study of Two Intervention Programmes for Teaching Soccer to School-Age Students. Sports, 7(3). https://doi.org/10.3390/sports7030074

García-Ceberino, J. M., Gamero, M. G., González-Espinosa, S., García-Rubio, J., \& Feu, S. (2018). Estudio de la carga externa de las tareas para la enseñanza del balonmano en función del género de los profesores en formación. E-balonmano.com: Revista de Ciencias del Deporte, 14(1), 45-54. https://doi.org/10.25267/rev_eureka_ensen_divulg_cienc.2018.v15.i3.3602

Ibáñez, S. J., Feu, S., \& Cañadas, M. (2016). Sistema integral para el análisis de las tareas de entrenamiento, SIATE, en deportes de invasión. E-balonmano.com: Revista de Ciencias del Deporte, 12(1), 3-30. https://doi.org/10.4321/s1578-84232012000200008 


\title{
Comparative analysis of interlimb asymmetry in a RSA Test in basketball players
}

\author{
DAVID MANCHA-TRIGUERO , CARLOS D. GÓMEZ-CARMONA, DAVID GARCÍA-SANTOS, JAVIER \\ GARCÍA-RUBIO, SERGIO J. IBÁÑEZ \\ Group GOERD, Faculty of Sports Science, University of Extremadura, Spain
}

\begin{abstract}
Due to the training processes, the athlete occasionally develops asymmetries between limbs which can cause a deficit in performance or even injury. Therefore, in this paper, 63 basketball players were analysed (U'14 $n=15 ; U^{\prime} 16 n=25 ; U^{\prime} 18 n=23$ ) to know if they had asymmetries between limbs in the different sprints of a RSA test. To this end, each athlete performed a RSA test $5 \times 14$ metres and the analysis differentiated between laterality of the support either right or left. The results show that there are no significant differences between sprints performed based on the same laterality of the support. However, there are significant differences in the variables when the analysis is made comparing right versus left step cycles. The data show that the athletes are well trained and that the effort made does not affect the athlete when the analysis is carried out comparing the same laterality. However, there are differences when the analysis compares laterality. This may be due to the lack of bilateral work and to the predominance of one side over the other what, in short efforts, does not affect the final performance. Keywords: Asymmetry; Interlimb; Laterality; Basketball; RSA Test.
\end{abstract}

Corresponding author. Facultad de Ciencias del Deporte. Avd. De la Universidad s/n. Cáceres (Cáceres). CP: 10003, Spain. E-mail: dmanchat@alumnos.unex.es

Supplementary Issue: Spring Conferences of Sports Science. International Seminar of Physical Education, Leisure and Health, 17-19 June 2019. Castelo Branco, Portugal.

JOURNAL OF HUMAN SPORT \& EXERCISE ISSN 1988-5202

(C) Faculty of Education. University of Alicante.

doi:10.14198/jhse.2019.14.Proc4.82 


\section{INTRODUCTION}

Basketball is an invasion team sport, dynamic and of an intermittent nature formed by fast and short moves where changes in speed and direction are produced and where the jumps are an integral part of the demands of the game (Zaric et al., 2018). The dynamics of the sport linked to the tactical component gives the game unpredictability and a changing environment. This unpredictability forces the athlete to have a wide repertoire of gestures, behaviours and moves that will be made during the competition in order to defeat the opponent. To achieve this great repertoire of gestures, behaviours and moves, during the training process the athlete must face a continuous learning with the objective of experiencing the greatest number of possible options as an athlete (Mancha-Triguero et al., 2018).

Occasionally, athletes limit their possibilities causing physical imbalances called asymmetries that affect the performance of the athlete (Bishop et al., 2018). These asymmetries are defined as the difference in function or performance of two homologous limbs (Bishop et al., 2018). The purposes of this study were to describe the support of the sprint in a RSA Test. Also, to knowing if there are differences between the supports of the same laterality in different sprints and between supports between different laterality.

\section{MATERIAL AND METHODS}

This research is framed within the studies of empirical nature of a quasi-experimental type as it attempts to examine the differences between groups and relations between tests. Within this type, it follows a design of non-equivalent groups in order to characterize the performance of basketball players through a physical fitness test.

\section{Participants}

The sample of participants in the study is made up of 63 basketball players (U'14 $n=15$; U'16n= 25; $U^{\prime} 18 n=23$ ). All the participants have participated in the national championship.

\section{Measures}

Each athlete has been equipped with an inertial device WIMU ${ }^{\mathrm{TM}}$ (Realtrack Systems, Almeria, Spain) to perform the RSA 5x14 metres test with 30 seconds of rest. The software SPro (Realtrack Systems) has been used to analyse the data. The independent variable was laterality of step cycles (right or left) and the depend variables analysed were: i) Step time, ii) Contact time, iii) Fly time, iv) Acceleration Maximum (Acc Max.).

\section{Procedures}

First, the athlete did a warm-up similar to the one he had previously done in a competition. Subsequently, each player performed the test individually. Once finished, the data was analysed and filtered according to the laterality of the supports (made with the right or left foot). Having done the analysis, the average value of the variables analysed for each sprint and by laterality was calculated.

\section{Analysis}

Firstly, a descriptive analysis (Mean and SD) was carried out, followed by a comparative analysis to know the difference between the variables according to laterality (right or left). Finally, a Repeated Measures test was performed to know if there were differences in the variables analysed throughout the 5 sprints. 


\section{RESULTS}

Table 1 shows the significant differences of the analysed variables according to the laterality of the step cycles. Table 2 shows the descriptive result of the variables (mean and ST).

Table 1. Inferential results of the analysed variables according to the laterality of the step cycles

\begin{tabular}{lcccccccc}
\hline & \multicolumn{2}{c}{ Contact time } & \multicolumn{2}{c}{ Step time } & \multicolumn{2}{c}{ Fly time } & \multicolumn{2}{c}{ Acc Max } \\
\hline & Mauchly's W & Sig. & Mauchly's W & Sig. & Mauchly's W & Sig. & Mauchly's W & Sig. \\
\hline Right & 0.717 & $0.019^{*}$ & 0.866 & 0.481 & 0.534 & $0.000^{*}$ & 0.605 & $0.000^{*}$ \\
Left & 0.608 & $0.001^{*}$ & 0.876 & 0.55 & 0.625 & $0.001^{*}$ & 0.81 & 0.186 \\
\hline
\end{tabular}

Table 2. Descriptive results from analysed variables

\begin{tabular}{|c|c|c|c|c|c|c|}
\hline & & $\mathrm{N}$ & Media Right & Media Left & ST Right & ST Left \\
\hline \multirow{4}{*}{$\begin{array}{l}\text { 言 } \\
\text { के }\end{array}$} & Step Time & 63 & 262.66 & 262.11 & 17.64 & 16.15 \\
\hline & Contact time & 63 & 192.19 & 191.42 & 16.97 & 17.25 \\
\hline & Fly time & 63 & 70.47 & 70.69 & 13.97 & 11.66 \\
\hline & Acc Max & 63 & 2.83 & 2.87 & 0.66 & 0.69 \\
\hline \multirow{4}{*}{ 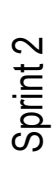 } & Step Time & 63 & 267.48 & 266.80 & 18.91 & 17.90 \\
\hline & Contact time & 63 & 196.43 & 194.88 & 21.54 & 21.92 \\
\hline & Fly time & 63 & 71.05 & 71.92 & 13.89 & 14.47 \\
\hline & Acc Max & 63 & 2.80 & 2.80 & 0.62 & 0.61 \\
\hline \multirow{4}{*}{$\begin{array}{l}\text { m } \\
\text { 롱 } \\
\text { के }\end{array}$} & Step Time & 63 & 264.52 & 265.25 & 18.32 & 17.86 \\
\hline & Contact time & 63 & 195.93 & 195.40 & 20.16 & 21.26 \\
\hline & Fly time & 63 & 68.59 & 69.85 & 13.94 & 13.58 \\
\hline & Acc Max & 63 & 2.90 & 2.87 & 0.72 & 0.70 \\
\hline \multirow{4}{*}{$\begin{array}{l}\text { 壱 } \\
\text { के } \\
\text { के }\end{array}$} & Step Time & 63 & 265.76 & 265.99 & 17.53 & 19.87 \\
\hline & Contact time & 63 & 197.84 & 197.12 & 19.48 & 21.73 \\
\hline & Fly time & 63 & 67.92 & 68.88 & 14.21 & 13.87 \\
\hline & Acc Max & 63 & 2.82 & 2.84 & 0.70 & 0.72 \\
\hline \multirow{4}{*}{ 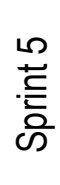 } & Step Time & 63 & 264.44 & 265.71 & 16.52 & 18.14 \\
\hline & Contact time & 63 & 195.37 & 194.36 & 18.44 & 18.16 \\
\hline & Fly time & 63 & 69.07 & 71.35 & 14.89 & 14.02 \\
\hline & Acc Max & 63 & 2.81 & 2.83 & 0.69 & 0.71 \\
\hline
\end{tabular}

\section{DISCUSSION}

The purposes of this study were to know the differences between the analysed variables that are related to each sprint step cycle, to compare such results according to laterality and to know if these differences caused an asymmetry in the athlete that could affect performance or have risk of injury. Once the sample has been analysed, there are significant differences in the variables when the analysis is made comparing with the step cycle of the right leg and those obtained with the left one. These differences do not reach the minimum levels to be considered asymmetry (> 10\%) (Gustavsson et al., 2006). 


\section{CONCLUSIONS}

The results show that the athletes are well trained and that the effort made does not cause an excessive muscle fatigue that affects the weakest limb. The sprint performed by the athletes is of short duration and the one with a high ratio of work-rest. It would be interesting to perform the test by modifying the sprint distance and the rest time.

\section{REFERENCES}

Bishop, C., Read, P., Lake, J., Chavda, S., \& Turner, A. (2018). Interlimb asymmetries: understanding how to calculate differences from bilateral and unilateral tests. J Strength Cond Res, 40(4), 1-6. https://doi.org/10.1519/ssc.0000000000000371

Gustavsson, A., Neeter, C., Thomeé, P., Silbernagel, K. G., Augustsson, J., Thomeé, R., \& Karlsson, J. (2006). A test battery for evaluating hop performance in patients with an ACL injury and patients who have undergone ACL reconstruction. Knee Sur Sport $\operatorname{Tr} A$, 14(8), 778-788. https://doi.org/10.1007/s00167-006-0045-6

Mancha-Triguero, D., González-Espinosa, S., García-Santos, D., \& Ibáñez, S. J. (2018). Evolución y relación de las variables pedagógicas en entrenamientos formativos de un equipo de baloncesto. En Baena, A. (Ed.), Metodologías Ciencias del Deporte (pp.171-179). Granada, España: Wanceulen. https://doi.org/10.5232/ricyde2019.05805

Zarić, I., Dopsaj, M., \& Marković, M. (2018). Match performance in young female basketball players: Relationship with laboratory and field tests. Int J Perf Anal Spor, 18(1), 90-103. https://doi.org/10.1080/24748668.2018.1452109

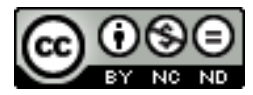

This work is licensed under a Attribution-NonCommercial-NoDerivatives 4.0 International (CC BY-NC-ND 4.0). 


\title{
Analysis of the action of penalty and double- penalty in football for blind people
}

\author{
JOSÉ MARTÍN GAMONALES , KIKO LEÓN-GUZMÁN, DAVID MANCHA-TRIGUERO, JESÚS MUÑOZ- \\ JIMÉNEZ \\ Grupo de Optimización del Entrenamiento y el Rendimiento Deportivo, Universidad de Extremadura, \\ Cáceres, Spain
}

\begin{abstract}
The aim of the present study was to analyse the differences between the penalties and double penalties in Football at 5 for blind people of the World Cup of 2014 and the 2016 Paralympic Games. A total of 127 penalties and doubles penalties for both championships will be analysed. A descriptive analysis of the differences between the variables proposed in the study was made in relation to the type of shooting. The result shows the relevance of the execution of penalties and double-penalties in football for the blind. There are significant differences between penalties and doubles penalties related to the variables Game phase, Moment of the shot and Final score. Therefore, penalty actions have not the highest probability of getting a score than double - penalty actions, despite the fact that the shot is executed from less distance. Keywords: Football 5-a-side; Disability; Performance analysis; Observational methodology.
\end{abstract}

Corresponding author. Avda de la Universidad s/n, Facultad de Ciencias del deporte, 10003, Cáceres, Spain.

E-mail: josemartingamonales@gmail.com

Supplementary Issue: Spring Conferences of Sports Science. International Seminar of Physical Education, Leisure and Health, 17-19 June 2019. Castelo Branco, Portugal.

JOURNAL OF HUMAN SPORT \& EXERCISE ISSN 1988-5202

(c) Faculty of Education. University of Alicante.

doi:10.14198/jhse.2019.14.Proc4.82 


\section{INTRODUCTION}

Football 5-a-side for blind people (Fa5) begins to manifest itself in the scientific literature (Gamonales, 2017; Gamonales, León-Guzmán, Muñoz-Jiménez, González-Espinosa \& Ibáñez, 2018). However, there are few references related to the analysis of sports performance (Gamonales, Muñoz-Jiménez, León \& Ibáñez, 2018a), that have been characterizing for the diverse of the documents (articles from scientific journals, academic papers, scientific publications and meetings, books, patent documents and book chapters).

In football, the penalty action is a game action where only two players are involved: shooter and goalkeeper (Palao, López-Montero \& López-Botella, 2010). However, in football to 5 for blind people (hereinafter, Fa5) three people are involved: shooter, goalkeeper, and guide. Therefore, is a specific action of this sport, never studied before. The objective of this research was to analyse the differences between the penalties and double-penalties in Fa5 in the 2014 World Championships and 2016 Paralympic Games.

\section{MATERIAL AND METHODS}

\section{Participants}

This research is positioned within observational descriptive studies of longitudinal and associative type, that examining the differences between two or more variables (Ato, López \& Benavente, 2013). The sample corresponds to the total of penalties and double-penalties ( $n=127)$, executed during the World Championship of Fa5 of 2014 ( $n=104)$ and of the Paralympic Games of 2016 in the modality of Fa5 (n=23).

\section{Measures}

The variables used in this research were defined precisely by Gamonales, Muñoz-Jiménez, León-Guzmán \& Ibáñez (2018b).

\section{Procedures}

To know the differences between penalties and double-penalties in Fa5, the IOLF5C instrument was used (Gamonales, León-Guzmán et al., 2018). A coder training process was applied in order to confirm that the data are valid and reliable to be used for the investigation. The training of the coders was divided into four stages: preparatory stage, stage of selection of the coders, stage of formation of the observers, and, finally, reliability stage with a Kappa value above to 0.95 . (Gamonales et al., 2018b). After the coders training, all the penalties and double-penalties of the championships $(n=127)$ are registered individually, which allowed obtaining all the records to perform the pertinent statistical analysis.

\section{Analysis}

A descriptive and inferential analysis was carried out, using Chi-square $(x 2)$, to obtain the relationship between the variables, measuring its intensity with the Cramer $V$ statistic $(\varphi \mathrm{C})$ and the Corrected Typified Residues (ASR).

\section{RESULTS}

Table 1 shows the significant differences between penalty and double-penalty, in relation to the variables raised in the study of $\mathrm{Fa} 5$. 
Table 1. Inferential analysis between type penalties and dependent variables of the study

\begin{tabular}{|c|c|c|c|c|c|c|}
\hline \multirow{2}{*}{ Variables } & \multicolumn{6}{|c|}{ Penalty and double-penalty } \\
\hline & $x^{2}$ & gl. & Sig. & $\varphi c$ & Sig. & Association level \\
\hline Type of competition & 3.658 & 1 & 0.056 & 0.170 & 0.056 & --- \\
\hline Game phase & 49.397 & 5 & 0.000 & 0.624 & 0.000 & Low \\
\hline Moment of the launch & 102.093 & 9 & 0.000 & 0.897 & 0.000 & Low \\
\hline Situation of the equipment & 0.936 & 2 & 0.626 & 0.086 & 0.626 & --- \\
\hline Final score & 17.879 & 2 & 0.000 & 0.375 & 0.000 & low \\
\hline Orientation technique & 3.095 & 3 & 0.377 & 0.156 & 0.377 & --- \\
\hline Release type & 4.850 & 2 & 0.088 & 0.195 & 0.088 & --- \\
\hline Body area & 0.357 & 1 & 0.550 & 0.053 & 0.550 & --- \\
\hline Type of beating & 4.870 & 2 & 0.088 & 0.196 & 0.088 & --- \\
\hline Release result & 7.906 & 5 & 0.088 & 0.250 & 0.088 & --- \\
\hline
\end{tabular}

\section{DISCUSSION}

The results show three associations between the dependent variables on the study and the type of penalty. Specifically, the results of the final score variable is relevant because, according to the Fa5 rules, the penalty is a more serious infraction and is considered with a greater probability of getting a goal than the throws of double penalty. However, the results show that this greater probability of achieving success does not exist.

\section{CONCLUSIONS}

There are no differences in the result of the action between the penalty or double penalty in Fa5. Therefore, penalty actions have not a highest probability of getting a score than double - penalty actions, despite the fact that the launch occurs from more distance. However, it would be advisable to increase the sample including other championships.

\section{REFERENCES}

Ato, M., López, J.J., \& Benavente, A. (2013). A classification system for research designs in psychology. Anales de Psicología, 29(3), 1038-1059. https://doi.org/10.6018/analesps.29.3.178511

Gamonales, J.M. (2017). Fútbol a 5 para personas ciegas como contenido de Educación Física. Revista Profesional de Investigación, Docencia y Recursos Didácticos, 80(3), 66-70.

Gamonales, J.M., León-Guzmán, K., Muñoz-Jiménez, J., González-Espinosa, S., \& Ibáñez, S.J. (2018). Validation of the IOLF5C instrument for the efficacy of shooting on goal in football for the blind. Revista Internacional de Medicina y Ciencias de la Actividad Física y el Deporte, 18(70), 361-381. https://doi.org/10.15366/rimcafd2018.70.010

Gamonales, J.M., Muñoz-Jiménez, J., León-Guzmán, K., \& Ibáñez, S.J. (2018a). Football 5-a-side for individuals with visual impairments: a review of the literature. European Journal of Adapted Physical Activity, 11(1), 1-19. https://doi.org/10.5507/euj.2018.004

Gamonales, J.M., Muñoz-Jiménez, J., León-Guzmán, K., \& Ibáñez, S.J. (2018b). Entrenamiento y confiabilidad entre observadores en el análisis del fútbol para ciegos. RETOS, Nuevas Tendencias en Educación Física, Deporte y Recreación, 34(2), 155-161. https://doi.org/10.15366/rimcafd2018.70.010 
Palao, J.M., López-Montero, M., \& López-Botella, M. (2010). Relationship between efficacy, laterality of foot strike, and shot zone of the penalty in relation to competition level in soccer. of the penalty in relation to competition level in soccer. International Journal of Sport Science, 19(6), 153-166. https://doi.org/10.5232/ricyde2010.01905

\section{(c) $(\mathrm{B})(\mathrm{EY}$}

This work is licensed under a Attribution-NonCommercial-NoDerivatives 4.0 International (CC BY-NC-ND 4.0). 


\title{
Loopboard: Device for acrobatics trainning in sliz sports
}

\author{
KIKO LEÓN-GUZMÁN $\square$, JOSÉ MARTÍN GAMONALES, CARLOS DAVID GÓMEZ-CARMONA, JESÚS \\ MUÑOZ-JIMÉNEZ \\ Grupo de Optimización del Entrenamiento y el Rendimiento Deportivo, Universidad de Extremadura, \\ Cáceres, Spain
}

\begin{abstract}
The support devices for sports training in Sports Sliz have as their mission the development of the technique of the athlete, as well as a positive and specific impact on physical qualities. In the present work an innovative device patented by the University of Extremadura, known as "Device for training of acrobatics in sliding sports" (Spain, 2018. P201830091), as well as the possible applications and sports modalities that is being developed can create. Keywords: Sport patent; Acrobatics training; Sliz sports.
\end{abstract}

Corresponding author. Avda de la Universidad s/n, Facultad de Ciencias del deporte, 10003, Cáceres, Spain.

E-mail: josemartingamonales@gmail.com

Supplementary Issue: Spring Conferences of Sports Science. International Seminar of Physical Education, Leisure and Health, 17-19 June 2019. Castelo Branco, Portugal.

JOURNAL OF HUMAN SPORT \& EXERCISE ISSN 1988-5202

(c) Faculty of Education. University of Alicante.

doi:10.14198/jhse.2019.14.Proc4.82 


\section{INTRODUCTION}

The development and evolution of the physical-sporting practice is influenced, to a great extent by the different advances in equipment, devices, procedures or systems that improve and study the different physical-sport modalities (Gamonales, 2015; León-Guzmán, Muñoz-Jiménez, Gamonales \& Pérez, 2013). The impact of science and technology in this field is increasingly evident, and its influence is shown as a key factor in the development of any sport physical activity (Bagnara \& Pacheco, 2011). The scientific, creative and innovative capacity of researchers needs a guarantee that "novelties" are disclosed to the scientific community and transferred to society through the granting of patents and their possible exploitation. (González-Montesinos \& Fernández-Santos, 2012). These products, the result of scientific, creative and innovative capacity, in most cases require a registry that guarantees its novelty through the Spanish Patent Office (Gamonales, León \& Muñoz, 2017). Therefore, the use of devices and systems that improve the results of training and / or safety in physical-sporting activities is shown as one of the main and most promising fields of research in Physical Activity and Sports Sciences.

\section{OBJETIVE}

To present an innovative device developed by its authors and protected by the presentation of the patent by the University of Extremadura as "Device for acrobatics training in Sliz sports" (Spain, 2018. P201830091) (Fig 1).

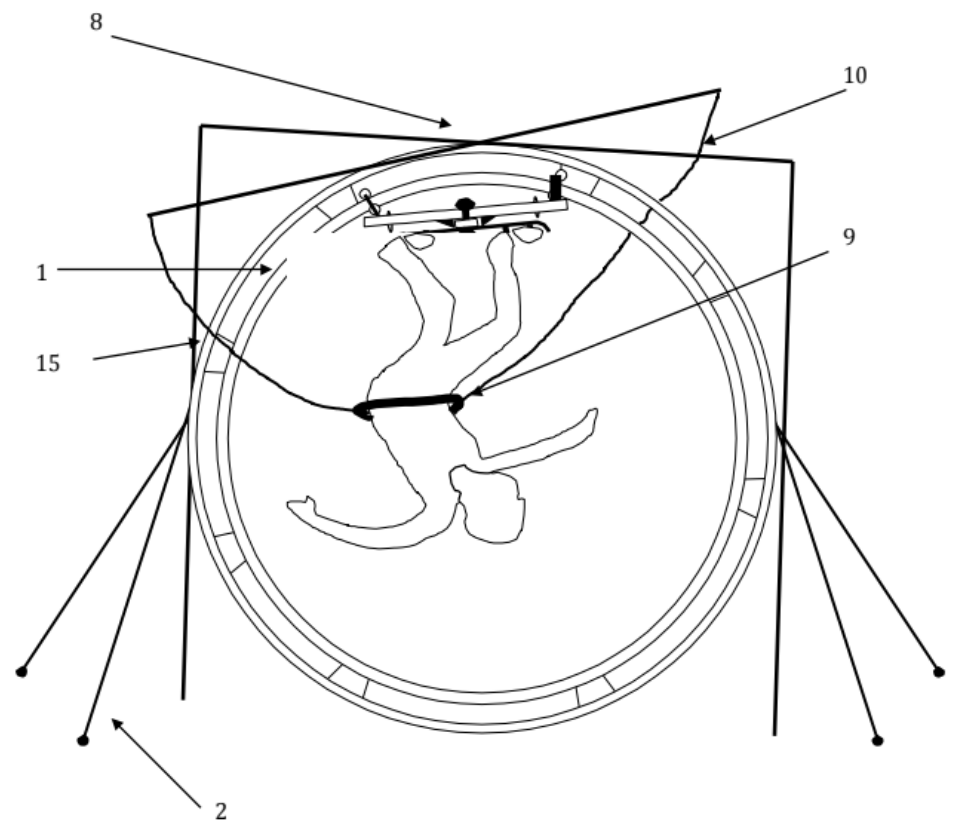

Figure 1. Device for acrobatics training in Sliz sports.

\section{MATERIAL AND METHODS}

The device is oriented to the training of vestibular and proprioceptive sensations through inversions and turns within the so-called Sliz Sports (León-Guzmán, 2001; León-Guzmán \& Parra-Boyero, 2001). It has been devised in order to respond to the needs of coaches looking for new options for their training. It was developed after an exhaustive search, in which no commercialized product of similar characteristics was found. It 
provides the essential characteristics of novelty and multiple advantages in its use with respect to devices used for the same training purposes.

\section{RESULTS AND DISCUSSION}

The applicability is multiple. Its circular configuration allows to work the technique and rotate in different axes with a single device. It allows the trainer to design varied tasks, to work both the physical condition, the technique, as well as a very specific work of sensations in the different corporal axes.

\section{CONCLUSIONS}

"Device for acrobatics training in Sliz sports" is a new sport patent that allows to improve the results of the training by providing specific solutions in acrobatic training and allowing coaches a useful resource to fully develop the abilities of their athletes.

\section{REFERENCES}

Bagnara, I., \& Pachecho, I. (2011). El desarrollo tecnológico en las actividades físicas y el deporte. Efdeportes: Revista Digital, 159(1), 10-15.

Gamonales, J.M. (2015). Popular Jibbing: dispositivo innovador para la práctica segura de los Deportes de Sliz. I Maratón de Investigadores Jóvenes. Universidad de Extremadura, Cáceres (España). https://doi.org/10.25145/c.educomp.2018.16.005

Gamonales, J.M., León-Guzmán, K., \& Muñoz,-Jiménez J. (2017). Herradura Soccer: equipamiento patentado de entrenamiento multifunción para fútbol. IV International Conference on Team Sports, Sevilla (España).

González-Montesinos, J., \& Del Rosario, J. (2012). Origen y evolución de las patentes y marcas en biomecánica deportiva. Revista Internacional de Ciencias del Deporte, 30(8), 295-323. https://doi.org/10.5232/ricyde2012.03001

León-Guzmán, K. (2001). Deportes de Sliz. Equilibrio y acrobacia. Clasificación. Efdeportes: Revista Digital, 159(1), 10-15.

León-Guzmán, K., \& Parra-Boyero, M. (2001). Nuevas tendencias deportivas: Deportes de Sliz. Revista Digital EFDeportes, 6(30), 1-3.

León-Guzmán, K., Muñoz-Jiménez, J., Gamonales, J.M., \& Pérez, D. (2013). Bottlefit: dispositivo innovador para la práctica del acondicionamiento físico acuático. Aplicabilidad y ventajas. I Congreso Internacional de Innovación Deportiva en un Marco Transfronterizo. Universidad de Extremadura, Cáceres (España). https://doi.org/10.25145/c.educomp.2018.16.005

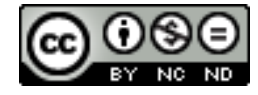

This work is licensed under a Attribution-NonCommercial-NoDerivatives 4.0 International (CC BY-NC-ND 4.0). 


\title{
Health education in patients with rheumatoid arthritis: A pilot education program
}

\author{
WENDELL MATTHEUS AMÂNCIO DA SILVA ${ }^{1}$, LILIAN REGIANI MERINE² ${ }^{2}$ LARISSA ZANETTI THEIL ${ }^{2}$, \\ PATRICIA WILKENS CHAVES ${ }^{1}$, JENNIFER LETÍCIA FERREIRA ${ }^{1}$, ELDER NASCIMENTO PEREIRA ${ }^{1}$, \\ ALINE SALIHA ALENCAR OLIVEIRA ${ }^{2}$ \\ 1 Universidade Federal do Amazonas (UFAM), Brazil \\ 2Universidade de Coimbra (UC), Portugal
}

\begin{abstract}
Rheumatoid arthritis is an inflammatory, systemic, chronic and autoimmune disease, characterized by joint damage, deformities, loss of function, and systemic complications that affect the lungs, heart and other organs. Health education consists of actions directed centrally to the change of the behaviour of the individuals focusing on their lifestyle being that these activities are concentrated in educational activities. The objective of this experience report was to implement a health education program aimed at patients with rheumatoid arthritis, providing the development of personal skills, autonomy, knowledge about the disease and self-care. Establishing a correlation between teaching, research and university extension. The educational program was developed in a university hospital in the city of Manaus, and used active and playful methodologies to propagate and transmit knowledge to individuals with rheumatoid arthritis. A positive performance of at least $80 \%$ was observed in all the questions asked. The program demonstrated good receptivity in the community. Keywords: Rheumatoid arthritis; Health education; Public health.
\end{abstract}

Corresponding author. Federal University of Amazonas (UFAM), Brazil.

E-mail: wendellmattheus@gmail.com

Supplementary Issue: Spring Conferences of Sports Science. International Seminar of Physical Education, Leisure and Health, 17-19 June 2019. Castelo Branco, Portugal.

JOURNAL OF HUMAN SPORT \& EXERCISE ISSN 1988-5202

(c) Faculty of Education. University of Alicante.

doi:10.14198/jhse.2019.14.Proc4.82 


\section{INTRODUCTION}

The World Health Organization (WHO) defines Rheumatoid Arthritis (RA) as an inflammatory, systemic, chronic and autoimmune disease, characterized by joint damage, deformities, loss of function, systemic complications, affecting the lungs, heart and other organs. (Passos, 2016; Costa, et al. 2014; Unk, 2014; \& Gomes, et al., 2017).

Although there is no consensus on the etiology of RA, it is known to be a multifactorial disease, in which the development of the disease can be favoured by different interactions of social and cultural, environmental, genetic and infectious agents, in which the destruction of cartilage and bones due to inflammation and synovial hypertrophy is identified. (Almeida, et al., 2015; \& Lopes, 2010). Health education consists of actions directed centrally to the change of the behaviour of the individuals focusing on their lifestyle being that these activities are concentrated in educational activities. Carvalho et al. Showed in their work with the group "Orientation of Individuals Affected by Rheumatic Diseases" that the participants showed an increased interest in attendance, reduction of pain, greater sense of competence, greater capacity to cope with the disease, increased socialization and perception of quality of life. (Carvalho, Noordhoek \& Silva, 2006). From the above, the objective of this project was to implement a health education program aimed at patients with air, providing the development of personal skills, autonomy, knowledge about the disease and self-care. establishing a correlation between teaching, research and university extension.

\section{MATERIAL AND METHODS}

The extension program "Health Education of Individuals with Rheumatoid Arthritis", of the Federal University of Amazonas, counts as an education project in rheumatological diseases, which were carried out by students of the Physiotherapy course of the Faculty of Physical Education and Physiotherapy of the Federal University of Amazonas FEFF-UFAM, under the supervision of a professor with institutional approval of PROEXT UFAM Extension and Interiorization Department.

\section{Participants}

Sixty-seven patients, of both sexes, with a mean age of 47 years residing in Manaus / AM, participated. The patients were invited to participate in the education group, which happened once a week, totalling 10 meetings, lasting one hour and thirty minutes each, in the Rheumatology Department of the Araújo Lima Ambulatory of the University Hospital Getúlio Vargas (HUGV) of the Federal University of Amazonas (UFAM).

\section{Measures}

The results were measured through a satisfaction questionnaire prepared by the program's own team, composed of 10 objective questions, in order to evaluate the quality and acceptance of the methodologies applied in the education program.

\section{Procedures}

The active methodologies covered during the meetings were: ludic lectures using comic drawings on pathophysiology, signs and symptoms, medications and importance of treatment, true and false games, interactive quiz and conversation wheel. The sessions were preliminarily elaborated and interactive, providing the participation of the volunteers, not being a formal teaching encounter. At the end of the meetings were held the protocol of exercises proposed by the team. 


\section{Analysis}

At the end of each meeting, a satisfaction questionnaire was elaborated by the program team, composed of objective questions, in order to evaluate the quality and acceptance of the methodologies applied in the education program.

\section{RESULTS}

According to the satisfaction questionnaire, an average of $88.51 \%$ of the participants involved in the project classified the methods covered in the program as "Good", 9.46\% classified the active methodologies involved in the project as "Regular" and only $1.79 \%$ rated the methods with "Bad".

\section{DISCUSSION}

The result showed that there was great acceptance of the community that participated in the education program, demonstrating that there is interest of the population in participating in activities and educational programs such as this in outpatient clinics and hospitals, which is a productive moment for patients and their families.

\section{CONCLUSIONS}

The education of the patient with rheumatoid arthritis is an important part of the treatment, allowing the understanding of his disease, for a better self-care. According to the evaluation of the satisfaction questionnaire prepared by the program team, there was an acceptance by the community of the methodologies used in the HUGV education program. The results obtained in this program make us seek new teaching strategies, approaches and evaluation through specific and validated instruments, since the program is a new project in the development phase, which showed great receptivity of the community.

\section{REFERENCES}

Almeida, P.H.T.Q, Pontes T.B, Matheus J.P.C et al (2015). Terapia ocupacional na artrite reumatoide: 0 que o reumatologista precisa saber?. Revista Brasileira de Reumatologia, 55(3):272-280. https://doi.org/10.1016/j.rbr.2014.07.008

Carvalho, M.G.R, Noordhoek, J e Silva, M.C.O. (2006). Grupo de Orientação a Indivíduos. Acometidos por Doenças Reumáticas: Espaço Educativo e Terapêutico. Revista Brasileira de Reumatologia, 46(2):134-136. https://doi.org/10.1590/s0482-50042006000200008

Costa, J.O, Almeida, A.M, Guerra, A.A et al. (2014) Tratamento da artrite reumatoide no Sistema Unico de Saude, Brasil: gastos com infliximabe em comparacao com medicamentos modificadores do curso da doenca sinteticos, 2003 a 2006. Cadernos de Saúde Pública, 30(2):283-295. https://doi.org/10.1590/0102-311x00017913

Gomes, R.K.S., Pires, F.A., Nobre, M.R.C., et al. (2017). Impacto da artrite reumatoide no sistema público de saúde em Santa Catarina, Brasil: análise descritiva e de tendência temporal de 1996 a 2009. Revista Brasileira de Reumatologia, 57(3):204-209. https://doi.org/10.1016/j.rbr.2016.05.003

Lopes, A.L.B., (2010). Influência de polimorfismo dos genes TNF e PTPN22 sobre a artrite reumatoide e a tuberculose no Amazonas, Brasil. Passos, L.F.S., (2016). Artrite reumatoide: novas opções terapêuticas. Organização Pan-americana da Saúde/Organização Mundial da Saúde. Brasília, 01(15):01-07. Unk, J.A. \& Brasington R., (2014) Efficacy study of multimedia rheumatoid arthritis 
patient education program. Journal of the American Association of Nurse Practitioners, 26(7):370377. https://doi.org/10.1002/2327-6924.12064

\section{(c) (i) (3)}

This work is licensed under a Attribution-NonCommercial-NoDerivatives 4.0 International (CC BY-NC-ND 4.0). 


\title{
Voluntary sports clubs and the participation of young refugees with uncertain perspective of staying: Access barriers and challenges
}

\author{
JACQUELINE TUCHEL ${ }^{1}$, ALEXANDER HODECK ${ }^{2}$, TORSTEN SCHLESINGER ${ }^{3}$ \\ ${ }^{1}$ Chemnitz University of Technology, Germany \\ ${ }^{2} E B C$ Hamburg \& Chemnitz University of Technology, Germany \\ ${ }^{3}$ Ruhr-University Bochum, Germany
}

\begin{abstract}
Since the rapid rise in the number of refugees in Germany in 2015, voluntary sports clubs (VSCs) are increasingly being asked to contribute to integration through sports activities. Although VSCs play an important role in integration work, numerous studies show that migrants are still rather underrepresented in VSCs. In the case of young refugees with uncertain perspective of staying, there are additional challenges for both the VSCs and the refugees to enable them to participate. In order to ensure access and participation for refugees in VSCs, the question should be discussed, which structural and personal access barriers arise in this context to in order to be able to adequately address them in the long term. As part of a broader study conducted in Germany (in the federal state of Saxony) on the participation of refugees in VSCs, qualitative semi-structured interviews were conducted with participating VSCs $(n=13)$, social workers and heads of accommodation units $(n=6)$ and refugees $(n=10)$. Results show that, despite the interest on both sides, various individual and structural barriers exist. Both sides have partial fears of contact and insufficient information about offers and needs. Furthermore, the transport to the sports facility, obtaining the eligibility to play, the cost absorption of formal membership, insufficient resources (sports facilities and trainers) as well as the expectations of the VSCs (regular participation in training and competition), are central barriers. In addition solutions to the investigated problems will also be shown and discussed. Keywords: Voluntary sports clubs; Integration; Refugees; Access barriers; Challenges.
\end{abstract}

Corresponding author. TU Chemnitz, Institute of Human Movement Science and Health, Thüringer Weg 11, 09126 Chemnitz, Germany.

E-mail: jacqueline.tuchel@hsw.tu-chemnitz.de

Supplementary Issue: Spring Conferences of Sports Science. International Seminar of Physical Education, Leisure and Health, 17-19 June 2019. Castelo Branco, Portugal.

JOURNAL OF HUMAN SPORT \& EXERCISE ISSN 1988-5202

(c) Faculty of Education. University of Alicante.

doi:10.14198/jhse.2019.14.Proc4.82

S1514 | 2019| Proc4 | VOLUME 14

C 2019 University of Alicante 


\section{INTRODUCTION}

Voluntary Sports Clubs (VSCs) are increasingly required to provide appropriate opportunity structures to allow refugees to participate in social life. Through their primarily physical interactions and the globally uniform rules, they can make a significant contribution to the integration of all sections of the population. Nonetheless, the primary purpose of VSCs is to satisfy the interests of its members, not the integration of specific groups, which also requires additional resources (Horch 1992). Compared to the population without migration background, immigrants are already underrepresented in VSCs anyway (e.g. Mutz/ Burrmann 2015). However, the VSCs, social workers and refugees articulate additional challenges in the integration of refugees with an uncertain perspective of staying, which were raised as part of the project "Sport for and with Refugees" (Anderson et al. 2019).

\section{MATERIAL AND METHODS}

\section{Participants}

As part of a broader study conducted in Germany (federal State of Saxony), refugees $(n=10)$, trainers/board members $(n=13)$ as well as social workers/heads of accommodation facilities $(n=6)$ were interviewed.

\section{Measures}

The data was collected by using semi-structured interviews. The questions focused on the organization of the sports activities of the refugees, the cooperation with the VSCs and the challenges in this context.

\section{Procedures}

The interviews were accomplished in the VSCs or in the accommodation units by trained scientific researchers and completely transcribed afterwards. The interviews with the refugees were translated simultaneously by an interpreter. All data were collected in 2016/2017.

\section{Analysis}

The data was analysed on the basis of Mayring's (2008) qualitative content analysis, because this approach allows a high level of intersubjective comprehensibility and comparability.

\section{RESULTS}

The analyses show that refugees are generally very interested in sports activities, particularly because of the cramped living conditions in the initial reception facilities and clearing houses. Access to VSCs, however, is made more difficult by a lack of information and contact opportunities, as well as transport problems to the VSCs. Furthermore, some of the refugees do not have suitable sports clothing and equipment. In addition, the refugees first have to get to know the host society and attend official appointments, so that they are often only able to attend the training on an irregular basis. Since the assumption of the costs of membership also entails bureaucratic burden, this way is perceived as disproportionate due to the uncertainty of staying. The lack of language skills, the "foreign" body and movement cultures, fear of stigmatisation and traditional gender roles (e.g. prohibitions, clothing regulations) are further burden. However, these uncertainties often go hand in hand with the desire to be accompanied during the first encounter with the VSC, which additionally increase the workload of social workers. The social workers and VSCs also note that some sports are completely unknown and address refugees' needs, and therefore, has sometimes only little meaning. In addition, the social workers have to spend a considerable amount of time to coordinate the interests and needs of refugees and the unstructured offers of the VSCs. Additionally there is a strong shortage of trainers and sports facilities 
and additional participants aggravate this situation. Since the VSCs mainly participate in competitions, they want long-term athletes, who regularly participate in training programs. The complications of obtaining permission to participate in regular competitions are reluctant to be tackled with only a short stay perspective. For unaccompanied minor refugees, the admission procedure must also be handled by the guardian. The needs of the refugees are hardly known to the VSCs and the contact data (complicated by decentralized accommodation of refugees) is missing. Furthermore, there are uncertainties in terms of the aspects of insurance and liability and the payment of membership fees. In this respect as they implement special conditions for refugees, there are problems with justification for their own (possibly socially weak) members. Some trainers feel uncertain due to a lack of foreign language skills and low intercultural experiences and are reluctant to deal with traumatic experiences of the refugees.

\section{DISCUSSION}

Some of the recognized barriers to access and problems do not only affect refugees with uncertain perspectives, but generally people with a migration background (e.g. Schlesinger et al. 2018). Both on the VSCs' side as well as on the refugee side, there are personal and structural hurdles that require a specific sports program that allows refugees with an uncertain perspective of staying to get to know a variety of sports activities without having to attend regularly or even be formal club members. These offers should take place close to the accommodation unit of refugees and at the same time don't overuse the resources of the VSCs.

\section{CONCLUSIONS}

In summary, there are a number of obstacles that affect the sport participation of refugees VSCs. Especially for refugees with an uncertain perspective existing club structures are too inflexible. The membership expectations of VSCs represent a significant barrier to their accessibility. The facilitation of organizing lowthreshold contact and access must be the focus of the efforts.

\section{REFERENCES}

Anderson, A., Dixon, M. A., Oshiroa, K. F., Wicker, P. Cunningham, G. C., \& Heere, B. (2019). Managerial perceptions of factors affecting the design and delivery of sport for health programs for refugee populations. Sport Management Review, 22, 80-95. https://doi.org/10.1016/j.smr.2018.06.015

Horch, H.-D. (1992). Geld, Macht und Engagement in freiwilligen Vereinigungen: Grundlagen einer Wirtschaftssoziologie von Non-Profit-Organisationen. Berlin: Duncker \& Humblot.

Mayring, P. (2015). Qualitative Inhaltsanalyse: Grundlagen und Techniken. Weinheim Basel: Beltz Verlag.

Mutz, M., \& Burmann, U. (2015). Zur Beteiligung junger Migrantinnen und Migranten am Vereinssport. In U. Burmann, M. Mutz, \& U. Zender (Eds.), Jugend, Migration und Sport: kulturelle Unterschiede und die Sozialisation zum Vereinssport. Wiesbaden: Springer. https://doi.org/10.1007/978-3-658$\underline{06397-9 \_3}$

Schlesinger, T., Klostermann, C., Hayoz, C., \& Nagel, S. (2018). Potenziale und Grenzen der Teilhabe und Integration von Menschen mit Migrationshintergrund im organisierten Sport. In A. Schneider, J. Köhler, \& F. Schumann (Eds.), Fairplay im Sport: Beiträge zur Wertedebatte und den ethischen Potenzialen. https://doi.org/10.1007/978-3-658-21342-8_4 


\section{(c) (i) (3)}

This work is licensed under a Attribution-NonCommercial-NoDerivatives 4.0 International (CC BY-NC-ND 4.0). 


\title{
In-season internal training load quantification of an under-17 European male soccer team: Starters versus Non-starters
}

\author{
RAFAEL OLIVEIRA1 ${ }^{1,2,3,4}$, JOÃO M. BRITO ${ }^{1,2,3}$, ALEXANDRE MARTINS ${ }^{1}$ \\ ${ }^{1}$ Sports Science School of Rio Maior, Polytechnic Institute of Santarém, ESDRM-IPS, Rio Maior, Portugal \\ ${ }^{2}$ Research Centre in Sport Sciences, Health Sciences \& Human Development, CIDESD, Vila Real, Portugal \\ ${ }^{3}$ Research Centre on Quality of Life, CIEQV, Santarém, Portugal \\ ${ }^{4}$ Department of Sports Sciences, University of Beira Interior, UBI, Covilhã, Portugal
}

\begin{abstract}
The aim of this study was to compare internal training load (ITL) of starters versus non-starters from the same under-17 soccer team. Rated of perceived exertion was collected and then multiplied by training or match duration (s-RPE) from twenty-three soccer players. The study analysed a total of 12 matches and 44 training sessions during the in-season with ANOVA with repeated measures. The main results showed that training duration presented significant differences between starters and non-starters for match-day minus 5 (MD-5, 80.6 vs $90.7 \mathrm{~min}, \mathrm{p}=0.000$ ) and match-day (MD, 64.2 vs $52.7 \mathrm{~min}, \mathrm{p}=0.036$ ), respectively. Regarding s-RPE, there were significant differences between starters and non-starters for MD-5 (442.4 vs 581.8 a.u., $p=0.000$ ) and MD (515.9 vs 366.1 a.u., $p=0.000$ ), respectively. This study present relevant additional ITL data for nonstarters in order to become a "starter player". Keywords: Soccer training; Internal training load; Training load; RPE; Monitoring.
\end{abstract}

Corresponding author. Sports Science School of Rio Maior, Av. Dr. Mário Soares nº 110, 2040-413, Rio Maior, Portugal. E-mail: rafaeloliveira@esdrm.ipsantarem.pt

Supplementary Issue: Spring Conferences of Sports Science. International Seminar of Physical Education, Leisure and Health, 17-19 June 2019. Castelo Branco, Portugal.

JOURNAL OF HUMAN SPORT \& EXERCISE ISSN 1988-5202

(c) Faculty of Education. University of Alicante.

doi:10.14198/jhse.2019.14.Proc4.82 


\section{INTRODUCTION}

The knowledge of internal training load (ITL) helps coaches to prevent increased levels of fatigue, and higher risk of illness and injury (Jones et al., 2017). By monitoring ITL, coaches can design a more effective individual and group training periodization (Malone et al., 2015).

Assessing ITL is recommended because determines the training outcome experienced from a specific external load may vary depending on specific contextual factors either between or within athletes. Therefore, this should be used as primary measure when monitoring athletes. An example that supports this rationale is associated the training status that is not fixed during a season and thus influence the ITL experienced (Impellizzeri et al., 2018). Consequently, starters and non-starters might demonstrate different physiological adaptations across time (Dalen \& Loras, 2019). For under-17 players, the adaptations across time would perhaps be more important given that matches are an important part of their soccer-specific practice and matches only occur once a week. Thus, the aim of the study was to compare ITL between starters versus non-starters from the same team during the in-season 2017-2018.

\section{MATERIAL AND METHODS}

\section{Participants}

Twenty-three male under-17 soccer players belonging to a Portuguese team with a mean \pm SD age, height and mass of $15.9 \pm 0.2$ years, $173.7 \pm 0.1 \mathrm{~cm}$ and $65.5 \pm 4.6 \mathrm{~kg}$. Inclusion criteria were regular participation in most of the training sessions ( $80 \%$ of weekly training sessions); the completion of at least 60 minutes in three consecutive matches.

\section{Measures}

Each individual RPE value was multiplied by the session duration to generate a session-RPE (s-RPE) value. All explanations of this measure were reported in previous study (Oliveira et al., 2019).

\section{Procedures}

The in-season phase was analysed in relation to the number of days away from the competitive one-match week (i.e., match day minus). In a week with only one match, the team typically trained five days a week (match day [MD] minus [-]; MD-5; MD-4; MD-3; MD-2). This approach was used by Malone et al. (2015).

All participants were familiarised with the training protocols prior to the investigation and gave their written consent to be included in the project. The study was conducted according to the requirements of the Declaration of Helsinki and was approved by Ethics Committee of the Research Centre for Sports Sciences, Health and Human Development, Vila Real, Portugal.

TL data were collected over a 12-week period of competition where occurred 12 matches and 44 training sessions during the 2017-2018 annual season. Only data from training sessions were considered. Data from rehabilitation or additional training sessions of recuperation were excluded. This study did not influence or alter the training sessions in any way. Training and match data collection for this study was carried out at the soccer club's outdoor training pitches. A total of 1226 individual training observations were collected during the study. Total minutes of training sessions included warm-up, main phase and slow down phase plus stretching. 


\section{Analysis}

Data were analysed using SPSS version 22.0 (SPSS Inc., Chicago, IL) for Windows statistical software package. Initially, descriptive statistics were used to describe and characterize the sample. Shapiro-Wilk and the Levene tests were used to assumption normality and homoscedasticity, respectively. ANOVA was used with repeated measures with Bonferroni post hoc, once variables obtained normal distribution (ShapiroWilk $>0.05$ ) to compare days away from the competitive match fixture. Also, it was used ANOVA Friedman and Mann-Whitney tests were used for the variables that not obtained normal distribution to compare different moments. Results were significant with $p \leq 0.05$. Data are represented as mean \pm SD.

\section{RESULTS}

Regarding training duration, there were significant differences between starters and non-starters for MD-5 (10.06, $p=0.000$ ) and $\operatorname{MD}(11.51, p=0.036)$. Also, there were significant differences for training duration between all training sessions and match $(p=0.000)$. Regarding s-RPE, there were significant differences between starters and non-starters for MD-5 (-139.36, $p=0.000)$ and MD $(149.79, p=0.000)$. Also, there were significant differences for training duration between all training sessions and match $(p<0.05)$. The following table 1 shows all data for squad average.

Table 1. Internal Training Load Data during the MD minus for squad average, Mean \pm SD

\begin{tabular}{lcccccc}
\hline & MD-5 & MD-4 & MD-3 & MD-2 & MD-1 & MD \\
\hline TD (min), starters & $80.6 \pm 3.2$ & $95.1 \pm 2.2$ & $102.9 \pm 0.4$ & $92.6 \pm 1.6$ & $X$ & $64.2 \pm 7.6$ \\
TD (min), non-starters & $90.7 \pm 5.1$ & $97.5 \pm 3.6$ & $104.9 \pm 3.3$ & $94.6 \pm 3.4$ & $X$ & $52.7 \pm 15.4$ \\
S-RPE (au), starters & $442.4 \pm 45.4$ & $637.6 \pm 34.3$ & $763.9 \pm 36.5$ & $565.8 \pm 43.6$ & $X$ & $515.9 \pm 75.0$ \\
S-RPE (au), non-starters & $581.8 \pm 55.0$ & $644.7 \pm 62.0$ & $746.0 \pm 62.5$ & $575.1 \pm 42.6$ & $X$ & $366.1 \pm 93.3$
\end{tabular}

$M D$ - = matchday minus (5. 4. 3. 2. 1); $X=$ day-off; $T D=$ training duration; min = minutes; s-RPE = session rating of perceived effort; $a u=$ arbitrary units.

\section{DISCUSSION}

The main finding of this study highlights that in-season physical ITL was similar between starters and nonstarters. There was only one day of the week with higher values for starters (MD) and one day with higher values for non-starters (MD-5). The data presented are in opposition to the study of Impellizzeri et al. (2005) that suggested that longer individual match playing time might favour those starters in improvement and maintenance of physical capacities relevant for soccer performance. However, our study proved that ITL can be similar in training sessions regarding the intensity. Even so, junior players could have different adaptations across time because the matches are an important part of their soccer-specific practice in order they improve real match situation (Impellizzeri et al., 2005). The present study does not provide information regarding distances covered at higher speed that are associated with explosive actions of the match and therefore, does not confirm if the ITL applied was enough to compensate the non-played matches and to reduce the training status of non-starters (Dalen \& Loras, 2019).

\section{CONCLUSIONS}

The main conclusion indicates that is possible to provide additional ITL with similar training duration for nonstarters in order to develop their status to become a "starter player", especially in MD-5. Also, the study provides relevant data to balance the differences in a team between starters and non-starters. 


\section{REFERENCES}

Dalen, T. \& Loras, H. (2019). Monitoring Training and Match Physical Load in Junior Soccer Players: Starters versus Substitutes. Sports, 7, 70. https://doi.org/10.3390/sports7030070

Impellizzeri, F., Marcora, S. \& Coutts, A. (2018). Internal and External Training Load: 15 Years On. Int J Sports Physiol Perform, (In Press). https://doi.org/10.1123/ijspp.2018-0935

Impellizzeri, F.M.; Rampinini, E.; Marcora, S.M. (2005). Physiological assessment of aerobic training in soccer. J Sports Sci, 23, 583-592. https://doi.org/10.1080/02640410400021278

Malone, J., Di Michele, R., Morgans, R., Burgess, D., Morton, J. \& Drust, B. (2015). Seasonal TrainingLoad Quantification in Elite English Premier League Soccer Players. Int J Sports Physiol Perform, 10, 489-497. https://doi.org/10.1123/ijspp.2014-0352

Oliveira, R., Brito, J., Martins, A., Mendes, B., Calvete, F., Carriço, S., Ferraz, R., Marques, M. (2019). In-season training load quantification of one-, two- and three-game week schedules in a top European professional soccer team. Physiol Behav, 201, 146-156. https://doi.org/10.1016/j.physbeh.2018.11.036 


\title{
Pre-season and in-season internal training load quantification of one-week schedules in under-17 European soccer team
}

\author{
RAFAEL OLIVEIRA1 ${ }^{1,2,3,4}$, JOÃO M. BRITO ${ }^{1,2,3}$, ALEXANDRE MARTINS ${ }^{1}$ \\ ${ }^{1}$ Sports Science School of Rio Maior, Polytechnic Institute of Santarém, ESDRM-IPS, Rio Maior, Portugal \\ ${ }^{2}$ Research Centre in Sport Sciences, Health Sciences \& Human Development, CIDESD, Vila Real, Portugal \\ ${ }^{3}$ Research Centre on Quality of Life, CIEQV, Santarém, Portugal \\ ${ }^{4}$ Department of Sports Sciences, University of Beira Interior, UBI, Covilhã, Portugal
}

\begin{abstract}
The aim of this study was to compare internal training load (ITL) of starters versus non-starters from the same under-17 soccer team. Rated of perceived exertion was collected and then multiplied by training or match duration (s-RPE) from twenty-three soccer players. The study analysed a total of 2 matches and 8 training sessions during the in-season with ANOVA with repeated measures. The main results showed higher values in pre-season for training duration (103.3 vs $90.0 \mathrm{~min}, p=0.000$ ) in MD-5 and (103.7 vs $96.7 \mathrm{~min}, p>0.05$ ) in MD-3 than in-season, but lower values for training duration (103.2 vs $112.0 \mathrm{~min}, \mathrm{p}=0.013$ ) in MD-4 and (84.0 vs $97.2 \mathrm{~min}, p=0.000$ ) in MD-2 than in-season. s-RPE was higher in pre-season than in-season (725.5 vs 481.3 a.u., $p=0.000$ ) in MD-5 and (755.8 vs 655.2 a.u., $p=0.031$ ) in MD-3 but lower (705.2 vs 821.3 a.u., $p=0.002$ ) in MD-4 and (476.0 vs 559.0 a.u., $p=0.020$ ) in MD-2 than in-season. This study present relevant additional ITL data for pre-season and in-season that could be used to adjust periodization in under-17 soccer players. Keywords: Soccer training; Internal load; Training load, Periodization, RPE, Monitoring.
\end{abstract}

Corresponding author. Sports Science School of Rio Maior, Av. Dr. Mário Soares nº 110, 2040-413, Rio Maior, Portugal. E-mail: rafaeloliveira@esdrm.ipsantarem.pt

Supplementary Issue: Spring Conferences of Sports Science. International Seminar of Physical Education, Leisure and Health, 17-19 June 2019. Castelo Branco, Portugal.

JOURNAL OF HUMAN SPORT \& EXERCISE ISSN 1988-5202

(c) Faculty of Education. University of Alicante.

doi:10.14198/jhse.2019.14.Proc4.82 


\section{INTRODUCTION}

As an index of perception of exertion of internal training load (ITL), rated of perceived exertion (RPE) is an integration of afferent neural signals from various physiological systems to the brain (Abbiss et al., 2015). Gaudino et al. (2015) used the training duration multiplied by the RPE to generate the session-RPE (s-RPE) and reported that s-RPE was significantly correlated with the highspeed running distance, number of impacts, and accelerations in elite soccer players. In addition, Impellizzeri et al. (2018) recommended to use ITL as primary measure when monitoring athletes because it determines the training outcome experienced from a specific external load applied. This is an easier and low-cost tool that could be used during an entire season. According to Bompa and Haff (2009), pre-season phase consists in the period before the beginning of competitions and its goal is to promote physical, technical and psychological base. Generally, this phase is associated by an increase in training volume during approximately 6 weeks. Then, competition phase, known as in-season phase, consists in high-intensity period of training and matches during approximately 40 weeks. The emphasis of this phase is to allow soccer players to give the best performance in soccer matches by keeping sport-specific fitness, developing technical and tactical soccer actions, and reducing fatigue (Bompa \& Haff, 2009). Therefore, the aim of this study was to analyse ITL between pre-season versus in-season and between player positions from the same team during the in-season 2017-2018.

\section{MATERIAL AND METHODS}

\section{Participants}

Twenty-one male under-17 soccer players belonging to a Portuguese team with a mean \pm SD age, height and mass of $16.0 \pm 0.2 y e a r s, 173.9 \pm 0.1 \mathrm{~cm}$ and $65.3 \pm 4.2 \mathrm{~kg}$, respectively, participated in this study. They consisted in four central defenders (CD), three wide defenders (WD), six central midfielders (CM), three wide midfielders (WM) and three strikers (ST). Inclusion criteria were regular participation in most of the training sessions (100\% of weekly training sessions).

\section{Measures}

Each individual RPE value was multiplied by the session duration to generate a session-RPE (s-RPE) value. All explanations of this measure were reported in previous study (Oliveira et al., 2019a, 2019b).

\section{Procedures}

The weeks from pre-season and in-season phases were analysed in relation to the number of days away from the competitive one-match week (i.e., match day minus) where the team trained four days a week (match day [MD] minus [-]; MD-5; MD-4; MD-3; MD-2) with one match-day (MD) (Oliveira et al. 2019a). Data from rehabilitation or additional training sessions of recuperation were excluded. This study did not influence or alter the training sessions in any way. Total minutes of training sessions included warm-up, main phase and slow down phase plus stretching. All participants were familiarized with the training protocols prior to investigation. This study was conducted according to the requirements of the Declaration of Helsinki and was approved by the institution's research ethics committee.

\section{Analysis}

Data were analysed using SPSS version 22.0 (SPSS Inc., Chicago, IL) for Windows statistical software package. Initially, descriptive statistics were used to describe and characterize the sample. Shapiro-Wilk and the Levene tests were used to assumption normality and homoscedasticity, respectively. ANOVA with repeated measures was used with Bonferroni post hoc, once variables obtained normal distribution (ShapiroWilk $>0.05$ ) to compare days away from the competitive match fixture and player positions. Also, it was used 
ANOVA Friedman and Mann-Whitney tests were used for the variables that not obtained normal distribution to compare different scenarios. Results were significant with $p \leq 0.05$.

\section{RESULTS}

In training duration from pre-season, there were significant differences between all player positions for MD5 and MD-4 ( $p<0.000$ ). Also, there were significant differences between CD vs ST ( 61.3 vs $36.7 \mathrm{~min}, p=0.034$ ), WD vs ST (71.3 vs $36.7 \mathrm{~min}, p=0.004)$, CM vs ST (62.2 vs $36.7 \mathrm{~min}, p=0.015)$, WM vs ST (80.0 vs $36.7 \mathrm{~min}$, $p=0.000$ ). Regarding s-RPE, there were significant differences between CM vs WM (914.7 vs 784.0 a.u., $p=0.038$ ) and CM vs ST (914.7 vs 746.7 a.u., $p=0.005$ ) in MD-4. Also, there were significant differences between CD vs WD (428.8 vs 521.7 a.u., $p=0.07$ ), WD vs ST (521.7 vs 273.3 a.u., $p=0.03$ ), CM vs WM (452.7 vs 640.0 a.u., $p=0.010$ ) and CM vs ST (452.7 vs 273.3 a.u., $p=0.014$ ) in MD.

In training duration from in-season, there were significant differences between all player positions for MD-5 $(p<0.000)$. No differences were found for any other training or match duration. No differences were found regarding s-RPE between any training session or match.

Training duration was higher in pre-season than in-season (103.3 vs $90.0 \mathrm{~min}, \mathrm{p}=0.000)$ in MD-5, (103.7 vs $96.7 \mathrm{~min}, \mathrm{p}>0.05)$ in MD-3. When comparing pre-season with in-season, training duration was lower in preseason than in-season (103.2 vs $112.0 \mathrm{~min}, \mathrm{p}=0.013$ ) in MD-4 and ( $84.0 \mathrm{vs} 97.2 \mathrm{~min}, \mathrm{p}=0.000$ ) in MD-2. No differences were found for training duration when comparing pre-season with in-season in MD. s-RPE was higher in pre-season than in-season (725.5 vs 481.3 a.u., $p=0.000$ ) in MD-5, (755.8 vs 655.2 a.u., $p=0.031$ ) in MD-3. When comparing pre-season with in-season, s-RPE was lower in pre-season than in-season $(705.2$ vs 821.3 a.u., $p=0.002$ ) in MD-4 and (476.0 vs 559.0 a.u., $p=0.020$ ) in MD-2. No differences were found for $\mathrm{s}-\mathrm{RPE}$ when comparing pre-season with in-season in MD.

\section{DISCUSSION}

The main finding of this study highlights that pre-season presented higher values of ITL than in-season in MD-5 and MD-3, however in-season presented higher values of ITL than pre-season in MD-4 and MD-2. These finding are not in line with periodization proposed by Bompa and Haff (2009) where pre-season phase should have higher values in all training sessions. Another finding is related to the player positions comparisons. Pre-season revealed that ST performed training session with lower durations than other positions. Also, CM and WM presented higher values when compared to other positions. However in inseason phase there were no differences between player positions for training duration or S-RPE which is in line with Oliveira et al. (2019b). Possible explanations could be associated to the volatile construct of s-RPE that can be dissociated from the physiological process as reported in Oliveira et al. (2019b).

\section{CONCLUSIONS}

The most relevant conclusion indicates that is still not well characterized pre-season and in-season phases. Our data provides additional information regarding ITL that could be used to adjust periodization to under -17 soccer players. 


\section{REFERENCES}

Abbiss, C.R., Peiffer, J.J., Meeusen, R. \& Skorski, S. (2015). Role of ratings of perceived exertion during self-paced exercise: What are we actually measuring? Sports Med, 45, 1235-1243. https://doi.org/10.1007/s40279-015-0344-5

Bompa, T. \& Haff, G. (2009). Periodization, Theory and Methodoly of Training. Human Kinetics. 5th Edition.

Gaudino, P., laia, F.M., Strudwick, A.J., Gawkins, R., Alberti, G., Atkinson, G. \& Gregson, W. (2015). Factors influencing perception of effort (session-RPE) during elite soccer training. Int J Sports Physiol Perform, 10, 860-864. https://doi.org/10.1123/ijspp.2014-0518

Oliveira, R., Brito, J., Martins, A., Mendes, B., Calvete, F., Carriço, S., Ferraz, R., Marques, M. (2019a). In-season training load quantification of one-, two- and three-game week schedules in a top European professional soccer team. Physiol Behav, 201, 146-156. https://doi.org/10.1016/j.physbeh.2018.11.036.

Oliveira, R., Brito, J., Martins, A., Mendes, B., Ferraz, R., Marques, M. (2019b). In-season internal and external training load quantification of an elite European soccer team. Plos One, 14(4), e0209393. https://doi.org/10.1371/journal.pone.0209393

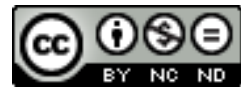

This work is licensed under a Attribution-NonCommercial-NoDerivatives 4.0 International (CC BY-NC-ND 4.0). 


\title{
Bullying among medical students: Integrative literature review
}

\author{
FERNANDA NATAL ALVES ${ }^{1}$, CAROLINA MILHIM BARCELLOS ${ }^{1}$, PRISCILLA DOS REIS OLIVEIRA ${ }^{1}$, \\ LILIAN CRISTINA GOMES DO NASCIMENTO ${ }^{1}$, BEATRIZ PEREIRA², MARTA ANGÉLICA IOSSI SILVA ${ }^{3}$, \\ JORGE LUIZ DA SILVA 1 \\ ${ }^{1}$ University of Franca, Brazil \\ 2University of Minho, Portugal \\ 3 University of São Paulo, Brazil
}

\begin{abstract}
This integrative literature review aimed to identify the prevalence and characteristics of bullying among medical students. The bibliographic survey was carried out in six databases: LILACS, PsycINFO, PubMed, SciELO, SCOPUS and Web of Science. In all bases the crossings of keywords were carried out: bullying AND medical students; bullying AND medical school. Only articles published in the last 10 years and published in Portuguese, Spanish and English were selected. Among the 111 identified articles, nine have met the inclusion criteria and were analysed. All of them were published in the last five years (2014-2018). The quantitative method was the most used (77.8\%). The prevalence of bullying ranged from $12.3 \%$ to $98.69 \%$, with average percentage of $39.1 \%$. The most prevalent aggression was verbal, followed by physical and cyberbullying. Verbal aggression was the most practiced by both sexes. Men practiced more physical aggression toward women. The places where the aggressions occurred most frequently were in the classroom, social networks, university exit, restrooms and cafeterias. When there was some help to the victim, it was performed by friends, most of the time. It is concluded that bullying has a high prevalence among medical students. Thus, better identification of bullying by the university, training of teachers and the provision of a support network and student orientation are fundamental to prevent and reduce this occurrence. Keywords: Bullying; Medical students; Medical school; Integrative literature review.
\end{abstract}

Corresponding author. University of Franca. Avenida Dr. Armando Salles de Oliveira, 201, zip code: 14404-600, Franca, São Paulo, Brazil.

E-mail: nanda.alves89@gmail.com

Supplementary Issue: Spring Conferences of Sports Science. International Seminar of Physical Education, Leisure and Health, 17-19 June 2019. Castelo Branco, Portugal.

JOURNAL OF HUMAN SPORT \& EXERCISE ISSN 1988-5202

(c) Faculty of Education. University of Alicante.

doi:10.14198/jhse.2019.14.Proc4.82 


\section{INTRODUCTION}

School bullying refers to acts among peers characterized by intention, repetitiveness and imbalance of power among students. These acts can be physical (e.g., hitting, kicking, pushing), verbal (e.g., calling names, swearing at the victim, laughing), or relational (e.g., socially isolating the victim, spreading rumours, or manipulating relationships) (Silva et al., 2016). Although bullying is considered a phenomenon during infancy and adolescence, it can also be present in the interaction of university students. To suffer, to practice and to testify the bullying at the university can cause sensations of fear and insecurity, besides producing negatives effects in the mental health, academic performance and quality of life of the university students. These effects can be higher in the medical students, for being a course that requires a lot of pressure and generally produces high levels of stress when compared to other courses (Pagnin \& Queiroz, 2015). Hence, the objective of this study is to identify the prevalence and the characteristics of the bullying in the students of medicine.

\section{MATERIAL AND METHODS}

This project is an integrating review literature, performed by the following steps: 1) Identification of the topic and performing of a search question; 2) establishment of inclusion and exclusion criteria; 3 ) definition of the information to be extracted from the studies; 4) evaluation of the included studies; 5 ) interpretation of the results and; 6) presentation of knowledge synthesis (Mendes et al. 2008).

The bibliographic survey was carried out in the month of October 2018 in six databases: LILACS, PsycINFO, PubMED, SciELO, SCOPUS and Web of Science. In all databases the crossing of the keywords were carried out by: bulling AND medical students; bullying AND medical school. The guiding question of the bibliographic search was: What is the prevalence and characteristics of bullying among medical students? It was elaborated according to PVO strategy (Population, Variables and Outcomes).

Publications of the last ten years (2009-2018) were included, with the purpose of recovering the most recent production on the subjects investigated. Only articles were selected, because they were evaluated more rigorously before being published. In addition, only articles with a specific focus on the subjects investigated and published in Portuguese, Spanish and English were inserted.

The process of search and bibliographic selection was carried out independently by two reviewers. The divergences between the searchers were discussed until a consensus could be found. For the selection of the articles found in the bibliographic search, it was carried out a reading of the titles and abstract. In this stage, those who didn't meet the search question and the previously defined inclusion criteria were excluded.

In order to support the work of the reviewers, a spreadsheet in the Excel program was composed by the following data: article title, authors, author's institution, year of publication, name of the journal, place of research, study objective, method, main results and conclusions.

\section{RESULTS}

Among the 111 identified articles, nine met the inclusion criteria and were analysed. All of them were published in the last five years (2014-2018). The quantitative method was the most used (77.8\%). The prevalence of bullying ranged from $12.3 \%$ to $98.69 \%$, with a mean percentage of $39.1 \%$. The most prevalent aggression was verbal, followed by physical and cyberbullying. Verbal aggression was the most practiced by 
both sexes. The men practiced more physical aggression toward women. The main perpetrators were peers and residents. The places where the aggressions occurred most frequently were the classroom, social networks, university exit, restrooms and cafeterias. When there was some help to the victim, it was performed by friends, most of the time. Students considered that there was not much to do, they generally ignored the aggression or avoided the aggressor.

\section{DISCUSSION}

This review evidenced the existence of high rates of bullying among medical students, indicating the need to study this phenomenon in depth and to adopt academic policies and strategies to prevent and reduce this phenomenon in the university (Reyes et al., 2015). It was also identified the need to increase the awareness of medical students about the harmful aspects of bullying, aiming to improve the coexistence between them (Silva-Villarreal et al., 2013). Similarly, it is important to look for ways to improve the students, residents and physicians' communication skills, especially those with more aggressive communication styles in order to reduce the occurrence of verbal bullying (Lazarus et al., 2016). As men and women practice and suffer bullying differently, it is critical that the interventions consider gender differences. The scarcity of studies identified in the literature search indicates the need for more research on the subject.

\section{CONCLUSION}

It is concluded that bullying is prevalence among medical students. Thus, better identification of bullying by the university, training of teachers and the provision of a support network and student orientation are fundamental to prevent and reduce its occurrence.

\section{REFERENCES}

Lazarus, J. L., Hosseini, M., Kamangar, F., Levien, D. H., Rowland, P. A., Kowdley, G. C., \& Cunningham, S. C. (2016). Verbal aggressiveness among physicians and trainees. J Surg Educ., 73(4):756-60. https://doi.org/10.1016/i.jsurg.2016.03.005

Mendes, K. S., Silveira, R. C. C. P., \& Galvão, C. M. (2008). Revisão integrativa: método de pesquisa para a incorporação de evidências na saúde e na enfermagem. Texto \& Contexto - Enferm., 17(4), 758-764. https://dx.doi.org/10.1590/S0104-07072008000400018

Pagnin D., \& Queiroz V. (2015). Comparison of quality of life between medical students and young general populations. Educ Health, 28(3), 209-212. https://doi.org/10.4103/1357-6283.178599

Reyes, L. C., Barrera Sanchez, L. F., \& Ospina Diaz, J. M. (2015). Caracterización del bullying en estudiantes de medicina de Tunja, Boyacá. Revista Virtual Universidad Católica del Norte, 45, 101 112.

Silva, J. L., Oliveira, W. A., Braga, I. F., Farias, M. S., Silva Lizzi, E. A., Gonçalves, M. F. C., Silva, M. A. I. (2016). The effects of a skill-based intervention for victims of bullying in Brazil. Int J Environ Res Public Health, 13, 1042-1052. https://doi.org/10.3390/ijerph13111042

Silva-Villarreal, S., Castillo, S., Eskildsen, E., Vidal, P., Mitre, J., Quintero, J. (2013). Prevalencia de bullying en estudiantes de los ciclos básicos y preclínicos de la carrera de medicina de la Universidad de Panamá. Arch Med, 9(4), 1-8. https://doi.org/10.15517/rc_ucr-hsjd.v3i10.12781

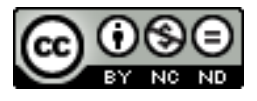

This work is licensed under a Attribution-NonCommercial-NoDerivatives 4.0 International (CC BY-NC-ND 4.0). 


\title{
Perceived motivational climate and goal orientation in soccer athletes: A longitudinal perspective
}

\author{
MARCIO DOMINGUES
}

CPES - ULHT, Portugal

\begin{abstract}
The study aims to understand the differences in motivation to help individuals, independently of their abilities to reach their full potential. The central point aims to study behaviour variability more than predicting performance outcomes. The purpose of the study was to characterize the clubs motivational climate and goal achievement regarding their coaches and athletes and to analyse the effects of time and context in these variables. We also intended to examine the variation in results associated with the training environment of latent variables resulting from the TEOSQ and PMCSQ. We will sample 120 football youngsters with ages 12-18 years old, coaches of each team with a longitudinal perspective. To assess the problem we will apply two questionnaires (TEOSQ and PMCSQ) with descriptive statistics and t-test and we also carried out analysis of content. There is a relationship between the approach undertaken by the coach and the sport context and the motivational climate variables. Keywords: Context; Football; Goal achievement; Perceived motivational climate.
\end{abstract}

Corresponding author. CPES - ULHT, Portugal.

E-mail: marcio.domingues@live.com.pt

Supplementary Issue: Spring Conferences of Sports Science. International Seminar of Physical Education, Leisure and Health, 17-19 June 2019. Castelo Branco, Portugal.

JOURNAL OF HUMAN SPORT \& EXERCISE ISSN 1988-5202

(c) Faculty of Education. University of Alicante.

doi:10.14198/jhse.2019.14.Proc4.82 


\section{INTRODUCTION}

Knowing the significance that football has in sport practice and participation in Portugal, it is important to analyse the effects of its involvement in youth athletes, in order to verify and comprehend the phenomenon that contribute to its development. As Roberts (2001) points out, the comprehension and development of motivation constitutes one of the central concerns of the human conduct, being it a very misunderstood subject. More and more, sport organizations look at youth formation as a pathway to the professional career in a global scale. In effect, in Europe and other countries, sport talent development programs have been generalized, delivered to sport organizations since sub-9 to sub 19 (Stratton, Reilly, Williams and Richardson, 2004). Past research in sport domain has shed evidence to the fact that perceptions of competencies demonstrated comprehend perceptions of the objective or subjective success or failure (Roberts \& Duda, 1992). The involvement through ego orientation, requires the presence of others that can be capable of valuing the performances (Roberts, 2001). What relation exists between goal achievement and the objectives of team sport, motivational climate, satisfaction, sources of satisfaction, realization strategies and perception of ability in team sports? The relation between perceived motivational climate and intrinsic motivation and self-efficacy, as well as goal achievement and motivational climate in the prediction of intrinsic motivation and self-efficacy were the objectives of Kavussanu and Roberts (1996). The purpose of this study is: (i) characterize the contexts of practice through the interviews to coaches of a professional club; (ii) analyse the effect of context of practice over the young players attitudes; (iii) analyse the effect of the context of practice in goal achievement; (iv) understand these variables in a longitudinal perspective through a time framework. The initial problem was to perceive the effect of the ecology of practice in football in goal achievement and motivational climate in young football players with ages between 12 and 18 years of age.

\section{MATERIAL AND METHODS}

\section{Participants}

a) Participants - 120 players of football with ages between 11 and 18 years old during 5 seasons;

b) Coaches -6 coaches from the groups analysed, the interviews will involve, the ecology of practice, perceived motivational climate, sports perspective and trajectories and social involvement.

\section{Measures}

a) The sport questionnaire in Goal Achievement (Ego/Task), is largely used internationally (Hanrahan \& Biddle, 2002). This questionnaire comes as a follow up of studies elaborated by Nicholls itself in the academic context, to create a motivational oriented scale. Chi and Duda (1995) tried to test simultaneous and independently the model assumed by TEOSQ. In sport settings, Duda and Nicholls, elaborated the original version of TEOSQ which contained 16 items, but ended up including only 13 (Duda \& Whitehead, 1998). In that sense, it was created to evaluate individual differences regarding task and ego motivational orientation, in a sport level and translated and validated to Portuguese by Fonseca and Biddle (1996).

b) Perceived Motivational Climate in Sport Questionnaire(PMCSQp; Fonseca \& Biddle, 1996). This questionnaire intends to evaluate the perception of athletes about the team's motivational climate, regarding two orientations (mastery climate and performance climate).

c) Interview to other significant - Semi-structured interview with content analysis. 


\section{Procedures}

Informed consent from parents which is mandatory when applying questionnaires to this particular study. The young athletes will be previously informed about the study and procedures to be taken. The questionnaires will be answered in a special room for the effect and they will last no longer than 30 minutes.

\section{Analysis}

SPSS v25 and content analysis.

\section{RESULTS}

Van-Yperen e Duda (1999) through their longitudinal study attempted to study the relation between goal achievement and beliefs of success in elite football players from the Netherlands. The researchers found a positive association between ego orientation and the belief that ability or innate talent are determinants of success. They found that task orientation is linked to beliefs that effort, team play and parental support contribute to goal achievement in football. It is worthwhile to consider a possible relation between results in the TEOSQ test and the assumption that talent selection is based as a recruitment machine with a national coverage.

\section{DISCUSSION}

The selected players can possess certain characteristics that meet the clubs philosophy and the rising to the competitive world. In fact, Pensgaard and Roberts (2002) refer the importance of task orientation in elite athletes, in the pathway that conducts to an elite culture, where we will find values such as persistence and hard work, something that meets the mental toughness reported by coaches. This construct demonstrates the impact of perceived motivational climate and goal achievement orientation in the creation of moral behaviour when we cross these data with the interviews (Domingues \& Gonçalves, 2013). The work that the elite club does around each player and the worries demonstrated with their sport and social behaviours predispose to a moral functioning and superior levels for social reasoning.

\section{CONCLUSIONS}

The study pretends to verify the inclusion of the variable age in a way as to ascertain the behaviours in goal achievement in posterior ages of the sport formation process; the study equates the possibility of verifying the constancy of the variables at play. The study allows the inclusion of perceived motivational climate and crossing the results with TEOSQ to verify which climate is dominant in the youth sport domain, that is, acknowledge the formative climate. Interviews to coaches in youth teams to evaluate how the selection of players is made, concept of formation, process of learning versus the emergency of results.

\section{REFERENCES}

Adelino, J., Vieira, J. \& Coelho, O. (2005). Caracterização da prática desportiva juvenil federada. Lisboa: Instituto do Desporto Portugal.

Chi, L. \& Duda, J.L. (1995). Multi-sample confirmatory factor analysis of the Task and Ego Orientation in Sport Questionnaire. Research Quarterly for Exercise and Sport, 66, 2, 91-98. https://doi.org/10.1080/02701367.1995.10762216

Domingues, M., \& Gonçalves, C. (2013) Managing Youth Sport in Declined Organizations. Studies in Sport Humanities, 14, 59-68. 
Duda, J. \& Whitehead, J. (1998). Measurements of goal perspectives in the physical domain". In Duda $\mathrm{JL}$ (ed.). Advances in Sport and exercise psychology measurement (pp. 21 - 48). Morgantown, WV: Fitness Information Technology.

Fonseca, A. \& Biddle, S. (1996). Estudo inicial para a adaptação dos Task and Ego Orientation in Sports Questionnaire (TEOSQ) à realidade Portuguesa. In Programa e Resumos da IV Conferência Internacional "Avaliação Psicológica: Forma e Contextos" e II Mostra de "Provas Psicológicas em Portugal". Minho: Universidade do Minho. https://doi.org/10.18803/capsi.v17.185-193

Kavussanu M, Roberts GC. (1996). Motivation in Physical Activity Contexts: The Relationship of Perceived Motivational Climate to Intrinsic Motivation and Self-Efficacy. In Journal of Sport \& Exercise Psychology, vol. 18 (3). https://doi.org/10.1123/jsep.18.3.264

Pensgaard A.M, Roberts GC. (2002). Elite athletes' experiences of the motivational climate: The coach matters. In Scandinavian Journal of Medicine \& Science in Sports, vol 12 (1),pp: 54-59. https://doi.org/10.1034/j.1600-0838.2002.120110.x

Roberts, G.C. (2001). Understanding the Dynamics of Motivation in Physical Activity: The Influence of Achievement Goals on Motivational Processes. Roberts GC (ed.). In Advances in Sport and Exercise (1-5). Champaign, Illinois: Human Kinetics.

Stratton, G., Reilly, T., Williams \& M., Richardson, D. (2004). Youth Soccer. From science to performance. Routledge. Taylor \& Francis group.

Van-Yperen NW, Duda JL. (1999). Goal orientations, beliefs about success, and performance improvement among young elite Dutch soccer players. In Scandinavian Journal Medicine \& Science in Sports, vol. 9 (6), pp: 358-364. https://doi.org/10.1111/j.1600-0838.1999.tb00257.x

\section{(9) $(\mathbb{Q} \Theta \Theta$}

This work is licensed under a Attribution-NonCommercial-NoDerivatives 4.0 International (CC BY-NC-ND 4.0). 


\title{
Bullying victimization and family interactions of Brazilian students: A mixed study
}

\author{
WANDERLEI A. OLIVEIRA ${ }^{1}$, JORGE L. SILVA², LIANDRA A. O. CAETANO², MARCELA A. \\ ZEQUINÃO2 ${ }^{2}$ MANOEL A. SANTOS², SIMONA C. S. CARAVITA², MARTA A. I. SILVA ${ }^{2}$ \\ 1 University of São Paulo, Brazil \\ 2University of Franca, Brazil \\ ${ }^{3}$ Santa Catarina State University, Brazil \\ ${ }^{4}$ Catholic University of the Sacred Heart, Italy
}

\begin{abstract}
Bullying victimization is a problem faced by many students. However, there are lacunas in Brazilian scientific production regarding the interface between bullying and family variables. Thus, this study aiming at analyses the relationship between the experience of victimization in the school and the quality of the family interactions of Brazilian students. It is a mixed study that included 2.354 students (50.6\% girls; $14.50 \pm 2.01$ years old) from 11 public schools. Data were collected by application of two scales (bullying and family interactions) and semi-structured interviews with a smaller sample subgroup $(n=55)$. Data analysis procedures included: descriptive statistics; analysis of variance; logistic regression; and thematic analysis of the qualitative data performed in Atlas.Ti software. The results showed a prevalence of $10.1 \%$ of victims of bullying among participants. In the combined analysis of data, it was identified that students not involved in bullying situations had better family interactions when compared with the victimized students. Negative communication and physical punishment in the family increased the possibility of students being targeted by offenders. On the other hand, a greater reference of parental supervision was related to lower reports of victimization. The study offers contributions because it reveals the reality of a developing country to the international scientific community regarding the analysis of bullying victimization. Keywords: Peer-group; Victimization; Violence; School health; Family relationships.
\end{abstract}

Corresponding author. Avenue Bandeirantes, 3900, 14040-902, EERP Room 75, Ribeirão Preto, São Paulo, Brazil.

E-mail: wanderleio@hotmail.com

Supplementary Issue: Spring Conferences of Sports Science. International Seminar of Physical Education, Leisure and Health, 17-19 June 2019. Castelo Branco, Portugal.

JOURNAL OF HUMAN SPORT \& EXERCISE ISSN 1988-5202

(c) Faculty of Education. University of Alicante.

doi:10.14198/jhse.2019.14.Proc4.82 


\section{INTRODUCTION}

Bullying is a problem that affects the health and development of school-age adolescents worldwide (Oliveira, 2017). There is a complex dynamic in the development of bullying situations and different subgroups of student participation have already been identified as aggressors, observers, victim-aggressors and victims (Olweus, 2013). In this study we are interested in the subgroup of victims who, in general, are not able to interrupt bullying alone. They also have a lower sense of belonging to the school context, as well as suffer the consequences of victimization in the teaching-learning process and in their overall health (Olweus, 2013; Oliveira, 2017). Moreover, in Brazil, bullying is still little studied and researches focuses in the diagnosis or the characterization of the phenomenon, revealing the need to advance in the perspective of new approaches, including context variables, and that could be related to their occurrence in the schools (Oliveira, 2017). Therefore, the main objective of this study was to analyse the relationship between the experience of victimization in the school and the quality of the family interactions of Brazilian students.

\section{MATERIAL AND METHODS}

It was a mixed study that gathered quantitative and qualitative data.

\section{Participants}

A total of 2.354 students ( $50.6 \%$ girls; $14.50 \pm 2.01$ years old) of 11 public schools were included. Of this total, 55 ( $46.5 \%$ girls; $15 \pm 2$ years old) were randomly selected for the qualitative research step.

\section{Measures}

Two scales (Cunha; Weber; Steiner Neto 2011; Weber; Salvador; Brandenburg, 2011) and semi-structured interviews were used to obtain the data.

\section{Procedures}

This study was realized between August and October 2014. The students answered the scales collectively and the interviews were individual, fully recorded and transcribed.

\section{Analysis}

Data analysis procedures included: descriptive statistical analysis; analysis of variance; logistic regression; and thematic analysis of the qualitative data (Bardin, 2011), which was performed in Atlas.Ti software.

\section{RESULTS}

Data analysis revealed that $10.1 \%(n=237)$ of the participants were victimized. These students presented high averages in the victimization scales and lower frequencies in the aggressiveness scales. The girls reported more cases of victimization than the boys, however, male sex was more associated with the group of victims (OR: $1.57 ; p<0.001)$. The dimensions of the quality of family interactions "physical punishment" (OR: 1.26, $p<0.01)$ and "negative communication" (OR: 1.81, $p<0.01)$ were significantly associated with the victims, indicating that their occurrence in the domestic environment increased the chance of victimization. These quantitative results were supported by qualitative analyze. 


\section{DISCUSSION}

The data from this study are in accordance with studies that have shown that families of bullying victims are generally less functional than families of students without bullying involvement (Oliveira et al., 2017). In addition, it is observed that repeated experiences in the family microsystem lead children and adolescents to internalize patterns of behaviours and attitudes that will induce social behaviour in the peer group (PATTON et al. 2013). This theoretical perspective may explain, in part, the data presented. In order to expand this discussion, the complete research can be verified in studies published elsewhere (Oliveira et al., 2018).

\section{CONCLUSIONS}

The number of students victimized, not only in this study, is worrying. This public, specifically, requires new directions in the field of intervention and prevention. It was also clear that anti-bullying interventions need to expand their focus beyond the school environment and encompass the family microsystem, including parents and responsible for the children or adolescent.

\section{FUNDING}

Preparation of this manuscript was supported by grants FAPESP (São Paulo Research Foundation) Process 2014/13062-7 and in part by the Coordenação de Aperfeiçoamento de Pessoal de Nível Superior - Brazil (CAPES) - Finance Code 001.

\section{REFERENCES}

Bardin, L. (2011). Análise de conteúdo. São Paulo.

Cunha, J. M., Weber, L. N. D., \& Steiner Neto, P. (2011). Escala de Vitimização e Agressão entre Pares (EVAP). In L. N. D. Weber \& M. A. Dessen (Eds.), Pesquisando a família. Instrumentos para coleta e análise de dados (pp. 103-114). Curitiba: Juruá.

Oliveira, W. A. (2017). Relações entre bullying na adolescência e interações familiares: do singular ao plural. (Tese de Doutorado), Universidade de São Paulo, Ribeirão Preto. https://doi.org/10.11606/t.22.2017.tde-26092017-212918

Oliveira, W. A., Silva, J. L., Santos, M. A., Hayashida, M., Caravita, S. C. S., \& Silva, M. A. I. (2018). Interações familiares de estudantes em situações de bullying. Jornal Brasileiro de Psiquiatria, 67(3), 151-158. https://doi.org/10.1590/0047-2085000000204

Oliveira, W. A., Silva, J. L., Sampaio, J. M. C., \& Silva, M. A. I. (2017). Students' health: an integrative review on family and bullying. Ciência \& Saúde Coletiva, 22, 1553-1564. https://doi.org/10.1590/1413-81232017225.09802015

Olweus, D. (2013). School bullying: development and some important challenges. Annual Review of Clinical Psychology, 9(1), 751-780. https://doi.org/10.1146/annurev-clinpsy-050212-185516

Patton, D. U., Hong, J. S., Williams, A. B., \& Allen-Meares, P. (2013). A review of research on school bullying among African American youth: An ecological systems analysis. Educational Psychology Review, 25(2), 245-260. https://doi.org/10.1007/s10648-013-9221-7

Weber, L. N. D., Salvador, A. P. V., \& Brandenburg, O. J. (2011). Escalas de Qualidade na Interação Familiar. In L. N. D. Weber \& M. A. Dessen (Eds.), Pesquisando a família. Instrumentos para coleta e análise de dados (pp. 61-72). Curitiba: Juruá. https://doi.org/10.7213/psicolargum.v26i52.20005 


\section{(c) (i) (3)}

This work is licensed under a Attribution-NonCommercial-NoDerivatives 4.0 International (CC BY-NC-ND 4.0). 


\title{
Habitual physical activity patterns of pre-school children from Bragança
}

\author{
PEDRO MAGALHÃES ${ }^{1}$, EDUARDA COELHO ${ }^{2,3}$, PEDRO FORTE $^{2,4}$, CATARINA VASQUES $^{1,5}$ \\ ${ }^{1}$ Escola Superior de Educação, Instituto Politécnico de Bragança (IPB), Portugal \\ ${ }^{2}$ Centro de Investigação em Desporto, Saúde e Desenvolvimento Humano (CIDESD), Portugal \\ 3 Universidade de Trás-os-Montes e Alto Douro (UTAD), Portugal \\ ${ }^{4}$ Instituto Superior de Ciências Educativas do Douro (ISCE Douro), Portugal \\ ${ }^{5}$ Centro de Investigação em Educação Básica (CIEB), Portugal
}

\begin{abstract}
The increase in the prevalence of overweight in children is commonly associated with their low levels of physical activity (PA). In fact, young people who have higher levels of PA over several years tend to have lower rates of overweight than their fewer active peers. Thus, it becomes of the utmost importance to promote the practice of regular PA among children. In this context, the aims of this study were to compare the number of steps done by children during the weekdays with the weekend and to analyse the contribution of a schoolbase physical exercise (PE) session to the total number of daily steps performed by children. The sample comprised 105 pre-school children, from 3 to 6 years old (4.48 \pm 0.69$)$ from 5 kindergartens from Bragança. The PA evaluation was done through daily number of steps from New-Lifestyles NL-2000 pedometer. The mean number of steps taken by the children remained relatively constant during the week, decreasing only slightly on Friday. However, on Saturday and Sunday the decline was more pronounced. Significant differences were found between the number of steps on weekdays and weekend days $(p<0.001)$, with higher levels of PA on weekdays. PA was higher $(p<0.001)$ on the days with PE session than without PE session in kindergarten. Children of preschool age are more active during the weekdays than at the weekend. The implementation at school-based of PE session at pre-school level had a significant impact on daily PA levels of the children. Keywords: Physical activity; Pre-school children; Number of steps; Health promotion.
\end{abstract}

Corresponding author. Campus de Santa Apolónia, 5300-253 Bragança, Portugal.

E-mail: pmaga@ipb.pt

Supplementary Issue: Spring Conferences of Sports Science. International Seminar of Physical Education, Leisure and Health, 17-19 June 2019. Castelo Branco, Portugal.

JOURNAL OF HUMAN SPORT \& EXERCISE ISSN 1988-5202

(C) Faculty of Education. University of Alicante.

doi:10.14198/jhse.2019.14.Proc4.82

VOLUME 14 | Proc4 | 2019 | S1537 


\section{INTRODUCTION}

The scientific community is unanimous about the importance of habitual physical activity (PA) as a factor promoting healthy lifestyles (Brown et al., 2004). However, children currently tend to spend most of their free time in sedentary activities (Reilly et al., 2004).

Low levels of PA associated with excess caloric intake are the main causes of obesity in children (Baba et al., 2009). Since childhood and youth are seen as the age groups in which PA habits are acquired and consolidated (Shephard, 1990), it is extremely important to implement intervention programs with PA at these ages. In this context, the main objectives of this study were to compare the number of steps done by children during the weekdays with the weekend days, and to analyse the contribution of a school-base physical exercise (PE) session to the total number of daily steps performed by children. We explored PA differences between days with PE session and days without PE session, and the PA patterns comparing weekdays with weekend days.

\section{MATERIAL AND METHODS}

\section{Participants}

The sample comprised 105 pre-school children, between 3 and 6 years old $(4.46 \pm 0.65)$ from 5 kindergartens from Bragança. From these children, 58 were also evaluated during a school-based PE session.

\section{Measures}

About the anthropometry measurements, height was measured with a portable stadiometer SECA (model 213) and weight using TANITA scale (model 885). Height and weight were used to calculate children's body mass index (BMI) (kg/m2). PA evaluation was done through daily number of steps from New-Lifestyles NL2000 pedometer.

\section{Procedures}

Parents were asked for an informed consent before any intervention and the study was authorized by the school authorities. The pedometers were placed on children at the kindergarten. They used it during all day for seven consecutive days, except when doing any activity in the water and during sleeping time.

We implemented in each kindergarten two sessions of PE/week, lasting 50 minutes, with the responsibility of a physical education teacher.

\section{Analysis}

A paired t-test was used to compare the changes in children's PA during the weekdays (Monday to Friday) and weekend days (Saturday and Sunday) and between days with and without PE session.

\section{RESULTS}

Table 1 shows the mean values, standard deviation and range for age and anthropometric characteristics of the children. 
Table 1. Participant characteristics

\begin{tabular}{lcc}
\hline & Mean \pm Standard Deviation & Range \\
\hline Age $($ years $)$ & $4.48 \pm 0.69$ & $3-6$ \\
Height $(\mathrm{m})$ & $1,09 \pm 0.06$ & $0.96-1.25$ \\
Weight $(\mathrm{kg})$ & $19.54 \pm 3.99$ & $13.70-32.50$ \\
BMI $\left(\mathrm{kg} / \mathrm{m}^{2}\right)$ & $16.41 \pm 2.00$ & $12.72-23.34$ \\
Percentile & $57.38 \pm 32.37$ & $1-99$ \\
\hline
\end{tabular}

Figure 1 demonstrates a stable pattern of PA levels over the first four weekdays, only decreasing on Friday. On Saturday and Sunday, the PA level decreases more sharply. There were no differences between sexes for the number of steps for all evaluated days.

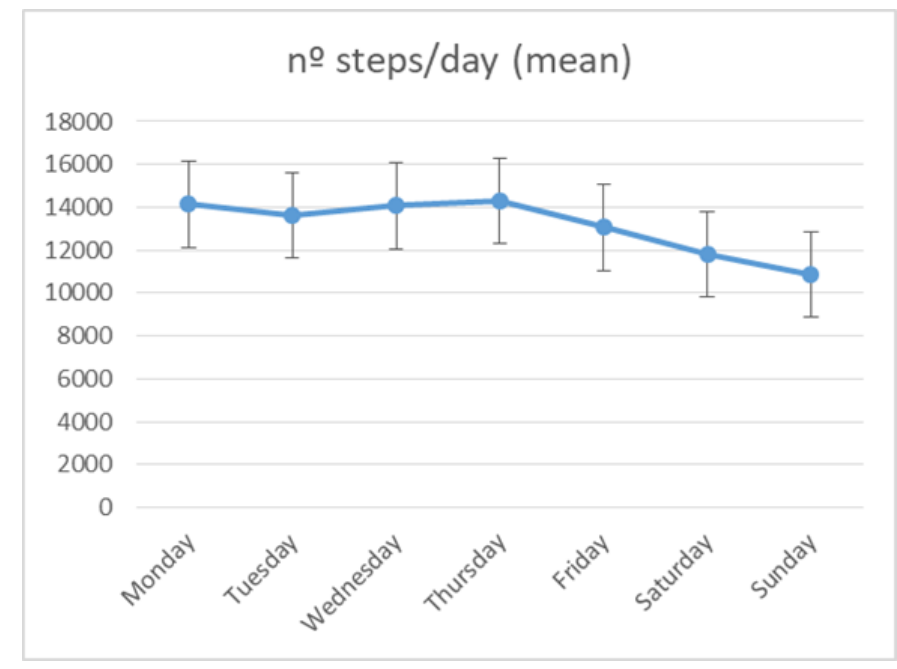

Figure 1. Physical activity pattern (expressed in mean values of steps numbers) by day of the week.

There were significant differences between the number of steps on weekdays and at weekend $(p<0.001)$, with higher levels of PA on weekdays. PA was higher $(p<0.001)$ on the days with PE session than without.

Table 2. Comparation of physical activity pattern (expressed in $n^{0}$ steps) between weekdays and weekend days, and between days with PE session and days without PE session

\begin{tabular}{lccc}
\hline & $\mathrm{n}$ & $\mathrm{M} \pm \mathrm{SD}$ & $\mathrm{p}$ \\
\hline $\mathrm{n}^{0}$ steps weekday/day & 105 & $13998.74 \pm 3608.73$ & 0.00 \\
$\mathrm{n}^{0}$ steps weekend/day & 105 & $11542.90 \pm 3990.99$ & \\
$\mathrm{n}^{0}$ steps with PE session/day & 58 & $15405.14 \pm 5836.21$ & 0.00 \\
$\mathrm{n}^{0}$ steps without PE session/day & 58 & $12277.12 \pm 4455.86$ & \\
$\mathrm{n}^{0}$ steps /PE session & 24 & $2535.33 \pm 751.71$ & \\
\hline
\end{tabular}
$P E$ - physical exercise.

\section{DISCUSSION}

The mean number of steps taken by children at all days of the week and weekend achieved the threshold of 9000 steps per day recommended for this age group to be considered as sufficiently active (Vale et al., 2015). However, it must be considered that this number of steps represents a minimum and not optimal threshold 
of PA levels for this age group. We also observed that the implemented session of PE contributed to a significant increase in the average number of daily steps performed by the children ( $2535.33 \pm 751.71$ steps).

\section{CONCLUSIONS}

Children of preschool age are more active during the week than at the weekend. The implementation at school-based of PE session at pre-school level had a significant impact on daily PA levels of the children.

\section{REFERENCES}

Baba, R., Koketsu, M., Nagashima, M., \& Inasaka, H. (2009). Role of exercise in the prevention of obesity and hemodynamic abnormalities in adolescents. Pediatr Int., 51(3), 359-363. https://doi.org/10.1111//.1442-200x.2008.02729.x

Brown, D. W., Brown, D. R., Heath, G. W., Balluz, L., Giles, W. H., Ford, E. S., et al. (2004). Associations between physical activity dose and health-related quality of life. Med Sci Sports Exerc, 36(5), 890896. https://doi.org/10.1249/01.mss.0000126778.77049.76

Reilly, J. J., Jackson, D. M., Montgomery, C., Kelly, L. A., Slater, C., Grant, S., et al. (2004). Total energy expenditure and physical activity in young Scottish children: mixed longitudinal study. Lancet, 363(9404), 211-212. https://doi.org/10.1016/s0140-6736(03)15331-7

Shephard, P. J. (1990). Exercice, Fitness and Health: a Consensus of Current Knowledge (Vol. II). Champaind: Human Kinetics.

Vale, S., Trost, S., Duncan, M., Mota, J. (2015). Step based physical activity guidelines for preschoolaged children. Preventive Medicine 70, 78-82. https://doi.org/10.1016/i.ypmed.2014.11.008

\section{(2) $\odot \Theta \Theta$}

This work is licensed under a Attribution-NonCommercial-NoDerivatives 4.0 International (CC BY-NC-ND 4.0). 


\title{
The (In)discipline: Playtherapy as prevention
}

\author{
BRUNO TRINDADE 1,3 , RICARDO POCINHO22, JOÃO SERRANO3,4, RUI PAULO33,4, MARIA JOSÉ \\ CONDE $^{5}$ \\ ${ }^{1}$ Câmara Municipal de Castelo Branco, Portugal \\ ${ }^{2}$ Escola Superior de Educação e Ciências Sociais, Instituto Politécnico de Leiria, Portugal \\ ${ }^{3}$ Escola Superior de Educação, Instituto Politécnico de Castelo Branco, Portugal \\ ${ }^{4}$ Sport, Health and Exercise Research Unit (SHERU), Portugal \\ 5 Universidad de Salamanca, Spain
}

\begin{abstract}
The use of a methodology based on a non-formal education, in which the practice of play activities is valued, is in accordance with the methodologies recommended for the education of the future. It is up to the Technicians of Sociocultural Animation to apply the theoretical foundations in the educational and social aspects of the children, with the aim of promoting and developing their capacities and, consequently, their growth as a person. This study, carried out in a school context, proposes to know better the role of Sociocultural Animation in the education of the children and, through the obtained results, to enable the educational community about the importance that the Sociocultural Animation has in the educational future. Participated 609 students and 36 teachers from the School Grouping, $1^{\circ}$ St Cycle School. The questionnaire "Adaptation of the Program Implementation Assessment Scale" was administered to collect information from students and teachers regarding the importance and impact of socio-cultural animation in the school context. From the descriptive statistical analysis it was possible to verify that students and teachers recognize the importance of non-formal education to promote school success and prevent social exclusion, and contributes to a more harmonious development of children, which makes them more autonomous and happier, influencing the well-being of the educational communities in which they are inserted. Keywords: Sociocultural animation; Child education; Non-formal education; Growth.
\end{abstract}

Corresponding author. Campus de Santa Apolónia, 5300-253 Bragança, Portugal.

E-mail: pmaga@ipb.pt

Supplementary Issue: Spring Conferences of Sports Science. International Seminar of Physical Education, Leisure and Health, 17-19 June 2019. Castelo Branco, Portugal.

JOURNAL OF HUMAN SPORT \& EXERCISE ISSN 1988-5202

(C) Faculty of Education. University of Alicante.

doi:10.14198/jhse.2019.14.Proc4.82

VOLUME 14 | Proc4 | 2019 | S1541 


\section{INTRODUCTION}

The traditional school, based on a formal and standardized education, cannot compete, in terms of attraction, with the abundance of technological offerings of modern society. This is just one of the problems that, directly or indirectly, contribute to the incorrect attitudes that children show in relation to school and at school, from demotivation to aggressive behaviours. It is urgent to prevent risky behaviours in our children, since they may have consequences in society. It is fundamental to intervene politically and socially, to change the mentality of the school community in relation to the school. In turn, the school has to accompany the changing society, in constant transformation. The play activity is the most instinctive means for the child's learning, at the level of behavioural and social rules. It is through this activity that she has the opportunity to experience new sensations, establish social bonds, access knowledge, "learn to learn" and overcome obstacles. Play activity will thus have effects on the development of the child. Animators have a relevant role, since they potentiate forms of learning, balancing them with their fun, as well as providing activities through play: stimulating the playful in several ways; by structured games, with their resources or free games, through their own creativity and imagination. These are aspects that favour the process of socialization. It is within this scope that the present study arises: to verify if non-formal education, with activities of socio-cultural animation, has a positive impact on the way students experience education, promoting school success, socialization. Socio-cultural animation must constitute itself as an educational offer in the curricular structure of the educational system.

\section{MATERIAL AND METHODS}

Sociocultural Animation in primary schools should be seen as a priority strategy to promote socialization and prevention of social exclusion as well as other problems that are inherent to it (eg school drop-out). The purpose of this study was to analyse the importance of socio-cultural activities for 1st cycle students, as well as for teachers, and to verify the existing perception about the impact of socio-cultural animation in the school context. More specifically, it was intended to study students' perceptions about the preventive impact that sociocultural animation might have on school problems (e.g. social exclusion, school dropout, indiscipline, bullying).

\section{Participants}

This study had the participation of 609 students, studying in the elementary school of the Nuno Álvares School Grouping, located in Castelo Branco, Portugal. The students were between 6 and 10 years old and were distributed between the 1 st year and the 4 th year of schooling. This study had, also, the participation of 36 teachers from the school group. All participants agreed to individual participation in this research study, thus respecting the informed consent necessary and inherent in any type of field study. Authorization was sought from the parents.

\section{Measures}

The collection of information to be used to analyse the study object was performed through the administration of a questionnaire - Adaptation of the Program Implementation Evaluation Scale (Jardim \& Pereira, 2006). The students and the teachers should respond according to a Likert scale, with a rating from 1 (Bad) to 5 (Very good).

\section{Analysis}

The statistical analysis was performed with the SPSS (Statistical Package for the Social Sciences) version 22.0 for Windows, and involved measures of descriptive statistics and inferential statistics. 


\section{RESULTS}

The results show that the students attribute importance to the activities developed by Sociocultural Animation $(68.3 \%)$, which, in the opinion of $66.1 \%$ of the students, contribute to their enjoyment of the school, to the point that $67.9 \%$ who would like Sociocultural Animation to be part of the school curriculum. It is also worth noting that the activities of Sociocultural Animation help to combat violence and indiscipline (58.8\%). The objective of Promoting Sociocultural Animation as an educational offer was the one that obtained the best quotation, with $77.8 \%$ of the students considering that the achievement of this objective was Very Good. In this context it is also possible to verify the relevance of the animation activities in the intervals between (75\%) and their relationship with the decrease in situations of violence $(72.2 \%)$.

With regard to teachers, the results lead one to conclude that the majority agree that the activities of animation contribute to decrease the rate of violence in the school space (80.6\%). In this group, $75 \%$ of the respondents are of the opinion that the Sociocultural Animation should be included in the school curriculum, being established its relationship with the fight against situations of violence and indiscipline $(80.6 \%)$.

\section{DISCUSSION}

The results show that the students have a very positive appreciation of the sociocultural animation in the school, noting that the activities promoted have a preventive impact of school dropout, aggressive and deviant behaviour within the school and makes it possible to combat school violence.

The results obtained in our study confirm the fundamental role that Sociocultural Animation has in the school context. With this study, we have demonstrated that Sociocultural Animation contributes to a more harmonious development of children, which makes them more autonomous and happier, influencing the wellbeing of the educational communities in which they are inserted. According to Trilla (1998, p.262), "animation is considered as an important instrument for coexistence and participation. In it are present both the sociological dimension and the pedagogical, the communitarian and the therapeutic ". Thus, as part of the curricular structure as an educational offer, Sociocultural Animation would be contributing to the progress of future society in general. According to Babo (2010, p.14), "education associated with socio-cultural animation contributes to greater values related to socialization, freedom, sharing of knowledge and learning by doing," which is in line with the field study we have undertaken. For Ribeiro $(2002$, p.56) "Playing is a means of expression, it is a way of integrating with the environment that surrounds it". Taking into account that Playing is not only a necessity of the child, it is above all an inherent action of the human being and that in the present day in our society the word "therapy" is associated with the idea of benefit or something that does well to human being, talk about play therapy, PlayTherapy, is very pertinent. It allows to provide resources and stimuli to children, so that, with their intrinsic motivation, they can gain essential skills, through exploratory activities, imitating daily actions, which allow a connection to thinking and a natural acquisition of values.

\section{CONCLUSION}

With this study, we have demonstrated that Sociocultural Animation contributes to a more harmonious development of children, which makes them more autonomous and happier, also influencing the well-being of the educational communities in which they are inserted. Sociocultural animation at school seems to have a positive impact on the prevention of deviant behaviour and school failure, preventing school drop-out and social exclusion. These activities allied to the school context promote interaction and social union, the promotion of adequate social behaviours and the interest and school motivation. The students and teachers 
mentioned the importance of the activities of socio-cultural animation in the educational context, revealing their importance in the curricular program.

\section{REFERENCES}

Babo, A. (2010). A actividade lúdica: um instrumento de inclusão da criança com necessidades educativas especiais. In M. S. Lopes, \& M. S. Peres (Coord.), Animação sociocultural e necessidades educativas especiais (pp.14-22). Chaves: Intervenção Associação para a Promoção e Divulgação Cultural. https://doi.org/10.22533/at.ed.29219150110

Ribeiro, P. (2002). Jogos e brinquedos tradicionais. In: Santos, S. M. P. (Org,). Brinquedoteca: o lúdico em diferentes contextos. 7. ed. Petrópolis: Vozes.

Jardim, J., \& Pereira, A. (2006). Competências pessoais e sociais: guia prático para a mudança positiva. Porto: Edições Asa.

Trilla, J. (1998). Animação Sociocultural Teoria, programas e Âmbitos, Instituto Piaget, Lisboa, 14-20.

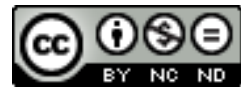

This work is licensed under a Attribution-NonCommercial-NoDerivatives 4.0 International (CC BY-NC-ND 4.0). 


\title{
Students' opinion on Physical Education and School: An association with academic performance
}

\author{
HELDER LOPES ${ }^{1,2}$, ÉLVIO GOUVEIA ${ }^{1,3}$, ADILSON MARQUES ${ }^{4}$, BRUNA R. GOUVEIA ${ }^{3,5}$, CATARINA $^{2}$ \\ FERNANDO ${ }^{1,2}$, JOÃO PRUDENTEE ${ }^{1,2}$, ANA L.CORREIA ${ }^{1}$, RICARDO ALVES ${ }^{1}$, ANA RODRIGUES ${ }^{1}$ \\ ${ }^{1}$ Department of Physical Education and Sports, University of Madeira, Portugal \\ ${ }^{2}$ CIDESD - Research Center in Sports Sciences, Health Sciences and Human Development, Portugal \\ ${ }^{3}$ LARSYS, Interactive Technologies Institute, Portugal \\ ${ }^{4}$ Interdisciplinary Center for the Study of Human Performance, FMH - University of Lisbon, Portugal \\ 5 Institute of Health Administration, IP-RAM; ESE São José de Cluny, Portugal
}

\begin{abstract}
The aims of this study were: (i) to characterize students' opinion on Physical Education (PE) and on the School and (ii) to analyze the associations between opinion about PE, gender, age, and academic performance. The sample was composed by 599 students (264 boys and 335 girls), aged 10 to 22 years (14.76 \pm 2.42 ). A subsample of 154 students (76 boys and 78 girls, $14.33 \pm 2.14$ ) were evaluated in academic performance. The students' opinion about PE and the School was evaluated through a questionnaire with a Likert scale response. The academic performance assessment was based on the curricular evaluation at the end of the 3rd period. The Kolmogorov-Smirnov test was used to test the normality of the distributions of the variables. Student's t-test was used to determine the differences between groups in variables with normal distribution and Pearson correlations to study the associations. The students' opinion regarding PE was mostly positive $(70 \%)$. Lower values were found in relation to the opinion on School $(40.8 \%)$. The opinion about PE was influenced by the grade achieved in the subject, but was not influenced by the overall academic performance. With increasing age, boys like PE and School less and less. Further research on the reasons underlying the results obtained can contribute to the acknowledgment of $P E$ as a powerful instrument to dynamize changes in the pedagogical process in Schools. Keywords: School; Physical education; Academic performance; Change.
\end{abstract}

\footnotetext{
Corresponding author. Department of Physical Education and Sports, University of Madeira, Campus da Penteada, 9020-105 Funchal, Portugal.

E-mail: hlopes@staff.uma.pt

Supplementary Issue: Spring Conferences of Sports Science. International Seminar of Physical Education, Leisure and Health, 17-19 June 2019. Castelo Branco, Portugal.

JOURNAL OF HUMAN SPORT \& EXERCISE ISSN 1988-5202

(C) Faculty of Education. University of Alicante.

doi:10.14198/jhse.2019.14.Proc4.82
} 


\section{INTRODUCTION}

The education system is still rooted in the factory paradigm and faces some difficulty meeting the challenges posed by a constantly changing society (Cândido, 2012; Lopes et al. 2014). A coherent change strategy requires that students' perceptions are taken into account (Lopes et al. 2018). Considering that Physical Education (PE) can be a pole that attracts change, the present study aims to: (i) characterize the students' opinions on the PE and the School and (ii) analyze the associations between the opinion on PE and gender, age, review of the school and school performance.

\section{MATERIAL AND METHODS}

\section{Participants}

The sample consisted of 599 children and adolescents ( 264 girls and 335 boys), aged 10 to 22 years (14.76 \pm 2.42 ).

The students belonged to a public education network in the municipality of Funchal. A subsample of 154 subjects ( 76 boys and 78 girls), with a mean age $14.33 \pm 2.14$ years, were evaluated in regard to academic performance.

\section{Measures}

The students' opinion about PE and the School was evaluated through a questionnaire, with a Likert scale of 1 (I do not like anything at all) to 5 (I really like it). The academic performance was determined based on the curricular notes at the end of the 3rd period, the average of the disciplines and PE, Mathematics and Portuguese courses were analysed separately.

\section{Procedures}

The present study is part of a larger project called "Physical Education in Schools of the Autonomous Region of Madeira" (EFERAM-CIT). Participants were informed about the aims of the study and written informed consent was obtained from their legal guardians. The study received Ethical approval from the Scientific Committee of the Faculty of Physical Education and Sports at the University of Madeira (Reference: ACTA N.77 - 12.04.2016).

\section{Analysis}

Initially, descriptive statistics were used to characterize the sample. Normality of the distribution for all variables was tested using the Kolmogorov-Smirnov test. The academic performance was standardized considering the different classification scales used in the 3rd cycle (from 1 to 5 values) and in the secondary (from 1 to 20 values). An independent-samples t-test was used to determine the differences between groups and Pearson correlations were calculated to study the associations. Statistical procedures were performed in SPSS software version 25.0 and the significance level adopted was $5 \%$.

\section{RESULTS}

Most participants "like a lot" (43.6\%) or "really like" (33.4\%) the PE course, although $8.5 \%$ dislike or consider that this discipline is indifferent (15.5\%). On average, boys reported that they liked PE more than girls (4.23 \pm 0.924 vs $3.78 \pm 1.00)(t(597)=5.598, p<0.001)$. A more negative perception of the PE was associated with increasing age $(r=-0.138 ; p=0.028)$ only in boys. 
Regarding the students' opinions about the school, it was found that $37.5 \%$ consider that the school is to them indifferent, and roughly 1 in 5 said that did not like the school (21.6\%). The remaining $40.8 \%$ said they liked the School. No gender differences were found in relation to opinion about school. A more negative perception about the School was associated with increasing age $(r=-0.151 ; p=0.045)$, in boys only.

This study identified an association between PE opinion and: (i) school opinion ( $r=0.151, p=0.001$ ), and (ii) the PE subject score ( $r=0.263, p=0.022$ for girls $r=0.381, p=0.001$ in boys). No significant associations were identified between the students' opinion about PE and the curricular average or the assessments in Mathematics and Portuguese courses ( $p>0.05)$.

\section{DISCUSSION}

The fact that the students' opinion about PE are mostly positive (70\%) and the opinion about school in less favourable (only $40.8 \%$ ) is in line with previous evidence in similar populations and contexts (Lopes et al., 2018).

Liking or disliking PE is not significantly influenced by the fact that students have a higher or lower overall academic performance. The same happens with the two nuclear disciplines in scientific-humanistic curricula (Portuguese and Mathematics). These results trigger the discussion about whether or not PE evaluation should count to the average for access to higher education, while helping to demystify the idea that good students do not like PE. The fact that older boys enjoy PE and School less and less corroborates previous results obtained by Lopes et al. (2018). This may be an indicator that PE can be underachieving its goal in regard to promoting a lifelong taste for physical activity and sports.

\section{CONCLUSIONS}

We conclude that the students' opinion about PE is more favourable, when compared to the opinion about the School. Older boys tend to have a less positive opinion about PE and School. A positive opinion on PE is associated with a better view of school, among both boys and girls. The opinion about PE is positively associated with the PE grade, but not with overall academic performance (in Portuguese and Mathematics). In this context, PE can be a powerful tool in transforming the pedagogical process in a School that must adapt to the challenges of a rapidly changing society.

\section{REFERENCES}

Cândido, F. (2012). Ponto de vista sobre os sistemas educativos e a perspectiva de mudança paradigmática. Rev. psicopedag. 29(88) 77-78.

Lopes, H., Gouveia, É., Marques, A., Gouveia, B., Fernando, C., Vicente, A., Correia, A., Alves, R. \& Rodrigues, A. (2018). A perceção dos alunos sobre a Escola e sobre a Educação Física. Journal of Sport Pedagogy and Research 4(2) 42.

Lopes, H., Prudente, J., Vicente, A. \& Fernando, C. (2014). Uma Mudança Coerente no Ensino Superior - A Ferramenta Processo Pedagógico. Revista da Sociedade Científica de Pedagogia do Desporto, 1(5), 55-60.

\section{@९@}

This work is licensed under a Attribution-NonCommercial-NoDerivatives 4.0 International (CC BY-NC-ND 4.0). 


\title{
The curricular identity of Physical Education: New perspective
}

\author{
ELISIANE MEDIANEIRA MORO TOLIO ${ }^{1}$, CLEONICE TEREZINHA FERNANDES $^{2} \square$, FABRÍCIO BRUNO \\ CARDOSO $^{3}$, CILENE MARIA LIMA ANTUNES MACIEL 4 \\ ${ }^{1}$ Mestrado em Ensino - UNICIIFMT - IFMT, Cuiabá, Mato Grosso, Brazil \\ 2Universidade de Cuiabá - UNIC, Mato Grosso, Brazil \\ ${ }^{3}$ Faculdade CENSUPEG, Rio de Janeiro, Brazil \\ ${ }^{4}$ Universidade de Cuiabá - UNIC, Mato Grosso, Brazil
}

\begin{abstract}
Since its establishment as a compulsory curricular component, the area of Physical Education in Brazil, has gone through several changes that made difficult the structuring of its curricular identity; as evidenced by its presence as a member of the language area in the new National Common Curricular Base, fact that has caused controversy among researchers/educators. Considering this scenario, the role of Physical Education in schools has been questioned as a curricular component for the student formation. Assuming that health education will present good results, the research proposal is to analyse the need to promote a curricular complementation for teachers in the area of Physical Education. The research will involve academics and professors of Pedagogy from a private university and the ones of Physical Education from the Federal University of Mato Grosso campus, whose participation will occur through individual and collective interviews (focus groups) integrated and by area. It will also be carried out, the analysis of the curricular proposals of the mentioned courses, classic and systematic bibliographic review, and observation of Physical Education classes in the public school system of Barra do Garças municipality in Mato Grosso state. It is expected that the participating educators will point out the difficulties found in the teaching practice of Physical Education and perhaps the need for a complementary training, through continuing education focused on health education, which would give a new horizon to Physical Education as a curricular component. Keywords: Health education; Continuing education; Teaching.
\end{abstract}

Corresponding author. Universidade de Cuiabá - UNIC, Mato Grosso, Brazil.

E-mail: cleo terezinha@hotmail.com

Supplementary Issue: Spring Conferences of Sports Science. International Seminar of Physical Education, Leisure and Health, 17-19 June 2019. Castelo Branco, Portugal.

JOURNAL OF HUMAN SPORT \& EXERCISE ISSN 1988-5202

(c) Faculty of Education. University of Alicante.

doi:10.14198/jhse.2019.14.Proc4.82 


\section{INTRODUCTION}

In the course of its history as a compulsory curricular component, Physical Education has undergone several influences. The consequences of these interferences led the discipline to be understood as an exclusively practical activity and turned solely to the body culture of movement, without leading the individual to form a critical and functional reading of the movement concerning physical and cognitive development. It will be necessary to go over the theories and pedagogical approaches that made the Physical Education as it is known today to understand its lack of identity. It is also observed in the teachers pedagogical practice, a certain hybridism or blend, reflections of the initial formation, at times forming bachelor's degree holders, at times more concerned with the motor development; at times prioritizing sports, at times health practices. Following the same path, the curricular proposal of the Pedagogy course provides a very limited amount of time for the discipline of Physical Education - when it is included - and these are professionals who teach Physical Education classes in Early Childhood Education and in the early years of Elementary Education; the pedagogues are the ones responsible for promoting/stimulating in children the main opportunities for motor development and also raising awareness about the interrelation between life habits, body care, physical activity and healthy life; in the present approach, this is understood as a crucial period for the development of positive values and attitudes through present and future life. The objective of this research is to propose/clarify possibilities for curricular reorganization and possible subjects for continuing education for Physical Education teachers, unveiling a new role for the professional in Brazilian schools; within the educational structure, it is emphasized that there is no longer an exclusive view on the culture of the human movement in classes, but, fundamentally, goals aligned with health education; therefore, through the selection, organization and availability of content, prioritize experiences that can provide learners with not only situations that make them more active and healthy children and young people, but, above all, that offer opportunities for the development of a critical sense, and sufficient knowledge to choose an active and healthy lifestyle also as adults. Proposing activities that emphasize health education, may be a new way to look at Physical Education as a curricular component, considering the new habits made possible by the technological society, including children in the midst of cognomotor development.

Therefore, the purpose of this research is to organize a set of methodological strategies for continuing education among educators, which contemplates health education related to the practices of school physical activity, so the discipline of Physical Education will lead students to realize the importance of adopting a healthy lifestyle by making it a daily, voluntary and conscious action for life.

\section{MATERIAL AND METHODS}

Applied research of qualitative descriptive and exploratory approach.

\section{Participants}

Students who graduated in Pedagogy from a private university and in Physical Education from the Federal University of Mato Grosso, who work in fifteen public schools of Barra do Garças municipality in Mato Grosso state.

\section{Measures}

i) Bibliographic research and Systematic Review on Health Education practices in schools (by which professionals and how and if it happens) in the last 5 years in Brazil; ii) Documentary analysis (curricular proposals present in the Physical Education program of Federal University of Mato Grosso and in the 
Pedagogy program of a private university); iii) Individual and collective interviews (focus group) for Physical Education teachers from 15 public schools of Barra do Garças; and iv) Class observation.

\title{
Procedures
}

It will be built a proposal for continuing education that includes health education: physical activity practice, healthy eating, implications of drug abuse, insufficient sleep, technology addiction, knowledge of the child's and adolescent's brain.

\begin{abstract}
Analysis
Analysis method by AC - Bardin Content Analysis (2016). It will be also used the qualitative software (IRAMUTEQ).
\end{abstract}

\section{RESULTS}

Accepting the challenge of developing a viable program of curricular complementation, specifically in Physical Education with emphasis on health education, is to lead the search for an identity for Physical Education as a more significant and essential curricular component in the individual's formation, potentializing protection behaviour and minimizing risk behaviour.

\section{DISCUSSION}

If reconsidered, the training of Physical Education professionals would have a more significant contribution in the formation of the individual. In this context, it is essential to understand that in the classrooms, in the sports courts or in other educational spaces, the teachers are the ones who assume the educational process, as fundamental elements to execute any curricular proposal; still they can go beyond what is set beforehand in the programs, since they are not mere executors of the curriculum, rather, they are mediators, interpreters, authors and curriculum builders in action. Considering Physical Education, as a curricular component linked to the political pedagogical project in schools, when it takes a stance on health condition, it also becomes an inclusive discipline, distancing itself from practices that privilege the most talented in sports, broadening the excluding understanding of body culture embracing minorities and cultural diversity. It is crucial to note that the "Physical Education class cannot become a discourse on the body culture of movement, risking the loss of the richness of its specificity" (Betti and Zuliani, 2002, p. 75). According to Guedes (1999) we find ourselves in a society where children, young people and adults cannot manifest attitudes, values, information and skills that allow them to adopt an active and healthy lifestyle due to deficiencies in their educational background. Undoubtedly, reversing this situation demands urgent change in the focus of the school physical education programs, allowing health-related physical activity aspects to prevail.

Following this path, probably, Physical Education as a discipline of the school curriculum, will be able to find its true role in the formation of the individuals, for the benefit of their own health, so that the professionals of the area can act in a more effective and valued way in our society.

\section{CONCLUSIONS}

Study in progress. The intention is to promote and lay the basis for reflections on the initial training of educators in the area of Physical Education, as Health Education, and, consequently, elaborate a new structure for this important curricular component. 


\section{REFERENCES}

Bardin L. (2016). Análise de Conteúdo. Lisboa:70.

Betti, M.; Zuliani, L. R. (2002). Educação física escolar: uma proposta de diretrizes pedagógicas. 2002 Revista Mackenzie da educação física e esporte, 1, (1). 73- 81. https://doi.org/10.1590/s180755092011000500011

Guedes, D. P. (1999). Educação para a saúde mediante programas de Educação Física escolar. Motriz, $5,(1)$.

Sacristán, J. G. (2000). O currículo: uma reflexão sobre a prática. 3 ed. Porto Alegre: ArtMed. 


\title{
The effect of combining general warm-up with specific warm-up in bench press performance
}

\author{
PEDRO P. NEVES ${ }^{1}$, ANA R. ALVES ${ }^{2}$, DANIEL A. MARINHO ${ }^{1}$, RICARDO FERRAZ1 ${ }^{1}$ Mª $^{a}$. HELENA GIL ${ }^{1}$, \\ LUÍS B. FAÍL ${ }^{1}$, ANTÓNIO C. SOUSA ${ }^{1}$, MÁRIO C. MARQUES ${ }^{1}$, HENRIQUE P. NEIVA ${ }^{1}$ \\ ${ }^{1}$ CIDESD, University of Beira Interior, Covilhã, Portugal \\ ${ }^{2}$ CIDESD, Polytechnic Institute of Beja, Beja, Portugal
}

\begin{abstract}
The aim of the current study was to determine the effect of a specific warm-up vs general followed by a specific warm-up in the bench press exercise performance. Fourteen subjects, aged between 19 and 43 years (mean \pm SD: $26.2 \pm 6.9$ years, $176.5 \pm 6.96 \mathrm{~cm}$ in height, $79.11 \pm 7.36 \mathrm{~kg}$ in weight) performed three sets of six repetitions of bench press exercise ( $80 \% 1$ repetition maximum: $1 \mathrm{RM}$ ) after a specific warm-up or a general warm-up followed by a specific warm-up, randomly. Mean propulsive velocity (MPV) and mean propulsive power (MPP) was assessed using a linear velocity transducer (T-Force System; Ergotech, Murcia, Spain). The results showed no significant differences between conditions in the maximal values of MPV ( $p=$ $0.37, d=0.07)$ and MPP $(p=0.51, d=0.05)$, however, it seems that protocol $B$ tend to produce better overall results. In conclusion, results evidenced that the general warm-up does not interfere in the subsequent strength training performance, highlight that specific warm-up could be as efficient as the inclusion of a general warm-up before strength training. Keywords: Warm-up; Strength; Specific warm-up; General warmup.
\end{abstract}

Corresponding author. Department of Sports Sciences, University of Beira Interior; Convento de Santo António, 6201 - 001 (Covilhã) Portugal.

E-mail: pedroneves93@hotmail.com

Supplementary Issue: Spring Conferences of Sports Science. International Seminar of Physical Education, Leisure and Health, 17-19 June 2019. Castelo Branco, Portugal.

JOURNAL OF HUMAN SPORT \& EXERCISE ISSN 1988-5202

(c) Faculty of Education. University of Alicante.

doi:10.14198/jhse.2019.14.Proc4.82 


\section{INTRODUCTION}

Different warm-ups strategies are usually implemented to maximize overall body functioning, as well as to prevent potential injuries (Gil et al., 2019; Silva et al., 2018). However, regarding the strength training performance, this seems to be quite questionable due to the lack of information on this issue (Simão, 2003). The usual warm-ups used by coaches and personal trainers consisted in a general warm-up followed by a specific warm-up. The specific warm-up usually comprised specific exercises and/or movements that will be later applied in the training session, but with lower external loads (Simão et al., 2004). Additionally, the general warm-up often consisted of aerobic exercises to increase body temperature, allowing greater metabolic efficiency (Robergs \& Robergs, 2002). Unfortunately, the amount of research that investigate the importance of a general warm-up and/or a specific warm-up for strength performance is restricted and somewhat ambiguous. Therefore, the purpose of the present study was to compare the acute effects of a specific warm-up versus a general warm-up followed by a specific warm-up in the bench-press performance.

\section{MATERIAL AND METHODS}

\section{Participants}

Fourteen male subjects aged 19 to 43 years (mean \pm SD: $26.2 \pm 6.9$ years, $176.5 \pm 6.96 \mathrm{~cm}$ in height, 79.11 $\pm 7.36 \mathrm{~kg}$ ), healthy and active, volunteered to participate in the current study.

\section{Measures}

Each subject performed two protocols (A: protocol with specific warm-up and B: protocol with a general warmup, followed by a specific warm-up), randomly, in two different sessions. The maximal, minimal, and mean values of MPV (mean propulsive velocity) and MPP (mean propulsive power) were assessed.

\section{Procedures}

One week before the implementation of protocols, each subject was evaluated regarding individual repetition maximum (1RM) in bench press exercise. These values were recorded and used as reference for the determination of external loads during warm-ups and training. The protocol A consisted in six repetitions with $32 \% 1 \mathrm{RM}$, rest $1 \mathrm{~min}$ and then another 6 reps with $64 \% 1 \mathrm{RM}$. The protocol $\mathrm{B}$ consisted on 10 minutes of low-intensity progressive running (starting with 2 min walking, 8 min slow running, and up to $70 \%$ of the maximal heart rate reserve), followed by specific warm-up of protocol A. After warm-ups (5minutes), each participant completed 3 sets of 6 repetitions with 80\% 1RM (3 minutes of interval between).

\section{Analysis}

The normality of all distributions was verified by the Shapiro-Wilk tests and the parametric statistical analysis was adopted. To compare the 2 trials, Student's paired t-tests and Cohens' $d$ measures $(p \leq 0.05)$ were calculated.

\section{RESULTS}

Table 1 shows the values of mean and standard deviation (Mean \pm SD) and significance values of the variables: mean propulsive velocity, mean maximum and minimum propulsive velocity, mean propulsive power and maximum and minimum propulsive mean power, extracted from the application of protocols $A$ and B. 


\begin{tabular}{ccccc}
\hline & $\mathrm{A}$ & $\mathrm{B}$ & P-value & Cohen's d \\
\hline MPV & $0.47 \pm 0.13$ & $0.48 \pm 0.15$ & 0.37 & 0.07 \\
MPP & $236.92 \pm 65.12$ & $240.27 \pm 63.55$ & 0.51 & 0.05 \\
MPV Máx & $0.54 \pm 0.12$ & $0.55 \pm 0.14$ & 0.47 & 0.07 \\
MPV Mín & $0.34 \pm 0.17$ & $0.36 \pm 0.17$ & 0.43 & 0.11 \\
MPP Máx & $275.27 \pm 72.89$ & $275.65 \pm 63.70$ & 0.97 & 0.01 \\
MPP Mín & $172.73 \pm 76.15$ & $176.59 \pm 64.93$ & 0.68 & 0.05 \\
\hline \multicolumn{5}{c}{ MPV: mean propulsive velocity, MPP: mean propulsive power. }
\end{tabular}

\section{DISCUSSION}

The aim of the present study was to understand the effect of two warm-up protocols in a bench press training set. Our results showed no significant differences between the two protocols assessed. Yet, it seems that protocol B tend to produce better overall results, except for MPP Min. The current results corroborate with those presented by Simão et al. (2004), that also verified no significant differences between aerobic warmup and specific warm-up, however, $60 \%$ of the subjects performed better after specific warm-up. Nevertheless, in the study of Young (2003), significant differences were found between aerobic warm-up and aerobic warm-up followed by specific exercises, with better performances after the aerobic warm-up. Our results tend to evidence that the general warm-up when added to specific warm-up did not result in better performances. Thus, it is suggested that the general component of warm-up may not be performed, without compromising mechanical propulsive velocity and power in the bench press exercise. This, perhaps, would allow to avoiding the accumulation of fatigue or even delaying the onset of fatigue during strength training (Hajoglou, 2005).

\section{CONCLUSIONS}

No differences were observed between warm-up protocols. Our results evidenced that the general warm-up applied in protocol $B$ does not interfere in the subsequent strength training performance, highlight that specific warm-up is as efficient as the inclusion of a general warm-up before a strength training set.

\section{FUNDING}

This work is supported by national funding through the Portuguese Foundation for Science and Technology, I.P., under project UID/DTP/04045/2019.

\section{REFERENCES}

Gil, M.H., Neiva, H.P., Sousa, A.C., Marques, M.C., \& Marinho, D.A. (2019). Current approaches on warming up for sports performance: a critical review. Strength and Conditioning Journal. (in press). https://doi.org/10.1519/ssc.0000000000000454

Hajoglou, A., Foster, C., De, J. K., Lucia, A. L. E. J. A. N. D. R. O., Kernozek, T. W., \& Porcari, J. P. (2005). Effect of warm-up on cycle time trial performance. Medicine and Science in Sports and Exercise, 37(9), 1608-1614. https://doi.org/10.1249/01.mss.0000177589.02381.0a

Robergs, R. A., \& Roberts, S. O. (2002). Princípios fundamentais de fisiologia do exercício para aptidão, desempenho e saúde. In Princípios fundamentais de fisiologia do exercício para aptidão, desempenho e saúde. https://doi.org/10.31219/osf.io/k6hrm 
Silva, L. M., Neiva, H. P., Marques, M. C., Izquierdo, M., \& Marinho, D. A. (2018). Effects of warm-up, post-warm-up, and re-warm-up strategies on explosive efforts in team sports: A systematic review. Sports Medicine, 48(10), 2285-2299. https://doi.org/10.1007/s40279-018-0958-5

Simão, R. (2003). Fundamentos fisiológicos para o treinamento da força e da potência. Phorte editora.

Simão, R., Senna, G., Nassif, L., Leitão, N., Arruda, R., Priore, M., ... \& Polito, M. D. (2004). Influência dos diferentes protocolos de aquecimento na capacidade de desenvolver carga máxima no teste de 1RM. Fitness \& performance journal, (5), 261-265. https://doi.org/10.3900/fpj.3.5.261.p

Young, W. B., \& Behm, D. G. (2003). Effects of running, static stretching and practice jumps on explosive force production and jumping performance. Journal of sports medicine and physical fitness, 43(1), 21-27. 


\title{
The importance of workplace health management in the context of skills shortage in small and medium-sized companies
}

\author{
LUISA HENTE $1 \square$, ALEXANDER HODECK² ${ }^{2}$, TORSTEN SCHLESINGER ${ }^{3}$ \\ ${ }^{1}$ Chemnitz University of Technology, Germany \\ ${ }^{2}$ EBC Hamburg \& Chemnitz University of Technology, Germany \\ ${ }^{3}$ Ruhr-University Bochum, Germany
}

\begin{abstract}
Demographic change is leading to changes and challenges in the world of work. The result of that phenomenon is a shortage of skilled workers and an upward shift in the age structure of employees. Therefore, companies are faced with the challenge of both attracting young qualified junior staff and keeping the ageing staff in the company for as long as possible. A prolongation of employability can be achieved through systematic workplace health management (WHM). In addition, WHM can increase the attractiveness of the company. This study examines the importance and characteristics of WHM in connection with demographic change and the shortage of skilled workers in small and medium-sized enterprises (SMEs) in rural areas of the eastern part of Germany. German SMEs $(n=244)$ in the federal state of Saxony (region: Ore Mountains) were surveyed by telephone using a standardized questionnaire on their activities in the area of WHM. The findings show that the shortage of skilled workers is a considerable problem for the majority of the surveyed companies. Although the companies assess WHM as increasingly important, only a few of the companies have implemented a systematically WHM (10.1\%) or health-promoting individual measures $(28.3$ $\%$ ) in their company. For more than $60 \%$ of the companies, health-promoting measures have so far played no role at all. Bivariate analyses, however, show structural differences. The findings indicate that there is still a discrepancy between the importance of WHM on the one hand and the implementation of health-promoting measures on the other. Keywords: Workplace health management (WHM); Small and medium-sized enterprises (SME); Demographic change; Skills shortage.
\end{abstract}

Corresponding author. TU Chemnitz, Institut für Angewandte Bewegungswissenschaften. Thüringer Weg 11, 09126 Chemnitz, Germany.

E-mail: luisa.hente@hsw.tu-chemnitz.de

Supplementary Issue: Spring Conferences of Sports Science. International Seminar of Physical Education, Leisure and Health, 17-19 June 2019. Castelo Branco, Portugal.

JOURNAL OF HUMAN SPORT \& EXERCISE ISSN 1988-5202

(C) Faculty of Education. University of Alicante.

doi:10.14198/jhse.2019.14.Proc4.82 


\section{INTRODUCTION}

The labour market has been transformed through the demographic change in Germany over the last decades. Demographic factors such as the declining number of inhabitants, the rising average age of the population, the lowered birth rate and the migration processes led to a substantial transformation within the work force. As a result, a deficiency regarding qualified junior staff and a changed age structure of the work force can be stated (Bellmann \& Hübler, 2014; Richenhagen, 2006). The situation in less urbanised regions with a lower population density and lower economic standard is much more difficult than in metropolitan areas, where still enough work force is available. As a consequence, especially small and medium-sized enterprises (SMEs) in rural areas are confronted with increasingly scarce human resources. The challenge for companies is to find enough qualified junior staff and to keep the (aging) workforce as long as possible in the company. Following Kieckbusch and Marquardt (2013) a prolongation of employability can be achieved through systematic workplace health management (WHM). In addition, WHM helps to enlarge the attractiveness of a company which increases the chance of recruiting new young workers and reduces the turnover of work force at the same time (Winter \& Grünewald, 2016). This study examines the importance of WHM in connection with demographic change and the shortage of skilled workers in SMEs in rural areas of the eastern part of Germany. This area is still underdeveloped compared to the German economic standard and more influenced by demographic changes than urban regions.

\section{MATERIAL AND METHODS}

\section{Participants}

The sample of the study consists of 244 German SMEs within the Ore Mountains (a rural area of the federal state of Saxony), randomly selected via a freely accessible database. Executive directors or people responsible for human relations were interviewed. Within the sample $47.1 \%$ are micro-enterprises (less than 10 employees), $34.0 \%$ are small enterprises (10 - 49 employees) and $18.9 \%$ are medium-sized enterprises (50 - 249 employees).

\section{Measures}

The companies were questioned by a standardized questionnaire via telephone. The questionnaire consisted of three parts: general company data; current state, motivation and goals regarding to WHM; networking. The questionnaire was checked by a pre-test. Mostly closed questions with a four-point Likert scale were used.

\section{Procedures}

The data were collected within six months in $2018 / 2019$ by trained scientific telephone interviewers in a Computer Assisted Telephone Interviewing (CATI) laboratory.

\section{Analysis \\ Descriptive and bivariate analyses were executed. SPSS was used to analyse the data.}

\section{RESULTS}

Lack of work force is already an important problem among more than half of the investigated companies. Employees who are getting older are currently less important. Only $31.6 \%$ of the sample identify a problem regarding the high age of their work force. On the one hand, SMEs believe that WHM is gaining importance. The reasons for this include the shortage of skilled workers (M: 2.9, SD: 1,0; $1=$ does not agree at all; $4=$ does agree totally) and demographic change (M: $3.1, \mathrm{SD}: 1,0)$. On the other hand, only $10.1 \%$ implement a 
systematic WHM. At least $28.3 \%$ already offer health orientated activities within the company. About $60 \%$ of the companies do not deal with health management at all. The size of the company affects the implementation of health-promoting measures significantly $\left(X^{2}=9,522 ; p=0,009\right)$. Micro-enterprises implement less health activities than small enterprises and medium-sized ones.

\section{DISCUSSION}

Regarding the results of this study, the lack of work force is the most important problem among SMEs in rural eastern German areas with regard to demographic change. Despite the insight that WHM plays an important role to deal with challenges of scare human resources the current state of implementation of WHM is disproportionately developed. There is a clear discrepancy between the perceived importance of WHM and the implemented activities in that field. Structural difference regarding the size of companies are congruent to existing studies (Hollederer \& Wießner, 2014). It can be assumed, that companies face some problems, which prevent them from implementing WHM. It might be more relevant to micro-enterprises. There are some relevant limitations to this study. The results are only meaningful for one specific area in Germany. Therefore, the outcomes can only give first insights to the importance of WHM regarding to skills shortage and older employees in rural areas.

\section{CONCLUSIONS}

In summary, the shortage of skilled workers is a problem for companies and therefore, that the importance of health promotion is growing. Nevertheless, only few SMEs have managed the successful implementation of workplace health promotion. Further studies should focus on these problems and the underlying factors, which force or hinder SMEs to implement WHM.

\section{REFERENCES}

Bellmann, L., \& Hübler, O. (2014). Skill Shortages in German Establishments. Journal of Economics and Statistics, 234(6), 800-826.

Hollederer, A., \& Wießner, F. (2014). Verbreitung und Entwicklung der Betrieblichen Gesundheitsförderung in Deutschland - Ergebnisse einer Wiederholungsbefragung im IAB Betriebspanel 2012. Das Gesundheitswesen, 76(08/09). https://doi.org/10.1055/s-0034-1386928

Kieckbusch, M., \& Marquardt, B. (2013). Trends für die Personalfunktion in 2020. In H.-G. Dachrodt, W. Koberski, V. Engelbert, \& G. Dachrodt (Eds.), Praxishandbuch Human Resources: Management Arbeitsrecht - Betriebsverfassung (pp. 77-90). Wiesbaden: Springer Gabler. https://doi.org/10.1007/978-3-658-00586-3

Richenhagen, G. (2006). Demografischer Wandel in der Arbeitswelt - Stand und Perspektiven in Deutschland. Gesundheits- Und Sozialpolitik, 60(5-6), 53-58. https://doi.org/10.5771/1611-58212006-5-6-53

Winter, W., \& Grünewald, C. (2016). BGM als Stellschraube von Arbeitgeberattraktivität. In B. Badura, A. Ducki, H. Schröder, J. Klose, \& M. Meyer (Eds.), Fehlzeiten-Report 2016: Unternehmenskultur und Gesundheit - Herausforderungen und Chancen (pp. 225-235). https://doi.org/10.1007/978-3$\underline{662-49413-4 \_20}$

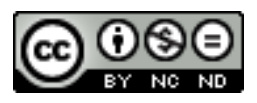

This work is licensed under a Attribution-NonCommercial-NoDerivatives 4.0 International (CC BY-NC-ND 4.0). 


\title{
Case study of the programs for soccer teaching of two teachers in training versus the Tactical Game Approach model
}

\author{
MARÍA G. GAMERO , JUAN M. GARCÍA-CEBERINO, SEBASTIÁN FEU, ANTONIO ANTÚNEZ, LUIS \\ CÓRDOBA \\ Optimization of Training and Sports Performance Research Group (GOERD), Department of Didactics of \\ Music, Plastic and Body Expression, University of Extremadura, Badajoz, Spain
}

\begin{abstract}
Regarding sports planning, it is important to know the different teaching-learning models that a teacher has at their disposal. The objective of this study was to analyse the pedagogical variables of the tasks designed by the pre-service teachers for the teaching of school football in order to compare these variables later with those used in a teaching program based on the Tactical Game Approach. 130 teaching tasks (pre-service teachers $n=101$, TGA $n=29$ ) were categorized with the Integral System for the Analysis of the Training Tasks (SIATE). The following variables were analysed: game situation, goalkeeper presence, game phase, content type I and II, specific content, medium of teaching and opposition level. A descriptive and inferential analysis was carried out using the Chi-Square test, Cramer's V test and the Corrected Typified Waste. The results show significant differences $(p<.05)$ in the design of the variables that teachers plan with respect to those designed in the tactical-based program except for the content type variable II. These differences are more evident in the game situation variables and specific content $(V c>, 500, p=, 000)$. Keywords: Planning; Pre-service teachers; Pedagogical variables; SIATE.
\end{abstract}

Corresponding author. Faculty of Education (office 0.1 ground-floor, main building), Avda. de Elvas S/N 06006, Badajoz, Spain.

E-mail: mgamerob@alumnos.unex.es

Supplementary Issue: Spring Conferences of Sports Science. International Seminar of Physical Education, Leisure and Health, 17-19 June 2019. Castelo Branco, Portugal.

JOURNAL OF HUMAN SPORT \& EXERCISE ISSN 1988-5202

(C) Faculty of Education. University of Alicante.

doi:10.14198/jhse.2019.14.Proc4.82 


\section{INTRODUCTION}

In the PE, sport is a curricular content and, like the rest of curricular contents, requires planning through the development of teaching units (González-Espinosa, Ibáñez, \& Feu, 2017). The reality shows the need to analyse the process of sports training for its optimization in the initial stages, which are the basis of the learning for the following stages (Cañadas and lbañez, 2010).

\section{MATERIAL AND METHODS}

This study is covered by an associative design of a comparative and transversal type (Ato, López, \& Benavente, 2013).

\section{Participants}

The participants in this study were two teachers in pre-service phase belonging to the degree of Physical Education of the University of Extremadura. The sample of the study was formed by the teaching-learning tasks that are part of the two didactic units planned by the pre-service teachers, and the tasks that cover the teaching program operating in the TGA method (Garcia-Ceberino, Feu \& Ibanez, 2019). Finally, a total of 130 tasks were analysed (perfesor $1 n=53$, teacher $2 n=48$, program TGA $n=29$ ).

\section{Measures}

The variables of the study were the pedagogical variables that define a task: teaching method, playing situation, goalkeeper presence, game phase, medium of teaching, type of content I, content type II, specific content, opposition level.

\section{Procedures}

In the first place, the participants of the study, teachers in the pre-service phase, were asked to plan a didactic unit for the teaching of the sport of football invasion. Subsequently, two of the teaching units prepared by the teachers were categorized. For their categorization, the SIATE instrument (Ibáñez, Feu \& Cañadas, 2016) was used and was carried out by an external encoder with specific training in the subject of the study.

\section{Analysis}

In the first place, a descriptive analysis was carried out to know the characteristics of the sample. Subsequently, an inferential analysis was carried out through the Chi-Square test ( $\mathrm{x} 2)$, the Cramer V coefficient (Vc). Moreover, the Corrected Typified Residues (RTC) of the contingency tables were used to interpret the relationship and the degree of association between the pedagogical variables studied (Field, 2009).

\section{RESULTS}

The descriptive analysis of the data shows which are the most used categories in each of the pedagogical variables per teacher. The teacher 1 and teacher 2 tend to design tasks that are framed in a traditional methodology, using: the simple exercise, the situations of $1 \mathrm{vs} 0$ and $2 \mathrm{vs} 0$, the technical gestures, without the presence of a goalkeeper, in the attack phase. 
Table 1. Inferential results with the Chi-square test and degree of association as a function of the variables

\begin{tabular}{lccccccc}
\hline & $\boldsymbol{x}^{\mathbf{2}}$ & $\boldsymbol{g l}$ & $\boldsymbol{p}$ & $\boldsymbol{f}$ & $\boldsymbol{p}$ & $\boldsymbol{V}_{\boldsymbol{c}}$ & $\boldsymbol{p}$ \\
\hline Situation of game & 82.559 & 48 & $.001^{*}$ & 71.590 & $.000^{*}$ & .564 & $.001^{*}$ \\
Goalkeeper presence & 6.689 & 2 & $.035^{*}$ & 8.040 & .016 & .227 & $.035^{*}$ \\
Phase of game & 27.222 & 6 & $.000^{*}$ & 21.775 & $.000^{*}$ & .324 & $.000^{*}$ \\
Type of content I & 57.008 & 18 & $.000^{*}$ & 55.228 & $.000^{*}$ & .468 & $.000^{*}$ \\
Type of content II & 4.516 & 6 & .607 & 4.999 & .577 & .247 & .607 \\
Specific content & 140.527 & 72 & $.000^{*}$ & 108.313 & $.000^{*}$ & .735 & $.000^{*}$ \\
Medium of teaching & 46.624 & 16 & $.000^{*}$ & 46.311 & $.000^{*}$ & .423 & $.000^{*}$ \\
Level of opposition & 20.356 & 6 & $.002^{*}$ & 21.496 & $.000^{*}$ & .280 & $.002^{*}$ \\
\hline
\end{tabular}

\section{DISCUSSION}

The objective of this study was to analyse the tasks designed by the professors in the pre-service phase according to the pedagogical variables in order to be, later, compared with the variables of the tasks positioned in the TGA method. The results show the existence of statistically significant differences $(p<.05)$ in the design of the tasks proposed by the teachers according to the different pedagogical variables except for the content type II variable, because in both cases the defines is worked through tactical behaviour and not through technical gestures.

\section{CONCLUSIONS}

Teachers in the pre-service phase use mixed teaching methods in their teaching units (combining the TGA with the ID), leaning more towards traditional teaching by using the different pedagogical variables to provide more isolated tasks and decontextualized from the real game.

\section{REFERENCES}

Ato, M., López, J. J., y Benavente, A. (2013). Un sistema de clasificación de los diseños de investigación en psicología. Anales de Psicología, 29(3), 1038-1059. https://doi.org/10.6018/analesps.29.3.178511

Cañadas, M., e Ibáñez, S. J. (2010). La planificación de los contenidos de entrenamiento de baloncesto en equipos de iniciación. E-balonmano.com: Revista de Ciencas del Deporte, 6(1), 49-65. https://doi.org/10.6018/293641

Field, A. (2009). Discovering statistics using SPSS (3a ed.). London: Sage Publications Ltd.

Garcia-Ceberino, J. M., Feu, S., \& Ibanez, S. J. (2019). Comparative Study of Two Intervention Programmes for Teaching Soccer to School-Age Students. Sports (Basel, Switzerland), 7(3). https://doi.org/10.3390/sports7030074

Ibáñez, S. J., Feu, S., \& Cañadas, M. (2016). Sistema integral para el análisis de las tareas de entrenamiento, SIATE, en deportes de invasión. E-Balonmano.com: Revista de Ciencias del Deporte, 12(1), 3-30. https://doi.org/10.4321/s1578-84232012000200008 


\section{(c) (i) (3)}

This work is licensed under a Attribution-NonCommercial-NoDerivatives 4.0 International (CC BY-NC-ND 4.0). 


\title{
Social and personal skills in Physical Education: teachers and students' preception about an intervention program
}

\author{
RICARDO ALVES ${ }^{1}$, ANA RODRIGUES ${ }^{1}$, HÉLIO ANTUNES ${ }^{1}$, ANA LUÍSA CORREIA ${ }^{1}$, ÉLVIO \\ GOUVEIA ${ }^{1,2}$, HELDER LOPES 1 \\ 1 University of Madeira, Portugal \\ 2ITI / LARSYS, Portugal
}

\begin{abstract}
The development of social and personal skills in schools, specifically in Physical Education classes, is fundamental to face the mission of Education and the demands of today's society. In this context, we tried to assess how students and teachers perceived such competences through an intervention program carried out in Physical Education classes. For this purpose, a qualitative study was developed in which 15 focus groups were created, between 4 and 6 students of both genders and aged between 14 and 16 years old $(14,83 \pm 0,59)$. Six physical education teachers were also interviewed. The focus group and interviews were conducted on the basis of a semi-structured script and were guided by trained researchers. The students were submitted to a 20-hour intervention program, based on the Model of Sports Education, with guidance and supervision of teachers, who had training and support of the research team, through weekly meetings and class follow-up. Firstly, the groups and the interviews were transcribed by trained investigators, being that, the transcription of the interviews was fully made (verbatim). The NVivo10 software was used to organize and categorize the transcripted data. The collected data show that both students and teachers manifested a high perception regarding the development of several social and personal skills in the implemented program, which allowed us to conclude that the Physical Education classes could contribute, effectively, to the improvement of social competences and personal needs of the students. Keywords: Social and personal skills; Intervention; Physical education; Teachers; Students.
\end{abstract}

Corresponding author. University of Madeira, Portugal.

E-mail: ricardo.alves@flow.com.pt

Supplementary Issue: Spring Conferences of Sports Science. International Seminar of Physical Education, Leisure and Health, 17-19 June 2019. Castelo Branco, Portugal.

JOURNAL OF HUMAN SPORT \& EXERCISE ISSN 1988-5202

(c) Faculty of Education. University of Alicante.

doi:10.14198/jhse.2019.14.Proc4.82 


\section{INTRODUCTION}

The work of personal and social skills - fundamental for the formation and development of capable, productive, autonomous and happy citizens - finds, in Physical Education classes, a potential ally in the sense of concretizing such transformations.

In this context, it was intended, with the present work, to characterize the perception of students and teachers about the development of social and personal skills through an intervention program in Physical Education classes.

\section{MATERIAL AND METHODS}

\section{Participants}

Fifteen focus groups, consisting of 4 to 6 students of both genders and between the ages of 14 and 16 (14.83 \pm 0.59 ), participated in this study. Six physical education teachers ( 5 males and 1 female), aged between 22 and 55 years $(33.83 \pm 13.98)$ were also interviewed.

\section{Measures}

The qualitative research paid particular attention on the realization of focus groups for the students and interviews to the teachers that stimulated the intervention program. The focus group and the interviews were based on a semi-structured guide, which was tested in a pilot study, where adjustments were made in aspects considered ambiguous. The design and development of the script were made in order to enable the students and teachers to know about the development of social skills. The focus groups and the interviews were conducted by trained researchers and recorded through an audio-visual record, ranging from 15 to 30 minutes. The qualitative research was only carried out in a single moment and at the end of the application of the intervention program.

\section{Procedures}

This study is part of a wide project called EFERAM-CIT (Physical Education in the Schools of the Autonomous Region of Madeira - Understanding, Intervening, Transforming) developed through a partnership between the University of Madeira and the Regional Secretary of Education. The participation of the subjects was authorized through the informed consent of the students' caregivers and the teachers themselves. The students took part of an intervention program with a duration of 20 hours, with theoretical support in the Sports Education Model (Siedentop, 1994), being promoted situations of collaborative and cooperative work, with orientation and supervision of the teacher. The teachers were trained and supported by the research team, through weekly meetings and class follow-up. At the same time, documentation was provided to support the development of the sessions. At the end of the intervention program, the evaluations were developed through a focus group (students) and interview (teachers).

\section{Analysis}

Firstly, the focus group and the interviews were transcribed by trained researchers, being made the total transcription of all of the interviews (verbatim). Subsequently the same transcription was verified by another member of the team. In order to organize and categorize the data of the transcriptions the software NVivo10 was used. Based on the purpose of the study, focus group guide and transcription content, a topic of deductive analysis (contributions from the intervention program for the development of social skills) was created. 


\section{RESULTS}

When asked about the social and personal skills developed with the intervention program, the students reported a high number of competences, such as a sense of responsibility $(60 \% ; n=9)$, "I think we gained more responsibility, in other words, we had to make decisions as a team and they were not always approved by everyone, but we also had to know how to change so that they reached the whole group." (Focus Group B). The capacity to develop cooperative work $(53.3 \%, n=8)$, communication capacity $(33.3 \%, n=5)$, critical attitude $(33.3 \%, n=5),(26.7 \%, n=4)$, organizational skills $(26.7 \%, n=4)$, self-confidence $(26.7 \%, n=4)$ and interpersonal relations $(26,7 \% ; n=4)$.

Just like the students, teachers reported a high number of competences, but cooperation work is the most highlighted competence $(100 \%, n=6)$, followed by interpersonal relations $(66.7 \%, n=4)$, communication capacity $(50 \%, n=3)$, autonomy and responsibility $(33.3 \%, n=2)$. In this context a teacher states: "At the skill levels I felt that they developed competences such as autonomy, - because they had to work, they had to be autonomous, they had to do; creativity, because they were forced to construct equipment, emblems and all this promoted the creativity; but also teamwork; the organization competence itself, because there were even groups that spoke in budgets, discussed among themselves, in other words, they had the need to discuss, to speak in budgets, to ponder how much it would be, that is, part of this work was also developed by the students; responsibility too. These were more: autonomy, responsibility, team spirit, cooperation."

\section{DISCUSSION}

The collected data show that both students and teachers manifested a high perception, regarding the intervention program used, in the development of various social and personal skills. This high perception evidences the recognition and potential that Physical Education classes can have for the students' personal formation and social development.

Cooperation was strongly enriched, both by students and teachers, which highlights the importance of this type of interventions in student classes inserted in a society where individualism is evident.

\section{CONCLUSIONS}

Physical Education classes can contribute, in an effective way, to the improvement of the social and personal competences of the students. This potential is recognized by both students and teachers and can be concretized through the dynamization of approaches that focus on these components.

\section{REFERENCES}

Siedentop, D. (1994). Sport education: Quality physical education through positive sport experiences. Champaign, IL: Human Kinetics.

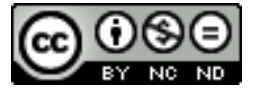

This work is licensed under a Attribution-NonCommercial-NoDerivatives 4.0 International (CC BY-NC-ND 4.0). 


\title{
Is $\mathrm{VO}_{2}$ kinetics influenced by swimming intensity in maximal and supramaximal velocities in young female swimmers?
}

\author{
TIAGO ALMEIDA ${ }^{1,2}$, DALTON M. PESSOA FILHO 2 , MÁRIO ESPADA ${ }^{1,3}$, JOANA REIS ${ }^{1,4}$, ASTOR \\ SIMIONATO ${ }^{2}$, LEANDRO SIQUEIRA ${ }^{2}$, ANDREI SANCASSANI ${ }^{2}$, JOÃO OLIVEIRA ${ }^{2}$, FRANCISCO ALVES ${ }^{1}$ \\ ${ }^{1}$ CIPER - Faculty of Human Kinetics, University of Lisbon, Portugal \\ 2 UNESP, São Paulo State University, Brazil \\ ${ }^{3}$ School of Education, Polytechnic Institute of Setúbal, Portugal \\ ${ }^{4}$ Universidade Europeia, Lisbon, Portugal
}

\begin{abstract}
The aim of the present study was to determine if $\mathrm{VO}_{2}$ Kinetics $\left(\mathrm{VO}_{2} \mathrm{~K}\right)$ and specifically, the time constant $(\tau)$ from rest to maximal and supramaximal velocities is influenced by the swimming intensity. Thirteen well trained female swimmers performed a discontinuous incremental test for maximal oxygen uptake ( $\left.\mathrm{VO}_{2 \text { peak }}\right)$ and maximal aerobic velocity (MAV) assessment and, two 100-m transitions for MAV (100MAV) and all out sprint $(100 \mathrm{MT})$. In the $100-\mathrm{m}$ tests, maximal rise of $\mathrm{VO}_{2}\left(\mathrm{VO}_{2 r i s e}\right)$, oxygen deficit at the onset of exercise $\left(\mathrm{O}_{2 \text { InitialDef }}\right)$ and $\mathrm{VO}_{2} \mathrm{~K}$ were determined. All tests were conducted using a breath-by-breath apparatus (K4b², Cosmed, Italy) connected to a swimming snorkel (new-AquaTrainer ${ }^{\circledR}$, Cosmed, Italy) for pulmonary gas sampling and an underwater visual pacer for velocity control. $\mathrm{VO}_{2 \text { peak }}$ at the incremental test was significantly higher than the $\mathrm{VO}_{2 \text { rise }}$ of both 100 -m tests. Swimmers presented a significantly faster $\mathrm{VO}_{2} \mathrm{~K}$ response (lower

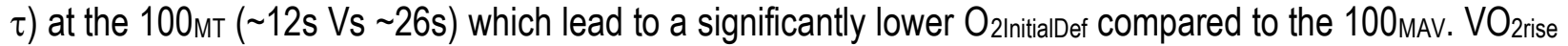
and $\tau$ were correlated between tests $(r=0.71, p<0.01$ and $r=0.59, p<0.05$, respectively). Based on our results it seems that the $\mathrm{VO}_{2} \mathrm{~K}$ is influenced by the exercise intensity at maximal and supramaximal intensities, getting faster as the exercise becomes more demanding. Furthermore, the capacity of attaining higher oxidative rates could be related to the physiological characteristics of the swimmers, since those who were more efficient in a physiological point of view (faster kinetics with higher $\mathrm{VO}_{2}$ ) in one test were those who presented better results in the other. Keywords: Swimming; VO2 kinetics; Supramaximal Intensity; Female swimmers; Youth athletes.
\end{abstract}

Corresponding author. CIPER - Faculty of Human Kinetics, University of Lisbon, Portugal.

E-mail: tiagofalmeida.w@gmail.com

Supplementary Issue: Spring Conferences of Sports Science. International Seminar of Physical Education, Leisure and Health, 17-19 June 2019. Castelo Branco, Portugal.

JOURNAL OF HUMAN SPORT \& EXERCISE ISSN 1988-5202

(c) Faculty of Education. University of Alicante.

doi:10.14198/jhse.2019.14.Proc4.82 


\section{INTRODUCTION}

The evaluation of oxygen uptake kinetics $\left(\mathrm{VO}_{2} \mathrm{~K}\right)$ is an important aspect of human performance, allowing a better understanding of human bioenergetics (Jones and Burnley, 2009). In swimming, studies have associated faster $\mathrm{VO}_{2} \mathrm{~K}$ to better performance (Espada et al., 2015), however the characteristics that may influence the $\mathrm{VO}_{2} \mathrm{~K}$ response are controversial since several studies reported that the $\mathrm{VO}_{2} \mathrm{~K}$ remains remarkably constant as the intensity increases (Pessoa Filho et al., 2012; Espada et al., 2015; Reis et al., 2017), while others, showed an acceleration of the $\mathrm{VO}_{2} \mathrm{~K}$ in maximal trials (Rodríguez et al., 2003; Sousa et al, 2013). The aim of this study was to analyse the $\mathrm{VO}_{2} \mathrm{~K}$ response between maximal (but constant) and supramaximal (without pacing strategies imposed) intensities in order to test if the increment of the intensity will change the $\mathrm{VO}_{2} \mathrm{~K}$ parameters.

\section{MATERIAL AND METHODS}

\section{Participants}

Thirteen well trained female swimmers (age $15.0 \pm 1.2 \mathrm{yr}$.; mass $56.0 \pm 6.9 \mathrm{~kg}$; height $163.1 \pm 6.9 \mathrm{~cm}$ ) that regularly competed at state or national championships, volunteered to participate in this study and were fully familiarized with the equipment and with the test procedures that were used in the study prior to the beginning of the test sessions. The study was approved by the local University Ethical Committee and conducted in accordance with the 1975 Declaration of Helsinki.

\section{Measures}

All swimmers completed three testing sessions in a 25 -meters swimming pool, separated by at least 48 hours: firstly, swimmers performed a discontinuous incremental test, composed by 6 sets of 250-m, plus 1 set of 200-m at maximal intensity (Espada et al., 2015), in order to access the maximal oxygen uptake (VO2peak) and the maximal aerobic velocity (MAV), the minimal velocity at which the $\mathrm{VO}_{2 \text { peak }}$ was reached; secondly, and in randomized order, swimmers performed two 100-m tests, one at the MAV (100MAV) and another in a maximal trial mode $(100 \mathrm{MT})$, to access the maximal rise of $\mathrm{VO}_{2}\left(\mathrm{VO}_{2 \text { rise }}\right), \mathrm{VO}_{2} \mathrm{~K}$ parameters (time delay, time constant and amplitude) and the oxygen deficit at the onset of exercise $\left(\mathrm{O}_{2 \text { nititaldeff }}\right.$.

\section{Procedures}

For $\mathrm{VO}_{2}$ data collection a telemetric portable breath-by-breath gas analyser ( $\mathrm{K}^{\mathrm{b}}{ }^{\mathrm{b}}$, Cosmed, Italy), connected to the swimmers by a swimming snorkel (new-AquaTrainer ${ }^{\circledR}$, Cosmed, Italy), was used (Reis et al., 2017). An underwater visual pacer (PACER 2 SWIM $^{\circledR}$, KulzerTEC, Portugal) set the velocity for the incremental and 100 mav tests. For $\mathrm{VO}_{2 \text { peak }}$ determination a $30 \mathrm{~s}$ moving average of data was used, while at the $100 \mathrm{~m}$ tests, given the shorter duration of the exercise 15 seconds moving average were applied for $\mathrm{VO}_{2 \text { rise }}$ determination. The $\mathrm{VO}_{2} \mathrm{~K}$ parameters were calculated, according to the following equation:

$$
\dot{\mathrm{V}} \mathrm{O}_{2}(\mathrm{t})=\dot{\mathrm{V}} \mathrm{O}_{2 \text { base }}+\mathrm{A}\left(1-\mathrm{e}^{-(\mathrm{t}-\mathrm{td}) / \mathrm{r}}\right)
$$

Where $\mathrm{VO}_{2(t)}$ represents the relative $\mathrm{VO}_{2}$ at a given time; $\mathrm{VO}_{2 \text { base }}$ represents the $\mathrm{VO}_{2}$ at rest, which was calculated as the average of the first 30 seconds of the last minute before the start of the exercise (after 10 minutes of passive rest); TD, $\tau$, and $A$, represent the time delay, the time constant (time that is needed to complete $\sim 63 \%$ of the $\mathrm{VO}_{2}$ response) and the amplitude of the exponential response of the $\mathrm{VO}_{2}$. 


\section{Analysis}

Normality of data was evaluated by the Shapiro-Wilk test. Paired t-test was used to check the differences between the 100-m tests and Pearson's linear correlation coefficient was used in order to establish the significant associations between physiological measures. Statistical significance was accepted at $p<0.05$.

\section{RESULTS}

The $\mathrm{VO}_{2 \text { peak }}\left(53.3 \pm 4.3 \mathrm{ml} . \mathrm{kg}^{-1} . \mathrm{min}^{-1}\right)$ of the incremental test was significantly higher than the $\mathrm{VO}_{\text {2rise }}$ of both $100-\mathrm{m}$ tests. MAV $\left(1.20 \pm 0.06 \mathrm{~m} . \mathrm{s}^{-1}\right)$ was significantly lower than the $100 \mathrm{MT}$ velocity. The swimmers physiological responses at the $100-\mathrm{m}$ tests (table 1) showed a significantly higher $\mathrm{VO}_{2}$ rise, with faster $\mathrm{VO}_{2} \mathrm{~K}$

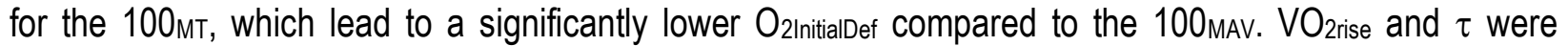
significantly correlated between tests ( $r=0.71, p<0.01$ and $r=0.59, p<0.05$, respectively).

Table 1. Mean \pm SD of swimmers physiological responses at the $100-m$ tests. *statistical differences to the 100 mav test

\begin{tabular}{lcc}
\hline Variable & $100 \mathrm{MAV}$ & $100 \mathrm{MT}$ \\
\hline time $(\mathrm{s})$ & $82.9 \pm 4.0$ & $75.1 \pm 5.2^{*}$ \\
$\mathrm{~V}\left(\mathrm{~m} \mathrm{~s}^{-1}\right)$ & $1.21 \pm 0.06$ & $1.34 \pm 0.09^{*}$ \\
$\%$ to $\mathrm{MAV}(\%)$ & $100.7 \pm 1.3$ & $111.8 \pm 5.4^{*}$ \\
$\mathrm{VO}_{2 \text { rise }}\left(\mathrm{ml} . \mathrm{kg} \cdot \mathrm{min}^{-1}\right)$ & $46.0 \pm 4.8$ & $49.6 \pm 5.1^{*}$ \\
$\%$ to VO peak $(\%)$ & $86.3 \pm 5.6$ & $93.1 \pm 5.1^{*}$ \\
$\mathrm{~A}\left(\mathrm{ml}^{\mathrm{k}} \mathrm{kg}{ }^{-1} \cdot \mathrm{min}^{-1}\right)$ & $40.4 \pm 5.6$ & $40.9 \pm 4.7$ \\
$\mathrm{td}(\mathrm{s})$ & $11.2 \pm 3.3$ & $11.7 \pm 3.5$ \\
$\tau(\mathrm{s})$ & $26.3 \pm 7.9$ & $11.6 \pm 2.3^{*}$ \\
$\mathrm{O}_{2 \text { nitialDef }}\left(\mathrm{mlO}_{2}\right)$ & $1406.3 \pm 666.8$ & $953.0 \pm 234.9^{*}$ \\
\hline
\end{tabular}

\section{DISCUSSION}

When comparing a swimming set at the MAV with a maximal trial it seems that there exists a clear acceleration on the $\mathrm{VO}_{2} \mathrm{~K}$ in the latter, expressed by the time constant ( 26s $\mathrm{Vs} \sim 12 \mathrm{~s}$, respectively). Our results contradict other studies that reported similar time constants at different intensities around the maximal lactate steady state (Espada et al., 2015) or between the moderate, heavy and severe domains (Pessoa Filho et al., 2012; Reis et al., 2017), however, they are in line with studies performed at maximal intensities or when swimmers are able to choose their own pacing strategies (Rodriguez et al., 2003; Sousa et al., 2013). Jones et al. (2008) demonstrated that in a fast-start strategy, compared with the even-pace or with the slow-start strategy, the $\mathrm{VO}_{2}$ increased more rapidly towards its peak in the first seconds of exercise, rising the oxidative contribution to the energy turnover which lead to a spare of the anaerobic capacity across the transition to higher intensity exercises, as observed with our values of $\mathrm{O}_{2 \text { InitialDef. }}$

\section{CONCLUSIONS}

Contrarily to what seems to occur with submaximal intensities, the $\mathrm{VO}_{2} \mathrm{~K}$ seemed to be influenced by the exercise intensity at maximal and supramaximal velocities, getting faster as the exercise becomes more demanding. Also, higher oxidative rates could be associated with specific physiological characteristics of the swimmers, since fast kinetics and higher oxygen uptakes seem to be related between both $100-\mathrm{m}$ tests. 


\section{REFERENCES}

Espada, M., Reis, J., Almeida, T., Bruno, P., Vleck, V., \& Alves, F. (2015). Ventilatory and Physiological Responses in swimmers below and above their maximal lactate steady state, J Strength Cond Res. 29(10): 2836-43. https://doi.org/10.1519/jsc.0000000000000504

Jones, A., \& Burnley, M. (2009). Oxygen uptake kinetics: An underappreciated determinant of exercise performance. Int J Sports Physiol Perform, 4(4), 524-532. https://doi.org/10.1123/ijspp.4.4.524

Jones, A., Wilkerson, D., Vanhatalo, A., \& Burnley, M. (2008). Influence of pacing strategy on 02 uptake and exercise tolerance. Scand J Med Sci Sports, 18, 615-626. https://doi.org/10.1111/i.1600$\underline{0838.2007 .00725 . x}$

Pessoa Filho, D., Alves, F., Reis, J., Greco, C., \& Denadai, B. (2012). VO2 kinetics during heavy and severe exercise in swimming. Int J Sports Med. 33(09), 744-748. https://doi.org/10.1055/s-00311299753

Reis, J. F., Millet, G. P., Bruno, P. M., Vleck, V., \& Alves, F. B. (2017). Sex and exercise intensity do not influence oxygen uptake kinetics in submaximal swimming, Front Physio, 8. https://doi.org/10.3389/fphys.2017.00072

Rodríguez, F., Keskinen, K., Malvela, M., \& Keskinen, O. (2003). Oxygen uptake kinetics during free swimming: A pilot study. Biomechanics and Medicine in Swimming, IX, 379-384.

Sousa, A., Jesus, K., Vilas-Boas, J., \& Fernandes, R. (2013). Oxygen uptake kinetics at moderate and extreme swimming intensities. Rev. Bras Med Esporte, 19(3), 186-190.

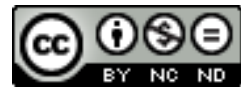

This work is licensed under a Attribution-NonCommercial-NoDerivatives 4.0 International (CC BY-NC-ND 4.0). 


\title{
Training and leadership profile in adapted sport coaches and the implication in athletes with intellectual disabilities
}

\author{
PEDRO PIRES ${ }^{1}$, HELENA MESQUITA², MARCO BATISTA ${ }^{3}$, SAMUEL HONÓRIO³ ${ }^{3}$, JOÃO SERRANO³, \\ TIAGO MACHADO ${ }^{1}$, SÉRGIO IBÁÑNEZ ${ }^{4}$ \\ ${ }^{1}$ Instituto Politécnico de Castelo Branco, Portugal \\ 2Instituto Politécnico de Castelo Branco / CICS.NOVA SHERU, Portugal \\ ${ }^{3}$ Sport, Health \& Exercise Reseach Unit (SHERU), Instituto Politécnico de Castelo Branco, Portugal \\ ${ }^{4}$ Universidad de Extermadura / GOERD, Spain
}

\begin{abstract}
The coach role has been widely studied, and it has become a ruling factor in achievement of more knowledge, and by improving their competence. However, most studies are related to regular sports, making it essential to develop the same process in adapted sports. The present study aims to create a project that intends to establish a coaching profile in the field of Adapted Sport through the perspectives of coaches and athletes with Intellectual Disability (ID). Quantitative methods will be used as resource. We will question coaches through questionnaires for the mentoring profile (CPQ; SCDS; SCLS; SCPS) whereas for the leadership profile we will use the Sports Leadership Scale (ELD). As for the athletes they will be questioned through: BRSQ; BPNSS; SWLS and PANAS and Leadership Scale for Sports - LSS - Perception Version and Preferences). The results obtained in the described process, will serve to develop out the construction of a profile of training and performance for the coaches in the area of adapted sport. Keywords: Adapted Sport; Adapted sport coach and athletes with intellectual disabilities.
\end{abstract}

Corresponding author. Escola Superior de Castelo Branco (Instituto Politécnico de Castelo Branco), Rua Prof. Dr Faria de Vasconcelos, 6000-266 Castelo Branco, Portugal.

E-mail: pedroruiinespires@gmail.com

Supplementary Issue: Spring Conferences of Sports Science. International Seminar of Physical Education, Leisure and Health, 17-19 June 2019. Castelo Branco, Portugal.

JOURNAL OF HUMAN SPORT \& EXERCISE ISSN 1988-5202

(c) Faculty of Education. University of Alicante.

doi:10.14198/jhse.2019.14.Proc4.82 


\section{INTRODUCTION}

Studies developed in this field have become important in the dissemination of knowledge, especially on the necessary knowledge that coaches must obtain to effectively apply in their job (Cushion, Armour \& Jones, 2003). In the case of coaches who intervene in the area of Adapted Sport, this knowledge is also essential, but it is vital to consider contextual aspects such as: accessibility of facilities, suitability of means of transport, assembly and handling of material, among others. These premises are crucial for the coach to adapt the training process and program, according to the degree of functionality of his/her athletes (MacDonald, Beck, Erickson \& Cotê, 2015). One of the obstacles to the evolution and progression of adapted sport is related to the lack of knowledge and lack of specific training from the professionals that intervene with this population (Moran \& Block, 2010; Roberton, Bucks, Skinner, Allison, \& Dunlop, 2011).

\section{MATERIAL AND METHODS}

The main goal of the project is to assess the profile and leadership style of the adapted sports coaches working with ID athletes. In addition to the mentioned aspects, it is essential to understand the extent to which previous assumptions influence the degree of self-determination (satisfaction of basic psychological needs and motivation) and subjective well-being (satisfaction with life and positive and negative affects) of these athletes.

\section{Participants}

It is expected the group to be consisted of 50 coaches (Special Olympics Portugal members and teachers of boccia sport school) and approximately 100 athletes.

\section{Measures}

For this project to be develop, we pretend to use the following methods:

To the coach's profile evaluation we chose the Coach Profile Questionnaire (CPQ); Sport Coach Decision Style (SCDS); Sport Coach Learning Style (SCLS); Sport Coach Planning Style (SCPS) and to the leadership profile we used the Leadership Scale for Sports (LSS- Auto perception version). For the athletes the questionnaires applied will be: Leadership Scale For Sports (LSS- Perception and Preferences Version), the Behavioural Regulation in Sport Questionnaire (BRSQ); Basic Psychological Needs in Exercise Scale (BPNES); Satisfaction With Life Scale (SWLS) and the Positive and Negative Affects Scale (PANAS).

\section{Procedures}

In order to inquire the athletes, in addition to the investigator, we will use licensed professionals specialized in adapted sports, to carry out the application of the questionnaire. These applicators will be subjected to training, in order to understand the contents of the questionnaires and the forms of adaptation for their application. Prior to the application on the large sample, some tests will be performed in order to correct and change possible gaps. Applicants will read the questionnaire statements to the respondents, who will answer through a pictographic scale developed for this purpose.

As for coaches, the procedure of collecting data will be done through questionnaires directly handed out by the investigator. 


\section{Analysis}

The data analysis process will be carried out in three phases: 1 - Inferential analysis made with comparisons, in order to analyse the relationship between the trainer and the sportsman and also to characterize the sample through parametric or non-parametric tests; 2- to make correlations, being again essential to evaluate the characteristics of the sample, and thus the use of parametric or non-parametric parameters will be essential; 3 - to understand which variables are predictors of others, we will use the simple linear regression test.

\section{RESULTS}

With the results we intend to analyse both the profile of leadership and coaches of Adapted Sport that work with athletes with ID, evaluate the degree of self-determination and subjective well-being of athletes and understand how the coach profile and the leadership profile relates with the self-determination values and subjective well-being of athletes.

\section{DISCUSSION}

The coach is responsible for optimizing and maximizing the potential of the team and/or the athlete, which requires constant decision-making on several of aspects and factors (Cushion et al., 2003). Based on this premise the results discussion will be evaluated according to the training profile, self-perception of the leadership profile and athletes' perception, analysis and preferences in relation to the coach's leadership styles. We also intend to verify if would exist: relationship between the variables of the leadership profile of the coach and athletes' perceptions and preferences of the leadership styles; if there is a relationship between the coach's profile and the variables of self-determination and well-being of the athletes and also analyse which variables of the coach's profile are strong predictors of self-determination and subjective well-being of the athletes.

\section{CONCLUSIONS}

This project in the field of Adapted Sport aims to draw a training profile for Coaches, who work with athletes with ID. This profile will help to improve training models for specific coaching courses and for better schooling of adapted sports technicians. Besides this conclusive aspect of the work, the research will also be concerned on allowing athletes with Intellectual Disability to have an opinion and express themselves.

\section{REFERENCES}

Cushion, C., Armour, K., \& Jones, R. (2003). Coach education and continuing professional development: experience and learning to coach. Quest, 55, 215-230. https://doi.org/10.1080/00336297.2003.10491800

MacDonald, J., Beck, K., Erickson, K., \& Cotê, J. (2015). Understanding Sources of Knowledge for Coaches of Athletes with Intellectual Disabilities. Journal of Applied Research in Intellectual Disabilities, 29(3), 242-249. https://doi.org/10.1111/jar.12174

Moran, E., \& Block, E. (2010). Barriers to Participation of Children with Disabilities in Youth Sports. Teaching Exceptional Children Plus, 6(3), 1-13.

Roberton, T., Bucks, S., Skinner, C., Allison, T., \& Dunlop, A. (2011). Barriers to Physical Activity in Individuals with Spinal Cord Injury: A Western Australian Study. Australian Journal of Rehabilitation Counselling, 17, 74-88. https://doi.org/10.1375/irc.17.2.74. 


\section{(c) $\underset{\mathrm{EY}}{\mathrm{NO}} \mathrm{\Theta}$}

This work is licensed under a Attribution-NonCommercial-NoDerivatives 4.0 International (CC BY-NC-ND 4.0). 


\title{
Adapted sports: An experience for initial skills development of sport professionals
}

\author{
PEDRO PIRES 1 , HELENA MESQUITA², MARCO BATISTA ${ }^{3}$, SAMUEL HONÓRIO ${ }^{3}$, JOÃO SERRANO³, \\ TIAGO MACHADO ${ }^{1}$, SÉRGIO IBÁÑEZ ${ }^{4}$ \\ 1 Instituto Politécnico de Castelo Branco, Portugal \\ 2 Instituto Politécnico de Castelo Branco / CICS.NOVA/ SHERU, Portugal \\ ${ }^{3}$ Sport, Health \& Exercise Research Unit (SHERU), Instituto Politécnico de Castelo Branco, Portugal \\ ${ }^{4}$ Universidad de Extremadura / GOERD, Spain
}

\begin{abstract}
The lack of specific training in Adapted Sports in Portugal is an obvious reality and according to this context, the Polytechnic of Castelo Branco, through their School of Education presents in the study plan of the bachelor's degree of Sports and Physical Activity, the specialization of Sport for People with Disabilities. One of the Curricular Units (CU) included in the study plan is Adapted Sports, where several practical and collaborative methodologies are used. A questionnaire was address to 30 students with the purpose of evaluating the teacher's performance and the importance of functional and practical dynamism of this Curricular Unit. The teaching methodologies used are highly appreciated by the students, as confirmed by the results of the curricular unit evaluation (mean 5.62) as well as the teacher's performance (mean 5.66), for a maximal evaluation of 6 . This $\mathrm{CU}$ also aims to sensitize the entire academic community to Adapted Sports, in order to develop workshops and continuous training in this field. Keywords: Adapted sports curricular unit; Evaluation of the study plan; Adapted sport.
\end{abstract}

Corresponding author. Escola Superior de Castelo Branco (Instituto Politécnico de Castelo Branco), Rua Prof. Dr Faria de Vasconcelos, 6000-266 Castelo Branco, Portugal.

E-mail: pedroruiinespires@gmail.com

Supplementary Issue: Spring Conferences of Sports Science. International Seminar of Physical Education, Leisure and Health, 17-19 June 2019. Castelo Branco, Portugal.

JOURNAL OF HUMAN SPORT \& EXERCISE ISSN 1988-5202

(C) Faculty of Education. University of Alicante.

doi:10.14198/jhse.2019.14.Proc4.82 


\section{INTRODUCTION}

Given the importance of Adapted Sport for people with disabilities, the professionals' role is to maximize the potential of their athletes, which requires specific training (Cushion et al., 2003). For Moran and Block, (2010) one of the main obstacles to the sport's evolution is the lack of technical training, often acquired only in professional courses, seminars and workshops (Timson-Katschis and North, 2008).

In this context, in order to solve this problem, the Polytechnic of Castelo Branco, through their School of Education decided to include in its sports bachelor's degree the specialty of Sport for People with Disabilities.

In this degree, at the end of the 1st year, students choose to do one out of two specialties (minor): Sports for People with Disabilities or Sports for Children and Elderly. For students who choose the first one, in the 2nd year / 1st semester the CU has a more theoretical component (Adaptive Motor Activity, Special Educational Needs), whereas in the second semester a more practical component (Adapted Sports and Didactics of Adapted Physical Activities) and in the 3rd and last year in the CU of Project and Practical Intervention I and $\mathrm{II}$, an internship is performed that allows the contact with this type of population.

This study aims to demonstrate the importance of the functional and practical dynamism of the Adapted Sports curricular unit.

\section{MATERIAL AND METHODS}

The teaching methodologies used are structured according to the objectives of this curricular unit, since that due to the methodological diversity, with variability according to the characteristics of the class, the students will be involved in diverse and enriching approaches, which will enable them to acquire planned competences and achieve the proposed objectives.

\section{Participants}

This study had 30 students who attended the Adapted Sports curricular unit of this bachelor's degree between 2016 and 2018.

\section{Measures}

At the end of this curricular unit, students were invited to fill out a questionnaire, with the goal of evaluating two dimensions: General Evaluation of the Curricular Unit and Evaluation of the Teacher Performance. This questionnaire consisted of 17 items that, throughout a 6-point Likert scale, varying between Totally Disagree (1) and Absolutely Agree (6), allows the student to evaluate these dimensions.

\section{Procedures}

To participate in this process, students had to attend between $80 \%$ and $100 \%$ of the classes taught, whose attendance register was performed by the teacher in the $\mathrm{CU}$ computer platform.

\section{Analysis}

After the data serialization, they were later analysed for descriptive statistics. According to Hill and Hill (2005): "a descriptive statistic summarizes some of the characteristics of one or more variables provided by a data sample" (p.192). 


\section{RESULTS}

The questionnaire allows to evaluate the teachers' performance in the curricular unit. Regarding the CU, the items evaluated were: Knowledge Acquisition; Skills development; Improvement of critical sense; Future Skills Development; Adequacy of CU; Sequence of contents; Bibliography; Materials of support. Regarding the performance of the teacher they were evaluated through the items: Preparation; Availability; Relationship; Exhibition; Organization; Exemplification. The final results indicate that the overall satisfaction level regarding the CU had a final mean score of 5.62 and the overall satisfaction level regarding the teacher's performance had a final mean score of 5.66 .

\section{DISCUSSION}

The results reveal the level of the students' satisfaction about the functional and practical dynamism of the mentioned CU, being highlighted in the evaluation the support material which was the most scored item. In the teacher evaluation the items are: availability, relationship and organization. The aspects above mentioned are essential for the student motivation, which should be stimulated by the teacher, so that the teaching method develops autonomy processes (Biggs and Tang, 2007). According to this, the learning progression must focus on what the student can do, while developing cooperative work with the purpose of acquiring transversal skills, essential for the development of teamwork (Esteves, 2010). It is also worth mentioning that students are asked to present oral presentations in several sports, scientific posters and a practical dynamization of an activity with disabled athletes for the academic community. There were 24 oral presentations and scientific posters, several lectures and 4 practical dynamizations (Table Tennis, Boccia, Goalball and Seated Volleyball).

\section{CONCLUSIONS}

At the Adapted Sports CU learning methodologies are essential, both for academic and professional success. We can conclude that students are very satisfied with the functioning and dynamism of the curricular unit and with the performance of the teacher, given that the weighted mean of the two dimensions evaluated are 5.62 and 5.66 respectively for a maximum of 6 .

\section{REFERENCES}

Biggs, J., \& Tang, C. (2007). Teaching for quality learning at university - What the student does ( $3^{\mathrm{a}}$ ed.). England: McGraw Hill Education/Open University Press.

Cushion, C., Armour, K., \& Jones, R. (2003). Coach education and continuing professional devel opment: experience and learning to coach. Quest, 55, pp. 215-230. https://doi.org/10.1080/00336297.2003.10491800

Esteves, M. (2010). Sentidos da inovação pedagógica no Ensino Superior. In C. Leite, Sentidos da pedagogia no Ensino Superior.Porto: ClIE/Livpsic, pp. 45-61.Hill, M. \& Hill, A. (2005). Investigação por Questionário. Lisboa: Edições Sílabo.

Moran, E., \& Block, E. (2010). Barriers to Participation of Children with Disabilities in Youth Sports. Teaching Exceptional Children Plus, 6(3), pp. 1-13.

Timson-Katchis, M., \& North, J. (2010). UK CoachTracking Study Sports Coach. Leeds, UK. 


\section{(c) $\underset{\mathrm{EY}}{\mathrm{NO}} \mathrm{\Theta}$}

This work is licensed under a Attribution-NonCommercial-NoDerivatives 4.0 International (CC BY-NC-ND 4.0). 


\title{
Perceived barriers and physical activity levels in older adults: The role of education
}

\author{
CLÁUDIA VAZ ${ }^{1}$, NUNO SERRA ${ }^{1}$, ERMELINDA MARQUES ${ }^{1,2}$, AGOSTINHA CORTE $^{1,2}$, CAROLINA VILA- \\ $\mathrm{CHÃ} \tilde{A}^{1,3}$ \\ ${ }^{1}$ Research Unit for Inland Development, Polytechnic Institute of Guarda, Portugal \\ ${ }^{2}$ Center for Health Technology and Services Research, Portugal \\ ${ }^{3}$ Research Center in Sports Sciences, Health Sciences and Human Development, CIDESD, Portugal
}

\begin{abstract}
Regular practice of physical activity (PA) is well known as an important factor to improve quality of life and promote independent ageing. However, most of the elderly does not accomplish the minimal levels of PA. Developing evidence to understand why older adults are not engaging in PA is an area that needs attention. Thus, the aim of this study was to explore how educational level influences perceived barriers and PA levels in the elderly. 234 older adults $(75.17 \pm 8.3 \mathrm{yr}$.) were recruited from health centres, day centres, social centres and other public spaces from Guarda, Portugal. A sociodemographic questionnaire, the Yale Physical Activity Survey for older adults (YPAS- PT) and a questionnaire on barriers to PA were applied. Participants were divided in 2 groups according to educational level: $\leq 4$ th Grade $(G 2=117)$ and $>4$ th Grade $(G 3=71)$. A chi-square test was used to determine whether perceived barriers are dependent on educational level. $U$ test Mann-Whitney was applied to investigate the influence of education on PA dimensions. Adverse weather, the existence of injuries/disabilities and believing to be already active enough were the most prevalent barriers to PA. Older adults with higher education level are more physically active and spend less time sitting than those with lower level of education. This study reveals that literacy is an important factor to increase PA levels. Although the most relevant barriers are similar among groups, the prevalence differs by educational level. Keywords: Perceived barriers; Physical activity; Older adults; Educational level.
\end{abstract}

Corresponding author. Instituto Politécnico da Guarda. Av. Francisco Sá Carneiro, n. 50; 6300-Guarda, Portugal.

E-mail: cvilacha@ipg.pt

Supplementary Issue: Spring Conferences of Sports Science. International Seminar of Physical Education, Leisure and Health, 17-19 June 2019. Castelo Branco, Portugal.

JOURNAL OF HUMAN SPORT \& EXERCISE ISSN 1988-5202

(c) Faculty of Education. University of Alicante.

doi:10.14198/jhse.2019.14.Proc4.82 


\section{INTRODUCTION}

Despite the importance of regular physical activity (PA) to promote independent ageing, most of elderly does not reach the minimal levels of PA (European Commission, 2018). Only 22\% of Portuguese older adults are physically active, and those with lower levels of education are less likely to participate in physical activities (Lopes et al., 2017). Most studies report poor health/disabilities, lack of company, and believing to be already active enough, as the most prevalent barriers to PA (Booth et al., 2002; Hirayama, 2006). Understanding why older adults are not engaging in PA is an area that needs attention, thus the aim of this study is to explore the influence of education on perceived barriers and levels of PA among older adults.

\section{MATERIAL AND METHODS}

\section{Participants}

The sample was composed of 232 older adults, recruited from health centres, day centres and other public spaces from Guarda, Portugal (130 women, 102 men), with ages between 60 and 96 (75.32 \pm 8.2 years).

\section{Measures}

A sociodemographic questionnaire was used to get data about age and educational level. The Yale Physical Activity Survey for Older Adults (YPAS- PT) was used to determine the levels of PA (Machado et al., 2016). A questionnaire on barriers, adapted from Hirayama (2006), composed by 29 possible barriers was applied, with a Likert scale from 1 (totally disagree) to 5 (totally agree).

\section{Procedures}

The volunteers signed an informed consent form, then the questionnaires were applied by the researchers in a single moment at the recruitment sites. After collecting the sociodemographic data, the sample was divided in 2 educational levels: lower or equal to 4th Grade $(G 1=163)$ and higher than 4th Grade $(G 2=71)$.

\section{Analysis}

Descriptive statistics were expressed as percentages and mean \pm SD. A chi-square test was used to determine if the perceived barriers, listed as the most or second-most important, are dependent of the educational level (G1 and G2). PA dimensions were computed as described by Machado et al. (2016). $U$ test Mann-Whitney was applied to investigate the influence of educational levels on PA dimensions.

\section{RESULTS}

$58.2 \%$ of the participants reported "the weather is adverse" as an important barrier to PA. The 2nd and 3rd most relevant barriers were "I have an injury or incapacity" (45.3\%) and "I am already active enough" (43.1\%). Figure 1 shows the results on most prevalent barriers to PA, revealing an influence of educational level.

Results on YPAS-PT PA dimensions are described in Table 1. Older adults with higher education level are more physically active (summary index values) and spend less time sitting (sitting index). 


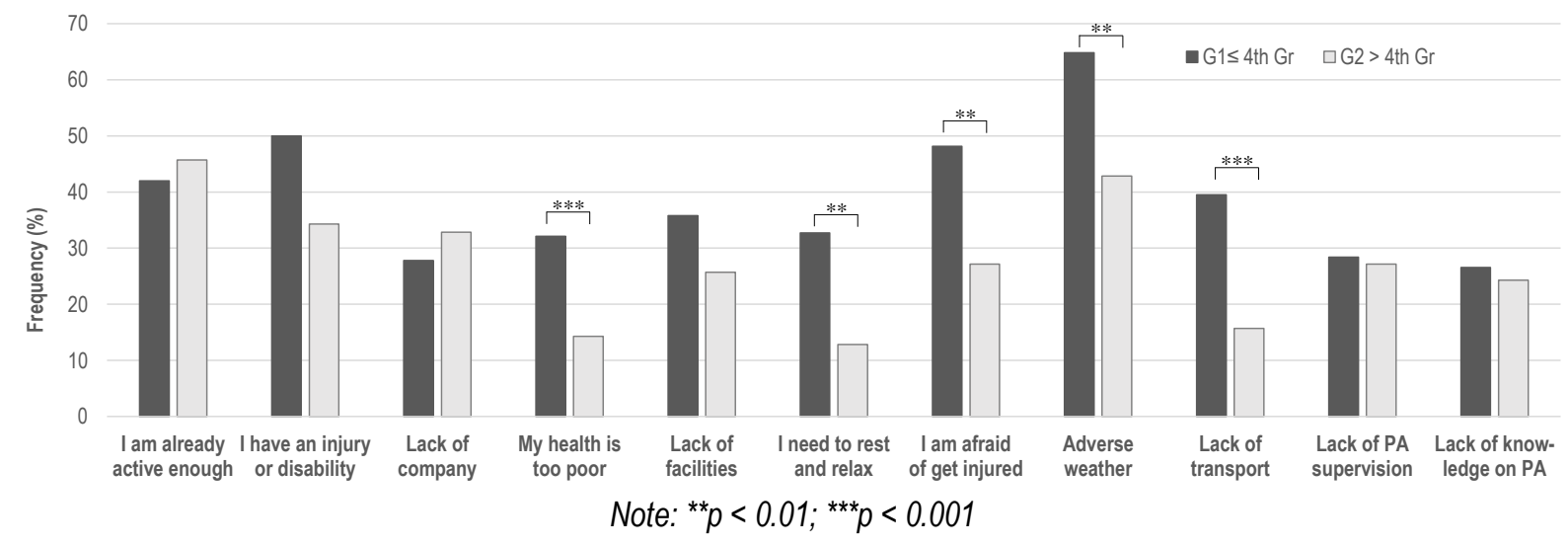

Figure 1. Barriers to physical activity according to educational level.

Table 1. Mean and SD of the YPAS- PT PA dimensions by educational level

\begin{tabular}{ccccccc}
\hline $\begin{array}{c}\text { PA } \\
\text { dimensions }\end{array}$ & $\begin{array}{c}\text { Summary } \\
\text { index }\end{array}$ & $\begin{array}{c}\text { Vigorous } \\
\text { index }\end{array}$ & $\begin{array}{c}\text { Walking } \\
\text { index }\end{array}$ & $\begin{array}{c}\text { Moving } \\
\text { index }\end{array}$ & $\begin{array}{c}\text { Standing } \\
\text { index }\end{array}$ & $\begin{array}{c}\text { Sitting } \\
\text { index }\end{array}$ \\
\hline $\mathrm{G} 1$ & $35.48 \pm$ & $6.69 \pm$ & $12.20 \pm$ & $8.69 \pm$ & $6.16 \pm$ & $2.29 \pm$ \\
& 25.14 & 14.31 & 14.26 & 3.42 & 2.14 & 0.96 \\
$\mathrm{G} 2$ & $39.86 \pm$ & $8.93 \pm$ & $14.51 \pm$ & $9.13 \pm$ & $5.70 \pm$ & $1.96 \pm$ \\
& 24.31 & 14.84 & 11.76 & 3.80 & 2.47 & 0.73 \\
$p$ value & 0.050 & 0.115 & 0.022 & 0.505 & 0.193 & 0.019 \\
\hline & & & ${ }^{*} p \leq 0.05$. & & &
\end{tabular}

\section{DISCUSSION}

The main results showed that educational level influences how older adults perceive barriers to PA, as well as PA levels. "Adverse weather" was considered the main barrier by more than $50 \%$ of the sample, followed by health-related barriers. These results were more pronounced in the lowest educational level group. Also, this group was the most inactive. To our best knowledge, there are no studies investigating the influence of educational level on perceived barriers to PA. In general, the results are in accordance with international studies, referring to physical and health factors as main barriers (Booth et al., 2002). Adverse weather was only mentioned in Hirayama's study (2006). Regarding to PA levels, our findings are in line with Lopes et al. (2017), in which the individuals with the lowest education level were the less active group.

\section{CONCLUSIONS}

This study provides evidence that literacy is an important factor to increase PA levels. Although the most relevant barriers are similar between groups, the prevalence differs between educational levels. Once the main barriers to PA are categorised, strategies can be drawn both to reduce barriers and to propose and develop more motivating PA programs for the elderly.

\section{ACKNOWLEDGMENT}

Study supported by FEDER funds through COMPETE2020 (POCI-01-0145-FEDER-023811). 


\section{REFERENCES}

Booth, M. L., Bauman, A., \& Owen, N. (2002). Perceived barriers to physical activity among older Australians. J Aging Phys Act, 10, 271-80. https://doi.org/10.1123/japa.10.3.271

European Commission (2018). Special Eurobarometer 472: Sport and physical activity Report. https://doi.org/10.2766/483047

Hirayama, M. S. (2006). Atividade física e doença de Parkinson: mudança de comportamento, autoeficácia, barreiras percebidas e qualidade de vida (Master's thesis, Unesp). Retrieved from: https://repositorio.unesp.br/handle/11449/87372

Lopes, C., Torres, D., Oliveira, A., Severo, M., Alarcão, V., Guiomar, S., ... Ramos, E. (2017). Inquérito Alimentar Nacional e de Atividade Física, IAN-AF 2015-2016: Relatório de resultados. ISBN: 978989-746-181-1.

Machado, M., Tavares, C., Moniz-Pereira, V., Andre, H., Ramalho, F., Veloso, A., \& Carnide, F. (2016). Validation of YPAS-PT - The Yale Physical Activity Survey for Portuguese Older People. Science Journal of Public Health, 4(1), 72-80. https://doi.org/10.11648/j.sjph.20160401.20 


\title{
Supervised vs. non-supervised physical activity: The impact on functional fitness in older adults
}

\author{
CLÁUDIA VAZ ${ }^{1}$, VITOR REZENDE ${ }^{2}$, NUNO SERRA ${ }^{1}$, ERMELINDA MARQUES ${ }^{1,3}$, MARIA FONSECA $^{4}$, \\ CAROLINA VILA-CHÃ 1,5 \\ ${ }^{1}$ Research Unit for Inland Development, Polytechnic Institute of Guarda, Portugal \\ ${ }^{2}$ Federal Institute of Education, Science and Technology of the Southeast of Minas Gerais, Brazil \\ ${ }^{3}$ Center for Health Technology and Services Research, Portugal \\ ${ }^{4}$ Guarda City Council, Portugal \\ ${ }^{5}$ Research Center in Sports Sciences, Health Sciences and Human Development, CIDESD, Portugal
}

\begin{abstract}
Literature has systematically shown that appropriate designed physical activity (PA) programs can have positive effects on the functional fitness in the elderly. Such programs can be implemented with or without supervision. The aim of the present study was to evaluate the differences between two modes of PA practice (supervised vs. non-supervised) on functional and aerobic fitness in older adults. 97 older adults (71.01 \pm $6.03 \mathrm{yr}$.) were recruited from Guarda+65 program, parish council, and health centres from Guarda, Portugal. A sociodemographic questionnaire was applied and the volunteers were divided in 3 groups according to PA practice: supervised ( $N=44)$; non-supervised $(\mathrm{N}=17)$; and control group ( $\mathrm{N}=36)$. Functional assessments included balance, $4 \mathrm{~m}$ walking and chair stand 5 times tests (Short Physical Performance Battery). Aerobic endurance was determined by the 2-min step test (Fullerton's Functional Fitness Test). Kruskal-Wallis test was applied to investigate the differences between groups in all evaluated variables. The supervised group showed the best results in SPPB tests and a trend for superior performance in the 2-min step test. Significant differences were observed between supervised and control groups in SPPB score, $4 \mathrm{~m}$ gait speed and chair stand test. Despite non-supervised PA group presented better performance results than control group, no statistical differences were found. Results suggest that supervised physical activity programs are relevant to maximize functional performance in the elderly. Keywords: Physical activity supervision; Older adults; Functional fitness.
\end{abstract}

Corresponding author. Instituto Politécnico da Guarda. Av. Francisco Sá Carneiro, n. 50; 6300-Guarda, Portugal.

E-mail: cvilacha@ipg.pt

Supplementary Issue: Spring Conferences of Sports Science. International Seminar of Physical Education, Leisure and Health, 17-19 June 2019. Castelo Branco, Portugal.

JOURNAL OF HUMAN SPORT \& EXERCISE ISSN 1988-5202

(c) Faculty of Education. University of Alicante.

doi:10.14198/jhse.2019.14.Proc4.82

S1582 | 2019| Proc4| VOLUME 14

(C) 2019 University of Alicante 


\section{INTRODUCTION}

As older adults are growing, both in number and in age (European Commission, 2017), a new challenge for society arises. Scientific literature is systematically showing that physical active older adults have better physical function and longer life expectancy than those who are sedentary (Keysor, 2003). Physical activity (PA) programs can be delivered with or without supervision. However, a recent systematic review has shown that older adults benefit from supervised PA programs in terms of functional performance, when compared with completely non-supervised practice (Lacroix et al., 2017). Nevertheless, some studies revealed positive effects on functional fitness variables for both intervention modes, when compared to an inactive control group (Tsekoura et al., 2018). Therefore, the aim of this study is to evaluate the effects of supervised versus non-supervised PA on functional fitness parameters in older adults.

\section{MATERIAL AND METHODS}

\section{Participants}

The sample was composed of 97 older adults (59 women, 38 men, $71.01 \pm 6.03$ years), recruited from Guarda+65 (a senior PA program), parish council, and health centres from Guarda, Portugal.

\section{Measures}

A sociodemographic questionnaire was used to obtain data on sex, age and PA practice (type, frequency per week, duration by session). Physical function was assessed using the Short Physical Performance Battery (SPPB): static balance, $4 \mathrm{~m}$ gait speed, chair-stand 5 times (Guralnik et al., 1994). Aerobic endurance was also evaluated using the 2-min step test from the Fullerton's Functional Fitness Test (Rikli \& Jones, 1999).

\section{Procedures}

Before conducting the research, all participants were informed about the assessment protocols and gave their written consent. The tests were applied by the researchers at sports laboratory of Polytechnic Institute of Guarda (LABMOV). Volunteers were then divided in three distinct groups according to their answers about the nature of PA practice: supervised $(N=44)$; non-supervised $(N=17)$; and control group $(N=36)$.

\section{Analysis}

Descriptive statistics were expressed as mean \pm SD in the text and mean \pm SE. Kruskal-Wallis test was applied to investigate the differences between supervised PA, non-supervised PA, and control (no PA) groups in functionality and aerobic endurance variables.

\section{RESULTS}

The effects of the PA practice mode on functional fitness components are shown in Figure 1. The supervised group showed the best results in all parameters evaluated in SPPB tests (static balance, gait speed in $4 \mathrm{~m}$, time to stand from a chair 5 times), when compared to control group.

In the 2-min step test, a trend for better results were observed in the supervised group (107.91 \pm 3.69$)$, when compared to non-supervised $(97.12 \pm 3.64)$ and control group (102.00 \pm 3.71$)$, however no statistical differences were observed between groups. 
(A)

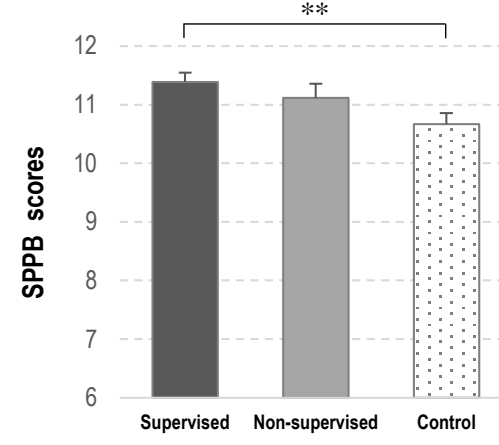

(B)

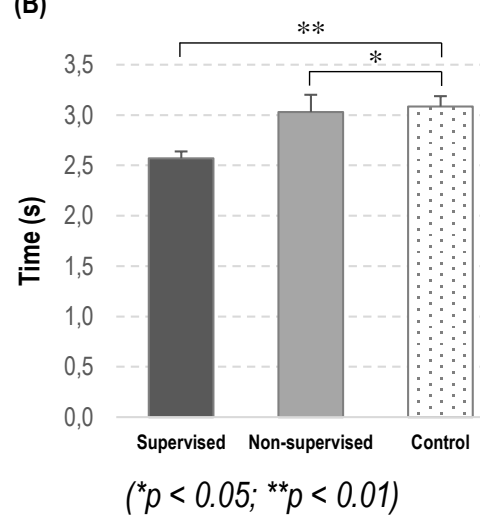

(C)

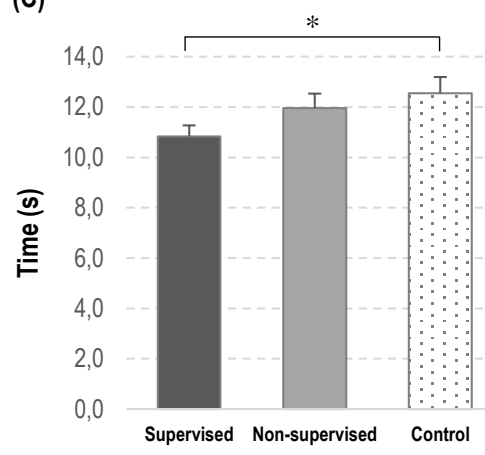

Figure 1. Functional fitness by PA group: (A) SPPB score; (B) 4 m walking test; (C) Chair stand 5 times.

\section{DISCUSSION}

The results showed that PA practice positively influences functionality in independent older adults, in comparison with those who are inactive (Keysor, 2003; Tsekoura et al., 2018). These findings were more pronounced in the supervised group, what suggests that supervised PA programs may be superior compared with non-supervised PA in improving measures of balance and muscle strength in the elderly (SPPB tests). Performance in aerobic endurance did not differ significantly between groups, although tendencies are observed in favour of the supervised group. Regarding to normative data for the 2-min step test, by age and sex (Rikli \& Jones, 1999), the participants of this study are mostly classified above the 75th (for men) and 50th percentiles (for women).

\section{CONCLUSIONS}

The findings of this study showed positive effects of physical activity on functional fitness in older adults. Older adults are capable of responding to a PA program whether is supervised or developed with no supervision. Both approaches can be effective, however supervised exercise seems to be superior in all functional fitness variables.

\section{FUNDING}

Study supported by FEDER funds through COMPETE2020 (POCl-01-0145-FEDER-023811).

\section{REFERENCES}

European Commission (2017). The 2018 Ageing Report: Underlying Assumptions and Projection Methodologies. https://doi.org/10.2765/286359

Guralnik, J.M., Simonsick, E.M., Ferrucci, L., Glynn, R.J., Berkman, L.F., Blazer, D.G., ... Wallace, R.B. (1994). A short physical performance battery assessing lower extremity function: association with self-reported disability and prediction of mortality and nursing home admission. J Gerontol, 49(2), M85-94. https://doi.org/10.1093/geroni/49.2.m85

Keysor, J. (2003). Does late-life physical activity or exercise prevent or minimize disablement? A critical review of the scientific evidence. Am J Prev Med, 25(3 Suppl 2), 129-36. https://doi.org/10.1016/s0749-3797(03)00176-4 
Lacroix, A., Hortobágyi, T., Beurskens, R., \& Granacher, U. (2017). Effects of Supervised vs. Unsupervised Training Programs on Balance and Muscle Strength in Older Adults: A Systematic Review and Meta-Analysis. Sports Med, 47(11), 2341-2361. https://doi.org/10.1007/s40279-017$\underline{0747-6}$

Rikli, R. E., \& Jones, C. J. (1999). Functional Fitness Normative Scores for Community-Residing Older Adults, Ages 60-94. J Aging Phys Act, 7, 162-181. https://doi.org/10.1123/japa.7.2.162

Tsekoura, M., Billis, E., Tsepis, E., Dimitriadis, Z., Matzaroglou, C., Tyllianakis, M., ... Gliatis, J. (2018). The Effects of Group and Home-Based Exercise Programs in Elderly with Sarcopenia: A Randomized Controlled Trial. J Clin Med, 7(12), 480. https://doi.org/10.3390/jcm7120480 


\title{
Playfulness in education: A systematic review
}

\author{
ANA LOURENÇO $\checkmark$, BEATRIZ PEREIRA \\ Universidade do Minho, Research Centre on Child Studies, IE, Portugal
}

\begin{abstract}
Play should be present in every dimension of children's lives as it is crucial to their healthy and happy development. Moreover, the right to play is not only closely connected to the right to health but also to the right to education. The balance between formal education and the time for leisure and rest brings, nowadays, several questions and the recognition that play has positive impact to children's educational development should be clearly stated. Play should be a reality in educational contexts, either in pre-school or primary school environments, mainly through a playful spirit that connects professionals and children, giving space for matters like creativity, cooperation and freedom to arise. So, the need to work with educational professionals to help them achieve their role as play advocates is a challenge that should be carried out. Playfulness, as the predisposition to play, is an individual variable that can be worked out with professionals and so we collected data on the relationships in research between playfulness and education, stating that playfulness is a mean to achieve better outcomes for children, namely on their school achievements and health indicators, but also for professionals, namely stronger motivation and commitment. We developed a systematic review in different databases (B-On, Scopus and Science Direct) between 2013-2019, in English and Portuguese, ending up with 17 articles for analysis. One of the most relevant ideas is that more research is needed as a playful environment and attitude has deep impact in children's learning and overall development. Keywords: Education; Health promotion; Playfulness; Right to play; Systematic review; Training.
\end{abstract}

Corresponding author. Instituto de Educação, Uni. Minho-Campus do Gualtar. Rua da Universidade, 4710-057 Braga, Portugal.

E-mail: $\underline{a}$ mrl@hotmail.com

Supplementary Issue: Spring Conferences of Sports Science. International Seminar of Physical Education, Leisure and Health, 17-19 June 2019. Castelo Branco, Portugal.

JOURNAL OF HUMAN SPORT \& EXERCISE ISSN 1988-5202

(c) Faculty of Education. University of Alicante.

doi:10.14198/jhse.2019.14.Proc4.82 


\section{INTRODUCTION}

Play is stated in the Convention on the Rights of the Child but evidence shows that children nowadays are seeing this right compromised: they have more and more of their day spent in formal educational settings that don't leave time for one of their biggest needs: time and space to play (Amado and Almeida, 2017).

We cannot forget that play is the most natural way for children to express themselves and crucial for children's health and learning: "play and recreation are essential to the health and well-being of children and promote the development of creativity, imagination, self-confidence, self-efficacy, as well as physical, social, cognitive and emotional strength and skills" (UN Committee on the Rights of the Child, 2013, p. 4).

The exaggerated thrive to success and the (incorrect) idea that play is not a serious matter have a deep impact on children's overall development (Neto and Lopes, 2017). Research has been reflecting on the links between fewer opportunities to play and higher rates of child's obesity and also on the arise of mental health issues like depression and anxiety in childhood (Goldstein, 2012; Miller and Almon, 2009).

The United Nations, in their General Comment nr.17 on the right to play, recommend that professionals receive training on how to create an environment where children can freely play and see their right fulfilled (Atkinson et al., 2017). So, a key matter in promoting the right to play can be each one's own playfulness, an understudied characteristic that can have a deep impact in all matters in education (Pinchover, 2017).

The present study aims to collect data on the relationships that have been found in research since 2013 between playfulness and education, stating that playfulness can be a mean to achieve better outcomes for children, namely on their school achievements and overall health indicators, but also for professionals, namely stronger motivation and commitment.

\section{MATERIAL AND METHODS}

\section{Participants}

We consulted 3 sources (B-on, Scopus and Science Direct) and found, respectively, 55, 66 and 43 articles. After the exclusion criteria described below, we ended up with 17 articles.

\section{Measures}

The search for articles was made with the keyword's "playfulness" and "education", in the subjects' terms, in the timeframe 2013-2019 for articles in English and Portuguese. Considering that this is an understudied relationship and the studies connecting these two areas are recent, we chose to focus on this timeframe, including also some studies from the current year.

\section{Procedures}

After the analysis of the title where we had 42 articles, the following step was reading the each abstract. We found several articles connected either to gamification, nature play, health contexts or high education and so these were taken out and are the exclusion criteria $(n=19)$. The main criteria for inclusion was articles regarding children in pre-school and primary school and articles focusing on playfulness as a professional competence for educators. Also, duplicated articles were taken out $(n=6)$. 


\section{Analysis}

The articles were analysed with NVivo software, with the following prior categories for analysis: keywords, objectives, methodology, participants, measures, findings and future research. PRISMA 2009 checklist was used as an orientation for the current systematic review.

\section{RESULTS}

The themes that arose from the analysis, by order of expression, were: the relationship between creativity and playfulness, learning facilitated by playfulness, the impact of educator's characteristics of playfulness on children's playfulness, adult-child interaction as promoter of playfulness, playfulness in children and its measures and finally, the role of families in playfulness and education.

\section{DISCUSSION}

The place for play in education is not a comfortable one: its value as promoter of success and healthier and happier school environments is not assumed by everyone. Research shows that the disposition to play, playfulness, is a key element for education. Experiences were playfulness is assumed as a key component in the educational area (e.g. Norway) are well succeeded and should inspire others around the world.

From the findings of the current review, we can state that playfulness is a characteristic that can be worked on and several researches show that it cannot only be improved along time, but also is permeable to intervention. The greater added value these kinds of interventions bring is to improve each one's well-being, personally and professionally, for adults and children.

\section{CONCLUSIONS}

Nicholson and Shimpi (2015) sum up extremely well the importance of the relationship between playfulness and education: "if we want early childhood professionals to develop as courageous advocates, articulating the value of play for children's learning, development, and well-being in socio-political contexts where play is increasingly marginalised and devalued, we must begin by supporting them to reclaim play in their own lives" (p. 1614). Another key point is to see playfulness through children's eyes: the child-adult interaction in education can be the difference between well succeeded and not succeeded school involvement.

More research is needed as a playful environment and attitude has deep impact in children's lives.

\section{REFERENCES}

Amado, J., \& Almeida, A. C. (2017). Políticas públicas e direito de brincar das crianças. Laplage Em Revista, 3(1), 101. https://doi.org/10.24115/s2446-6220201731237p.101-116

Goldstein, J. (2012). Play in children's development, health and well-being. Brussels: Toy Industries of Europe.

Miller, E. \& Almon, J. (2009). Crisis in the Kindergarten Why Children Need to Play in School. Available at http://www.allianceforchildhood.org/publications

Neto, C. \& Lopes, F. (2017). Brincar em Cascais. Cascais: CERCICA.

Nicholson, J., \& Shimpi, P. M. (2015). Guiding future early childhood educators to reclaim their own play as a foundation for becoming effective advocates for children's play. Early Child Dev Care, 185(10), 1601-1616. https://doi.org/10.1080/03004430.2015.1013538 
Pinchover, S. (2017) The Relation between Teachers' and Children's Playfulness: A Pilot Study. Front. Psychol. 8:2214. https://doi.org/10.3389/fpsyg.2017.02214

UN Committee on the Rights of the Child (2013). General comment No. 17 on the right of the child to rest, leisure, play, recreational activities, cultural life and the arts (art. 31). CRC/C/GC/17.

\section{(c) $(\mathrm{B})(\mathrm{EY}$}

This work is licensed under a Attribution-NonCommercial-NoDerivatives 4.0 International (CC BY-NC-ND 4.0). 


\title{
The 2019 Special Olympics World Games experience: Perspective of athletes who participated
}

\author{
RAQUEL CORREIA ${ }^{1} \triangle$, PEDRO PIRES ${ }^{1,2}$, ANABELA ANTUNES ${ }^{1}$, JOÃO GIL ${ }^{1}$, NOEL GONÇALVES ${ }^{1}$, \\ PEDRO PIO ${ }^{1}$, RAFAELA LOURO1 ${ }^{1}$ RICARDO ZORRO ${ }^{1}$ \\ ${ }^{1}$ Associação Portuguesa de Pais e Amigos do Cidadão Deficiente Mental (APPACDM) de Castelo Branco, \\ Portugal \\ 2Instituto Politécnico de Castelo Branco, Portugal
}

\begin{abstract}
The Special Olympics World Games are considered a life changing event by the people who participated in this event. The present work aims to describe the experience of participating in the SO World Games, by the perspective of the athletes. For this purpose, two athletes were interviewed by an Inclusive Research Group, using photo-elicitation interview. The participants considered the experience very enriching and with memorable moments. Keywords: Special Olympics; Athletes with intellectual disabilities and photoelicitation interviews.
\end{abstract}

Corresponding author. Rua Frente ao Hospital Amato Lusitano, 6000-109 Castelo Branco, Portugal.

E-mail: raquel.a.correia@gmail.com

Supplementary Issue: Spring Conferences of Sports Science. International Seminar of Physical Education, Leisure and Health, 17-19 June 2019. Castelo Branco, Portugal.

JOURNAL OF HUMAN SPORT \& EXERCISE ISSN 1988-5202

(c) Faculty of Education. University of Alicante.

doi:10.14198/jhse.2019.14.Proc4.82 


\section{INTRODUCTION}

Sports are important for people with intellectual disabilities (ID); however the experience of the Special Olympics (SO) World Games has rarely been examined (Werner, 2015). Participation in the World Games is a unique experience that includes travelling to a different country, be in touch with a new culture, competing at an international level, and being close to media interest (Dowling et al., 2012). This year the SO World Games were held in Abu Dhabi, between 14th and 22th of March. This completion included 7000 athletes from 170 countries. From Portugal, participated 31 athletes in different sports. The present work aims to describe the experience of the SO World Games from the perspective of the athletes.

\section{MATERIAL AND METHODS}

\section{Participants}

The participants were two athletes who participated in the Special Olympics World Games in Abu Dhabi, between 8th and 22th of March 2019. Athlete 1 was female, 24 years old and competed in Swimming, 50m backstroke, and $50 \mathrm{~m}$ freestyle. Athlete 2 was male, 31 years old and competed in Judo. These athletes are from the Occupational Activities Centre of the Associação Portuguesa de Pais e Amigos do Cidadão Deficiente Mental (APPACDM) from Castelo Branco, Portugal. They also are members of the Inclusive Research Group from the referred service provider. This research are held by this Inclusive Research Group.

\section{Measures}

For the purpose, photo-elicitation interview will be used, where photographs are used to evoke comments, memories and dialogue (Banks, 2007).

\section{Procedures}

The study is developed by the Inclusive Research Group from APPACDM Castelo Branco, which is composed by 6 people with ID and a clinical psychologist. The athletes who participated in the SO World Games chose the photographs from the most important moments. Then the other participants of the group asked questions related to the photographs.

\section{Analysis}

The interviews were recorded and transcribed verbatim. For data analyses the NVivo 11 software was used. Using this software, categories were created and associated with direct quotations from the participants, which is an effective way to preserve the "voice" of persons with ID in the manuscripts produced from qualitative research (Irvine, 2010). The transcription and coding was using a process of inductive coding (Thomas, 2006).

\section{RESULTS}

The photographs chosen by the participants were related with the following moments: competition, contact with other cultures, medal ceremony, social moments, flight, and return to Portugal. The medal ceremony was very referred by the athletes as a moment to remember the whole course. The reception at the airport, when they came back, was another important moment, because they felt valued by the country. 


\section{DISCUSSION}

Through the photo-elicitation interviews it was possible to access data about the persons present in the photographs, the moments in which they were taken and the feelings present and evoked.

\section{CONCLUSIONS}

In conclusion, the athletes enjoyed their experience in the SO World Games and considered that had a positive impact in their lives.

The photo-elicitation interview it is a very rich and promising data collection method, since it allows exploring the perceptions of the participants in an in-depth way.

\section{REFERENCES}

Banks, M. (2007). Using visual data in qualitative research. London: Sage.

Irvine, A. (2010). Conducting qualitative research with individuals with developmental disabilities: Methodological and ethical considerations. Developmental Disabilities Bulletin, 38(1/2), 21-34.

Thomas, D. (2006). A general inductive approach for analyzing qualitative evaluation data. American Journal of Evaluation, 27(2), 237-246. https://doi.org/10.1177/1098214005283748

Werner, S. (2015). Athletes' parents', and siblings' experiences from the Special Olympics World Games. Journal of intellectual \& developmental disability, 40 (2), 167-178. https://doi.org/10.3109/13668250.2015.1010148.

\section{(9)}

This work is licensed under a Attribution-NonCommercial-NoDerivatives 4.0 International (CC BY-NC-ND 4.0). 


\title{
The importance of sports to the Inclusive Research Group of APPACDM Castelo Branco
}

\author{
RAQUEL CORREIA ${ }^{1} \triangleleft$, ANABELA ANTUNES ${ }^{1}$, JOÃO GIL ${ }^{1}$, NOEL GONÇALVES ${ }^{1}$, PEDRO PIO ${ }^{1}$, \\ RAFAELA LOURO1', RICARDO ZORRO1, PEDRO PIRES ${ }^{1,2}$ \\ ${ }^{1}$ Associação Portuguesa de Pais e Amigos do Cidadão Deficiente Mental (APPACDM) de Castelo Branco, \\ Portugal \\ 2Instituto Politécnico de Castelo Branco, Portugal
}

\begin{abstract}
The ways of looking and doing research are diversifying and get as close as possible to the real contexts, thus allowing new audiences to be included, always maintaining the necessary accuracy. People with intellectual disabilities (ID) are one of the populations that are starting to contribute to research, as active participants. The main aim of this work is to explain the beginning of an inclusive research project that is studying the importance of sports to the quality of life of people with ID. The participants of the project, six people with ID, supported by a clinical psychologist, are very motivated with the weekly sessions and they enjoy learning about research. Keywords: Inclusive research; Intellectual disabilities; Adapted sports.
\end{abstract}

Corresponding author. Rua Frente ao Hospital Amato Lusitano, 6000-109 Castelo Branco, Portugal.

E-mail: raquel.a.correia@gmail.com

Supplementary Issue: Spring Conferences of Sports Science. International Seminar of Physical Education, Leisure and Health, 17-19 June 2019. Castelo Branco, Portugal.

JOURNAL OF HUMAN SPORT \& EXERCISE ISSN 1988-5202

(c) Faculty of Education. University of Alicante.

doi:10.14198/jhse.2019.14.Proc4.82 


\section{INTRODUCTION}

Inclusive research has emerged as a new research paradigm that encompasses a number of forms of conducting research (Walmsley \& Johnson, 2003), all of which focus on researching with people with intellectual disabilities (ID) rather than about them (Strnadová \& Cumming, 2014). This type of research also has many advantages for its participants, namely the development of cognitive and decision-making skills. The aim of this work is to describe the implementation process of an inclusive research project in a day care service provider in Portugal that will study the theme of sports.

\section{MATERIAL AND METHODS}

\section{Participants}

The participants of the project are six people with ID and a clinical psychologist that is also a Ph.D. student. The participants with ID attend the occupational centre of a day care service provider and their ages are between 24 to 48 years old, 4 men and 3 women.

\section{Measures}

This project is based on an action research methodology. Through the process the participants will decide the steps that will be done for all phases of the research, in a co-construction process. To gather the information about the importance of sports, the participants will interview the athletes of the service provider.

\section{Procedures}

The group gathers weekly in a Higher Education School. The sessions started with a first activity named "a morning in the Science Laboratory". This session had the aim of knowing the research process, its phases and characteristics. It's important that all the elements of an inclusive research group learn research skills, so the group could work efficiently (Strnadová et al., 2014).

\section{Analysis}

The satisfaction of the participants is evaluated weekly, through the session report. The interviews of the other athletes will be analysed using content analysis.

\section{RESULTS}

The study is in progress. All the people with ID are active participants in sports activities, so they chose and agreed with the theme. They already had the change to do two oral communications in conferences and wrote a book chapter about the beginning of that experience. These experiences were very important to the people with ID, since they can feel that they are able to participate actively in different research practices and motivate them to proceed.

\section{DISCUSSION}

At the moment, the participants are initializing their scientific study, about the importance of sports to the quality of life to people with ID. They are very motivated and give ideas to proceed with the research. All the work results from the exchange of ideas, dialogue and research that occurs in the weekly sessions, with all elements of the group. 


\section{CONCLUSIONS}

People with ID should have the opportunity to participate in inclusive research projects because their perspective can provide an enriching contribution to research. In all the process is important to apply different and active methods, and use accessible language. It is an important research decision to give voice to persons with ID and learn from their perspectives and opinions.

\section{REFERENCES}

Strnadová, I., \& Cumming, T. (2014). People with intellectual disabilities conducting research: New directions for inclusive research. Journal of Applied Research in Intellectual Disabilities, 27, 1-2. https://doi.org/10.1111/jar.12075

Strnadová, l., Cumming, T., Knox, M., Parmenter, T., \& Welcome to Our Class Research Group (2014). Building an inclusive research team: The importance of team building and skills training. Journal of Applied Research in Intellectual Disabilities, 27, 13-22. https://doi.org/10.1111/jar.12076

Walmsley, J., \& Johnson, K. (2003). Inclusive research with people with learning disabilities: Past, present and futures. London: Jessica Kingsley.

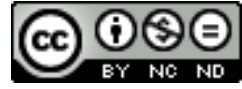

This work is licensed under a Attribution-NonCommercial-NoDerivatives 4.0 International (CC BY-NC-ND 4.0). 


\title{
Adapted sports: Curricular traineeship
}

\author{
ANTÓNIO FAUSTINO1,2 $\triangle$, FILIPE GONÇALVES², JOSÉ MARTINHO², PEDRO PIRES² \\ ${ }^{1}$ Instituto Politécnico de Castelo Branco, SHERU, Portugal \\ 2Instituto Politécnico de Castelo Branco, Portugal
}

\begin{abstract}
The licence in Sports and Physical Activity from the ESE - IPCB integrates the minor of Sports for the Disabled in its plan of studies. In the scope of the curricular units of Project and Practical Intervention I and II, the students should go through a stage that will afford them an advantage as to what an adapted sports technician needs in order to practice his/ her profession. This study is focused on the topic of self-assessment and reflection of the student as an adapted sports technician trainee as to leading the sessions and their planning through comparison of key-points referring to objectives, exercise description, exercise representation and their timing, practice plans and self-assessment tables for practice and theory, filled out at the end of each session of the three categories comprised in the training: Swimming, Table Tennis and Boccia. The comparison between all of the practice plans and assessment charts corresponding to the students' own self-assessment of the sessions during the training allow for the analysis of the occurring progress. Keywords: Traineeship; Adapted sports; Students; Athletes with IDD.
\end{abstract}

Corresponding author. Escola Superior de Castelo Branco (Instituto Politécnico de Castelo Branco), Rua Prof. Dr Faria de Vasconcelos, 6000-266 Castelo Branco, Portugal.

E-mail: a.faustino@ipcb.pt

Supplementary Issue: Spring Conferences of Sports Science. International Seminar of Physical Education, Leisure and Health, 17-19 June 2019. Castelo Branco, Portugal.

JOURNAL OF HUMAN SPORT \& EXERCISE ISSN 1988-5202

(c) Faculty of Education. University of Alicante.

doi:10.14198/jhse.2019.14.Proc4.82 


\section{INTRODUCTION}

The curricular stage allows the student to ease the transition from superior education to the world of work and will work as an increase of tools for future employment opportunities (Ryan et al, 1996). The benefits of a traineeship aren't only the integration of the individual in the work market or the perfecting of professional skills, but it is also relevant for personal development, as is the case of developing social and interpersonal skills (Caires \& Almeida, 1996) and the increase of levels of maturity and self-confidence of the students (Amaral et al., 1996; Vieira et al., 2011). The curricular traineeship teaches the students how he/ she can adapt the contents and theoretical concepts in real problems in the work context and so, begin to build their own style of action (Ryan et al., 1996).

\section{MATERIAL AND METHODS}

Two students carried out traineeship activities in the Adapted Sports Section of the APPACDM (Associação de Pais e Amigos do Cidadão Deficiente Mental of Castelo Branco, working in the categories of Swimming, Boccia, and Table Tennis. The construction of an annual plan by the students was initially agreed upon among the members involved in the training process, which constituted a first step of the planning and preparation of the teaching.

\section{Participants}

The training consisted of accompanying sixteen athletes, spread over three categories: two participated in two categories simultaneously; four participated in Swimming; six participated in Table Tennis and eight participated in Boccia. The challenged participating athletes had three different types of issues (Intellectual Development Disorder; Trisomy 21/ Down Syndrome and Cerebral Palsy).

\section{Measures}

In the course of the training, there were observations, in competition, of the six different athletes, in the form of video recording and later analysis of points marked, suffered, services and reception to the services, in each set.

\section{Procedures}

The self-assessment of the students was done in three assessment moments: the initial phase with six sessions; intermediate with seven sessions, and final with another seven sessions. At these three moments, the changes of the leading of the sessions and the improvement of the practice plans were marked in percentages.

\section{Analysis}

The analysis applied to the data from the assessment tables, SWOT analysis and changes to the practice plans have the prime function of describing the progress of the trainee student in the construction of the practice plans and leading of the sessions, both as to theoretical and as to practical aspects. The analysis done for the observation of the practice plans focused on the objectives, description of the exercises, representation of the exercises and their length. On the other hand, the analysis done for the observation of the progress of leading the sessions was divided into two parts: theory and practice. The theoretical part encompassed the sequence of description of the exercises, the language used, the use of institutional images, the material used and, lastly, the definition of general and specific objectives. The practical part encompassed the explanation of the exercises, the organization of the practice, the organization of the 
material, the organization of the exercises, the use of correct language and the feedback given at the session by the students to the institution's athletes.

\section{RESULTS}

The exercise description in Swimming and the exercise representation in Table Tennis were the aspects that needed to be corrected and improved. As to Boccia, the description of exercises and objectives of the practice plans were the aspects to be improved. In a total of twenty observations in each of the four key-points, one can observe a percentage of twenty-five percent relative to the aspects that should be improved and corrected in the categories of Swimming and Table Tennis and a percentage of fifty percent relative to the same aspects defined for the success of the practice plan. As to the aspects analysed for the observation of the progress of the leading of the sessions, the changes were more visible in a more global aspect. In a total of twenty observations of the six aspects that were analysed, there was a change of about $83.3 \%$ in all the theoretical aspects in the categories of swimming and table tennis, except in the material used, while in Boccia the number was $66.7 \%$, relative to all the changes except the material used and definition of specific objectives. Lastly, the last aspects analysed were the practical ones, where one saw a change in all the aspects in the categories of Table Tennis and Swimming and four in the category of Boccia, with a change percentage of $66.7 \%$ in the explanation of the exercises, organization of the class, feedback and organization of the exercises.

\section{CONCLUSIONS}

The curricular stage is considered an important component of the academic education process, in which the student prepares him/herself for the introduction in the work market by participating in real work situations, i.e., placing students in real work situations, in practical situations where they can begin to practice their profession and the inherent functions (Melo Silva, 2003) (Rocha de Oliveira \& Piccinini, 2012). In order to progress during the curricular stage, the students' reflection is fundamental, exploring their experience with the objective to concretize a new understanding and create a dialogue between thinking and doing, through which one becomes more able (Boud et al., 1985) At the end of the stage, there was conclusively an improvement in all the necessary aspects for the control of the practices. These aspects were justified in the analysis of all the reflections made at every practice of the categories included in the curricular traineeship.

\section{REFERENCES}

Amaral, M., Moreira, M. \& Ribeiro, D. (1996). O papel do supervisor no desenvolvimento do professor reflexivo: estratégias de supervisão. In: Alarcão, l. (Org.), Formação reflexiva de professores: estratégias de supervisão. Porto: Porto Editora. https://doi.org/10.11606/d.8.2017.tde-07082017194408

Boud, D., Keogh, R., \& Walker, D. (1985). Reflection: Turning experience into learning. London, Kogan Page.

Caires, S. \& Almeida, L. (1996). Avaliação de estágios curriculares: contributos para a operacionalização de uma escala. In: Almeida, L., Araújo, S., Gonçalves, M., Machado, C. \& Simões, M. (Orgs). Avaliação Psicológica: formas e contextos (Vol. IV). Braga: Apport.

Melo Silva, L. (2003). Formação do psicólogo: a contribuição da orientação profissional. Revista de Psicologia da Vetor Editora, 4 (1), pp. 42-53. 
Rocha de Oliveira, S. \& Piccinini (2012). Uma análise sobre a inserção profissional de estudantes de administração no Brasil. RAM, Revista de Administração Mackenzie, 13 (2), pp. 44-75. https://doi.org/10.1590/s1678-69712012000200003

Ryan, G., Toohey, S. \& Hughes, C. (1996). The purpose, value and structure of the practicum in higher education: a literature review. Higher Education, 31, pp. 355-377. https://doi.org/10.1007/bf00128437

Vieira, D., Caires, S. \& Coimbra, J. (2011). Do ensino superior para o trabalho: contributo dos estágios para a inserção profissional. Revista Brasileira de Orientação Profissional, 12 (1), pp. 29-36. https://doi.org/10.26707/1984-7270/2019v19n2p133 


\title{
The observation: Adapted table tennis
}

\author{
FILIPE GONÇALVES ${ }^{1} \triangle$, ANTÓNIO FAUSTINO ${ }^{1}$, JOSÉ MARTINHO11,PEDRO PIRES ${ }^{1,2}$ \\ ${ }^{1}$ Instituto Politécnico de Castelo Branco, Portugal \\ 2Instituto Politécnico de Castelo Branco/ APPACDM de Castelo Branco, Portugal
}

\begin{abstract}
The degree of Sports and Physical Activity of the ESE - IPCB presents in its study plan, the minor of Sports for the Disabled, where in the scope of the curricular units of Projects and Practical Intervention I and II the students must complete a stage that propels them an added value in what is the practice of the profession of an adapted sports technician. The study focuses on the elaboration and application of an observation sheet of the table tennis games to athletes with DID coincident in the stage of this modality, in Special Olympic competitions. In the observation sheets constructed it is intended to analyse how marked, suffered points, services and the reception occurred. The way the points suffered / scored are analysed is whether they occurred from diagonal right, parallel right, left diagonal and left parallel. On the other hand, services and reception are evaluated on a four-level scale ranging from insufficient to very good. The analysis of the performance observation of the athletes in the test allows to analyse their strengths and weaknesses, so that the training is optimized at the individual level. Keywords: Internship; Table tennis adapter; Students; Athletes with DID.
\end{abstract}

Corresponding author. Escola Superior de Castelo Branco (Instituto Politécnico de Castelo Branco), Rua Prof. Dr Faria de Vasconcelos. 6000-266 Castelo Branco, Portugal.

E-mail: filipe.8h@ipcb.pt

Supplementary Issue: Spring Conferences of Sports Science. International Seminar of Physical Education, Leisure and Health, 17-19 June 2019. Castelo Branco, Portugal.

JOURNAL OF HUMAN SPORT \& EXERCISE ISSN 1988-5202

(c) Faculty of Education. University of Alicante.

doi:10.14198/jhse.2019.14.Proc4.82 


\section{INTRODUCTION}

Observing is something more than looking, it is capturing different meanings through visualization (Sarmento, 2004). The observable is strictly human and space-temporal, which is collected by observation emerges from the need for knowledge, but also by the understanding of its particular actions (Sarmento, 2004).

However, before an observation is made it is necessary to take into account a set of factors that influence the quality of observation, answering questions such as what and who to observe, how to observe, when to observe, why to observe, what to observe, and under what conditions (Sanchez, 2015). In Physical Education, as in sports training contexts, observation is an essential capacity for the use, being central to the analysis and evaluation of the performance of students or athletes, and as such, in the teacher's own activity (Aranha, 2007).

The study focuses on the elaboration and application of an observation sheet of the table tennis games to athletes with DID coincident in the stage of this modality, in Special Olympic competitions.

\section{MATERIAL AND METHODS}

Two students perform internship activities in the Adapted Sport Section of APPACDM in Castelo Branco, developing work with the Table Tennis modality. It was initially agreed among the members involved in the internship process, the preparation and application by the students of a table of analysis to the athletes coinciding with their internship, in competition, and of evaluation sheets, in order to optimize the training.

\section{Participants}

The stage consisted of monitoring of the process of six athletes, Table tennis practitioners. Athletes with disability status presented a single typology - DID, which is defined by the American Association on Intellectual and Development Disabilities (AAIDD) as a disability that appears before the age of 18 and is characterized by significant limitations, which may be at the level of intellectual functioning or adaptive behaviour, which normally encompasses many social skills and day-to-day practices. The athletes who are competing in the Special Olympics, whose mission is to provide athletic training throughout the year and athletic competition for children and adults with intellectual disabilities.

\section{Measures}

In the course of the training, there were observations, in competition, of the six different athletes, in the form of video recording and later analysis of points marked, suffered, services and reception to the services, in each set.

\section{Procedures}

The students' observation was carried out in two competitions. From these two evaluations, the data were withdrawn, in order to optimize the individual training of each athlete.

\section{Analysis}

The observations elaborated and applied to the performance of the athletes in the test have the main function of describing to the trainee student the evolution of the athletes in the modality, in the points gained, lost, services and reception to them. In addition to this process, an evaluation form was drawn up in two moments that evaluate on a scale of 0 to 3 (does not execute, execute, perform well) basic, technical and tactical aspects. 
The analyses of the observation of the evolution of the athletes in the points won and lost during the competition, are analysed if they were in beats of diagonal right, parallel right, left diagonal and left parallel. Following the observation, it was also analysed on an insufficient scale (it loses the point per service action I cannot make the reception), enough (performs the service but loses the point with the course of the game I makes the reception but loses the point with the course of the game), good (can do the service but wins the point with the course of the game / can make the reception, but loses the point with the course of the game) and very good (can do the service and win point / can make the reception in the service and earn point) of the services and reception to them.

For the analysis of the above, we used the measure of central tendency, the average, in order to observe how points were made, lost, services and reception to services.

\section{RESULTS}

The two evaluation sheets applied to the training of athletes with fifteen topics that evaluate their basics and their technical - tactical performance, were extracted from the data that five of the athletes obtained the average of that executes the modality and the remaining one, that performs well the mode. This evaluation form allows the trainee student to help analyse the performance and, compared to the final evaluation form, the evolution of the athletes who, despite all of them having raised the level, four of them maintained the level of performance of the modality and two of them who performed well.

Data taken from athletes' observation of the marked points score, is of greater mean in the diagonal and parallel rights. On the other hand the points suffered are more related to diagonal and parallel lefts, which allows me to conclude the stronger side and what needs to be more trained. In terms of the services and their receptions in the test, the averages had more importance in the scale of good and then, in the scale of sufficient, which has the positive aspect that the athletes gain points, but of the negative aspect that the majority of them you get or lose with the course of the game.

\section{CONCLUSIONS}

In this sense, it seems to be unanimous accepted that the control and evaluation of training is a key role both for the reformulation of planning and for the enhancement of the athletes' sport performance, in view of the objectives previously set, in view of the sporting season (Gaspar \& Santos, 2004), as well as the acceptance and importance of the specialized areas related to sport sciences, more specifically Game Analysis, which is now widely recognized as being essential in the understanding and evolution of performance (Carling, Reilly, \& Williams, 2009). In order to have an evolution in the training of athletes, it is essential to observe them both in the test and also in the training, so that these are optimized according to the individuality of the athlete.

From the data collected in the test we found that in the marked points the mean is greater in the diagonal and parallel rights and that the points suffered result more from left diagonal and parallel. On the services and their reception, it was verified that they were classified as good and sufficient, and the losses occur in the ball exchanges. At the end of the training it was concluded that with the observations and evaluations made for an individual optimization of the training, there was an improvement in the individual performance of the athletes. 


\section{REFERENCES}

Aranha, A. (2007). Observação de aulas de Educação Física. Vila Real: Universidade de Trás-os-Montes e Alto Douro. https://doi.org/10.24140/issn.1645-7250.rle38.03

Carling, C., Reilly, T. \& Williams, A. M. (2009). Performance Assessment For Field Sports. London and New York: Routledge - Taylor \& Francis Group.

Gaspar, P. \& Santos, C. (2004). Avaliação e controlo do treino em ténis: um estudo exploratório em treinadores de ténis. Revista Portuguesa de Ciências do Desporto. Vol.4. $n^{0} 2$ (suplemento), pp. 211-233. https://doi.org/10.5628/rpcd.05.02.184

Sanchez, F. (2015). Que esconde tu rival? : FutbolDLibro.

Sarmento, P. (2004). Pedagogia do desporto e observação. Cruz Quebrada, Lisboa: Edições FMH. 


\title{
Changing elderlies strength levels with a four months multicomponent training program
}

\author{
MADALENA PÁSSARO 14 , PEDRO FORTE ${ }^{2,3}$, ANTÓNIO M MONTEIRO1,3 \\ ${ }^{1}$ Instituto Politécnico de Bragança (IPB), Portugal \\ 2Instituto Superior de Ciências Educativas do Douro (ISCE Douro), Portugal \\ ${ }^{3}$ Research Center in Sports Sciences, Health Sciences and Human Development (CIDESD), Portugal
}

\begin{abstract}
The multicomponent training seems to positively influence elderly's physical fitness. It is unclear the effects of this type of training with less intervention time in strength levels. Thus, the aim of this study was to assess the effects of a multicomponent training program with four months in elderly's strength levels. The sample of this study was composed by 30 subjects with $69.30( \pm 5.45)$ years old and $71.80( \pm 8.97) \mathrm{Kg}$. Body weight was assessed with a bioimpedance balance. The knee flection and extension, arm curl and abduction were assessed with a strain gauge in kilograms of force (Kgf). There were no significant differences between the two moments. However, the mean body weight, knee flection and extension strength levels increased. A four months multicomponent training program seem to be insufficient to improve strength levels in elderlies. Keywords: Strength; Elderlies; Multicomponent training.
\end{abstract}

Corresponding author. Campus de Santa Apolónia - 5300-253, Bragança, Portugal.

E-mail: mada.passaro@gmail.com

Supplementary Issue: Spring Conferences of Sports Science. International Seminar of Physical Education, Leisure and Health, 17-19 June 2019. Castelo Branco, Portugal.

JOURNAL OF HUMAN SPORT \& EXERCISE ISSN 1988-5202

(C) Faculty of Education. University of Alicante.

doi:10.14198/jhse.2019.14.Proc4.82 


\section{INTRODUCTION}

Elderlies physical fitness seems to be determinant for daily life activities and quality of life. Thus, exercise programs are regularly prescribed to improve older people physical fitness (Marsh et al., 2009). The multicomponent training seems to positively influence elderly's physical fitness. However, the literature is scarce about the benefits of multicomponent training in strength levels (Nelson et al.,2004).

Different studies assessed the eight multicomponent training effects in body composition and physical fitness with a duration of eight months (Monteiro, Alves \&Forte, 2019). It is unclear the effects of this type of training with less intervention time. Thus, the aim of this study was to assess the effects of a multicomponent training program with four months in elderly's strength levels.

\section{MATERIAL AND METHODS}

\section{Participants}

The sample of this study was composed by 30 subjects with $69.30( \pm 5.45)$ years old and $71.80( \pm 8.97) \mathrm{Kg}$. Among them, fourteen were males and sixteen were females. All the procedures were in accord to the Helsinki's declaration regarding human research. A written consent by the parents or tutors was obtained beforehand.

\section{Measures}

Body weight was assessed with a bioimpedance balance (Tanita, BC-601, USA). The knee flection and extension, arm curl and abduction were assessed with a strain gauge in kilograms of force (Kgf).

\section{Procedures}

For body weight assessment the subjects wear light clothes and without shoes. Before the strength evaluations the subjects performed a 10 minutes walking warm up, combined with upper limbs rotations, elevations, flexions and extensions. The strength levels were assessed with a strain gauge fixed to the machines arms at $60^{\circ}$. The subjects performed three repetitions and the highest value was selected. Between repetitions, each subject rested 2 minutes. The multicomponent training was characterized by three sessions per week with exercises of resistance, strength, coordination and balance. Each session volume was sixty minutes (Monteiro et al., 2018).

\section{Analysis}

The Kolmogorov-Smirnov test allowed to assess the sample normality. T-Test assessed the statistical differences between groups. The significance level was $5 \%$.

\section{RESULTS}

Table 1 present the mean, standard deviation $( \pm S D)$, and the comparison between the baseline and after four months of multicomponent training in weight and strength levels.

There were no significant differences between the two moments. However, the mean body weight, knee flection and extension strength levels increased. 
Table 1. Mean, standard deviation $( \pm S D)$, and the comparison between the baseline and after four months of multicomponent training in weight and strength levels

\begin{tabular}{|c|c|c|c|c|}
\hline Variables & $\begin{array}{c}\text { Baseline } \\
\text { Mean }( \pm \text { SD) }\end{array}$ & $\begin{array}{c}\text { Post-Training } \\
\text { Mean }( \pm \text { SD) }\end{array}$ & $\mathrm{T}$ & $p$ \\
\hline Weight & $71.80( \pm 8.97)$ & $71.83( \pm 9.08)$ & -0.138 & 0.891 \\
\hline Knee flection & $13.82( \pm 6.40)$ & $14.07( \pm 6.05)$ & -0.518 & 0.608 \\
\hline Knee extension & $33.39( \pm 12.77)$ & $33.45( \pm 12.62)$ & -0.119 & 0.906 \\
\hline Arm curl & $13.82( \pm 4.36)$ & $13.68( \pm 4.46)$ & 0.494 & 0.625 \\
\hline Arm abduction & $6.78( \pm 2.58)$ & $6.38( \pm 2.66)$ & 1.641 & 0.112 \\
\hline
\end{tabular}

\section{DISCUSSION}

This study aimed to assess the effects of a multicomponent training program with four months in elderly's strength levels. The results shown that there were no significant differences between the baseline and after four months of intervention. That might be explained by the lack of specificity of multicomponent training in strength capacity (Carvalho et al., 2010). In fact, multicomponent training aim to train resistance, strength, coordination and balance in a single session (Carvalho et al., 2010; Monteiro et al., 2018). Even more, four months of multicomponent training might not be enough to improve strength levels by multicomponent training.

\section{CONCLUSIONS}

A four months multicomponent training program seem to be insufficient to improve strength levels in elderlies. It is recommended to assess the effect of different multicomponent training programs duration in strength level.

\section{REFERENCES}

Marsh, A. P., Miller, M. E., Rejeski, W. J., Hutton, S. L., \& Kritchevsky, S. B. (2009). Lower Extremity Muscle Function After Strength or Power Training in Older Adults. Journal of Aging and Physical Activity, 17(4), 416-443. https://doi.org/10.1123/japa.17.4.416

Nelson, M. E., Layne, J. E., Bernstein, M. J., Nuernberger, A., Castaneda, C., Kaliton, D., Hausdorff, J., Judge, J. O., Buchner, D. M., Roubenoff, R., \& Fiatarone Singh, M. A. (2004). The effects of multidimensional home-based exercise on functional performance in elderly people. J Gerontol A Biol Sci Med Sci, 59(2), 154-160. https://doi.org/10.1093/gerona/59.2.m154

Monteiro, A. M., Alves, E., \& Forte, P. (2019). An eight months multicomponent training effect in elderly's functional fitness. Motricidade, 15, 24-24.

Carvalho, J., Marques, E., Soares, J. M., \& Mota, J. (2010). Isokinetic strength benefits after 24 weeks of multicomponent exercise training and a combined exercise training in older adults. Aging Clin Exp Res. https://doi.org/10.1007/bf03324817

Monteiro, A. M., Rodrigues, F., Forte, P. \& Carvalho, J. (2018). The influence of an eight month multicomponent training program in edlerlies gait and bone mineral mass. In Proceedings of the 4th IPLeiria's International Health Congress (No. 18 (Suppl 2), pp. 52-52). BMC Health Services Research. https://doi.org/10.1186/s12913-018-3444-8 


\section{(c) $\underset{\mathrm{EY}}{\mathrm{NO}} \mathrm{\Theta}$}

This work is licensed under a Attribution-NonCommercial-NoDerivatives 4.0 International (CC BY-NC-ND 4.0). 


\title{
Bone mineral density and muscle strenght in elderly: A cross-sectional study
}

\author{
SÍLVIA ROCHA-RODRIGUES ${ }^{1,2} \triangle$, BRUNO SILVA ${ }^{1,3}$, MIGUEL CAMÕES ${ }^{1,3}$, LUÍS PAULO \\ RODRIGUES ${ }^{1,3}$, PEDRO BEZERRA ${ }^{1,3}$ \\ ${ }^{1}$ Instituto Politécnico de Viana do Castelo, Escola Superior Desporto e Lazer de Melgaço, Viana do Castelo, \\ Portugal \\ 2Laboratory of Metabolism and Exercise, Research Centre for Physical Activity, Health and Leisure (CIAFEL), \\ Faculty of Sport, University of Porto, Portugal \\ ${ }^{3}$ Research Center in Sports Sciences, Health Sciences and Human Development (CIDESD), Portugal
}

\begin{abstract}
Background: Although studies have addressed the age-related changes in bone mineral density (BMD), data associated BMD and muscle strength with age in both genders is scarce. Thus, we aimed to analyse the BMD of proximal femur of elderly women and men and their association with muscle strength. Methods: From an initial sample composed of 269 elderly recruited in the district of Viana do Castelo, 58 participants (women, $79.3 \pm 6.2 \mathrm{y}$ and men, $79.53 \pm 5.89 \mathrm{y}$ ) were randomly selected for determination of BMD by dual-energy X-ray absorptiometry (DXA). The values of bone mineral content (BMC), bone area, and BMD (BMC/bone area, $\mathrm{g} / \mathrm{cm} 2)$ were determined. The isometric knee extension strength was also determined. Results: Women had lower total BMD (1.04 vs.1.16, 95\%Cl: [1.11,1.21]; $\mathrm{P}<0.01 ; \mathrm{ES}=0.99)$ than men, but no changes in proximal femur BMC were found. There was a significant age-sex interaction for BMD and knee extension measure,

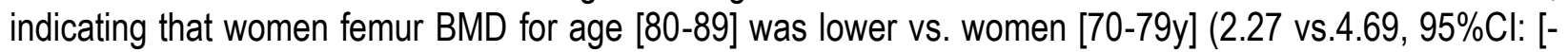
$0.19,-0.001]$; $P=0.03)$. Total BMD (1.11 vs. $0.99,95 \% \mathrm{Cl}:[0.08,0.26]$ in men was higher vs. women [80-89y]. Knee extension was lower in women [80-89y] (18.54, vs.26.0, 95\%Cl:[-10.07,-4.81]; $\mathrm{P}<0.001)$ vs. women [70-79y] and higher in men (30.69 vs.18.56; 95\%Cl: [8.52-15.74]) compared with women with same age. A moderate correlation were found between total $\mathrm{BMD}$ was positively associated with knee extension strength $(r=0.268, P=0.042)$. Conclusions: In spite of the small sample, data demonstrate the association between $\mathrm{BMD}$ and knee extension strength, suggesting that muscle strength may have a positive effect on attenuating age-associated BMD loss. Keywords: Aging; Femur; Knee extension.
\end{abstract}

Corresponding author. Escola Superior Desporto e Lazer de Melgaço; Complexo Desportivo e de Lazer Comendador Rui Solheiro, 4960-320 Melgaço, Viana do Castelo, Portugal.

E-mail: silviadarocharodrigues@gmail.com

Supplementary Issue: Spring Conferences of Sports Science. International Seminar of Physical Education, Leisure and Health, 17-19 June 2019. Castelo Branco, Portugal.

JOURNAL OF HUMAN SPORT \& EXERCISE ISSN 1988-5202

(C) Faculty of Education. University of Alicante.

doi:10.14198/jhse.2019.14.Proc4.82

S1608 | 2019| Proc4| VOLUME 14

(C) 2019 University of Alicante 


\section{INTRODUCTION}

Bone tissue is affected by the aging process with significant and progressive alterations in composition and architecture that progressively can lead to osteoporosis (Demontiero et al., 2012). Most investigations of agerelated changes of BMD reported that these alterations are more prevalent in women during the postmenopausal transition and in old age (Hernlund et al., 2013). On the other hand, muscle strength has been suggested to be a crucial predictor of BMD, indirectly indicating osteoporotic fracture risk (Rikkonen et al., 2012). Previous reports supported an association between hip abductor strength and isometric knee extension and proximal femur BMD (Pasco et al., 2015; Stasi et al., 2018). Although a large number of studies have addressed the age-related changes in BMD (Demontiero et al., 2012; Hernlund et al., 2013), little is known regarding association between BMD and muscle strength with age in both genders. Therefore, we aimed to analyse the bone mineral density of proximal femur of elderly women and male as well as their association with muscle strength.

\section{MATERIAL AND METHODS}

\section{Participants}

From an initial sample composed of 269 non-institutionalized elderly recruited in the district of Viana do Castelo, 58 participants (women, $79.3 \pm 6.2$ years and men, $79.53 \pm 5.89$ years) were randomly selected for determination of BMD.

\section{Measures}

Based on the General Physical Activity Questionnaire, participants were divided into i) physically active (PA; $n=196$ ) for at least 150 min of moderate physical activity per week or ii) non-physically active (N-PA; n=73) groups. Muscle strength was by isometric knee extension strength was assessed three times for each limb and the maximal performance was used for analysis. Bone mineral density was determined by dual-energy $X$-ray absorptiometry (DXA). The equipment was regularly calibrated, and the values of bone mineral content (BMC), bone area, and BMD (BMC/bone area, $\mathrm{g} / \mathrm{cm} 2$ ) were obtained.

\section{Analysis}

Associations between BMD measures and physical fitness tests were examined using Pearson's correlation coefficient for parametric and nonparametric samples, respectively. We applied the Mann-Whitney $U$ test (nonparametric distribution) to compare continuous variables between groups. Additionally, we calculated effect sizes (ES) as Cohen's d using the pooled standard deviation of the random effects. To determine the effects of age and sex on each outcome, we performed two-way analysis of variance. If the two-way ANOVA indicated a significant age effect and either a sex effect or age-sex interaction, then one-way ANOVAs were used: a) to assess the effect of age on each sex separately, followed by Tukey post-hoc testing if the age effect was significant; and $b$ ) to assess the effect of sex at each age. Significance level was defined as $p<$ 0.05 .

\section{RESULTS}

Generally, women had lower total BMD (1.04, 95\%Cl: [0.98,1.09] vs. 1.16, 95\%Cl:[1.11,1.21]; $\mathrm{P}<0.01$; ES= $0.99)$, proximal femur $(4.57,95 \% \mathrm{Cl}$ : [0.5,1.19] vs. $5.24,95 \% \mathrm{Cl}$ : [0.71,0.84]; $\mathrm{P}<0.001 ; \mathrm{ES}=1.0)$ than men, but no changes were observed in the proximal femur $\mathrm{BMC}(3.69,95 \% \mathrm{Cl}$ : [2.5,4.88] vs. $4.09,95 \% \mathrm{Cl}$ : [3.66,4.52]; $P>0.05, E S=0.17$ ). There was a significant age-sex interaction (by two-way ANOVA) for BMD and isometric knee extension measures, indicating that women and men aged somewhat differently. Women proximal 


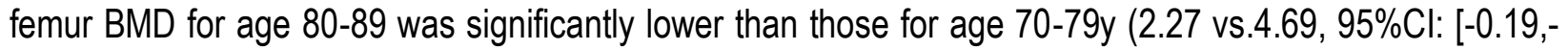
$0.001]$; $P=0.03)$. Total BMD (1.11 vs. $0.99,95 \% \mathrm{Cl}$ : $[0.08,0.26]$ in men was higher than women for age $80-$ $89 y$. Isometric knee extension was lower in women aged 80-89y (18.54, vs.26.0, 95\% Cl:[-10.07,-4.81]; $\mathrm{P}<0.001)$ vs. women $(70-79 \mathrm{y})$ and higher in men $(30.69$ vs.18.56; $95 \% \mathrm{Cl}$ : [8.52-15.74]) compared with women with same age. A moderate correlation were found between total BMD was positively associated with knee extension strength $(r=0.468, P=0.032)$.

When classified the participants according to their physical activity or inactivity, we found no alterations on the total BMD $(1.1,95 \% \mathrm{Cl}:[1.05,1.13]$ vs.(1.08, 95\% Cl:[0.91,1.18]; $\mathrm{P}>0.05$, ES=0.19), proximal femur BMD (0.89, 95\%Cl:[0.66,0.91] vs. 0.96, 95\% Cl:[0.57,1.08]; $\mathrm{P}>0.05$, $E S=-0.58)$ and $\mathrm{BMC}(4.59,95 \% \mathrm{Cl}:[2.98,4.76]$ vs.4.25, 95\%Cl:[2.96,4.71]; $\mathrm{P}>0.05, \mathrm{ES}=0.06)$ between groups.

\section{DISCUSSION}

The present study demonstrates that women loss more BMD with age compared with men. Moreover, BMD was associated with knee extension strength, suggesting that muscle strength of lower limbs may have a positive effect on attenuating age-associated BMD loss. However, with advancing age, older adults often use more muscle groups of the upper limbs in daily activities instead of muscle groups of the lower extremities, which is associated preferentially with low levels of physical activity (Takagi et al., 2015). This condition may lead to an age-associated changes in BMD in both women and men at skeletal sites, in women the decrease occurs predominantly after the menopause (Trombetti et al., 2016). In this line, we found that women showed lower values of BMD than men that was associated with poor knee extension strength. Indeed, several studies have postulated that muscle strength in lower limbs plays, in part, an important role to attenuate BMD loss (Pasco et al., 2015; Stasi et al., 2018). Accordingly, Rikkonen et al. (2012) reported that muscle mass and strength were independently associated with BMD in postmenopausal women and thus, important for maintaining BMD in the normal (healthy) range. In the clinical practice context, these findings support the value and utility of isometric knee extension strength as a measure of muscle strength in elderly.

\section{CONCLUSIONS}

In spite of the small sample, data demonstrate the association between BDM and knee extension strength, suggesting that muscle strength may have a positive effect on attenuating age-associated BDM loss.

\section{REFERENCES}

Demontiero, O., Vidal, C., and Duque, G. (2012). Aging and bone loss: new insights for the clinician. Ther Adv Musculoskelet Dis, 4(2), 61-76. https://doi.org/10.1177/1759720x11430858

Hernlund, E., Svedbom, A., Ivergard, M., Compston, J., Cooper, C., Stenmark, J., McCloskey, E. V., Jonsson, B., and Kanis, J. A. (2013). Osteoporosis in the European Union: medical management, epidemiology and economic burden. A report prepared in collaboration with the International Osteoporosis Foundation (IOF) and the European Federation of Pharmaceutical Industry Associations (EFPIA). Arch Osteoporos, 8, 136. https://doi.org/10.1007/s11657-013-0136-1

Pasco, J. A., Holloway, K. L., Brennan-Olsen, S. L., Moloney, D. J., and Kotowicz, M. A. (2015). Muscle strength and areal bone mineral density at the hip in women: a cross-sectional study. BMC musculoskeletal disorders, 16, 124-124. https://doi.org/10.1186/s12891-015-0586-2 
Rikkonen, T., Sirola, J., Salovaara, K., Tuppurainen, M., Jurvelin, J. S., Honkanen, R., and Kroger, H. (2012). Muscle strength and body composition are clinical indicators of osteoporosis. Calcif Tissue Int, 91(2), 131-138. https://doi.org/10.1007/s00223-012-9618-1

Stasi, S., Papathanasiou, G., Chronopoulos, E., Galanos, A., Papaioannou, N. A., and Triantafyllopoulos, I. K. (2018). Association between abductor muscle strength and functional outcomes in hip-fractured patients: a cross-sectional study. J Musculoskelet Neuronal Interact, 18(4), 530-542.

Takagi, D., Nishida, Y., and Fujita, D. (2015). Age-associated changes in the level of physical activity in elderly adults. J Phys Ther Sci, 27(12), 3685-3687. https://doi.org/10.1589/jpts.27.3685

Trombetti, A., Reid, K. F., Hars, M., Herrmann, F. R., Pasha, E., Phillips, E. M., and Fielding, R. A. (2016). Age-associated declines in muscle mass, strength, power, and physical performance: impact on fear of falling and quality of life. Osteoporos Int, 27(2), 463-471. https://doi.org/10.1007/s00198-0153236-5 


\title{
Effects of a multicomponent exercise program with duration of 12 weeks on the quality of life in breast cancer survivors
}

\author{
PEDRO PINTO1 14 , PEDRO FORTE ${ }^{2,3}$, ANTÓNIO M MONTEIRO1,3 \\ ${ }^{1}$ Instituto Politécnico de Bragança (IPB), Portugal \\ 2Instituto Superior de Ciências Educativas do Douro (ISCE Douro), Portugal \\ ${ }^{3}$ Research Center in Sports Sciences, Health Sciences and Human Development (CIDESD), Portugal
}

\begin{abstract}
The aim of this study was to verify the changes induced by the multicomponent exercise program with duration of 12 weeks on quality of life in breast cancer survivors. The sample of this study consisted of 7 female subjects with an average of $64( \pm 8.6)$ years who volunteered for this investigation and presented breast cancer pathology diagnosed in the clinical history. The exercise program had a duration of 12 consecutive weeks and a weekly frequency of three days. The duration of the exercise was 60 continuous minutes and the exercise used were from the multicomponent method, combining aerobic, resistance, flexibility and balance training. The variables of healthy-related quality of life questionnaire EORTC QLQ-C30 and QLQ-BR23 were evaluated on the baseline and after 12 weeks. The data followed a descriptive and statistical analysis related to the results obtained in the various subscales of the EORTC QLQ-C30 and QLQBR23 on the baseline and 12 weeks. From baseline to 12 weeks, the most increased values were $24( \pm 16.5)$ points in the role function; $11( \pm 5.3)$ in the emotional function. In the symptomatic scales, the most improved symptoms were fatigue with a reduction of $20( \pm 3.5)$ points; $14( \pm 3.8)$ in the pain scale, $16( \pm 4.8)$ on breast symptoms, and $19( \pm 10.9)$ points on arm symptoms. The results suggest that the multicomponent exercise program in this study didn't have significant differences on the health-related quality of life subscales in breast cancer survivors. Keywords: Breast cancer; Quality of life; Exercise.
\end{abstract}

Corresponding author. Rua Dr. António Granjo n¹ Bragança, Portugal.

E-mail: pedrompinto22@gmail.com

Supplementary Issue: Spring Conferences of Sports Science. International Seminar of Physical Education, Leisure and Health, 17-19 June 2019. Castelo Branco, Portugal.

JOURNAL OF HUMAN SPORT \& EXERCISE ISSN 1988-5202

(c) Faculty of Education. University of Alicante.

doi:10.14198/jhse.2019.14.Proc4.82 


\section{INTRODUCTION}

The classification of "cancer survivor" indicates any person who had or has cancer from the time of diagnosis (Marzorati et al., 2017). The treatment is essentially centred on the objective of curing the disease and in the prevention of its metastic reappearance (Devin et al., 2019). This evidence emphasizes the importance of research on the development of strategies to improve quality of life, reduce the risk of recurrence, probability of contracting other diseases and prolong the survival of this population (Lee et al., 2016).

\section{MATERIAL AND METHODS}

Initial assessments were performed in January 2019 , and the post-intervention evaluation was performed in April 2019. Inclusion criteria were: 1) be diagnosed as breast cancer patients; 2) designated for definitive surgery (any type); 3) designated for chemotherapy, radiotherapy, hormonal therapy, or a combination of these treatments after surgery.

\section{Participants}

The sample of this study consisted of 7 female subjects. The volunteers had an average of $64( \pm 8.6)$ years.

\section{Measures}

The assessment of quality of life related to Health was performed using the third version of the questionnaire of the European Organization Research Treatment of Cancer, Quality of life of the patient with cancer (EORTC QLQ-C30) and breast cancer (QLQ-BR23) (Aaronson et al., 1993).

\section{Procedures}

The subjects that volunteered for this investigation presented breast cancer pathology diagnosed in the clinical history. The questionnaires were submitted on the baseline and after 12 weeks by the investigators in the facilities of Polytechnic Institute of Bragança, Portugal. The sessions of 60 minutes were constituted by: 1) 5-8 minutes of general warming, 2) aerobic exercises (15-20 min); 3) 1 to 3 series of muscular endurance exercises involving the main muscular groups, such as knee flexors/extensors, abductors and adductors of the shoulder, elbow flexors/extensors; 4) Static and dynamic balance training for 5-8 min; 5) 5 min of return to the calm. The intensity was maintained in 12-14 on the scale of subjective perception of Borg exertion (1998).

\section{Analysis}

The data followed a descriptive and statistical analysis using the Statistical Package for Social Sciences (SPSS) software, version 23.

\section{RESULTS}

From baseline to 12 weeks, the most increased values were $24( \pm 16.5)$ points in the role function; $11( \pm 5.3)$ in the emotional function. In the symptomatic scales, the most improved symptoms were fatigue with a reduction of $20( \pm 3.5)$ points; $14( \pm 3.8)$ in the pain scale, $16( \pm 4.8)$ on breast symptoms, and $19( \pm 10.9)$ points on arm symptoms. However, we didn't found significant differences on the variables of this study. 
Table 1. Baseline and 12 weeks health-related quality of life subscales (EORTC QLQ-C30/BR23) of breast cancer survivors

\begin{tabular}{lcccc}
\hline Variables QLQ-C30 & Baseline $(\mathbf{n}=\mathbf{7}) \mathbf{M}(\mathbf{\pm S D})$ & 12 weeks $(\mathbf{n}=\mathbf{7}) \mathbf{M}(\mathbf{\pm S D})$ & $\mathbf{U}$ & $\mathbf{P}$ \\
\hline Global Health Status (QOL) & $60.71( \pm 17.8)$ & $57.1( \pm 11.1)$ & 20.500 & 0.620 \\
Physical Function (PF) & $83.8( \pm 9.3)$ & $84.7( \pm 11.9)$ & 29.500 & 0.535 \\
Role Function (RF) & $59.5( \pm 33.1)$ & $83.3( \pm 16.6)$ & 35.500 & 0.165 \\
Emotional Function (EF) & $67.8( \pm 23.2)$ & $78.5( \pm 17.9)$ & 31.000 & 0.456 \\
Cognitive Function (CF) & $76.1( \pm 18.8)$ & $80.9( \pm 11.5)$ & 29.000 & 0.620 \\
Social Function (SF) & $83.2( \pm 21.6)$ & $71.3( \pm 34.3)$ & 20.000 & 0.620 \\
Fatigue (FA) & $46( \pm 24.3)$ & $26.9( \pm 27.8)$ & 14.500 & 0.209 \\
Pain (PA) & $46.2( \pm 24.4)$ & $32.1( \pm 28.2)$ & 17.000 & 0.383 \\
\hline Variables QLQ-BR23 & & & & \\
\hline Breast Symptoms (BRBS) & $23.8( \pm 17.9)$ & $7.14( \pm 13.1)$ & 9.500 & 0.053 \\
Arm Symptoms (BRAS) & $32.9( \pm 27.6)$ & $13.88( \pm 16.7)$ & 13.500 & 0.165 \\
\hline
\end{tabular}

\section{DISCUSSION}

The variables related to quality of life improved after the intervention of the exercise. These results are consistent with Naumann et al. (2012), who reported an increase of more than 10 points in the variables of quality of life, in breast cancer survivors, after 9 weeks of exercise. In the present study, the exercise induced a marked decrease in the symptoms of fatigue and pain, which is in line with the results of the literature, stating that exercise can help reduce fatigue during and after the treatments (Naumann et al., 2012).

\section{CONCLUSIONS}

The results of the present study suggest that supervised multicomponent exercise program for 12 weeks didn't have significant differences on the health-related quality of life subscales in breast cancer survivors.

\section{REFERENCES}

Aaronson, N. K., Ahmedzai, S., Bergman, B., Bullinger, M., Cull, A., Duez, N. J., Filiberti, A. (1993). The European Organization for Research and Treatment of Cancer QLQ-C30: a quality-of-life instrument for use in international clinical trials in oncology. J Natl Cancer Inst, 85(5), 365-376. https://doi.org/10.1093/inci/85.5.365

Devin, J. L., Hill, M. M., Mourtzakis, M., Quadrilatero, J., Jenkins, D. G., \& Skinner, T. L. (2019). Acute high intensity interval exercise reduces colon cancer cell growth. The Journal of physiology. https://doi.org/10.1113/jp277648

Lee, C. E., Von Ah, D., Szuck, B., \& Lau, Y. K. (2016). Determinants of physical activity maintenance in breast cancer survivors after a community-based intervention. In Oncology nursing forum (Vol. 43, No. 1, pp. 93-102). https://doi.org/10.1188/16.onf.43-01ap

Marzorati, C., Riva, S., \& Pravettoni, G. (2017). Who is a cancer survivor? A systematic review of published definitions. Journal of Cancer Education, 32(2), 228-237. https://doi.org/10.1007/s13187016-0997-2

Naumann, F., Munro, A., Martin, E., Magrani, P., Buchan, J., Smith, C., Piggott, B. (2012). An individualbased versus group-based exercise and counselling intervention for improving quality of life in breast cancer survivors. A feasibility and efficacy study. Psychooncology, 21(10), 1136-1139. https://doi.org/10.1002/pon.2015 


\section{(c) $\underset{\mathrm{EY}}{\mathrm{NO}} \mathrm{\Theta}$}

This work is licensed under a Attribution-NonCommercial-NoDerivatives 4.0 International (CC BY-NC-ND 4.0). 


\title{
How well do muscular strength and endurance predict cognitive function in youth?
}

\author{
ANA LUÍSA CORREIA ${ }^{1}$, ÉLVIO R. GOUVEIA ${ }^{1,2}$, ADILSON MARQUES $^{4}$, BRUNA GOUVEIA ${ }^{2,3}$, ANA J. \\ RODRIGUES ${ }^{1}$, RICARDO ALVES ${ }^{1}$, HELDER LOPES ${ }^{1}$, MATTHIAS KLIEGEI ${ }^{5}$, ANDREAS ILHE ${ }^{5}$ \\ ${ }^{1}$ Department of Physical Education and Sport, University of Madeira, Funchal, Portugal \\ 2LARSYS/ITI, Portugal \\ ${ }^{3}$ IASáude IP., RAM / Saint Joseph of Cluny Higher School of Nursing, Funchal, Portugal \\ ${ }^{4}$ FMH / Centro de Investigação em Saúde Pública, Escola Nacional de Saúde Pública, Universidade Nova \\ de Lisboa, Lisboa, Portugal \\ ${ }^{5}$ Center for the Interdisciplinary Study of Gerontology and Vulnerability, Department of Psychology, University \\ of Geneva, Geneva, Switzerland
}

\begin{abstract}
Background: Muscular strength and endurance (MSE) have been correlated to healthy physical and mental condition throughout the lifespan. Objective: The main objective of this study was to investigate how well do MSE predict cognitive function in youth, after controlling for age. Methods: The sample comprised 302 students (157 girls and 145 boys), mean age 15.7 years (SD = 2.7, range 9.9-20.6), from the 5th to the 12th year, in 5 public schools. Cognitive Telephone Screening Instrument (COGTEL) as used to access cognitive function. MSE of students were assessed using the hand grip test (static strength) and sit-ups (trunk strength) from EUROFIT battery. Bivariate correlations and hierarchical multiple regression analyses were performed on the data analysis. Results: COGTEL was positively related to static strength $(r=.282, p<.001)$ and trunk strength tests scores ( $r=.268, p<.001)$. Age explained $5.9 \%$ of the variance in COGTEL (step 1). After entry MSE at step 2, the total variance explained by the model as a whole was $12 \%$. In the final model, both age and MSE composite score were statistically significant, with the MSE recording a higher beta value (beta=.264, $p<.001$ ) than age (beta=.136, $p=.034$ ). Conclusion: this study reinforces that better MSE significantly correlates to higher performance in cognitive function. Moreover, MSE predict cognitive function in youth in the same proportion as age does. Keywords: Health-related physical fitness; Cognitive performance; Youth; Physical education.
\end{abstract}

Corresponding author. Department of Physical Education and Sport, University of Madeira, Funchal, Portugal.

E-mail: anacorreia@staff.uma.pt

Supplementary Issue: Spring Conferences of Sports Science. International Seminar of Physical Education, Leisure and Health, 17-19 June 2019. Castelo Branco, Portugal.

JOURNAL OF HUMAN SPORT \& EXERCISE ISSN 1988-5202

(c) Faculty of Education. University of Alicante.

doi:10.14198/jhse.2019.14.Proc4.82 


\section{INTRODUCTION}

Muscular strength and endurance (MSE) are considered important health-related physical fitness components, since there is a positive well-known relationship with a healthy physical and mental condition throughout the lifespan (Mintjens al., 2018). There is some evidence that MSE have been related to improvements in bone mass, glucose tolerance, musculotendinous integrity, the ability to performed the everyday activities (which is related to perceived quality of life and self-efficacy among other indicators of mental health), fat-free mass and resting metabolic rate (ACSM, 2018). Despite the fact that there is a remarkable amount of research reporting a positive relationship between muscular strength and physical health, much less consistent are the relationships between cognitive function MSE (Chu et al., 2016). The present study relaunches this question, aiming to quantifying how well MSE predict cognitive function in youth. To contribute to a deeper understanding of these relationships, the main objective of this study was to investigate how well MSE predict cognitive function in youth, after controlling for age.

\section{MATERIAL AND METHODS}

\section{Participants}

The sample comprised 302 students (157 girls and 145 boys), mean age 15.7 years (SD $=2.7$, range 9.920.6), from the 5 th to the 12 th year in 5 public schools.

\section{Measures}

Cognitive Telephone Screening Instrument (COGTEL): was used to assess cognitive functioning. The COGTEL consists of 6 subtests covering prospective memory, verbal short- and long-term memory, working memory, verbal fluency, and inductive reasoning (Kliegel et al. 2007). The assessments were conducted in private rooms of each school, by trained research personnel.

MSE: were assessed using the hand grip test (static strength) and sit-ups (trunk strength) from EUROFIT battery (Research CES., 1993). The two physical fitness tests were administered during physical education classes. For analyses, we calculated a composite score.

\section{Procedures}

Participants were part of the research project entitled "Physical Education in Schools from Autonomous Region of Madeira" (EFERAM-CIT). The assessments occurred between October and November of 2017. Participants were informed about the objectives of the study and written informed consent was obtained from their legal guardians. The study received Ethical approval from the Scientific Committee of the Faculty of Physical Education and Sports at the University of Madeira (Reference: ACTA N.77 - 12.04.2016).

\section{Analysis}

First, we inspected bivariate correlations of COGTEL with age, static strength and trunk strength. Second, hierarchical multiple regression analyses were conducted to investigate the amount of variance in COGTEL that was explained by MSE, simultaneously controlling for the influence of age. All analyses were performed using SPSS (version 23.0).

\section{RESULTS}

An inspection of bivariate correlations showed that COGTEL was positively related to static strength ( $r=.282$, $p<.001)$ and trunk strength tests scores $(r=.268, p<.001)$. In the hierarchical multiple regression analyses, 
age was entered at step 1 explaining $5.9 \%$ of the variance in COGTEL. After entry of MSE at step 2, the total variance explained by the model as a whole was $12 \%, F(2,259)=64.13, p<.001$. MSE explained an additional $5,8 \%$ of the variance in COGTEL, after controlling for age, $\mathrm{R}$ squared change $=.058(1,259)=$ $17.12, p<.001$. In the final model, both age and MSE were statistically significant, with the MSE recording a higher beta value (beta=.264, $p<.001$ ) than age (beta=.136, $p=.034$ ).

\section{DISCUSSION}

This study adds some evidence about the positive association between MSE and cognitive function, even after controlling for age effect. Although there are some studies that corroborate the positive association between muscular strength and cognitive function (Chu et al., 2016), when those relationships are controlled for potential covariates such as age, the associations were weakened (Kao et al., 2017). One novel aspect of the present study is that we demonstrated, that MSE explained approximately $6 \%$ of the variance in cognitive function. Interestingly, age also explained $6 \%$ of the variance in cognitive function. Moreover, evaluating each of the independent variable, in order of importance, MSE made the strongest (26\%) unique contribution, when the overlapping effect of age was statistically removed. Since MSE are important modifiable health-related fitness components, school policies aiming to increase physical activity focus on promoting MSE, independently of age, may increase cognitive function. These results offer important practical contributions that should be taken into consideration by schools' administrators and teachers. For example, the increasing of time in schools allocated to sports activities, in particular activities that promote health related fitness components, such as MSE, may be associated to increased cognitive function and as consequence, academic achievement.

\section{CONCLUSIONS}

This study reinforces that better MSE significantly correlates to higher performance in cognitive function. Moreover, MSE predict cognitive function in youth in the same proportion as age does.

\section{REFERENCES}

American College of Sports Medicine. ACSM's Guidelines for Exercise Testing and Prescription, 10th Edition, Philadelphia: Lippincott Williams \& Wilkins.2018.

Chu CH, Chen FT, Pontifex MB et al. (2016). Health-related physical fitness, academic achievement, and neuroelectric measures in children and adolescents, Int $\mathrm{J}$ Sport Exerc Psychol. https://doi.org/10.1080/1612197x.2016.1223420

Council of Europe Committee for the Development of Sport. EUROFIT: handbook for the EUROFIT tests of physical fitness. Strasbourg: Council of Europe, 1993.

Kao SC, Westfall DR, Parks AC et al. Muscular and aerobic fitness, working memory, and academic achievement in children. Med Sci Sports Exerc 2017; 49(3):500-508. https://doi.org/10.1249/mss.0000000000001132

Kliegel M, Martin M, Jäger T. Development and validation of the Cognitive Telephone Screening Instrument (COGTEL) for the assessment of cognitive function across adulthood. J Psychol. 2007; 32:147-170. https://doi.org/10.3200/jrlp.141.2.147-172

Mintjens S, Menting MD, Daams JG et al. Cardiorespiratory Fitness in Childhood and Adolescence Affects Future Cardiovascular Risk Factors: A Systematic Review of Longitudinal Studies. Sports Med 2018; 48(11):2577-2605. https://doi.org/10.1007/s40279-018-0974-5 


\section{(c) $\underset{\mathrm{EY}}{\mathrm{NO}} \mathrm{\Theta}$}

This work is licensed under a Attribution-NonCommercial-NoDerivatives 4.0 International (CC BY-NC-ND 4.0). 


\title{
Orienteering sport and environmental education: A theoretical review
}

\author{
ALLANA JOYCE SOARES GOMES SCOPEL $1,2,3$ \\ ${ }^{1}$ Federal Institute of Education, Science and Technology of Ceará, Brazil \\ 2State University of Maringá, Brazil \\ ${ }^{3}$ Centre for Social Studies, University of Coimbra, Portugal
}

\begin{abstract}
This theoretical review aims to discuss the Environmental Education (EE) conceptual essence on the available orienteering literature. The bibliographical search identified 92 articles in English, Portuguese and Spanish, of which five were fully analysed. The analysis revealed lack of theoretical consistency about EE. Keywords: Physical education; Education; Environment; Review.
\end{abstract}

Corresponding author. Instituto Federal de Educação, Ciência e Tecnologia do Ceará - IFCE - Campus de Fortaleza: Av. Treze de Maio, 2081, Benfica. Fortaleza - CE - Brasil.

E-mail: allana@ifce.edu.br

Supplementary Issue: Spring Conferences of Sports Science. International Seminar of Physical Education, Leisure and Health, 17-19 June 2019. Castelo Branco, Portugal.

JOURNAL OF HUMAN SPORT \& EXERCISE ISSN 1988-5202

(C) Faculty of Education. University of Alicante.

doi:10.14198/jhse.2019.14.Proc4.82 


\section{INTRODUCTION}

The Environmental Education (EE) has been argued in the several educational contexts. The evidence that human-environment relationships were responsible for the social and environmental crisis led to calls for $E E$ (Edwards, 2013). However, this research field is also marked by theoretical-practical and political divergences.

In the physical education studies this issue is present in the debate about corporal practices in integration with nature and its relationship with environmental education and leisure. The orienteering as a sport practice, for example, has been increasingly investigated in the last ten years. Despite several authors are studying the relationship between orienteering practice and school education, there is a gap of knowledge about the association between orienteering practice and EE. Thus, this theoretical review aims to discuss the Environmental Education conceptual essence on the available orienteering literature.

\section{MATERIAL AND METHODS}

This is a descriptive and analytical bibliographic research.

\section{Participants (sample)}

Initially the bibliographical search has founded 92 articles, after reading their title and abstract 89 studies were eliminated based on the exclusion criteria. After full reading, five works were selected.

\section{Measures}

The systematic search was made by the Google Scholar. This web search engine was chosen because we aimed to analyse articles not only from the Physical Education data bases but reunite several disciplines which are studying the EE and Orienteering practice relationship.

\section{Procedures}

The search was made by keyword combinations: "orienteering sport" was used associated with the terms: "environmental education", "ecopedagogy", "sustainable education", "education for sustainability" "Education for sustainable development" all in English, Portuguese and Spanish.

The inclusion criteria for studies were: full text articles published since 2009 in academic journal; written in Portuguese, English or Spanish; Discussion on the relationship between environmental education and orienteering practice. Graduation Final Work; dissertations, thesis, book chapters, conference papers and article with no discussion on environmental education were excluded.

\section{Analysis}

The selected papers were altogether read, the discussion on EE-Orienteering relationship were extracted and analysed based on the theoretical framework.

\section{RESULTS}

The five articles analysed were published in 2011, 2013, 2014, 2017, 2018, respectively in five different journals: Non-Formal Education (Uruguay), Physical Education (Brazil), Local Sustainable Development (Spain), Baltic Journal of Sport \& Health Sciences (Lithuania), Environmental Education Sergipe Journal (Brazil). 
For Queiroz and Martins (2018) the EE is critical, dialectical, threefold (built by teaching, research and extension) and relates to social and environmental issues. Nascimento, Souza e Andrade (2014) understand the EE as interdisciplinary, holistic, non-reductionist, related to respect, responsibility, affectivity and intimacy. According to Alpuy and Méndez (2011) the EE for sustainable development improves human-environment relationships. Other authors believe that EE promotes respect, protection and sensibilization about nature. Yıldız et al. (2017) believe that nature education or outdoor education can be educational about the environment and orienteering practice in outdoor camp can promote environment sensitivity.

In addition, the orienteering practice it was presented as a teaching methodology for the EE (Queiroz \& Martins, 2018) that can provide environmental problems perception and motivates to propose alternatives (Nascimento, Souza, \& Andrade, 2014). Carmona et al. (2013) understand the orienteering sport as an escape from the urban environment and an interdisciplinary practice that influences respect, sensibilization, protection and conscience about rights and duties. For Alpuy and Méndez (2011) the orienteering provides natural environment contact, encourages the education of values and environmental respect.

\section{DISCUSSION}

It is remarkable that for some authors EE has a critical theoretical framework, but it was mostly related to escape and a preservationist ideal. The problem of this approach is a fragmented view about the EE, based on a discourse that appeals to the conservation and preservation of a distant nature and disregards the urban environment and the body itself as an important part of this nature and contributes to the distaining of humanenvironment relations. (Rodrigues \& Gonçalves Junior, 2009).

\section{CONCLUSIONS}

This review has been shown that the studies about the relationship between orienteering practice and EE are scarce. Furthermore, the articles analysed revealed lack of theoretical consistency about EE. Considering the limitations of this study, additional investigations needs to be conducted for the continuity of the debate.

\section{REFERENCES}

Alpuy, C. M., \& Méndez, F. V. (2011). El valor educativo del deporte "orientación." Enfoques, Revista de Educación No Formal, 2(1688 - 7743), 139-146. Retrieved from https://www.mec.gub.uy/innovaportal/file/16122/1/enfoques_2_web.pdf

Carmona, E. K., Begossi, T. D., Soares, S. S., \& Mazo, J. Z. (2013). O Esporte De Orientação : Possibilidades E Perspectivas. Educação Física Em Revista - EFR, 7(3), 19-27. Retrieved from https://portalrevistas.ucb.br/index.php/efr/article/view/4366

Edwards, J. (2013). Towards effective socially critical environmental education: stories from primary classrooms. Environmental Education Research, 19(2), 258-259. https://doi.org/10.1080/13504622.2012.736477

Nascimento, D. G., Souza, R. L. F., \& Andrade, F. A. V. (2014). A prática do desporto orientação: uma proposta metodológica para a educação ambiental no ensino médio. Revista Desarrollo Local Sostenible DELOS, 7(18), 1-23. Retrieved from http://www.eumed.net/rev/delos/19/desportoorientazao.pdf

Queiroz, E. D. de, \& Martins, G. dos S. (2018). Por uma educação ambiental tríplice: a dialética do ensinar, pesquisar e extensionar no Parque Natural Municipal de Nova Iguaçu, RJ. Revista 
Sergipana de Educação Ambiental (REVISEA), 5(6), 19-29. https://doi.org/10.14295/ambeduc.v22i1.6223

Rodrigues, C., \& Gonçalves Junior, L. (2009). Ecomotricidade: sinergia entre educação ambiental, motricidade humana e pedagogia dialógica^ipt. Motriz Rev. Educ. Fís. (Impr.), 15(4), 987-995. https://doi.org/10.5016/3273

Yıldız, K., Güzel, P., Çetinöz, F., \& Beşikçi, T. (2017). Outdoor camp effects on athletes: orienteering example. Baltic Journal of Sport \& Health Sciences, 1(104), 55-64. https://doi.org/10.33607/bjshs.v1i104.18 


\title{
Evaluation of the quality of life of schoolchildren of the EJA in the Municipality of Itacoatiara, Amazonas
}

\author{
EZEQUIAS SERRÃO PIMENTA $\triangleleft$, ROSANA ALBUQUERQUE, MARCELA DANTAS XIMENES \\ Universidade do Estado do Amazonas, Brazil
}

\begin{abstract}
Interest in the topic Quality of Life (QOL) has increased significantly in the last decades, it is a concept that involves parameters in several areas. Among the studies on QoL in the educational area, few have discussed how the health and QoL of students have been affected by environmental, behavioural and organizational factors. The objective of this study was to analyse the QOL related to the health of the students of the Education of Youths and Adults in the municipality of Itacoatiara / AM - Brazil. The sample used was 84 students aged 18 to 59 years ( $M=24.47 \pm 7.53)$. The data collection was done in meetings, with application of QV SF-36 questionnaire. The study project was approved by the ethics committee of the State University of Amazonas. The results showed that the selected sample presented a satisfactory level of QoL, above $60 \%$ in 6 domains (Functional Capacity, Limitation by Physical Aspects, Pain, Vitality, Social Aspects and Mental Health). However, in 2 domains (General Health and Limitation for Emotional Aspects), the values were below $60 \%$, classifying them as regular, unexpected results for these domains, given the low average chronological age of the sample of 24.47 years. It is understood that establishing or measuring the level of QoL of a specific individual or groups, becomes fundamental to the understanding of the real situation in which these individuals are, aiming at the end to improve the QoL levels of the individuals surveyed. Keywords: Health; Quality of life (QOL); SF-36; Schoolchildren; EJA.
\end{abstract}

Corresponding author. Universidade do Estado do Amazonas, Brazil.

E-mail: kiaspimenta@gmail.com

Supplementary Issue: Spring Conferences of Sports Science. International Seminar of Physical Education, Leisure and Health, 17-19 June 2019. Castelo Branco, Portugal.

JOURNAL OF HUMAN SPORT \& EXERCISE ISSN 1988-5202

(c) Faculty of Education. University of Alicante.

doi:10.14198/jhse.2019.14.Proc4.82 


\section{INTRODUCTION}

Interest in the topic of Quality of Life (QOL) has increased significantly in the last decades, it is a concept that involves parameters in several areas and everything that relates to the human being, its culture and its environment. The objective of this study was to analyse the quality of life related to the health of schoolchildren in the education of young people and adults (education for those who did not have access or continuity of studies in primary and secondary education in their own age) in the municipality of Itacoatiara. The main objective of this study was to evaluate the Quality of Life (QoL) of youths and adults. Through MOS SF-36, it is possible to address some physical, social and psychological pathological factors and their interferences on the quality of life, also contributing in the search for more appropriate interventions.

\section{MATERIAL AND METHODS}

\section{Participants}

A total of 84 schoolchildren ( 42 males and 42 females) aged 18 to 59 years ( $M=24.47 \pm 7.53$ ) participated in the study, regularly using the EJA of the State School Teacher Maria Ivone de Araújo Leite, linked to Regional Coordination of Education of Itacoatiara (CREI / SEDUC), located in the Itacoatiara municipality, located in the metropolitan region of Manaus, in the east-central part of the State of Amazonas, Brazil. According to CREI / SEDUC, the total number of students enrolled in the EJA in 2017 was 834, the participating sample was $10 \%$ of the total value of this population.

\section{Measures}

The SF-36 generic self-administered questionnaire was used, consisting of 36 items grouped into 8 domains: Functional Capacity (10 items), Limitation by Physical Aspects (4), Pain (2), General Health Status, Vitality (4), Social Aspects (2), Limitation for Emotional Aspects (3), Mental Health (5), and one more question of comparative evaluation between current health conditions and those of a year ago, this question does not receive punctuation. To evaluate their results, a score was given for each question, later they were transformed into a scale (Raw Scale) of 0 (worse health) to 100 (better health).

\section{Procedures}

The research project was approved by the Ethics Committee of the State University of Amazonas. For the data collection, the legal authorizations (Consent Form) with CREI / SEDUC were requested, the students were invited to participate, authorizing their participation through the signing of the Informed Consent Term. The SF-36 questionnaire was applied and filled out by the students in meetings held during 2 weeks in the theoretical / practical classes of Physical Education, always in the presence of the class teacher.

\section{Analysis}

The database was generated in Excel spreadsheet version 7. The analysis was constituted in three moments: sorting, data classification and final analysis. Statistical package version 10 was used for the statistical calculations. The statistics used were: Average, Minimum, Maximum and Standard Deviation.

\section{RESULTS}

Regarding the classification of QL domains by age group, in practically all variables statistics the men's score is higher than the women's score (except for the items General Health Status and Social Aspects in the 2029 age group), in general, men assessed their Quality of Life (QL) more positively by comparing women (Table 1). 
Table 1. SF-36 Statistics QoL Scale by age group

\begin{tabular}{|c|c|c|c|c|}
\hline Variables & Sex & $\begin{array}{l}15 \text { to } 19 \text { Years } \\
M \pm S D\end{array}$ & $\begin{array}{c}\text { Age Group } \\
20 \text { to } 29 \text { Years } \\
M \pm S D\end{array}$ & $\begin{array}{l}30 \text { to } 59 \text { Years } \\
M \pm S D\end{array}$ \\
\hline \multirow{6}{*}{ Limitation by Physical Aspects } & M & $18.67 \pm 0.49$ & $23.81 \pm 2.82$ & $42.11 \pm 8.05$ \\
\hline & F & $15.99 \pm 15.67$ & $23.50 \pm 3.19$ & $30.67 \pm 1.15$ \\
\hline & M & $83.33 \pm 20.38$ & $87.86 \pm 17.00$ & $92.22 \pm 8.33$ \\
\hline & $F$ & $51.52 \pm 40.04$ & $78.93 \pm 14.23$ & $66.67 \pm 35.12$ \\
\hline & M & $56.25 \pm 30.39$ & $70.24 \pm 31.24$ & $63.89 \pm 18.16$ \\
\hline & $\mathbf{F}$ & $45.03 \pm 21.18$ & $65.18 \pm 34.92$ & $50.00 \pm 43.30$ \\
\hline \multirow{2}{*}{ Pain } & M & $83.00 \pm 18.14$ & $77.81 \pm 24.34$ & $68.33 \pm 13.77$ \\
\hline & $\mathbf{F}$ & $47.55 \pm 32.07$ & $67.11 \pm 23.60$ & $50.00 \pm 15.82$ \\
\hline \multirow{2}{*}{ General Health Status } & M & $59.92 \pm 13.79$ & $58.00 \pm 13.81$ & $65.33 \pm 17.54$ \\
\hline & $\mathrm{F}$ & $38.06 \pm 25.37$ & $59.46 \pm 15.32$ & $61.33 \pm 8.14$ \\
\hline \multirow{2}{*}{ Vitality } & M & $64.17 \pm 28.27$ & $68.33 \pm 19.32$ & $73.33 \pm 8.05$ \\
\hline & $\mathbf{F}$ & $44.48 \pm 27.01$ & $66.43 \pm 23.21$ & $61.67 \pm 23.09$ \\
\hline \multirow{2}{*}{ Social Aspects } & M & $72.92 \pm 30.07$ & $68.45 \pm 29.74$ & $73.61 \pm 23.75$ \\
\hline & $\mathrm{F}$ & $49.76 \pm 24.16$ & $71.88 \pm 25.60$ & $54.17 \pm 14.43$ \\
\hline \multirow{2}{*}{ Limitation by Emotion Aspects } & M & $52.73 \pm 26.42$ & $63.47 \pm 37.87$ & $59.22 \pm 27.79$ \\
\hline & $\mathrm{F}$ & $44.58 \pm 16.09$ & $55.93 \pm 40.63$ & $55.53 \pm 50.51$ \\
\hline \multirow{2}{*}{ Mental Health } & M & $65.67 \pm 32.34$ & $70.29 \pm 21.67$ & $42.11 \pm 19.30$ \\
\hline & $\mathrm{F}$ & $48.51 \pm 27.24$ & $69.21 \pm 22.09$ & $30.67 \pm 6.11$ \\
\hline
\end{tabular}

\section{DISCUSSION}

In this study it was noticed that the mean values of most domains exceed $60 \%$ on average, which classifies the QoL level of this sample as satisfactory, considering the mean age of 24 years. Only 2 domains had their mean values below 60\%: General Health Status $(\mathrm{M}=59.25 \%)$ and Emotional Aspects Limitation ( $\mathrm{M}=$ $53.93 \%$ ). The gender issue of the respondents with respect to the mean age, for men was 26.67 years, and for women 22.68 years a difference of approximately 4 years of age. Regarding the classifications of the domains of QOL by age group, we see that the mean of the functional capacity domain was higher in the age range of "30 to 59 years" for both men and women.

\section{CONCLUSIONS}

According to the study objectives, the sample presented a satisfactory level of Quality of Life (QoL), being these individuals theoretically less exposed to some types of pathologies. When we compared domains by age group, we noticed that age did not influence a better evaluation in relation to health and physical wellbeing, since the older ones evaluated more positively compared to the younger ones, when we referred to the social and psychological domains there was a between age groups. Comparing genders, men evaluated their Quality of Life (QL) more positively in relation to women.

\section{REFERENCES}

Ciconelli, R. M.; Ferraz, M. B.; Santos, W.; Meinão, I.; Quaresma, M. R. Translation into the Portuguese language and validation of the generic questionnaire for quality of life evaluation SF-36 (Brasil SF36). Brazilian Journal of Rheumatology, São Paulo, v.39, n.3, p.143-50, 1999. 


\section{(c) $\underset{\mathrm{EY}}{\mathrm{NO}} \mathrm{\Theta}$}

This work is licensed under a Attribution-NonCommercial-NoDerivatives 4.0 International (CC BY-NC-ND 4.0). 


\title{
Is performance in basketball referees affected by gender?
}

\author{
D. GARCÍA-SANTOS , M. REINA, S. GONZÁLEZ-ESPINOSA, J. GARCÍA-RUBIO, S.J. IBÁÑEZ \\ Grupo de Optimización del Entrenamiento y el Rendimiento Deportivo, Universidad de Extremadura, \\ Cáceres, Spain
}

\begin{abstract}
The workload performances have an important role in decisions making of basketball referees. But it is not known if the gender influences this load. Therefore, the aim of this study focuses on knowing the existing differences according to the gender in workload during competition. The participants were nine international basketball referees (six male and three female). In each game participated three referees. Fifteen matches were registered during U-16 Female EuroBasket. The variables analysed were: Accelerations, Decelerations, Speed, Distance, Distance in different intensities (walking, jogging, running and sprinting) and Player Load. Each referee was equipped with WIMUPROTM inertial device with Ultra-Wide Band systems. For the statistical analysis the U-Mann-Whitney test was used. Only there were significant differences in Acc/min and Dis/min between men and women. In conclusion, the gender should not be predictive on competitive workload of basketball referees, because both of them must perform similar movements according to the game. Keywords: Basketball; Referees; Gender; Workload; Competition.
\end{abstract}

Corresponding author. Grupo de Optimización del Entrenamiento y el Rendimiento Deportivo, Universidad de Extremadura. Avda de la Universidad s/n, Facultad de Ciencias del deporte, 10003, Cáceres, Spain.

E-mail: dgarcianu@alumnos.unex.es

Supplementary Issue: Spring Conferences of Sports Science. International Seminar of Physical Education, Leisure and Health, 17-19 June 2019. Castelo Branco, Portugal.

JOURNAL OF HUMAN SPORT \& EXERCISE ISSN 1988-5202

(c) Faculty of Education. University of Alicante.

doi:10.14198/jhse.2019.14.Proc4.82 


\section{INTRODUCTION}

Exists numerous studies to analysed the performance load during competition in basketball players, but there are few investigations related to the referees. These demands of internal and external load acquire great importance in decision-making, since they must maintain an adequate physical condition to be able to accurately assess the different situations that occur in a match (Ahmed, Davison \& Dixon, 2017). For this reason, the objective of this paper focuses on knowing the differences between gender in external load basketball referees during an international U-16 competition.

\section{MATERIAL AND METHODS}

\section{Participants}

The study sample consisted of nine international FIBA referees ( 3 women and 6 men). They refereed FIBA U16 Women's European Championship 2017. Each referee participated in five games. All of them were informed of the experimental protocol and its possible benefits.

\section{Measures}

The independent variable was gender. The variables used to describe the workload basketball referee were:

- Accelerations (ACC) and Decelerations (DEC): Speed and directions changes made during the game. In this case, was recorded the total number, per minute and maximum accelerations and decelerations.

- Speed (S): The rate of change of distance travelled with respect to time. The average and maximum speed were calculated.

- Distance (Dis): The full length of a match. It was classified according to intensity: walking (0-6 km/h), jogging $(6-12 \mathrm{~km} / \mathrm{h})$, running $(12-18 \mathrm{~km} / \mathrm{h})$ and sprinting $(18-21 \mathrm{~km} / \mathrm{h})$.

- Player Load (PLTM): The neuromuscular load of each referee (Cormack, Mooney, Morgan \& McGuigan, 2013). It is an objective load measurement, validated and calculated from accelerometer signal in the 3 axes (Schelling \& Torres, 2016).

\section{Procedures}

For recording of variables were used a WIMUPROTM inertial devices with Ultra-Wide-Band (UWB) technology. Each inertial device was placed 20 minutes before starting the game on the top of the referee's back within a specific anatomical harness. The UWB system was adjusted to the measurements of the pitch before competition, placing six radiofrequency antennas around the court.

\section{Analysis}

The S-PRO software was used to obtain the data. The U-Mann-Whitney test was performed to know the differences between gender. The statistical program used was SPSS (IBM Corp. Released 2017. IBM SPSS Statistics for Windows, Version 21.0, Armonk, NY: IBM Corp.). The degree of significance ( $p \leq .05)$ was calculated accurately with the Monte Carlo method, with $99 \%$ confidence intervals.

\section{RESULTS}

The results of the study show that there are significant differences in Acc/min ( $p=.017)$ and Dis/min ( $p=.034)$, being always higher in women. 


\begin{tabular}{|c|c|c|c|c|c|}
\hline \multirow{3}{*}{ Variables } & \multirow{3}{*}{ U de Mann-Whitney } & \multirow{3}{*}{ Z } & \multicolumn{3}{|c|}{ Sig. Monte Carlo (bilateral) } \\
\hline & & & \multirow{2}{*}{ Sig. } & \multicolumn{2}{|c|}{ Confidence Interval $(99 \%)$} \\
\hline & & & & $\mathrm{L}$ & U \\
\hline Accelerations (n) & 197.000 & -.674 & $.507^{\mathrm{b}}$ & .494 & .519 \\
\hline $\operatorname{Acc} / \mathrm{min}\left(\mathrm{m} / \mathrm{s}^{2}\right)$ & 125.000 & -2.408 & $.017^{\mathrm{b}}$ & .014 & .020 \\
\hline $\operatorname{AccMax}\left(\mathrm{m} / \mathrm{s}^{2}\right)$ & 215.500 & -.229 & $.827^{\mathrm{b}}$ & .817 & .836 \\
\hline Decelerations (n) & 191.000 & -.819 & $.410^{b}$ & .398 & .423 \\
\hline $\operatorname{Dec} / \mathrm{min}\left(\mathrm{m} / \mathrm{s}^{2}\right)$ & 151.000 & -1.782 & $.074^{b}$ & .068 & .081 \\
\hline $\operatorname{DecMax}\left(\mathrm{m} / \mathrm{s}^{2}\right)$ & 219.000 & -.144 & $.893^{b}$ & .885 & .901 \\
\hline SpeedMax $(\mathrm{km} / \mathrm{h})$ & 188.500 & -.879 & $.393^{b}$ & .380 & .405 \\
\hline SpeedMed (km/h) & 221.000 & -.096 & $.925^{b}$ & .918 & .932 \\
\hline PlayerLoad & 156.500 & -1.649 & $.098^{b}$ & .090 & .106 \\
\hline PlayerLoad/min & 202.500 & -.543 & $.596^{b}$ & .583 & .608 \\
\hline Distance (m) & 172.000 & -1.276 & $.204^{b}$ & .193 & .214 \\
\hline Distance (m/min) & 138.000 & -2.095 & $.034^{b}$ & .029 & .039 \\
\hline Dis $0-6 \mathrm{~km} / \mathrm{h}$ & 205.000 & -.482 & $.641^{b}$ & .629 & .654 \\
\hline Dis $6-12 \mathrm{~km} / \mathrm{h}$ & 166.000 & -1.421 & $.156^{b}$ & .146 & .165 \\
\hline Dis $12-18 \mathrm{~km} / \mathrm{h}$ & 220.000 & -.120 & $.911^{b}$ & .904 & .918 \\
\hline Dis $18-21 \mathrm{~km} / \mathrm{h}$ & 202.000 & -.554 & $.594^{b}$ & .581 & .606 \\
\hline
\end{tabular}

\section{DISCUSSION}

External load variables allow establish a performance profile during competition. Considering the results obtained, it is possible that there are only differences in the Acc/min and Dis/min because the referees must perform similar movements during the game (García-Santos, Gamonales, León \& Muñoz, 2017). The importance of analysing these variables is centred on the fact that these are determinants in the game and the referees must develop a great capacity to adapt to the needs of the game (Delaney, Cummings, Thornton \& Duthie, 2017). In addition, they are variables that affect the fatigue of referees, regardless of the gender, as they are related to high intensity actions (Hoppe et al., 2017).

\section{CONCLUSIONS}

Pursuant to the results obtained, basketball referees present significant differences according to gender in Acc/min and Dis/min, being higher in women. These results show the importance of analysing the movements of the referee, regardless of gender, to adjust to competition and referees requirements. Furthermore, knowing these variables allows to establish training programs according to the profile of the game.

\section{REFERENCES}

Ahmed, H., Davison, G., \& Dixon, D. (2017). Analysis of activity patterns, physiological demands and decision-making performance of elite Futsal referees during matches. International Journal of Performance Analysis in Sport, 17(5), 737-751. https://doi.org/10.1080/24748668.2017.1399321

Cormack, S. J., Mooney, M. G., Morgan, W., \& McGuigan, M. R. (2013). Influence of neuromuscular fatigue on accelerometer load in elite Australian football players. International Journal of Sports Physiology and Performance, 8(4), 373-378. https://doi.org/10.1123/ijspp.8.4.373 
Delaney, J. A., Cummins, C. J., Thornton, H. R., \& Duthie, G. M. (2017). Importance, reliability and usefulness of acceleration measures in team sports. Journal of Strength \& Conditioning Research. https://doi.org/10.1519/jsc.0000000000001849

García-Santos, D., Gamonales, J. M., León, K., \& Muñoz, J. (2017). A case study: Characterization of the physiological, kinematic and neuromuscular demands of handball referee during competition. $E$ Balonmano.com: Revista de Ciencias del Deporte, 13(3), 207-216.

Hoppe, M. W., Baumgart, C., Slomka, M., Polglaze, T. y Freiwald, J. (2017). Variability of Metabolic Power Data in Elite Soccer Players During Pre-Season Matches. Journal of Human Kinetics, 58(1), 233-245. https://doi.org/10.1515/hukin-2017-0083

Schelling, X., \& Torres, L. (2016). Accelerometer Load Profiles for Basketball-Specific Drills in Elite Players. Journal of Sports Science \& Medicine, 15(4), 585. https://doi.org/10.1249/01.mss.0000487540.00343.1a 


\title{
Associations of physical activity with body composition and aerobic capacity in adults with Down syndrome
}

\author{
ANA RODRIGUES ${ }^{1} \square$, HÉLIO ANTUNES ${ }^{1}$, RICARDO ALVES ${ }^{1}$, DUARTE SOUSA ${ }^{1}$, HELDER LOPES ${ }^{1,2}$, \\ JOÃO PRUDENTE1,2 \\ ${ }^{1}$ Department of Physical Education and Sport, Faculty of Social Sciences, University of Madeira, Portugal \\ ${ }^{2}$ CIDESD, Portugal
}

\begin{abstract}
The characterization of physical activity profile and the relation with health indicators is still an issue to explore in populations with Down Syndrome. With the development of this study we intend to: (i) describe the levels of physical activity, aerobic capacity and body composition of participants, considering gender and age, (ii) analyse the association between physical activity levels and aerobic capacity and body composition. In this study participated 26 subjects (16 males and 10 females) with Down syndrome, aged between 19 and 41 years (27.33 \pm 11.93$)$. All participants were evaluated in physical activity using a pedometer (one week), aerobic capacity (AAHPERD, 1998), weight and height. Reduced levels of physical activity (4073.71 \pm 1217.69 steps/day) and aerobic capacity (3.09 \pm .90 minutes) were observed among participants. The prevalence of obesity was $36.4 \%$ and $13.6 \%$ overweight, $50 \%$ of the participants presented the recommended weight. However, none of the analysed parameters shows differences between sexes ( $p>.05)$, nor association between age and the indicators evaluated ( $p>.05)$. Physical activity has been shown to be associated with: (i) body mass index ( $r=-.747, p<.001)$ and (ii) with cardiorespiratory capacity $(r=.458$, $p=.019)$. It is necessary to develop programs and policies to promote physical activity in this population, in order to prevent the development of various pathologies. Keywords: Down syndrome; Physical activity; Aerobic capacity; Adults.
\end{abstract}

Corresponding author. Department of Physical Education and Sport, Faculty of Social Sciences, University of Madeira. Campus da Penteada, 9020-105 Funchal, Portugal.

E-mail: anajar@staff.uma.pt

Supplementary Issue: Spring Conferences of Sports Science. International Seminar of Physical Education, Leisure and Health, 17-19 June 2019. Castelo Branco, Portugal.

JOURNAL OF HUMAN SPORT \& EXERCISE ISSN 1988-5202

(c) Faculty of Education. University of Alicante.

doi:10.14198/jhse.2019.14.Proc4.82 


\section{INTRODUCTION}

The promotion of physical activity has been one of the main concerns of several health care organizations, as a strategy to prevent the development of several pathologies. The relationship between physical activity and various health indicators is widely reported in the literature (Bouchard, Blair, \& Haskell, 2018). However, the studies that analyse the beneficial effects of physical activity on health in people with Down syndrome are still limited (Mendonca, Pereira, \& Fernhall, 2010). In this context, with the development of this work we intend to: (i) describe the levels of physical activity, aerobic capacity and body composition of participants, considering gender and age; (ii) To analyse the association between physical activity levels with aerobic capacity and body composition.

\section{MATERIAL AND METHODS}

\section{Participants}

In this study participated 26 subjects (16 males and 10 females) with Down syndrome, aged between 19 and 41 years (27.33 \pm 11.93$)$. All participants have Physical Education classes, 2 to 3 session 60 minutes per session (total 120 to 180 minutes/week).

\section{Measures}

All participants were assessed in Physical Activity using a pedometer (Yamax Digiwalkers SW-700), worn for seven days. All participants were also evaluated for weight, height, body mass index and aerobic capacity (AAHPERD, 1998).

\section{Procedures}

Participants were invited to this study, through informed consent signed by the tutor. After the informed consent was collected, all participants were evaluated in body composition and aerobic capacity. The application and supervision of the pedometer use were carried out by the research team in collaboration with teachers and tutors.

\section{Analysis}

Descriptive statistics (mean and standard deviation) were used to characterize the sample in the variables under study. The gender difference in the variables under study where tested using the Mann-Whitney test. Spearman correlations were used to study the relationship between age and study variables and between physical activity, aerobic capacity and body composition. The statistical software used was SPSS version 25.0 and the level of significance was $5 \%$.

\section{RESULTS}

Table 1. Characterization of the sample in the study variables: analysis by sex

\begin{tabular}{lcccc}
\hline & Total & Men & Females & $\mathrm{p}$ \\
\hline Height $(\mathrm{cm})$ & $155.04 \pm 13.06$ & $159.56 \pm 12.87$ & $147.80 \pm 10.14$ & 0.020 \\
Weight $(\mathrm{kg})$ & $64.18 \pm 14.26$ & $65.85 \pm 14.56$ & $61.78 \pm 14.32$ & 0.647 \\
BMI $\left(\mathrm{kg} / \mathrm{m}^{2}\right)$ & $28.04 \pm 15.38$ & $26.92 \pm 4.79$ & $29.67 \pm 6.06$ & 0.262 \\
Aerobic capacity (min) & $3.09 \pm 0.90$ & $3.15 \pm 0.88$ & $2.98 \pm 0.96$ & 0.660 \\
Physical Activity (steps. Day) & $4073.71 \pm 1217.69$ & $4458.05 \pm 1320.95$ & $3458.76 \pm 728.49$ & 0.053 \\
\hline
\end{tabular}


On average each participant performs $4073.71 \pm 1217.69$ steps/day. The prevalence of obesity was $36.4 \%$ and $13.6 \%$ overweight, $50 \%$ of the participants presented the recommended weight. In aerobic fitness on average the participants perform the test in $3.09 \pm .90$ minutes. However, none of the analysed parameters shows differences between sexes ( $p>.05)$, nor association between age and the indicators evaluated ( $p>.05)$.

Physical activity has been shown to be associated with: (i) body mass index ( $r=-.747, p<.001$ ); (ii) with cardiorespiratory capacity $(r=.458, p=.019)$.

\section{DISCUSSION}

It is observed that the participants present reduced levels of physical activity, aerobic fitness and high prevalence of overweight and obesity. Similar results are reported in other studies regarding physical activity (Shields, Plant, Warren, Wollersheim, \& Peiris, 2018),, aerobic fitness (Pitetti, Climstein, Campbell, Barrett, \& Jackson, 1992) and overweight (Stancliffe et al., 2011). In fact, the literature reports the reduced levels of physical activity among adults with Down syndrome, since only one in three adults with intellectual disabilities were sufficiently active to achieve health benefits (Temple, Frey, \& Stanish, 2006).

Reduced levels of physical activity are worrying, considering that they represent an increased risk for the development of several pathologies (Bouchard et al., 2018), since that people with Down syndrome have an increased risk of developing them (Sobey, Judkins, C. P. Sundararajan, Phan, Drummond, \& Srikanth, 2015). Our research confirms this aspect when we verify the association between physical activity levels, aerobic capacity and body composition, and reduced levels of physical activity are associated with reduced levels of aerobic capacity and high body mass index. The development of studies that deepen physical activity characteristics such as intensity should also be the target of future investigations.

\section{CONCLUSIONS}

Levels of physical activity and aerobic capacity were found to be low among people with Down syndrome. In this context, it is necessary to develop programs and policies to promote physical activity in this population, namely by the relationship with other health indicators such as aerobic capacity and body composition.

\section{REFERENCES}

Bouchard, C., Blair, S. N., \& Haskell, W. (2018). Physical Activity and Health (2th Eds). Champaign: Human Kinetics.

Mendonca, G., Pereira, F., \& Fernhall, B. (2010). Reduced exercise capacity in persons with Down syndrome: Cause, effect, and management. Therapeutics and Clinical Risk Management, 6, 601610. https://doi.org/10.2147/tcrm.s10235

Pitetti, K. H., Climstein, M., Campbell, K. D., Barrett, P. J., \& Jackson, J. A. (1992). The cardiovascular capacities of adults with Down syndrome: a comparative study. Medicine \& Science in Sports \& Exercise, 24(1), 13-19. https://doi.org/10.1249/00005768-199201000-00004

Shields, N., Plant, S., Warren, C., Wollersheim, D., \& Peiris, C. (2018). Do adults with Down syndrome do the same amount of physical activity as adults without disability? A proof of principle study. Journal of Applied Research in Intellectual Disabilities: JARID, 31(3), 459-465. https://doi.org/10.1111/jar.12416 
Sobey, C. G., Judkins, C. P. Sundararajan, V., Phan, T. G., Drummond, G. R., \& Srikanth, V. K. (2015). Risk of Major Cardiovascular Events in People with Down Syndrome. PLoS One, 10, E0137093. https://doi.org/10.1371/journal.pone.0137093

Stancliffe, R. J., Lakin, K. C., Larson, S., Engler, J., Bershadsky, J., Taub, S., ... Ticha, R. (2011). Overweight and Obesity Among Adults With Intellectual Disabilities Who Use Intellectual Disability/Developmental Disability Services in 20 U.S. States. American Journal on Intellectual and Developmental Disabilities, 116(6), 401-418. https://doi.org/10.1352/1944-7558-116.6.401

Temple, V. A., Frey, G. C., \& Stanish, H. I. (2006). Physical Activity of Adults with Mental Retardation: Review and Research Needs. American Journal of Health Promotion, 21(1), 2-12. https://doi.org/10.4278/0890-1171-21.1.2 


\title{
Differences on body composition and biochemical parameters between practitioners and non- practitioners of soccer
}

\author{
ELIANE A. CASTRO1,2 $\triangle$, SÍLVIA A. HALLAK ${ }^{1}$, LÍGIA R. MOSCARDINI ${ }^{1}$, DANIEL DOS SANTOS ${ }^{1}$ \\ 1 Universidade de Franca (UNIFRAN), Brazil \\ 2LFE Research Group, Universidad Politécnica de Madrid (UPM), Spain
}

\begin{abstract}
Amateur soccer players often present characteristics and current problems of sedentary lifestyle, such as excessive levels of adiposity and an increase in cardiovascular risk factors. The objective of this study was to compare body composition and biochemical parameters between practitioners and non-practitioners of soccer. Thirty-eight healthy men were divided into two groups: soccer practitioners, who trained three times a week for at least one year ( $n=26 ; 22.1 \pm 4.5$ years) and non-soccer practitioners ( $n=12 ; 22.5 \pm 3.8$ years). Body weight, height, total cholesterol, high-density lipoprotein, low-density lipoprotein, very-low-density lipoprotein, fasting glucose and C-reactive protein were measured and body mass index (BMI) and body fat percentage were calculated. All assessments were realized in the University of Franca - Brazil. For mean comparison T-test and Mann Whitney test were employed according data distribution. Significant differences were observed between soccer practitioners and non-soccer practitioners, respectively, for fat percentage $(14.3 \pm 4.8 \%$ vs $21.2 \pm 7.5 \% ; p=.005)$ and fasting glucose $(82.9 \pm 6.9 \mathrm{mg} / \mathrm{dL}$ vs $89.8 \pm 8.7 \mathrm{mg} / \mathrm{dL} ; p=.005)$. No differences were observed for any other variable between two groups. Soccer practitioners presented a more favourable body composition and fasting glucose profile than their non-practicing counterparts, although fasting glucose was normal levels for both groups. Football practice could help maintain adequate levels of body fat in healthy young adults. Keywords: Physical activity; Exercise; Body fat percentage; Fasting glucose; Football players; Sedentary lifestyle.
\end{abstract}

Corresponding author. Rua dos Pracinhas, 2000, ap. 01, Residencial Paraíso, 14403-160, Franca, SP, Brasil.

E-mail: elianeaparecidacastro@gmail.com

Supplementary Issue: Spring Conferences of Sports Science. International Seminar of Physical Education, Leisure and Health, 17-19 June 2019. Castelo Branco, Portugal.

JOURNAL OF HUMAN SPORT \& EXERCISE ISSN 1988-5202

(c) Faculty of Education. University of Alicante.

doi:10.14198/jhse.2019.14.Proc4.82

S1636 | 2019| Proc4| VOLUME 14

C 2019 University of Alicante 


\section{INTRODUCTION}

The benefits of physical activity and exercise, used as tools to maintain a high health status, are highly documented in the literature, and exercise is considered the major drugs universal, with lower side effects and costs for public health (Booth et al., 2012; Lavie et al., 2017; Lobelo et al., 2014; Nunan et al., 2013). However, amateur soccer players often present characteristics and current problems of sedentary lifestyle, such as excessive levels of adiposity and an increase in cardiovascular risk factors (Pontes et al., 2006). Therefore, the objective of this study was to compare body composition and biochemical parameters between practitioners and non-practitioners of soccer.

\section{MATERIAL AND METHODS}

Descriptive and cross-sectional study, with quantitative approach for data analysis.

\section{Participants}

Thirty-eight healthy men were divided into two groups: soccer practitioners ( $n=26 ; 22.1 \pm 4.5$ years) and nonsoccer practitioners ( $n=12 ; 22.5 \pm 3.8$ years). The included practitioners trained three times a week for at least one year.

\section{Measures}

Body weight and height were measured and body mass index (BMI) was calculated as body weight/height2. Body fat percentage was obtained by Falkner formula composed of the triceps, subscapular, suprailiac and abdominal skinfolds. Total cholesterol (TC), high-density lipoprotein (HDL), low-density lipoprotein (LDL), very-low-density lipoprotein (VLDL), fasting glucose and C-reactive protein were measured by standard procedures.

\section{Procedures}

This study was realized between February and October 2018 in the University of Franca - Brazil. All assessments will be conducted at similar physical, physiological and climatic times and conditions for all individuals.

\section{Analysis}

Data are presented as mean $\pm S D$. For mean comparison T-test and Mann Whitney test were employed according data distribution. All analyses were conducted in SPSS-PC v20 (IBM SPSS Statistics, Armonk, NY USA), and level of significance was set at .05.

\section{RESULTS}

Significant differences were observed between soccer practitioners and non-soccer practitioners, respectively, for fat percentage (14.3 $\pm 4.8 \%$ vs $21.2 \pm 7.5 \% ; p=.005)$ and fasting glucose $(82.9 \pm 6.9 \mathrm{mg} / \mathrm{dL}$ vs $89.8 \pm 8.7 \mathrm{mg} / \mathrm{dL} ; \mathrm{p}=.005)$. No differences were observed for any other variable between two groups ( $p>05$; soccer practitioners and non-soccer practitioners, respectively: body weight $=72.8 \pm 9.6 \mathrm{~kg}$ vs $75.4 \pm 18.2 \mathrm{~kg}$; height $=1.8 \pm .06 \mathrm{~m}$ vs $1.8 \pm .06 \mathrm{~m} ; \mathrm{BMl}=22.7 \pm 2.9 \mathrm{~kg} / \mathrm{m} 2$ vs $24.1 \pm 4.6 \mathrm{~kg} / \mathrm{m} 2 ; \mathrm{TC}=150.4 \pm 32.8 \mathrm{mg} / \mathrm{dL}$ vs $157.5 \pm 35.4 \mathrm{mg} / \mathrm{dL} ; \mathrm{HDL}=46.6 \pm 8.8 \mathrm{mg} / \mathrm{dL}$ vs $51.7 \pm 9.6 \mathrm{mg} / \mathrm{dL} ; \mathrm{LDL}=88.6 \pm 28.8 \mathrm{mg} / \mathrm{dL}$ vs $88.2 \pm 26.9 \mathrm{mg} / \mathrm{dL}$; VLDL $=15.2 \pm 6.8 \mathrm{mg} / \mathrm{dL}$ vs $17.6 \pm 11.3 \mathrm{mg} / \mathrm{dL} ;$ triglycerides $=76.1 \pm 34 \mathrm{mg} / \mathrm{dL}$ vs $88.2 \pm 56.6 \mathrm{mg} / \mathrm{dL}$ and $\mathrm{C}$ reactive protein $=1.6 \pm 1.9 \mathrm{mg} / \mathrm{dL}$ vs $7.2 \pm 21.7 \mathrm{mg} / \mathrm{dL}$ ). 


\section{DISCUSSION}

Soccer practitioners presented a more favourable body composition and fasting glucose profile than their non-practicing counterparts, although fasting glucose was normal levels for both groups. Our results showed that football practice could help maintain adequate levels of body fat in healthy young adults. In this line, studies have also indicated that recreational soccer seems to stimulate musculoskeletal, metabolic and cardiovascular adaptations, reducing the risk of developing lifestyle diseases (Krustrup et al., 2010). The influence of this sport on biochemical parameters should be evaluated in older populations, where the impact of age on these variables is greater.

\section{CONCLUSIONS}

Studies that characterize health profiles of amateur soccer players, as well as provide comparisons between practitioners and non-practitioners, are extremely important for a more conscious practice that takes into account the individual characteristics and that approach the sport to the fundamental concepts of health promotion.

\section{FUNDING}

This study was financed in part by the Coordenação de Aperfeiçoamento de Pessoal de Nível Superior Brasil (CAPES) - Finance Code 001.

\section{REFERENCES}

Booth, F. W., Roberts, C. K. \& Laye, M. J. (2012). Lack of exercise is a major cause of chronic diseases. Comprehensive Physiology, 2(2), 1143-1211. https://doi.org/10.1002/cphy.c110025

Lavie, C. J., Archer, E. \& Lee, D. C. (2017). Persistent physical activity translating to persistent reduction in mortality. European Journal of Preventive Cardiology, 24(15), 1612-1614. https://doi.org/10.1177/2047487317730462

Lobelo, F., Stoutenberg, M. \& Hutber, A. (2014). The Exercise is Medicine Global Health Initiative: a 2014 update. British Journal of Sports Medicine, 48(22), 1627-1633. https://doi.org/10.1136/bjsports-2013$\underline{093080}$

Nunan, D., Mahtani, K. R., Roberts, N. \& Heneghan, C. (2013). Physical activity for the prevention and treatment of major chronic disease: an overview of systematic reviews. Systematic Reviews, 10(2), 56. https://doi.org/10.1186/2046-4053-2-56

Pontes, L. M. D., Sousa, M. D. S. C. D. \& Lima, R. T. D. (2006). Dietetic profile, nutritional status and prevalence of central obesity in recreative soccer practitioners. Revista Brasileira de Medicina do Esporte, 12(4), 201-205.

Krustrup, P., Aagaard, P., Nybo, L., Petersen, J., Mohr, M. \& Bangsbo, J. (2010). Recreational football as a health promoting activity: a topical review. Scandinavian Journal of Medicine \& Science in Sports, 20(Suppl 1), 1-13. https://doi.org/10.1111/j.1600-0838.2010.01108.x

\section{(ㅇ) $(\mathbb{\Theta \Theta} \Theta$}

This work is licensed under a Attribution-NonCommercial-NoDerivatives 4.0 International (CC BY-NC-ND 4.0). 


\title{
Which factors are related with coaches' perception of young soccer players competence: Physical fitness, motor coordination or specific skill?
}

\author{
VÍTOR P. LOPES ${ }^{1} \square$, ÁLVARO FORTUNATO², CELINA GONÇALVES ${ }^{1}$ \\ ${ }^{1}$ Research Center in Sports Sciences, Health Sciences and Human Development (CIDESD) and Instituto \\ Politécnico de Bragança, Portugal \\ ${ }^{2}$ Research Centre in Physical Activity, Health and Leisure and Instituto Politécnico de Bragança, Portugal
}

\begin{abstract}
A large number of youth sports programs in sport clubs focus on results. However, the success of young practitioners depends on several factors, as generic and specific motor competence that develops with the practice. The sample was boys ( $\mathrm{N}=111)$ with 10.5(2.48) years of age, who practiced soccer regularly, and their coaches ( $\mathrm{N}=17)$. Boys were evaluated on physical fitness (multistage aerobic test), motor coordination (KTK) and in specific soccer skills (passing and receiving, juggling the ball, dribbling, and kicking to the goal). A bioimpedance scale were used to determine percent body fat. The coaches were asked about the future success (achieving high performance) of each of their athletes. Multiple regression was performed to identify the variables that predicted the coaches' perception of the competence of young soccer players. Results show that only physical fitness test (cardiovascular fitness) was associated with coaches' perception of young soccer players competence. In conclusion, cardiovascular fitness was associated with the perception that coaches have of their young soccer players competence, but the specific skill levels and motor coordination were not. Keywords: Motor competence; Youth sport programs; Motor skills; Soccer; Physical fitness.
\end{abstract}

Corresponding author. Research Center in Sports Sciences, Health Sciences and Human Development (CIDESD), and Instituto Politécnico de Bragança, Campus de Santa Apolónia, 5300-253 Bragança, Portugal.

E-mail: vplopes@ipb.pt

Supplementary Issue: Spring Conferences of Sports Science. International Seminar of Physical Education, Leisure and Health, 17-19 June 2019. Castelo Branco, Portugal.

JOURNAL OF HUMAN SPORT \& EXERCISE ISSN 1988-5202

(C) Faculty of Education. University of Alicante.

doi:10.14198/jhse.2019.14.Proc4.82 


\section{INTRODUCTION}

Many youth sports programs in sport clubs aim to develop sporting excellence and focus on results, with the tangible success of a sports program (Galatti, et al., 2016). often related to the club's ability to produce good athletes within specific sporting disciplines. However, the success of young practitioners depends on several factors, namely the generic and specific motor competence that develops with the practice (Galatti, et al., 2016).

The present study aims to understand if physical fitness, specific skill levels, and motor coordination, are associated with the perception of coaches about young soccer players competence.

\section{MATERIAL AND METHODS}

\section{Participants}

Participants were $\mathrm{N}=111$ boys with $10.5(2.48)$ years of age, who practiced soccer regularly in youth training clubs, and their coaches $(\mathrm{N}=17)$.

\section{Measures}

Boys were evaluated on physical fitness (multistage aerobic test), motor coordination (KTK) and in specific soccer skills (passing and receiving, juggling the ball, dribbling, and kicking to the goal). Percent body fat was determined with a bioimpedance scale. The coaches were asked about the future success (achieving high performance) of each of their athletes, having into account four parameters: tactic ability, motor skills, physical aptitude, and psychological skills.

\section{Coaches' perceptions}

The coaches were asked about the future success (achieving high performance) of each of their athletes, having into account four parameters: tactic ability, motor skills, physical fitness, and psychological skills. For each parameters each coach responded in a 3-point scale: (1) Will not succeed, (2) Could succeed, (3) Will succeed. The result is the sum of the points assigned in the four parameters.

\section{Analysis}

Pearson correlation were calculated between coaches' perceptions and each performance test. Multiple regression was used to identify the variables that predicted the coaches' perception of the competence of young soccer players. The performance results in specific soccer skills were previously transformed into zscores and the results of all the skills' tests were summed to obtain an indicator of soccer skill performance.

\section{RESULTS}

Table 1. Descriptive statistics of coaches' perceptions in the four parameters: tactic ability, motor skills, physical fitness, and psychological skills

\begin{tabular}{lcc} 
& Mean & SD \\
\hline Physical fitness & 2.1 & .6 \\
Motor skills & 2.1 & .6 \\
Psychological skills & 2.1 & .7 \\
Tactic ability & 2.1 & .7 \\
Total & 8.42 & 1.9 \\
\hline
\end{tabular}


Table 1 shows the descriptive statistics of coaches' perceptions in the four parameters: tactic ability, motor skills, physical fitness, and psychological skills.

Table 2 shows the Person correlation between coaches' perceptions and each performance test. Motor coordination test results (KTK) has no significant correlation with coaches' perception. Specific soccer skills have a significant correlation but negative with coaches' perception. Multistage Fitness Test have the highest correlation with coaches' perception.

Table 2. Person correlation between coaches' perceptions and each performance test

\begin{tabular}{lcc}
\hline & & Coaches' perceptions \\
\hline Specific soccer skills & $\mathrm{r}$ & $-.263^{*}$ \\
KTK & $\mathrm{N}$ & 93 \\
& $\mathrm{r}$ & .109 \\
Multistage Fitness Test & $\mathrm{N}$ & 102 \\
& $\mathrm{r}$ & $.321^{* *}$ \\
\hline
\end{tabular}

${ }^{*}$ significant for $p<0.05$

${ }^{* *}$ significant for $p<0.001$

Multiple regression results show that only cardiovascular fitness (Multistage Fitness Test) predict coaches' perception of young soccer players competence.

\section{DISCUSSION}

The main goal of this study was to find out if physical fitness, specific skill, and motor coordination, were associated with the perception of coaches about young soccer players competence and their future success. The results shows that only cardiovascular fitness predicts the coaches' perception, nor generic motor coordination nor specific motor skills significantly predict coaches' perception.

These results suggest that most coaches have a better understanding of the cardiovascular fitness of young athletes. Eventually this fact derives from the susceptibility of the coaches to be in tune with this parameter, to the detriment of others that also assume important relevance. It is pertinent that coaches develop the ability to perceive that other factors, such as those associated with motor coordination and specific gaming skills, should be the subject of systematic reflection.

\section{CONCLUSIONS}

In conclusion, the perception that coaches have of their young soccer players competence was associated with cardiovascular fitness, but not with specific skill levels and motor coordination. This suggests that coaches need to better now their young soccer player's competence and not only focus on results. Other important aspect it is that Coaches' perceptions could be influenced by their experience (Santos et al., 2010), for this reason could be interest include this variable in the future investigation (e.g. education, training programs of the coaches). 


\section{REFERENCES}

Galatti, L.; Scaglia, A.; Bettega, O.; \& Paes, R. (2016). Coaches' perceptions of youth players' development in a professional soccer club in Brazil: paradoxes between the game and those who play. Sports Coaching Review, 5(2), 174-185. https://doi.org/10.1080/21640629.2016.1201359

Santos, S; Mesquita, I; Graça, A.; \& Rosado, A. (2010). Coaches' perceptions of competence and acknowledgement of training needs related to professional competences. Journal of Sports Science and Medicine, 9, 62-70.

\section{(c) $(\mathrm{B})(\mathrm{EY}$}

This work is licensed under a Attribution-NonCommercial-NoDerivatives 4.0 International (CC BY-NC-ND 4.0). 


\title{
Sports attitudes of young people practicing orienteering: The influence of the additional practice of another sport
}

\author{
TADEU CELESTINO ${ }^{1,2,3} \triangle$, HUGO SARMENTO ${ }^{4,5}$, ARTUR SANTOS ${ }^{4}$, ANTONINO PEREIRA ${ }^{2,3}$ \\ ${ }^{1}$ Agrupamento de Escolas de Nelas, Portugal \\ 2Escola Superior de Educação, Portugal \\ ${ }^{3}$ Centro de Estudos em Educação, Tecnologia e Saúde (CI\&DETS), Instituto Politécnico de Viseu, Portugal \\ ${ }^{4}$ Centro de Investigação do Desporto e da Actividade Física, Faculdade de Ciências do Desporto e Educação \\ Física, Universidade de Coimbra, Portugal \\ ${ }^{5}$ Spertlab, Faculdade de Motricidade Humana, Universidade de Coimbra, Portugal
}

\begin{abstract}
The processes of sports socialization imply the adoption of attitudes, norms and identity behaviours of the contexts where they are developed. Despite the formative potential of sports orienteering, there are still few investigations about values and ethics in this modality. Thus, the purpose of this preliminary study was to explore the association of the sports attitudes of young orienteers with the practice of another modality. The sample consisted of 201 orienteering athletes, aged 13 to 18 years, 115 boys and 86 girls. Of these, 96 practiced orienteering exclusively and 105 practiced another modality besides orienteering in the context of school and federative sports. For the data collection, the SAQ-Sport Attitudes Questionnaire (Gonçalves, et al., 2006) was used to evaluate the sports attitudes. SPSS 11 software was used to perform the statistical analysis, namely measures of central tendency and dispersion and student $t$ test (independent). The results show that there are no statistically significant differences in athletic attitudes among athletes who are exclusively practicing orienteering compared to those who, in addition to orienteering, practice another sporting modality in the various dimensions of SAQ. Nevertheless, in the dimension associated with "antisports", athletes practicing other modalities present higher means showing a greater propensity for adopting unsportsmanlike behaviours in sports practice. Thus, greater assertiveness is needed in the adoption of strategies to promote ethical attitudes in the various contexts of sports practice. Keywords: Ethics; Values; Sports training; Young sport.
\end{abstract}

\footnotetext{
Corresponding author. AE Nelas - Rua Eng. Alberto Cardoso de Vilhena -3520-090, Portugal.

E-mail: titta2323@hotmail.com

Supplementary Issue: Spring Conferences of Sports Science. International Seminar of Physical Education, Leisure and Health, 17-19 June 2019. Castelo Branco, Portugal.

JOURNAL OF HUMAN SPORT \& EXERCISE ISSN 1988-5202

(c) Faculty of Education. University of Alicante.

doi:10.14198/jhse.2019.14.Proc4.82
} 


\section{INTRODUCTION}

The processes of sports socialization imply the adoption of distinctive attitudes, norms and identity behaviours of the contexts in which they develop. Thus, it is very common for athletes to assimilate, in particular, the subculture of the sport or club (Donnely \& Young, 2001).

Orienteering, as a sporting modality, has been gradually gaining its space as a sport that promotes the ideals of the multidimensional formation of individuals in their different contexts of practice. In this sense Celestino, Sarmento, Santos and Pereira (2018a), identify a generalized acceptance of the pro-social attitudes (convention and commitment) and rejection of antisocial attitudes (cheating and anti-sportsmanship) toward orienteering practice. In the same way, differences in the positive dimensions are identified, particularly in the younger echelons, and as the competitive echelon rises, levels of affiliation to the positive attitudes associated with convention and commitment increases.

Nevertheless, the same authors identify that boys, compared to girls, are those that are more willing to practice cheating and anti-sports activities in orienteering (Celestino, Sarmento, Santos \& Pereira, 2018b). In summary, this evidence suggests that there may be pedagogical weaknesses in the teaching and coaching processes. As such, it is imperative to reinforce, in an assertive way, the systematization and sensitization of all to values and sports ethics.

Despite the formative potential of orienteering sport, there is still little research on values and ethics in this modality. Thus, the purpose of this preliminary study was to explore the association of the sports attitudes of young orienteers with the practice of another sport.

\section{MATERIAL AND METHODS}

\section{Participants}

The sample consisted of 201 orienteering athletes, 115 boys and 86 girls, aged between 13 and 18 years. 96 practice only orienteering and 105 practice other modality besides orienteering in the context of school and federative sports.

\section{Measures}

For data collection, the SAQ-Sport Attitudes Questionnaire (Gonçalves et al., 2006) was used to evaluate the sports attitudes.

\section{Procedures}

The questionnaires were applied between March and April 2018, at the beginning and end of school sports gatherings and at the weekends of orienteering Portuguese Cup competitions. They were administered after consent of the legal guardians of the athletes and followed by a brief explanation under their filling.

\section{Analysis}

For the treatment of the data, SPSS version 11 software was used to carry out the statistical analyses, namely measures of central tendency and dispersion and student $t$ test. 


\section{RESULTS}

The results show that there are no statistically significant differences in the different dimensions of the SAQ, among the athletic attitudes of athletes who practiced orienteering only in comparison with those who, in addition to orienteering, practice other sports.

However, in the dimension associated to "anti-sports", athletes practicing other modalities have higher means, which reveals a greater propensity for adopting negative and unethical behaviours in sports practice.

\section{DISCUSSION}

The results obtained are in line with those of other studies, where in general, young people in these age groups adopt positive and pro-social attitudes towards sports (Gonçalves, 2013).

However, a gap of studies is identified that evaluate the effect of the practice of other sport modalities in what is the main modality.

In fact, when the type of sport practiced is evaluated, the evidence suggests that young people who practice individual sports have a higher development of ethical values than those who practice collective sports (Carneiro et al., 2013).

\section{CONCLUSIONS}

In the context of orienteering, the results suggest that this group of young practitioners has been consolidating the adoption of positive attitudes imbued with the values of sports ethics. Therefore, it is deduced that the cumulative practice of another sport does not appear to have significant interference for the adoption of negative attitudes in the practice of orienteering.

However, young practitioners who cumulatively develop other sports practices are more susceptible to adopting unsportsmanlike attitudes.

In sum, these results demonstrate not only the need for more research on this subject, but also highlight the need for greater assertiveness in the adoption of strategies that promote pro-ethical attitudes in sports in the various contexts of practice.

\section{REFERENCES}

Donnely, P., Young, K. (2001). The construction and confirmation of identity in sport subcultures. In Andrew Y.; \& Merrill, M. (Ed.). Comtemporary issues in sociology of sport (pp. 399-412). Champaign: Human Kinetics.

Gonçalves, C.E., (2013). Desportivismo e desenvolvimento de competências socialmente positivas. Lisboa: Edições Afrontamento.

Gonçalves, C. E., Silva, M. J. C., Chatzisarantis, N., Lee, M. J., \& Cruz, J. (2006). Tradução e validação do SAQ (Sports Attitudes Questionnaire) para jovens praticantes desportivos portugueses com idades entre os 13 e os 16 anos. Revista Portuguesa de Ciências do Desporto, 6(1), 38-49. https://doi.org/10.5628/rpcd.06.01.38 
Carneiro, D., Silva, E., Pereira, F., Santos, J., Silva, P., \& Gomes, T. (2013). Atitude desportivas dos jovens em função do tipo de modalidade praticada. In A. Pereira, F. Mendes, A. Figueiredo (Eds.), Livro de Resumos "Seminário Ética no Desporto: Move-te por Valores" (p.37). Viseu: Centro de Estudos em Educação, Tecnologias e Saúde - Instituto Politécnico de Viseu. https://doi.org/10.24873/j.rpemd.2018.06.223

Celestino, T., Sarmento, H., Santos, A., \& Pereira, A. (2018a). Atitudes desportivas dos jovens praticantes de orientação: a influência do contexto. Journal of Sport Pedagogy \& Research, 4(2), 11.

Celestino, T., Sarmento, H., Santos, A., \& Pereira, A. (2018b). Atitudes desportivas dos jovens praticantes de orientação: a influência do género e do nível competitivo. In $2 .^{\circ}$ Fórum REDESPP Desporto. Livro de Atas (pp.110). Leiria: REDESPP.

\section{(c) $(\mathrm{B})(\mathrm{EY}$}

This work is licensed under a Attribution-NonCommercial-NoDerivatives 4.0 International (CC BY-NC-ND 4.0). 


\title{
The thinking process of Football coaches: The training factors
}

\author{
GONÇALO CARVALHO ${ }^{1,2} \square$, JOSÉ F. RODRIGUES ${ }^{1,2}$ \\ ${ }^{1}$ Escola Superior de Desporto de Rio Maior, Instituto Politécnico de Santarém, Portugal \\ ${ }^{2}$ Centro de Investigação em Qualidade de Vida, IPSantarém/IPLeiria, Portugal
}

\begin{abstract}
This research is a qualitative study with focus on the thinking process of the Football coaches, related to the training factors. We interviewed ten coaches, with recent experience in the First and Second Portuguese Professional Football Leagues. The data collected was treated using the MAXQDA 2018® software. The analysis performed highlights that all participants indicate work the training factors in an integrated manner. They assume the importance of psychological factors for a constant sport performance. We are led to conclude that the approach to the training factors is related to the thinking process of the coach, materialized through the planning set, in the thoughts and interactive decisions, as well as in their beliefs and reflexions. Keywords: Coach; Football; Training factors; Sport performance; Thought.
\end{abstract}

Corresponding author. Sport Sciences School of Rio Maior - Polytechnic Institute of Santarém, Portugal.

E-mail: gfscarvalho@gmail.com

Supplementary Issue: Spring Conferences of Sports Science. International Seminar of Physical Education, Leisure and Health, 17-19 June 2019. Castelo Branco, Portugal.

JOURNAL OF HUMAN SPORT \& EXERCISE ISSN 1988-5202

(c) Faculty of Education. University of Alicante.

doi:10.14198/jhse.2019.14.Proc4.82 


\section{INTRODUCTION}

The coach responsibility in the improvement and development of a team, managing to obtain positive results, is universally recognized. Therefore, the study of the Training Factors (technical, tactical, physical, psychological) is relevant to understand how these are weighed by the coach in the performance of his duties.

The coach, guiding professional of sport training, applies his own knowledge in the execution of his functions. To accomplish his own challenges, the coach reveals skills resulting from the mobilisation, production and use of relevant knowledge, scientific, organizational, pedagogical and technical-practical (Rosado \& Mesquita, 2007).

This study involved interviews with ten coaches, professionals of reference in the Portuguese football scenario. It is in line with the researchers findings that highlight the influence of the coach in the quality of the sport experience (Rocha \& Silva, 2018) and highlight the importance of the research, that studies the thinking process of football coaches (Pinheiro et al., 2018), in search of an improvement of the training.

We underpin in this qualitative study two main objectives: i) to gather relevant information related to the subject; II) identify the options/decisions of the coaches in the approach to training factors, throughout the season.

\section{MATERIAL AND METHODS}

\section{Participants}

The study was guided by research questions that inquired the relevance and meaning attributed to training factors and sport performance, given the experiences of the professional coaches. To accomplish it, ten football coaches were included, with current or recent roles in senior men's teams, competing in a professional league in our country (I and II League). The coaches' participants are male, aged between 35 and 57 and have ten or more years of experience leading teams in different clubs, with experience in different age groups and in different competitive levels.

\section{Measures}

In this qualitative study, we have chosen only one data collection instrument: the semi-structured interview. We take into account the nature, the objectives and the research questions. This instrument has been previously tested and validated by two experts, researchers in Sport Sciences.

\section{Procedures}

The process of organizing, processing and analysing data was carried out after the transcription of the interviews and followed the steps that characterize the content analysis. A "floating reading" was performed, followed by a detailed analysis of the material. After, were followed the procedures of coding and categorizing and, subsequently, the operationalization of the results treatment, process in which inference and interpretation were used (Bardin, 1977). We used the MAXQDA 2018® software.

\section{Analysis}

Established the relationship between the questions of the interview and the variables in study, we considered 5 codes for the Training Factors (Integrated, Physical, Psychological, Technical and Tactical) and 4 for the Sport Performance (Multifactorial, Oscillating, Constant, Meaningless). 


\section{RESULTS}

The study allowed us to identify the existence of some influences for decision-making/methodological options, with respect to the intervention of the variables considered in the research. (Table 1).

The Training Factor most indicated by the coaches was the Integrated and the Sport Performance most valued was the Constant. It is noteworthy that, of the 46 records obtained from the ten interviews, the code Technical in the variable Training Factors is the only one in which no occurrence of speech was observed with the interviewees. Nevertheless, that code was considered once it had been admitted as a possibility of response by the participants.

Table 1. Results of the coding process (frequency units)

\begin{tabular}{clcc}
\hline \multirow{2}{*}{ Variables } & Codes & \multicolumn{2}{c}{ Frequency Units } \\
\cline { 3 - 4 } & Integrated & 11 & Total \\
\hline \multirow{3}{*}{ Training Factors } & Psychological & 9 & $44 \%$ \\
& Physical & 1 & $36 \%$ \\
& Tactical & 4 & $16 \%$ \\
& Technical & 0 & $0 \%$ \\
& Multifactorial & 3 & $13 \%$ \\
& Oscillating & 2 & $10 \%$ \\
& Constant & 14 & $67 \%$ \\
& Meaningless & 2 & $10 \%$ \\
\hline
\end{tabular}

\section{DISCUSSION}

This analysis allowed us to prove what was pointed out by Carvalhal et al. (2014), Azevedo (2011), Ferraz (2013), among others, regarding the perspective of work in an integrated way, taken in relation to the training factors. The relevance attributed to psychological factors (Coach J: "I believe that the psychological factors, naturally, are evidenced in everything"; Coach E: "Everything is fundamental but the head commands everything") crosses with the theoretical perspective of Garganta $(2015$, p. 5), who shows us how "(...) it does not seem viable a positive and modern football, thought and carried out by distorted or aged minds".

The search for a constant sport performance, mentioned by the ten participants (Coach I: "The player must always be well, always on fire") aligns with the recommended by the most recent theories (Carvalhal et al., 2014), in order to maintain a constant (and maximum) level of the players sport performance.

\section{CONCLUSIONS}

The conclusions we present report to the objectives of the study and are evidenced in the data obtained through the semi-structured interview performed to the ten coaches participating in this research. The aforementioned data led us to conclude that the coaches involved: a) adopt an integrated work of the different training factors; b) seek constant sport performance.

The fact that the psychological training factors are appropriated by the participants with some emphasis, take us to a relationship with the coaches thinking process, namely at the level i) of the influence of the characteristics of the coach (social, cultural, mental) in his decision-making; ii) of coaches taking decisions 
in complex and uncertain contexts, taking into account their thoughts and judgments; iii) of coaches assuming, in their daily and professional function, the assumptions planning, thoughts and interactive decisions, theories and beliefs.

\section{REFERENCES}

Azevedo, J. (2011). Por dentro da táctica. Estoril: Prime Books.

Bardin, L. (1977). Análise de conteúdo.Lisboa: Edições 70.

Carvalhal, C., Lage, B., \& Oliveira, J. (2014). Futebol - Um saber sobre o saber fazer (2a edição). Estoril: Prime Books.

Ferraz, F. (2013). A importância da Periodização Tática no futebol moderno. Uma breve abordagem às metodologias de treino utilizadas em Portugal. Treino Científico, (14), 14-16.

Garganta, J. (2015). Revisitando as oficinas do futebol, atrás do palco. Treino Científico, (25), 3-5.

Pinheiro, V., Belchior, D., Sousa, P., \& Santos, F. (2018). Manual para treinadores de futebol de excelência. (1 ${ }^{\mathrm{a} E}$ dição). Estoril: Prime Books.

Rocha, T., \& Silva, M. (2018). O papel do treinador na sociedade. In J. Rodrigues et al (Eds), Desporto, Desenvolvimento e Bem-estar - Fórum Politécnico \#4. REDESPP, IPSantarem-ESDRM, 65-68.

Rosado, A., \& Mesquita, I. (2007). A formação para ser treinador. Em Congresso Internacional de Jogos Desportivos (Vol. 1). Acedido em 7 de Julho, 2018, em http://areas.fmh.utl.pt/ arosado/afpsr.pdf

\section{(9)}

This work is licensed under a Attribution-NonCommercial-NoDerivatives 4.0 International (CC BY-NC-ND 4.0). 


\title{
Differences of Imagery ability between youth soccer and swimming practitioners
}

\author{
PEDRO DUARTE-MENDES ${ }^{1,2} \triangle$, DANIEL A. MARINHO ${ }^{3,4}$, JOÃO SERRANO1,2, RUI PAULO 1,2, \\ ANTÓNIO FAUSTINO ${ }^{1,2}$, ANDRÉ RAMALHO ${ }^{1,2}$, JOÃO PETRICA ${ }^{1,2}$ \\ ${ }^{1}$ Department of Sports and Well-being, Polytechnic Institute of Castelo Branco, Castelo Branco, Portugal \\ ${ }^{2}$ SHERU - Sport, Health \& Exercise Research Unit, Polytechnic Institute of Castelo Branco, Castelo Branco, \\ Portugal \\ ${ }^{3}$ Department of Sport Sciences, University of Beira Interior, Covilhã, Portugal \\ ${ }^{4}$ Research Centre in Sport Sciences, Health Sciences and Human Development (CIDESD), Covilhã, Portugal
}

\begin{abstract}
Imagery (IM) is one of the most popular psychological techniques used by athletes and coaches to improve performance at competition level. Some authors refer that individuals with good movement IM ability learn better and improve their physical performances. Therefore, the purpose of this study was to verify the differences and the magnitude of effects on the imagery ability and three modalities (Visual Internal= Vl; Visual External $=\mathrm{VE} ; \mathrm{K}=$ Kinesthesic) between youth soccer (team sport) and swimming (individual sport) practitioners. Sixty-two soccer practitioners and twenty-seven swimming practitioners from the Castelo Branco district volunteered to participate in this study. The Movement Imagery Questionnaire MIQ - 3 Portuguese version (Mendes et al., 2016) was used to assess imagery ability. Descriptive statistics (mean \pm standard deviation) was performed for all variables under analysis. Data normality was tested using the Kolmogorov-Smirnov. Mann-Whitney and T-Test for Independent sample were used to verify the differences between groups. The magnitude of the effects was also calculated. The results suggest differences between groups $(p<0,05)$ in all analysed variables $(\mathrm{MIQ}-3, \mathrm{VI}, \mathrm{VE}, \mathrm{K})$. The soccer practitioners reveal better scores in the total MIQ-3 Scores and in all subscales. Keywords: Imagery ability; Team sports; Individual sports.
\end{abstract}

Corresponding author. Department of Sports and Well-being, Polytechnic Institute of Castelo Branco, Rua Prof. Dr. Faria de Vasconcelos, 6000-266 Castelo Branco, Portugal.

E-mail: pedromendes@ipcb.pt

Supplementary Issue: Spring Conferences of Sports Science. International Seminar of Physical Education, Leisure and Health, 17-19 June 2019. Castelo Branco, Portugal.

JOURNAL OF HUMAN SPORT \& EXERCISE ISSN 1988-5202

(c) Faculty of Education. University of Alicante.

doi:10.14198/jhse.2019.14.Proc4.82 


\section{INTRODUCTION}

Imagery (IM) is one of the most popular psychological techniques used by athletes and coaches to improve performance at competition level. In general, IM can be defined as the mental creation or recreation of an experience from information stored in the memory, without physical movement. The IM ability can be evaluated in three different modalities such as the kinesthetic, internal visual and external visual, to get better results in its evaluation and application processes (Cumming \& Williams, 2013). Martin, Moritz and Hall (1999) claim that individuals with good movement IM ability learn better and improve their physical performances. According to Williams and Cumming (2012), the individual differences in IM ability may be related to genetic differences and to experience in sport. Therefore, the purpose of this study was verifying the differences and the magnitude of effects on the imagery ability and three modalities (Visual Internal= VI; Visual External =VE; K=Kinesthesic) between soccer (team sport) and swimming (individual sport) young practitioners. Based on the review of literature, we expect that there will be differences between the groups and that the soccer group presents better results.

\section{MATERIAL AND METHODS}

\section{Participants}

Sixty-two soccer practitioners (age $=16,4 \pm 1,34 ;$ male $=31$, female $=1$ ) and twenty-seven swimming practitioners (age $=15,7 \pm 2,29$; male $=11$, female $=16$ ) from Castelo Branco district. All the subjects were federated in their sport activities and had at least 3 hours of weekly practice. All the participants signed the consent form according to the Declaration of Helsinki.

\section{Measures}

This study was a cross-sectional analysis, whereby subjects performed The Movement Imagery Questionnaire MIQ - 3 Portuguese version (Mendes et al., 2016), assessed in a single session. This instrument evaluates the imagery ability with three subscales to assess kinaesthetic, internal and external visual imagery.

\section{Procedures}

The instrument was applied in similar places and settings for all participants, in a room with the maximum number of five athletes and all subject completed the questionnaire individually. The right environment was provided so that the athletes could be concentrated while completing the questionnaire. Data were collected anonymously to guarantee its confidentiality, making sure it would not be individually passed on to third parties.

\section{Analysis}

Descriptive statistics (mean \pm standard deviation) was performed for all variables under analysis. Data normality was tested using the Kolmogorov-Smirnov. Mann-Whitney and T-Test for Independent sample were used to verify the differences between groups. The magnitude of the effects was also calculated (Cohen d): 0-0.2, trivial; 0.21-0.6, small; 0.61-1.20, moderate; 1.21-2.0, big; > 2.0, very big.

\section{RESULTS}

The results suggest differences between groups in all analysed variables (Table 1). The soccer practitioners reveal better scores in the total MIQ-3 Scores and in all subscales. 
Table 1. Descriptive statistic, differences between groups, and effect size

\begin{tabular}{|c|c|c|c|c|c|c|c|c|}
\hline MIQ-3 & Sport & $\mathbf{N}$ & $\mathbf{M}$ & sd & $p$ & $\eta^{2}$ & $d$ & $\mathrm{C} \mid 90 \%$ \\
\hline Total_K & $\begin{array}{c}\text { Soccer } \\
\text { Swimming }\end{array}$ & $\begin{array}{l}62 \\
27\end{array}$ & $\begin{array}{l}23.4 \\
19.7\end{array}$ & $\begin{array}{l}3.1 \\
4.1\end{array}$ & $0.000^{* a}$ & 0.17 & 0.094 (trivial) & -- \\
\hline Total_VI & $\begin{array}{l}\text { Soccer } \\
\text { Swimming }\end{array}$ & $\begin{array}{l}62 \\
27\end{array}$ & $\begin{array}{l}25.2 \\
21.1\end{array}$ & $\begin{array}{l}2.4 \\
4.0\end{array}$ & $0.000^{* b}$ & -- & 1.371 (big) & 0.956-1.786 \\
\hline Total_VE & $\begin{array}{c}\text { Soccer } \\
\text { Swimming }\end{array}$ & $\begin{array}{l}62 \\
27\end{array}$ & $\begin{array}{l}24.8 \\
22.0\end{array}$ & $\begin{array}{l}2.7 \\
4.3\end{array}$ & $0.004^{* b}$ & -- & 0.841 (moderate) & $0.841-1.234$ \\
\hline Total_MIQ3 & Soccer & 62 & 73.3 & 6.9 & $0.000^{* a}$ & 0.216 & 1.05 (moderate) & --- \\
\hline
\end{tabular}

\section{DISCUSSION}

The aim of our study was to verify the differences and the magnitude of effects on the imagery ability and the three modalities (VI, VE, K) between youth soccer (team sport) and swimming (individual sport) practitioners. Our findings suggest that youth soccer practitioners have better scores in imagery ability when compared with youth swimming practitioners. In the subscales, the soccer practitioners present better results in visual internal modality and the swimming practitioners in the visual external modality. Cumming and Williams (2013) refer that the ability to generate and control images is present in all individuals but to varying degrees, and that the life experiences can influence the ability as a result of the development of the motor process. Some studies have been conducted showing the characteristics of athletes' imagery use across different sports, and individual versus team sports, but few have studied the imagery ability. Previous studies reported that team sports use more imagery than those individual sports (Hall et al.,1998). Our results are consistent with the literature that suggests that person's capability to generate and control images is partly fixed and partly modifiable, with the former being reflected by the developmental changes occurring as a result of maturation and life experience (Williams \& Cumming, 2012).

\section{CONCLUSIONS}

The present results suggest that youth soccer practitioners have better scores in imagery ability and in the three modalities when compared with youth swimming practitioners. These results are important to help sport professionals in the use of imagery interventions, taking in account the imagery ability.

\section{REFERENCES}

Cumming, J., \& Williams, S. (2013). Introducing the revised applied model of deliberate imagery use for sport, dance, exercise, and rehabilitation. Movement \& Sport Sciences - Science \& Motricité, 82, 6981. https://doi.org/10.1051/sm/2013098

Hall, C., Mack, D., Paivio, A. \& Hausenblas, H. (1998). Imagery use by athletes: Development of the Sport Imagery Questionnaire. Int J Sport Psychol, 29, 73-89. https://doi.org/10.1037/t52953-000

Martin, K., Moritz, S. \& Hall, C. (1999). Imagery Use in Sport: A Literature Review and Applied Model. J Appl Sport Psychol,13 (3), 245-268. https://doi.org/10.1123/tsp.13.3.245

Mendes, P., Marinho, D., Petrica, J., Silveira, P., Monteiro, D., \& Cid, L. (2016). Tradução e Validação do Movement Imagery Questionnaire - 3 (MIQ-3) com Atletas Portugueses. Motri, 12(1), 149-158. https://doi.org/10.6063/motricidade.7006 
Williams, S., \& Cumming, J. (2012). Athletes' ease of imaging predicts their imagery ability and observational learning use. Sychol Sport Exerc, 13(4), 363-370. https://doi.org/10.1016/.psychsport.2012.01.010

\section{(c) (i) (3)}

This work is licensed under a Attribution-NonCommercial-NoDerivatives 4.0 International (CC BY-NC-ND 4.0). 


\title{
Comparison of physical fitness between young and middle-aged adults
}

\author{
DINEIA A. LUCAS ${ }^{1} \unlhd$, FERNANDA SILVA ${ }^{1}$, ANDRÉ RAMALHO ${ }^{1,2}$, RUI PAULO ${ }^{1,2}$, JOÃO SERRANO ${ }^{1,2}$, \\ PAULO SILVEIRA ${ }^{1,2}$, PEDRO DUARTE-MENDES ${ }^{1,2}$ \\ ${ }^{1}$ Department of Sports and Well-being, Polytechnic Institute of Castelo Branco, Castelo Branco, Portugal \\ ${ }^{2}$ SHERU - Sport, Health \& Exercise Research Unit, Polytechnic Institute of Castelo Branco, Castelo Branco, \\ Portugal
}

\begin{abstract}
Aging is characterized by a loss of Physical Fitness. There is a progressive decline of skeletal muscle performance from young adulthood to middle age compromising Strength, Flexibility and Aerobic Capacity. Understanding changes in Physical Fitness during middle age provide relevant information to professionals. The purpose of this study was to analyse differences in Aerobic Capacity $\left(\mathrm{VO}_{2 \mathrm{max}}\right)$, and upper and lower limb strength and flexibility between young and middle-aged adults. One hundred and eleven adults (young adults: $\mathrm{n}=58$; middle-aged adults: $\mathrm{n}=53$ ) volunteered to participate in this study. Each participant performed 3 trials of Squat Jump Test (SJ) followed by 3 trials of Handgrip (HG), Seat and Reach (SR), Behind Back Reach (BBR) and Chester Step Test (CST). The variables under analysis were SJ power (SJ_P), HG, SR, $\mathrm{BBR}$ and $\mathrm{VO}_{2 \text { max. }}$. Data normality was tested using Kolmogorov-Smirnov. Mann-Whitney and T-Test for Independent samples were used to verify differences between groups. Results reveal no significant differences between groups in BBR $(p=0.026, d=-0.786)$. However, Young Adults revealed higher values in HG $(p=0.045, \eta 2=0.036, d=0.338)$, SJ_P $(p=0.032, \eta 2=0.410, d=0.416), S R(p=0.000, \eta 2=0.013$, $d=0.231)$ and $\mathrm{VO}_{2 \max }(p=0.000, \eta 2=0.199, d=0.998)$. We can conclude that there is a significant loss of $\mathrm{VO}_{2 m a x}$, upper and lower limb strength and lower limb flexibility from young adulthood to middle age. Keywords: Young adults; Middle-aged adults; Physical fitness; Aging.
\end{abstract}

\footnotetext{
Corresponding author. Department of Sports and Well-being, Polytechnic Institute of Castelo Branco, Rua Prof. Dr. Faria de Vasconcelos, 6000-266 Castelo Branco, Portugal.

E-mail: lucasdineia@gmail.com

Supplementary Issue: Spring Conferences of Sports Science. International Seminar of Physical Education, Leisure and Health, 17-19 June 2019. Castelo Branco, Portugal.

JOURNAL OF HUMAN SPORT \& EXERCISE ISSN 1988-5202

(C) Faculty of Education. University of Alicante.

doi:10.14198/jhse.2019.14.Proc4.82
} 


\section{INTRODUCTION}

Aging is a life-long process characterized by a progressive loss of Physical Fitness (Kirkwood, 2017). Sure enough, there is a continuous loss of skeletal muscle performance compromising Strength, Flexibility and Aerobic Capacity over time (Hollenberg et al., 2006). Moreover, Physical Fitness is associated with higher quality of life among older adults (Kirkwood, 2017).

Understanding which Physical Fitness parameters decline faster during middle age provide relevant information to Sports and Health professionals who may optimise exercise prescription in order to attenuate the downfall of Physical Fitness among middle-aged adults.

Therefore, the purpose of this study was to analyse the differences in $\mathrm{VO}_{2 m a x}$, upper and lower limb strength and upper and lower limb flexibility between young and middle-aged adults. Based in the review of literature we expected significantly higher scores in young adults for all parameters.

\section{MATERIAL AND METHODS}

\section{Participants}

One hundred and eleven adults (young adults: $n=58$; age $=28.69 \pm 6.44$ years old, height $=1.67 \pm 0.09$ m, body mass $=69.63 \pm 13.46 \mathrm{~kg}$; middle-aged adults: $n=53 ;$ age $=50.09 \pm 6.21$ years old, height $=1.64$ $\pm 0.09 \mathrm{~m}$, body mass $=73.06 \pm 14.59 \mathrm{~kg}$ ) volunteered to participate in this study.

\section{Measures}

This study was a cross-sectional analysis, whereby subjects performed a Squat Jump (SJ) from a contact mat using the Chronojump system (Chronojump-Boscosystem, Spain), followed by a HG using a manual dynamometer designed by Lafayette $\Theta$, a SR, a BBR and CST on a using a Polar $\mathrm{H} 10$ heart rate sensor designed by Polar®.

\section{Procedures}

After arriving facilities, ratters gave participants standardized instructions about tests procedures. Each participant performed 3 trials of the SJ with 1 minute of rest between each trial and 3 minutes of rest prior commencing the subsequent tests. Afterwards, 3 trials of HG, SR and BBR were performed in a randomassigned order, allowing 30 seconds of rest between trials and 2 minutes delay between the aforementioned tests. Finally, participants underwent a CST consisting of 5 stages, each of 2 minutes duration. The step cadence was set with a metronome increasing by 5 steps/min every 2 minutes. $\mathrm{VO}_{2 \max }$ was calculated with the CST Calculator, and the highest values of SJ, HG, SR and BBR were considered for analysis. Dependent variables were SJ_P, HG, SR, BBR and $\mathrm{VO}_{2 m a x}$. Being $m$ the body mass $(\mathrm{kg})$, and $\mathrm{h}$ the falling height $(\mathrm{m}), \mathrm{P}$ was calculated as follows (Fox and Mathews, 1974):

$$
P=\sqrt{4.9} * 9.8 * m * \sqrt{h} \mathrm{~W}
$$

\section{Analysis}

Descriptive statistics (mean \pm standard deviation) were performed for all variables under analysis. Data normality was tested using Kolmogorov-Smirnov test. Mann-Whitney and T-Test for Independent samples 
were used to verify differences between groups. Effects' magnitude (Cohen d) were also calculated and classified as follows (Lakens, 2013) : small $(d=0.2)$; moderate $(d=0.5)$; large $(d=0.8)$.

\section{RESULTS}

Although we did not find significant differences between groups in BBR, Young Adults reveal higher values in HG, SJ_P, SR and VO2max. Detailed results are listed in Table 1.

Table 1. Descriptive statistics (Mean \pm SD), differences between groups (p), and effect size ( $\eta 2, d, I C 90 \%)$.

\begin{tabular}{|c|c|c|c|c|c|c|}
\hline & Age Group & Mean \pm SD & $p$ & $\eta^{2}$ & $d$ & $\mathrm{Cl} 190 \%$ \\
\hline Handgrip (kg) & $\begin{array}{l}\text { Young Adults } \\
\text { Middle-Aged }\end{array}$ & $\begin{array}{l}33.22 \pm 13.31 \\
28.59 \pm 15.13\end{array}$ & $0.045^{\star a}$ & 0.036 & 0.388 & --- \\
\hline Squat Jump Power & Young Adults & $720.86 \pm 188.9$ & & & & \\
\hline (W) & Middle-Aged & $\begin{array}{c}643.14 \pm \\
249.09\end{array}$ & $0.032^{* a}$ & 0.410 & 0.416 & --- \\
\hline $\begin{array}{l}\text { Seat and Reach } \\
(\mathrm{cm})\end{array}$ & $\begin{array}{l}\text { Young Adults } \\
\text { Middle-Aged }\end{array}$ & $\begin{array}{l}33.13 \pm 8.07 \\
27.18 \pm 6.96\end{array}$ & $0.000^{* *_{a}}$ & 0.013 & 0.231 & -- \\
\hline $\begin{array}{l}\text { Behind Back Reach } \\
\text { (cm) }\end{array}$ & $\begin{array}{l}\text { Young Adults } \\
\text { Middle-Aged }\end{array}$ & $\begin{array}{c}1.35 \pm 6.51 \\
-2.08 \pm 9.71\end{array}$ & $0.226^{b}$ & -- & $\begin{array}{c}- \\
0.786\end{array}$ & $\begin{array}{r}-1.11-- \\
0.462\end{array}$ \\
\hline $\mathrm{VO}_{2 \max }(\mathrm{ml} / \mathrm{kg} / \mathrm{min})$ & $\begin{array}{l}\text { Young Adults } \\
\text { Middle-Aged }\end{array}$ & $\begin{array}{c}43.26 \pm 8.99 \\
36.22 \pm 5.87\end{array}$ & $0.000^{* *_{a}}$ & 0.199 & 0.998 & -- \\
\hline
\end{tabular}

* $p<0,05$; a Mann-Whitney Test; $b$ T test for independent samples; $\eta 2$ - Eta squared; $d$ - effect size; $\mathrm{Cl}$ = Confidence Interval.

\section{DISCUSSION}

Our findings suggest that young adults perform better in $\mathrm{HG}, \mathrm{SJ}, \mathrm{SR}$ and $\mathrm{VO}_{2 \max }$ when compared with middleaged adults. Fleg et al. (2005) have also verified a decrease of $\mathrm{VO}_{2 \max }$ from young adulthood to middle age. Furthermore, Lee et al. (2015) verified a progressive loss of upper and lower limb strength. Considering lower limb flexibility, Holland et al. (2002) also referred a significant loss of range of motion from young adulthood to middle age. However, the results do not reveal significant differences in BBR between groups. The lack of differences of upper limb flexibility between groups can be attributed to sports practice among subjects since physical activity is associated with significantly lower age-related loss of upper limb flexibility (Holland et al., 2002).

\section{CONCLUSIONS}

In short, there is a significant loss of $\mathrm{VO}_{2 \max }$, upper and lower limb strength and lower limb flexibility from young adulthood to middle age. In order to attenuate the downfall of Physical Fitness among middle-aged adults, Sports and Health professionals should consider these findings when prescribing exercise.

\section{REFERENCES}

Fleg, J.L., Morrell, C.H., Bos, A.G., Brant, L.J., Talbot, L.A., Wright, J.G., and Lakatta, E.G. (2005). Accelerated longitudinal decline of aerobic capacity in healthy older adults. Circulation, 112(5), 674682. https://doi.org/10.1161/circulationaha.105.545459 
Fox, E.L., and Mathews, D.K. (1974). Interval Training: Conditioning for Sports and general fitness. Philadelphia: Saunders Company.

Hollenberg, M., Yang, J., Haight, T.J., and Tager, I.B. (2006). Longitudinal changes in aerobic capacity: implications for concepts of aging. J Gerontol A Biol Sci Med Sci, 61(8), 851-858. https://doi.org/10.1093/gerona/61.8.851

Holland, G.J., Tanaka, K., Shigematsu, R., and Nakagaichi, M. (2002). Flexibility and physical functions of older adults: a review. J Aging Phys Act, 10(2), 169-206. https://doi.org/10.1123/japa.10.2.169

Kirkwood, T.B.L. (2017). Why and how are we living longer? Exp Physiol, 102(9), 1067-74. https://doi.org/10.1113/ep086205

Lakens, D. (2013). Calculating and reporting effect sizes to facilitate cumulative science: a practical primer for t-tests and ANOVAs. Front Psychol, 4, 863. https://doi.org/10.3389/fpsyg.2013.00863

Lee, Y., Chang, L., Chung, W., Lin, T., and Shiang, T. (2015). Does functional fitness decline in accordance with our expectation? - a pilot study in healthy female. BMC Sports Sci Med Rehabil, 717. https://doi.org/10.1186/s13102-015-0012-y 


\title{
Adherence to physical activity guidelines and body composition in elderly people using objective measurements
}

FERNANDA M. SILVA ${ }^{1} \triangle$, JOÃO PETRICA ${ }^{1,2}$, JOÃO SERRANO1,2, RUI PAULO ${ }^{1,2}$, ANDRÉ RAMALHO ${ }^{1,2}$, DINEIA A. LUCAS ${ }^{1}$, JOSÉ PEDRO FERREIRA ${ }^{3}$, PEDRO DUARTE-MENDES 1,2

${ }^{1}$ Department of Sports and Well-being, Polytechnic Institute of Castelo Branco, Castelo Branco, Portugal

${ }^{2}$ SHERU - Sport, Health \& Exercise Research Unit, Polytechnic Institute of Castelo Branco, Castelo Branco, Portugal

${ }^{3}$ Research Unit for Sport and Physical Activity (CIDAF), University of Coimbra, Portugal

\begin{abstract}
The number of overweight and obese older adults is increasing. The aim of this paper was to verify the effects and differences regarding body composition between two groups of elderly people: those who met (active) and those who did not meet (non-active) the Global Recommendations on Physical Activity for Health. This cross-sectional study sample included 71 elderly individuals (72.44 \pm 5.68 years old), both male and female, divided in two groups: the group of active individuals $(\mathrm{N}=34)$ and the group of non-active individuals $(\mathrm{N}=37)$. Physical activity time was assessed using the ActiGraph ${ }^{2}$ GT1M Accelerometer. A Body Composition Analyser Inbody $270 \circledR$, a stadiometer SECA (Germany, Hamburg) and a flexible measuring tape (Rosscraft $($ ) were hence used to assess anthropometric values. In order to analyse data, descriptive and inferential statistics were performed. Mann-Whitney test and T-test were used to compare groups. Inference methods based on effect size (d-Cohen) were also used. Significant differences were found between groups, especially regarding BMI $(p=0.048)$, fat mass $(p=0.019)$ and body fat percentage $(p=0.004)$ levels. Results suggest that promoting MVPA in the elderly might have a positive influence on their body composition, leading to a decrease in adiposity indicators. Keywords: Ageing; Accelerometery; Fat mass; Adiposity; Elderly people; Physical activity.
\end{abstract}

Corresponding author. Department of Sports and Well-being, Polytechnic Institute of Castelo Branco, Rua Prof. Dr. Faria de Vasconcelos, 6000-266 Castelo Branco, Portugal.

E-mail: geral.fernandasilva@gmail.com

Supplementary Issue: Spring Conferences of Sports Science. International Seminar of Physical Education, Leisure and Health, 17-19 June 2019. Castelo Branco, Portugal.

JOURNAL OF HUMAN SPORT \& EXERCISE ISSN 1988-5202

(c) Faculty of Education. University of Alicante.

doi:10.14198/jhse.2019.14.Proc4.82 


\section{INTRODUCTION}

Aging is followed by changes in body composition, generally leading to a decrease in muscle mass and an increase in total and central adiposity (Reinders et al., 2016). These changes are common in elderly and are related to numerous health risks (e.g., hypertension, hyperlipidaemia, diabetes, functional decline) (Santanasto et al., 2017). It is recommended that elderly participate in at least 30 minutes of moderate-to vigorous-intensity activity (MVPA) $(\geq 3 \mathrm{METs}$ ) per day in order to achieve health benefits and prevent obesity (World Health Organization [WHO], 2010). Subjects who do not achieve the recommended levels of MVPA are considered to be physically inactive (Sedentary Behaviour Research Network, 2012). Studies have confirmed the positive impact of moderate-to-vigorous-intensity physical activity on body composition in elderly (Pelclová et al., 2012; Gába et al., 2009). In fact, it is known that most active subjects have lower average body adiposity values (Pelclová et al., 2012; Gába et al., 2009). The aim of this paper was to verify the effects and differences regarding body composition between two groups of old people: those who met (active) and those who did not meet (non-active) with the Global Recommendations on Physical Activity for Health (WHO, 2010).

\section{MATERIAL AND METHODS}

\section{Participants}

This cross-sectional study sample included 71 elderly individuals (72.44 \pm 5.68 years old), both male and female. Participants were divided (through the accelerometry record) into an active group if they completed 30 minutes daily MVPA ( $N=34)(\overline{\mathrm{x}} \mathrm{SD}, 70.79 \pm 4.94$ years $)$ and into an inactive group if they did not complete the 30 minutes daily MVPA ( $N=37$ ) ( $\overline{\mathrm{x} \pm S D}, 73.95 \pm 5.95$ years). The sample recruitment was carried out in senior universities and health promotion fairs. All subjects signed an informed consent form.

\section{Measures}

Physical activity time was assessed using the ActiGraph® GT1M Accelerometer. A Body Composition Analyser Inbody $270 \AA$, a stadiometer SECA® (Germany, Hamburg) and a flexible measuring tape (Rosscraft(囚) were hence used to assess body composition.

\section{Procedures}

The accelerometer was utilized during waking hours for at least five consecutive days. The devices were activated on the first day at 5.00 a.m., and data was recorded in 15 -s epochs. The study included the results from participants with at least three valid days (including one weekend day) and 10h of utilization time per day. MVPA was defined as $\geq 2020$ counts/ min (Troiano et al., 2008). Weight, fat mass (FM) and body fat percentage were measured by bioelectric impedance balance. Waist circumference (WC) was measured using a flexible measuring tape. Body mass index (BMI) and waist-to-height ratio (WHtR) were calculated.

\section{Analysis}

Descriptive statistics (mean \pm standard deviation) was performed for all variables under analysis. Data normality was tested using Kolmogorov-Smirnov test. Mann-Whitney test and T-test were used to compare groups. Effect Size (Cohen d) was also calculated and classified as suggested by Hopkins et al. (2009): 00.2 , trivial; $0.21-0.6$, small; $0.61-1.20$, moderate; $1.21-2.0$, big; > 2.0, very big. 


\section{RESULTS}

There were significant differences between groups in BMI ( $p=0.048$; $d=-0.48$, small effect), $F M(p=0.019$; $d=-0.57$, small effect) and body fat percentage ( $p=0.004 ; d=-0.71$, moderate effect), being the active group the one with lower mean adiposity values.

\section{DISCUSSION}

Our results are congruent with those presented by Gába et al. (2009) and Pelclová et al. (2012), where it was found that the most active women had lower mean adiposity values. According to the same authors, adherence to the Global Recommendations of Physical Activity for Health is extremely important in elderly people either to improve the quality of life as well as from the point of view of public health.

\section{CONCLUSIONS}

Evidence suggests that adherence to these guidelines might have a positive influence on elderly body composition, leading to a decrease in adiposity values.

\section{REFERENCES}

Gába, A., Pelclová, J., Pridalová, M., Riegerová, J., Dostálová, I., and Engelová, L. (2009). The evaluation of body composition in relation to physical activity in 56-73 year old women: a pilot study. Acta Gymnica, 39(3), 21-30.

Hopkins, W., Marshall, S., Batterham, A., and Hanin, J. (2009). Progressive statistics for studies in sports medicine and exercise science. Med Sci Sports Exerc, 41(1), 3-12. https://doi.org/10.1249/mss.0b013e31818cb278

Pelclová, J., Gába, A., Tlucáková, L., and Póspiech, D. (2012). Association between physical activity (PA) guidelines and body composition variables in middle-aged and older women. Arch Gerontol Geriatr, 55(2), 14-20. https://doi.org/10.1016/j.archger.2012.06.014

Reinders, I., Visser, M., and Schaap, L. (2016). Body weight and body composition in old age and their relationship with fragility. Curr Opin Clin Nutr Metab Care, 20(1), 11-15. https://doi.org/10.1097/MC0.0000000000000332

Santanasto, A., Goodpaster. B., Kritchevsky, S., Milijkovic, I., Satterfield, S., Schwartz, A., ... Newman, A. (2017). Body composition Remodeling and Mortality: The Health Aging and Body Composition Study. J Gerontol A Biol Sci Med Sci, 72(4), 513-519. https://doi.org/10.1093/gerona/glw163

Sedentary Behaviour Research Network (2012). Letter to the editor: Standardized use of the terms "sedentary" and "sedentary behaviours." Appl Physiol Nutr Metab, 37(3), 540-542. https://doi.org/10.1139/h2012-024

Troiano, R., Berrigan, D., Dodd, K., Mâsse, L., Tilert, T., and Mcdowell, M. (2008). Physical Activity in the United Sates Measured by accelerometer. Med Sci Sports Exerc, 40(1), 181-188. https://doi.org/10.1249/mss.0b013e31815a51b3

World Health Organization (2010). Global Recommendations on Physical Activity for Health. Geneva: World Health Organization. Retrieved from: http://apps.who.int/iris/bitstream/10665/44399/1/9789241599979_eng.pdf 


\section{(c) (i) (3)}

This work is licensed under a Attribution-NonCommercial-NoDerivatives 4.0 International (CC BY-NC-ND 4.0). 


\title{
Correlation between pulmonary function and aerobic capacity in middle-aged adults
}

\author{
DINEIA A. LUCAS ${ }^{1}$, FERNANDA M. SILVA ${ }^{1}$, RUIPAULO ${ }^{1,2}$, JOÃO PETRICA ${ }^{1,2}$, ANDRÉ RAMALHO $^{1,2}$, \\ JOÃO ROCHA ${ }^{1,2}$, PEDRO DUARTE-MENDES ${ }^{1,2}$ \\ ${ }^{1}$ Department of Sports and Well-being, Polytechnic Institute of Castelo Branco, Castelo Branco, Portugal \\ 2SHERU - Sport, Health \& Exercise Research Unit, Polytechnic Institute of Castelo Branco, Castelo Branco, \\ Portugal
}

\begin{abstract}
There seems to be an association between aerobic capacity and pulmonary function. Nevertheless, this correlation remains unclear in middle-aged adults populations. Therefore, the purpose of this study was to verify the correlation between pulmonary function and aerobic capacity in middle-aged adults populations. Forty-two middle-aged adults (male: $n=19$; female: $n=23$ ) volunteered to participate in this study. Each participant performed one Chester Step Test (CST) followed by 3 to 8 spirometric trials. The variables under analysis were maximal aerobic capacity $\left(\mathrm{VO}_{2 \mathrm{max}}\right)$, predicted forced vital capacity (FVC\%), predicted forced expiratory volume in $1 \mathrm{sec}$ (FEV1\%), and predicted peak expiratory flow (PEF\%). Data normality was tested using the Kolmogorov-Smirnov, and Spearman's rank correlation coefficient $(r)$ and coefficient of determination ( $\mathrm{r} 2$ ) were used to verify correlations between $\mathrm{VO}_{2 \max }$ and $\mathrm{FVC} \%$, $\mathrm{FEV} 1 \%$ and $\mathrm{PEF} \%$. Even though we did not find a significant correlation between $\mathrm{VO}_{2 \max }$ and $\mathrm{FVC} \%(r=0.076, r 2=0.041, p=0.632)$, we verified a positive correlation between $\mathrm{VO}_{2 \max }$ and $\mathrm{FEV} 1 \%(r=0.389, \mathrm{r} 2=0.143, p=0.011)$ and also between $\mathrm{VO}_{2 \max }$ and PEF\% ( $\left.r=0.405, r 2=0.098, p=0.008\right)$. Careful consideration should be given to aerobic exercise prescription since it should be done with the right purpose. Keywords: Middle-aged adults; Pulmonary function; Aerobic capacity; $\mathrm{VO}_{2 m a x}$; Spirometry.
\end{abstract}

Corresponding author. Department of Sports and Well-being, Polytechnic Institute of Castelo Branco, Rua Prof. Dr. Faria de Vasconcelos, 6000-266 Castelo Branco, Portugal.

E-mail: lucasdineia@gmail.com

Supplementary Issue: Spring Conferences of Sports Science. International Seminar of Physical Education, Leisure and Health, 17-19 June 2019. Castelo Branco, Portugal.

JOURNAL OF HUMAN SPORT \& EXERCISE ISSN 1988-5202

(c) Faculty of Education. University of Alicante.

doi:10.14198/jhse.2019.14.Proc4.82 


\section{INTRODUCTION}

Spirometry is deemed as the most useful assessment of pulmonary function (Azeredo, 2002) and FVC\%, FEV1\% and PEF\% are accurate parameters to consider (Miller et al., 2005). $\mathrm{VO}_{2 m a x}$ is generally considered the best measure of aerobic capacity (Solomon et al., 2018) and CST protocols are considered highly reliable to measure $\mathrm{VO}_{2 \max }$ (Sykes and Roberts, 2004). Both pulmonary function and $\mathrm{VO}_{2 \max }$ decline gradually after peaking between the age of 20 and 30 years (Guénard and Rouatbi, 2002 ; Fleg et al., 2005). Although there seems to be an association between aerobic capacity and pulmonary function (Zavorsky and Smoliga, 2017), to the best of our knowledge there are no studies analysing the aforementioned association in healthy middleaged adults populations. Therefore, the purpose of this study was to verify the correlation between pulmonary function and $\mathrm{VO}_{2 \max }$ in healthy middle-aged adults.

\section{MATERIAL AND METHODS}

\section{Participants}

Forty-two middle-aged adults (male: $\mathrm{n}=19$; age $=51.89 \pm 4.62$ years old, height $=1.68 \pm 0.07 \mathrm{~m}$, body mass $=78.85 \pm 10.58 \mathrm{~kg}$; female: $\mathrm{n}=23$; age $=53.09 \pm 5.26$ years old, height $=1.57 \pm 0.05 \mathrm{~m}$, body mass $=68.82 \pm 22.95 \mathrm{~kg}$ ) volunteered to participate in this study and signed an informed consent form.

\section{Measures}

This study utilized a cross-sectional analysis, whereby participants underwent a spirometric test using a microQuark spirometer designed by Cosmed®, followed by a CST on a $30 \mathrm{~cm}$ tall step device using a Polar $\mathrm{H} 10$ heart rate sensor designed by Polarß.

\section{Procedures}

After arriving facilities, ratters gave participants standardized instructions about tests procedures. Each participant performed a minimum of 3 and a maximum of 8 valid spirometric trials with a rest period $\geq 1 \mathrm{~min}$ between manoeuvres (Miller et al., 2005). Afterwards, participants underwent a CST consisting of 5 stages, each of 2 minutes duration. The step cadence was set with a metronome, starting at $15 \mathrm{steps} / \mathrm{min}$ and increasing by 5 steps/min every 2 minutes. If $85 \%$ of predicted maximum heart rate (Jackson et al., 1990) was achieved before the end of the 5 stages, the test would be interrupted (Sykes and Roberts, 2004). $\mathrm{VO}_{2 \max }$ was calculated with the CST Calculator (Sykes, 2005), and the highest values of $\mathrm{FVC} \%$, FEV1\% and PEF\% (Miller et al., 2005) were considered for analysis.

\section{Analysis}

Descriptive statistics (mean \pm standard deviation) were performed for all variables under analysis. Data normality was tested using the Kolmogorov-Smirnov, and Spearman's rank correlation coefficient $(r)$ and coefficient of determination ( $\mathrm{r} 2$ ) were used to verify correlations between $\mathrm{VO}_{2 \max }$ and $\mathrm{FVC} \%$, $\mathrm{FEV} 1 \%$ and PEF\%. The strength of the correlation was classified as follows (Mukaka, 2012): very high $(0.90<r<1.00)$; high $(0.70<r<0.90)$; moderate $(0.50<r<0.70)$; low $(0.30<r<0.50)$; little $(0.10<r<0.30)$.

\section{RESULTS}

Although there was no significant correlation between $\mathrm{VO}_{2 \max }$ and $\mathrm{FVC} \%(r=0.076, \mathrm{r} 2=0.041, p=0.632)$, we verified a positive correlation between $\mathrm{VO}_{2 \max }$ and $\mathrm{FEV} 1 \%(r=0.389, \mathrm{r} 2=0.143, p=0.011)$ and also between $\mathrm{VO}_{2 \max }$ and $\mathrm{PEF} \%(r=0.405, \mathrm{r} 2=0.098, p=0.008)$. 


\section{DISCUSSION}

Our findings suggest that $\mathrm{VO}_{2 \max }$ is not significantly correlated with $\mathrm{FVC} \%$. However, the results reveal a positive correlation between $\mathrm{VO}_{2 m a x}$ and $\mathrm{FEV} 1 \%$, and also between $\mathrm{VO}_{2 m a x}$ and $\mathrm{PEF} \%$. Previous work is concurrent with our findings since authors have not found correlation between $\mathrm{VO}_{2 m a x}$ and $\mathrm{FVC}$ (Zavorsky et al., 2010), but between VO2max and FEV1 (Camargo et al., 2011) and between $\mathrm{VO}_{2 \text { max }}$ and PEF (Moradians et al., 2016). However, caution should be taken when comparing results since previous literature focused either on pathological populations or younger and older adults. Therefore, sports and health professionals should consider aerobic exercise prescription with the aim of increasing FEV1 and PEF on middle-aged adults populations.

\section{CONCLUSIONS}

In short, results confirm that $\mathrm{VO}_{2 m a x}$ is significantly correlated with $\mathrm{FEV} 1$ and $\mathrm{PEF}$, but not with $\mathrm{FVC}$ in middleaged adults. Careful consideration should be given to aerobic exercise prescription since it should be done with the right purpose.

\section{REFERENCES}

Azeredo, C.A. (2002). Fisioterapia Respiratória Moderna (4th Ed.). São Paulo: Manole.

Camargo, A.A., Justino, T., De Andrade, C.H., Malaguti, C., and Dal Corso, S. (2011). Chester step test in patients with COPD: reliability and correlation with pulmonary function test results. Respir Care, 56(7), 995-1001. https://doi.org/10.4187/respcare.01047

Fleg, J.L., Morrell, C.H., Bos, A.G., Brant, L.J., Talbot, L.A., Wright, J.G., and Lakatta, E.G. (2005). Accelerated longitudinal decline of aerobic capacity in healthy older adults. Circulation, 112(5), 674682. https://doi.org/10.1161/circulationaha.105.545459

Guénard, H., and Rouatbi, S. (2002). Physiological aspects of the decline of pulmonary function with age. Rev Mal Respir 19(2), 230-240. PMID: 12040323.

Jackson, A.S., Blair, S.N., Mahar, M.T., Wier, L.T., Ross, R.M., and Stuteville, J.E. (1990). Prediction of functional aerobic capacity exercise testing. Med Sci Sports Exerc, 22(6), 863-870. https://doi.org/10.1249/00005768-199012000-00021

Miller, M., Hankinson, J., Brusasco, V., Burgos, F., Casaburi, R., Coates, A., ... Wanger, J. (2005). Standardization of spirometry. Eur Respir J, 26(2), 319-338. https://doi.org/10.1183/09031936.05.00034805

Moradians, V., Rahimi, A., Moosavi, J.S.A., Khorasani, S.F.S., Mazaherinejad, A., Mortezazade, M., and Raji, H. (2016). Effect of Eight-Week Aerobic, Resistive, and Interval Exercise Routines on Respiratory Parameters in Non-Athlete Women. Tanaffos, 15(2), 96-100. PMCID: PMC5127621.

Mukaka, M.M. (2012). A guide to appropriate use of Correlation coefficient in medical research. Malawi Med J, 24(3), 69-71. PMID: 23638278.

Sykes, K., and Roberts, K. (2004). The Chester Step Test - a simple yet effective tool for the prediction of aerobic capacity. J Physiother, 90(4), 183-188. https://doi.org/10.1016/i.physio.2004.03.008

Sykes, K. (2005).The Chester Step Test (2nd Ed.). Liverpool, UK: Assist Creative Resources.

Solomon, A., Borodulin, K., Ngandu, T., Kivipelto, M., Laatikainen, T., and Kulmala, J. (2018). Self-rated physical fitness and estimated maximal oxygen uptake in relation to all-cause and cause-specific mortality. Scand J Med Sci Sports, 28(2), 532-540. https://doi.org/10.1111/sms.12924 
Zavorsky, G.S., Wilson, B., Harris, J.K., Kim, D.J., Carli, F., and Mayo, N.E. (2010). Pulmonary diffusion and aerobic capacity: is there a relation? Does obesity matter?. Acta Physiol, 198(4), 499-507. https://doi.org/10.1111/j.1748-1716.2009.02059.x

\section{(c) $(\mathrm{E}) \Theta$}

This work is licensed under a Attribution-NonCommercial-NoDerivatives 4.0 International (CC BY-NC-ND 4.0). 


\title{
Exploring relative age effect and maturity status on physical performance of school-age children
}

\author{
GABRIEL VILAS BOAS $1 \triangleleft$, DIEGO ARAÚJO1', JORGE AREDE², P. ESTEVES ${ }^{2,3}$, NUNO LEITE $^{1,2}$ \\ ${ }^{1}$ University of Trás-os-Montes and Alto Douro, Portugal \\ ${ }^{2}$ Research Center in Sports Sciences, Health Sciences and Human Development, CIDES, Portugal \\ ${ }^{3}$ Polytechnic Institute of Guarda, Portugal
}

\begin{abstract}
The purpose of this study was to verify the association between the date of birth and the maturity status on physical performance among young children at school-level. Data was collected from 454 students, ages ranging between 5.7 and 10.5 years, from 4 schools of public-school system, in the north of Portugal. The students were divided into four relative age quarters according to their month of birth (Q1, Q2, Q3 and Q4). Two fitness tests were performed in order to evaluate their agility and vertical jump performances. Bivariate correlations were used to examine the associations between maturity status and physical performance. The results in the CMJ test indicated a moderate increase in performance in the students born in Q1 and Q3 $(0,32$ and 0,37 respectably). As for the results in the agility test show a moderate decrease in times (better performance) in the students born in Q1, Q2 and Q3 $(-0,34,-0,35$ and $-0,34$ respectably). Interestingly the values corresponding to the students born in Q4 show a smaller correlation, meaning that jump and agility performance has a smaller correlation to students born in the Q4. Keywords: Maturity; Physical activity; Talent development.
\end{abstract}

Corresponding author. University of Trás-os-Montes and Alto Douro, Portugal.

E-mail: gabrielvilasboas10@gmail.com

Supplementary Issue: Spring Conferences of Sports Science. International Seminar of Physical Education, Leisure and Health, 17-19 June 2019. Castelo Branco, Portugal.

JOURNAL OF HUMAN SPORT \& EXERCISE ISSN 1988-5202

(C) Faculty of Education. University of Alicante.

doi:10.14198/jhse.2019.14.Proc4.82 


\section{INTRODUCTION}

Annual age-grouping in sports is a very well stablished procedure where athletes are separated in cohorts by chronological age. In many cases, this leads to a biased distribution of athletes' birth dates as a result of an advantage of the older individual with respect to the youngest in the same category, leading to a phenomenon called Relative Age Effect (RAE) (Cobley et al. 2009). This effect is explained by the possibility to access more opportunities for training and competing, together with incentives and feedbacks that increase the likelihood of becoming a professional athlete, or at least a better athlete. On the other hand, those that are younger (born closely before the cut-off date) are not as mature as their fellows and seldom don't get the same opportunities, which may cause a motivational decline and even practice dropout (Gómez-López et al., 2017). However, few studies have been made on the analysis of RAE within public-school system. This study aimed to verify if there is any association between birth-date children and maturational stages of school agedchildren in relation to their physical performance.

\section{MATERIAL AND METHODS}

\section{Participants}

Our sample was composed by 454 students, in between 5.7 and 10.5 years of age (standard deviation: \pm 1.17), from 4 schools integrating public-school system, in the north of Portugal. The birth dates of all participants were collected and then divided into four relative age quarters according to their birth months: January to March - quarter 1 (Q1); April to June - quarter 2 (Q2); July to September - quarter 3 (Q3), and October to December - quarter 4 (Q4).

\section{Measures}

The biological maturity status was assessed by the age at peak height velocity (APHV) (Mirwald et al., 2002; Muiller et al., 2017).

\section{Procedures}

Two fitness tests were performed in order to evaluate the agility and jump performances. Agility was evaluated through the pro agility test (Harman, et al., 2000). while the vertical jump performance was evaluated through the Counter-Movement Jump Test.

\section{Analysis}

We applied bivariate correlations to examine associations among maturity status and each item of the physical tests. Correlation coefficients was interpreted using the following thresholds (Hopkins et al., 2009): trivial $(r<0.1)$, small $(0.1<r<0.3)$, moderate $(0.3<r<0.5)$, large $(0.5<r<0.7)$, very large $(0.7<r<0.9)$, and nearly perfect $(r>0.9)$. All calculations were made using SPSS procedures (SPSS for MacOS release 10.0).

\section{RESULTS}

We found that a higher level of performance in the CMJ test was associated to the students born in Q1 and Q3 $(0,32$ and 0,37$)$. Interestingly the values corresponding to the students born in Q4 show a smaller level of association. As for the results in the agility test, a moderate association between the performance and the students born in Q1, Q2 and Q3 (-0,34, -0,35 and -0,34 respectably) was noticed. In contrast, for the students born in the Q4, the association was smaller. 


\section{CONCLUSIONS}

This study suggests that the children born in the last quartile of the year (Q4) appear to exhibit a lower value of physical performance. With regard to the vertical jump, the children born in the quartile 1 and 3 showed a moderate correlation between jump height and maturity status. A negative association was observed between agility performance (time) and maturity status, especially for children born in the quartiles 1,2 and 3. These results conform to previous research by showing that children with greater chronological age tend to exhibit greater sport success predominantly at younger ages (Hancock, 2017; \& Leonardo, 2018).

\section{REFERENCES}

Cobley, S., Baker, J., Wattie, N., and McKenna, J. (2009) Annual age-grouping and athlete development: a meta-analytical review of relative age effects in sport. Sports Med. 2009;39(3):235-256. https://doi.org/10.2165/00007256-200939030-00005

Gómez-López, M. (2017) Relative age effect in handball players of Spain. Journal of Physical Education \& Sport. 17, 2, 705-711.

Hancock, D. J. (2017) Female relative age effects and the second-quartile phenomenon in young female ice hockey players. Psychology of Sport and Exercise. 32, 12-16, ISSN: 14690292. https://doi.org/10.1016/j.psychsport.2017.05.002

Harman, E., Garhammer, J. and Pandorf, C. (2000) Administration, scoring and interpretation of selected tests. In: Baechle TR, Earle RW, eds. Essentials of strength and conditioning. Champaign.

Leonardo, L., Lizana, C., Krahenbühl, T., and Scaglia, A. (2018). Relative age effect affects the time of competitive participation in male handball athletes aged up to 13 years. Retos. 195-198.

Mirwald, R., Baxter-Jones, A., Bailey, D., and Beunen, G. (2002). An assessment of maturity from anthropometric measurements. Medicine and science in sports and exercise. 34. 689-94. https://doi.org/10.1097/00005768-200204000-00020

Muiller, L., Gonaus, C., Perner, C., Muiller, E. and Raschner, C. (2017) Maturity status influences the relative age effect in national top level youth alpine ski racing and soccer. PLoS ONE 12, e0181810. https://doi.org/10.1371/journal.pone.0181810

\section{@) $\odot \Theta \Theta$}

This work is licensed under a Attribution-NonCommercial-NoDerivatives 4.0 International (CC BY-NC-ND 4.0). 


\title{
Push-ups with hands or feet on unstable surface: Does it affects muscle activation and ground reaction forces?
}

\author{
ANDREIA NICOLAU1', LUÍS RIBEIRO¹, FILIPE CONCEIÇÃO2, PEDRO FONSECA², NUNO SERRA, \\ CAROLINA VILA-CHÃ $\tilde{A}^{1,3}$ \\ ${ }^{1}$ Research Unit for Inland Development, Polytechnic Institute of Guarda, Portugal \\ 2Laboratório de Biomecânica da Universidade do Porto (LABIOMEP), Portugal \\ ${ }^{3}$ Research Center in Sports Sciences, Health Sciences and Human Development, CIDESD, Portugal
}

\begin{abstract}
Unstable surfaces (US) are often used to introduce instability into conventional push-up (PU) exercise. The

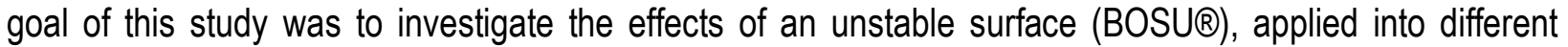
locations (under the hands or feet), on the muscle activation patterns and ground reaction forces (GRF) production. Twelve healthy male subjects ( $22 \pm 3.9$ years), were selected and performed $3 \mathrm{PU}$ conditions: standard PU, PU with US under hands and PU with US under feet. Surface EMG signals were collected from agonist, antagonist and stabilizer muscles. GRF was collect by using force plates embedded on the floor and kinematic variables was evaluated with a Motion Analysis 3D system. Vertical GRF (Fz) was significantly higher in the PU feet than in the other conditions $(P>0.05)$. However the highest values in the anteroposterior (Fx) and mediolateral (Fy) GRF were found in the PU hands ( $P>0.001$, in all comparisons). Muscle activation of the triceps and biceps significantly increased with US addition, while no changes were observed in the pectoralis major. Increased external oblique activation were only observed in the PU feet. On the other hand, PU hand, induced a substantial decrease of the serratus activation. The results showed that the addition of unstable surfaces produced changes on the activation pattern of agonist, antagonist muscles, and shoulder and trunk stabilizers muscles. Such changes were differently affected, whether instability was applied to the hands or to feet. Keywords: Push-up; Unstable surface; Muscle activity; Kinetic.
\end{abstract}

Corresponding author. Research Unit for Inland Development, Polytechnic Institute of Guarda, Portugal.

E-mail: cvilacha@ipg.pt

Supplementary Issue: Spring Conferences of Sports Science. International Seminar of Physical Education, Leisure and Health, 17-19 June 2019. Castelo Branco, Portugal.

JOURNAL OF HUMAN SPORT \& EXERCISE ISSN 1988-5202

(c) Faculty of Education. University of Alicante.

doi:10.14198/jhse.2019.14.Proc4.82 


\section{INTRODUCTION}

Push-up (PU) exercise is one of the simplest and popular exercise used by fitness and sports practitioners to improve strength and resistance of the upper body. A variety of exercise modes have been explored in order to create different training stimuli, including the application of unstable surfaces in the hands or/and in feet. Several studies showed that PU with unstable surfaces (under the hands, where force application is made), changes the muscle activation of both agonists and antagonists and stabilizers muscles (Chou, 2011; Lehman, Gilas, \& Patel, 2008). However, few studies have investigated the effects of unstable surfaces under feet (corresponding to torque fulcrum) either on the muscle activation or force production. In addition, a recent review indicated that studies on kinetics-based quantification on PU are scarce (Dhahbi et al., 2018). Therefore, the aim was to investigate the effects of applying an unstable surface, either under the hands or under the feet, on the muscle activation and ground reaction forces production.

\section{MATERIAL AND METHODS}

\section{Participants}

Twelve healthy male subjects $(22 \pm 3.9$ years, $72 \pm 7.8 \mathrm{~kg}, 176 \pm 0.06 \mathrm{~cm})$, familiar with push-up exercise were included in the study.

\section{Measures}

Surface EMG signals from agonists (sternal pectoral major, triceps brachii and anterior deltoid), antagonists (latissimus drosi and biceps brachii) and stabilizers (serratus anterior, superior trapezius, external oblique and erector spinae) muscles were collected at $1000 \mathrm{~Hz}$ with single differential active electrodes (Trigno ${ }^{\mathrm{TM}}$ Wireless EMG - Delsys $\left.{ }^{\circledR}\right)$. For the acquisition of kinematic variables it was used a system of 8 cameras at $200 \mathrm{~Hz}$ (Motion Analysis 3D - Qualisys). Kinetic data at $2000 \mathrm{~Hz}$ was collect using force plates embedded on the floor (Bertec, Worthington), under the hands. All data recorded were synchronized with Qualisys system.

\section{Procedures}

The subjects were instructed to perform the push-up movements, at 2-second rate for descent and ascent phase of the push-up cycle (maintained by a $60-\mathrm{Hz}$ metronome). Three variants of PU were accomplished for each subject: standard PU [standard PU (S)] PU with hands [PU Hands (H)] or the feet [PU Feet (F)] on an unstable surface (BOSU $\otimes)$. Reflective markers on specific landmarks were attached to torso, hip and upper limbs segments.

\section{Analysis}

All marker trajectories were identified using Qualisys software and skeletal representation was generated in Visual 3D (C-motion, Inc.).Signal analysis was preformed using MATLAB (MathWorks, Inc) routine. The raw EMG signals were full-wave rectified and filtered. The Average Rectified Values (ARV) of the EMG signals from each muscle was computed and normalized in respect to the mean value of the $4 \mathrm{ARV}$ maximum values found in 1st 3 repetitions. Smoothing of ground reaction forces (GRF) and kinematic parameters was performed by a 4th order low-pass Butterworth filter with a cut off of $50 \mathrm{~Hz}$ and $4 \mathrm{~Hz}$, respectively. A one-way ANOVA with repeated measures was performed. Pairwise comparisons were performed with the StudentNewman-Keuls post hoc test when ANOVA was significant. The significance level was set to $P<0.05$.

\section{RESULTS}

Table 1, shows the results on GRF for all PU conditions. PU feet produced the highest vertical force (Fz). 
Table 1. Mean \pm SD ground reaction forces (FZ-vertical; FX- anteroposterior; Fy- mediolateral) during pushup conditions.

\begin{tabular}{lcccl}
\hline & Standard(S) & PU Hands(H) & PU Feet(F) & P value \\
\hline FZmax & $717.8 \pm 74.2$ & $708.2 \pm 71.7$ & $732.7 \pm 61.9^{*}$ & ${ }^{*} \mathrm{~F} \neq \mathrm{H} ; P<0.05$ \\
FXmax & $112.4 \pm 22.0$ & $126 \pm 27.8$ & $47.7 \pm 14.5^{*} \dagger$ & ${ }^{*} \mathrm{~F} \neq \mathrm{H}, P<0.001 ; \mathrm{F} \neq \mathrm{S}, P<0.001$ \\
FYmax & $16.8 \pm 7 . \S^{*}$ & $31.9 \pm 10.2^{\ddagger}$ & $24.4 \pm 6.3$ & $\S \mathrm{S} \neq \mathrm{H}, P<0.001 ;{ }^{*} \mathrm{~S} \neq \mathrm{F}, P<0.001 ; * \mathrm{H} \neq$ \\
& & & $\mathrm{F}, P<0.01$ \\
FX range & $77.6 \pm 27.9$ & $101.9 \pm 21.9 \neq$ & $65.6 \pm 12.6$ & $* \mathrm{*} \neq \mathrm{F} ; P<0.001 ;{ }^{*} \mathrm{H} \neq \mathrm{S} ; P<0.001 ;$ \\
& & ${ }^{*}$ & & \\
Fy range & $29.8 \pm 10.2$ & $54.8 \pm 20.4^{*}$ & $37.4 \pm 10.6$ & $* \mathrm{H} \neq \mathrm{F} ; P<0.001 ;{ }^{*} \mathrm{H} \neq \mathrm{S} ; P<0.001 ;$ \\
\hline
\end{tabular}

The highest anteroposterior (Fx) and mediolateral (Fy) forces were produced in the PU Hands. No significant differences were observed on elbow angle amplitude (S: $100.5 \pm 9.8 ; \mathrm{H}: 99.0 \pm 8.6 ; \mathrm{F}: 98.7 \pm 5.4 ; \mathrm{P}=0.71$ ) and maximum angular velocity (S: $1.12 \pm 0.15 ; \mathrm{H}: 1.03 \pm 0.2 ; \mathrm{F}: 1.13 \pm 0.13 ; \mathrm{P}=0,11)$. Results on muscle activation are shown in the Figure 1.

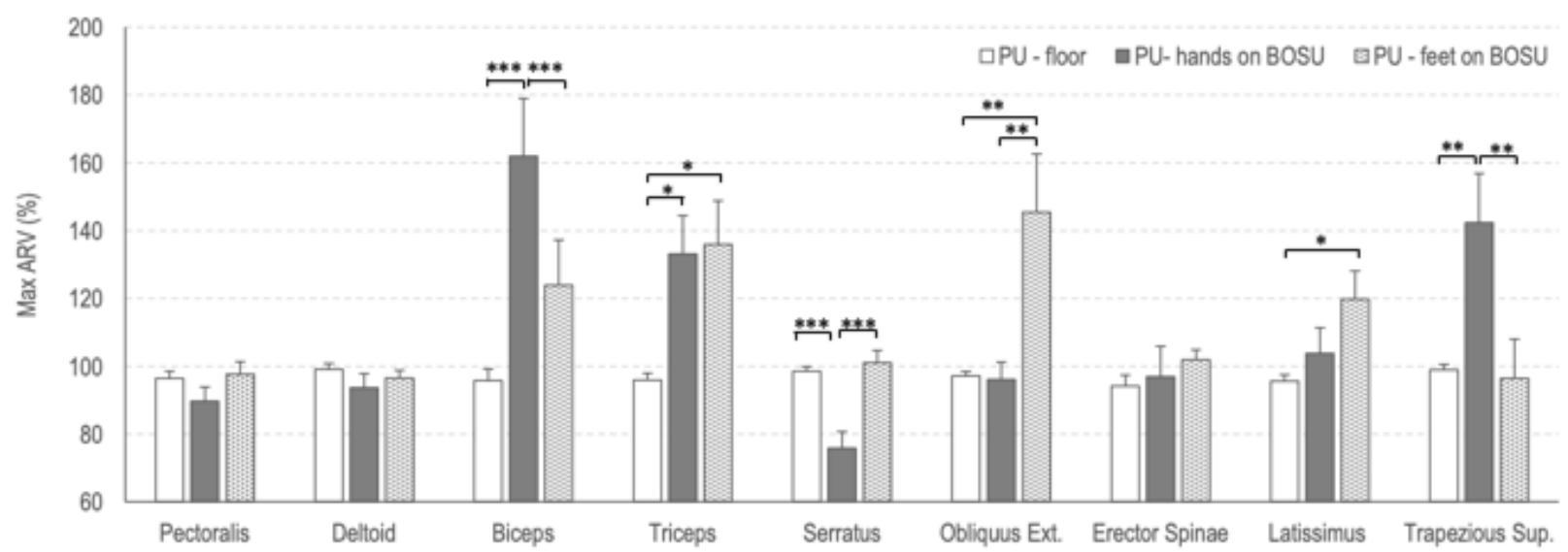

Figure 1. Maximum Average Rectified Value (ARV) of agonists, antagonists and stabilizer muscles during all PU conditions.

\section{DISCUSSION}

The addition of unstable surfaces, both under the hand and the feet, produced changes on recruitment pattern and ground reaction forces production. Such changes were differently affected, whether instability was applied to the hands or to the feet. Contrary to Lehman et al. (2008) results, where feet elevation increased scapula-thoracic muscle activity in our study PU feet did not affect substantially the groups muscles, but increased oblique external activity. Activation of scapula-thoracic muscles activity were, in contrast, affected by the application of US under the hands.

\section{CONCLUSIONS}

The results showed that the addition of unstable surfaces, both under the hand or the feet, produced different changes on the activation pattern of agonist and antagonist muscles, as well as of shoulder and trunk stabilizers muscles. Moreover, differences in the ground reaction forces production between PU conditions were also noticed. Such changes were differently affected, whether instability was applied to the hands or to feet. 


\section{REFERENCES}

Chou, P. P.-H. (2011). Journal of Medical and Biological Engineering, 31(3), 161-8.

Dhahbi, W., Chaabene, H., Chaouachi, A., Padulo, J., Behm, D. G., Cochrane, J., et al. (2018). Kinetic analysis of push-up exercises: a systematic review with practical recommendations. Sports Biomechanics, 00(00), 1-40. https://doi.org/10.1080/14763141.2018.1512149

Lehman, G. J., Gilas, D., \& Patel, U. (2008). An unstable support surface does not increase scapulothoracic stabilizing muscle activity during push up and push up plus exercises. Manual Therapy, 13(6), 500-506. https://doi.org/10.1016/..math.2007.05.016 


\title{
Correlation between vertical stiffness and agility performance in sport students
}

\author{
PEDRO DUARTE-MENDES ${ }^{1} \triangle$, RUI PAULO ${ }^{1,2}$, DINEIA A. LUCAS ${ }^{1}$, CAROLINA LIBÓRIO ${ }^{1}$, JOÃO \\ AFONSO ${ }^{1}$, JOÃO ROCHA ${ }^{1,2}$, SAMUEL HONÓRIO1,2, MARCO BATISTA ${ }^{1,2}$ \\ ${ }^{1}$ Department of Sports and Well-being, Polytechnic Institute of Castelo Branco, Castelo Branco, Portugal \\ 2SHERU - Sport, Health \& Exercise Research Unit, Polytechnic Institute of Castelo Branco, Castelo Branco, \\ Portugal
}

\begin{abstract}
Physical performance is highly dependent on Agility and global Stiffness of the lower limb, particularly in activities involving running, jumping, hopping and change of direction. Nevertheless, whether or not Agility Performance is significantly influenced by vertical Stiffness $(k)$ remains unclear. Therefore, the purpose of this study was to verify the correlation between $k$ and Agility Performance. Thirty-eight college students (male: $n=30$; female: $n=8$ ) volunteered to participate in this study. Each participant performed 3 trials of Drop Jump Test (DJ) followed by 2 trials of Pro Agility Test (PA), Hexagon Test (H) and Four Corner Agility Test (FC). The variables under analysis were k (kN.m) and time (seg) of Pro Agility Test (tPA), Hexagon Test (tH) and Four Corner Agility Test (tFC). Data normality was tested using the Kolmogorov-Smirnov, and Spearman's rank correlation coefficient $(r)$ and coefficient of determination ( $r 2$ ) were used to verify correlations between $\mathrm{k}$ and tPA, tH and tFC. Results reveal no significant correlation between $\mathrm{k}$ and $\mathrm{tPA}(\mathrm{r}=$ $-0.242, r 2=0.030, p=0.143)$ nor between $k$ and $\mathrm{tH}(r=0.029, r 2=0.002, p=0.864)$, although there is a negative and low correlation between $k$ and tFC $(r=-0.475, r 2=0.019, p=0.003)$. We can conclude that careful consideration should be given to the most appropriate assessment of Stiffness. Keywords: Agility; Stiffness; Vertical stiffness; Performance.
\end{abstract}

\footnotetext{
Corresponding author. Department of Sports and Well-being, Polytechnic Institute of Castelo Branco, Rua Prof. Dr. Faria de Vasconcelos, 6000-266 Castelo Branco, Portugal.

E-mail: pedromendes@ipcb.pt

Supplementary Issue: Spring Conferences of Sports Science. International Seminar of Physical Education, Leisure and Health, 17-19 June 2019. Castelo Branco, Portugal.

JOURNAL OF HUMAN SPORT \& EXERCISE ISSN 1988-5202

(c) Faculty of Education. University of Alicante.

doi:10.14198/jhse.2019.14.Proc4.82
} 


\section{INTRODUCTION}

Agility is the rapid whole-body movement with change of direction or speed in response to a stimulus, being an extremely important characteristic of sports performance (Paul et al., 2015). Understanding the potential determinants of Agility provide the coach with important information which may better inform the training process (Triplett, 2012). Vertical Stiffness (k) describes the vertical displacement of the centre of mass in response to vertical ground reacting during a task performed in the sagittal plane (Latash and Zatsiorsky, 1993). Since greater Stiffness would increase the utilisation of elastic energy (Maloney and Fletcher, 2018), there is growing evidence that sports performance is highly influenced by global Stiffness of the lower limb, particularly in activities involving running, jumping, hopping and change of direction (Maloney et al., 2017). However, whether or not Agility Performance is significantly influenced by $k$ remains unclear (Pruyn et al., 2014). Therefore, the purpose of this study was to verify the correlation between $k$ and Agility Performance. Based in the review of literature we expected a relation between $\mathrm{k}$ and Agility Performance in sport students.

\section{MATERIAL AND METHODS}

\section{Participants}

Thirty-eight college sports students (male: $n=30$; age $=21.07 \pm 2.19$ years old, height $=1.74 \pm 0.05 \mathrm{~m}$, body mass $=69.97 \pm 8.34 \mathrm{~kg}$; female: $n=8$; age $=21.00 \pm 1.41$ years old, height $=1.59 \pm 0.06 \mathrm{~m}$, body mass $=59.95 \pm 14.12 \mathrm{~kg}$ ) volunteered to participate in this study.

\section{Measures}

This study utilized a cross-sectional analysis, whereby subjects performed a Drop Jump (DJ) from a contact mat using the Chronojump system (Chronojump-Boscosystem, Spain) and had their times in Pro Agility Test $(\mathrm{PA})$, Hexagon Test $(\mathrm{H})$ and Four Corner Agility Test (FC) assessed in a single session.

\section{Procedures}

After arriving facilities, ratters gave participants standardized instructions about tests procedures. Each participant performed 3 trials of the DJ with 1 minute of rest between each trial and 3 minutes of rest prior commencing agility tests (Secomb et al., 2015). Afterwards, 2 trials of PA, H and FC were performed in a random-assigned order, allowing 2 minutes of rest between trials and 10 minutes delay between the aforementioned tests (Triplett, 2012). Dependent variables were $\mathrm{k}$ (kN.m) and time (seg) of PA (tPA), $\mathrm{H}$ (tH), and FC (tFC). The higher value of $k$ and the lower times of each agility test were considered for analysis. Being $m$ the total body mass, Tc the ground contact time and Tv de flight time, $\mathrm{k}$ was calculated as follows (Dalleau et al., 2004):

$$
k=\frac{m * \pi(T v+T c)}{T c^{2}\left(\frac{T v+T c}{\pi}-\frac{T c}{4}\right)} \quad \mathrm{kN} . \mathrm{m}
$$

\section{Analysis}

Descriptive statistics (mean \pm standard deviation) were performed for all variables under analysis. Data normality was tested using the Kolmogorov-Smirnov. Spearman's rank correlation coefficient ( $r)$ and coefficient of determination ( $r 2$ ) were used to verify correlations between $\mathrm{k}$ and tPA, tH and tFC,. The strength of the correlation was classified as follows (Mukaka, 2012): very high $(0.90<r<1.00)$; high $(0.70<r<0.90)$; moderate $(0.50<r<0.70)$; low $(0.30<r<0.50)$; little $(0.10<r<0.30)$. 


\section{RESULTS}

Although there was no significant correlation between $k$ and tPA $(r=-0.242, r 2=0.030, p=0.143)$ and between $\mathrm{k}$ and $\mathrm{tH}(r=0.029, \mathrm{r} 2=0.002, p=0.864)$, we verified a negative and low correlation between $\mathrm{k}$ and tFC $(r=-0.475, r 2=0.019, p=0.003)$.

\section{DISCUSSION}

Our findings suggest that $\mathrm{k}$ is not significantly correlated with tPA nor tH. However, the results reveal a negative correlation between $\mathrm{k}$ and $\mathrm{tFC}$. This might be due to the differences between agility tests since PA and $\mathrm{H}$ essentially demand changes of direction, accelerations and deceleration, whereas $\mathrm{FC}$ is based on changes of direction speed while moving forward, side to side, and backwards (Triplett, 2012).It should be noted that the evidence indicating that increases in lower limb Stiffness are associated with heightened performance (Brazier et al., 2017) assessed lower limb Stiffness through leg Stiffness (lk), calculated during horizontal movements. In fact, when analysing k, Pruyn et al. (2014) concluded that k does not discriminate between Agility Performance. Therefore, Ik might be more correlated with Agility Performance than k.

\section{CONCLUSIONS}

In short, vertical Stiffness is not significantly correlated to Agility Performance involved in tasks requiring rapid whole-body movements with change of direction or speed. Careful consideration should be given to the most appropriate assessment of Stiffness on a team or individual basis.

\section{REFERENCES}

Brazier, J., Maloney, S., Bishop, C., Read, P. J., and Turner, A.N. (2019). Lower Extremity Stiffness: Consideration for Testing, Performance Enhancement and Injury Risk. J Strength Cond Res, 33(4), 1156-1166. https://doi.org/10.1519/jsc.0000000000002283

Dalleau, G., Beli, A., Viale, F., Lacour, J.R., and Bourdin, M. (2004). A Simple Method for Field Measurements of Leg Stiffness in Hoping. Int J Sports Med, 25(3), 170-176.. PMID : 15088239.

Latash, M.L., and Zatsiorskty, V.M. (1993). Joint Stiffness: Myth or reality?. Human Mov Sci, 12(6), 553692. https://doi.org/10.101670167-9457893990010-M

Maloney, S.J., and Fletcher, I.M. (2018). Lower limb stiffness in athletic performance: a critical review. Sports Biomech, 16(1), 1-22. https://doi.org/10.1080/14763141.2018.1460395

Maloney, S.J., Richards, J., Nixon, D.G.D., Lewis, J.H., and Fletcher, I.M. (2017). Do stiffness and asymmetries predict change of direction performance? J Sports Sci, 35(6), 547-556. https://doi.org/10.1080/02640414.2016.1179775

Mukaka, M.M. (2012). A guide to appropriate use of Correlation coefficient in medical research. Malawi Med J, 24(3), 69-71. PMID: 23638278.

Paul, D.J., Gabbett, T.J., and Nassis, G.P. (2016). Agility in Team Sports: Testing, Training and Factors Affecting Performance. Sports Med, 46(3), 421-442. https://doi.org/10.1007/s40279-015-0428-2

Pruyn, E.C., Watsford, M., and Murphy, A. (2014). The correlation between lower-body stiffness and dynamic performance. Appl Physiol Nutr Metab, 39, 1144-1150. https://doi.org/10.1139/apnm-2014$\underline{0063}$

Secomb, J.L., Nimphius, S., Farley, O.R., Lundgreen, L.E., Tran, T.T., and Sheppard, J.M. (2015). Correlations between Lower-Body Muscle Structure and, Lower-Body Strength, Explosiveness and 
Eccentric Leg Stiffness in Adolescent Athletes. J Sports Med Sci, 14(4), 691-697. PMID: 26664263. https://doi.org/10.1519/jsc.0000000000000858

Triplett, N. T. (2012). Speed and Agility. In: T. Miller, ed., NSCA's Guide to Tests and Assessments, 1st ed. Champaign, IL: Human Kinetics, pp. 253-274.

\section{(c) (i) $\ominus$}

This work is licensed under a Attribution-NonCommercial-NoDerivatives 4.0 International (CC BY-NC-ND 4.0). 


\title{
Parental involvement in health promotion programs during pre-school aged children: A systematic review
}

\author{
ANABELA REIS $\triangle$, TERESA VILAÇA, RAFAELA ROSÁRIO \\ Universidade do Minho, Portugal
}

\begin{abstract}
Behaviours and health habits are established early in childhood and parents are essential in the development of their children lifestyles. Based on this evidence, this paper aims to analyse the effectiveness of healthy lifestyles promotion programs in pre-school children that include a component of parental involvement. The questions that gave rise to this review were: (1) What healthy lifestyle promotion programs exists for preschool children? (2) What is the parental involvement in these programs? (3) What measures are used to assess the effectiveness of these programs in children lifestyles?. We developed a systematic review in different databases (Academic Search Complete, Science Direct, Business Source Complete, OAPEN Library, Science in context, Literature Resource Center, Directory of Open Access Journal) between 2010 and 2019, only in English. We included randomised controlled trials, including cluster-randomised controlled trials and cross-over trials, of any intervention primarily targeting health promotion programs lead by parents of children 3-6 years old. 21679 papers were found, and the first author screened titles and abstracts of identified papers; a second review author was involved, and 47 papers were analysed. The intervention programs with direct involvement of the parents have a greater impact on child's physical health, namely at the level of body mass index, physical activity and sedentary behaviour. The parental involvement during development, implementation and evaluation of the interventions improves their effective participation throughout the process. Keywords: Parental involvement; Healthy lifestyles; Preschool children.
\end{abstract}

Corresponding author. Instituto de Educação, Uni. Minho-Campus do Gualtar. Rua da Universidade, 4710-057 Braga, Portugal.

E-mail: adfonseca@gmail.com

Supplementary Issue: Spring Conferences of Sports Science. International Seminar of Physical Education, Leisure and Health, 17-19 June 2019. Castelo Branco, Portugal.

JOURNAL OF HUMAN SPORT \& EXERCISE ISSN 1988-5202

(c) Faculty of Education. University of Alicante.

doi:10.14198/jhse.2019.14.Proc4.82 


\section{INTRODUCTION}

At pre-school education, parents play a greater role in children social and physical environment and this constitutes an opportunity to intervene and to promote healthy lifestyles (Goldfield et al., 2012). In Portugal, the prevalence of overweight and obesity is about 32\% (Vale et al., 2015; Freitas et al., 2019), following the trends of other developed countries. The evidence shows that children with overweight at the age of 5 are four times more likely to become obese in adulthood, which reinforces the premise that habits and lifestyles acquired in childhood and adolescence are determinant throughout the life cycle (Berkman and Kawachi, 2014). For this reason, exploring the effectiveness of healthy lifestyle promotion programs, focusing on preschool children and with a component of parental involvement, analysing their impact on children's health, summarizing best practices, is essential to create more effective healthy lifestyles programs and to promote a more effective parental involvement.

\section{MATERIAL AND METHODS}

\section{Participants}

A systematic literature review about healthy lifestyle promotion programs for pre-school children that included the parental involvement component was developed, with studies from 2010 to 2019. In a first phase, the BOn platform was consulted (which includes databases such as Academic Search Complete, Science Direct, Business Source Complete) with the following keywords: "Parental Involvement"; "Healthy Lifestyle"; "Preschool Children"; "Preschool Intervention Program"; "Healthy Lifestyle Program".

\section{Measures}

Inclusion criteria was as follows: parental involvement; central focus on promoting healthy lifestyles (dietary intake and/or physical activity and/or sleep and/or sedentary behavior); pre-school children. Studies aimed at treatment of overweight and / or obesity or other diseases were excluded.

\section{Procedures}

To guide the process of literature search, data coding and data collection, we used the Preferred Reporting Items for Systematic Reviews and Meta-Analysis - PRISMA statement (Moher et al., 2009).

\section{Analysis}

The articles were analysed with the following prior categories: keywords, objectives, methodology, participants, measures, findings and future research. Extracted data were synthesized in table format.

\section{RESULTS}

In the research we found 21679 results, and after title and abstract screening 1253 papers were screened. According to our inclusion and exclusion criteria 47 papers were included in the revision. 34 studies were randomized controlled trials (including cluster) and 13 observational studies. Most of the studies included in this review used anthropometric data as the main outcome measure (McSweeney et al., 2017; Quick et al., 2018) and some used accelerometers to evaluate the 24 hours of movement (physical activity, sedentary behaviour and sleep) (Lee et al., 2019; De Craemer et al., 2016). Parental involvement component was associated with positive results in terms of children's lifestyles, particularly when using online strategies to mitigate some difficulties of parental involvement. Although some evaluation instruments were applied to parents, specific results of their participation during the programs were not detailed. 


\section{DISCUSSION}

The pre-school period is crucial for the establishment of certain behaviours and parents are considered important in modelling behaviour at this age, highlighting the need to develop healthy lifestyles promotion programs with parental involvement in pre-school. This involvement will be improved whether the intervention program is feasible to the real needs of the parents. To ensure its effectiveness, it is proposed that future parental involvement programs include a knowledge component (e.g. information about healthy lifestyles), the establishment of communication networks (e.g. to clarify doubts), online participation (e.g. activities to be developed at home) and communication between peer groups (e.g. creation of groups on social networks).

\section{CONCLUSIONS}

Based on this review it is evident the need to evaluate parental involvement during the development, implementation and evaluation of the intervention programs.

\section{REFERENCES}

Berkman, L., Kawachi, I., (2014). A historical framework for social epidemiology. In Glymour, M. (eds) (2014). Social Epidemiology. Nova lorque: Oxford University Press. https://doi.org/10.1093/med/9780195377903.003.0001

De Craemer, M., De Decker, E., Verloigne, M., De Bourdeaudhuij, I., Manios, Y., \& Cardon, G. (2016). The effect of a cluster randomised control trial on objectively measured sedentary time and parental reports of time spent in sedentary activities in Belgian preschoolers: the ToyBox-study. Int J Behav Nutr Phy, 13(1), 1-18. https://doi.org/10.1186/s12966-015-0325-y

Freitas, A.I., Moreira, C.,\&Santos, A. C. (2019).Time trends in prevalence and incidence rates of childhood overweight and obesity in Portugal: Generation XXI birth cohort. Int J Obesity, 43(2), 424427. https://doi.org/10.1038/s41366-018-0286-8

Goldfield, G. S., Harvey, A., Grattan, K. \& Adamo, K. B. (2012). Physical activity promotion in the preschool years: A critical period to intervene. Int J Env Res Pub He, 9, 1326-1342. https://doi.org/10.3390/ijerph9041326

Lee, R. E., Lorenzo, E., Szeszulski, J., Arriola, A., Bruening, M., Estabrooks, P. A., ... Todd, M. (2019). Design and methodology of a cluster-randomized trial in early care and education centers to meet physical activity guidelines: Sustainability via Active Garden Education (SAGE). Contemp Clin Trials, 77(December), 8-18. https://doi.org/10.1016/j.cct.2018.12.003

McSweeney, L., Araújo-Soares, V., Rapley, T., \& Adamson, A. (2017). A feasibility study with process evaluation of a preschool intervention to improve child and family lifestyle behaviours. Bmc Public Health, 17(1), 1-16. https://doi.org/10.1186/s12889-017-4167-1

Moher D, Liberati A, Tetzlaff J, Altman DG, The PRISMA Group (2009). Preferred Reporting Items for Systematic Reviews and Meta-Analyses: The PRISMA Statement. PLoS Med 6(7). https://doi.org/10.1371/journal.pmed.1000097

Quick, V., Martin-Biggers, J., Povis, G. A., Worobey, J., Hongu, N., \& Byrd-Bredbenner, C. (2018). Longterm follow-up effects of the HomeStyles randomized controlled trial in families with preschool children on social cognitive theory constructs associated with physical activity cognitions and behaviors. Contemp Clin Trials, 68(March), 79-89. https://doi.org/10.1016/j.cct.2018.03.006

Vale, S., Trost, S. G., Rêgo, C., Abreu, S., \& Mota, J. (2015). Physical activity, obesity status, and blood pressure in preschool children. J Pediatr, 167(1), 98-102. https://doi.org/10.1016/j.jpeds.2015.04.031 


\section{(c) (i) (3)}

This work is licensed under a Attribution-NonCommercial-NoDerivatives 4.0 International (CC BY-NC-ND 4.0). 


\title{
Motor development in children from 12 to 46 months: Influence of the variable "type of breastfeeding"
}

MIGUEL REBELO ${ }^{1} \triangleleft$, RUI PAULO ${ }^{2,3}$, DANIEL A. MARINHO4 ${ }^{4}$, PEDRO DUARTE-MENDES², JOÃO SERRANO2,3

${ }^{1}$ Department of Sport Sciences, University of Beira Interior, Covilhã, Portugal

${ }^{2}$ SHERU - Sport, Health \& Exercise Research Unit, Polytechnic Institute of Castelo Branco, Castelo Branco, Portugal

${ }^{3}$ Department of Sports and Well-being, Instituto Politécnico de Castelo Branco, Castelo Branco, Portugal

${ }^{4}$ Research Centre in Sport Sciences, Health Sciences and Human Development (CIDESD), Covilhã, Portugal

\begin{abstract}
Motor development presupposes a set of lifelong processes of change, these processes occur mostly during the first years of life, as such, it is important to know the different factors that influence the development of motor skills during childhood, for example, the type of breastfeeding. The objective of this study was to verify if there were differences in the development of motor skills (global and fine) comparing children who were breastfed or not breastfed. A total of 208 children of both sexes (33.22 \pm 5.98 months), the group of children breastfed with 106 children (33.59 \pm 4.92 months) and the non-breastfed group per mother's milk, for 98 children (32.81 \pm 6.95 months). Motor skills were assessed using the PDMS-2 scales. For the statistical analysis, the Kolmogorov-Smirnov test was used to test the normality, the Mann-Whitney test for independent samples. There were statistically significant differences in locomotion skills $(p=0.012)$, manipulation of objects $(p=0.042)$, fine manipulation $(p=0.000)$, visuo-motor integration $(p=0.000)$ and in Global Motricity $(p=0.026)$. Breast-fed infants presented, on average, better overall motor performance and children who were not breast-fed in fine motor skills. These results show that breast milk due to its nutritional, immunological, psychological and social benefits make the child more "strong" at the global level (corer, skip, lance, kick), since the children without breastmilk presented better results in fine motor skills probably from the early need they have in the initial manipulation of the fine movements when handling the bottle. Keywords: Motor development; Type of breastfeeding; PDMS-2.
\end{abstract}

Corresponding author. Department of Sport Sciences, University of Beira Interior, Covilhã, Portugal.

E-mail: miguelrebelo7@hotmail.com

Supplementary Issue: Spring Conferences of Sports Science. International Seminar of Physical Education, Leisure and Health, 17-19 June 2019. Castelo Branco, Portugal.

JOURNAL OF HUMAN SPORT \& EXERCISE ISSN 1988-5202

(c) Faculty of Education. University of Alicante.

doi:10.14198/jhse.2019.14.Proc4.82 


\section{INTRODUCTION}

Maternal milk is considered the adequate food for children in the first months of life, both nutritionally and immunologically as well as psychologically, providing an affective mother-child bond (Bosi and Machado, 2005). Breast milk is an ideal food for the healthy growth and development of the child, in this way we must implement good eating habits from birth to a good health maintenance. According to Levy and Bértolo (2013) there is currently "a worldwide consensus that their exclusive practice is the best way to feed children up to 6 months of age".

It is pertinent to understand at an early stage of development (from 12 to 46 months) which variables can influence the motor development of the child, this being, breastfeeding. Thus the objective of this study was to verify if there were differences in the motor skills (global and fine), comparing the children were breastfed by maternal bed of those who were not.

\section{MATERIAL AND METHODS}

\section{Participants}

A total of 208 children of both sexes ( $33.22 \pm 5.98$ months) participated. Two groups were created: the group of children who were breastfed, consisting of 106 children (33.59 \pm 4.92 months) and the group that was not breastfed by 98 (32.81 \pm 6.95 months). The following exclusion criteria were considered:

- Children who have been diagnosed with learning difficulties and / or developmental impairments;

- Children with some type of deficiency diagnosed;

\section{Measures}

The motor skills were evaluated using the scales of the PDMS-2 test battery (Saraiva \& Rodrigues, 2007). PDMS-2 stands out as one of the most recent instruments in the evaluation of child motor development (Folio \& Fewell, 2000). The PDMS-2 consists of 170 items grouped into five subtests that assessed Global Motricity (Posture Abilities, Locomotion Abilities and Object Handling Skills) and Fine Motricity (Fine Handling and Visuo-motor Integration Skills). Administration of PDMS-2 is individual and takes about 45 to 60 minutes, depending on the child's age. The scales are standardized for children and have an average value of 10 points $( \pm 3)$ for each test and the mean value of 100 points $( \pm 15)$ for motor quotients. To obtain information about the participants, an anamnesis form was created, in which information was collected on the type of breastfeeding.

\section{Procedures}

After approval by the institution of the data collection, a Free and Informed Consent Form was sent and requested the completion of the characterization form of the child, which allowed to select the subjects taking into consideration the exclusion requirements of the study.

All the norms and general procedures of administration of the scales mentioned by Folio and Fewell (2000) were followed. All ethical principles, norms and international standards relating to the Declaration of Helsinki and the Convention on Human Rights and Biomedicine were followed, respected and preserved (Tuckman, 2000). This project was approved by the Ethics Committee of the institution where the authors conduct their research (CE-UBI-Pj-2018-051: ID739). 


\section{Analysis}

For data analysis we used descriptive and inferential statistics. The Kolmogorov-Smirnov test was applied to test normality, with all variables not normal distribution, and the Mann-Whitney test for independent samples.

\section{RESULTS}

Breastfed children presented better results in locomotion skills ( $9,35 \pm 1,11$ vs. $8,95 \pm 1,06 ; p=0,012)$, object manipulation skills $(9,30 \pm 1,39$ vs. $8,72 \pm 1,66 ; p=0,042)$, visual-motor integration skills $(10,74 \pm 1,90$ vs. $9,99 \pm 2,16 ; p=0,000)$ and in global motricity $(103,18 \pm 7,49$ vs. $100,70 \pm 7,75 ; p=0,026)$ compared to those who were not breastfed. While the children who were not breastfed had better results in postural skills $(12,85$ $\pm 2,17$ vs. $12,36 \pm 2,16 ; p=0,185)$, fine manipulation skills $(12,04 \pm 1,92$ vs. $10,71 \pm 2,62 ; p=0,000)$ and in fine motor $(106,09 \pm 8,85$ vs. $104,39 \pm 11,81 ; p=0,264)$, when compared to those who were breastfed.

\section{DISCUSSION}

The decision to breastfeed that should be taken by the parents early, this decision can have important consequences for the cognitive and behavioural functioning of a child. After a thorough review of the subject, there were difficulties in finding breastfeeding investigations, mainly in this age group and to evaluate the motor skills. However, we can find in the study by McCrory and Murray (2012), a consensus, prevalent in epidemiological studies of great importance, in which children who were breastfed have higher levels in IQ tests and cognitive functioning than children who were fed by milk powder, but in our study breastfed children only show better global motor skills (locomotor skills and object manipulation). Thus, our results go according to the studies of Issaacs et al. (2010) where they observed differences in motor skills, comparing the type of breastfeeding.

\section{CONCLUSIONS}

We can conclude that in these children, those who were breastfed for breastmilk present better results in the global motricities, while children who were not breastfed for breastfeeding had better results in fine motor skills. These can be because breast milk because of its nutritional, immunological, psychological, social and economic benefits, make the child more "strong" at the global level (corer, jump, lance, kick), in turn the fact that children without breastmilk present better results in the fine motor of the early need that they have in the initial manipulation of the fine movements when handling baby bottle.

\section{REFERENCES}

Bosi, M., \& Machado, M. (2005). Amamentação: um resgate histórico, Cadernos Esp-Escola de Saúde Publica, V.1 - N.1 - Julho - Dezembro.

Folio, R., \& Fewell, R. (2000). Peabody Developmental Motor Scales-2. Austin: TX: Pro-Ed.

Isaacs, E. B., Fischl, B. R., Quinn, B. T., Chong, W. K., Gadian, D.G., Lucas, A. (2010). Impacto de leite materno sobre o quociente de inteligência, o tamanho do cérebro eo desenvolvimento da matéria branca. Pediatr.Res. 67, 357 - 362.

Levy, L., \& Bértolo, H. (2013). Manual de aleitamento materno. Comité Português para a UNICEF/Comissão Nacional Iniciativa Hospitais Amigos dos Bebés. Edição Revista.

McCrory, C., \& Murray, A. (2012). O efeito de amamentação em neuro desenvolvimento em infância. Matern. Criança J. Saúde. 
Saraiva, L., \& Rodrigues, L. (2007). Peadoby Developmental motor scale-2 (PDMS-2): definição e aplicabilidade no contexto educativo, clínico e científico. Viana do Castelo: Escola Superior de Educação, Instituto Politécnico de Viana do Castelo. https://doi.org/10.31014/aior.1993.01.01.14

\section{(c) (i) $\ominus$}

This work is licensed under a Attribution-NonCommercial-NoDerivatives 4.0 International (CC BY-NC-ND 4.0). 


\title{
Physiological responses at maximal aerobic swimming pacing in different distance-trials
}

\author{
TIAGO ALMEIDA ${ }^{1,3}$, DALTON M. PESSOA FILHO ${ }^{3}$, MÁRIO ESPADA $^{1,2}$, JOANA REIS ${ }^{1,4}$, ASTOR \\ SIMIONATO ${ }^{3}$, LEANDRO SIQUEIRA ${ }^{3}$, ANDREI SANCASSANI ${ }^{3}$, JOÃO OLIVEIRA ${ }^{3}$, FRANCISCO ALVES ${ }^{1}$ \\ ${ }^{1}$ CIPER - Faculty of Human Kinetics, University of Lisbon, Portugal \\ ${ }^{2}$ School of Education, Polytechnic Institute of Setúbal, Portugal \\ 3 UNESP, São Paulo State University, Brazil \\ ${ }^{4}$ Universidade Europeia, Lisbon, Portugal
}

\begin{abstract}
The aim of this study was to analyse the physiological responses of swimmers while performing different swimming distances at the maximal aerobic velocity (MAV) in order to understand the swimmers physiological impact along different levels of effort. Eight well trained swimmers performed a discontinuous incremental test for maximal oxygen uptake ( $\mathrm{VO}_{2 \text { peak }}$ ) and MAV assessment. Also, 3 different sets at the MAV, a timelimit session until exhaustion (TLim-MAV) and two distance trials of 100-m (100 mav) and 200-m (200 MAV) long, were performed. At the MAV tests, maximal rise of $\mathrm{VO}_{2}\left(\mathrm{VO}_{2 \text { rise }}\right)$, oxygen initial deficit $\left(\mathrm{O}_{2 \text { InitialDef }}\right)$ and $\mathrm{VO}_{2}$ kinetics $\left(\mathrm{VO}_{2} \mathrm{~K}\right)$ were determined. All tests were conducted using a breath-by-breath apparatus (K4b2, Cosmed, Italy) connected to a swimming snorkel (new-AquaTrainer ${ }^{\circledR}$, Cosmed, Italy) for pulmonary gas sampling and an underwater visual pacer for velocity control. $\mathrm{VO}_{2 \text { peak }}$ at the incremental test was significantly

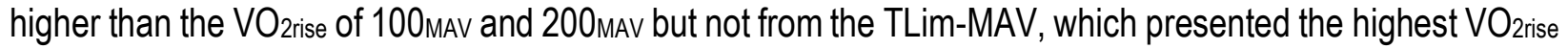
among the MAV tests. At the TLim-MAV swimmers were able to hold the intensity for $235.7 \pm 34.1 \mathrm{~s}$ performing $306.3 \pm 46.4 \mathrm{~m}$. The main finding of our work is that independently of the continuous rise of the $\mathrm{VO}_{2}$ along different swimming distances at the MAV, it does not affect the $\mathrm{VO}_{2} \mathrm{~K}$ response, which remained remarkably similar. The reference values of $\mathrm{VO}_{2 \text { rise }}$ for each swimming distance obtained in this study, as well as the $\sim 236$ seconds of swimmers capacity to hold the MAV, could be take into account by coaches for their daily training routines planning. Keywords: Maximal aerobic velocity; $\mathrm{VO}_{2}$ kinetics; $\mathrm{VO}_{2}$ slow component; Swimming; Youth athletes.
\end{abstract}

Corresponding author. CIPER - Faculty of Human Kinetics, University of Lisbon, Portugal.

E-mail: tiagofalmeida.w@gmail.com

Supplementary Issue: Spring Conferences of Sports Science. International Seminar of Physical Education, Leisure and Health, 17-19 June 2019. Castelo Branco, Portugal.

JOURNAL OF HUMAN SPORT \& EXERCISE ISSN 1988-5202

(C) Faculty of Education. University of Alicante.

doi:10.14198/jhse.2019.14.Proc4.82 


\section{INTRODUCTION}

The maximal aerobic velocity (MAV), that corresponds to the minimal velocity at which the maximal oxygen consumption ( $\mathrm{VO}_{2 \text { peak }}$ ) of an athlete occurs in an incremental test, is one of the most important variables of study in sports physiology since it combines exercise economy and $\mathrm{VO}_{\text {2peak }}$ into a single factor (Billat and Koralsztein, 1996). MAV, that can be easily accessed through the velocity of the swimmers 400 meters performance, or more precisely throughout the velocity of the 300 central meters of the 400 meters event, is being well related with performance and is usually used by coaches for training prescription (Espada et al., 2015). The aim of this study was to analyse the physiological responses of swimmers while performing different swimming distances at the MAV in order to understand the swimmers physiological impact along different levels of effort.

\section{MATERIAL AND METHODS}

\section{Participants}

Eight well trained swimmers, 6 males and 2 females (age $15.5 \pm 1.3 \mathrm{yr}$; mass $60.6 \pm 8.5 \mathrm{~kg}$; height $172.9 \pm$ $10.8 \mathrm{~cm}$ ), volunteered to participate in this study and were fully familiarised with the equipment and test procedures before the beginning of the test sessions. The criterion for swimmers participation was the regular participation at state or national championships. The study was approved by the local University Ethical Committee and conducted in accordance with the 1975 Declaration of Helsinki.

\section{Measures}

All swimmers performed 4 testing sessions separated by at least 48 hours: firstly, a discontinuous incremental test composed by 6 sets of 250-m, plus 1 set of 200-m at maximal intensity (Espada et al., 2015) was used to determine the $\mathrm{VO}_{2 \text { peak }}$ and $\mathrm{MAV}$; secondly, and in randomized order, swimmers performed 3 different sets at the MAV intensity, one time-limit session until exhaustion (TLim-MAV), and the other two with a previous defined distance of $100-\mathrm{m}(100 \mathrm{mav})$ and $200-\mathrm{m}(200 \mathrm{MAV})$ long. At the MAV tests the maximal heart rate (HR), rate of perceived exertion (PRE), maximal rise of the $\mathrm{VO}_{2}\left(\mathrm{VO}_{2 \text { rise }}\right), \mathrm{VO}_{2} \mathrm{~K}$ parameters (time delay (TD), time constant $(\tau)$ and amplitude $(\mathrm{A})$ and the oxygen deficit at the onset of exercise $\left(\mathrm{O}_{2 \text { nnitialef }}\right)$, were determined.

\section{Procedures}

All tests were conducted using a breath-by-breath apparatus ( $K 4 \mathrm{~b}^{2}$, Cosmed, Italy) connected to a swimming snorkel (new-AquaTrainer ${ }^{\circledR}$, Cosmed, Italy) for pulmonary gas sampling (Espada et al., 2012) and an underwater visual pacer (PACER 2 SWIM ${ }^{\circledR}$, KulzerTEC, Portugal) was used for setting the correct velocity of each test. For the maximal $\mathrm{VO}_{2}$ assessment, a $30 \mathrm{~s}$ moving average of the $\mathrm{VO}_{2}$ data was used for the incremental and 200 mav tests, while a sampling interval of $15 \mathrm{~s}$ was applied to the 100 mav test given it shorter duration. The $\mathrm{VO}_{2} \mathrm{~K}$ parameters were calculated, according to the following equation:

$$
\dot{V} O_{2}(t)=\left\{\begin{array}{lll}
\dot{V} O_{2 \text { base }} & \text { for } t<t d_{p} & \\
\dot{V} O_{2 \text { base }}+A_{p}\left(1-e^{-\left(t-t d_{p}\right) / \tau_{p}}\right) & \text { for } t d_{p} \leq t<t d_{s c} & \text { (primarycomponent) } \\
\dot{V} O_{2 \text { base }}+A_{p}\left(1-e^{-\left(t d_{s c}-t d_{p}\right) / \tau_{p}}\right)+A_{s c}\left(1-e^{-\left(t-t d_{s c}\right) / \tau_{s c}}\right) & \text { for } t \geq t d_{s c} & \text { (slow component) }
\end{array}\right.
$$

Where $\mathrm{VO}_{2(t)}$ represents the relative $\mathrm{VO}_{2}$ at a given time; $\mathrm{VO}_{2 \text { base }}$ represents the $\mathrm{VO}_{2}$ at rest; $\mathrm{TD}$, $\tau$, and $\mathrm{A}$, represent the time delay, the time constant (time that is needed to complete $~ 63 \%$ of the $\mathrm{VO}_{2}$ response) and the amplitude of the exponential response of the $\mathrm{VO}_{2} ; \mathrm{p}$ and sc represents the primary and secondary (slow component) exponential $\mathrm{VO}_{2}$ responses.

Analysis

VOLUME 14 | Proc4 | 2019 | S1687 
Normality of data was evaluated by the Shapiro-Wilk test. The differences between each session were tested for statistical significance using ANOVA for repeated measures with Bonferroni correction. Statistical significance was accepted at $p<0.05$.

\section{RESULTS}

At the incremental test the $\mathrm{VO}_{2 \text { peak }}$ and MAV were $60.5 \pm 2.1 \mathrm{ml} \cdot \mathrm{kg}^{-1} \cdot \mathrm{min}^{-1}$ and $1.31 \pm 0.06 \mathrm{~m}^{-1} \mathrm{~s}^{-1}$, respectively. $\mathrm{VO}_{2 \text { peak }}$ was significantly higher than the $\mathrm{VO}_{2 \text { rise }}$ of $100 \mathrm{MAV}$ and $200 \mathrm{MAV}$ but not from the TLim-MAV. At the TLim-MAV test swimmers were able to performed the intensity for $235.7 \pm 34.1$ seconds performing $306.3 \pm$ 46.4 meters. At the MAV tests (table 1), the TLim-MAV presented the higher $\mathrm{VO}_{2 \text { rise. The }} \mathrm{VO}_{2} \mathrm{~K}$ parameters did not show significantly differences at the primary component, while the slow component was just observed in the TLim-MAV test.

Table 2. Mean \pm SD of the swimmers physiological responses at the MAV tests. Statistical differences are identified by a, $b$ and $c$ for the 100MAV, 200MAV and TLim-MAV tests, respectively

\begin{tabular}{|c|c|c|c|}
\hline Variable & $100 \mathrm{MAV}$ & $200 \mathrm{MAV}$ & TLim-MAV \\
\hline $\mathrm{VO}_{\text {2rise }}\left(\mathrm{ml} \cdot \mathrm{kg}^{-1} \cdot \mathrm{min}^{-1}\right)$ & $52.0 \pm 2.9 c$ & $53.8 \pm 2.5^{c}$ & $60.1 \pm 1.8^{a, b}$ \\
\hline$\%$ to $\mathrm{VO}_{2 \text { peak }}(\%)$ & $86.1 \pm 5.2^{c}$ & $89.0 \pm 4.4^{c}$ & $99.4 \pm 2.4^{a, b}$ \\
\hline$A_{p}\left(\mathrm{ml} \cdot \mathrm{kg}^{-1} \cdot \mathrm{min}^{-1}\right)$ & $44.0 \pm 1.9$ & $44.2 \pm 3.9$ & $46.8 \pm 3.1$ \\
\hline $\operatorname{td}_{p}(s)$ & $12.1 \pm 2.5$ & $12.0 \pm 1.8$ & $11.7 \pm 3.1$ \\
\hline$\tau_{p}(s)$ & $22.8 \pm 9.3$ & $24.5 \pm 7.2$ & $23.7 \pm 7.0$ \\
\hline$A_{s c}\left(\mathrm{ml} \cdot \mathrm{kg}^{-1} \cdot \mathrm{min}^{-1}\right)$ & - & - & $3.9 \pm 2.0$ \\
\hline $\mathrm{Td}_{\mathrm{sc}}(\mathrm{s})$ & - & - & $156.7 \pm 29.3$ \\
\hline$\tau_{s c}(s)$ & - & - & $22.2 \pm 18.4$ \\
\hline $\mathrm{O}_{\text {2lnitialDef }}\left(\mathrm{mlO}_{2}\right)$ & $1583.7 \pm 508.0$ & $1620.8 \pm 414.7$ & $1667.7 \pm 402.8$ \\
\hline $\mathrm{HR}(\mathrm{bpm})$ & $159.2 \pm 11.3^{c}$ & $171.4 \pm 9.2$ & $185.8 \pm 8.7^{a}$ \\
\hline PRE (0-10 units) & $2.6 \pm 0.9 b, c$ & $5.8 \pm 2.0^{a}$ & $8.5 \pm 0.8^{a}$ \\
\hline
\end{tabular}

\section{DISCUSSION}

It is generally accepted that training with exercise intensities around the MAV could improve the athletes' aerobic parameters. The study of the physiological responses along different swimming distances could provide insightful information for a better planning of the training sets, especially for the high-intensity interval training (Sousa et al.,2018). Our results suggest that the main difference on the $\mathrm{VO}_{2}$ response occurs between the $100 \mathrm{MAV}$ and $200 \mathrm{mAV}$ in relation to the TLim-MAV test. Since the $\mathrm{VO}_{2} \mathrm{~K}$ parameters of the primary

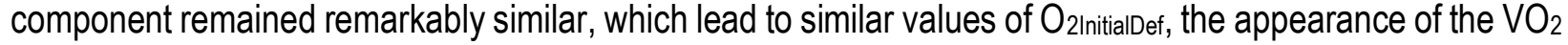
slow component at the $\sim 158$ s of the TLim-MAV test, which is usually associated to the effect of fatigue induced by the exercise, could justify the significantly higher $\mathrm{VO}_{2}$ found (Fernandes et al., 2003; Espada et al., 2015), while the 100mav and 200mav tests showed similar profiles between each other. Notwithstanding, swimmers reported significantly higher values of PRE at the 200 MAV compared to the 100mav test which indicates that some level of fatigue was already in the swimmers at that point.

\section{CONCLUSIONS}

The main finding of our work is that independently of the continuous rise of the $\mathrm{VO}_{2}$ along different swimming distances at the MAV, it does not affect the primary phase of $\mathrm{VO}_{2} \mathrm{~K}$ response. The reference values of $\mathrm{VO}_{2 \text { rise }}$ 
for each distance obtained in this study, as well as the $\sim 236$ seconds of swimmers capacity to hold the MAV, could be take into account by coaches for their daily training routines planning.

\section{REFERENCES}

Billat, V., \& Koralsztein, J. P. (1996). Significance of the velocity at VO2max and time to exhaustion at this velocity. Sports Med., 22(2), 90-108. https://doi.org/10.2165/00007256-199622020-00004

Espada, M., Reis, J., Almeida, T., Bruno, P., Vleck, V., \& Alves, F. (2015). Ventilatory and Physiological Responses in swimmers below and above their maximal lactate steady state, J Strength Cond Res. 29(10): 2836-43. https://doi.org/10.1519/jsc.0000000000000504

Fernandes, R. J., Cardoso, C. S., Soares, S. M., Ascensão, A., Colaço, P. J., \& Vilas-Boas, J. P. (2003). Time limit and VO2 slow component at intensities corresponding to VO2max in swimmers. Int J Sports Med., 24(8), 576-581. https://doi.org/10.1055/s-2003-43274

Sousa, A. C., Fernandes, R. J., Boas, J. P. V., \& Figueiredo, P. (2018). High-intensity Interval Training in Different Exercise Modes: Lessons from Time to Exhaustion. Int J Sports Med., 39(9), 668-673. https://doi.org/10.1055/a-0631-2682 


\title{
What do students think in physical education?
}

\author{
PAULO PEREIRA ${ }^{1} \triangle$, FÁTIMA BENTO², FERNANDO SANTOS ${ }^{1}$, ANTÓNIO CARDOSO ${ }^{1}$ \\ ${ }^{1}$ Polytechnic Institute of Porto, School of Higher Education, Portugal \\ ${ }^{2}$ Polytechnic Institute of Guarda, School of Higher Education, Communication and Sport, Portugal
}

\begin{abstract}
Based on a interpretative approach, this study aims to shed light on teaching processes within physical education. More specifically, this study explored students' thoughts during physical education classes. The participants were eight students in the ninth grade attending schools located at north of Portugal. Data was collected through interviews. The findings highlight that (a) students have more thoughts connected to the skill being taught before and during a task; (b) thoughts concerning how to execute a skill were more frequent during a task rather than before a task; (c) affective thoughts are more frequent before and during a task and seem to play a crucial role in students success; (d) thoughts non-related to a task are more frequent before students execute it. An increased awareness about students' thought processes may enable a more comprehensive understanding about how to create a favourable climate that is conducive to better learning outcomes. Keywords: Teaching; Reflection; Youth; Learning.
\end{abstract}

Corresponding author. Rua Dr. Roberto Farias 606, 4901-908 Porto, Portugal.

E-mail: pereira@ese.ipp.pt

Supplementary Issue: Spring Conferences of Sports Science. International Seminar of Physical Education, Leisure and Health, 17-19 June 2019. Castelo Branco, Portugal.

JOURNAL OF HUMAN SPORT \& EXERCISE ISSN 1988-5202

(c) Faculty of Education. University of Alicante.

doi:10.14198/jhse.2019.14.Proc4.82 


\section{INTRODUCTION}

Currently, one of the most relevant approaches to research in physical education considers the need to understand students' thoughts (Piéron, 1999). This research tendency alludes to the fact there is not a linear relationship between teacher behaviour and students' success (Walton-Fisette, 2010). Thus, there is a dual relationship: (a) one between teaching processes and students' thoughts; (b) the other between students' thoughts and learning or performance (Scrabis-Fletcher \& Silverman, 2017). Most research within physical education has stated that students' thoughts in classes are associated to high quality settings and students' success (Piéron, 1999; Locke \& Jensen, 1974). Therefore, this study explored students' thoughts during physical education classes.

\section{MATERIALS AND METHODS}

\section{Participants}

The participants were eight ninth grade students attending schools located at north of Portugal.

\section{Instruments}

Each student was interviewed five times during a 90-minute physical education class. In each interview students were asked about their thoughts in the moments prior and during a task.

\section{Procedure}

Before data collection, ethical clearance was attained to proceed with the present study.

\section{Data analysis}

A content analysis was conducted as all themes were defined deductively (Lee et al., 1992).

\section{RESULTS}

The findings show that before and during students' thoughts were focused on the skill being taught (see chart 1 for a detailed description of the results). Further, students focused more frequently on skill execution rather than an outcome. Prior to a task, affective components were also identified as a key part of students' thought processes. Further, students' thoughts concerning a specific skill were associated to students' success in a given task.

Chart 1. Students' thoughts in physical education sessions prior and during a motor task

\begin{tabular}{lcccc}
\hline \multirow{2}{*}{ Thoughts } & \multicolumn{2}{c}{ Before a task } & \multicolumn{2}{c}{ During a task } \\
\cline { 2 - 5 } Categories & $\mathrm{N}$ & $\%$ & $\mathrm{~N}$ & $\%$ \\
\hline Outcome & 8 & 20 & 9 & 22,5 \\
Technical skill & 12 & 30 & 16 & 40 \\
Negative self-assessment & 4 & 10 & 4 & 10 \\
Self-motivation & 5 & 12,5 & 2 & 5 \\
Task assessment & 4 & 10 & 6 & 15 \\
Non-related to a task & 7 & 17,5 & 3 & 7,5 \\
\hline TOTAL & 40 & 100 & 20 & 100 \\
\hline
\end{tabular}




\section{DISCUSSION}

These findings support the notion that students' thoughts are mainly task-related rather than affective which supports previous research in the field (Scrabis-Fletcher \& Silverman, 2017). Hence, the relationship between students' thoughts and their success may help guide teachers' interventions towards better learning outcomes in physical education. Affective variables seem to play a crucial in creating a climate conducive to better outcomes (Walton-Fisette, 2010).

\section{CONCLUSIONS}

The findings highlight that (a) students have more thoughts connected to the skill being taught before and during a task; (b) thoughts concerning how to execute a skill were more frequent during a task rather than before a task; (c) affective-related thoughts are more frequent before and during a task and seem to play a crucial role in students success; (d) thoughts non-related to a task are more frequent before students execute it. An increased awareness about students' thought processes may enable (a) appropriate instruction and feedback, (b) the creation of a positive climate that is conducive to learning, and (c) increase students awareness about a task and components of a skill.

\section{REFERENCES}

Lee, A., Landin, D., \& Carter, J. (1992). Student thoughts during tennis instruction. J Teach Phys Educ, 11(3), 256-267. https://doi.org/10.1123/itpe.11.3.256

Lee, A., \& Solmon, M. (1992). Cognitive conceptions of teaching and learning motor skills. Quest, 44(1), 57-71. https://doi.org/10.1080/00336297.1992.10484041

Locke, L., \& Jensen, M. (1974). Thought sampling: A study of student attention through self- report. Res. Q., 45(3), 263-275. https://doi.org/10.1080/10671315.1974.10615271

Piéron, M. (1999). Para una enseñanza eficaz de las actividades físico-deportivas. Barcelona: INDE.

Scrabis-Fletcher, K., \& Silverman, S. (2017). Student perception of competence and attitude in middle school physical education. Phys Educ, 74(1), 85-103. https://doi.org/10.18666/tpe-2017-v74-i16557

Walton-Fisette, J. (2010). Getting to Know Your Students. J Phys Educ Recr D, 81(7), 42-49. https://doi.org/10.1080/07303084.2010.10598508

\section{(9) $(\mathbb{Q} \Theta \Theta$}

This work is licensed under a Attribution-NonCommercial-NoDerivatives 4.0 International (CC BY-NC-ND 4.0). 


\title{
Leisure-time physical activity and food consumption among Brazilian university students
}

\author{
SANDRA FONSECA ${ }^{1} \triangle$ MICHELLE PONTE$^{2}$, MARIA ISABEL CARVALHAL ${ }^{1}$ \\ ${ }^{1}$ Research Center in Sports Sciences, Health Sciences and Human Development (CIDESD), Universidade \\ de Trás-os-Montes e Alto Douro (UTAD), Portugal \\ ${ }^{2}$ Centro Universitário INTA (UNINTA), Sobral, Ceará, Brazil
}

\begin{abstract}
The objective of this study was to explore the association between leisure-time physical activity and food consumption variables. The sample includes 324 university students from Ceará, Brazil, to whom the Isaq-A questionnaire was applied. Consumption of soft drinks, red meat and snacks is associated with physical inactivity, while consumption of fruits and vegetables is associated with physical activity. In conclusion, physical activity might be associated with the adoption of healthy lifestyles. Keywords: Leisure-time physical activity; Nutritional status; Food habits; University students.
\end{abstract}

Corresponding author. Universidade de Trás-os-Montes e Alto Douro, Vila Real, Portugal.

E-mail:sfonseca@utad.pt

Supplementary Issue: Spring Conferences of Sports Science. International Seminar of Physical Education, Leisure and Health, 17-19 June 2019. Castelo Branco, Portugal.

JOURNAL OF HUMAN SPORT \& EXERCISE ISSN 1988-5202

(C) Faculty of Education. University of Alicante.

doi:10.14198/jhse.2019.14.Proc4.82 


\section{INTRODUCTION}

The transition from high school to university is a critical time because it involves many behavioural changes with major implications for health. Some studies have documented unhealthy behaviours among university students, such as a decreased physical activity, increased smoking rates and alcohol consumption and an unhealthy diet (Wengreen \& Moncur, 2009). According to the World Health Organization (WHO, 2010) physical inactivity represents a high risk factor for overall mortality and there is a general perception that adults who opt for regular exercise also tend to adopt other healthy lifestyles. In this context, the objective of this research was to explore the associations between leisure-time physical activity and some food consumption variables.

\section{MATERIAL AND METHODS}

\section{Participants}

The sample includes 324 students from a university of northwest of Ceará, Brazil. To define the sample size and its representativeness, the proportion of students in each course was calculated by the ratio of the population to the number of students who met the eligibility criteria in each area.

\section{Measures}

Data were collected in 2017 with the application of the Isaq-A questionnaire validated for Brazilian university students (Sousa et al., 2013).

\section{Procedures}

A team of trained teachers applied the questionnaires to students who voluntarily enrolled in the project. Leisure-time physical activity was evaluated through questions related to physical leisure activities, weekly frequency and number of hours per day. The students were classified according to cut-off points for a weekly practice of physical activity: active $>150$ minutes and inactive $<150$ minutes.

\section{Analysis}

The logistic regression was performed to test the factors associated with physical activity prevalence.

\section{RESULTS}

Table 1. Logistic regression: Association between leisure-time physical activity and weekly food consumption

\begin{tabular}{|c|c|c|c|c|c|c|c|}
\hline & \multicolumn{2}{|c|}{ Active } & \multicolumn{2}{|c|}{ Inactive } & & & \\
\hline Weekly food consumption & Mean & DP & Mean & DP & $\mathrm{p}$ & OR & IC 95\% \\
\hline Fruit & 3.8 & 2.1 & 2.8 & 1.9 & $<0.01^{*}$ & 0.79 & {$[0.71-0.89]$} \\
\hline Vegetables & 3.3 & 2.4 & 2.7 & 2.1 & $0.02^{*}$ & 0.89 & {$[0.80-0.98]$} \\
\hline Red meat & 3.1 & 1.9 & 3.5 & 1.7 & $0.04^{*}$ & 1.14 & {$[1.01-1.28]$} \\
\hline Chicken with skin & 2.2 & 2.1 & 2.3 & 1.9 & 0.45 & & \\
\hline Salty snacks & 2.3 & 2.0 & 3.0 & 1.9 & $<0.01^{*}$ & 1.08 & {$[1.08-1.36]$} \\
\hline Soft drinks & 2.1 & 2.2 & 3.0 & 2.2 & $<0.01^{*}$ & 1.18 & {$[1.07-1.31]$} \\
\hline
\end{tabular}

$55.6 \%$ of university students practice leisure-time physical activity, of these $50.5 \%$ between 4 to 6 days/week and $66.7 \%$ more than 60 minutes/day; $44,4 \%$ are inactive. The linear regression (Table 1) indicates that consumption of red meat, snacks and soft drinks is associated with inactivity, and the risk is $1.18,1.14$ and 
1.08 times higher for those who consume these foods, respectively. The consumption of fruits and vegetables are preventive factors associated with practice of leisure-time physical activity. No statistically significant association was found between leisure-time physical activity and chicken consumption with skin.

\section{DISCUSSION}

Our results are in agreement with Wengreen and Moncu (2009) displaying unhealthy behaviours, such as high level of inactivity (44\%) and the consumption of unhealthy foods among university students. The consumption of unhealthy foods increases the chance of being physically inactive and, according to the WHO (2010), physical inactivity represents a high risk of general mortality. Poor diet and low levels of physical activity, during the university period, may represent risks for no communicable chronic diseases, such as obesity. The relationship between fruit and vegetables consumption and leisure-time physical activity is a preventive factor. The results suggest that college students with regular exercise also tend to adopt later other healthy lifestyles.

\section{CONCLUSIONS}

A high level of inactivity and inadequate eating habits was observed among students. The results support that the consumption of soft drinks, red meat and snacks is associated with physical inactivity, whereas the consumption of fruits and vegetables is associated to the practice of physical activity.

\section{REFERENCES}

Sousa TF, Fonseca AS, José HPM, Nahas MV. (2013). Validade e reprodutibilidade do questionário Indicadores de Saúde e Qualidade de Vida de Acadêmicos (Isaq-A). Arq Ciên Esporte. V1, n1:21.

Wengreen, H. \& Moncur, C. (2009). Change in diet, physical activity, and body weight among youngadults during the transition from high school to college. Nutrition Journal, 8-32. https://doi.org/10.1186/1475-2891-8-32

World Health Organization. (2010). Global Recommendations on Physical Activity for Health. WHO Library Cataloguing-in-Publication Data. ISBN 9789241599979.

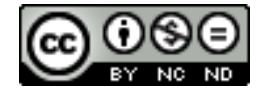

This work is licensed under a Attribution-NonCommercial-NoDerivatives 4.0 International (CC BY-NC-ND 4.0). 


\title{
Motor imagery and music: The influence of music on mental rotation of bodily-related pictures
}

\author{
FERNANDO CASTELLAR ${ }^{1}$, PEDRO DUARTE-MENDES ${ }^{2,3}$ \\ 1 University of Lisbon, Portugal \\ ${ }^{2}$ Department of Sports and Well-being, Polytechnic Institute of Castelo Branco, Castelo Branco, Portugal \\ ${ }^{3}$ SHERU - Sport, Health \& Exercise Research Unit, Polytechnic Institute of Castelo Branco, Castelo Branco, \\ Portugal
}

\begin{abstract}
The pursuit of excellence in sporting practices sheds light on the use of techniques beyond those that pertain to the physical training. Moreover, mental training is now considered a key aspect in an athlete's training routine, and as a consequence, mental imagery techniques have become popular among athletes and coaches since the mental practice of body movements reportedly improves the actual physical performance. One plausible explanation for this is the overlap between imagery and motor execution in terms of neural substrates activated in the pre-motor and motor cortical areas. Furthermore, music has been associated with enhancement in spatial-temporal reasoning, reflecting on performance in tasks that assess mental imagery processes, and this phenomenon is justified by the hypothesis of a direct cortical activation of areas responsible for spatial-temporal reasoning. It has also been reported that the motor system is actively involved in music processing, in which different patterns of neural activation in the pre-motor and motor cortical areas are elicited according to the rhythmic complexity of a stimulus, strengthening the hypothesis that musical stimuli may be responsible for improvement in motor imagery processes. To investigate this hypothesis, 30 Sports Science students completed a motor imagery-related cognitive task - a mental rotation of bodily-related pictures task - after exposure to three different stimuli (silence, music with complex rhythm and music with simple rhythm). Results showed no differences either in mental rotation performance, casting doubt on the hypothesis of improvement in imagery processes after music listening. Keywords: Functional equivalence hypothesis; Motor imagery; Mental rotation.
\end{abstract}

Corresponding author. University of Lisbon, Portugal.

E-mail: fernandocastellar@campus.ul.pt

Supplementary Issue: Spring Conferences of Sports Science. International Seminar of Physical Education, Leisure and Health, 17-19 June 2019. Castelo Branco, Portugal.

JOURNAL OF HUMAN SPORT \& EXERCISE ISSN 1988-5202

(C) Faculty of Education. University of Alicante.

doi:10.14198/jhse.2019.14.Proc4.82 


\section{INTRODUCTION}

Cumming and Williams (2013) argue that the study of imagery is the centre pillar of applied Sports Psychology, since mental techniques used by athletes play a role in the self-regulation of thoughts, feelings and behaviours. Additionally, the evidence of some shared activation in neural substrates involved with imagery of movement and those of motor planning gives rise to the Functional Equivalence Hypothesis (Jeannerod, 1994), a hypothesis which provides mental imagery of motor movements (motor imagery) with some functional effects on motor behaviour and how the mind plans and produces skilled actions and movements, ultimately proposing that, through these neural pathways, imagery can improve performance.

As far as the relationship between mental imagery and cognitive performance is concerned, Rauscher et al. (1993) reported subjects' performance enhancement in cognitive tasks related to mental imagery after listening to music, and this enhancement was justified by the direct activation of cortical areas related to the subsequent task by the music. In line with this claim, neuroimaging studies (Grahn and Brett, 2007) have found out that different levels of neural activations in the pre-motor and motor areas are elicited according to the rhythmic complexity of a stimulus, whereas stimulus with simple rhythmic patterns elicited significantly stronger activation than stimulus with complex rhythmic patterns. Thus, this study investigates whether musical stimuli facilitate motor imagery processes and, consequently, the performance of a task that reliably assesses these processes and also relies on the neural activation of the pre-motor and motor areas: a mental rotation of bodily-related pictures (Hamada et al., 2018).

\section{MATERIAL AND METHODS}

\section{Participants}

Participants were 30 voluntary Sports Science students from Instituto Politécnico de Castelo Branco (24 males and 6 females), aged 18-25 years (M: 20.21 years, $S D=3.20$ years). All participants reported their hearing as normal. No participant had previous experiences with mental rotation tasks.

\section{Measures}

As criteria of exclusion, the MIQ-3 translated and adapted to European Portuguese (Mendes et al., 2016) was employed in order to assess participants' mental imagery abilities, yielding no exclusion. Response times and accuracy in mental rotation tasks were computed by E-Prime 2.0 Psychological Software.

\section{Procedures}

Participants gave their informed consent to participate in the experiment, which was approved by the ethics committee of the University of Lisbon. Participants were placed in groups of 5 subjects each ( 1 female per group) and were exposed to all three stimuli for three consecutive days. The order of stimuli by which participants were exposed was counterbalanced. For the musical conditions, participants listened to 8:25 minutes of either the original version of an instrumental piece with a steady and simple rhythmic pattern (Space Katzler, by Motorcitysoul), or to the edited version of the song, in which the rhythmic patterns varied to purposely make rhythm perception more difficult (Grahn and Brett, 2007) and, consequently, elicit weaker neural activation in the pre-motor and motor areas. Participants remained in silence for the same time under the silence condition. After exposure to the stimuli, participants carried out a mental rotation task of pictures of hands and feet in different degrees of inclination $\left(0^{\circ}, 30^{\circ}, 60^{\circ}, 90^{\circ}, 120^{\circ}, 150^{\circ}, 180^{\circ}\right)$. Participants had to indicate whether the hand/foot depicted on the screen belonged to the left/right part of the body by pressing either the left or right arrow keys of the keyboard to left or right stimulus, respectively. 


\section{Analysis}

Firstly, the Kolmogorov-Smirnov test was employed in order to check whether data distribution was normal or not normal. The results showed that distribution was not normal; hence, non-parametric tests (Friedman test and a Wilcoxon test) were applied to carry out further data analysis.

\section{RESULTS}

A Friedman test yielded no significant differences neither in Response Times nor in Accuracy after exposure to the three different conditions (silence, music with simple rhythm and music with complex rhythm) in mental rotation task: $X^{2}(2, N=30)=, 467, p>0,05$ for Response Times, and $X^{2}(2, N=30)=, 883, p>0,05$ for Accuracy.

\section{DISCUSSION}

In line with the claims by Rauscher et al. (1993) and Grahn and Brett (2007), it had been hypothesized that exposure to music that reportedly activates neural cortical areas involved in motor imagery processes and in the completion of a mental rotation task of bodily-related pictures would result in a better performance at the task when participants were exposed to music with simple rhythmic patterns, music with complex rhythmic patterns and silence, respectively. The results did not confirm the hypothesis, neither in Response times nor in Accuracy. In a study by Chabris et al. (1999) that disputes Rauscher et al.'s (1993) claim, it was hypothesized that instead of directly activating cortical areas responsible for improvement in mental/motor imagery processes, music could be seen as a vehicle that changes arousal/mood levels and, as a consequence of this alteration, could improve subjects' performance in tasks that assess mental/motor imagery processes. This claim should be thoroughly examined in future studies.

\section{CONCLUSIONS}

Results provided no support for the hypothesis that music improves mental/motor imagery processes since no differences in performance in mental rotation task of bodily-related pictures were found after participants' exposure to either musical stimuli or silence. More studies are needed in order to investigate whether music may improve mental/motor imagery processes in different circumstances and settings compared to those of the present study, and if so, the main reasons for such improvement.

\section{REFERENCES}

Chabris, C. F. (1999). Prelude or requiem for the 'Mozart effect'? Nature, 400(1), 826-828. https://doi.org/10.1038/23608

Cumming, J. \& Williams, S. (2013). Introducing the revised applied model of deliberate imagery use for sport, dance, exercise, and rehabilitation. Movement \& Sport Sciences, 82(1), 69-81. https://doi.org/10.1051/sm/2013098

Grahn, J.A, \& Brett, M. (2007). Rhythm and beat perception in motor areas of the brain. Journal of Cognitive Neuroscience, 19(5), 893-906. https://doi.org/10.1162/jocn.2007.19.5.893

Hamada, H., Matsuzawa, D., Sutoh, C., Hirano, Y., Chakrabort, S., Ito, H., Tsjui, H., Obata, T., Shimizu, E. (2018). Comparison of brain activity between motor imagery and mental rotation of hand tasks: a fMRI study. Brain Imaging and Behavior, 12(1), 1596-2006. https://doi.org/10.1007/s11682-017$\underline{9821-9}$

Jeannerod, M. (1994). The representing brain: neural correlates of motor intention and imagery. Behavioural and Brain Sciences, 17(1), 187-245. https://doi.org/10.1017/s0140525x00034026 
Mendes, P. (2016). Tradução e Validação do Movement Imagery Questionnaire - 3 versão Portuguesa $\mathrm{e}$ as habilidades de Imagery em atletas de modalidades distintas (Doctoral Dissertation). Universidade da Beira Interior, Covilhã, Portugal. https://doi.org/10.22355/exaequo.2017.36.10

Rauscher, F. H., Shaw, G. L., \& Ky, K. N. (1993). Music and spatial task performance. Nature, 365(1), 611. https://doi.org/10.1038/365611a0

\section{(c)}

This work is licensed under a Attribution-NonCommercial-NoDerivatives 4.0 International (CC BY-NC-ND 4.0). 


\title{
Study of differences in motor coordination, comparing individuals with eutrophic and overweight, with Intellectual Disability
}

\author{
BRUNO TABORDA ${ }^{1} \triangle$, RUI PAULO ${ }^{1,2}$, HENRIQUE NEIVA ${ }^{3,4}$, JOÃO ROCHA ${ }^{1,2}$, ANDRÉ RAMALHO $^{1,2}$, \\ PEDRO DUARTE-MENDES ${ }^{1,2}$ \\ ${ }^{1}$ SHERU - Sport, Health \& Exercise Research Unit, Instituto Politécnico de Castelo Branco, Portugal \\ ${ }^{2}$ Department of Sports and Well-being, Instituto Politécnico de Castelo Branco, Portugal \\ ${ }^{3}$ Department of Sport Sciences, University of Beira Interior, Portugal \\ ${ }^{4}$ Research Center in Sport Sciences, Health Sciences and Human Development (CIDESD), Portugal
}

\begin{abstract}
The aim of the current study was to analyse and compare the motor coordination between eutrophic and overweight individuals with intellectual disability. Forty-eight 48 subjects were analysed regarding the body composition using the bio-impedance scale Inbody270. The battery of Körperkoordinationstest Fur Kinder (KTK) tests was applied to assess motor coordination. Then, the participants were allocated according to the BMI level. The Eutrophic group included those with BMI up to $24.9 \mathrm{~kg} / \mathrm{m}^{2}$ and the Overweight group, the subjects with BMl above $25 \mathrm{~kg} / \mathrm{m}^{2}$. There were significant differences $(p \leq 0.05)$ between the two groups of the sample in all KTK battery tasks, with the eutrophic group recording better results than the overweight group. In conclusion, individuals with intellectual disability, with BMI less than or equal to 25 (eutrophic), seem to be present better performance in motor coordination tasks compared to the overweight group. Keywords: Motor coordination; KTK; Physical activity; Intellectual disability.
\end{abstract}

Corresponding author. Av. Pedro Álvares Cabral, nº 126000-084 Castelo Branco, Portugal.

E-mail: taborda bruno@hotmail.com

Supplementary Issue: Spring Conferences of Sports Science. International Seminar of Physical Education, Leisure and Health, 17-19 June 2019. Castelo Branco, Portugal.

JOURNAL OF HUMAN SPORT \& EXERCISE ISSN 1988-5202

(c) Faculty of Education. University of Alicante.

doi:10.14198/jhse.2019.14.Proc4.82 


\section{INTRODUCTION}

According to Luckasson et al. (2002) intellectual disability (ID) is an intellectual subject alongside the average. Therefore, it is concomitant with interdisciplinarity in two or more areas of specialization such as: communication, personal autonomy, at home and in life, health and safety, social and academic skills, leisure and work. Studies by Temple et al. (2014) and Hamilton et al. (2007) also report a trend towards increased overweight and obesity and sedentary behaviour of ID groups. Furthermore, people should have access to a choice for a healthy lifestyle. Therefore, the referred factors can interfere in the amount of cardiovascular diseases and in the reduction of the probability of people with ID (Ferreira et al., 2017).

\section{MATERIAL AND METHODS}

This study is based on a quantitative typology, which is a cross-sectional study, since a single data collection is performed. Regarding design, this is an exploratory study with an "ex post facto" design, as it tries to establish cause-effect relationships. It proceeds between reality and past circumstances, which cannot be manipulated or controlled by the investigator.

\section{Participants}

In this study, individuals with mild intellectual disabilities, male and female, totalling 48 individuals ( $N=48$ ), with an average age of $27.33 \pm 8.42$, participated. The sample was divided into two groups, the first being the "Eutrophic - subjects with BMl up to $24.9 \mathrm{~kg} / \mathrm{m}^{2 "}$ and the "Overweight - subjects with BMl above $25 \mathrm{~kg} /$ $\mathrm{m}^{2 \prime}$. Participants fulfilled inclusion criteria, being individuals diagnosed with intellectual disability, individuals over 12 years of age and without cardiovascular limitations.

\section{Measures}

The body mass was weighted in kilograms to 100 grams using a digital scale, SECA 708 (Germany, Hamburg) to the approximation of hundredths. According to the technique described by the Council of Europe, two evaluations were carried out and the arithmetic average was calculated between them. Stature or total body height values were measured in millimetres by a SECA (Germany, Hamburg) stadiometer. BMI is determined using the formula: $\mathrm{BMI}=$ Body mass $(\mathrm{Kg}) /$ Height $(\mathrm{m}) 2$. The test used to assess motor coordination was the Körperkoordinationstest Fur Kinder (KTK), which consists of four tasks.

\section{Procedures}

All the associations that collaborated in the study received a request with the detailed explanation of the study. After this phase, a given data entry was accepted and agreed. As far as the study participants are concerned, only those with mild intellectual disability were included in the sample. In this way, satisfying the requirements, the informed consent term was fulfilled, following the Declaration of Helsinki.

\section{Analysis}

Data analysis was performed using SPSS (v.23.0). Subsequently, descriptive statistics were performed. To verify the normality of the data distribution, the "Shapiro-Wilk" test was used. This way, it was possible to observe that only the variable "Monopedal Jumps" presents non-normal distribution, with a normal distribution in all other variables. For the inferential analysis, the "Mann-Whitney $U$ test" (non-parametric for non-normal distribution) was used, and the "t-test" (parametric for normal distribution) was used to verify if there were differences between the two groups. For these tests, the level of significance was set for alpha $<0.05$ and alpha $<0.01$. The method of inferences based on the magnitude of the effects was also performed. The 
ranges for classifying the magnitude of effects (d Cohen) were as follows: 0-0.2, trivial; 0.21-0.6, small; 0.611.2, moderate, 1.21-2.0, large; > 2.0, very large (Hopkins et al., 2009).

\section{RESULTS}

Analysing the values of the variable "Balance Beam" we verified that there are statistically significant differences $(p=0.001)$ in the comparisons between the two groups of the sample, with the normal BMl group showing better results. Regarding the variable "Transfer of Platforms", there are statistically significant differences ( $p=0.001$ ) between the two groups, with the BMl Overweight group presenting worse results. Comparing now the variable "Jumps", we found that there are statistically significant differences $(p=0.002)$ between the groups and, in turn, the normal BMI group presented better results than the BMI Overweight. Finally, in the Total Score variable we showed statistically significant differences $(p=0.000)$ between the two groups, and once again, the group of BMI Overweight presented worse results.

\section{DISCUSSION}

As in other populations, there also appears to be evidence of increased functionality presented by individuals with normal BMI, either in the average values of the battery or in the maximum values reached (Gomes, Carletti, \& Perez, 2014). In a second phase of our inferential analysis we verified that in studies with ID of the same order, it was also verified that individuals with overweight BMI had worse physical fitness results, as in the case of Sousa et al. (2015). In their study, it was concluded that adults with overweight ID had higher values of body fat percentage and lower muscle strength / endurance levels than eutrophic ones. Still according to our research, Frey \& Chow (2006), in their study, found that overweight is minimally associated with aerobic conditioning and muscle strength in young people with mild ID.

\section{CONCLUSIONS}

It is concluded with the present study that, in the comparison between the eutrophic and overweight groups for the variables "Balance Beam", "Monopedal Jumps", "Jumps lateral", "Transfer platforms" and "final score" presents higher values and, in this case, better performances from the point of view of motor coordination. We also checked the effect size of our variables, presenting moderate values for "Balance Beam", "SingleHeel Jumps", "Side Jumps", "Platform Transfer" and a large value for the final score.

\section{REFERENCES}

Ferreira, A. P., Oliveira, L. d., Soutoa, E. C., Seron, B. B., \& Greguol, M. (2017). Indicadores Antropométricos e Pressão Arterial de Adultos com Deficiência Intelectual. Jornal Health Science, 160-164. https://doi.org/10.17921/2447-8938.2017v19n3p160-164

Frey, G., \& Chow, B. (2006). Relationship between BMI, physical fitness, and motor skills in youth with mild intellectual disabilities. International Journal of Obesity, 861-867. https://doi.org/10.1038/s.i.jo.0803196

Gomes, K. B., Carletti, L., \& Perez, A. J. (2014). Desempenho em teste cardiopulmonar de adolescentes: peso normal e excesso de peso. Revista Brasileira de Medicina do Esporte, 195-199. https://doi.org/10.1590/1517-86922014200301879

Hamilton, S., Hankey, C. R., Miller, S., Boyle, S., \& Melville, C. A. (2007). A review of weight loss interventions for adults with intellectual disabilities. obesity reviews, 339-345. https://doi.org/10.1111/i.1467-789x.2006.00307.x 
Hopkins, W. G., Marshall, S. W., Batterham, A. M., \& Hanin, J. (2009). Progressive Statistics for Studies in Sports Medicine and Exercise Science. Medicine \& Science in Sports \& Exercise, 3-12. https://doi.org/10.1249/mss.0b013e31818cb278

Luckasson, R., Borthwick-DUFFY, S., Buntinx, W., Coulter, D., Craig, E., Reeve, A.,...Tasse, M. (2002). Mental retardation: Definition,classification, and systems of supports. Washington DC: Association on Mental Retardation.

Sousa, G. R., Pinto, M. G., Seeber, J. R., \& Silva, D. A. (2015). Associação do estado nutricional com aptidão física relacionada à saúde em adultos com deficiência intelectual. Revista Brasileira de Educação Físisca Esporte, 543-550. https://doi.org/10.1590/1807-55092015000400543

Temple, V. A., Foley, J. T., \& Lloyd, M. (2014). Body mass index of adults with intellectual disability. Journal of Intellectual Disability Research participating in Special Olympics by world region, 277-284. https://doi.org/10.1111/jir.12011 


\title{
Validity and applicability of a web and mobile application to control the physical recovery of athletes
}

\author{
ELTO LEGNANI ${ }^{1,2} \triangle$, CLAUDIO OLTMANN ${ }^{1}$, TIAGO A. ANDRADE ${ }^{1}$, CAIO ANDRADE ${ }^{1}$, MÁRCIO JOSÉ \\ KERKOSKI ${ }^{2}$, ROSIMEIDE F.S. LEGNANI ${ }^{3}$ \\ ${ }^{1}$ Graduate Program in Biomedical Engineering (PPGEB), Federal Technological University of Paraná, \\ (UTFPR), Curitiba, Brazil \\ ${ }^{2}$ Department of Physical Education (DAEFI), Federal Technological University of Paraná (UTFPR), Curitiba, \\ Brazil \\ ${ }^{3}$ Universidade Estadual de Ponta Grossa, Departamento de Educação Física, Ponta Grossa, Paraná
}

\begin{abstract}
The advancement of physical training in sports has greatly increased with athletes' physical recovery. The present study was used to develop a web application and a mobile application to control the recovery of training loads through Total Quality Recovery (TQR) and was divided into four phases: 1) elaboration, 2) development, 3) validation and 4) application of the TQR scale in soccer and futsal athletes. In the development of the web and mobile applications specifically software's and were submitted to the evaluation of a committee of professionals in the area of sports training, considering 14 criteria for evaluation. The last phase of the project included the application of the TQR scale in a group of 105 male athletes of the collective futsal and soccer modalities, for a period of 4 consecutive weeks, 3 times a week, totalling 12 training sessions. In the validation process of the web version and mobile application, the experts evaluated 14 criteria's on a scale from "excellent" to "very poor". None of the items evaluated by the experts received a bad or very bad concept. The items that received the best rankings by the majority of the specialists were items 9,10 and 11 , that is, between $80 \%$ and $90 \%$ (excellent). The results generated from the web and mobile applications provided important elements about the recovery of each training session. With these data it is possible to prescribe the individualization of the training loads more appropriate to each athlete, helping in the prevention of injuries and the overtraining. Keywords: Web application; Mobile application; Training recovery.
\end{abstract}

Corresponding author. Graduate Program in Biomedical Engineering (PPGEB), Federal Technological University of Paraná, (UTFPR), Curitiba, Brazil.

E-mail: elto@utfpr.edu.br

Supplementary Issue: Spring Conferences of Sports Science. International Seminar of Physical Education, Leisure and Health, 17-19 June 2019. Castelo Branco, Portugal.

JOURNAL OF HUMAN SPORT \& EXERCISE ISSN 1988-5202

(c) Faculty of Education. University of Alicante.

doi:10.14198/jhse.2019.14.Proc4.82

S1704 | 2019| Proc4| VOLUME 14

C 2019 University of Alicante 


\section{INTRODUCTION}

The physical training aims to increase athletes' performance in a systematic, complex and dynamic way (Kentta \& Hassmen, 1998). The systematization of this process consists of a balance between the adequate distribution of training loads and sufficient recovery, seeking to find the ideal performance peaks, avoiding the unwanted adaptations known as: overtraining syndrome, staleness or burnout. (Bishop, Jones, \& Woods, 2008) (Borresen \& Lambert, 2008). Frequent advances in various areas of sports training such as nutrition, materials and equipment industry, technology and scientific technical knowledge have made increase the physical training in volume (quantitative), intensity (qualitative), and dramatically change the physical recovery variables in athletes. This became a concern for technicians, physical trainers, physiologists and sports scientists. Since these are intervening factors in the training and there is a very thin line between positive results and negatives caused by the stress of training, especially in athletes who are always in the body's psychophysical limit (Nakamura, Moreira, \& Aoki, 2010). In order to avoid this situation, in addition to biochemical, physiological and immunological indications, other less costly strategies can be used (Oltmann, Andrade \& Legnani, 2018), they are the psychophysiological indicators, which present good indicators of reliability. For example, mood swings coincide with increases and decreases in training loads, being highly replicable, correlating with physiological markers. In the researched literature, there are still few effective methods to monitor physical recovery, minimizing the risk of injury and aiding in the prevention of staleness and overtraining states. The present study had two objectives: development and validation of a web and mobile application to monitor the control to monitor the physical recovery of athletes during a period of training.

\section{MATERIAL AND METHODS}

\section{Participants}

For the validation of the application were selected 10 scientists from the training area. For the applicability stage of the system, a group of 105 male athletes from the futsal collective modalities were selected, with ages ranging from 12 to 17 years.

\section{Measures}

For the validation process of the TQR web and mobile version, 10 experts evaluated 14 criteria on a scale of "excellent", "very good", "good", "poor" and "very poor". The TQR scale was applied to a group of athletes of the collective modalities of futsal.

\section{Procedures}

The present study comprised the development and validation of a web application and a mobile application to monitor the control of retraining of training loads through Total Quality Recovery (TQR). The project was approved by the Human Research Ethics Committee of the Federal Technological University of Paraná under the number in the CAAE: 68706216.2.0000.5547 and was divided into 4 (four) phases: 1) elaboration, 2) development, 3) validation 4) application. In the development of web and mobile applications were developed using specific software's. The web application allocated to a company specialized in web hosting, can be accessed, free of charge through the link: www.e-trimp.com.br.

\section{RESULTS}

None of the items rated by experts received bad or very bad concept. The items that received the best rankings by the majority of the specialists were items 9,10 and 11 , that is, between $80 \%$ and $90 \%$ (excellent), 
$92 \%$ of the evaluators attributed a very good or excellent concept for all evaluated items. These indicators support the validity of web and mobile systems in controlling the physical recovery of soccer players. The web application allowed to collect several parameters inherent to the internal and external training loads, through the volume of training in hours and minutes for each session, the types of training applied to each cycle and the monitoring of the training loads. Data were collected from the athletes' recoveries at each session, and from this information, graphs were generated that correlated the recovery with the TQR items, in addition to identifying the points that are contributing or hindering their recovery.

\section{DISCUSSION}

The application was validated by the qualified professionals of the area. Also the application integrated different areas that need integration. Technological innovation, the web and mobile application for the physical recovery management of athletes, can help in the elimination of geographical and temporal barriers regarding the management of training sessions. The program can also provide instant feedback to athletes and coaches about training recovery processes, especially through a versatile, affordable and inexpensive application.

\section{CONCLUSIONS}

The web and mobile application e-TRIMP proved to be effective for the collection of important data regarding the recovery of the athletes. It provided several parameters for coaches' decision-making before the next training session, allowing individual training loads to be tailored to each athlete, assisting in injury prevention and overtraining.

\section{REFERENCES}

Bishop, P. A., Jones, E., \& Woods, A. K. (2008). Recovery from training: a brief review: brief review. Journal of Strength and Conditioning Research, 22(3), 1015-1024. https://doi.org/10.1519/JSC.0b013e31816eb518

Borresen, J., \& Lambert, M. I. (2008). Autonomic control of heart rate during and after exercise : measurements and implications for monitoring training status. Sports Medicine (Auckland, N.Z.), 38(8), 633-646. https://doi.org/10.2165/00007256-200838080-00002

Kentta, G., \& Hassmen, P. (1998). Overtraining and recovery. A conceptual model. Sports Medicine (Auckland, N.Z.), 26(1), 1-16. https://doi.org/10.2165/00007256-199826010-00001

Nakamura, F., Moreira, A., \& Aoki, M. (2010). Monitoramento da carga de treinamento: A percepção subjetiva do esforço da sessão é um método confiável? In Revista da Educação Física/UEM (Vol. 21). https://doi.org/10.4025/reveducfis.v21i1.6713

Oltmann, C., A. Andrade, T., \& Legnani, E. (2018). Métodos de monitoramento e controle da recuperação física em atletas: estudo de revisão. Anais Do V Congresso Brasileiro De Eletromiografia $E$ Cinesiologia E X Simpósio De Engenharia Biomédica. https://doi.org/10.29327/cobecseb.78850

\section{(9) $(\mathcal{Q} \Theta \Theta$}

This work is licensed under a Attribution-NonCommercial-NoDerivatives 4.0 International (CC BY-NC-ND 4.0). 


\title{
Motor imagery and music: A function of arousal?
}

\author{
FERNANDO CASTELLAR ${ }^{1} \triangle$, PEDRO DUARTE-MENDES ${ }^{2,3}$ \\ ${ }^{1}$ University of Lisbon, Portugal \\ ${ }^{2}$ Department of Sports and Well-being, Polytechnic Institute of Castelo Branco, Castelo Branco, Portugal \\ ${ }^{3}$ SHERU - Sport, Health \& Exercise Research Unit, Polytechnic Institute of Castelo Branco, Castelo Branco, \\ Portugal
}

\begin{abstract}
The study of imagery has been a central pillar in the Sports Psychology field, since athletes have reported the use of imagery techniques in order to mentally practice body movements and, therefore, improve the actual physical performance. Similarly, music plays a quintessential role in many sports as its influence on athletes' motivation, concentration, confidence and anxiety levels before and during the sporting practice has been widely reported and studied. The application of imagery and music combined has been reported to yield improvement in sporting performance. As a possible explanation, certain types of music have been considered as an element that decreases arousal levels in subjects, promoting a more effective use of imagery prior to performance. However, it has also been reported that subjects had better performance at tasks that assessed mental imagery processes after arousal increase after subjects' exposure to arousing music. Thus, this study has investigated whether two distinct musical stimuli (simple and complex music) affected arousal levels in participants, and consequently the performance in a mental imagery of body parts (henceforth: motor imagery) cognitive task, namely mental-rotation of bodily-related pictures. 30 Sports Science students completed this mental rotation of body pictures task after exposure to three different stimuli (silence, complex music and simple music). Although results showed arousal levels decrease after subjects were exposed to both complex music and silence, this decrease did not yield any effect on performance, casting doubt on the hypothesis that arousal decrease facilitates motor imagery processes. Keywords: Mental imagery; Motor imagery; Mental rotation; Subjective arousal.
\end{abstract}

Corresponding author. University of Lisbon, Portugal.

E-mail: fernandocastellar@campus.ul.pt

Supplementary Issue: Spring Conferences of Sports Science. International Seminar of Physical Education, Leisure and Health, 17-19 June 2019. Castelo Branco, Portugal.

JOURNAL OF HUMAN SPORT \& EXERCISE ISSN 1988-5202

(c) Faculty of Education. University of Alicante.

doi:10.14198/jhse.2019.14.Proc4.82

VOLUME 14 | Proc4 | 2019 | S1707 


\section{INTRODUCTION}

Although the influence of both imagery and music on sporting performance have been thoroughly examined within the Sports Psychology literature (Kuan et al. 2018), the field lacks studies in which imagery and music are used concomitantly at pre-intervention treatment prior to sporting performances. In a 12-week treatment in which elite shooters combined the use of different types of music and a motor imagery script, Kuan et al. (2018) found out that participants who listened to relaxing music had more improvement at dart-throwing tasks compared to their counterparts who listened to arousing music or no music at all. The authors claim that this difference in performance enhancement between groups is due to greater decrease in arousal by participants exposed to relaxing music compared to the other groups. On the other hand, studies from Cognitive Psychology have reported that the enhancement in performance in cognitive tasks that assess mental imagery processes (i.e. Paper-folding and cutting, mental rotation of pictures, etc.) was due to arousal increase and enjoyment of the stimulus (Husain et al, 2012). Thus, by selecting musical stimuli with high and low levels of load information (simple music and complex music, respectively) that were expected to elicit different arousal levels in participants (Kriger, 1989), this study investigated how the exposure to musical stimuli and silence would influence participants' level of arousal and, then, their performances in a cognitive task that assesses motor imagery processes - a mental rotation of body parts-picture (Hamada et al., 2018).

\section{MATERIAL AND METHODS}

\section{Participants}

Participants were 30 voluntary Sports Science students from Instituto Politécnico de Castelo Branco (24 males and 6 females), aged 18-25 years (M: 20.21 years, $S D=3.20$ years). All participants reported their hearing as normal. No participant had previous experiences with mental rotation tasks.

\section{Measures}

As criteria of exclusion, the MIQ-3 translated and adapted to European Portuguese (Mendes et al., 2016) was employed in order to assess participants' mental imagery abilities, yielding no exclusion. Arousal levels were assessed both before and after participants were exposed to musical stimulus by employing the SelfAssessment Manikin - SAM (Bradley and Lang, 1994). Response times and accuracy in mental rotation tasks were computed by E-Prime 2.0 Psychological Software.

\section{Procedures}

Participants gave their informed consent to participate in the experiment, which was approved by the ethics committee of the University of Lisbon. Participants were placed in groups of 5 subjects each ( 1 female per group) and were exposed to all three stimuli for three consecutive days. The order of stimuli to which participants were exposed was counterbalanced. For the musical conditions, participants listened to 8:25 minutes of either the original version of an instrumental piece with a steady and simple rhythmic pattern (Space Katzler, by Motorcitysoul), or to the edited version of the song, in which the rhythmic patterns varied to purposely make the piece as a high-information-load one (Kiger,1989). Participants remained in silence for the same time under the silence condition. Also, participants were asked to create mental images about their own bodies and imagine how they would physically react to the music they were listening to or to the silence (i.e. imagine if they were dancing). Both before and after exposure to musical stimulus, participants responded to the SAM (Bradley and Lang, 1994) in order to have their arousal levels assessed at these two different times. After exposure to the stimuli, participants carried out a mental rotation task of pictures of hands and feet in different degrees of inclination $\left(0^{\circ}, 30^{\circ}, 60^{\circ}, 90^{\circ}, 120^{\circ}, 150^{\circ}, 180^{\circ}\right)$. Participants had to 
indicate whether the hand/foot depicted on the screen belonged to the left/right part of the body by pressing either the left or right arrow keys of the keyboard to left or right stimulus, respectively.

\section{Analysis}

Firstly, The Kolmogorov-Smirnov test was employed in order to check whether data distribution was normal or not normal. The results showed that distribution was not normal; hence, non-parametric tests (Friedman test and a Wilcoxon test) were applied to carry out further data analysis.

\section{RESULTS}

A Friedman test yielded no significant differences neither in Response Times nor in Accuracy after exposure to the three different conditions (silence, music with simple rhythm and music with complex rhythm) in mental rotation task: $X^{2}(2, N=30)=, 467, p=0,792$ for Response Times, and $X^{2}(2, N=30)=, 883, p=0,643$. for Accuracy. A Wilcoxon test yielded significant decreases in arousal levels after participants were exposed to complex music and silence: $Z=-2,381, p=0,017$, median score rating $=5.00$ before and 4.00 after exposure to silence, and $Z=-2,325, p=0,020$, median score rating $=5.00$ before and 4.00 after exposure to complex music. However, the Wilcoxon test yielded no significant changes in arousal levels after participants were exposed to simple music: $Z=-1,827, p=0.69$, median score rating $=5.00$ before and 4.00 after exposure to simple music.

\section{DISCUSSION}

In line with the claims by Kuan et al (2018) and Kriger (1989), it had been hypothesized that exposure to music with low-information load (simple music) would result in participants' greater decrease in arousal levels and, consequently, a better performance in the task when participants were exposed to simple music than when exposed to high-information load music (complex music) and silence, respectively. The results did not confirm the hypothesis, suggesting that: 1) the hypothesis that music with low-information load decreases arousal compared to music with high-information load does not hold true, and 2) arousal decrease does not necessarily facilitate the motor imagery process. As there was no report of arousal increase, the hypothesis that arousal increase facilitates motor imagery processes remains to be clarified.

\section{CONCLUSIONS}

Results provided no support for the hypothesis that improvement in mental/motor imagery processes is a product of arousal increase/decrease, since changes in arousal levels did not influence performance in a mental rotation task of bodily-related pictures. Thus, more studies are needed in order to investigate whether music may improve mental/motor imagery processes in different circumstances and settings compared to those of the present study, and if so, the main reasons for such improvement.

\section{REFERENCES}

Bradley, M. M., \& Lang, P. J. (1994). Measuring emotion: The Self-Assessment Manikin and the semantic differential. Journal of Behavior Therapy and Experimental Psychiatry, 25(1), 49-59. https://doi.org/10.1016/0005-7916(94)90063-9

Hamada, H., Matsuzawa, D., Sutoh, C., Hirano, Y., Chakrabort, S., Ito, H., Tsjui, H., Obata, T., Shimizu, E. (2018). Comparison of brain activity between motor imagery and mental rotation of hand tasks: a 
fMRI study. Brain Imaging and Behavior, 12(1), 1596-2006. https://doi.org/10.1007/s11682-017$\underline{9821-9}$

Husain, G., Thompson, W. F., \& Schellenberg, E. G. (2002). Effects of musical tempo and mode on arousal, mood, and spatial abilities. Music Perception, 20(1), 151-171. https://doi.org/10.1525/mp.2002.20.2.151

Kiger, D. (1989). Effects of music information load on a reading comprehension task. Perceptual and Motor Skills, 69(1), 531-534. https://doi.org/10.2466/pms.1989.69.2.531

Kuan G, Morris T, Kueh Y., and Terry P. (2018) Effects of Relaxing and Arousing Music during Imagery Training on Dart-Throwing Performance, Physiological Arousal Indices, and Competitive State Anxiety. Frontiers in Psychology, 9(1), 1-12. https://doi.org/10.3389/fpsyg.2018.00014

Mendes, P. (2016). Tradução e Validação do Movement Imagery Questionnaire - 3 versão Portuguesa e as habilidades de Imagery em atletas de modalidades distintas (Doctoral Dissertation). Universidade da Beira Interior, Covilhã, Portugal. https://doi.org/10.22355/exaequo.2017.36.10

\section{@(@) $\Theta \Theta$}

This work is licensed under a Attribution-NonCommercial-NoDerivatives 4.0 International (CC BY-NC-ND 4.0). 


\title{
Effects of a physical exercise program on body composition and functional physical fitness in the elderly
}

\author{
ANDRÉ RAMALHO ${ }^{1,2} \unlhd$, RUI PAULO ${ }^{1,2}$, JOÃO ALEGRIA ${ }^{1,2}$, PEDRO DUARTE-MENDES ${ }^{1,2}$, JOÃO \\ SERRANO ${ }^{1,2}$, PAULO SILVEIRA ${ }^{1,2}$, JOÃO PETRICA ${ }^{1,2}$ \\ ${ }^{1}$ SHERU - Sport, Health \& Exercise Research Unit, Instituto Politécnico de Castelo Branco, Portugal \\ ${ }^{2}$ Department of Sports and Well-being, Instituto Politécnico de Castelo Branco, Portugal
}

\begin{abstract}
The aging process causes innumerable changes in elderly people daily routines, affecting their functionality, mobility, health, and depriving them of an autonomous and healthy life, debilitating their quality of life. This study aims to verify the effects of the practice of a physical exercise program on body composition and functional fitness of elderly people. Participated in our study 40 elderly people divided into two groups. The experimental group ( $N=20$ ) subjected to structured physical activity and the control group $(N=20)$, that didn't practice any structured physical activity. We used the battery Functional Fitness Test, and the evaluation of the measures of body composition (body mass index, waist circumference, percentage of fat mass, bone mass and muscular mass). In the comparison between Baseline and Follow-up the control group presents differences in the strength and resistance of the lower limbs, strength and resistance of the upper limb, flexibility of the lower limb, agility, flexibility of the upper limb, Muscular Mass and Bone Mineral, with worse values in the follow-up. Regarding the experimental group, in the comparison between Baseline and Followup, we observed differences in flexibility of the lower limb, flexibility of the upper limb, Muscle Mass, \% Fat Mass and Bone Mineral, with worse values in Follow-up. In conclusion, it was verified that in both groups there was involution in the results, from baseline to Follow-up, but this involution was more severe in the control group. Keywords: The elderly; Ageing; Physical activity; Physical abilities.
\end{abstract}

Corresponding author. Rua Prof. Dr. Faria de Vasconcelos 6000-266 Castelo Branco, Portugal.

E-mail: andre.ramalho@ipcb.pt

Supplementary Issue: Spring Conferences of Sports Science. International Seminar of Physical Education, Leisure and Health, 17-19 June 2019. Castelo Branco, Portugal.

JOURNAL OF HUMAN SPORT \& EXERCISE ISSN 1988-5202

(c) Faculty of Education. University of Alicante.

doi:10.14198/jhse.2019.14.Proc4.82 


\section{INTRODUCTION}

The evaluation of the functional aptitude of elderly individuals is thus important, as it is possible to find ways to prevent or delay the onset of physical fragility that occur in advanced ages. Health is not only the absence of diseases, but also the presence of certain levels of physical and functional capacity of the organism, which are the physiological bases of the physical and psychic well-being of the human being. Muscular strength can be understood as the ability of humans to overcome or oppose resistance through their muscular structure (Mazo, Lopes, \& Benedetti, 2009). The motor ability flexibility is an essential component of physical fitness because it is considered a capability that allows the accomplishment of motor actions, with great breadth and harmony of movement, maintaining the physiological joint stability. Physical mobility, agility, speed and balance are a set of motor abilities that allow the individual to change the position of the body or the direction of a movement with time efficiency (Mazo et al., 2009). Body composition values have been changing over the years, where physical exercise prescription, together with a healthy diet program, are significant indicators for an optimal weight at levels of quality of life and health of the human being (McArdle et al., 2008). The objective of the present study was to investigate the effect of a physical exercise program on body composition and functional physical fitness in institutionalized and non - institutionalized elderly.

\section{MATERIAL AND METHODS}

\section{Participants}

In this longitudinal study 40 elderly people participated. After selecting the participants (for convenience), with the application of a sociodemographic questionnaire, the elderly were allocated in 2 groups. The control group, with 20 elderly people who did not participate in the supervised exercise program, maintaining the life cycle, and the experimental group, comprised of 20 elderly people who started participating in the supervised physical exercise program.

\section{Measures}

The data were collected through the Tanita BC-601 Tetrapolar bioimpedance balance, which allowed to evaluate the following variables: Body Mass Index (BMI), Skeletal Muscle Mass (SMM), Fat Mass (FM) and $\%$ Body Fat $\%(\% \mathrm{BF})$, according to the recommendations of Heyward and Stolarczyk (2000). The Waist circumference (WC) was measured using a tape measure (Rosscraft) measuring two meters and a resolution of $1 \mathrm{~mm}$. For the evaluation of Functional Physical Fitness, the Functional Fitness Test battery of Rikli and Jones (2013) was used.

\section{Procedures}

All ethical and legal human research principles have been considered (Helsinki Declaration). An initial evaluation was performed to both groups and after six months a second evaluation was carried out. The experimental group was submitted to a program consisting of physical activity classes, biweekly, with sessions of 60 minutes, with multivariate and moderate intensity exercises. The program lasted 6 months.

\section{Analysis}

Regarding the statistical analysis, using the SPSS 23.0 Software, we verified the distribution of data normality (Shapiro-Wilk test). For the variables that presented a non-normal distribution, we used non-parametric statistics, using the Man-Withney $\mathrm{U}$, for the remaining variables, with normal distribution, we used the t-test. The level of significance was set for alpha $<0.05$ and alpha $<0.01$. The method of inferences based on the magnitude of the effects was also performed. The ranges for classifying the magnitude of effects ( $d$ Cohen) 
were as follows: 0-0.2, trivial; 0.21-0.6, small; 0.61-1.2, moderate, 1.21-2.0, large; > 2.0, very large (Hopkins et al., 2009).

\section{RESULTS}

In the control group, in the comparison between Baseline and Follow-up, statistically significant differences $(p \leq 0.05)$ were observed regarding the strength and resistance of the lower limbs $(p=0.00)$, limb strength and resistance $(p=0.00)$, flexibility of the lower limb $(p=0.00)$, agility $(p=0.00)$ and flexibility of the upper $\operatorname{limb}(p=0.00)$. In this way, the control group showed significant losses in functional aptitude between the two moments. Regarding body composition, we observed statistically significant differences $(p \leq 0.05)$ in the variables muscle mass $(p=0.00)$ and bone mineral $(p=0.00)$. Here too, the worst values were recorded in the follow-up and moderate magnitudes were recorded. Regarding the experimental group, in the comparison between Baseline and Follow-up, we only found statistically significant differences $(p \leq 0.05)$ regarding functional physical fitness, lower limb flexibility $(p=0.00)$, and flexibility of the upper $\operatorname{limb}(p=0.00)$. The worst values were recorded in the follow-up and moderate magnitudes of effect were recorded. In this group, we found statistically acceptable differences $(p \leq 0.05)$ in the variables muscle mass $(p=0.00)$, percentage of fat mass $(p=0.00)$ and bone mineral $(p=0.00)$. Here too, the worst values were recorded in the follow-up and moderate magnitudes were recorded.

\section{DISCUSSION}

Despite the decrease in limb strength with age, this change can be attenuated with exercise. This decrease in strength is more pronounced when we associate old age with physical inactivity and physical inactivity. Eckert (1993), as a rule, will be slower, with progressive aging, since there will be a longer reaction time and consequently a slower movement speed. The same phenomenon occurred in the control group where the best results were recorded in the baseline. The reduction in balance, postural instability and falls interfere with walking parameters and the quality of life of the elderly (Sanglard et al., 2004). There was also an involution in the bone mineral in both groups. Nóbrega et al. (1999) report that the reduction of bone mineral, when more pronounced, characterizes osteoporosis, predisposing to the occurrence of fractures, as well as changes in articular cartilage and biomechanics, which impair the locomotor function and flexibility, hindering movement. There was also an involution in the body composition variables. The plausible explanation for a higher energy expenditure associated with exercise does not fit with our results, evidenced by the principle that the combination of diet and regular physical activity forms the most effective means of weight control (Slentz et al., 2004).

\section{CONCLUSIONS}

In conclusion, it was verified that in both groups there was involution in the results, from baseline to followup, but this involution was more severe in the control group.

\section{REFERENCES}

Eckert, H. (1993). Desenvolvimento motor. ( $3^{\mathrm{a}}$ ed.). São Paulo: Manole. Heyward V., \& Stolarczyk, L. (2000). Avaliação da Composição Corporal Aplicada. São Paulo: Manole. Mazo, G., Lopes, M., \& Benedetti, T. (2009). Atividade Física e o idosos: Concepção Gerontológica (3a ed.). Porto Alegre: Sulina. 
McArdle, W., Katch, F., \& Kacth, V. (2008). Fisiologia do exercício: energia, nutrição e desempenho humano ( $6^{\mathrm{a}}$ ed.). Rio de Janeiro: Guanabara Koogan.

Nóbrega, A., Freitas, E., Oliveira, M., Leitão, M., Lazzoli, J., Nahas, R., ... Rose, E. (1999). Posicionamento Oficial da Sociedade Brasileira de Medicina do Esporte e da Sociedade Brasileira de Geriatria e Gerontologia: Atividade Física e Saúde do Idoso. Revista Brasileira de Medicina do Esporte, 5, 207-211. https://doi.org/10.1590/s1517-86921999000600002

Rikli, R., \& Jones, C. (2013). Development and validation of criterion-referenced clinically relevant fitness standards for maintaining physical independence in later years. Gerontologist, 53, 255-267. https://doi.org/10.1093/geront/gns071

Sanglard, R., Henriques, G., Ribeiro, A., Corrêa, A., \& Pereira, J. (2004). Alterações dos parâmetros da marcha em função das queixas de instabilidade postural e quedas em idosos. Fitness e Performance, 3, 149-156. https://doi.org/10.3900/fpj.3.3.149.p

Slentz, C., Duscha, B., Johnson, J., Ketchum, K., Aiken, L., Samsa, G., ... Kraus, W. (2004). Effects of the amount of exercise on body weight, body composition, and measures of central obesity: STRRIDE--a randomized controlled study. Archives of internal medicine, 164, 31-39. https://doi.org/10.1001/archinte.164.1.31

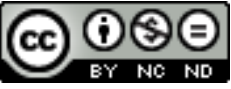

This work is licensed under a Attribution-NonCommercial-NoDerivatives 4.0 International (CC BY-NC-ND 4.0). 


\title{
Quality of life of Nursing students: Relationship with the level of Physical Activity
}

\author{
MARINELA PINTO1, AMÂNCIO CARVALHO² \\ ${ }^{1}$ Centro Hospitalar de Trás-os-Montes e Alto Douro EPE, Unidade de Vila Real, Portugal \\ 2Escola Superior de Saúde, Universidade de Trás-os-Montes e Alto Douro, Centro de Investigação em \\ Estudos da Criança, Portugal
}

\begin{abstract}
Currently there is an increase in the interest of the scientific community in studying the Level of Physical Activity (LPA) of university students, given that this stage of life is where the lifestyles are consolidated that contribute to Quality of Life $(\mathrm{QL})$ and health. It is intended to analyse the relationship between the QL and the LPA of Portuguese nursing students. Descriptive-correlational study, cross-sectional, of quantitative approach, with a sample of 289 Nursing students. A questionnaire was applied, which included the WHOQOL-Bref scale and the International Physical Activity Questionnaire. Data were processed using SPSS software (24.0), with descriptive and inferential statistics. The level of significance was $5 \%$. In the total sample $(n=289)$, the majority were female $(77.0 \%)$ and were in the $20-21$ age group $(40.5 \%)$. The average of the Global QL was of $63.48 \pm 10.64$ points and $41.9 \%$ of the students was in the Moderate LPA. The Global QL did not differ significantly among students with different LPA (ANOVA: $p \geq 0,419$ ). The Global QL of the student sample may be considered good and the LPA moderated, being very similar to the values obtained by other studies. There was no relationship between QL and LPA, as opposed to other studies. It will be important that higher education establishments can extend their training and cover not only the technical and scientific issues, but also take a close look at the health, promoting physical activity of their students. Keywords: Quality of life; Physical activity; Diagnosis of health situation; Student health.
\end{abstract}

Corresponding author. Rua das Pintas, $n^{\circ}$ 5, Bairro da Laverqueira, Lordelo, 5000-225 Vila Real, Portugal.

E-mail: amancioc@utad.pt

Supplementary Issue: Spring Conferences of Sports Science. International Seminar of Physical Education, Leisure and Health, 17-19 June 2019. Castelo Branco, Portugal.

JOURNAL OF HUMAN SPORT \& EXERCISE ISSN 1988-5202

(C) Faculty of Education. University of Alicante.

doi:10.14198/jhse.2019.14.Proc4.82 


\section{INTRODUCTION}

Quality of Life (QL) is considered a multidimensional construct, involving the physical, psychological, social and environmental dimensions (The WHOQOL Group, 1995). The National Program for the Promotion of Physical Activity (DGS, 2016), encompasses the university setting, among the spaces in which it will be necessary to promote a physical and socio-cultural environment, facilitating more PA and less sedentary time. This program states that in Portugal in 2008, physical inactivity was responsible for $8.4 \%$ of deaths due to cardiovascular diseases, $10.5 \%$ for Type 2 Diabetes Mellitus, $14.2 \%$ for breast cancer and $15.1 \%$ for colon cancer. It is within the scope of this problematic that our study emerges that aims to analyse the relationship between the QL and the level of PA (LPA) of nursing students.

\section{MATERIAL AND METHODS}

Descriptive-correlational study, transversal and quantitative approach.

\section{Participants}

The population was made up of all undergraduate Nursing students attending a health school in the north of Portugal, aged over 18, estimated at 375 students. The non-random and convenience sample included 289 students, about $77.1 \%$ of the population. The majority of students $(n=289)$ were female $(77.9 \%)$, were in the $20-21$ age group ( $40.5 \%$ ) and belonged to the middle class (39.4\%). The mean age was $21.29 \pm 4.398$ years.

\section{Measures}

In the collection of data we used a self-questionnaire, consisting of three parts: i) sociodemographic characterization; ii) LPA through the International Physical Activity Questionnaire (IPAQ short version), validated for 12 countries, including Portugal, by Craig et al. (2003) (criterion validity from mild to moderate with correlation value $r=0.33$ ). This questionnaire classifies individuals into three PA levels: Low/Inactive, Moderate and High; iii) Evaluation of QL through the WHOQOL Bref, validated for the Portuguese population, by Vaz Serra et al. (2006). It consists of 26 items and four domains, including a facet about the General QL. The instrument has good internal consistency for the 26 items of 0.92 .

\section{Procedures}

In order to carry out the study, authorization was requested from the Ethics Committee of the Higher Education Institution, which received a favourable opinion (Opinion no. 37/2017). The ethical principles were respected, including free and informed consent, confidentiality of data and anonymity. The researchers contacted the teachers in each class, and the date of data collection was scheduled. On the scheduled date they went to school and distributed and collected the questionnaires below.

\section{Analysis}

The data treatment was performed through the Software Statistical Package for the Social Sciences (SPSS version 24.0). We used the descriptive and inferential statistics, having applied Student's $t$ test and ANOVA.

\section{RESULTS}

Of the total sample, the largest group of students (41.9\%) had moderate LPA and the lowest percentage of students had a high LPA (24.6\%). Regarding the global QL, the students presented a positive and reasonable perception $(63.48 \pm 10.64)$. There were no significant statistical differences between the mean of the global QL score of students with different levels of PA (ANOVA: $p>0.419$ ). 


\section{DISCUSSION}

In the present study it was verified that the majority of students, of both sexes, had moderate PA (41.9\%). This result is corroborated by the study by Esteves, Vieira, Brás, O'Hara and Pinheiro (2017), conducted at the University of Beira Interior (UBI) and University of Madeira (UMa), with university students, in which the authors found that at the UBI, $42 \%$ of the students had a moderate LPA and at UMa about $38 \%$. The percentage of UBI students is almost the same as in the present study. In terms of QL, our results are in agreement with those obtained by Langame et al. (2016), in which 492 students participated (Brasil), where they found that regarding the global QL, it also presented a positive mean (72.5 \pm 18.2$)$, higher than the present study. In the present study there were no statistically significant differences between the global QL and different LPA. However, these results do not corroborate the reviewed literature which points out that the higher the LPA, the higher the QL scores (Mendes-Netto, Silva, Costa and Raposo, 2012).

\section{CONCLUSIONS}

The LPA of the students in the sample is predominantly moderate. However, it is noteworthy that a third of the students were enrolled in the low/inactive LPA, perhaps explained by the enormous curricular load of the course. On the other hand, the Global QL of the students in the sample can be said to be good. We did not find any relation between the global QL and LPA, perhaps due to the reduced intensity of the PA practiced by this sample. This higher education institution should promote PA in its students, since one-third have low LPA or are inactive as a measure to improve their QL.

\section{REFERENCES}

Craig, C., Marshall, A., Sjostrom, M., Bauman, A., Booth, M., Ainsworth, B., Oja, P. (2003). Internacional physical activity questionaire: 12-country reliability and validity. Medicine and Science in Sports and Exercise, 35(8), 1381-1395. https://doi.org/10.1249/01.mss.0000078924.61453.fb

Direção-Geral da Saúde (DGS, 2016). Programa Nacional para a Promoção da Atividade Física. A saúde dos Portugueses 2016. Lisboa: DGS. https://doi.org/10.1590/s0034-89102002000200022

Esteves, D., Vieira, S., Brás, R., O'Hara, K., \& Pinheiro, P. (2017). Nível de atividade física e hábitos de vida saudável de universitários portugueses. Revista Iberoamericana de Psicología del Ejercicio y el Deporte, 12(2), 261-270. Retrieved from http://www.redalyc.org/html/3111/311151242009/

Langame, A. P., Neto, J- A. C., Melo, L. N. B., Castelano, M. L., Cunha, M., \& Ferreira, R. E. (2016). Qualidade de vida do estudante universitário e o rendimento académico. Revista Brasileira em Promoção da Saúde, 29(3), 313-325. https://doi.org/10.5020/18061230.2016.p313

Mendes-Netto, R. S., Silva, C. S., Costa, D., \& Raposo, O. F. F. (2012). Nível de atividade física e qualidade de vida de estudantes universitários da área de saúde. Revista de Atenção a Saúde, 10(34), 47-55. https://doi.org/10.13037/rbcs.vol10n34.1802

The WHOQOL Group (1995). The World Health Organization Quality of Life assessment (WHOQOL): Position paper from the World Health Organization. Soc. Sci. Med., 41(10):1403-1409. https://doi.org/10.1016/0277-9536(95)00112-k

Vaz Serra, A., Canavarro, M.C., Simões, M., Pereira, M., Gameiro, S., Quartilho, M.J., Rijo, D., Carona, C., \& Paredes, T. (2006). Estudos psicométricos do instrumento de avaliação da qualidade de vida da Organização Mundial de Saúde (WHOQOL-Bref) para Português de Portugal. Psiquiatria Clínica, 27(1): 41-49. https://doi.org/10.14417//p.627 


\section{(c) (i) (3)}

This work is licensed under a Attribution-NonCommercial-NoDerivatives 4.0 International (CC BY-NC-ND 4.0). 


\title{
Motor skills in childhood: From the family perceptions to the practices of the children
}

\author{
ISABEL CABRITA CONDESSA
}

Centro de Investigação em Estudos da Criança, Universidade do Minho, Faculdade de Ciências Sociais e Humanas, Universidade dos Açores, Portugal

\begin{abstract}
The school has a prominent place in the implementation of life habits that promote Physical Activity (PA), allowing children to acquire a motor skill repertoire that will streamline their future practices. Promoting early PA in children's lives prevents them from becoming young and adults with greater possibility of comorbidities associated with sedentary behaviours and overweight. There is that many families still do not set an example or incentive for motor practice, creating barriers to children's performance. With this study we tried to relate the perceptions of family kindergarten/pré school $(\mathrm{KC})$ and primary/ 1st cycle schools (PC) children competence in some typical and unique motor skills in childhood, and perception of a little group of children. The methodology used involved the application of a questionnaire survey, carried out to family members and adapted to a group of children from the 1st cycle. The analysis of the data will be made with treatment descriptive (percentage) comparative (chi square) and correlate (ró spearman) was carried out through 25.0 SPSS version and that allowed us to reach some final considerations. We have noted some differences, especially in what refers to the perceptions of the practices of "childhood routine" such as jumping (rope and shaft), balancing and moving equipment (roller with skates and bicycle) and water (swimming) and climbing trees or walls. As a reinforcement of this analysis, we have crossed data obtained about the perception of 1st cycle children's of their competence in these abilities and we can saw a low performance in in jumping rope. Keywords: Childhood; Motor skills; Perceptions; Families; Children.
\end{abstract}

Corresponding author. Rua da Mãe de Deus 9501-855 Ponta Delgada, Portugal.

E-mail: maria.id.condessa@uac.pt

Supplementary Issue: Spring Conferences of Sports Science. International Seminar of Physical Education, Leisure and Health, 17-19 June 2019. Castelo Branco, Portugal.

JOURNAL OF HUMAN SPORT \& EXERCISE ISSN 1988-5202

(c) Faculty of Education. University of Alicante.

doi:10.14198/jhse.2019.14.Proc4.82 


\section{INTRODUCTION}

According to Seabra $(2017$, p.15), the school is considered the ideal place for the implementation of Physical Activity (PA) Program. The children have a lot of their working time in school, what motivates them to be stimulated to maintain an active living environment. In fact, school plays an important role in the physical and motor education of children and, for it we try to understand, the family awareness how they had capacity ability to moved and performer in motor skills to consolidate their role in the future practice in PA.

"Good Experiences" at school and with the family in the weekend, through different motor skills in childhood, may also improve motor skills, which can influence later participation in PA and in sportive activities. Childhood movement skills like running, jumping, throwing, catching, cycling, etc. are the essential for the acquisition of sportive skills that can be applied later in life, such as sporting, recreational and physical activity pursuits. (Goldfield, Harvey, Grattan \& Adamo, 2012).

\section{MATERIAL AND METHODS}

\section{Participants}

Participated in the study one hundred and ninety individuals, 172 families in the majority parents [family of 106 kindergarten/preschool children (KC) and 66 primary/1st cycle (KP)] and 18 children of the primary/1 st cycle of this group (6-8 years old) of some public schools in the same region.

Parents believe that it is important to develop PA with their students $(90.9 \%)$ and believe in the role of the school, not only in general in the practice of Physical Education (PE), but also in the 1st Cycle through initiation to Sports at school. They emphasize the importance for physical and psychomotor practice for the youngest (KC) and, for the elderly (CP) relieve the health, giving priority for all children the outdoor activities over the weekend.

\section{Measure}

We used a questionnaire with different parts, in the last part a group of open and closed questions, allowed to characterize the practice of PA and the perception of motor performance of the student, using a scale with 4 items: 1 - do not perform; 2 - perform with difficulty; 3 - perform well; 4 - perform very well. We adjusted the IV part of the questionnaire and applied to a group of these children of the 1st and 2 nd year of the 1 st cycle (6-8 years), using the same questions but using four emojis.

\section{Procedures}

The family members completed a direct questionnaire completion and the children were supported in reading the questions. For the analysis we associated in two: a. Bad performance perception - do not perform / perform with difficulty; b. Good performance perception - perform well / perform very well.

\section{Analysis}

The analysis of the data will be made with treatment descriptive $(n, \%)$ and non-parametric statistic comparative (Chi Square - X2) and correlate (Ro Spearman - $\rho$ ) was carried out through 25.0 SPSS's and allowed us to reach some final considerations between family perception of some motor skills learning in the childhood. The significance level for analyse was maintained at $p \leq .05$. 


\section{RESULTS}

In fact, in a first descriptive analysis and observing the graph 1, we note that a higher percentage of KC relatives refer as a higher capacity of their students a good performance: 1 st - "to kick a ball", 2nd - "to run", 3.rd - "to swim", while the relatives of the PCs mention: 1st - "to run", 2nd - "to kick a ball", 3rd - "to ride a bike".

For the first ones, "to jump rope" and "to roll skating" are the most problematic skills for learning, and, for the second ones is "to jump axis" and "to roll skating". Older children (PC) have a similar perception - greater "to kick a ball" and worse " to jump axis ", but they differ because they consider themselves better - "to ride a bike" and "to swim" but more worrying because they have a low perception jumping rope.

We determined some relations $(\rho)$ and some differences $(X 2)$ regarding the perceptions of family members regarding the motor domain of their students in different practices in the usual motor skills of childhood. There were some links between the children's ages and the "to jump the rope" domain ( $\rho=.389$ a $p \leq .001)$, "to ride a bike" ( $\rho=.234$ a $p \leq .01)$ and "to run" ( $\rho=.212$ a $p \leq .01)$.

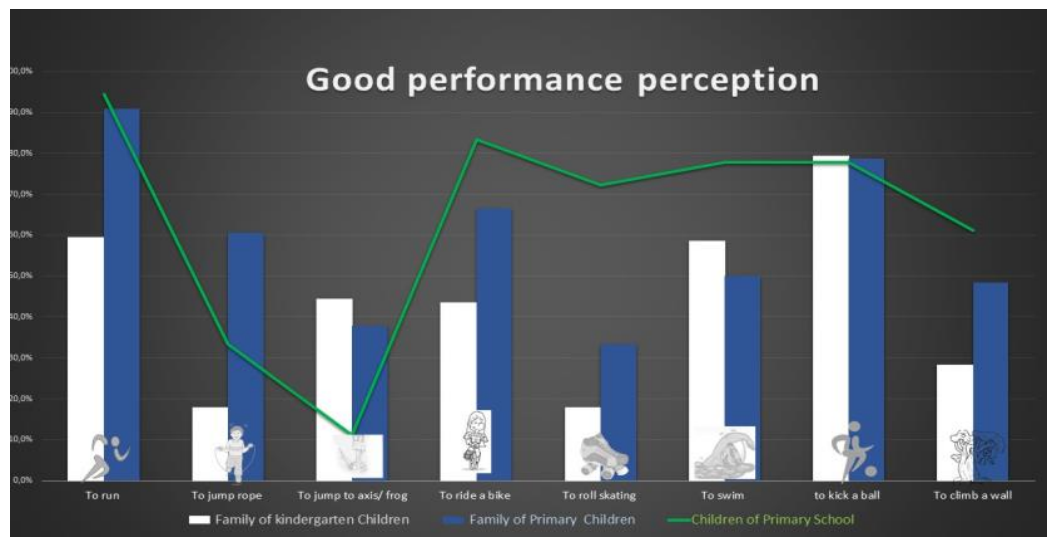

Graphic 1. Good performance perception.

We also observed some differences between the perceptions of "good performance" in these motor skills of the relatives of the smallest children (KC) and of the larger ones (PC), with emphasis on "to run" and "to jump rope" ( $p=.000)$, followed by "to roller skating" ( $p=.005)$. In general, and without no differences be tween the ages of their learners, according to family members, is easier "to kick a ball" and more difficult "to jump a rope", "to climb a wall" and "to roll skating". It is to register that we find a low perception of competence in "to jump the rope" in small group of children of the 1st cycle.

\section{DISCUSSION}

Concerning the perceptions of family members about the motor skills of their children, they are very supportive and affirm that they promote opportunities for their practices over the weekend, which is in line with Seabra (2017: 14) when he said that the promotion of AF depends of behavioural issues, in reducing barriers that limit their participation, here the motivation and safety guaranteed by family members is fundamental. The proficiency of children in various relevant motor skills is related with their experience physical and their parents showed to know their greatest weaknesses. We wish to given young children 
different and simple intervening activities with an ideal environment for the promotion of motor skills with associated benefits health (Goldfield et al, 2012).

\section{CONCLUSIONS}

Family of preschool and primary children of public school considered the relevant role of school in motor development and performance gains in motor skills typical of childhood.

"To kick a ball", "to run" are more developed abilities in these children and they believe that there are a great difficulty of children in "to roller skating", "to jump axis" and "to climb a wall / tree ". However, it is in "to jump rope" that children of the 1 st cycle have less confidence and perception from less success than their family.

\section{REFERENCES}

Cordeiro, N. \& Condessa, I. (2016). A perceção de pais sobre as rotinas, a prática de atividade física e a proficiência motora dos seus filhos. In Serrano, J. \& Petrica, J (Editores). Estudos em Desenvolvimento Motor da Criança IX, pp.24-31. Castelo Branco: IPCB.

Goldfield, G. S., Harvey, A., Grattan, K., \& Adamo, K. B. (2012). Physical activity promotion in the preschool years: a critical period to intervene. International journal of environmental research and public health, 9(4), 1326-1342. https://doi.org/10.3390/ijerph9041326

Seabra, A. (2017). A Atividade Física em crianças e adolescentes. Um comportamento decisivo para um estilo de vida saudável. Revista Factores de Risco, 44 (12), 9 - 20.

\section{(c) $(7) \ominus$}

This work is licensed under a Attribution-NonCommercial-NoDerivatives 4.0 International (CC BY-NC-ND 4.0). 


\title{
Association between body composition and functional physical fitness in the elderly population
}

\author{
JOÃO MARTINS ${ }^{1,2} \triangle$, RUIPAULO ${ }^{1,2}$, ANDRÉ RAMALHO ${ }^{1,2}$, PEDRO DUARTE-MENDES ${ }^{1,2}$, FERNANDA \\ SILVA ${ }^{1,2}$, JOÃO SERRANO ${ }^{1,2}$ \\ ${ }^{1}$ SHERU - Sport, Health \& Exercise Research Unit, Instituto Politécnico de Castelo Branco, Castelo Branco, \\ Portugal \\ ${ }^{2}$ Department of Sports and Well-being, Instituto Politécnico de Castelo Branco, Castelo Branco, Portugal
}

\begin{abstract}
The main objective of this study went to analyse the correlation between the variables of body composition and variables of functional physical fitness in the elderly population. The sample included 114 subjects $(N=$ 114) with a mean age of $73.98 \pm 6.855$. The participants, coming from the district of Castelo Branco, proposed to the study after being informed by local institutions. In order to evaluate the Body Composition, an Inbody 270 bioimpedance scale was used to obtain the values of Skeletal Muscle Mass, Fat Mass and percentage of body fat. The height was measured with a stadiometer and the BMI was calculated. For physical fitness, was used the motor fitness test Rikli and Jones (1999). When correlating the variables of body composition and physical fitness it was concluded that the better the body condition the better the physical capacity. Keywords: Elderly; Body composition; Physical fitness.
\end{abstract}

Corresponding author. Avenida Av. Pedro Álvares Cabral, n 12, 6000-084 Castelo Branco, Portugal.

E-mail: joao.farinha.64@gmail.com

Supplementary Issue: Spring Conferences of Sports Science. International Seminar of Physical Education, Leisure and Health, 17-19 June 2019. Castelo Branco, Portugal.

JOURNAL OF HUMAN SPORT \& EXERCISE ISSN 1988-5202

(c) Faculty of Education. University of Alicante.

doi:10.14198/jhse.2019.14.Proc4.82 


\section{INTRODUCTION}

It is consensual that grip strength and gait speed decrease with aging, demonstrating that muscle mass decreases as age increases and fat mass increases, negatively affecting muscle function and justifying, in part, functional limitations (Woo, Leung, \& Kwok, 2007). Other studies indicate that the amount of fat mass negatively affects physical performance and lean mass has a direct association with strength (Sternfeld, Ngo, Satariano, \& Tager, 2001). We think it is a pertinent study because it allows to know the state of the physical fitness of the elderly in one of the oldest areas of Europe.

The objective of this study is to analyse the correlation/association between the variables of body composition and variables of functional physical fitness in the elderly population. The pertinence of this study focuses on the need to perceive the influence of physical fitness on body composition.

\section{MATERIAL AND METHODS}

\section{Participants}

The participants of the study were selected with the cooperation of associations from the municipality of Castelo Branco, indicated by relatives or known persons, with 114 subjects $(N=114)$ aged between 65 and 93 years old. The mean age of the study was 73.98 years and a standard deviation of 6,855 , all participants were Caucasian.

\section{Measures}

For the study was used a bioimpedance scale Inbody 270. A portable stadiometer was used to measure height.

For manual hold was used the Lafayette 78010 dynamometer.

Physical fitness tests of the Rikli and Jones test battery (1992) were used to obtain the results of physical fitness (Baptista \& Sardinha, 2005).

\section{Procedures}

The participants of the study were all over 65 years old and all subscribed the term of informed consent, following the Declaration of Helsinki (2008).

\section{Analysis}

In the first analysis was verified the normality of the sample (Kolmogorov-Smirnov). It was found that the variables BMI, waist perimeter and fat mass showed normal distribution and was used the Pearson parametric correlation test. Spearman's non-parametric correlation test was used for non-normal distribution tests.

We adopted a level of significance of $5 \%$ and also verified the magnitude-effect values, according to Wiersma and Jurss (2003): 0.90 to 1.00 "Very high"; 0.70 to 0.90 "High"; 0.50 to 0.70 "Moderate"; 0.30 to 0.50 "Low"; 0.10 to 0.30 "Small". Was also used the coefficient of determination (R2). 


\section{RESULTS}

There is a high positive correlation between the variables BMI-FM and FM- $\% B F(0.7 \leq r \leq 0.9)$ and in them we can also observe that $\mathrm{R} 2$ (coefficient of determination) is high, indicating that $74 \%$ of the FM variance can be attributed to $\mathrm{BMI}$ and $73 \%$ of the $\% \mathrm{BF}$ variance can be attributed to $\mathrm{FM}$.

The correlation between lift/sit and arm flexion was highlighted as having a high positive correlation $(0.7 \leq r \leq$ $0.9)$.

\section{DISCUSSION}

Our investigation contains several results that suggest that the higher the skeletal muscle mass and the lower the fat mass the better was the performance in the physical condition tests. When comparing the different variables, a good physical performance of the upper limbs was visible when the lower limbs also obtained good values.

Through this study we can observe that physical fitness has influence on body composition. Through a similar study Dhana, et al. (2016) demonstrated a positive correlation between the variables FM and BMI. The correlation between the variables FM-\%BF was positive high, being the one that had higher value in the present study, Gomez-Peralta, et al. (2018) obtained a similar correlation between the two variables.

The correlation of the sit/lift variable and arm flexion is high positive, Herman, et al. (2005) conducted a study that demonstrates that there is a positive correlation between limb strength, so when better outcomes in upper limbs better outcomes of lower limbs, as our study demonstrates.

Through this study we can observe that physical fitness has influence on body composition which will provide a better quality of life for the elderly.

This cross-sectional study may present some gaps such as the fact that it is performed at a single time lapse and does not determine a cause/effect relationship, therefore we suggest the use of a longitudinal study where physical fitness and body composition characteristics were verified over a given time.

\section{CONCLUSIONS}

With our study we conclude that the better the physical condition the better the physical capacity. Correlations among body composition indicators include high positive correlations between the variables BMI-FM and \%BF-FM. In the correlations between body composition variables and physical fitness tests there is only one positive increase, this being between S/L-AF.

We believe that the present study was relevant for the evaluation of a greater number of variables of body composition and physical fitness, in order to delineate a more complete and adjusted body composition profile and physical fitness, analysing the relationship between these variables.

\section{REFERENCES}

Baptista, F. \& Sardinha, L. (2005). Avaliação da Aptidão Física e do Equilíbrio de Pessoas Idosas Baterias de Fullerton. Cruz Quebrada: Faculdade de Motricidade Humana. 
Dhana, K., Koolhas, C., Schoufour, J., Rivadeneira, F., Hofman, A., Kavousi, M., \& Franco, O. (22 de março de 2016). Maturitas. Association of anthropometric measures with fat and fat-free mass in the elderly: The Rotterdam study, pp. 98-99. https://doi.org/10.1016/i.maturitas.2016.03.018

Gomez-Peralta, F., Abreu, C., Cruz-Bravo, M., Alcarria, E., Gutierrez-Buey, G., Krakauer, N., \& Krakauer, J. (2018). Diabetology \& Metabolic Syndrome. Relationship between“a body shape index (ABSI)" and body composition in obese patients with type 2 diabetes, pp. 4-6. https://doi.org/10.1186/s13098018-0323-8

Herman, S., Kiely, D., Leveille, S., O’Neill, E., Cyberey, S., \& Bean, J. (2005). Upper and Lower Limb Muscle Power Relationships in Mobility-Limited Older Adults. Journal of Gerontology: Medical Sciences, 60A(4), 478-479. https://doi.org/10.1093/gerona/60.4.476

Sternfeld, B., Ngo, L., Satariano, W., \& Tager, I. (30 de Outubro de 2001). Associations of Body Composition with Physical Performance and Self-reported Functional Limitation in Elderly Men and Women. American Journal of Epidemiology, 116-120. https://doi.org/10.1093/aje/kwf023

Woo, J., Leung, J., \& Kwok, T. (7 de Julho de 2007). BMI, Body Composition, and Physical Functioning in Older Adults. Obesity, 15, 1887-1889. https://doi.org/10.1038/oby.2007.223

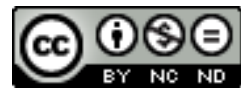

This work is licensed under a Attribution-NonCommercial-NoDerivatives 4.0 International (CC BY-NC-ND 4.0). 


\title{
Correlation between sedentary behavior, physical activity and lung function in the elderly
}

\author{
FERNANDA M. SILVA ${ }^{1}$, DINEIA A. LUCAS ${ }^{1}$, RUI PAULO 1,2, ANDRÉ RAMALHO1,2, DIOGO \\ MONTEIRO ${ }^{3,4}$, LUÍS CID ${ }^{3,4}$, BRUNO TRAVASSOS ${ }^{3,5}$, PEDRO DUARTE-MENDES ${ }^{1,2}$ \\ ${ }^{1}$ Department of Sports and Well-being, Polytechnic Institute of Castelo Branco, Castelo Branco, Portugal \\ 2SHERU - Sport, Health \& Exercise Research Unit, Polytechnic Institute of Castelo Branco, Castelo Branco, \\ Portugal \\ ${ }^{3}$ Research Centre in Sport Sciences, Health Sciences and Human Development (CIDESD), Portugal \\ ${ }^{4}$ Sport Sciences School of Rio Maior, Polytechnic Institute of Santarém (ESDRM-IPSantarém), Portugal \\ ${ }^{5}$ Department of Sport Sciences, University of Beira Interior, Covilhã, Portugal
}

\begin{abstract}
Aging is associated with the loss of Lung Function, resulting in anatomical and functional changes. Identifying the modifiable determinant factors of pulmonary function enables to prevent obstructive respiratory disease development or progression. The purpose of this study was to verify the correlation between Sedentary Behaviour (SB), Light Physical Activity (LPA), Moderate to Vigorous Physical Activity times (MVPA) with the predicted values of forced vital capacity (FVC\%), forced expiratory volume in 1 sec (FEV1\%) and peak expiratory flow (PEF\%) in the Elderly. Seventy-seven elderly subjects (age $=71.9 \pm 5.50$ years old ; bodyweight $=70.74 \pm 12.96 \mathrm{~kg}$; height $=157.29 \pm 9.97 \mathrm{~cm}$ ) volunteered to participate in this study. Sedentary Behaviour and Physical Activity times were assessed with a ActiGraph® GT1M accelerometer. Spirometric Values were assessed with a Cosmed® Microquark spirometer. Results do not reveal significant correlations between SB, LPA, MVPA with FVC\%, FEV1\% and PEF\% in the Elderly. Sports and health professionals should continue in their efforts to find the modifiable determinant factor of LF in the Elderly. Keywords: Aging, Accelerometry; Spirometry; Physical activity; Sedentary behaviour.
\end{abstract}

Corresponding author. Department of Sports and Well-being, Polytechnic Institute of Castelo Branco, Rua Prof. Dr. Faria de Vasconcelos, 6000-266 Castelo Branco, Portugal.

E-mail: f.m.a.s 298@hotmail.com

Supplementary Issue: Spring Conferences of Sports Science. International Seminar of Physical Education, Leisure and Health, 17-19 June 2019. Castelo Branco, Portugal.

JOURNAL OF HUMAN SPORT \& EXERCISE ISSN 1988-5202

(C) Faculty of Education. University of Alicante.

doi:10.14198/jhse.2019.14.Proc4.82 


\section{INTRODUCTION}

Lung function is known to be a morbidity and mortality predictive factor depending on nervous system function, respiratory muscles strength and pulmonary dimensions (Fatima et al., 2013). Forced vital capacity (FVC) and forced expiratory volume in $1 \mathrm{sec}$ (FEV1) both starts declining during life's third decade in about 25 to $30 \mathrm{ml}$ each year (Miller, 2010). Furthermore, there is a tendency towards decreasing of peak expiratory flow (PEF) throughout life (Janssens, 2005). Aging effects increase the risk of pulmonary disease (Lowery et al., 2013). Therefore, it seems to be mandatory to identify the modifiable determinant factors of lung function in order to prevent obstructive respiratory disease development or progression. Since there are few studies about the correlation between the SB or PA with lung function in healthy subjects, the purpose of this study was to verify the correlation between SB, LPA, MVPA with FVC\%, FEV1\% and PEF\% in the Elderly.

\section{MATERIAL AND METHODS}

\section{Participants}

Seventy-seven elderly subjects (age $=71.9 \pm 5.50$ years old ; bodyweight $=70.74 \pm 12.96 \mathrm{~kg}$; height $=$ $157.29 \pm 9.97 \mathrm{~cm}$ ) volunteered to participate in this study. Sample recruitment was carried out in senior universities and health promotion fairs. Inclusion criteria were: age $\geq 65$ years old, Caucasian ethnicity, absence of clinical symptoms of severe respiratory or cardiac disease, non-smokers and physical independency. All subjects signed an informed consent form.

\section{Measures}

Sedentary Behaviour (counts min-1<100) and Physical Activity times were assessed using the ActiGraph® GT1M Accelerometer. Spirometric values were assessed using the Cosmed® MicroQuark spirometer.

\section{Procedures}

After arriving facilities, ratters gave participants standardized instructions about tests procedures. FVC\%, $\mathrm{FEV} 1 \%$ and $\mathrm{PEF} \%$ were obtained through a minimum of 3 and a maximum of 8 valid spirometric trials with a rest period $\geq 1$ min between forced expiratory manoeuvres (Miller et al., 2005). All spirometric trials were performed during early morning period with participants sitting with a correct posture and wearing a nose clip. Assessors gave clear instructions about forced expiratory manoeuvres since they should be acceptable and reproduceable according to American Thoracic Society (ATS) and European Respiratory Society (ERS) guidelines (Miller et al., 2005). Finally, participants were instructed to utilize the accelerometer during waking hours for at least five consecutive days in order to assess SB, LPA and MVPA times. The devices were activated on the first day at $5.00 \mathrm{a}$.m. and data was recorded at 15 -s epochs. This approach required at least three valid days (including one weekend day) and $10 \mathrm{~h}$ of utilization per day.

\section{Analysis}

Descriptive statistics (mean \pm standard deviation) were performed for all variables under analysis. Data normality was tested using the Kolmogorov-Smirnov test. Spearman's rank or Pearson's correlation coefficient (r) were used to verify correlations between SB, LPA, MVPA, FVC $\%$, FEV1\% and PEF\%. The strength of the correlation was classified as follows (Mukaka, 2012): very high $(0.90<r<1.00)$; high $(0.70<$ $r<0.90)$; moderate $(0.50<r<0.70)$; low $(0.30<r<0.50)$; little $(0.10<r<0.30)$. 


\section{RESULTS}

Although there was no significant correlation between variables under analysis ( $p \geq 0.05)$, we found a positive relationship between SB and PEF\%, AFMV and FEV1\%, and AFMV and PEF\%. Results are listed in table 1.

Table 1. Correlation between variables under analysis, significance level ( $p$ ) and correlation coefficient ( $r$ )

\begin{tabular}{|c|c|c|c|c|}
\hline & & $\begin{array}{c}\text { FVC } \\
\text { (\%pred.) }\end{array}$ & $\begin{array}{c}\text { FEV1 } \\
\text { (\%pred) }\end{array}$ & $\begin{array}{c}\text { PEF } \\
\text { (\%pred) }\end{array}$ \\
\hline SB - Sedentary Behaviour (min/d) & $r$ & $-0.061^{\mathrm{b}}$ & $-0.077^{b}$ & $\begin{array}{c}-0.102^{b} \\
0.379\end{array}$ \\
\hline LPA - Light Physical Activity (min/d) & $r$ & $0.071 \mathrm{a}$ & $0.022^{a}$ & $0.005^{a}$ \\
\hline MVPA - Moderate to Vigorous Physical Activity & $\begin{array}{l}p \\
r \\
p\end{array}$ & $\begin{array}{l}0.539 \\
0.063^{a} \\
0.587\end{array}$ & $\begin{array}{l}0.846 \\
0.141^{a} \\
0222\end{array}$ & $\begin{array}{l}0.962 \\
0.104^{a} \\
0.369\end{array}$ \\
\hline
\end{tabular}

\section{DISCUSSION}

Our results suggest that SB and PA are not significantly related with spirometric values. However, correlation direction is concurrent with previous work since authors have found a positive association between PA and FEV1, whereas SB was negatively associated with FEV1 (Jakes et al., 2002; Orlieb et al. 2014). Our statistical non-significance might be due to different methods used by authors, particularly as regards to PA subjective measurement methods (Jakes et al., 2002) and to different cut-off values used to set PA intensity (Orlieb et al., 2014). This study is a contribution to literature since there are few evidences with regard to the correlation between SB and PA with Lung Function in the Elderly.

\section{CONCLUSIONS}

Results did not confirm a significant correlation between SB, PA and Lung Function. Sports and health professionals should continue in their efforts to find the modifiable determinant factor of LF in the Elderly.

\section{REFERENCES}

Fatima, S., Rehman, R., Saifullah, \& Khan, Y. (2013). Physical activity and its effect on forced expiratory volume. J Pak Med Assoc, 63, 310-312.

Jakes, R., Day, N., Patel, B., Khaw, K., Oakes, S., Luben, R., ... Wareham, N. (2002). Physical Inactivity is Associated with Lower Forced Expiratory Volume in 1 Second European Prospective Investigation into Cancer-Norfolk Prospective Population Study. Am J Epidemiol, 156(2), 139-147. https://doi.org/10.1093/aje/kwf021

Janssens, J. (2005). Aging of the Respiratory System: Impact on Pulmonary Function Tests and Adaptation to Exertion. Clini in Chest Med, 26, 469-484. https://doi.org/10.1016/i.ccm.2005.05.004

Lowery, E., Brubaker, A., Kuhlmann, E., \& Kovacs, E. (2013). The aging lung. Clin Interv Aging, 8, 14891496. https://doi.org/10.2147/cia.s51152

Miller, M. (2010). Structural and Physiological Age-Associated Changes in Aging Lungs. Semin Respir Crit Care Med, 31(5), 521-527. https://doi.org/10.1055/s-0030-1265893

Miller, M., Hankinson, J., Brusasco, V., Burgos, F., Casaburi, R., Coates, A., ... Wanger, J. (2005). Standardization of spirometry. Eur Respir J, 26, 319-38. 
Mukaka M.M. (2012). A guide to appropriate use of Correlation coefficient in medical research. Malawi Med J, 24(3), 69-71. PMID: 23638278.

Ortlieb, S., Dias, A., Gorzelniak, L., Nowak, D., Karrasch, S., Petrs, A., ... KORA Study Group (2014). Exploring patterns of accelerometry-assessed physical activity in elderly people. Int J Behav Nutr Phys Act, 11(28), 2-10. https://doi.org/10.1186/1479-5868-11-28

\section{(c) $(\mathrm{B})(\mathrm{EY}$}

This work is licensed under a Attribution-NonCommercial-NoDerivatives 4.0 International (CC BY-NC-ND 4.0). 


\title{
Study of the relationship between global motor skills, fine motor skills and age
}

\author{
VIVIAN CORTE $1 \square$, RUI PAULO ${ }^{1,2}$, MIGUEL REBELO1 ${ }^{1}$, ANDRÉ RAMALHO1,2, PEDRO DUARTE- \\ MENDES ${ }^{1,2}$, JOÃO SERRANO ${ }^{1,2}$ \\ ${ }^{1}$ SHERU - Sport, Health \& Exercise Research Unit, Instituto Politécnico de Castelo Branco, Portugal \\ ${ }^{2}$ Department of Sports and Well-being, Instituto Politécnico de Castelo Branco, Portugal
}

\begin{abstract}
Several studies (Melo, 2011; Gaul \& Issarter, 2016) have developed their investigations as they prepare for age as an important variable in the child's motor development. In the present study we seek to study the motor performance level of children of both genders aged 18-44 months. This way, the study aimed to know if there is a correlation between Global Motor Skills, Fine Motor Skills and Age. For this purpose, we selected a convenience sample of the non-probabilistic type, which involved a total of 128 children of both genders. Peabody Developmental Motor Scales - Second Edition (PDMS-2) was used to evaluate children's performance (Folio \& Fewell, 2000). The collection of the data was done according to the protocol, following scrupulously the norms of application and respecting the routines of the institutions. Data correlation test (Kolmogorov-Smirnov test), using Spearman's nonparametric correlation test. We also used the method of inferences in data magnitude (d-Cohen) (Hinkle, Wiersma \& Jurss, 2003) and adopted a significance level of .005. Overall, moderate correlations and between ages and motor generations, fine and correlated, between global abilities and motor skills. Therefore, as age increases, there is an improvement in the results of global and fine motor skills. Keywords: Motor development; Motor skills; Peabody Developmental Motor Scales-2.
\end{abstract}

Corresponding author. Av. Pedro Álvares Cabral, $n^{\circ}$ 126000-084 Castelo Branco, Portugal.

E-mail: vivian corte@live.com.pt

Supplementary Issue: Spring Conferences of Sports Science. International Seminar of Physical Education, Leisure and Health, 17-19 June 2019. Castelo Branco, Portugal.

JOURNAL OF HUMAN SPORT \& EXERCISE ISSN 1988-5202

(c) Faculty of Education. University of Alicante.

doi:10.14198/jhse.2019.14.Proc4.82 


\section{INTRODUCTION}

In today's world, Motor Development can be defined as the "area of study of motor behaviour that is concerned with knowledge of the processes of change, in an adaptive view, and in a broad temporal dimension - throughout life" (Barreiros \& Krebs, 2007, p.7). This study aims to know and analyse the existing correlations between global motor skills, fine motor skills and age. Focusing on the need to know if there is any relationship between transcribed variables, for a possible understanding analysis in the development process, making it easier to properly plan and design intervention strategies.

\section{METHODS}

This study is based on a quantitative correlational typology, being a cross-sectional study, since only a single data collection was performed.

\section{Participants}

A total of 128 children between the ages of 18 and 44 months of both sexes participated in the study. All children were included, except for children who were diagnosed with learning difficulties and / or developmental impairments, and children with some type of deficiency diagnosed.

\section{Measures}

Peabody Developmental Motor Scales - Second Edition (PDMS-2) was used to collect information on the motor profile (Folio \& Fewell, 2000). The PDMS-2 scales are, as already mentioned, one of the instruments most used in the field of motor evaluation. Recently reviewed, it allows to evaluate the performance of the fine and global motor skills of children up to 71 months.

\section{Procedures}

We have followed all ethical principles, international norms and standards relating to the Helsinki Declaration and the Convention on Human Rights and Biomedicine have been followed, respected and preserved (Tuckman, 2000). After obtaining the approval of the institution, and a term of consent, was sent and requested the completion of the characterization form for the child. After the informed consent term and the child's characteristics sheet were duly filled in, the instrument / battery was applied to the children who fulfilled the inclusion requirements in the study.

\section{Analysis}

Using SPSS (v.23.0), the data distribution (Kolmogorov-Smirnov test) was verified, confirming that all variables had a non-normal distribution, using the non-parametric correlation test of Spearman. A significance level of $5 \%$ was adopted and we also investigated the magnitude-effect values, according to Hinkle, Wiersma and Jurss (2003): 0.90 to 1.00 "Very high"; 0.70 to 0.90 "High"; 0.50 to 0.70 "Moderate"; 0.30 to 0.50 "Low"; 0.10 to 0.30 "Small". The coefficient of determination ( $r 2$ ) was also used, which refers to the proportion of variance shared between two variables.

\section{RESULTS}

A positive low correlation $(0.3 \leq r \leq 0.5)$ between the variable Age and the variables Postural Skills and Locomotion Skills, a small positive correlation $(0.1 \leq r \leq 0.3)$ between the Age variable and the Global Motor Skills variable $(r 2=7.3 \%)$, statistically accepted $(p \leq 0.05)$. 
We also observed a moderate positive correlation $(0.5 \leq r \leq 0.7)$ between the Age variable and the variables Fine Motor Skills and Fine Manipulation Skills $(r 2=29 \%)$, and a small positive correlation $(0.1 \leq r \leq 0.3)$ among the variables Age and Ability Visuo-motor integration, statistically accepted ( $p \leq 0.05)$.

A positive low correlation $(0.3 \leq r \leq 0.5)$ was found between the variables Global Motor Skills, Fine Motor Skills $(r 2=9.4 \%)$. and the Visio-motor Integration Skills, and a low positive correlation $(0.3 \leq r \leq 0.5)$ between the variables Statistically Accepted Fine Motor Skills, Posture Skills and Locomotion Skills $(p \leq 0.05)$.

\section{DISCUSSION}

In the correlation between Age and Global Motor Skills, we found in a study (Child Care Health Development, 2017) a positive correlation, demonstrating that with increasing age, overall motor skills also tend to increase / improve. These results are in line with the results we have found. Regarding the correlation between Age and Fine Motor Skills, we found a low positive correlation. Also in the study by Gaul and Issarter (2016), who examined the level of Fine Motor Ability, they found a higher performance in older children. In the correlation between Age and Posture and Locomotion Skills, our study shows low positive correlations, evidencing an improvement of these abilities with increasing age. In a study (Melo, 2011), it was observed that age only influenced the locomotion variable, confirming that the quantitative and qualitative changes in movement occur due to several factors (Gallahue \& Ozmun, 2003).

\section{CONCLUSIONS}

We conclude that there is a tendency for positive (mostly low and small) correlations between the variables Age, HMG and HMF, emphasizing an improvement / development of these abilities as children grow. It would be likely to find "stronger" correlations by meeting the fundamentals of Motor Development. Any changes in the life routine of these children, not always with "appropriate" stimuli, may determine these "weak" correlations, evidencing a greater influence of the maturation variable to the detriment of the psychomotor stimulation.

\section{REFERENCES}

Barreiros, J., \& Krebs, R. (2007). Desenvolvimento motor: a delimitação de uma subárea disciplinar. In J. Barreiros, R. Cordovil \& S. Carvalheiro (Eds.), Desenvolvimento Motor da Criança (pp. 7-23). Lisboa: Edições FMH.

Chil Care, H. \&. (2017). Child Care Health \& Development.

Gallahue, D. \& Ozmun, J. (2003). Compreendendo o Desenvolvimento Motor. Bebês, crianças, adolescentes e adultos. ( $2^{\mathrm{a} e d .) . ~ S a ̃ o ~ P a u l o: ~ P h o r t e ~ E d i t o r a . ~}$

Gaul, D., \& Issartel, J. (2016). Fine motor skill proficiency in typically developing children: On or off the maturation track? Hum Mov Sci, 46, 78-85. https://doi.org/10.1016/j.humov.2015.12.011

Hinkle, D. E., Wiersma, W., \& Jurs, S. G. (1998). Applied Statistics for Behavioural Sciences (5th ed.). Chicago, IL: Rand McNally College Publishing.

Folio, R. \& Fewell, R. (2000). Peabody Developmental Motor Scales: Examiner's Manual (2a ed.). Austin, Texas: Pro-ed.

Melo, A. (2011). Influência das variáveis biológicas e socioculturais no perfil motor de Crianças dos 36 aos 71 meses (Dissertação de mestrado, Universidade de Trás os Montes e Alto Douro). https://doi.org/10.25145/.j.pasos.2018.16.017

Tuckman, B. (2000) Manual de Investigação em Educação Lisboa, Fundação Calouste Gulbenkian. 


\section{(c) (i) (3)}

This work is licensed under a Attribution-NonCommercial-NoDerivatives 4.0 International (CC BY-NC-ND 4.0). 


\title{
Functional exercise vs aquafit for seniors
}

\author{
JOÃO PETRICA ${ }^{1,2} \triangleleft$, JORGE SANTOS ${ }^{1,2}$, RUI PAULO $^{1,2}$, ANDRÉ RAMALHO $^{1,2}$, SAMUEL HONÓRIO $^{1,2}$, \\ MARCO BATISTA ${ }^{1,2}$, ANTÓNIO FAUSTINO ${ }^{1,2}$, JOÃO ROCHA ${ }^{1,2}$, JOÃO SERRANO ${ }^{1,2}$ \\ ${ }^{1}$ Instituto Politécnico de Castelo Branco, Portugal \\ ${ }^{2}$ SHERU - Sport, Health \& Exercise Research Unit, Portugal
}

\begin{abstract}
Elderly people practicing physical activity has increased but it is still insufficient compared to those who are sedentary. The benefits that an active lifestyle can provide are known. So, it is increasingly important to understand which physical activities bring more benefits for the elderly. The aim of the present study was to determine if there were changes in the results in the pre-test and post-test performed to the elderly after 3 months of intervention on Aquafit and Functional Exercise. It is a case study carried out in one of the most deserted and aged regions of Europe and, therefore the sample is small, but they are elderly people who need very much of intervention: $n=20$ persons, over 65 years old, from the district of Castelo Branco. They were divided into two groups: 10 who practiced hydro gymnastics and 10 who practiced functional exercise. For data collection were used the Senior Fitness Test battery, The sphygmomanometer \& the Freestyle Optium Neo. T-test or the Wilcoxon test \& the probability of ,05 were used to make the comparisons. The results showed that there were statistically significant differences in the values of the Heart Rate, Raise and Sit, Sit and Reach tests, Reach Behind the Back and Walk 6 minutes between pre and post-test. The elderly practicing Functional Exercise presented better values in the physiological parameters and in the variables of the physical condition: Flexibility, Lift and Seat, Speed and Strength. Elderly practicing Aquafit presented better values in the variable resistance. Keywords: Physical condition; Physical activity; Aquafit; Functional Exercise; Senior.
\end{abstract}

Corresponding author. Instituto Politécnico de Castelo Branco, Portugal.

E-mail: i.petrica@ipcb.pt

Supplementary Issue: Spring Conferences of Sports Science. International Seminar of Physical Education, Leisure and Health, 17-19 June 2019. Castelo Branco, Portugal.

JOURNAL OF HUMAN SPORT \& EXERCISE ISSN 1988-5202

(c) Faculty of Education. University of Alicante.

doi:10.14198/jhse.2019.14.Proc4.82 


\section{INTRODUCTION}

Physical activity has been gaining strength as the best treatment for old age. As functions are increasingly compromised as well as autonomy and functional independence, arises the need to provide the elderly with physical, emotional, social and intellectual stimuli that are achieved through the practice of physical activity (Petrica \& Gomes, 2015).

The Aquafit has often be used in physical activity programs for seniors by the ease in overcoming the weight of the body with much less effort for the joints since the impact at this level is decreased and contracted by the force of thrust, because the support given by the aquatic environment makes the movement easier to perform, allowing an increase of the capacity self-sufficiency (Case,2001), and Serrano et al (2017), consider that this practice could help a more active and healthy aging.

Some studies have shown that this modality has a great impact on physical fitness, with changes in body composition (Queirós \& Barbosa, 2005), strength, flexibility, mobility (Santos and Morouço, 2015) and balance (Honório et al. 2016).

However there are studies that evoke the advantages of practicing other activities. Nunes \& Santos (2009), for example, found that a group that worked on the base of the walk was superior in tests of strength of lower limbs and of aerobic capacity, compared with those of the Aquafit. On the other hand, D'Elia (2017) presents the concept of "Functional Training" as pulling, pushing, stabilizing, lifting, throwing, running or jumping, making the body a means to produce efficient movements, improving performance and preventing injuries. This idea is reinforced by Stocco (2017) who says that this type of activity complemented with aerobic neuromuscular stimuli in proprioception, agility and motor coordination, taking into account the improved control and stability, prepare better movements for the day-to-day.

With this study we intend to evaluate the level of physical condition and associated physiological parameters to determine if there is evolution in the results of pre-test and post-test in elderly practicing aquafit and practicing functional exercise and to see if the practice of one provides better physical condition than the practice of the other, after three months of intervention.

\section{MATERIAL AND METHODS}

\section{Participants}

The sample, obtained in one of the oldest and most depopulated areas of Europe, the Pinhal area of the Castelo Branco District in Portugal, consisted of 20 people, 14 females and 6 males, \pm 74.74 years old, all practitioners of Physical Activity, twice a week, divided into 10 users of the Functional Exercise class of Cernache do Bonjardim and 10 users of the Hidro Senior group of Vila de Rei.

\section{Measures}

In order to study the physical condition of the elderly, the Senior Fitness Test battery (Rickli \& Jones, 2008) was used, the heart rate and blood pressure were measured through the automatic sphygmomanometer OMRON M6 Comfort, following the indications suggested by Marques et al. (2012) and to evaluate the glycemia was used the Freestyle Optium Neo, following the procedure indicated by ESEP (2012). Weight and height were measured using a scale and tape and the body mass index was calculated.

The protocol of application of each of the tests was followed so that the values were rigorous. 


\section{Procedures}

Two data collections were performed, one at the beginning (pre-test) and one at the end (post-test), from a period in which the elderly benefited from a 3-month physical activity program with two weekly sessions, in which one group practiced aquafit and the other functional exercise. It should be noted that the participation of the study was carried out voluntarily and all participants signed the Free and Informed Consent Term.

\section{Analysis}

For the statistical treatment of the data, we used the Shapiro-Wilk normality test (for groups with less than 30 participants) and the paired T- tests when normality was checked, or the Wilcoxon non-parametric test to compare two samples when this was not the case. A statistical significance level of 0.05 was considered.

\section{RESULTS AND DISCUSSION}

The results generally show that there was evolution in most of the parameters obtained, explaining the efficacy of the two types of activity in the elderly intervention.

\begin{tabular}{lccc}
\hline & \multicolumn{3}{c}{ Group } \\
\hline Card. Freq. & $\mathbf{N}$ & hydro senior & Functional Exercise \\
Glycemia & 10 & $0.472^{\mathrm{b}}$ & $0.749^{\mathrm{b}}$ \\
Sys. Blood Pres. & 10 & $0.575^{\mathrm{a}}$ & $0.575^{\mathrm{a}}$ \\
Dia. Blood Pres. & 10 & $0.102^{\mathrm{b}}$ & $1.000^{\mathrm{a}}$ \\
Stand up \& sit & 10 & $0.666^{\mathrm{a}}$ & $0.177^{\mathrm{b}}$ \\
Forearm flexion & 10 & $0.252^{\mathrm{a}}$ & $0.02 \mathrm{~b}^{*}$ \\
Sitting \& reaching & 10 & $0.152^{\mathrm{a}}$ & $0.227^{\mathrm{b}}$ \\
Sitting \& walking & 10 & $0.908^{\mathrm{b}}$ & $0.021^{\mathrm{b}^{*}}$ \\
Reach behind back & 10 & $0.076^{\mathrm{a}}$ & $0.049^{\mathrm{b}^{*}}$ \\
walk 6 min. & 10 & $0.280^{\mathrm{b}}$ & $0.083^{\mathrm{b}}$ \\
Weight & 10 & $0.009^{* \mathrm{~b}}$ & $0.540^{\mathrm{b}}$ \\
Body mass index & 10 & $1.000^{\mathrm{b}}$ & $0.058^{\mathrm{b}}$ \\
\hline
\end{tabular}

Note: a) Wilcoxon test b)Peer t-test ${ }^{*}$ Sig $<0.05$

However, the application of the statistical tests to realize the extent to which the evolution was statistically significant, showed that the group that benefited from work on the basis of the functional exercise, registered significant differences in a greater number of variables than the group of aquafit.

\section{CONCLUSIONS}

The elderly practicing functional exercise presented better values in the physiological parameters and in the variables of the physical condition: Flexibility, Raise and Sit, Speed and Strength. Elderly practitioners of aquafit presented better values in the variable of the physical condition: resistance.

This study seems to give favourable indication to the work based on the Functional Exercise not neglecting the advantages of the use of aquafit for people with articular problems. 


\section{REFERENCES}

Case L. (2001). Aquagym ejercicios rutinas y programas de ejercicios acuaticos. Barcelona: Hispano Europeia.

D'Elia L. (2017). Guia Completo de Treinamento Funcional. São Paulo: Phorte.

ESEP (2012). Intervenção de enfermagem: Colher sangue para pesquisa de glicémia capilar. Intervenções e Prescrições, 1-2.

Honório S., Batista M., Paulo R., Mendes P., Santos J., Serrano J., Petrica J. \& Martins, J. (2016). Análise do Equilíbrio e da Amplitude Articular dos Segmentos Corporais em Gerontes. Revista Kairós Gerontologia, 19(4), out-Dez, pp. 89-110.

Marques, Marques A., Revige G., Marques I., Silva A., Cunha G., Fonseca V., \& Lobato J. (2012). Validação do dispositivo automático de medição de pressão arterial, OMRON M6 Confort. Saúde e Tecnologia, 47-54.

Nunes M. \& Santos S. (2009). Avaliação Funcional de idosos em três programas de atividade física: caminhada, hidroginástica e Lian Gong. Revisão Ciencias do Desporto, 150-159. https://doi.org/10.5628/rpcd.09.02-03.150

Petrica J. \& Gomes M. (2015). A Influência de um Programa de Jogos Tradicionais nos Níveis de Aptidão Física da Mulher Idosa. Em E-Balonmano, Vol.11, Suplemento 2, 171-172.

Queirós T. \& Barbosa T. (2005). Manual prático de atividades aquáticas e Hidroginástica. Lisboa: Xistarca, Promoções e Public.

Rickli R. \& Jones C. (2008). Testes de aptidão física para idosos. Barveri, SP: Manole.

Santos I. \& Morouço P. (2015). Benefícios da Hidroginástica na Aptidão Física de Idosos. Inter. Congress of Phys. Activity, 94.

Serrano J., Farinha C., Petrica J., Mesquita H., Paulo R., Mendes P. \& Faustino A. (2017). Níveis de Aptidão Física de idosos praticantes de hidroginástica. XIII SIEFLAS, 85.

Stocco M. (2017). Treino funcional para idosos. PR: Londrina.

\section{(9) $(\mathcal{Q} \Theta \Theta$}

This work is licensed under a Attribution-NonCommercial-NoDerivatives 4.0 International (CC BY-NC-ND 4.0). 


\title{
Effects of a senior exercise program on functional capacity in institutionalized elderly in the municipality of Mação
}

\author{
JORGE SANTOS ${ }^{1} \triangle$, JOÃO PETRICA ${ }^{1}$, MARCELO MENDES ${ }^{2}$, JOÃO SERRANO ${ }^{1}$, PAULO SILVEIRA $^{1}$, \\ LUIS MAIA ${ }^{3}$ \\ ${ }^{1}$ SHERU (Sport, Health and Exercise Research Unit), Instituto Politécnico de Castelo Branco, Portugal \\ ${ }^{2}$ Escola Superior de Eduçação de Castelo Branco, Instituto Politécnico de Castelo Branco, Portugal \\ 3 Universidade da Beira Interior, Departamento de Psicologia e Educação, Covilhã, Portugal
}

\begin{abstract}
The aim of this study was to study the effects of a Senior Gymnastics Program (SGP), with duration of 12 weeks 2 times per week, on the functional capacity of the elderly in the municipality of Mação, through the application of the Senior Fitness Test (SFT). On the other hand, to perceive how Functional Capacity evolved in the elderly who did not benefit from the program. We tried to verify if an SGP, specific for this age group, caused significant effects in both genres in the improvement of the functional capacity of the institutionalized elderly. It was sought to evaluate the evolution of the functional capacity (FC) of the institutionalized elderly. who did not benefit from SGP. A quantitative, experimental, analytical and longitudinal research was carried out. The sample consisted of 2 groups of elderly institutionalized in the House of the Santa Casa da Misericórdia de Mação, one that we designated as experimental (GE) with 13 individuals (10 women and 3 men $87,15 \pm 6,09$ ) who carried out the 12 week program and one that we denominated sedentary group (GS) with 10 ( 7 women and 3 men79,6 $\pm 6,45$ individuals who did not benefit from the program. As a conclusion, the experimental group showed statistically significant differences in all SFT battery tests, except for the 8 foot Up and Go again ( $p \geq 0.06$ ). Keywords: Physical activity; Functional capacity; Aging; Institutionalized Elderly; Senior Gymnastics Program.
\end{abstract}

Corresponding author. Rua da Zebreira $n^{\circ} 81$ - 6060-257 Ladoeiro, Portugal.

E-mail: jorgesantos@ipcb.pt

Supplementary Issue: Spring Conferences of Sports Science. International Seminar of Physical Education, Leisure and Health, 17-19 June 2019. Castelo Branco, Portugal.

JOURNAL OF HUMAN SPORT \& EXERCISE ISSN 1988-5202

(c) Faculty of Education. University of Alicante.

doi:10.14198/jhse.2019.14.Proc4.82

VOLUME 14 | Proc4 | 2019 | S1739 


\section{INTRODUCTION}

The objective of this work is to make known the reality that exists in the municipality of Mação, a municipality in the interior of the country, in a very old area, with respect to functional capacity. According to INE (2011), in Portugal, about $19 \%$ of the Portuguese population is 65 years old or more. The aging rate has increased from 102 in 2001 to 129 in 2011, which means that there are currently 129 elderly people per 100 young people. By 2050, the percentage of the elderly will be $25 \%$, when, for the first time in history, there will be more people over 65 than young people. In terms of population structure according to WHO (2002), in 2050, Portugal will be the 4th country of the 25 of the EU with the highest percentage of elderly and with a lower percentage of active population and $6^{\circ}$ with greater dependence on the elderly population, surpassed only by the Spain, Italy, Greece, Germany and Belgium. Thus, it is essential to include in the daily routine of the elderly the practice of regular and well-oriented AF, where it is essential to carry out an exercise program specifically aimed at this target audience, with the main objectives of socializing and maintaining mobility. and autonomy. (Serrano, Faustino, Rato, Petrica, Paulo, Mendes and Batista, 2015). This study seeks to know the reality of a specific area of the country, such as for example the study of Mira (2016), which sought to know the state of PA in the elderly of Melgaço and cross-border population of Neves, Arbo, Crecente, Cañiza and Padrenda. Thus, we sought to know how the functional capacity of two groups evolved, one that benefited from a 12-week Senior Gymnastics Program, and another that did not benefit from this program.

\section{MATERIAL AND METHODS}

\section{Participants}

The sample selected for the accomplishment of this study is a sample for convenience, constituted by 23 institutionalized elderly people attending the Elderly Home of SCM de Mação. After selecting the sample and applying the test battery by Rikli and Jones (1999; 2001), the subjects were divided into 2 groups: GE (experimental group) $-n=13$ elderly people 10 females and 3 males who participated in the Senior Gymnastics Program; SGP, ${ }^{-} \mathrm{x}=87.15 \pm 6.09$ years old - Group without Physical Activity Practice $n=10$ elderly people 7 females and 3 males who did not participate in the Senior Gymnastics Program, ${ }^{-} x=79.6$ \pm 6.45 ; Inclusion criteria: Participation in at least $90 \%$ of SGP classes; For all SFT tests; Have been present at the meeting and sign the informed consent form; Be over 65 years old; Do not make any formal AF prior to the PGS; Institutionalized at SCM Mação. Exclusion criteria: Present an attendance of less than $90 \%$ to the SGP classes; Inability / impossibility to perform any of the SFT tests; Perform formal AF beyond the SGP.

\section{Measures}

Data was recorded in a spreadsheet. In order to compare and verify whether or not there was evolution, we used, as already mentioned, two evaluation moments with a 12-week interval between them, which allowed us to compare the results before and after the SGP. Still, with regard to the collection of data, it was only carried out after all ethical and anonymity principles were assured, in such a way that a number was assigned to each participant. The instrument used was the test battery of Rikli and Jones (1999; 2001). The order of application of the tests was as follows: ${ }^{10} 30$ s Chair Stand, $2^{\circ} \mathrm{Arm}-\mathrm{Curl}, 3^{\circ} \mathrm{Chair}$ Sit-and-Reach, $4^{\circ} 8$-Foot Upand-Go, $5^{\circ}$ Back Scratch, $6^{\circ}$ 6-Minute Walk and finally IMC.

\section{Procedures}

What we want to know is whether a SGP with a duration of three months of structured PA offered on a permanent basis, with an average of 45 to 50 minutes in duration and frequency of 2 times a week, applied to a group of institutionalized your FC To do this, we used the test battery of Rikli and Jones $(1999 ; 2001)$ in two moments: one before the beginning of the program (pre-test) and one second at the end of the program 
(post-test). In both, the data was collected in order to explain and compare the information collected from each of the variables.

\section{Analysis}

Statistical Package for the Social Sciences (SPSS) - Version 24.0 and Microsoft Excel 2013 were used. The level of significance was set at $a=0.05$ (95\% confidence interval). All the descriptive statistics were performed, followed by the groups' characterization as well as the comparison between them. Groups were also be characterized by gender as well as compared. For the comparison, first check the normality, through statistical tests.

\section{RESULTS}

In the pre-test, the results obtained by the GS show the values obtained by the males and females groups. In the pre-test yet, the results obtained by the GE, show the values obtained by both genders are shown in table 1.

Table 1. Pre-test results in both groups

\begin{tabular}{|c|c|c|c|c|c|c|c|c|}
\hline & \multicolumn{3}{|c|}{ Pré - Test } & \multirow[b]{2}{*}{ GE } & \multicolumn{4}{|c|}{ Post- Test } \\
\hline & & GS & & & & GS & GE & \\
\hline & Male & Female & Male & Female & Male & Female & Male & Female \\
\hline IMC $\left(\mathrm{kg} / \mathrm{m}^{2}\right)$ & 27.07 & 27.41 & 29.29 & 29.27 & 27.34 & 27.87 & 28.73 & 28.57 \\
\hline 30s Chair Stand & 4.33 & 6 & 5 & 4.9 & 3 & 4.71 & 7.67 & 7.9 \\
\hline Arm-Curl & 9 & 9.86 & 9 & 7.4 & 6.67 & 7.29 & 15.33 & 12.3 \\
\hline Chair Sit-and-Reach (cm) & -12.67 & -5.86 & -9.67 & -10.8 & -15 & -9.71 & -6 & -5.75 \\
\hline 8-Foot Up-and-Go (seg) & 26.85 & 19.56 & 17.19 & 23.24 & 30.7 & 22.42 & 15.37 & 21.62 \\
\hline Back Scratch $(\mathrm{cm})$ & -42.33 & -43.43 & -46.67 & -37 & -48.33 & -46.29 & -34.67 & -27.8 \\
\hline 6-Minute Walk (m) & 278.33 & 342.86 & 340 & 309.5 & 235 & 303.57 & 401.67 & 361 \\
\hline
\end{tabular}

In the same table yet, the results obtained by gender and by group are found after the 12 weeks of the SGP, the SFT test battery was again performed.

\section{DISCUSSION}

For GE, we verified that there are significant differences in the initial and final functional capacity of institutionalized elderly people who were given a specific program of 12 weeks of senior gymnastics, as shown in table 2, except for Sitting, walking 2.44 meters and returning to sit.

Table 2. Presentation of the values obtained in GE after application of the t-student or the Wilcoxon test

\begin{tabular}{lcc}
\hline Tests & $\mathbf{t}$ & Sig. \\
\hline IMC 1 e 2 a) & 3.78 & $0.003^{2}$ \\
Arm-Curl 1 e 2 a) & -12.74 & $<0.001^{2}$ \\
Back Scratch 1 e 2 a) & -4.28 & $0.001^{2}$ \\
6 Minute Walk 1 e 2 a) & -9.37 & $<0.001^{2}$ \\
8-Foot Up-and-Go 1 e 2 b) & -1.85 & $0.064^{1}$ \\
Chair Sit-and-Reach 1 e 2 b) & -2.50 & $0.012^{2}$ \\
30s Chair Stand 1 e 2 b) & -3.20 & $0.001^{2}$ \\
\hline
\end{tabular}
Legend: a) Student's t-test; b) Wilcoxon test; 1 - Does not present DES; 2 - Displays OFF 
Table 3. Presentation of the values obtained in study of Mira (2016) after and before fitness program

\begin{tabular}{lcc}
\hline \multicolumn{1}{c}{ Tests } & After & Before \\
\hline IMC (kg/m ${ }^{2}$ ) & $25.35 \pm 12.87$ & $26.65 \pm 3.28$ \\
30s Chair Stand & $14 \pm 6.52$ & $14.5 \pm 6.89$ \\
Arm-Curl & $19 \pm 5.27$ & $20 \pm 6.83$ \\
Chair Sit-and-Reach (cm) & $4.5 \pm 10.14$ & $5 \pm 11.09$ \\
8-Foot Up-and-Go (seg) & $7.38 \pm 4.09$ & $6.55 \pm 2.13$ \\
Back Scratch (cm) & $-19 \pm 12.62$ & $-17 \pm 12.47$ \\
6-Minute Walk (m) & $482 \pm 110.13$ & $481 \pm 101.15$ \\
\hline
\end{tabular}

Comparing our results with those obtained by Mira (2016), we found that these did not follow their trend since, after applying a fitness program, slightly worse results were observed comparing the initial and final moments, with the exception of IMC and 6 Minutes' walk.

\section{CONCLUSIONS}

We conclude that, with the application of PGS, there were significant differences in the FC of the institutionalized elderly in the municipality of Mação. We also concluded that the physical fitness levels of the elderly who participated in SGP improved significantly, with the exception of the sitting test, walked 2.44 meters and returned to sit, but also improved. In addition, we conclude that the elderly who were part of the GS showed a decrease in their abilities, presenting worse results compared to the initial moment. It is important to point out that this decrease occurred in both genders.

\section{REFERENCES}

Instituto Nacional de Estatística, [INE], (2011), Resultados definitivos Censos 2011. Consultado a 8/08/2017 através de http://www.agap.pt/images/userfiles/files/20Censos2011 res definitivos.pdf

Mira, C. (2016). Atividade física no idoso de Melgaço e população transfronteiriça de Neves, Arbo, Crecente, Cañiza e Padrenda. Viana do Castelo: Escola Superior de Desporto e Lazer do IP de Viana do Castelo. https://doi.org/10.5628/rpcd.05.01.77

Organização Mundial da Saúde, [OMS], (2002). Global forum for health research: The 0/90 report on health research. Genebra: Organização Mundial da Saúde. https://doi.org/10.7476/9788575413982

Rikli, R. \& Jones, C. (1999). Development and validation of a functional fitness test for community Residing older adults. Journal of Aging and Physical Activity, 7 (2), 129-161. https://doi.org/10.1123/japa.7.2.129

Serrano, J.; Faustino, A.; Rato, V.; Petrica, J.; Paulo, R.; Mendes, P.\& Batista, M. (2015). O perfil comum dos programas de atividade física das academias séniores. Revista de Ciencias del Desporte, 11, 135-136.

\section{(9) $\odot \Theta \Theta$}

This work is licensed under a Attribution-NonCommercial-NoDerivatives 4.0 International (CC BY-NC-ND 4.0). 


\title{
Comparison of physical fitness tests and special judo fitness test performance and classificatory tables development for juvenile and cadet male athletes
}

\author{
LUÍS MONTEIRO1,4 , RUI VELOSO ${ }^{2,4}$, ANA HORMIGO ${ }^{4}$, ABEL LOURO ${ }^{4}$, PAULA SALDANHA $^{4}$, TIAGO \\ SILVA 3,4, PAULO NOGUEIRA 4 \\ ${ }^{1}$ Universidade Lusófona de Humanidades e Tecnologias, Faculdade de Educação Física e Desporto, \\ Portugal \\ 2Universidade Lusófona do Porto, Portugal; Faculdade de Desporto da Universidade do Porto, Portugal \\ 3 Universidade de Lisboa, Faculdade de Motricidade Humana, Portugal \\ ${ }^{4}$ Federação Portuguesa de Judo, Portugal
}

\begin{abstract}
The aims of this work were to compare the performances of juvenile and cadet male judo athletes and to develop classificatory tables to these judo-specific tests for national and international-level of Portuguese athletes from these ages. Data collected during training camps (2017-2018) of male juvenile and cadet judo from Clubs and National team of Portugal were analysed, totalizing 430 individual results of Special Judo Fitness Test (SJFT), and Physical Fitness Tests (PFT), Beeps Test (BT), VO2máx, Sit-ups 1 min, Push-ups, Hand Grip Strength (HGS), Squat Jump (SJ), Countermovement Jump (CMJ), Standing Broad Jump (SBJ) and Sit and Reach (S\&R) respectively. Cadets performed better than juvenile in the SJFT, with higher number of throws in $A, B, C(P<0.01)$ for all comparisons final heart rate $(P=0.05)$, and index $(P<0.001)$, as well as higher performance in the PFT $(P<0.001)$. Considering these differences, tables were established by age groups, using percentile values. Thereby, each of the parameters of the SJFT (number of throws, heart rate after and 1 min after the test, and SJFT index), as well as absolute results in PFT, were classified by age (juvenile and cadet). These classificatory tables can be useful for judo coaches to monitor specific physical performance during different phases of periodization process and individualize training programs for successful long-term athletic development. Keywords: Judo; Physical fitness; Field tests; Reference values; Performance; Young athletes.
\end{abstract}

Corresponding author. Universidade Lusófona de Humanidades e Tecnologias, Faculdade de Educação Física e Desporto, Portugal.

E-mail: luis.monteiro@ulusofona.pt

Supplementary Issue: Spring Conferences of Sports Science. International Seminar of Physical Education, Leisure and Health, 17-19 June 2019. Castelo Branco, Portugal.

JOURNAL OF HUMAN SPORT \& EXERCISE ISSN 1988-5202

(C) Faculty of Education. University of Alicante.

doi:10.14198/jhse.2019.14.Proc4.82 


\section{INTRODUCTION}

Judo is a combatffighting sport characterized by a huge variety and quantity of technical and tactical elements which require the whole body and are expressed through great variability in what concerns intensity and intermittence (Crnogorac, 2010). For that reason, the judo combat dynamic is in everlasting change. Besides, in order to succeed in judo athletes need to develop various physical abilities where power and anaerobic capacity, power and aerobic capacity, maximum isometric strength and dynamics, muscular power and resistant strength can be included (Franchini et al., 2011). In this context, the several studies concluded (Ceylan and Balci, 2018; Agostinho et al., 2018) that the SJFT (Cumming et al., 2017) that general anthropometric and maturation degree features, as well as the results obtained in a battery of general tests may discriminate athletes of different levels and make it possible to predict the young judoists performance. In view of the above, the aim of our study was to create scoreboards based on the PFT and morphological assessment results, both for juveniles and cadets, in order to contribute for an improvement of the training and talent detection process.

\section{MATERIAL AND METHODS}

\section{Participants}

Four hundred and thirty male judo athletes from Clubs and National Team of Portugal, were evaluated. Athletes age, height, sitting height, body mass BMl and fat, were as following (mean \pm standard deviation): male juvenile - $13.5 \pm 1.0$ years, $1.63 \pm .08 \mathrm{~m}, 1.26 \pm 0.01 \mathrm{~m}, 55.4 \pm 14.2 \mathrm{~kg}, 20.5 \pm 3.5 \mathrm{~kg} / \mathrm{m} 2,11.4 \pm 5.5(\%)$; male cadets $-15.7 \pm 0.6$ years, $1.70 \pm 0.08 \mathrm{~m}, 1.35 \pm 1.0 \mathrm{~m}, 61.6 \pm 11.5 \mathrm{~kg}, 20.1 \pm 4.9 \mathrm{~kg} / \mathrm{m} 2,9.6 \pm 2.9(\%)$.

\section{Measures and Procedures}

To evaluate the Physical Fitness (PF) the athletes performed the $\mathrm{BT}$ ( $\mathrm{VO}_{2 \text { máx }}$ ), Sit-ups (1 min) Push-ups (1 $\mathrm{min})$, HGS (kg) using a dynamometer (Takei Physical Fitness Test, TKK 5001), SJ (cm), CMJ (cm) was evaluated according to the Bosco protocol, and measured using Chronojump measurement technology (Bosco System, Globus, Italy). SBJ $(\mathrm{cm})$ and S\&R $(\mathrm{cm})$. To the SJFT, participants performed the original recommendations. Briefly, the SJFT is divided into three active periods (A, $15 \mathrm{sec} ; \mathrm{B}$ and $\mathrm{C}, 30 \mathrm{sec}$ ) with 10$\mathrm{sec}$ intervals between them. During each period, the athlete being evaluated throws 2 partners (separated from each other by a distance of $6 \mathrm{~m}$ ) as many times as possible using the ippon-seoi-nage technique. Immediately after and 1 min after completion of the test, the athletes heart rate was measured using a heart rate monitor (Polar® RS400).

\section{Analysis}

All analyses were performed using the SPSS software (Version 23.0, IBM SPSS, Chicago, IL), and the significance was set at $5 \%$. Participants were stratified by age group (juvenile, 13-14 years old and cadets, 15-16 years old). Basic descriptive statistics (mean and standard deviation) were calculated for all the variables, which also were examined for normality by the Shapiro-Wilk test and homogeneity of variances by the Levene test. Analysis of variance (ANOVA) was performed to test the differences between the two groups. For each test scales percentile values were adopted to establish the following categories, as suggested by Agostinho (2018): excellent, highest 5\%; good, next 15\%; regular, middle 60\%; poor, next lowest $15 \%$; very poor, lowest $5 \%$. 


\section{RESULTS}

Cadets performed better than juvenile in the SJFT, with higher number of throws in $A, B, C(P<0.01)$ for all comparisons final heart rate $(P=0.05)$, and index $(P<0.001)$, as well as higher performance in the $P F T(P<$ 0.001). Considering these differences, tables were established by age groups, using percentile values. Thereby, each of the parameters of the SJFT (number of throws, heart rate after and 1 min after the test, and SJFT index), as well as absolute results in PFT, were classified by age (juvenile and cadet). Table 1 and Table 2 presents the PF classificatory table for male juvenile and cadet group; and Table 3 and Table 4 presents the SJFT table, respectively for male juvenile and cadet group.

Table 1. Physical Fitness classificatory table for male juvenile judo athletes

\begin{tabular}{|c|c|c|c|c|c|c|c|c|c|c|}
\hline Level & $\begin{array}{l}\text { BT } \\
\left(n^{0}\right)\end{array}$ & $\begin{array}{l}\mathrm{VO}_{2 m a ́ x} \\
\left(\mathrm{ml} . \mathrm{kg}^{-}\right. \\
\left.\text {1. } \mathrm{min}^{-1}\right)\end{array}$ & $\begin{array}{c}\text { Sit-ups } \\
1 \text { min } \\
\left(n^{0}\right)\end{array}$ & $\begin{array}{c}\text { Push- } \\
\text { ups } \\
\left(n^{0}\right)\end{array}$ & $\begin{array}{c}\text { Right } \\
\text { HGS } \\
(\mathrm{kg})\end{array}$ & $\begin{array}{l}\text { Left } \\
\text { HGS } \\
(\mathrm{kg})\end{array}$ & $\begin{array}{l}\text { SJ } \\
(\mathrm{cm})\end{array}$ & $\begin{array}{l}\text { CMJ } \\
(\mathrm{cm})\end{array}$ & SBJ (m) & $\begin{array}{c}S \& R \\
(0=26 \mathrm{~cm})\end{array}$ \\
\hline Excellent & $\geq 96$ & $\geq 51$ & $\geq 60$ & $\geq 69$ & $\geq 48.1$ & $\geq 45.2$ & $\geq 29.1$ & $\geq 35.6$ & $\geq 2.17$ & $\geq 41.5$ \\
\hline Good & $81-95$ & $46-50$ & $48-59$ & $51-68$ & $38-48$ & $35-45$ & $25-29$ & $27-35$ & $1.95-2.15$ & $34-41.0$ \\
\hline Regular & $73-80$ & $44-45.8$ & $44-47$ & $40-50$ & $32-37$ & $32-34$ & $22-24$ & $26-28$ & $1.87-1.92$ & $29-32.7$ \\
\hline Poor & $54-72$ & $37-43.2$ & $40-43$ & $28-39$ & $25-31$ & $24-31$ & $19-21$ & $26-23$ & $1.70-1.86$ & $25.5-28.9$ \\
\hline $\begin{array}{l}\text { Very } \\
\text { Poor }\end{array}$ & $\leq 53.8$ & $\leq 36.8$ & $\leq 39.0$ & $\leq 27.6$ & $\leq 24.8$ & $\leq 23.2$ & $\leq 19.0$ & $\leq 22.7$ & $\leq 1.69$ & $\leq 25.4$ \\
\hline
\end{tabular}

Table 2. Physical Fitness classificatory table for male cadet judo athletes

\begin{tabular}{|c|c|c|c|c|c|c|c|c|c|c|}
\hline Level & $\begin{array}{l}\mathrm{BT} \\
\left(\mathrm{n}^{0}\right)\end{array}$ & 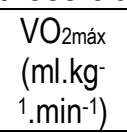 & $\begin{array}{c}\text { Sit-ups } 1 \\
\min \left(n^{0}\right)\end{array}$ & $\begin{array}{l}\text { Push- } \\
\text { ups }\left(n^{0}\right)\end{array}$ & $\begin{array}{c}\text { Right } \\
\text { HGS (kg) }\end{array}$ & $\begin{array}{l}\text { Left } \\
\text { HGS } \\
(\mathrm{kg})\end{array}$ & $\begin{array}{l}\text { SJ } \\
(\mathrm{cm})\end{array}$ & $\begin{array}{l}\mathrm{CMJ} \\
(\mathrm{cm})\end{array}$ & $\mathrm{SBJ}(\mathrm{m})$ & $\begin{array}{c}\text { S\&R } \\
(0=26 \\
\mathrm{cm})\end{array}$ \\
\hline Excellent & $\geq 100$ & $\geq 52$ & $\geq 70$ & $\geq 78$ & $\geq 54$ & $\geq 51$ & $\geq 33$ & $\geq 37$ & $\geq 2.25$ & $\geq 46$ \\
\hline Good & $90-99$ & $49-52$ & $59-70$ & $61-77$ & $47-53$ & $45-50$ & $28-32$ & $32-36$ & $\begin{array}{l}2.09- \\
2.24\end{array}$ & $39-45$ \\
\hline Regular & $79-90$ & $45-48.7$ & $50-58$ & $55-60$ & $40-45$ & $39-44$ & $24-27$ & $30-32$ & $\begin{array}{l}1.99- \\
2.08\end{array}$ & $34-38$ \\
\hline Poor & $71-78$ & $43-45.1$ & $45-50$ & $45-54$ & $35-39$ & $34-38$ & $22-24$ & $25-29$ & $1.85-1.8$ & $30-33$ \\
\hline $\begin{array}{l}\text { Very } \\
\text { Poor }\end{array}$ & $\leq 70$ & $\leq 42.8$ & $\leq 44.4$ & $\leq 44.2$ & $\leq 34.02$ & $\leq 33.4$ & $\leq 21.7$ & $\leq 25.0$ & $\leq 1.84$ & $\leq 29.5$ \\
\hline
\end{tabular}

Table 3. Special Judo Fitness Test classificatory table for female cadet judo athletes

\begin{tabular}{ccccc}
\hline Level & $N^{0}$ of throws & HR after (beats/min) & HR 1 min after (beats/min) & Index \\
\hline Excellent & $\geq 28$ & $\geq 191$ & $\geq 174$ & $\geq 12.95$ \\
Good & $25-27$ & $184-190$ & $146-172$ & $14.8-12.64$ \\
Regular & $23-23$ & $181-184$ & $141-145$ & $15-70-14.79$ \\
Poor & 22 & $171-180$ & $133-140$ & $19.5-15.67$ \\
Very Poor & $\leq 21$ & $\leq 170$ & $\leq 132$ & $\leq 19.30$ \\
\hline
\end{tabular}

$H R$, heart rate

Table 4. Special Judo Fitness Test classificatory table for male cadet judo athletes

\begin{tabular}{ccccc}
\hline Level & $\mathrm{N}^{0}$ of throws & HR after (beats/min) & HR 1 min after (beats/min) & Index \\
\hline Excellent & $\geq 30$ & $\leq 172$ & $\leq 133$ & $\leq 11.38$ \\
Good & $28-29$ & $184-173$ & $147-134$ & $11.3-12.7$ \\
Regular & $26-27$ & $185.4-192$ & $159-148$ & $12.80-13.7$ \\
Poor & $24,5-25$ & $191.6-199$ & $168-160$ & $13.7-15.9$ \\
Very Poor & $\leq 24.2$ & $\geq 200$ & $\geq 169.7$ & $\geq 15.96$ \\
\hline \multicolumn{4}{r}{ HR, heart rate }
\end{tabular}




\section{DISCUSSION}

The main findings of the present study were: (a) cadets performed better in PFT (higher values in physical parameters) and SJFT (higher number of throws in A, B, C, and lower final heart rate and index); (b) body mass was higher and fat (\%) was lower in cadets than in juveniles with different into all performance variables. Concerning the differences in performance between age groups (juvenile and cadet), and considering the values of SJFT in cadets, are similar to the results of the study of Agostinho (2018). In fact, juveniles also presented lower mean and peak power during BT, HGS and explosive strength compared to cadet judo athletes. Studies with grappling combat sports athletes (Agostinho, 2018) suggested that differences in aerobic capacity and maximal isometric strength between adolescents and adults are probably related to maturational aspects. Practitioners working with youth should use relevant monitoring and assessment tools, control the progress and individualize training programs for successful long-term athletic development (Lloyd et al., 2016): (a) promote the benefits of a lifetime of healthy physical activity, and (c) prevent and/or minimize injuries from sports participation for all boys and girls. This is the first study that compared the performance in the SJFT between juvenile and cadet male judo athletes.

\section{CONCLUSIONS}

In conclusion, Cadets performed better than Juveniles, in the PFT and in the SJFT. Additionally, in order to contribute for an improvement of the training and talent detection process, and the 5-grade scale proposed in this study and used as a reference to guide young athletes evaluation, training, and during the rehabilitation processes.

\section{REFERENCES}

Agostinho, M., Junior, J., Stankovic, N., Escobar-Molina, R., \& Franchini, E. (2018). Comparison of special judo fitness test and dynamic and isometric judo chin-up tests performance and classificatory tables development for cadet and junior athletes. J Exerc Rehabilit, 14(2), 244-252. https://doi.org/10.12965/jer.1836020.010

Ceylan, B., \& Balci, S. (2018). The Comparison of Judo-Specific Tests. J Martial Arts, 18(4), 54-62.

Crnogorac, B. (2010). Transformation of Basic and Especific Motor Status Of Elite Judo Players Under The Influence Of Innovated Trainig Model. Sport Sci, 3(3), $27-31$.

Cumming, S., Lloid, R., Oliver, J., Eisenmmann, J., \& Malina, R. (2017). Bio-Banding in Sports: Application to Competittion, Talent Identification, and Strength and Conditioning of Youth Athletes. Strength Cond J, 39(2), 34-47. https://doi.org/10.1519/ssc.0000000000000281

Franchini, F., Del Vecchio, F., Matsushigue, K., \& Artioli, G. (2011). Physiological Profiles of Elite Judo Athletes. Sports Med, 41(2),147-166. https://doi.org/10.2165/11538580-000000000-00000

Lloyd, R., Cronin, J., Faigenbaum, A., Haff, G., Howard, R., Kraemer, W., Micheli, L., Myer, G., \& Oliver, J. (2016). National Strength and Conditioning Association position statement on long-term athletic $\begin{array}{lllll}\text { development. J Strength } \quad \text { Cond } & \text { Res, }\end{array}$ https://doi.org/10.1519/jsc.0000000000001387

\section{(ㅇ) $(\mathbb{\Theta \Theta} \Theta$}

This work is licensed under a Attribution-NonCommercial-NoDerivatives 4.0 International (CC BY-NC-ND 4.0). 


\title{
Postural stability and handgrip strength in the older adults: Differences between fallers and non- fallers
}

\author{
CAROLINA VILA-CHÃ $1,2 \triangle$, CLÁUDIA VAZ ${ }^{1}$, ERMELINDA MARQUES ${ }^{1,3}$, AGOSTINHA CORTE ${ }^{1,3}$, NUNO \\ SERRA ${ }^{1}$ \\ ${ }^{1}$ Research Unit for Inland Development, Polytechnic Institute of Guarda, Portugal \\ ${ }^{2}$ Research Center in Sports Sciences, Health Sciences and Human Development, CIDESD, Portugal \\ ${ }^{3}$ Center for Health Technology and Services Research, Portugal
}

\begin{abstract}
The aim of this study was investigated differences in postural stability and handgrip strength between faller and non-fallers older adults. 169 older adults $(76.5 \pm 7.5$ years), were recruited and were divided in fallers ( $\mathrm{n}$ $=58)$ and non-fallers $(n=111)$ groups according to their fall history. Postural stability measures were made in 3 different quite stance positions using a force platform. Total, anteroposterior (AP) and mediolateral (ML) displacement of centre of pressure (COP) were then computed. Finally, muscle strength was assessed with the handgrip test. The results showed that those adults who fell one or more times in a year, have lower handgrip strength $(-15.5 ; \mathrm{P}<0.001)$ and higher displacement of the COP (total and AP direction), than those that did not fell ( $P>0.05$ for the side-by-side stand and semi-stand positions). Moreover, associations between handgrip strength, total and AP COP displacement, and falls history were found. The present results indicates that control of balance in narrow base stances may be an important tool in identifying elderly fallers. The tests applied in the present study allowed to distinguish between fallers and non-fallers older adults, providing valuable information on this population which may help in the implementation of falls prevention and functional fitness protocols, contributing for promoting aging with quality of life. Keywords: Postural stability; Older adults; Handgrip strength; Centre of pressure.
\end{abstract}

Corresponding author. Instituto Politécnico da Guarda. Av. Francisco Sá Carneiro, n. 50; 6300-Guarda, Portugal.

E-mail: cvilacha@ipg.pt

Supplementary Issue: Spring Conferences of Sports Science. International Seminar of Physical Education, Leisure and Health, 17-19 June 2019. Castelo Branco, Portugal.

JOURNAL OF HUMAN SPORT \& EXERCISE ISSN 1988-5202

(c) Faculty of Education. University of Alicante.

doi:10.14198/jhse.2019.14.Proc4.82

VOLUME 14 | Proc4 | 2019 | S1747 


\section{INTRODUCTION}

It is expected that postural control changes with age due to several age-related alterations, such as loss of muscle strength and decreased proprioception (Ko \& Newell, 2016). Such deterioration may contribute to falls in older people, which can lead to serious consequences, including bone fractures, loss of overall quality of life and further dependence (Moreira et al., 2018). Several studies have addressed the assessment of this ability in order to identify elderly person risk of falling. However, impaired postural stability may not be revealed in a standard examination. Consequently, there is a crucial need to investigate postural instability, aiming to recognize older people who are at risk of falling (Ko \& Newell, 2016). Therefore, the aim of this study was to investigate if simple biomechanical tests can show differences between faller and non-fallers older adults. In addition, associations between falls history, muscle strength, and centre of pressure were explored.

\section{MATERIAL AND METHODS}

\section{Participants}

A total of 169 older people ( 93 women and 76 men), aged between 65 and 96 year old (76.5 \pm 7.5 years), were recruited in the Gmove+ cohort, which had a first phase composed by observational cross-sectional design to explore and characterize functional fitness and quality of life of the elderly population from Guarda council. This study was approved by local ethical committee of ULS Guarda (ref 11136.17-11-09).

\section{Measures}

The standing postural stability, on a firm surface, was measured with a force platform (Kistler, model 9260AA6, Switzerland) in the following feet positions: a) side-by-side stand (feet together); b) semi-tandem stand and; $\mathrm{C}$ ) tandem stand. Centre of pressure (COP), data during the stability tests were sampled at a frequency of $1000 \mathrm{~Hz}$ and was low-pass filtered at $5 \mathrm{~Hz}$. Total COP, mediolateral (ML) and anterior-posterior (AP) displacements were determined and then normalized to subjects height. The participants were also asked about episodes of falls through a customized questionnaire designed to identify whether participants experienced a fall during the last 12 months (Buchner et al., 1997). Handgrip strength was measured using a calibrated Jamar hand dynamometer (USA) as an indicative of general muscle function.

\section{Procedures}

The participants were introduced to each test and were allowed to do a pre-trial test for each standing position. The subjects were instructed to stand as still as possible on the force plate and to look straight ahead, with arms kept by the side of the body. Three trials of 20 seconds were performed for each task with 30 seconds of rest between them. After, the subjects completed the questionnaire about falls and performed the handgrip strength test. Participants were instructed to exert their maximal force 3 times by each hand, with one minute of rest in between.

\section{Analysis}

Based on the falls questionnaire responses, two groups were formed: fallers $(n=58)$ and non-fallers ( $n=$ 111). Mann-Whitney $U$ test was applied to investigate the differences between fallers and non-fallers regarding to COP variables and handgrip strength. Associations between variables were determined by Spearman statistical test. Descriptive statistics were expressed as mean $\pm S D$ in the text and mean $\pm S E$ in the figures. The significance level was set to $P<0.05$. 


\section{RESULTS}

No differences were observed between groups regarding age (fallers: $77.3 \pm 8.0$; non-fallers: $75.8 \pm 7.5 \mathrm{yrs}$.; $P=0,175)$. Results on COP displacement for fallers and non-fallers groups are shown in the Figure 1 . Fallers group showed increased COP displacement in both AP and ML directions, as well as in total COP displacement. However significant differences were only observed for Total and AP COP displacements in the side-by-side and semi-tandem stand position (Fig. 1). The maximum handgrip strength was significant greater for those who did not fall than for those that fell one or more times in a year ( $27.1 \pm 8.8$ and $22.8 \pm$ $8.4 \mathrm{~kg}$, respectively; $P<0.001)$. Weak and negative associations between falls history and total and AP COP displacement in the side-by-side and semi-tandem stand position was found $(0.286<r>0.180 ; P<0.05)$. Handgrip strength was moderately and negatively associated with falls history and all COP variables $(-0.502<$ $r>-0,326 ; P>0.01)$.
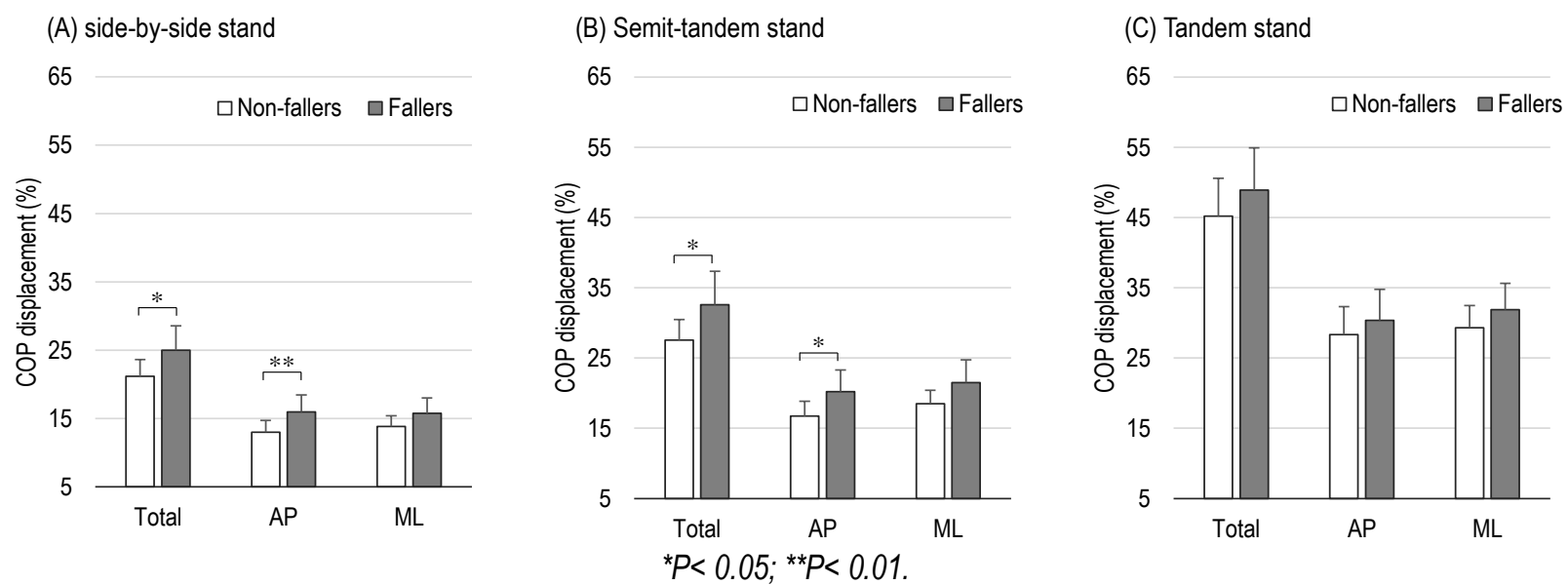

Figure 1. Mean \pm SE of the total, AP and ML COP displacements in the 3 stand positions, for the fallers and non-faller older adults.

\section{DISCUSSION}

The present study showed that those older adults that fell one or more times in the last 12 months have higher COP displacements during simple stand positions. Moreover this group also presented lower level of handgrip strength revelling lower functional capacity. The results are in accordance with Moreira et al. (2018), which reported lower functional capacity among fallers than non-faller older adults. A moderate and negative association was found between handgrip strength and COP variables investigated in this study, which might be considered an indicative of the strength importance to postural stability.

\section{CONCLUSIONS}

The findings of this study indicate that those older adults who have higher handgrip strength values had lower chance of presenting fall episodes. Postural control as measured through COP displacement during quite stance positions showed that fallers have increased COP displacement than non-fallers. Handgrip strength and COP displacement was associated to falls history. 


\section{FUNDING}

Study supported by FEDER funds through COMPETE2020 (POCI-01-0145-FEDER-023811).

\section{REFERENCES}

Buchner, D. M., Cress, M. E., De Lateur, B. J., Esselman, P. C., Margherita, A. J., Price, R., \& Wagner, E. H. (1997). The effect of strength and endurance training on gait, balance, fall risk, and health services use in community-living older adults. Journals of Gerontology - Series A, 52A (4), M218M224. https://doi.org/10.1093/gerona/52a.4.m218

Moreira, N. B., Rodacki, A. L. F., Pereira, G., \& Bento, P. C. B. (2018). Does functional capacity, fall risk awareness and physical activity level predict falls in older adults in different age groups? Archives of Gerontology and Geriatrics, 77, 57-63. https://doi.org/10.1016/i.archger.2018.04.002

Ko, J. H., \& Newell, K. M. (2016). Aging and the complexity of center of pressure in static and dynamic postural tasks. Neuroscience Letters. Neuroscience Letters, 610, 104-109. https://doi.org/10.1016/.neulet.2015.10.069 


\title{
Fine motor skills: An emergent competence in preschool age
}

\author{
LINDA SARAIVA ${ }^{1,4}$, FERNANDO SANTOS ${ }^{1,2}$, PEDRO GIL MADRONNA ${ }^{3}$, CÉSAR SÁ 1,4 \\ ${ }^{1}$ Polytechnic Institute of Viana do Castelo, School of Higher Education, Portugal \\ 2Polytechnic Institute of Porto, School of Higher Education, Portugal \\ 3 University of Castilla-La Mancha, Faculty of Education of Albacete, Spain \\ ${ }^{4}$ Research Centre on Child Studies, Institute of Education, University of Minho, Portugal
}

\begin{abstract}
Recent research has shown that low fine motor competence is related to difficulties in academic performance, problems in school adaptation and social behaviour during transition from preschool to primary school. It is clear that prevention or reduction of these negative effects is dependent on early identification of motor impairment and timely intervention. Therefore, the purposes of this study were: i) to assess fine motor competence of preschool children; ii) to explore the influence of gender on fine motor competence; and iii) to identify fine motor tasks that children can successfully achieve at the end of pre-school education. A total of 366 children (195 girls and 171 boys) aged 36 to 71 months $(53.0 \pm 9.6)$ were assessed by fine motor subscale of PDMS-2. The majority of the children reached expected performance in grasping and visualmotor integration skills at all age-groups. Significant differences were found between boys' and girls' performance. Girls presented higher scores than boys in Grasping and Visual-motor integration at 3 and 4 years of age, particularly in tasks such as grasping marker, unbuttoning button, buttoning button, colouring between lines and cutting. At the age of five, children still show difficulties in folding paper. The results of this study clearly reinforce the assumption that fine motor skills are an emergent competence in preschool age that depends on children's maturation but also on opportunities for stimulation which are provided in preschool and family environments. Keywords: Fine motor competence; Grasping; Visual-motor integration; PDMS-2; Preschool children.
\end{abstract}

Corresponding author. Escola Superior de Educação - IPVC, Av. Cap. Gaspar de Castro 513, 4901-908 Viana do Castelo, Portugal.

E-mail: lindasaraiva@ese.ipvc.pt

Supplementary Issue: Spring Conferences of Sports Science. International Seminar of Physical Education, Leisure and Health, 17-19 June 2019. Castelo Branco, Portugal.

JOURNAL OF HUMAN SPORT \& EXERCISE ISSN 1988-5202

(c) Faculty of Education. University of Alicante.

doi:10.14198/jhse.2019.14.Proc4.82

VOLUME 14 | Proc4 | 2019 | S1751 


\section{INTRODUCTION}

The Fine Motor development begins at a very early age. However it is at the preschool age that a set of skills emerges that constitute a prerequisite for various activities of daily living (e.g., dressing, writing, cutting, playing), as well as autonomy, self-esteem and academic achievement of the child (e.g., Gauls et al., 2016). Overall, research also reports that fine motor competence (FMC) is linked to school adaptation and social behaviour during the transition from preschool to primary school. FMC is a powerful predictor of school readiness, as well as a predictor of later academic achievement, especially in reading and mathematics (Pichford et al., 2016). Thus, it is crucial to develop efforts towards early identification of children with any type of motor impairments and conduct interventions in a timely manner. The purposes of this study were: i) to assess FMC of preschool children; ii) to explore the influence of gender on FMC; and iii) to identify fine motor tasks that children can successfully achieve at the end of pre-school education.

\section{MATERIAL AND METHODS}

\section{Participants}

366 typically developing children (171 males and 195 females), aged between three to five years (mean age $53.0 \pm 9.6$ ) were randomly selected from ten public preschools of Viana do Castelo. The legal guardians of the preschool children were informed about testing procedures, and corresponding written consent was obtained. Overall, the sample exhibited a balanced ratio of the participants according to sex and age.

\section{Measures}

Children's FMC was assessed using the Fine Motor Subscale of PDMS-2 (Folio \& Fewell, 2000), translated and adapted for the Portuguese population (Saraiva, Rodrigues \& Barreiros, 2011). The subscale is composed of two subtests: Grasping (26 items) and Visual-motor integration (72 items). For this study, only the raw scores of Grasping and Visual-Motor Integration were calculated.

\section{Procedures}

The PDMS-2 was administered according to manual guidelines (Folio \& Fewell, 2000). Each child was individually tested by the same experienced tester on PDMS-2 in a quiet area of the preschool settings. The procedures employed were in accordance with Helsinki's Declaration of Human Ethical guidelines (1975).

\section{Analysis}

Descriptive statistics were calculated. The Student's t-test was calculated to examine the differences on motor performance between boys and girls at each age group. The level of significance was set at .05 for all statistical tests. In addition, Cohen's d measure of effect size was calculated. Effect sizes of $<0.5,0.5-0.8$, and $>0.8$ were interpreted as small, moderate, and large, respectively (Cohen, 1988).

\section{RESULTS}

Table 1. Mean raw scores and standard deviations (M $\pm S D$ ) for each PDMS-2 subtest by sex and age group

\begin{tabular}{|c|c|c|c|c|c|c|c|c|c|}
\hline \multirow[b]{2}{*}{ Subtest } & \multicolumn{3}{|c|}{3 years } & \multicolumn{3}{|c|}{4 years } & \multicolumn{3}{|c|}{5 years } \\
\hline & Boys $(n=61)$ & Girls $(n=61)$ & $t$ & Boys $(n=56)$ & Girl $(n=76)$ & $t$ & Boys $(n=54)$ & Girls $(n=61)$ & $t$ \\
\hline Grasping a) & $48.2 \pm 2.8$ & $49.7 \pm 2.1$ & $-3.39^{\star \star \star}$ & $50.3 \pm 1.8$ & $51.2 \pm 1.1$ & $-3.35^{* * *}$ & $51.1 \pm 1.2$ & $51.5 \pm 0.7$ & -2.95 \\
\hline $\begin{array}{l}\text { Visual-motor } \\
\text { integration }{ }^{b}\end{array}$ & $122.9 \pm 6.9$ & $124.6 \pm 7.0$ & $-2.14^{*}$ & $134.3 \pm 6.2$ & $136.7 \pm 5.2$ & $-2.31^{*}$ & $139.7 \pm 4.3$ & $140.2 \pm 3.0$ & -.723 \\
\hline
\end{tabular}

Note: a) score range $0-52$; b) score range $0-144 ;{ }^{*} p<.05 ;{ }^{* * *} p \leq .001$; Girls presented a better performance on Grasping subtest (3 yrs: $d=.61 ; 4$ yrs: $d=.60$ ) and Visual-motor integration subtest ( 3 yrs: $d=.24$; 4yrs: $d=.42$ ). 


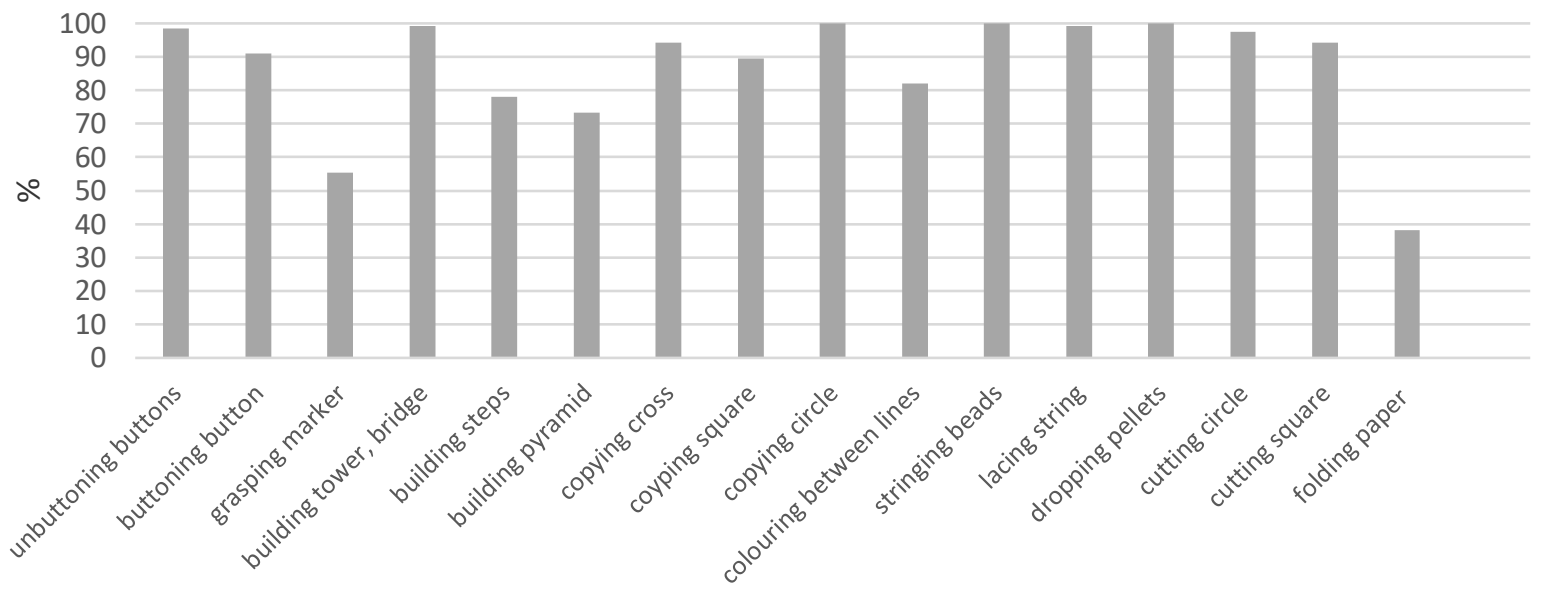

Figure 1. Success rates (\%) in fine motor tasks at the end of pre-school education

\section{DISCUSSION}

It is interesting to observe that girls' superiority in grasping and visual-motor integration skills decrease in older age cohorts. The differences between boys and girls in fine motor skills throughout preschool ages might be explained by school practices in which both genders are equally stimulated, but this hypothesis requires further research. The evidence of early gender differences in FMC is not new in literature (e.g., Gauls et al., 2016), suggesting strong early effects of biological variables in motor development and a progressive modulation effect of motor experiences across childhood. Most children of our sample have adequate fine motor proficiency for their age. However, the results indicate that at the age of five children still show difficulties in folding paper. This finding reinforces the need to encourage children to practice more folding paper skills in the pre-school settings. Based on our results, we can conclude that children at the end of pre-school education should be able to perform the following fine motor skills: building with blocks, cutting, copying, colouring lines, unbuttoning buttons, buttoning button, stringing beads, lacing string and grasping marker.

\section{CONCLUSIONS}

This study clearly reinforces the assumption that fine motor skills are an emergent competence in preschool age that depends on children's maturation but also on opportunities for stimulation which are provided in preschool and family environments.

\section{REFERENCES}

Cohen, J. (1988). Statistical power analysis for the behavioral sciences (2nd ed.). Hillsdale, NJ: Lawrence Earlbaum Associates.

Folio, R. \& Fewell, R. (2000). Peabody Developmental Motor Scales-2: Austin, TX: Pro-ed.

Gaul, D. \& Issartel, J. (2016). Fine motor skill proficiency in typically developing children: On or off the maturation track? Hum Movement Sci, 46, 78-85. https://doi.org/10.1016/.humov.2015.12.011

Pitchford, N. J., Papini, C., Outhwaite, L. A., \& Gulliford, A. (2016). Fine motor skills predict maths Ability better than they predict reading ability in the Early Primary School Years. Front Psychol, 7, 783. https://doi.org/10.3389/fpsyg.2016.00783 
Saraiva, L., Rodrigues, L. P., \& Barreiros, J. (2011). Adaptation and Validation of the Portuguese PDMS2 version: A study with Portuguese Preschoolers. Journal of Physical Education/UEM, 22, 4, 511 521.

\section{(c) $(9)\left(\Theta_{\mathrm{EY}}\right.$}

This work is licensed under a Attribution-NonCommercial-NoDerivatives 4.0 International (CC BY-NC-ND 4.0). 


\title{
Physical education in primary school: From perceptions to practices
}

\author{
LINDA SARAIVA ${ }^{1,3} \square$, VÂNIA CERQUEIRA ${ }^{1}$, FERNANDO SANTOS ${ }^{1,2}$, CÉSAR SÁ $^{1,3}$ \\ ${ }^{1}$ Polytechnic Institute of Viana do Castelo, School of Higher Education, Portugal \\ 2Polytechnic Institute of Porto, School of Higher Education, Portugal \\ 3 University of Castilla-La Mancha, Faculty of Education of Albacete, Spain
}

\begin{abstract}
The current importance of physical education on children's holistic development is indisputable. In addition to providing opportunities for learning basic motor skills, physical education has a key role in cognitive, emotional, affective and social development, while also fostering a healthy lifestyle. This study aimed at analysing the perceptions of pupils, legal guardians and generalist teachers concerning how physical education is operationalized in the school context. Participants were 14 generalist teachers, 279 legal guardians and 346 third and fourth grade pupils from five primary school settings located in Northern Portugal. Data was collected through a survey and documental analysis. The findings show that physical education. Further, physical education is not delivered within the curriculum and in some schools was replaced by extracurricular activities. This is cause for concern, as not all children attend extracurricular activities and do not have the opportunity to develop physical literacy. This undermines the call for a holistic development mandate. Hence, it is necessary to rethink our perspectives and practices towards physical culture within the school context. Keywords: Physical education; Primary school; Perceptions.
\end{abstract}

Corresponding author. Escola Superior de Educação - IPVC, Av. Cap. Gaspar de Castro 513, 4901-908 Viana do Castelo, Portugal.

E-mail: lindasaraiva@ese.ipvc.pt

Supplementary Issue: Spring Conferences of Sports Science. International Seminar of Physical Education, Leisure and Health, 17-19 June 2019. Castelo Branco, Portugal.

JOURNAL OF HUMAN SPORT \& EXERCISE ISSN 1988-5202

(c) Faculty of Education. University of Alicante.

doi:10.14198/jhse.2019.14.Proc4.82 


\section{INTRODUCTION}

The Physical Education (PE) programme is a key reference for the decision-making process in education, as it determines teachers' intervention towards students' holistic development by prioritizing motor skill development and personal and social development (Cruz et al., 1998). More specifically, PE should be guided by the need to develop motor, social and emotional skills. In primary school, PE is part of the curriculum and is also integrated within extracurricular curricular programs as "Physical and Sport Activities". It is important to highlight that PE is a compulsory subject (Bill $n^{0} 139 / 2012$ July 5 ) whereas extracurricular activities are not. However, many times, misguided conceptions seem to arise about PE's role (Carse, 2015; Carse et al., 2018). In this sense, it is relevant to understand the view of pupils, legal guardians and generalist teachers concerning this issue. Therefore, this study aimed to analyse the perceptions of students, legal guardians and generalist teachers concerning how physical education is operationalized within the school context.

\section{MATERIALS AND METHODS}

\section{Participants}

Participants were 14 generalist teachers, 279 legal guardians and 346 third and fourth grade pupils from five primary school settings located in Northern Portugal.

\section{Instruments}

Data was collected through three different surveys aiming to analyse the perceptions of pupils, legal guardians and generalist teachers. Documental analysis was also carried out (i.e., curricular programme and the schools' schedules).

\section{Procedures}

The surveys were previously tested through a pilot study and validated by three experts on PE. In the present study, an informed consent was obtained through the schools' director board and legal guardians.

\section{Analysis}

Concerning open-ended questions, a content analysis was performed through a deductive analysis. The remaining data was analysed through descriptive statistics (relative percentage).

\section{RESULTS}

All generalist teachers considered PE a compulsory and crucial subject. However, only $64.3 \%$ delivered PE to their students once a week, with sessions lasting between 45 to 60 minutes. On the other hand, several motives lead generalist teachers not to deliver PE to their pupils: (i) the subject should be delivered by other educational agent/PE teacher, (ii) PE is already delivered in extracurricular activities, and finally (iii) the primary school curriculum is too thick, as other subjects such as Portuguese and Mathematics are prioritized. Concerning who is responsible for delivering PE, $42.8 \%$ of generalist teachers believed PE should be delivered by a specialist teacher (i.e., PE teacher) and $28.6 \%$ mentioned that they should be assisted by a specialist teacher. Most of these teachers recognized their initial teacher training lacked depth, specifically in skating, swimming and gymnastics. The teachers identified "games" as the content that was more familiar and developed in PE classes. Regarding pupils' perceptions, PE was the subject they most liked, as games and swimming were identified as their preferred contents. For most pupils (73\%), PE is delivered by the PE teacher/teacher who delivers extracurricular activities once a week, with sessions lasting between 45 to 60 minutes. However, $91.5 \%$ alluded to the fact there should be an increase in the time given to this subject. 
Further, pupils acknowledged that PE helped them improve physical condition (17.3\%), health and well-being (17.1\%), and was a vehicle for learning sport-specific skills (14.9\%).

On the other hand, most legal guardians $(72.8 \%)$ believed PE was delivered once per week, with sessions lasting between 45 to 60 minutes, and by a PE teacher/"Expressions teacher". According to their perspectives, PE was important to children's' holistic development, as PE fosters physical and mental development, as well as providing health, values and individual skills.

\section{DISCUSSION}

It is possible to verify that pupils' and legal guardians' perceptions about who delivers PE is similar, as participants did not see the generalist teacher as responsible for delivering PE. On the other hand, most generalist teachers (64.3\%) claimed they did not deliver PE systematically and were not fulfilling the guidelines from bill n. ${ }^{0}$ 176/2014, December 12 (which states the number of hours that should be used for PE) neither the PE curriculum. Thus, 34.7\% of generalist teachers mentioned PE was delivered by another educational agent, which should not happen, as they hold this responsibility. Bill $n^{0} 139 / 2012$ mentions the possibility of having a PE teacher assisting generalist teachers. Nevertheless, generalist teachers still lead the teaching and learning process, as they know pupils' development needs better than an outside agent. While this assisted process is crucial, there is also the possibility for generalist teachers to develop an interdisciplinary approach and foster better outcomes.

\section{CONCLUSION}

Based on the participants' perceptions, we found that PE is not delivered systematically or intently, as in most schools it is not part of their schedule and, in some cases, is replaced by extracurricular activities. This is cause for concern, as not all children attend extracurricular activities and are not exposed to crucial developmental opportunities that may enable social, emotional and motor literacy, which may create barriers to a holistic development mandate. Hence, it is necessary to rethink our perspectives and practices towards physical culture within the school context, embedding PE materials in continuous teacher education programs and developing policies that align with these notions.

\section{REFERENCES}

Carse, N. (2015). Primary teachers as physical education curriculum change agents. Eur Phy Educ Rev., 21, 3, 309-324. https://doi.org/10.1177/1356336x14567691

Carse, N., Jess, M., \& Keay, J. (2018). Primary physical education: Shifting perspectives to move forwards. Eur Phy Educ Rev., 24, 4, 487-502. https://doi.org/10.1177/1356336x16688598

Cruz, S., Carvalho, L. D., Rodrigues, I., Mira, J., Fernandes, L. \& Brás, J. (1998). Manual de Educação Física: $1^{\circ}$ Ciclo do Ensino Básico ( $3^{a}$ ed.). Oeiras: Câmara Municipal de Oeiras.

\section{@ $\odot \Theta \Theta$}

This work is licensed under a Attribution-NonCommercial-NoDerivatives 4.0 International (CC BY-NC-ND 4.0). 


\title{
Physical fitness level of a population with mild cognitive impairment
}

\author{
CATARINA RONDÃO ${ }^{1,2}$, DULCE ESTEVES ${ }^{1,3}$, PAULA MOTA $^{3,4}$ \\ 1 Universidade da Beira Interior, Portugal \\ ${ }^{2}$ Câmara Municipal do Fundão, Portugal \\ ${ }^{3}$ Research Center in Sports Sciences, Health Sciences and Human Development (CIDESD), Portugal \\ ${ }^{4}$ Universidade de Trás-os-Montes e Alto Douro, Portugal
}

\begin{abstract}
Older people are the fastest growing group in the world, and they experience a progressive loss of cognitive and physiological functions, leading to cognitive impairments and loss of functional motricity. The maintenance of physical activity and good physical fitness is important for functional independence. Information available about the physical activity and sedentary behaviour of people with cognitive impairment is scarce. Therefore, this study aims examine the physical fitness level in older persons with mild cognitive impairment. The cross-sectional study included older people $\geq 65$ years of age. 36 nursing home residents (mean 79.36 years) from both genders participated in this study; all participants have mild cognitive impairment according to the Mini Mental State Examination. Physical fitness was assessed using the Rikli \& Jones Test. Statistical analyses were conducted with the statistical software SPSS. Results show that physical fitness in a population of older persons with mild cognitive impairment is very poor, with severe consequences on functional independence. Therefore, promoting an active lifestyle among this population should be a crucial concern, to maintain motor abilities to perform daily tasks. Keywords: Mild cognitive impairment; Physical fitness; Aging; Rikli \& Jones Test.
\end{abstract}

Corresponding author. Universidade da Beira Interior, Departamento de Ciências do Desporto, 6200 Covilhã, Portugal. E-mail: cr.sport11@gmail.com

Supplementary Issue: Spring Conferences of Sports Science. International Seminar of Physical Education, Leisure and Health, 17-19 June 2019. Castelo Branco, Portugal.

JOURNAL OF HUMAN SPORT \& EXERCISE ISSN 1988-5202

(c) Faculty of Education. University of Alicante.

doi:10.14198/jhse.2019.14.Proc4.82 


\section{INTRODUCTION}

Physical fitness is closely related to health-related quality of life among older people with dementia (Hesseberg et al., 2016), since it is essential to carry out daily task, to participate social life and to live autonomously (Donnezan et al., 2018). Prevention of decline in physical fitness is important since it is related to older person's independence and wellbeing. However, aging brings chronic diseases, cognitive impairment and hospitalization which have direct impact on physical fitness and ability to stay active (Hesseberg et al., 2016). Cognitive impairment may be associated with lower levels of physical fitness and become a serious threat to older people's independence and quality of life (Bárrios et al., 2013; Hesseberg et al., 2016; Pettersson et al., 2005).

Therefore, the main objective of this study was to examine Physical fitness of individuals with mild cognitive impairment, in order to evaluate the need of a specific Physical activity program that improve their functional fitness status.

\section{MATERIAL AND METHODS}

\section{Participants}

This investigation is a cross-sectional study in individuals with mild cognitive impairment. A total of 36 nursing home residents (mean 79.36 years) from both genders participated in this study. Inclusion criteria: all participants have mild cognitive impairment according to the Mini Mental State Examination (Guerreiro et al., 1994). Exclusion criteria: clinical diagnosis of advanced dementia syndrome; uncontrolled hypertension (BP> $160 / 90 \mathrm{mmHg}$ ); frequent hypoglycaemia; severe congestive heart failure; acute myocardial infarction in the last year; severe anaemia (HB <8 g / dl); severe respiratory disease; severe osteoporosis; sensory deficit (vision / hearing) that disables collaboration in the physical exercise program; severe psychiatric disorders.

\section{Measures}

Evaluation of cognitive impairment was made according to the Mini Mental State Examination (Guerreiro, et al., 1994). Physical fitness was assessed using the Rikli \& Jones Test (Rikli \& Jones, 1999).

\section{Procedures}

Participants were completely free to participate in the study, after the presentation of researchers' affiliation, investigation goals and guaranty of total confidentiality. An informed consent was signed by participant and relative. All evaluation procedures were made by a qualified professional: Mini Mental State Examination by a physiologist and Physical fitness by a sport professional, $\mathrm{PhD}$ student.

\section{RESULTS}

The physical fitness of individuals evaluated is presented on Figure 1, revealing that the level of physical fitness of patients with mild cognitive impairment is mainly too week or week.

\section{DISCUSSION}

The results show that the level of physical fitness of patients with mild cognitive impairment is low, revealing deficits in motor performance that may affect their level of independence and their ability to perform daily tasks. Literature shows results similar findings (Chang et al., 2016; Hesseberg et al., 2016; Song et al., 2018). These results support the need for a program of functional physical activity in these individuals, which slows 
the deterioration of their motor abilities and allows an improvement in aerobic capacity, balance, strength and generic motor skills.

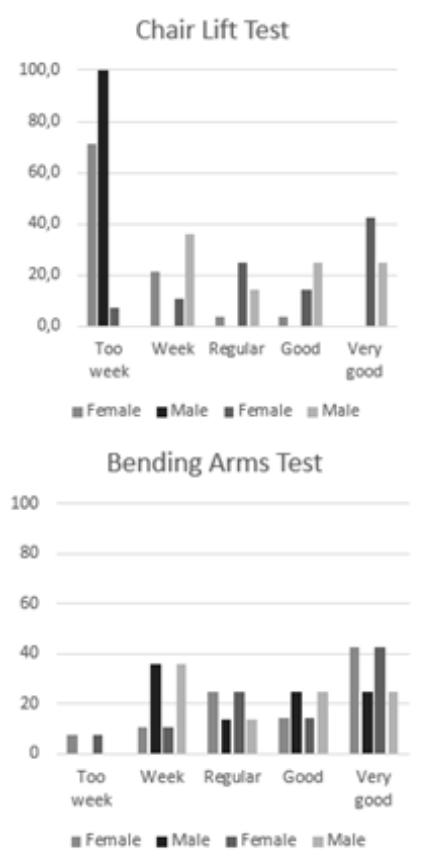

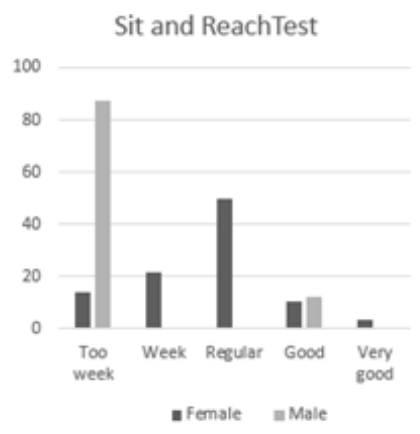

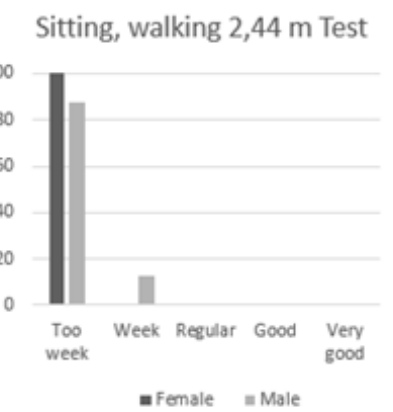

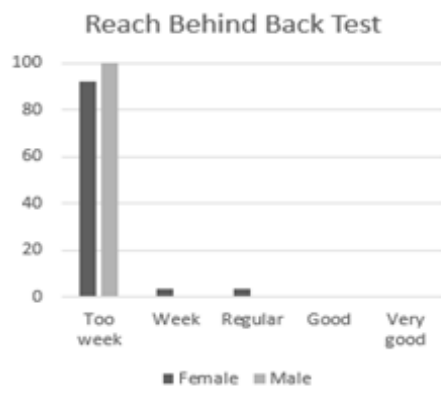

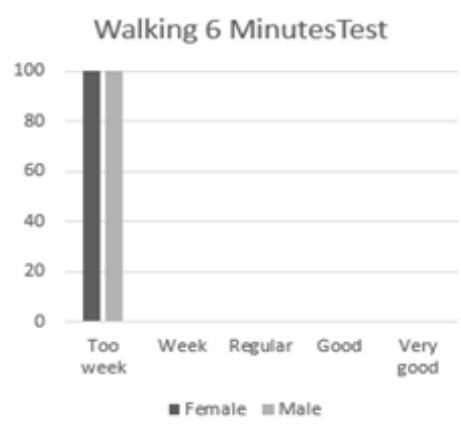

Figure 1. Physical fitness of individuals with mild cognition impairments, according to Rikli \& Jones Test

\section{CONCLUSIONS}

Based on these results, authors designed a specific physical activity program to individuals with mild cognitive impairment: MEMO_MOVE program.

\section{REFERENCES}

Bárrios, H., Narciso, S., Guerreiro, M., Maroco, J., Logsdon, R., \& de Mendonça, A. (2013). Quality of life in patients with mild cognitive impairment. Aging Ment Health, 17(3), 287-292. https://doi.org/10.1080/13607863.2012.747083

Chang, K. V., Hsu, T. H., Wu, W. T., Huang, K. C., \& Han, D. S. (2016). Association between sarcopenia and cognitive impairment: a systematic review and meta-analysis. J Am Med Dir Assoc., 17(12), 1164-e7. https://doi.org/10.1016/i.jamda.2016.09.013

Donnezan, L. C., Perrot, A., Belleville, S., Bloch, F., \& Kemoun, G. (2018). Effects of simultaneous aerobic and cognitive training on executive functions, cardiovascular fitness and functional abilities in older adults with mild cognitive impairment. Ment Health Phys Act., 15, 78-87. https://doi.org/10.1016/i.mhpa.2018.06.001

Guerreiro, M., Silva, A., Botelho, M., Leitão, O., Castro Caldas, A., \& Garcia, C. (1994). Adaptação à população portuguesa da tradução do Mini Mental State Examination (MMSE). Rev Port Neurol, 1(9), 9-10. 
Hesseberg, K., Bergland, A., Rydwik, E., \& Brovold, T. (2016). Physical fitness in older people recently diagnosed with cognitive impairment compared to older people recently discharged from hospital. Dement Geriatr Cogn Disord Extra, 6(3), 396-406. https://doi.org/10.1159/000447534

Pettersson, A. F., Olsson, E., \& Wahlund, L. O. (2005). Motor function in subjects with mild cognitive impairment and early Alzheimer's disease. Dement Geriatr Cogn Disord, 19(5-6), 299-304. https://doi.org/10.1159/000084555

Rikli, R. E., \& Jones, C. J. (1999). Development and validation of a functional fitness test for communityresiding older adults. J Aging Phys Act., 7(2), 129-161. https://doi.org/10.1123/japa.7.2.129

Song, D., Doris, S. F., Li, P. W., \& Lei, Y. (2018). The effectiveness of physical exercise on cognitive and psychological outcomes in individuals with mild cognitive impairment: A systematic review and metaanalysis. Int J Nurs Stud., 79, 155-164. https://doi.org/10.1016/j.jinurstu.2018.01.002

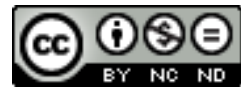

This work is licensed under a Attribution-NonCommercial-NoDerivatives 4.0 International (CC BY-NC-ND 4.0). 


\title{
The sports practice of karate in Portugal: Sociological analysis of the identities, ideologies, communities and cultures of the Portuguese karateka's (brown and black belts)
}

\author{
VÍTOR ROSA \\ CPES - Centro de Pesquisa e Estudos Sociais, ULHT, Portugal
}

\begin{abstract}
In the sociological analysis of the cultures of graduated karateka's, we intend to demonstrate that the mythical warrior traditions of Japan Samurai is present in a dominant way in karate globalization process, reflected in the training as an expression of Budô, constitutive by an eastern philosophy, an identity lifestyle, and private organizational involvements that states as a form of resistance to Western sports competition model, despite the existence of different uses and social provisions by practitioners. We set three hypotheses and construct an analysis model for its operation. We have used extensive methodology and the application of various techniques of social research: direct observation, participant observation, semi-directive interview and survey with a questionnaire. The universe was 244 practitioners of advanced karate (brown and black belts), at national level. Additionally, 31 interviews have been performed. The results show that there is an identity lifestyle among experienced practitioners of karate, although due to particular uses and social provisions, we have found some differentiation that presupposes our first hypothesis, but regardless of the styles adopted in practice, sex, age and education level. Keywords: Sport; Globalization; Martial arts; Karate; Culture.
\end{abstract}

Corresponding author. Universidade Lusófona de Lisboa, Campo Grande, 376, Edifício U, 1749-024 Lisboa, Portugal.

E-mail: vitor.rosa@ulusofona.pt

Supplementary Issue: Spring Conferences of Sports Science. International Seminar of Physical Education, Leisure and Health, 17-19 June 2019. Castelo Branco, Portugal.

JOURNAL OF HUMAN SPORT \& EXERCISE ISSN 1988-5202

(c) Faculty of Education. University of Alicante.

doi:10.14198/jhse.2019.14.Proc4.82 


\section{INTRODUCTION}

In the sociological analysis of the cultures of graduated karateka's, we intend to demonstrate that the mythical warrior traditions of Japan Samurai is present in a dominant way in karate globalization process, reflected in the training as an expression of Budô, constitutive by an eastern philosophy, an identity lifestyle, and private organizational involvements that states as a form of resistance to Western sports competition model, despite the existence of different uses and social provisions by practitioners.

\section{MATERIAL AND METHODS}

We set three hypotheses and construct an analysis model for its operation. We have used extensive methodology and the application of various techniques of social research: direct observation, participant observation, semi-directive interview and survey with a questionnaire.

\section{Participants}

The universe was 244 practitioners of advanced karate (brown and black belts), at national level. Additionally, in order to complete the information, 31 interviews have been performed.

\section{Measures}

In our model of analysis, we defined four dimensions ("Model of Disaggregated Analysis"), exposing a set of "classificatory concepts fit to measure", variables and indicators. In the first dimension, which we called "Social representations of karate" (D.1). In the second dimension, the "Lifestyles" (D.2). In the third, which we called "Cooperation / Demarcation" (D.3). Finally, in the fourth dimension, the "Profile of the karateka's" (D.4).

\section{Procedures}

As a first hypothesis, we assume that there is a style of identity life among experienced karate practitioners, even if, due to particular social uses and dispositions, there is some differentiation according to the style adopted in practice, regardless of gender, age and gender. schooling. In their analysis, we will analyse the involvement in karate (club membership and association), investment in karate in other sports, affinities with martial arts, identities and the relevance of karate in practitioners' lives, use of karate in the public space, values, gender and ethnic prejudices, food, health and well-being, the linguistic influence of Japanese, religion and the political-ideological spectrum. In the second hypothesis, we considered that the majority of experienced karate practitioners conceive of their practice as an expression of Budo, reproduced in a dominant way by the teaching agents of the sport through conventional fighting practices, images, symbols and values, regardless of style of karate, sex, age and schooling. In this hypothesis, the aim is to investigate the orientations of karate practice, the reasons for its practice, art, tradition and competition, techniques, learning and performances, images and symbols, the transmission of values and styles, authority and Physical violence. Finally, in the third hypothesis, we considered that the relations between experienced karate practitioners in the field of non-competition and sports competition have been characterized by tensions, resistance dynamics and conflicts, generating divisions among teaching agents, practice spaces or clubs and federative structure, regardless of karate style, gender, age and schooling. We will analyse cooperation and demarcation in karate, institutional relations, federation aspects and sports policy, sociability among karate, resistance and tensions in karate and conflicts. 


\section{Analysis}

We have made the analysis with the software SPSS: descriptive statistic, chi-square independence test, means, correlations, univariate analysis, comparing dichotomous variables, etc.

\section{RESULTS}

The results show that there is an identity lifestyle among experienced practitioners of karate, although due to particular uses and social provisions, we have found some differentiation that presupposes our first hypothesis, but regardless of the styles adopted in practice, sex, age and education level. The analysis of the information mobilized points out the existence of a character multidimensional of orientation and expression of Budô although conventional practical struggles, images, symbols and values, as it presupposes our second hypothesis, also regardless of karate styles, sex, age and education level. The data also point out that the relationships between the experience apprentices of karate of the non-competition and sport competition have been becoming characterized for tensions, resistance and conflicts dynamics, generators of scissions among teaching agents, practice spaces or clubs and federal structure, has affirmed by our third hypothesis, although the accomplished analysis allows us to conclude that the reading expressed from reality is reduced, and, this way, we can only partly affirm that the hypothesis is verified. This is affirmation of dynamics and interest and power within the karate field coexisting with various forms of cooperation, both contributing to the strengthening of the identity of the same.

\section{DISCUSSION}

Immersed in the specific historical, social and cultural contexts (East and West), karate is transformed and is in constant interaction and change. In this way, karate can be understood as a sport manifestation that gives expression to the intersection of cultures (Eastern and Western), in perpetual evolution, where it is possible to verify the strength lines, to grasp what is at stake and to understand its state gift.

\section{CONCLUSIONS}

To conclude, we may say that the Japanese samurai warrior tradition is present in the process of globalization of karate, translated into the concept of training as an expression of Budô. We could also affirm that, despite the different appreciation on the part of the practitioners of the uncompetitive and competitive side, this becomes an aggregator of an identity lifestyle based on Eastern values. Also, in spite of the diverse involvements in the clubs, associations and federation of the modality, there are dynamics of affirmation and resistance on the part of karateka of the non-sportive side facing the model of western sports competition, nevertheless, the axes of conflict found, reinforce the specific field of karate practices, since they provide it with an identity based on various forms of cooperation.

\section{REFERENCES}

Rosa, V. (2017). A prática desportiva do karaté em Portugal: análise sociológica sobre as identidades, ideologias, comunidades e culturas dos karatecas (cintos castanho e negro) portugueses. Tese de doutoramento. Lisboa: FEFD, ULHT.

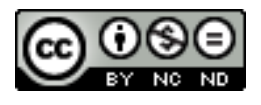

This work is licensed under a Attribution-NonCommercial-NoDerivatives 4.0 International (CC BY-NC-ND 4.0). 


\title{
Portuguese play report 2018: Children up to 10 years
}

\author{
RUI MENDES ${ }^{1}$, LARA NEVES ${ }^{2}$, FRANCISCO CAMPOS $^{3}$, ANA LOURENÇO ${ }^{4}$, MADALENA DIOGO 5 \\ ${ }^{1}$ Instituto Politécnico de Coimbra, Escola Superior de Educação - UNICID, I2A - ROBOCORP, CIDAF-UC, \\ Portugal \\ 2IPC-ESE, Mestrado em Jogo e Motricidade na Infância, Portugal \\ 3IPC: ESE - UNICID, I2A - ROBOCORP, Portugal \\ ${ }^{4}$ Instituto de Apoio à Criança, Sector da Actividade Lúdica, Portugal \\ ${ }^{5}$ Estrelas \& Ouriços, Portugal
}

\begin{abstract}
Spaces, social and family environments, standardized school educational context and possibilities of time for the child to play freely, have decreased in the last decades. This paper underline the main conclusions of "Portuguese Play Report 2018: children up to 10 years" developed by Mendes et al. (2019) to know and understand the importance of play for parents and also to know how children play in Portugal nowadays. 1466 parents (20-65 years, mean age 39 years old; female: $92.6 \%$ ) of children of both sexes, aged up to 10 years, resident in Portugal, including the Azores and Madeira, filled out an online questionnaire on the Google Forms digital platform. Using simple descriptive statistics analysis of data, only $6 \%$ of parents emphasize the effect of play on motor and physical development (6.0\%). Many participants (44.5\%) would like their children play more and, $69.7 \%$ highlight that the essential element for children to play more is their free time. Only $2.2 \%$ of children play in the street. These results suggest that it is relevant to implement public strategies to value play activity and implement conditions for children and families taking time to play. Keywords: Play; Child development; Physical activity; Leisure; Parents' engagement.
\end{abstract}

Corresponding author. Instituto Politécnico de Coimbra - Escola Superior de Educação; Rua Dom João III - Solum, 3030329 Coimbra, Portugal.

E-mail: rmendes@esec.pt

Supplementary Issue: Spring Conferences of Sports Science. International Seminar of Physical Education, Leisure and Health, 17-19 June 2019. Castelo Branco, Portugal.

JOURNAL OF HUMAN SPORT \& EXERCISE ISSN 1988-5202

(C) Faculty of Education. University of Alicante.

doi:10.14198/jhse.2019.14.Proc4.82 


\section{INTRODUCTION}

Play and physical activity games made by children by enjoyment and recreation, have undergone very significant changes in recent years in societies around the world: it reduces the number of spaces and possibilities for children to play freely. The goal of success, the intense rhythm of parents' lives, and the thought that play is not serious have dramatic consequences for children's healthy development (Neto \& Lopes, 2017; Dias \& Mendes, 2012). The relationship between the decrease in outdoor play and time of physical activity in obesity and, in terms of mental health, considering the emotional and behavioural benefits of playing the consequences of restraint of natural leisure activity and spontaneous development of the child in increasing childhood depression and anxiety (Goldstein, 2012; Miller \& Almon, 2009). The adult's attitude toward play influences the way children play around, especially their children (Whitebread, 2012). Our research (Mendes et al., 2019) aimed to know and understand the importance of playing for Portuguese parents and also to know how children play in Portugal nowadays.

\section{MATERIAL AND METHODS}

\section{Participants}

The study involved 1466 parents (20-65 years old, mean age 39 years, women: $92.6 \%$ ) of children of both sexes, aged up to 10 years, resident in Portugal, including the Azores and Madeira.

\section{Measures}

A questionnaire with 52 questions, the majority of multiple choice, previously validated by specialists.

\section{Procedures}

The questionnaire was filled out by participants on the Google Forms digital platform at https://goo.gl/forms/9tof1TTDkNYBtM3N2

\section{Analysis}

Data were analysed using simple descriptive statistics.

\section{RESULTS}

For many parents to play is important because it promotes the affective and emotional development of the child $(31.3 \%)$. The least valued items by the parents were the effect of playing on motor and physical development (6.0\%) and on the socialization of the child (1.8\%). Children play between 2 to 3 hours a day (25\%). Many participants (44.5\%) would like their children to play more. The majority $(69.7 \%)$ say that the essential element for children to play more is just their free time. It is at school that children play more $(53.8 \%)$. Only $2.2 \%$ of children play in the street. Most children receive many toys throughout the year (30.8\% more than 15 and $29.3 \%$ among 6 to 10 toys). The use of digital games, in tablets and smartphones, is common in $65.3 \%$ of children, with $21.6 \%$ of them already using their own devices. Most parents $(73.1 \%)$ report that children have already played with traditional Portuguese games during their playing time.

\section{DISCUSSION}

These results suggest that it's relevant to implement strategies to value play activity and its benefits. Improving time and spaces that can promote play and create the conditions for children to be able to experience risk safely (European Parliament, 2011). Taking time to play as a priority in family routines, 
promoting learning through play, or guiding the child without the temptation to dominate their decisions and types of play are important recommendations expressed in the most recent literature on this subject (Minnesota Children's Museum , 2016, IKEA, 2015 and 2017, LEGO, 2018, Real Play Coalition, 2019).

\section{CONCLUSIONS}

Trends about how children play in Portugal were identified. It's important to mobilize the population and train professionals for the importance of playing. It is also essential to implement strategies and public policies, as a social challenge of quality of life and well-being and educational and cultural purpose, to promote habits of free play for longer in public spaces and natural environments.

\section{REFERENCES}

Dias, G. \& Mendes, R. (2012). As potencialidades do jogo no desenvolvimento da criança. EFDeportes.com, 17, 173, Outubro.

European Parliament (2011). Resolution on early years learning in the european union. Brussel: EU.

Goldstein, J. (2012). Play in Children's Development, Health and Well-being. Brussel: Toy Industries of Europe.

IKEA (2015). The play report 2015: say yes to play!. IKEA.

IKEA (2017). Play report 2017: a spark of play everyday. IKEA.

LEGO Group (2018). Play well report 2018. LEGO.

Miller, E. \& Almon, J. (2009). Crisis in the kindergarten: why children need to play in school. MD: Alliance for Childhood.

Minnesota Children's Museum (2016). Play under pressure: surveying parents about children and the state of play. Minessota.

Mendes, R., Neves, L., Lourenço, A. \& Diogo, M. (2019, Abril). Portugal a brincar: relatório do brincar de crianças portuguesas até aos 10 anos - 2018. $1^{\text {a }}$ Conferência Estrelas \& Ouriços: Como brincam hoje as crianças portuguesas!. Cascais: Estrelas e Ouriços.

Neto, C. \& Lopes, F. (2017). Brincar em cascais. Cascais: CERCICA.

Real Play Coalition (2019). Value of play report. National Geographic, LEGO, UNILEVER \& IKEA.

Whitebread, D (2012). The importance of play: A report on the value of children's play with a series of policy recommendations. Brussel: Toy Industries of Europe.

\section{@ $\odot \Theta \Theta$}

This work is licensed under a Attribution-NonCommercial-NoDerivatives 4.0 International (CC BY-NC-ND 4.0). 


\title{
Prevalence of Methicillin - Resistant Staphylococcus Aureus in students of higher education
}

\author{
FRANCISCO RODRIGUES ${ }^{1} \triangle$, PATRICIA COELHO ${ }^{1}$, SÓNIA MATEUS ${ }^{2}$ \\ ${ }^{1}$ Qualidade de Vida no Mundo Rural (QRural), Sport, Health \& Exercise Unit (SHERU), Instituto Politécnico \\ de Castelo Branco, Portugal \\ ${ }^{2}$ Hospital Espirito Santos de Évora, Sport, Health \& Exercise Unit (SHERU), Instituto Politécnico de Castelo \\ Branco, Portugal
}

\begin{abstract}
Introduction: $S$ aureus is a bacterium that is part of the normal flora of many Human Beings, and in its most aggressive variant (MRSA) it can constitute a potential risk for the bearer and the People of their relationship. Objective: To evaluate the prevalence rate of $S$ aureus and MRSA; assess the main risk factors; evaluate the students' knowledge about the bacterium. Methods: Nasal zaragotoa harvest, with posterior laboratory analysis for bacterial identification and questionnaire. Results: In our study we had $35.23 \%$ positive cases for $S$ aureus. In the MRSA prevalence analysis we have $5.7 \%$ of positive cases. We found a higher prevalence in the men, with $41.7 \%$ positive cases, compared with the women (30.8\%). Concerning the MRSA incidence, this is higher in women (5.8\%) than in men (5.6\%). We can also see that the traineeship has an important role in the contamination. Conclusion: We concluded a high percentage of $S$ aureus carriers and an expected percentage of MRSA carriers. Keywords: S. aureus; MRSA; Nasal carrier.
\end{abstract}

Corresponding author. Escola Superior de Saúde Dr Lopes Dias, Portugal.

E-mail: franciscobrodrigues@ipcb.pt

Supplementary Issue: Spring Conferences of Sports Science. International Seminar of Physical Education, Leisure and Health, 17-19 June 2019. Castelo Branco, Portugal.

JOURNAL OF HUMAN SPORT \& EXERCISE ISSN 1988-5202

(c) Faculty of Education. University of Alicante.

doi:10.14198/jhse.2019.14.Proc4.82 


\section{INTRODUCTION}

Staphylococcus aureus (S.aureus) is a gram positive coccus, facultative aerobic, catalase and coagulase positive (Larkin et al. 2009). S. aureus is a major responsible for nosocomial infections (Freeman-Cook and Freeman-Cook, 2006). Can cause numerous diseases in humans: grows and damages the tissue at the site of infection but also release toxins into the bloodstream that can cause diseases in all of the body (Lencastre et al. 2007). Before the introduction of penicillin in the treatment of infections with S. aureus, the mortality rate was $80 \%$. However, shortly after starting treatment with this antibiotic, in the early forties, appeared the first strains resistant to penicillin. In 1960 about $80 \%$ of S. aureus were resistant to penicillin. In 1961 came the first strains resistant to these antibiotics due to the acquisition of the mecA gene (Lencastre et al. 2007). Currently it is estimated that every year in the US, approximately 400,000 hospital patients are infected by S. aureus, (100,000 die from complications) (Gomes and Westh 2006). With this study we attempted to measure the percentage of people with nasal carriage of $\mathrm{S}$. aureus in the student population of Higher Education of Health (these people are within a few years to provide care for hospitalized patients). We intend not only to know the percentage of nasal carriers but also see if the contact with hospital (through training) or specific formation on the proper way to wash hands or on the bacteria in question can contribute to the prevention of infection (DeLeo and Chambers, 2009).

\section{MATERIAL AND METHODS}

The nasal samples were collected with sterile cotton swabs. These samples have been preserved at ambient temperature until the inoculation in the culture medium. The culture medium used in this study was the Mannitol Salt Agar. After collecting the swab, it was readily inoculated in the culture medium, which was at room temperature and was placed in the oven at $37^{\circ} \mathrm{C}$ for $24 \mathrm{~h}$. After 24 hours we looked for characteristic colonies of S. aureus in the MSA. To confirm we made the catalase (ID Color Catalase, BioMerieux) and coagulase (Slidex Staph Plus, BioMerieux) tests. Colonies that prove to be positive for S. aureus were then transferred to the chromID MRSA medium (BioMerieux). The cases presented positive in the chromID MRSA were then sent to the department of clinical pathology of a Hospital, to confirm the results through automated antibiogram (Vitek @ 2). Knowledge about S. aureus infections and the theoretical formation on Hand Washing Protocols: Each student responded to an anonymous survey with which it was intended to examine three things: history and recent contact with the hospital environment, theoretical knowledge of the $S$. aureus infections and knowledge and application washing hands protocols.

\section{Participants}

For this study 88 students were randomly chosen from the Institution of Higher Education in the Health Area. Of these students 52 were female and 36 were male.

\section{Analysis}

Use the Statistical Package software for the Social Sciences (IBMSPSS v22). A descriptive analysis of all variables was performed. Non-parametric Chi-Square tests and Mann-Whitney test were used to relate.

\section{RESULTS}

In our study we had $35.23 \%$ positive cases and $64.77 \%$ negative cases. In the MRSA prevalence analysis we have $5.7 \%$ of positive cases. The other $94.3 \%$ were negative for MRSA nasal carriage. We found a higher prevalence in the men, with $41.7 \%$ positive cases, compared with the women $30.8 \%$. Concerning the MRSA incidence, this is higher in women (5.8\%) than in men (5.6\%). We can also see that the traineeship has an 
important role as the students that had have a traineeship in the past six months present a higher rate of $S$. aureus and MRSA against the students that haven't had traineeship. The same thing can be observer in the rows concerning the knowledge about infections caused by $\mathrm{S}$. aureus, where the incidence is higher in the students that had no knowledge in about this kind of infections. About the rows that concern the students that had have theoretical formation about Hand Washing Protocols, we have an higher incidence in the students with knowledge about this kind of protocols.

\section{DISCUSSION}

The results obtained from our study are within the expected values. We can see a greater focus on students who have had contact with the hospital, most likely due to the high percentage of existing carriers on these sites. According to a study (Mulqueen and Cafferty, 2007), a prevalence of $7.7 \%$ for MRSA was found in General Practitioners in Ireland. This proves that there is a high risk of infection in hospitals, not only in patients but also healthcare providers. In other article (Thompson, 2004), in which he studied the prevalence of MRSA in an intensive care unit we can see that the 1242 patients admitted to this unit, 68 of them have acquired MRSA during the time of internment, which just goes to show that this exist in hospitals, especially in a unit that should be as aseptic as possible. In the study (Lietzau and Sturmer, 2004) in which it sought to verify the percentage of patients who came to consult with GPs, found a prevalence of S. aureus by $24.3 \%$ and $0.7 \%$ for MRSA. This shows that not only contact with the hospitals that can make students acquire $\mathrm{S}$. aureus but also in contact with patients. But not all students had contact with the hospital and therefore the results presented are due to S. aureus in community acquired. In other study (Miller and Cook, 2009), which were tested inhabitants of northern Manhattan randomly chosen, the results for S. aureus and $25 \%$ of MRSA from $0.4 \%$. This demonstrates that this bacterium does not exist only in hospitals but also in the community. We can also see with our study that students with theoretical training in bacterial infections related to $\mathrm{S}$. aureus are less susceptible to contamination by these bacteria. As for students who have specific training on the proper way to wash their hands, that this training had a higher prevalence, perhaps due to failure to apply this knowledge.

\section{CONCLUSIONS}

We can conclude that there is a high percentage of nasal carriers of S. aureus in this Institution. We could also verify that the internship at a hospital is a risk factor with regards to contamination. We cannot say that this is the only factor, since there are others who may be influential and weren't studied.

\section{REFERENCES}

DeLeo, F. R. and H. F. Chambers (2009). "Reemergence of antibiotic-resistant Staphylococcus aureus in the genomics era." J Clin Invest 119(9): 2464-74. https://doi.org/10.1172/jci38226

Deurenberg, R. H. and E. E. Stobberingh (2009). "The molecular evolution of hospital- and communityassociated methicillin-resistant Staphylococcus aureus." Curr Mol Med 9(2): 100-15. https://doi.org/10.2174/156652409787581637

Gomes, A. R., H. Westh, et al. (2006). "Origins and evolution of methicillin-resistant Staphylococcus aureus clonal lineages." Antimicrob Agents Chemother 50(10): 3237-44. https://doi.org/10.1128/aac.00521-06

Freeman-Cook, K. Freeman-Cook, Deadly (2006) Diseases and Epidemics - Staphylococcus aureus Infections, Chelsea House Publishers, Philadelphia. 
Larkin, E. A., R. J. Carman, (2009). "Staphylococcus aureus: the toxic presence of a pathogen extraordinaire." Curr Med Chem 16(30): 4003-19. https://doi.org/10.2174/092986709789352321

Lencastre, H., D. Oliveira, et al. (2007). "Antibiotic resistant Staphylococcus aureus: a paradigm of adaptive power." Curr Opin Microbiol 10(5): 428-35.

Lietzau, S., T. Sturmer (2004). "Prevalence and determinants of nasal colonization with antibioticresistant Staphylococcus aureus among unselected patients attending general practitioners in Germany." Epidemiol Infect 132(4): 655-62. https://doi.org/10.1017/sh0950268804002341

Miller, M., H. A. Cook (2009). "Staphylococcus aureus in the community: colonization versus infection." PLoS One 4(8): e6708.

Mulqueen, J., F. Cafferty, (2007). "Nasal carriage of meticillin-resistant Staphylococcus aureus in GPs in the West of Ireland." Br J Gen Pract 57(543).

Thompson, D. S. (2004). "Methicillin-resistant Staphylococcus aureus in a general intensive care unit." J R Soc Med 97(11): 521-6. https://doi.org/10.1258/jrsm.97.11.521 


\title{
Risk factors and arterial hypertension
}

\author{
FRANCISCO RODRIGUES ${ }^{1} \triangle$, PATRICIA COELHO ${ }^{1}$, SÓNIA MATEUS ${ }^{2}$ \\ ${ }^{1}$ Qualidade de Vida no Mundo Rural (QRural), Sport, Health \& Exercise Unit (SHERU), Instituto Politécnico \\ de Castelo Branco, Portugal \\ ${ }^{2}$ Hospital Espirito Santos de Évora, Sport, Health \& Exercise Unit (SHERU), Instituto Politécnico de Castelo \\ Branco, Portugal
}

\begin{abstract}
Cerebrovascular risk factors are increasingly present in modern society. These include a range of pathologies in which some of them are already beginning to be considered a serious public health problem. The study of these factors and the impact they may have on the morbimortality of the population should be a source of high concern for health authorities. The main objective was to understand the cerebrovascular and cardiovascular risk factors present in the study population and their relationship with the presence of arterial hypertension. This cross-sectional, analytical and observational study was carried out in all municipalities in the district of Castelo Branco, in the interior region of Portugal. A total of 11316 individuals were studied, of which $55.6 \%$ belonged to the female gender $(n=6292)$ and $44.4 \%$ to the male $(n=5024)$. Of these $75.9 \%$ reported being sedentary, $63.5 \%$ were overweight and obese, $54.1 \%$ said they had a family history of hypertension and $41 \%$ had knowledge of having hypercholesterolemia. The results obtained demonstrate that there is a high prevalence of cerebrovascular and cardiovascular risk factors. Keywords: Prevalence; Risk factors; Arterial hypertension.
\end{abstract}

Corresponding author. Escola Superior de Saúde Dr Lopes Dias, Portugal.

E-mail: franciscobrodrigues@ipcb.pt

Supplementary Issue: Spring Conferences of Sports Science. International Seminar of Physical Education, Leisure and Health, 17-19 June 2019. Castelo Branco, Portugal.

JOURNAL OF HUMAN SPORT \& EXERCISE ISSN 1988-5202

(c) Faculty of Education. University of Alicante.

doi:10.14198/jhse.2019.14.Proc4.82 


\section{INTRODUCTION}

Hypertension is the most important risk factor for cerebrovascular and cerebrovascular diseases, especially in relation to the presence of acute myocardial infarction (AMI) and cerebrovascular accident (CVA), which in turn are important causes of morbidity and mortality around the world (Uva and Vitorino, 2014), (Perdigão and Rocha, 2011). In Portugal, about 40 thousand individuals die each year due to these two pathologies, in which about 20 thousand deaths / year occur due to stroke and 10 thousand deaths / year by EAM's (Macedo and Lima, 2007).

There are, however, other risk factors that may influence blood pressure values and consequently lead to the development of hypertension $(\mathrm{AH})$. Among the most commonly associated with this pathology, sedentary lifestyle, obesity, smoking, hypercholesterolemia, and Diabetes Mellitus (DM) (Ribeiro and Faria, 2013) and (Cordinhã and Paúl, 2009).

\section{MATERIAL AND METHODS}

In the collection of the sample, and according to defined inclusion criteria, individuals 18 years of age or older residing in the different municipalities, who were willing to voluntarily participate in the study and signed informed consent were considered.

\section{Participants}

After all the collected data were collected, a total of 11316 individuals were obtained, in which $55.6 \%$ were female $(n=6292)$ and $44.4 \%$ were male $(n=5024)$, aged 18 and 101 years, and an average of 58.12 , with a standard deviation of 17.96 years.

\section{Measures}

For the BMI study, the individuals were grouped according to the classification of the World Health Organization (WHO): having low weight $<18.5 \mathrm{~kg} / \mathrm{m} 2$; normal weight was $18.5-24.9 \mathrm{~kg} / \mathrm{m} 2$; excess weight is $25-29.9 \mathrm{~kg} / \mathrm{m}$ 2; and obesity when $>30 \mathrm{~kg} / \mathrm{m} 2$ (Mancia and Fagard, 2013).

\section{Procedures}

A questionnaire was used that was properly tested before being applied by the investigators. All questionnaires were answered in person.

\section{Analysis}

The collected data were inserted, analysed and treated with the statistical analysis program SPSS $®$ (Statistical Product and Service Solution) version 22.0. A simple descriptive analysis was used to characterize the sample and distribute the variables, and the absolute $(n)$ and relative (\%) frequencies were calculated for the qualitative variables. As for the quantitative variables, the mean, standard deviation and minimum and maximum values were calculated. A $p \leq 0.05$ and a $95 \%$ confidence interval were established as a criterion of statistical significance.

\section{RESULTS}

In the analysis of the risk factors studied by the questionnaire we found that the most prevalent were sedentary lifestyle, body mass index (> $25 \mathrm{~kg} / \mathrm{m} 2)$, family history of hypertension and hypercholesterolemia, with $75.9 \%, 63.5 \%, 54,1 \%$ and $41.0 \%$, respectively. It was also verified, in the course of this analysis, that 
$25.7 \%$ of the respondents had smoking habits and $16.0 \%$ of the individuals studied had Diabetes Mellitus. In the analysis of the answers given, it was also observed that $18.4 \%$ of the respondents reported having cerebrovascular and cerebrovascular diseases. Of these, the majority responded to the presence of Acute Coronary Syndrome (38.5\%); $28.4 \%$ reported having rhythm disturbances; $14.6 \%$ said they had a stroke and $8.6 \%$ had valvulopathies.

\section{DISCUSSION}

The study of cerebrovascular risk factors brings us important information about the health status of the general population. This type of investigation allows us to work with the population in a way that makes them aware of the risks inherent in their presence. In this study, when we analysed risk factors that could be associated with arterial hypertension, we found that the most prevalent were sedentary lifestyle, excess weight and obesity, the presence of a family history of hypertension and hypercholesterolemia, all statistically related to the presence of HTA with a $p<0.001$, which is in line with the results of the AMALIA study, which presents as the most prevalent risk factor the sedentary lifestyle with $65.3 \%$. In the relationship between the data we found with the AMALIA study, we found that hypercholesterolemia had a significantly lower percentage (19.7\%) (Perdigão and Rocha, 2011).

We also analysed the prevalence of smoking habits, cardiovascular diseases and DM, which presented, respectively, prevalence's of $25.7 \%, 18.4 \%$ and $16.0 \%$. Regarding the $18.4 \%$ of cerebrovascular and cerebrovascular diseases, $38.5 \%$ reported a history of acute coronary syndrome and a $14.6 \%$ history of stroke. In order to verify if there was a relation between these risk factors and the presence of hypertension, the chi-square test was performed, and it was verified that there was a high significance in all cases ( $p$ $<0.001)$. In the AMALIA study regarding DM and smoking, the prevalence was very low $(8.9 \%$ and $16.3 \%)$ (Perdigão and Rocha, 2011). In the study by Gus and Zaslavsky (2004), smoking had a higher prevalence to our study, namely, $34.0 \%$.

This study revealed results that confirm the high prevalence of cerebrovascular and cardiovascular risk factors in the population of Beira Baixa, it is evident that it is necessary to have alert and awareness measures for populations to control cerebrovascular and cardiovascular risk factors.

\section{CONCLUSIONS}

It is important to study the populations in order to assess the best means of communication and alert them.

\section{REFERENCES}

Cordinhã, A,C, Paúl, A. Fernandes, L. (2009). Obesidade Infantil e Hipertensão Arterial - a Realidade de uma População Pré-escolar. Acta Pediátrica Portuguesa. 40, 145-9.

Gomes, A.T. Coelho, P. Pereira, A. (2012). Prevalência de Hipertensão Arterial na População Adulta do Concelho da Covilhã. Revista Portuguesa de Hipertensão e Risco Cardiovascular. 32, 4-14. https://doi.org/10.11606/d.6.2010.tde-27012011-141644

Gus, I. Zaslavsky, C. Medinam C. Harzheim, E. (2004). Prevalência, Reconhecimento e Controle da Hipertensão Arterial Sistêmica no Estado do Rio Grande do Sul. Arquivos Brasileiros de Cardiologia. 83, 5, 424-8. https://doi.org/10.1590/s0066-782x2004001700009 
Macedo, M.E. Lima, M.J. Silva, A.O. Alcântara, P. Ramalhinho, V. Carmona, J. (2007). Prevalência, Conhecimento, Tratamento e Controlo da Hipertensão em Portugal. Estudo PAP. Revista Portuguesa de Cardiologia. 26, 1, 21-39.

Perdigão, C. Rocha, E. Duarte, J.S. Santos,.A. Macedo, A. (2011). Prevalência, Caracterização e Distribuição dos Principais Fatores de Risco Cardiovasculares em Portugal. Uma Análise do Estudo AMÁLIA. Revista Portuguesa de Cardiologia. 30, 4, 393-432.

Ribeiro, A.S. Faria, R.J. (2013). Prevalência de Factores de Risco Cardiovasculares e Lesão de OrgãoAlvo em Doentes com Hipertensão Arterial. Revista Portuguesa de Hipertensão e Risco Cardiovascular. 33, 4-7. https://doi.org/10.1016/j.repc.2018.09.011

Uva, M. Victorino, P. Roquette, R. Machado, A. Dias, C. (2014). Investigação epidemiológica sobre prevalência e incidência de hipertensão arterial na população portuguesa - uma revisão de âmbito. Revista Portuguesa de Cardiologia. 33, 7-8, 451-63. https://doi.org/10.1016/..repc.2014.02.012 


\title{
The importance of the electrocardiogram in the competitive pre-period
}

\author{
PATRICIA COELHO 14 , FRANCISCO RODRIGUES ${ }^{1}$, SÓNIA MATEUS ${ }^{2}$ \\ ${ }^{1}$ Qualidade de Vida no Mundo Rural (QRural), Sport, Health \& Exercise Unit (SHERU), Instituto Politécnico \\ de Castelo Branco, Portugal \\ ${ }^{2}$ Hospital Espirito Santos de Évora, Sport, Health \& Exercise Unit (SHERU), Instituto Politécnico de Castelo \\ Branco, Portugal
}

\begin{abstract}
The electrocardiogram is a safe and practical exam that allows the diagnosis of subclinical pathologies in asymptomatic athletes. In order to reduce false positives, it is of the utmost importance that this analysis be performed based on criteria specific to athletes. To analyse electrocardiograms according to the criteria of Seattle performed to athletes in the pre-competitive phase, in two consecutive years. A longitudinal observational study was performed in 14 athletes from different sport modalities who underwent a precompetitive evaluation in two consecutive years. The average age of the athletes was $24.5 \pm 4.9$ years, of which 13 were male $(92.9 \%)$ and 1 were female $(7.1 \%)$. According to the Seattle Criteria, electrocardiographic changes were recorded in 10 individuals $(71.4 \%), 6$ of which were considered physiological, 2 were borderline and 2 were pathological. In the relation between the two years there was an increase of electrocardiographic alterations. Most of the alterations found were classified as physiological according to the Seattle Criteria, although they could be considered pathological following non-specific criteria for athletes. The electrocardiographic analysis of these athletes allowed to track two athletes with alterations considered pathological according to the criteria of Seattle. Keywords: Electrocardiogram; Athletes; Seattle Criteria.
\end{abstract}

Corresponding author. Rua Prof. Sebastião Antonio Morão Correia L133 Rc dto, Portugal.

E-mail: patriciamcoelho@gmail.com

Supplementary Issue: Spring Conferences of Sports Science. International Seminar of Physical Education, Leisure and Health, 17-19 June 2019. Castelo Branco, Portugal.

JOURNAL OF HUMAN SPORT \& EXERCISE ISSN 1988-5202

(c) Faculty of Education. University of Alicante.

doi:10.14198/jhse.2019.14.Proc4.82 


\section{INTRODUCTION}

Regular and intense practice can lead to physiological cardiac changes, both at the structural and functional and electrical levels. The electrocardiogram (EKG) stands out as an increase in the ability to identify silent cardiac pathologies in asymptomatic athletes, since it is an examination that allows the detection of about 70 to $90 \%$ of the cases of hypertrophic cardiomyopathy, sometimes even before thickening of the ventricular wall and the earlier the diagnosis is made, the greater the possibility of effective preventive and therapeutic interventions (Prutkin and Drezner, 2017).

\section{MATERIAL AND METHODS}

This is a longitudinal observational study, in which the EKGs of young federated athletes were analysed during the pre-competitive phase. Each athlete, before completing the examination, followed the procedures of the laboratory, for which he completed a questionnaire regarding sports, personal and family history of heart disease, smoking and alcoholic habits. For the examinations and questionnaires to be used in this study, the researchers obtained the informed consent of each athlete.

\section{Participants}

The sample was selected according to the inclusion criteria; age $>18$ years, to be federated, to practice sports for at least 5 years and to have performed an electrocardiogram in two consecutive years in the ECG laboratory where the sample was collected.

In this way, 14 individuals were selected, of which $13(92.9 \%)$ were male and $1(7.1 \%)$ were females aged between 21 and 28 years and a mean of $24.5 \pm 4.95$ years.

\section{Measures}

The electrocardiograms were performed using the Schiller AT 101 equipment. For the interpretation of the exams, the conventional recommendations and the Seattle Criteria (Dores and Freitas, 2014) were used in order to properly classify the changes in athletes.

\section{Procedures}

For measurement of the waves and intervals of each EKG, a single measurement standard was followed. The electrical axis measured on the QRS in the DI and DII leads, the heart rate calculated by the 1500 method, the P wave lengths and amplitudes, PQ, QT interval and QTc calculated on the DII derivation of all electrocardiograms. The amplitude and duration of the QRS were measured in the leads where they were larger. We also collected the answers to the questionnaire, with a descriptive analysis.

\section{Analysis}

All collected data were entered, analysed and treated statistically through the software Statistical Package for the Social Sciences (IBM SPSS Statistics $®$ version 22).

The chi-square or Fisher's exact test was used for the relation between nominal variables. In the correlation between ordinal variables, the Kendall Tau $b$ test was used and to evaluate differences in the classification of the electrocardiographic alterations according to the different Wilcoxon test criteria. 


\section{RESULTS}

In the comparison between the two years it was observed that there was an increase in electrocardiographic changes between year 1 and year 2 by conventional EKG analysis criteria (57\% vs $64.3 \%$ ). There was an increase in cases with axis deviation, branch blocks and hemiblock marks between the two years. Most of the alterations found were considered physiological according to the criteria of Seattle. However, pathological changes were found in 2 athletes and in 2 other borderline patients.

\section{DISCUSSION}

The athletes participating in this study had an average of 9.64 years of sports practice and trained on average about 7 hours per week. According to the World Health Organization (WHO), adults are advised to take at least 150 minutes of moderate-intensity exercise a week. Within these time limits, individuals generally do not experience any side effects, and cardiac adaptations are often not classified as modifications observed in high-performance athletes, whether professional or amateur (Nobrega, 2013). According to the study by Ghorayeb and Giuseppe (2005), sinus bradycardia is characterized by cardiac frequencies lower than $60 \mathrm{bpm}$, which is present in $80 \%$ of highly trained athletes, and in this type of athletes normal heart rates up to $30 \mathrm{bpm}$ were considered. On average, the athletes did not present bradycardia at rest with the minimum value recorded in year 1 of $47 \mathrm{bpm}$ and year 2 of $45 \mathrm{bpm}$.

In year 1 our results show that two athletes presented deviation of the cardiac axis and in year 2 they suggest 2 new cases of axis deviation. Stein and Silveira (2011) report that a slight deviation of the axis to the right or left in athletes should not generate future research, unless there is a history of pulmonary disease or hypertension, respectively. According to Seattle's criteria (Dores and Freitas, 2014), the left axis deviation and the right deviation should be classified as borderline, so if isolated individuals do not need further evaluation, however, in the presence of two or more changes, they require a more in-depth study of the athlete. In our study, two of the studied athletes who presented axillary changes also presented other alterations. In this sense, these athletes were referred to the Sports Medicine.

From year 1 to year 2, although there have been some changes in the electrocardiographic patterns of the athletes, in general there was no worsening of the changes, and there was even a regression of the T wave inversion, which may be related to the persistence of juvenile pattern.

In the present study, EKG analysis was performed according to conventional criteria for electrocardiographic analysis, not specific for athletes, and according to the criteria of Seattle (Dores and Freitas, 2014), in order to adjust the classification of the changes and, together, to demonstrate the relevance of knowing the specific criteria for athletes in order to avoid an incorrect diagnosis and increase of false positives.

\section{CONCLUSIONS}

The inclusion of the ECG in the pre-competitive evaluation is essential to rule out the presence of pathological changes in athletes.

\section{REFERENCES}

Dores, H. Freitas, A. Mendes, M. (2014). Interpretação do eletrocardiograma do atleta: os 'Critérios de Seattle. Rev. Medicina Desportiva, 2, 5, 11-15. 
Ghorayeb, N. Dioguardi, G. S. Daher, D. J. Jardim, C. A. Baptista, C. A. Batlouni, M. (2005). O coração, o esporte, e o exercício físico. Rev Soc Cardiol. 15, 3, 97-102.

Lejderman, B. Sodré, E. G. S. Castro, I. Zimerman, L. I. Pimentel, M. (2013). Diretriz em Cardiologia do Esporte e do Exercício da Sociedade Brasileira de Cardiologia e da Sociedade Brasileira de Medicina do Esporte. 100, 2, 1-41. https://doi.org/10.5935/abc.2013s002

Prutkin, J.M. Drezner, J.A. (2017). Training and Experience Matter. Circulation: Cardiovascular Quality and Outcomes, 10, 8, 1-3.

Stein, R. Silveira, A. D. (2011). O eletrocardiograma do atleta. Revista da Sociedade de Cardiologia do Estado do Rio Grande do Sul. 21, 1-9. https://doi.org/10.29381/0103-8559/2019290182-7 


\title{
Excess weight and obesity in a region of the interior of Portugal
}

\author{
PATRICIA COELHO 14 , FRANCISCO RODRIGUES ${ }^{1}$, SÓNIA MATEUS ${ }^{2}$ \\ ${ }^{1}$ Qualidade de Vida no Mundo Rural (QRural), Sport, Health \& Exercise Unit (SHERU), Instituto Politécnico \\ de Castelo Branco, Portugal \\ ${ }^{2}$ Hospital Espirito Santos de Évora, Sport, Health \& Exercise Unit (SHERU), Instituto Politécnico de Castelo \\ Branco, Portugal
}

\begin{abstract}
Obesity is a serious public health problem that has gained great expression in Portugal. Through several studies it has been verified that there are more and more individuals with excess and weight and obesity and that this risk factor begins to have an increasing expression at younger ages. Our study aims to determine the prevalence of overweight and obesity in the adult population of the district of Castelo Branco through the collection of data in each county. It is a cross-sectional, analytical and observational study whose sample collection took place in all municipalities in the district of Castelo Branco, totalling a sample of 11316 individuals, of whom $55.6 \%$ belonged to the female gender $(n=6292)$ and $44,4 \%$ to males $(n=5024)$, with ages between 18 and 101 years, mean of 58.12 and standard deviation of 17.96 years. Of the total number of subjects in the study sample, $44.7 \%$ were overweight, $18.8 \%$ were obese, $0.9 \%$ were underweight, while $35.6 \%$ were within their normal range. The mean body mass index (BMI) was $26.62 \mathrm{~kg} / \mathrm{m} 2$, with a standard deviation of $4,11 \mathrm{~kg} / \mathrm{m} 2$, where the minimum BMI was 15.57 and the maximum was $50.78 \mathrm{~kg} / \mathrm{m} 2$. The results show that there is a high prevalence of overweight and obesity in the adult population of the region. Keywords: Prevalence; Obesity; Body mass index.
\end{abstract}

Corresponding author. Rua Prof. Sebastião Antonio Morão Correia L133 Rc dto, Portugal.

E-mail: patriciamcoelho@gmail.com

Supplementary Issue: Spring Conferences of Sports Science. International Seminar of Physical Education, Leisure and Health, 17-19 June 2019. Castelo Branco, Portugal.

JOURNAL OF HUMAN SPORT \& EXERCISE ISSN 1988-5202

(c) Faculty of Education. University of Alicante.

doi:10.14198/jhse.2019.14.Proc4.82 


\section{INTRODUCTION}

Obesity is a pathology of multifactorial origin, which tends to be increasingly present in modern societies, thus becoming an important marker of risk for cerebro-cerebrovascular pathologies. Considering the high morbimortality rate in Portugal and in the world, it is necessary to study the prevalence of cerebrovascular and cerebrovascular risk factors in order to be able to act in their re-education and prevention.

\section{MATERIAL AND METHODS}

The collection of the sample was carried out by the researchers in all the municipalities of the district of Castelo Branco according to inclusion criteria defined for the investigation. Individuals who agreed to participate in the study responded to a questionnaire that had been previously tested and signed an informed consent. This research was approved by an Ethics Committee.

\section{Participants}

After all the collected data were collected, a total of 11316 individuals were obtained, in which $55.6 \%$ were female $(n=6292)$ and $44.4 \%$ were male $(n=5024)$, aged 18 and 101 years, and an average of 58.12, with a standard deviation of 17.96 years.

\section{Measures}

For the BMl study, the individuals were grouped according to the classification of the World Health Organization (WHO): having low weight $<18.5 \mathrm{~kg} / \mathrm{m} 2$; normal weight was $18.5-24.9 \mathrm{~kg} / \mathrm{m} 2$; excess weight is $25-29.9 \mathrm{~kg} / \mathrm{m} 2$; and obesity when $>30 \mathrm{~kg} / \mathrm{m} 2$ (Mancia and Fagard, 2013).

\section{Procedures}

To determine the BMI of each individual, the weight was measured by means of a calibrated digital scale and the height confirmed by the official Portuguese identification card.

\section{Analysis}

The collected data were inserted, analysed and treated with the statistical analysis program SPSS $§$ (Statistical Product and Service Solution) version 22.0.

\section{RESULTS}

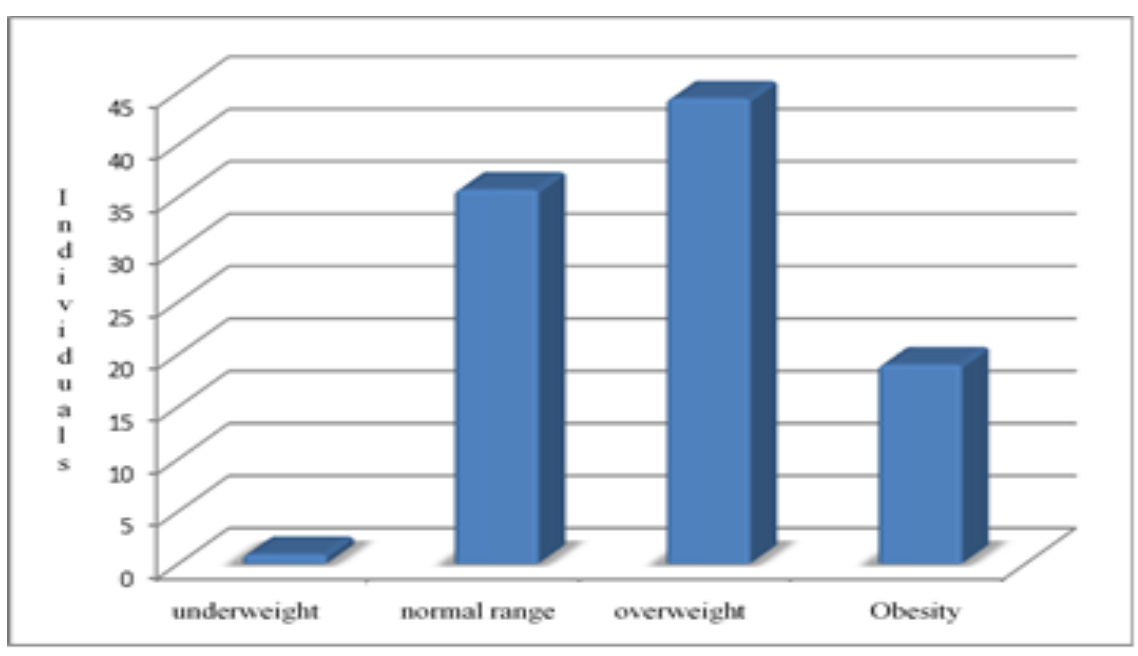


Of the total number of subjects studied, $44.7 \%$ were overweight, $18.8 \%$ were obese, $0.9 \%$ were underweight, while $35.6 \%$ had their weight within normal values, as can be check on the chart. The mean BMI was 26.62 $\mathrm{kg} / \mathrm{m} 2$, with a standard deviation of $4.11 \mathrm{~kg} / \mathrm{m} 2$, where the minimum BMI value was 15.57 and the maximum BMl was $50.78 \mathrm{~kg} / \mathrm{m} 2$. Thus, it is verified that the majority of respondents are overweight.

\section{DISCUSSION}

The constant evolution of modern societies and their adaptation to new habits of life are increasingly related to the increase in the prevalence of cardiovascular risk factors. Obesity is one of these risk markers and is increasingly present in populations. The picture of the National Health System of 2018 reveals that $53.3 \%$ of the Portuguese population in the central region is overweight or obese (Ministry of Health, 2108), a figure lower than that found by us that reveals that $63.5 \%$ of the population studied is overweight or obese, which can be explained by the known aging of the population of the interior, region where the sample was collected and which meets the report that states that 8 out of 10 elderly people have overweight. When we compare our results with other studies conducted at a national level, and do not differentiate the prevalence's between the coast and the interior, we realize that the prevalence found by us is always higher, as is the case of the AMALIA study that refers to a percentage of obesity was lower than in our study (9.3\%). However, when the overweight variable was analysed, the value was more concordant (42.3\%) (Perdigão and Rocha, 2011). Still comparing the reality we found with the results of a study by Silva and Petroski (2012) in the population of Florianopolis we found that the results show that the prevalence of overweight is $51.9 \%$ and that of obesity is $16,1 \%$ values very similar to those found by us. In all the mentioned studies, including ours, we verified that it is in the category of excess weight that is the greater percentage of individuals. It is described that the practice of physical exercise can have a positive influence on dyslipidaemia, insulin resistance, obesity and the presence of arterial hypertension or its prevention and that it is most advisable to have a regular exercise practice at least 3 times a week for a period of more than 30 minutes (Machado and Alves, 2010). According to Ruivo and Alcântara (2011) about $57.0 \%$ of the European population does not practice physical activity. Through our results, we verified a high prevalence of sedentary lifestyle in the studied sample $(75.9 \%)$. In the national portrait of 2018 it is mentioned that obesity is related to the lack of regular practice of physical activity, sport and / or programmed leisure, even saying that only $41.8 \%$ of the population practice some physical activity programmed (Ministry of Health , 2018). This fact, together with the aging population of the interior of Portugal, may explain the high prevalence rate of overweight and obesity found.

\section{CONCLUSIONS}

It is necessary to raise the awareness of the population about the need for exercise programs, better eating habits and the adoption of a healthy lifestyle.

\section{REFERENCES}

Machado, H. Alves, A. Tinoco, C. Gonçalves, C. Matos, C. Rego, D. (2010). Prevalência do diagnóstico de hipertensão arterial em pressoas sedentárias e em praticantes de exercício físico, na cidade do Porto. Acta Médica Portuguesa. 23,153-8. https://doi.org/10.11606/d.17.2015.tde-08102015-165014

Mancia, G. Fagard, R. (2013). ESH/ESC Guidelines for the Management of Arterial Hypertension. Journal of Hypertension. 31, 1281-357.

Ministério da Saúde (2018). Retrato da Saúde. Portugal. 
Perdigão, C. Rocha, E. Duarte, J.S. Santos, A. Macedo, A. (2011). Prevalência, Caracterização e Distribuição dos Principais Fatores de Risco Cardiovasculares em Portugal. Uma Análise do Estudo AMÁLIA. Revista Portuguesa de Cardiologia. 30, 4,393-432.

Ruivo, J. Alcântara, P. (2012). Hipertensão arterial e exercício físico. Revista Portuguesa de Cardiologia. 32, 2, 151-8. https://doi.org/10.1016/i.repc.2011.12.012

Silva, D. Petroski, E. Peres, M. (2012). Pré-hipertensão e hipertensão em adultos de Florianópolis: estudo de base populacional. Revista de Saúde Pública. 46, 6, 988-98. https://doi.org/10.1590/s0034-89102012000600008 


\title{
Venous insufficiency and sedentary job activity
}

\author{
SÓNIA MATEUS ${ }^{1} \triangleleft$, PATRICIA COELHO² ${ }^{2}$ FRANCISCO RODRIGUES ${ }^{2}$ \\ ${ }^{1}$ Hospital Espirito Santos de Évora, Sport, Health \& Exercise Unit (SHERU), Instituto Politécnico de Castelo \\ Branco, Portugal \\ ${ }^{2}$ Qualidade de Vida no Mundo Rural (QRural), Sport, Health \& Exercise Unit (SHERU), Instituto Politécnico \\ de Castelo Branco, Portugal
}

\begin{abstract}
In Europe venous insufficiency is a common pathology that affects $50 \%$ of women and $30 \%$ of men in working age, compromising their life quality, especially in daily tasks. Sedentary job activities may lead to the onset or worsening the disease. Evaluate the relationship between the presence of venous insufficiency and the type of postural position during a sedentary job activity. A cross-sectional observational study was carried out in 76 individuals of both sexes. One group had a sedentary job activity in a seated position (28 individuals) and the other had orthostatic position (46 individuals). The sample was collected within six months. The registry of information on risk factors and venous insufficiency according to the CEAP classification was carried out in a table prepared for this purpose. We obtained a sample of 44 women (58\%) and 32 men (42\%) with a mean age of 42.26 years +9.74 . According to the CEAP classification, venous insufficiency was present in $61.4 \%$ women $(p=0.001)$ and the orthostatic position presents a higher average order in the classification $(p=0.005)$. The sedentary job activity in orthostatic position contributes to a greater wear of the muscular pump, more easily conditioning the venous hemodynamic compromise. Keywords: Job; Sedentary; Venous insufficiency.
\end{abstract}

Corresponding author. Rua do Escoural n¹6, Bairro dos Álamos 7005-426 Évora, Portugal.

E-mail: soniamatildemateus@gmail.com

Supplementary Issue: Spring Conferences of Sports Science. International Seminar of Physical Education, Leisure and Health, 17-19 June 2019. Castelo Branco, Portugal.

JOURNAL OF HUMAN SPORT \& EXERCISE ISSN 1988-5202

(c) Faculty of Education. University of Alicante.

doi:10.14198/jhse.2019.14.Proc4.82 


\section{INTRODUCTION}

The pathologies of the venous system present high incidence and prevalence, affecting individuals of different ages and in active age. Venous insufficiency (VI) consists in venous difficulty return to the heart, due to an imbalance between the forces that favour and oppose the return (França and Tavares, 2003). The venous system is a capacitance system that is divided into a deep and superficial venous system, and both are exposed to hydrostatic pressures found in the human vascular system in the orthostatic position (Santos and Bonamino, 2003).

\section{MATERIAL AND METHODS}

Cross-sectional observational study performed through a representative sample of individuals with sedentary job activity in orthostatic or seated, which took place over six months. For all subjects researchers record information about job activity, the pathology clinical presence according to CEAP classification, blood pressure, glucose value and body mass index were evaluated. All individuals read and signed informed consent.

\section{Participants}

After we have used the inclusion criteria (individuals of both gender between 20 and 65 years old and sedentary job activity in orthostatic or seated position who are in the same job between 10 and 15 years, and who spend 8 hours in the same position), a sample was obtained with 76 individuals, 44 females (58\%) and 32 males ( $42 \%$ ) aged between 28 and 64 years and a mean of 42.26 years +9.74 . Of the total, 46 individuals reported job activity in orthostatic position and 28 in seated position.

\section{Measures}

The evaluation of the presence of the pathology and its clinical characterization was performed according to the CEAP classification (Leal and Mansilha, 2010). Blood pressure was measured according to the 2013 ESH / ESC guidelines (Mansia et al, 2013), the glucose value according to the American Diabetes Association (2018) and the calculation of body mass index according to DGS (2017).

\section{Procedures}

The application of CEAP classification was performed by experienced clinician and is divided into seven items. These are the clinical classification $(C)$, etiological $(E)$, anatomical $(A)$ and pathophysiological $(P)$ classification. (C0), telangietasias / reticular veins $(\mathrm{C} 1)$, varicose veins $(\mathrm{C} 2)$, edema $(\mathrm{C} 3)$, pigmentation or eczema (C4a), lipodermatosclerosis (C4b)), healed venous ulcer (C5) and finally active venous ulcer (C6). For blood pressure measurement a manual sphygmomanometer was used, to measure glucose we used a glucose monitor and On Call Plus test strips. To calculate the mass index, the weight and height were obtained. Information registration was carried out by the researchers in a table prepared for the purpose.

\section{Analysis}

Statistical analysis was performed using the statistical software program Statistical Package for the Social Sciences (IBM SPSS Statistics ${ }^{\circledR}$ version 22). A descriptive analysis of all variables was performed. The normal distribution of sample analysis the Kolmogorov-Smirnov test was performed, where it was verified that the body mass index and age variables follow a normal distribution.

Non-parametric Chi-Square and Mann-Whitney tests were used to relate the pathology whit the individual's working position and associated risk factors. 


\section{RESULTS}

In the body mass index classification, most individuals had normal parameters. VI compromises mainly individuals with normal weight and overweight with a percentage of $20.78 \%$, however, there is no statistically significant relation $(p=0.379)$. There is a significant relationship between the presence of $\mathrm{VI}$ and nonmodifiable risk factors such as female $(61.4 \%)(p=0.001)$ and family history $(67.6 \%)(p=0.001)$. According to the clinical classification of CEAP, individuals who have orthostatic as their job position are those with a higher average order $(p=0.005)$. Concerning the development of job activities commitment the average order is higher in the orthostatic position, with a statistically significant difference $(p=0.005)$.

\section{DISCUSSION}

The high incidence and prevalence of venous system diseases affects individuals of different ages and is considered a public health problem (France, 2003). In Portugal, an epidemiological study showed that the prevalence of VI was higher in female subjects, reaching the majority of women between 55 and 64 years. This correlates with some demographic characteristics of our study, where the relationship between the presence of $\mathrm{VI}$ and risk factors such as sex and family history showed statistically significant differences ( $p$ $=0.001$ and $p=0.001$ respectively). According to Medeiros and Mansilha (2012) it is now known that age, family history and sex are factors that contribute to the development of $\mathrm{VI}$. The fact that it is more frequent in the female sex may be a result of hormonal factors, but also because it is considered aesthetics matter being the sex that most seeks treatment. In this sample the presence of VI does not depend on the body mass index class. This fact is not in agreement with the literature and can be justified by the small number of observations. Currently, overweight is considered to impair blood changes due to increased adipose tissue, which increases blood stasis leading to the onset / worsening of the disease (Santos and Bonamino, 2009). Other risk factors such as hypertension and diabetes were studied without significant results.

According to the clinical classification of CEAP the individuals that presented a higher average order were those with orthostatic job position. According to Berenguer and Lins (2011) the maintenance of the orthostatic position, in $45 \%$ of the working time, is considered enough to provoke symptoms of fatigue and discomfort of the lower limbs, being the venous edema the first manifestation of the blood stasis. It can be said that edema is a very important factor in an individual life quality, causing discomfort, feeling of heavy legs, and can later evolve into VI (Belczak and Gody, 2008). This leads us to significant results $(p=0.005)$ also in the relation between the commitment to the development of job activities in individuals with this type of position and higher ordering in the clinical classification of CEAP. According to Berenguer and Lins (2011) these job activities can lead to the appearance / aggravation of VI by making muscular work more exhausting. For good maintenance of orthostatic position, constant levels of muscle contraction are necessary, compressing the blood vessels, which will easily provoke their fatigue leading to a decrease in their quality of life (Medeiros and Mansilhas, 2012).

\section{CONCLUSIONS}

Jobs performed in orthostatic position increase the influence of hydrostatic and gravitational pressure with muscle pump wear, hindering venous return, which can lead to VI, with a negative impact on quality of life. 


\section{REFERENCES}

American Diabetes Association (2018). Classification and Diagnosis of Diabetes: Standards of Medical Care in Diabetes-2018 Diabetes Care 2018 Jan; 41(Supplement 1): S13-S27. https://doi.org/10.2337/dc18-s002

Belczak, C., Gody, J., Ramos, R., Oliveira, M., Belczak, S., Caffaro, R. (2008). Influência do turno laboral na formação de edema dos membros inferiores em indivíduos normais. J Vasc Bras. Vol 7, №3. https://doi.org/10.1590/s1677-54492008000300007

Berenguer, F.A., Lins, D.A., Carvalho, S.C. (2011). Influência da posição ortostática na ocorrência de sintomas e sinais clínicos de venopatias de membros inferiores em trabalhadores de uma gráfica na cidade do Recife-PE. Revista. Brasileira Saúde ocupacional. São Paulo. 36 (123): 153-161. https://doi.org/10.1590/s0303-76572011000100016

Camolas, J., Gregório, M.J, Sousa, S.M., Graça, P. (2017). Obesidade: otimização da abordagem terapêutica no Serviço Nacional de Saúde. Programa Nacional para a Promoção da Alimentação Saudável. Direção-Geral de Saúde. https://doi.org/10.11606/d.89.2017.tde-25092010-123402

França, L.H. and Tavares, V. (2003). Insuficiência Venosa Crônica. Uma atualização. Artigo de revisão $\mathrm{J}$ VascBr. Vol. 2, No4.

Leal, J. e Mansilha, A. (2010). Como avaliar o impacto da doença venosa crónica na qualidade de vida. Artigo de revisão. Angiologia e Cirurgia Vascular. Vol. 6, № 4.

Mansia, G., Robert, F., Krzysztof, N., Josep, R., Alberto, Z., Michael, B., Thierry, C., Renata, C., Guy, B., Anna, D., Maurizio, G., Diederick, E.G., Tiny, J., Paulus, K., Sverre, E.K., Ste'phane, L., Athanasios, J.M., Peter, M.N., Luis, M.R., Roland, E.S., Per, A.S., Peter, S., Margus, V., Bernard, W. and Faiez, Z.(2013).ESH/ESC Guidelines for the management of arterial hypertension. The Task Force for the management of arterial hypertension of the European Society of Hypertension (ESH) and of the European Society of Cardiology (ESC). Journal of Hypertension. 31:1281-1357. https://doi.org/10.1201/b17072-61

Medeiros, J. and Mansilha, A. (2012). Estratégia Terapêutica na Doença Venosa Crónica. Artigo de revisão. Angiologia e Cirurgia Vascular.| Vol. 8, №3.

Santos, P. and Bonamino, M. (2003). Efeitos Cardiovasculares Agudos da Exposição ao Ambiente Microgravitacional. Arq Bras Cardiol. Vol. 80, No1: 105-115. https://doi.org/10.1590/s0066$\underline{782 \times 2003000100012}$

Santos, R., Profírio, G.J., Pitta, G. (2009). A diferença na qualidade de vida de pacientes com doença venosa cnónica leve e grave. J Vasc Bras . Vol. 8, No 2. https://doi.org/10.1590/s1677$\underline{54492009000200008}$

\section{(9) $(\mathbb{\Theta \Theta}$}

This work is licensed under a Attribution-NonCommercial-NoDerivatives 4.0 International (CC BY-NC-ND 4.0). 


\title{
Peripheral arterial systolic-diastolic velocities in athletes and non-athletes by Doppler ultrasound
}

\author{
SÓNIA MATEUS ${ }^{1} \checkmark$, PATRICIA COELHO², FRANCISCO RODRIGUES ${ }^{2}$ \\ ${ }^{1}$ Hospital Espirito Santos de Évora, Sport, Health \& Exercise Unit (SHERU), Instituto Politécnico de Castelo \\ Branco, Portugal \\ ${ }^{2}$ Qualidade de Vida no Mundo Rural (QRural), Sport, Health \& Exercise Unit (SHERU), Instituto Politécnico \\ de Castelo Branco, Portugal
}

\begin{abstract}
Intensive sports practice can result in negative consequences. Although the exercise is considered a benefit to the well-being and health of the human being. The form and intensity with which it is practiced can cause negative changes, namely at vascular level, such as changes in the wall and vascular hemodynamic, traumatic injuries, etc. Evaluate the relationship between arterial flow velocities of the lower limbs and the practice of intensive exercise through arterial Doppler ultrasound. Cross-sectional analytical study using arterial Doppler ultrasound of the lower limbs in 76 male subjects, 38 athletes and 38 non-athletes aged 18 to 30 years. The sample was collected over a period of six months in two football clubs in the central region. The mean age was $26 \pm 1.8$ years for non-athletes and $24 \pm 2.79$ years for athletes. There is no statistically significant relationship $(p=0.553)$ between smoking habits and if they are athletes our non-athletes. Regarding the results of the parameters evaluated by Doppler ultrasound the mean of the arterial resistance indices and mean of the systolic and diastolic velocities between the athletes and non-athletes, there were significant variations in some arteries. In this studied sample there is a greater predisposition for athletes to develop small arterial alterations of the lower limbs, however, they are not hemodynamic significant. Keywords: Arteries; Athletes; Doppler; Ultrasound; Velocity.
\end{abstract}

Corresponding author. Rua do Escoural n¹6, Bairro dos Álamos 7005-426 Évora, Portugal.

E-mail: soniamatildemateus@gmail.com

Supplementary Issue: Spring Conferences of Sports Science. International Seminar of Physical Education, Leisure and Health, 17-19 June 2019. Castelo Branco, Portugal.

JOURNAL OF HUMAN SPORT \& EXERCISE ISSN 1988-5202

(c) Faculty of Education. University of Alicante.

doi:10.14198/jhse.2019.14.Proc4.82 


\section{INTRODUCTION}

In general, all vascular risk factors affect and cause changes in the vascular wall, but each has a different degree and form of influence. In particular, we emphasize the modifiable risk factors that are associated with lifestyle, where exercise practice is usually a positive contribution due to good vasoconstriction during exercise and good post-exercise vasodilation. But in some particular situations intensive practice has negative effects. For example increased blood pressure during exercise increases the friction between the blood and the arterial wall, which can lead to a weakening of the wall and the formation of aneurysm in the long term. Fistula formation may also occur, aneurysms, occlusions or stenoses, embolism, etc. (Blomqvist and Saltin, 1983) In order to be able to evaluate the morphology and flow dynamics in the main arteries of the lower limbs, there are diagnostic tests, such as peripheral arterial Doppler ultrasound.

\section{MATERIAL AND METHODS}

An analytical cross-sectional study was performed on a sample of athletes and non-athletes, using lower limbs arterial Doppler ultrasound and filling a questionnaire related to sports and smoking habits. The sample was collected for six months at two football clubs in the central region.

\section{Participants}

After applying the defined inclusion criteria (male individuals athletes and/or non-athletes, aged between 18 and 30 years who accepted participate in the study and performed lower limbs arterial Doppler ultrasound), a sample was obtained with 76 individuals males being 38 athletes and 38 non-athletes aged between 18 and 30 years. The mean age for non-athletes were $26 \pm 1.8$ years and $24 \pm 2.79$ years for athletes.

\section{Measures}

To perform the Doppler ultrasound besides the physical space it was necessary an ultrasound technician and ultrasound with $7-12 \mathrm{MHrz}$ linear probe. In the peripheral arterial exam, three techniques were applied: ultrasound, pulsed Doppler and colour coded Doppler allowing visualizing the arterial lumen, to evaluate the Doppler spectrum morphology, measure the systolic (SV) and diastolic (DV) flow velocity and measure the resistance index (RI). Conventional recommendations were used to perform the tests and analyse their criteria according to Thrush and Hartshorne (1999).

\section{Procedures}

Individuals who practiced three times or more sports for week were considered athletes and individuals who did not practice once a week were considered non-athletes. A questionnaire and information of vascular risk factors, physical activity, number of exercise times and peripheral arterial Doppler ultrasound results, namely SV and DV in cm/s and RI of eight arteries in all individuals, were applied: common femoral arteries (CFA), superficial femoral arteries (SFA), deep femoral arteries (DFA) and popliteal arteries (PA).

\section{Analysis}

Statistical analysis was performed using the statistical software program Statistical Package for the Social Sciences (SPSS Statistics ${ }^{\circledR}$ version 22). The normality of the sample was verified through the Shapiro-Wilk test. The Chi-square test was performed in conjunction with the Fisher's exact test to correlate the smoking habits with athlete/non-athlete, Student- $T$ and Mann-Whitney tests were performed to verify if there were significant differences between the two groups regarding RI, SV and DV of the arteries of the lower limbs. 


\section{RESULTS}

Although there were more non-athletes (19 smokers) who smokes than athletes (15 smokers) and there was no significance (Fisher's $p=0.553$ ). Of the arteries studied, the relationship between arterial IR and athlete's or non-athlete was only statistically significant in the left DFA $(p=0.002)$ and left PA $(p=0.003)$. Regarding velocities: the arterial SV is higher in athletes in all arteries with the exception of left PFA. The highest SV in athletes and non-athletes was in the right SFA $(136 \mathrm{~cm} / \mathrm{s})$ in the athletes and left CFA $(121 \mathrm{~cm} / \mathrm{s})$ in the nonathletes and the lowest SV were in the PA $(36 \mathrm{~cm} / \mathrm{s}$ on the left in athletes and $29.3 \mathrm{~cm} / \mathrm{s}$ on the right in nonathletes). However, between the SV and the athlete or non-athlete, only significant differences were found in the VS of the left CFA ( $p=0.041)$; DV is also higher in all arteries in athletes. The highest DV in the athletes and non-athletes was in the left CFA $(33.4 \mathrm{~cm} / \mathrm{s})$ in the athletes and in the left SFA $(30.7 \mathrm{~cm} / \mathrm{s})$ in the nonathletes, as the minimum DV was in the PFA $(6.5 \mathrm{~cm} / \mathrm{s}$ left on athletes and $2.4 \mathrm{~cm} / \mathrm{s}$ on right non athletes). However, there was a statistically significant difference between the DV and the athlete in the left CFA $(p=0.013)$, the right PFA (0.004) and the right PA $(p=0.024)$.

\section{DISCUSSION}

Investigations in this area refer that pathological alterations suffered by the practice of high-level physical exercise, together with vascular risk factors potentiate its appearance. Smoking habits were studied, which did not reveal a correlation with being an athlete or non-athlete. According to Gaspar de Matos et al. (2003) young people who practice more physical activity report that they have tried but don't smoke regularly and young people who do not practice regular physical activity smoke regularly.

Regarding Doppler ultrasound results, there were no statistically significant differences in almost all studied arteries. Stebbings and Morse (2013) in a study that performed between two groups (trained and control) did not observe changes in the RI of the control group over time. Considering that athletes have larger diameters, it is normal to have an adjusted arterial RI (lower), according to the Poiseuille equation, the increase in arterial diameter causes a decrease in the resistance to the passage of blood flow, they are inversely proportional. There were also no statistically significant differences between the SV and DV in the athlete or not, in the great majority of the arteries. However, there were slight differences in SV and DV (within normal values) between the two groups, being slightly higher in the athletes. Which is accord to Schep and Bender (2001) (2002) who found significantly higher SV in athletes in reference limbs. Brum and Negrão (2004), in a study about acute and chronic physical exercise adaptations in the cardiovascular system, mention Forjaz and Tinucci (2000), who say that in dynamic exercises, as contractions are followed by joint movements, there is no mechanical obstruction of the blood flow, observing an increase of the sympathetic nervous activity, which is triggered by the activation of the central command, muscular mechanoreceptors and, depending on the intensity of the exercise, muscle metaboreceptors. In response to increased sympathetic activity, heart rate, systolic volume, cardiac output, and consequently flow velocities increase. In addition, the production of muscle metabolites promotes vasodilatation in the active musculature, generating a reduction in peripheral vascular resistance. In this way, the authors support the present study, because in the athletes the vascular resistances are smaller and the speeds higher than in the non-athletes (Forjaz and Tinucci, 2000). However, there are studies such as Currie, Thomas and Goodman (2009), where the authors point out that the changes caused by exercise are variable and can vary mainly with the type of exercise, the intensity, the time of practice. 


\section{CONCLUSIONS}

The practice of exercise may lead to small variations in the lower limb arteries, which are related to the adaptation that the vascular network suffers from the conditions imposed by the practice of the sport, varying with the time practiced and the intensity.

\section{REFERENCES}

Blomqvist. C.G, Saltin, B. (1983) Cardiovascular adaptations to physical training. Annu Rev Physiol [Internet]. Jan;45(55):169-89. https://doi.org/10.1146/annurev.ph.45.030183.001125

Brum, P.C., Negrão, C.E. (2004). Adaptações agudas e crônicas do exercício físico no sistema cardiovascular. Rev. paul. Educ. Fís. São Paulo, v.18, p.21-31.

Currie, K.D., Thomas, S.G., Goodman, J.M. (2009). Effects of short-term endurance exercise training on vascular function in young males. Eur J Appl Physiol. 107:211-218. https://doi.org/10.1007/s00421$\underline{009-1116-4}$

Gaspar de Matos, M., Gaspar, T. e Vitória, P. (2003). Comportamentos e atitudes sobre o tabaco em adolescentes portugueses fumadores. Psicologia, Saúde e Doenças. 4 (2), 205-219.

Matthew, J.; Fox, E.L. (1979). Bases Fisiológicas da Educação Física e dos desportos; 2. ed. São Paulo: Interamericana, cap. 9, p. 162-183.

Schep, G., Bender, M.H., Schmikli, S.L., Mosterd, W.L., Hammacher, E.R., Scheltinga, M. et al (2002). Recognising vascular causes of leg complaints in endurance athletes. Part 2: the value of patient history, physical examination, cycling exercise test and echo-Doppler examination. Int J Sports Med. 23(5):322e8. https://doi.org/10.1055/s-2002-33142

Schep, G., Bender, M.H., Schmikli, S.L., Wijn, P.F. (2001) Color Doppler used to detect kinking and intravascular lesions in the iliac arteries in endurance athletes with clau- dication. Eur $\mathrm{J}$ Ultrasound.14(2e3):129e40. https://doi.org/10.1016/s0929-8266(01)00154-9

Stebbings, G.K., Morse, C.I., McMahon, G.E., Onambele, G.L (2013). Resting arterial diameter and blood flow changes with resistance training and detraining in healthy young individuals. Journal of Athletic Training.48(2):209-219. https://doi.org/10.4085/1062-6050-48.1.17

Thrush, A., Hartshone,T. (2005) Peripheral Vascular Ultrasound. How, Why and When. Second Edition. Elsevier Churchill Livingstone.

\section{@ $\odot \Theta \Theta$}

This work is licensed under a Attribution-NonCommercial-NoDerivatives 4.0 International (CC BY-NC-ND 4.0). 


\title{
Pilates for elderly women: An improvement in functional mobility and balance
}

\author{
SAMUEL HONÓRIO ${ }^{1,2} \triangleleft$, MARCO BATISTA ${ }^{1,2}$, JOÃO ROCHA ${ }^{1,2}$, JOÃO PETRICA ${ }^{1,2}$, JORGE \\ SANTOS ${ }^{1,2}$, HELENA MESQUITA' ${ }^{1,2}$, JOÃO SERRANO ${ }^{1,2}$, DÉBORA SOUSA ${ }^{1}$, RAQUEL SILVA ${ }^{3}$ \\ 1 Instituto Politécnico de Castelo Branco, Portugal \\ ${ }^{2}$ SHERU - Sport, Health and Exercise Research Unit, Portugal \\ ${ }^{3}$ Faculty of Health Sciences, University Fernando Pessoa, Oporto, Portugal. Research Centre for \\ Anthropology and Health, University of Coimbra, Portugal. Scientific Commission of the Gymnastics \\ Federation of Portugal, Lisbon, Portugal
}

\begin{abstract}
This study intends to determine and relate the effects of a program of Pilates sessions in elderly women, comparing balance assessments by the Berg scale and physical mobility with the scale of Leighton for articular ampleness. Elderly evaluation was conducted by using a universal goniometer, the Berg scale, the Leighton scale and data collection was performed in two different moments. This study had 52 elderly women aged between 60 and 83 years. The participants had Pilates sessions of 30 minutes each, twice a week, for 6 months. The statistical analysis used were descriptive based on mean and standard deviation, statistical inference by applying the Wilcoxon and the effect size by Cohen's $d$ where we observed an increase in balance parameters and articular mobility of elderly practitioners, with improvements from considered "average" in the first evaluation to "above average" in the second evaluation. The implemented Pilates program proved to be extremely favourable, with significant improvements in all analysed variables with intermediate and large effect sizes. Keywords: Pilates; Elderly; Balance; Physical mobility; Berg Scale; Leighton Scale.
\end{abstract}

\footnotetext{
Corresponding author. Instituto Politécnico de Castelo Branco, Escola Superior de Educação. SHERU (Sport, Health and Exercise Research Unit). Portugal.

E-mail: samuelhonorio@ipcb.pt

Supplementary Issue: Spring Conferences of Sports Science. International Seminar of Physical Education, Leisure and Health, 17-19 June 2019. Castelo Branco, Portugal.

JOURNAL OF HUMAN SPORT \& EXERCISE ISSN 1988-5202

(c) Faculty of Education. University of Alicante.

doi:10.14198/jhse.2019.14.Proc4.82
} 


\section{INTRODUCTION}

Functional autonomy can be understood under the following aspects: autonomy of action is related to the physical independence or ability to perform day-to-day tasks; autonomy of will corresponds to individual selfdetermination; and autonomy of thought is the one that allows for the making of decisions and judgments. The preservation of these capacities influences the quality of life of the elderly (Emery, Serres, McMillan \& Côté, 2010). In this study only functional autonomy was evaluated in the first sense. The Pilates method consists of physical exercises whose main characteristic is resistance work and dynamic stretching, performed in conjunction with breathing and respecting the following principles: control, precision, centring, fluidity of movement, concentration and respiration (Anderson \& Specor, 2000; Sekendiz, Altuna, Korkusuza \& Akinb, 2007). The method is aimed at strengthening the muscles located in the centre of the body (abdominal, paravertebral, gluteal and pelvic muscles), named after powerhouse creator Joseph Pilates. The Pilates Method proposal can be an improvement in the quality of life of its practitioners, through an optimized condition of a new posture, developing greater mobility, balance and agility.

\section{MATERIAL AND METHODS}

\section{Participants}

A number of 52 elderly participants aged between 60 and 83 years (mean $74.48 \pm 7.36$ ) have participated. They practice Pilates sessions of 30 minutes each, twice a week for a period of 6 months. This research did not offer physical or psychological risks to participants and all ethical issues were respected. All participants gave their free and informed consent in writing.

\section{Measures}

For elderly evaluation a universal goniometer was used to measure the joints of the participants, namely the shoulder joint (extension, flexion, adduction and abduction) and the thigh/knee joint (flexion, adduction and abduction). The results were classified according to the Leighton (1955) table, a classification table widely used to refer each individual in terms of the total ampleness of joint actions, for several articulations. With this table, the classification of each joint was verified, classifying it in low, below average, average, above average and high. Balance was also evaluated through the Berg Balance Scale (Berg \& Norman, 1996), with the objective of evaluating the risk of fall, comprising a scale of 14 day-to-day tasks involving static and dynamic balance such as reaching, rotating, transferring, stand and get up. We only evaluated the tasks of "sitting to standing" and "standing".

\section{Procedures}

The elderly were subjected to a general evaluation (including age, weight and gender). It was necessary to use a universal goniometer and the results were compared with Leighton's table, which classifies the level of flexibility according to the degree obtained after the measurement, Marques (2003). The berg scale was applied after these evaluations in order to assess the risk of falls in the elderly and to relate this variable to their mobility. The evaluations were obtained in two moments between 6 months of Pilates practice.

\section{Analysis}

Descriptive statistics and the Wilcoxon test was applied for data analysis, for comparison between the two moments. Also, was performed the d Cohen's effect size to determine if this programme had significant practical effects on the variables under study. 


\section{RESULTS}

Table 1. Comparison between the two evaluations and the effect size of the variables under study

\begin{tabular}{|c|c|c|c|c|}
\hline Variables & Mean & sd & Sig. ${ }^{*}$ & d Cohen \\
\hline Shoulder Flexion/Extension (1st) & 229.67 & 18.10 & \multirow{2}{*}{0.001} & \multirow{2}{*}{$0.546^{*}$} \\
\hline Shoulder Flexion/Extension (2nd & 238.77 & 15.13 & & \\
\hline Shoulder Adduction/abduction ( 1 st) & 197.77 & 16.39 & \multirow{2}{*}{0.001} & \multirow{2}{*}{$0.541^{*}$} \\
\hline Shoulder Adduction/abduction (2nd) & 206.69 & 18.47 & & \\
\hline Thigh Flexion $(1$ st $)$ & 107.13 & 13.18 & \multirow{2}{*}{0.001} & \multirow{2}{*}{0.327} \\
\hline Thigh Flexion $\left(2^{\text {nd }}\right)$ & 111.98 & 16.32 & & \\
\hline Thigh abduction (1st) & 57.27 & 7.24 & \multirow{2}{*}{0.001} & \multirow{2}{*}{$1.68^{* * *}$} \\
\hline Thigh abduction (2nd $)$ & 73.71 & 11.75 & & \\
\hline Seating to standing ( 1 st) & 3.60 & 0.49 & \multirow{2}{*}{0.001} & \multirow{2}{*}{$1.15^{\star *}$} \\
\hline Seating to standing $\left(2^{\text {nd }}\right)$ & 4 & 0.00 & & \\
\hline Standing for $2 \min (1 \mathrm{st})$ & 3.35 & 0.81 & \multirow{2}{*}{0.001} & \multirow{2}{*}{$1.14^{* *}$} \\
\hline Standing for $2 \min \left(2^{\text {nd }}\right)$ & 4 & 0.00 & & \\
\hline
\end{tabular}

${ }^{*}$ Wilcoxon $p \leq 0.005 /{ }^{*} d$ Cohen intermediate effect $/{ }^{* *} d$ Cohen large effect $/{ }^{* * *} d$ Cohen very large effect

\section{DISCUSSION}

The Pilates group had improvements in articular mobility and balance, as found by Silva et al. (2008). It is known that the act of sitting to standing requires strengthening of the muscles of the lower limbs, as well as the postural muscles (spinal stabilizers), in addition to flexibility, balance and coordination. In the getting up test, improvements were seen as studied in Kolyniak et al. (2000). With regard to the standing up variable, besides flexibility, balance and coordination were favourable, as was observed in Hall et al. (1999). The studies of Hall et al. (1999) and Johnson et al. (2007) have shown that Pilates practice can also promote the improvement of the dynamic balance of the elderly, by strengthening their muscles.

\section{CONCLUSIONS}

The Pilates method proves to be a complete, very effective tool for quality of life and health purposes. The results show that the practice of this activity applied to the elderly group of this study, promoted a significant improvement in their functional performance. It can be concluded that a 6-months Pilates program improved functional mobility and body balance, probably by strengthening the muscles involved in postural control.

\section{REFERENCES}

Anderson, D. \& Spector, A. (2000). Introduction to Pilates-based rehabilitation. Orthopedics and Physical Therapy Clinics, 9, 385-410.

Berg, K. \& Norman, K. (1996) Functional assessment of balance and gait. Clinics in Geriatrics Medicine, 4, 705-723. https://doi.org/10.1016/S0749-0690(18)30197-6

Cohen, J. (1988). Statistical power analysis for the behavioral sciences (2. Auflage). New Jersey: Erlbaum.

Emery, K., Serres, S., McMillan, A., Côté, J. (2010). The effects of a Pilates training program on armtrunk posture and movement. Clinical Biomech, 25(2), 124-30. https://doi.org/10.1016/i.clinbiomech.2009.10.003 
Hall, D., Nichols, J., Aguilar, L., Larkam, E. (1999). Effects of Pilates-based-training on static and dynamic balance in an elderly population. Medicine Science Sports Exercise, 31(5), 375-388. https://doi.org/10.1097/00005768-199905001-01987

Kolyniak, I., Cavalcanti, S., Aoka, S. (2004). Avaliação isocinética da musculatura envolvida na flexão e extensão do tronco: efeito do método Pilates. Revista Brasileira Medicina Esporte,10(6), 487-90. https://doi.org/10.1590/S1517-86922004000600005

Johnson, G., Larsen, A., Ozawa, H., Wilson, C., Kennedy, K. (2007). The effects of Pilates-based exercises on dynamic balance in healthy adults. Journal of Bodywork Movement Therapy, 11(3),23842. https://doi.org/10.1016/i.jbmt.2006.08.008

Leighton, J. (1955). Instrument and technic for measurement of range of joint motion. Archives of Physical Medical Reabilitation, 36, 571 - 578

Marques, A. (2003). Manual de Goniometria - Ângulos articulares dos membros superiores e inferiores. São Paulo: Editora Manole.

Sekendiz, A., Altuna, O., Korkusuza, B. \& Akinb, S. (2007). Effects of Pilates exercise on trunk strength, endurance and flexibility in sedentary adult females. Journal of Bodywork and Movement Therapy, 11(4), 318-26. https://doi.org/10.1016/i.jbmt.2006.12.002

Silva, A., Almeida, G., Cassolhas, R., Cohen, M., Paccin, M., Tifik, S. (2008). Equilíbrio, coordenação e agilidade de idosos submetidos à prática de exercícios físicos resistidos. Revista Brasileira Medicina Esporte, 14(2),88-93. https://doi.org/10.1590/S1517-86922008000200001

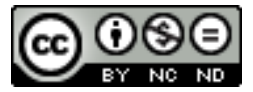

This work is licensed under a Attribution-NonCommercial-NoDerivatives 4.0 International (CC BY-NC-ND 4.0). 


\title{
Physical activity and subjective well-being in Health Sciences first-year students
}

\author{
SAMUEL HONÓRIO1,2 $\triangleleft$, MARCO BATISTA ${ }^{1,2}$, MARIA-RAQUEL G. SILVA ${ }^{3}$ \\ ${ }^{1}$ SHERU (Sport, Health and Exercise Research Unit), Instituto Polytechnic de Castelo Branco, Portugal \\ ${ }^{2} R E C l$ (Research, Education and Community Intervention), Portugal \\ ${ }^{3}$ Faculty of Health Sciences, University Fernando Pessoa, Oporto, Portugal. Research Centre for \\ Anthropology and Health, University of Coimbra, Portugal. Scientific Commission of the Gymnastics \\ Federation of Portugal, Lisbon, Portugal
}

\begin{abstract}
It is evident that physical activity implies several health benefits and is considered a fundamental component for improving quality of life and well-being. The main objective of the study was to identify the relationship between students who maintain a regular physical activity practice and how this aspect contributes to their well-being, namely life satisfaction and affections. A total of 177 students of both genders, aged between 18 and 30 years, enrolled in the first year of Health Sciences College degrees participated in this research. The data collection instrument used was a questionnaire, with the Positive and Negative Affective Scale (PANAS) and the Satisfaction with Life (SWLS). In addition to descriptive statistics, the Mann-Whitney test was used to compare groups (83 practitioners and 94 non-practitioners). Results showed that students, who maintain practicing PA with the entrance in College had more favourable values in well-being with significant differences in terms of their satisfaction with life $(p=0.04)$ and positive affections $(p=0.01)$. We conclude that students from Health Sciences College degrees who maintain a constant physical activity practice present better results in the analysed variables in relation to those who stopped practicing after they start attending the first year of College. Keywords: Students; Life satisfaction; Affections; Physical activity; Well-being.
\end{abstract}

Corresponding author. Instituto Politécnico de Castelo Branco, Escola Superior de Educação. SHERU (Sport, Health and Exercise Research Unit). Portugal.

E-mail: samuelhonorio@ipcb.pt

Supplementary Issue: Spring Conferences of Sports Science. International Seminar of Physical Education, Leisure and Health, 17-19 June 2019. Castelo Branco, Portugal.

JOURNAL OF HUMAN SPORT \& EXERCISE ISSN 1988-5202

(c) Faculty of Education. University of Alicante.

doi:10.14198/jhse.2019.14.Proc4.82 


\section{INTRODUCTION}

The practice of physical activity (PA) positively influences self-image and body satisfaction (Silva, 2014). Individuals with higher levels of mental well-being and health perception are more capable and motivated to be more active in their daily lives. Batista (2009) identifies the sense of well-being or satisfaction with life intimately linked to how the individual can cope and absorb the occurrence of episodes during his life. Subjective well-being is defined as experiencing a high level of positive affect and low level of negative affect, as well as, a high degree of satisfaction with life (Ryan \& Deci, 2007). Higher life satisfaction seems to be related to positive functioning indicators and lower satisfaction with life has been associated with greater depressive symptoms, interpersonal rejection and aggressive behaviours (Halbrook, 2012). Subjective wellbeing has been associated with a hedonic view of well-being that integrates dimensions of affection and satisfaction with life. It aims to understand the affective dimension (positive and negative affects) and the cognitive dimension (satisfaction with life) of the subjective evaluation that the subject makes of himself and his life (Lopes, 2012). The main objective of the study was to identify the relationship between students who maintain a regular physical activity practice and how this aspect contributes to their well-being, namely life satisfaction and affections.

\section{MATERIAL AND METHODS}

\section{Participants}

A number of 177 first-year students enrolled in Health School Sciences in Portuguese Universities, with ages between 18 and 30 (20.20 \pm 3.04 years old). They were divided into 2 groups ( 83 practitioners of PA and 94 non-practitioners).

\section{Measures}

For data collecting purposes and according to what was intended to be evaluated, two scales were used: the PANAS-Positive and Negative Affect Schedule (Watson, Clark \& Tellegen, 1988), a self-report questionnaire that consists of two scales to measure positive and negative effects, where each item is rated on a 5-point scale of 1 (not at all) to 5 (very much) and; the LSC-Life Satisfaction Scale (Diener, Emmons, Larsen \& Griffin, 1985). ) used to evaluate life satisfaction as a cognitive construct. This scale has 5 items and consists of indicating, through a 7-point Likert scale, that varies between Totally Disagree (1) and Absolutely Agree (7) the degree of satisfaction according to each item.

\section{Procedures}

Before starting data collection, subjects were informed about the scope and objectives of our study, as well as, the confidentiality of the individual data and results of the tests performed. Subjects participated freely in the study, giving their signed informed consent. The questionnaires were self-filled in our presence and returned at the same time, making them give it to us when they were completed. After questionnaires were processed, they were destroyed. This study was approved by the faculty Ethics Committee.

\section{Analysis}

Descriptive statistics are presented and the comparison between the practice and non-practice groups was assessed by the Mann-Whitney test. 


\section{RESULTS}

Table 1. Descriptive statistics and significance values from variables comparison by Mann-Whitney Test

\begin{tabular}{lccccc}
\hline & a Cronbach & Mean \pm SD & $\begin{array}{c}\text { Practitioners } \\
\text { Mean } \pm \text { SD }\end{array}$ & $\begin{array}{c}\text { Non-practitioners } \\
\text { Mean } \pm \text { SD }\end{array}$ & Sig. \\
\hline Satisfaction with life & .89 & $4.97 \pm 1.07$ & $5.15 \pm 1.01$ & $4.81 \pm 1.09$ & $0.04^{*}$ \\
Positive Affections & .87 & $2.93 \pm 0.61$ & $3.11 \pm 0.61$ & $2.76 \pm 0.56$ & $0.01^{* *}$ \\
Negative Affections & .82 & $2.08 \pm 0.62$ & $2.11 \pm 0.63$ & $2.04 \pm 0.61$ & 0.58 \\
\hline
\end{tabular}

Students who maintained the practice of PA through the first year in College had significantly higher values in life satisfaction $(P=0.04)$ and in production of positive affections $(P=0.01)$; however, no significant differences were observed for negative affections.

\section{DISCUSSION}

Well-being consists of a cognitive component, such as satisfaction with life, and an affective component (Marconcin, Alves, Dias \& Fonseca, 2010). Individuals, who achieve high levels of subjective well-being have a high satisfaction with life and moments of positive affection and with few moments of negative affection (Lyubomirsky, 2008; Rodriguez, 2009). In this study, students who practiced PA during the first year of College had higher levels of well-being compared to non-practitioners, as they obtained higher values of satisfaction with life and positive affections, as studied in Batista, Jimenez Castuera, Petrica, Serrano, Honório, Paulo and Mendes (2016). However, regarding negative effects, there were no significant differences corroborating the studies of Zanon and Hutz (2010) and Marconcin, Alves, Dias and Fonseca (2010).

\section{CONCLUSIONS}

We conclude that first-year students from Health Sciences who maintain a constant physical activity practice present better results in the analysed variables in relation to those who stopped practicing after they start attending the first year of college, being able to use this as a preventive and prophylactic measure as future health professionals.

\section{REFERENCES}

Batista, M. (2009). Bem estar psicológico - relação entre a prática de judo e auto conceito. Tese de Doutoramento, Universidad de Extremadura, Faculdad de Educación, Extremadura.

Batista, M.; Jimenez Castuera, R.; Petrica, J.; Serrano, J.; Honório, S.; Paulo, R.; Mendes, P. (2016). Self-determined motivation and well-being in Portuguese active adults of both genders. Journal: BMC Health Services Research. 16 (3), 103.

Diener, E., Emmons, R., Larsen, R. \& Griffin, S. (1985). The satisfaction with life scale. Journal of Personality Assessment, 49(1), 71-75. https://doi.org/10.1207/s15327752.jpa4901 13

Halbrook, M., Blom, L., Hurley, K., Bell, R. \& Holden, J. (2012). Relationship's among motivation, gender, and, cohesion in a sample of collegiate athletes. Journal of Sport Behavior, 35, 61-77.

Lopes, M. D. (2012). Hábitos de vida dos adolescentes: Género, ano de escolaridade e prática de atividade física. Dissertação de Mestrado, Faculdade de Motricidade Humana, Universidade Técnica de Lisboa, Lisboa. https://doi.org/10.32385/rpmgf.v28i5.10971

Lyubomirsky, S. (2008). La ciencia de la felicidad. Barcelona: Ediciones Urano. 
Marconcin, P. E., Alves, N. J., Dias, C., \& Fonseca, A. M. (2010). Bem-estar subjetivo e a prática de atividade desportiva em idosos alunos de universidades seniores do Porto. Passo Fundo, 7(3), 335345.

Rodriguez, A. (2009). Bienestar psicológico y autoconcepto físico. In A. G. Granmontagne, El Autoconcepto Físico: Psicología y Educación (pp. 193-205). Madrid: Ediciones Pirámide.

Ryan, R. \& Deci, E. (2007). Active Human Nature: Self-Determination Theory and the Promotion and Maintenance of Sport, Exercise, and Health. In M. Hagger \& N. Chatzisarantis (Eds.), Intrinsic Motivation and Self-Determination in Exercise and Sport (pp. 1-19). Human Kinetics: Champaign. https://doi.org/10.1016/i.psychsport.2007.06.003

Silva, J. F., Rosado, A. F., Silva, C. M. \& Serpa, S. (2014). Relação entre inteligência emocional, satisfação com a vida e prática desportiva. Revista Iberoamericana de Psicología del Ejercicio y el Deporte, 9(1), 93-109.

Singh, K. \& Jha, S. (2008). Positive and Negative Affect, and Grit as predictors of Happiness and Life Satisfaction. Journal of the Indian Academy of Applied Psychology, 34, 40-45.

Watson, D., Clark, L. A. \& Tellegen, A. (1988). Development and validation of brief measures of positive and negative affect: the PANAS scales. Journal of personality and social psychology, 54(6), 405 416. https://doi.org/10.1037//0022-3514.54.6.1063

Zanon, C. \& Hutz, C. (2010). Relações entre bem-estar subjetivo, neuroticismo, ruminação, reflexão e sexo. Gerais: Revista Interinstitucional de Psicologia, 2(2), 118-127.

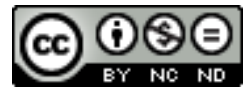

This work is licensed under a Attribution-NonCommercial-NoDerivatives 4.0 International (CC BY-NC-ND 4.0). 


\title{
Validation of the Intentionality Scale of being physically active in a Portuguese population
}

\author{
RUTH JIMÉNEZ 14 , MARTA LEYTON², MARCO BATISTA ${ }^{3}$ \\ ${ }^{1}$ Research Group on Didactic and Behavioral Analysis of Sport, Faculty of Sport Sciences, University of \\ Extremadura, Cáceres, Spain \\ ${ }^{2}$ Center for Sport Studies, University of Rey Juan Carlos, Madrid, Spain \\ ${ }^{3}$ Sport, Health and Exercise Research Unit (SHERU), Polytechnic Institute of Castelo Branco, Portugal
}

\begin{abstract}
The objective of our study was to validate the Intention to be physically active Scale to the Portuguese context, afterwards, to provide the questionnaire with concurrent validity through a correlation analysis between the variables of the levels of self-determined motivation and the intention to be physically active. The sample consisted of 308 subjects, aged between 15 and 29 years old (17.81 \pm 2.53 ). Five items evaluated the factor "Intention to be physically active". The overall results of the model indicated an optimal fit, as well as good concurrent validity. This study provided a valid and reliable scale to assess the intention to be physically active of the Portuguese population. The application of this scale is considered extremely useful especially in students. Keywords: Physical activity; Motivation; Scale.
\end{abstract}

Corresponding author. Research Group on Didactic and Behavioral Analysis of Sport. Faculty of Sport Sciences. University of Extremadura. Cáceres. Spain.

E-mail: ruthij@unex.es

Supplementary Issue: Spring Conferences of Sports Science. International Seminar of Physical Education, Leisure and Health, 17-19 June 2019. Castelo Branco, Portugal.

JOURNAL OF HUMAN SPORT \& EXERCISE ISSN 1988-5202

(c) Faculty of Education. University of Alicante.

doi:10.14198/jhse.2019.14.Proc4.82 


\section{INTRODUCTION}

The intention to be physically active, refers to the intention of a subject to continue practicing physical activity in the future, or more specifically, we could say that the intention is a predictor of behaviour and reflects the strength of the subjects to maintain said behaviours (Arias, Castejón, \& Yuste, 2013). Franco, Coterón, \& Gómez (2017) and Franco, Coterón, Gómez, \& Laura (2017), found a positive and significant correlation between the more self-determined motivation and the practice of physical activity in free time, which in turn were predictors of the intention to practice in the future. Therefore, the objective of the present study was to validate the Scale of Intentionality of Being Physically Active (EIFA) to the Portuguese context, and as a secondary objective it was intended to provide the EIFA with concurrent validity by means of a correlation analysis with the different types of self-determined motivation.

\section{MATERIAL AND METHODS}

\section{Participants}

308 people aged between 15 and 29 years (17.81 \pm 2.53$)$, Portuguese students, of whom 157 were men and 151 women took part of this study. The components of this sample group were selected by means of an intentional non-probabilistic sampling by conglomerate.

\section{Measures}

The items were extracted from the Scale of Intentionality to be Physically Active (Hein, Müür, \& Koka, 2004), and translated by a group of Portuguese experts. The questionnaire consists of 5 items, which evaluates a single factor, the intention to be physically active (e.g. "I am interested in the development of my physical form"). In order to find the concurrent validity, the level of self-determined motivation was measured by applying the Scale of the Locus Perceived of Causality in Physical Education (PLOC Scale). The Portuguese validation (Monteiro, Moutão, \& Cid, 2018) of the Perceived Locus of Causality Scale by Goudas, Biddle, \& Fox (1994) was used. It consists of 24 items, divided into 6 factors (intrinsic motivation, integrated, identified, introjected, external regulation and amotivation). The answers were collected on a Likert-type scale, whose score range is between 1 (Strongly disagree) and 5 (Strongly agree).

\section{Procedures}

Firstly, the online questionnaire was produced using the Google Form platform. Later we administered the questionnaires through different channels (WhatsApp, Facebook, Twitter), and within several Secondary Schools of Portugal.

\section{Analysis}

Descriptive values were obtained, with means and standard deviations, as well as the reliability values of the questionnaire factors. Each variable passed the normality tests through the Kolgomorov Smirnov test and the homogeneity of the variances through the Levene test (Field, 2013), which led to the use of parametric statistics. Subsequently, the confirmatory factor analysis (CFA) was carried out, in which a combination of indices was taken into account, as advised by Bentler (1995). For this reason, to determine the adjustment of the scale, the indicators recommended by Byrne (2008) were followed: $x 2, x 2 / g l, C F I$ (Comparative Fit Index), IFI (Incremental Fit Index) and SRMR (Standardized Root Mean Square Residual). Jöreskog \& Sörbom (1993) recommend that X2 / g.l. Present values below 2 , which indicates a very good fit of the model, while values below 5 are considered acceptable (Schumacker \& Lomax, 2004). In the incremental indexes (CFI, IFI), values greater than .90 are considered acceptable (Schumacker \& Lomax, 2004). The error rates RMSEA and SRMR must be less than .08 (Browne \& Cudeck, 1993; Hu \& Bentler, 1999). An internal 
consistency analysis was performed through Cronbach's alpha. Finally, a descriptive and concurrent validity analysis was carried out through an analysis of bivariate correlations. For this, the statistical package SPSS 19.0 was used. To verify if the structure of the respective factors, with their corresponding items, was correctly adjusted to the Portuguese context, confirmatory factor analysis (CFA) was carried out through the EQS 6.1 program.

\section{RESULTS}

\section{Confirmatory Factor Analysis}

The standardized factorial loads were all statistically significant $(p<.05)$. The overall results of the model indicated an optimal fit: $\mathrm{X} 2=18.76, p=.00, \mathrm{X} 2 \mathrm{~g} . \mathrm{I} .=3.15, \mathrm{CFI}=.93, \mathrm{IFI}=.93, \mathrm{GFI}=.92, \mathrm{SRMR}=.05$, RMSEA $=.06$.

\section{Internal Consistency Analysis}

The internal consistency of the Intention to be Physically Active factor presented a Cronbach's Alpha of .86, surpassing the criterion value of .70 established by Nunnally (1978) to establish an acceptable internal consistency. Also, all PLOC factors showed acceptable values of internal reliability (between .86 and .97). Concurrent Validity Analysis.

Table 1. Descriptive statistics and variable correlations

\begin{tabular}{|c|c|c|c|c|c|c|c|c|c|}
\hline & M & $S D$ & 1 & 2 & 3 & 4 & 5 & 6 & 7 \\
\hline $\begin{array}{l}\text { 1. Intention to be } \\
\text { Physically Active }\end{array}$ & 3.86 & 1.01 & - & $.165^{* *}$ & .103 & $.279^{* *}$ & $.305^{* *}$ &.-- & $.426^{\star *}$ \\
\hline 2. Intrinsic Motivation & 3.16 & 1.97 & - & - & $.863^{* *}$ & $.583^{* *}$ & $-.741^{* *}$ & $-.811^{* *}$ & $-.791^{* *}$ \\
\hline $\begin{array}{l}\text { 3. Integrated } \\
\text { Regulation }\end{array}$ & 3.56 & 1.69 & - & - & - & $.636^{* *}$ & $-.572^{* *}$ & $-.672^{* *}$ & $-.647^{* *}$ \\
\hline $\begin{array}{l}\text { 4. Identified } \\
\text { Regulation }\end{array}$ & 3.98 & 1.31 & - & - & - & - & $-.271^{* *}$ & $-.340^{* *}$ & $-.303^{* *}$ \\
\hline $\begin{array}{l}\text { 5. Introjected } \\
\text { Regulation }\end{array}$ & 3.31 & 1.42 & - & - & - & - & - & $.885^{* *}$ & $.839^{* *}$ \\
\hline 6. External Regulation & 3.08 & 1.59 & & - & - & - & - & - & $.948^{* *}$ \\
\hline 7. Amotivation & 3.13 & 1.41 & - & - & - & - & - & - & - \\
\hline
\end{tabular}

Note: ${ }^{*} p<.05 ;{ }^{* *} p<.01 ;$ M: Media; SD: Standard Deviation.

\section{DISCUSSION AND CONCLUSIONS}

The EIFA validated to Portuguese presented optimal results, in addition to adequate concurrent validity. Chicote-López, Abarca-Sos, Gallardo, \& García-González (2018) and Franco, Coterón, \& Gómez (2017) in a model of structural equations found positive and significant predictions between intrinsic motivation and the intention to be physically active. In conclusion, this study has enabled the Portuguese to be validated by the EIFA, proving that it is a valid and reliable instrument to assess the intention to be physically active in a Portuguese population between 15 and 29 years of age.

\section{FUNDING}

This study was carried out thanks to the contribution of the Ministry of Economy and Infrastructure of the Council of Extremadura, through the European Regional Development Fund. A way to make Europe. 
(GR18129). We would like to specify that the financial assistance is only for the Professor Ruth Jiménez Castuera.

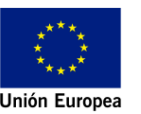

\section{JUNTA DE EXTREMADURA}

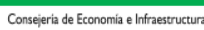

\section{REFERENCES}

Hein, V., Müür, M., \& Koka, A. (2004). Intention to be physically active after school graduation and its relationship to three types of intrinsic motivation. European Physical Education Review, 10, 5-19. https://doi.org/10.1177/1356336x04040618

Monteiro, D., Moutão, J. M., \& Cid, L. (2018). Validation of the Behavioural Regulation in Sport Questionnaire in Portuguese athletes. Revista de psicología del deporte, 27(1), 145-150. https://doi.org/10.1037/t68847-000

*To consult the complete bibliography of the work, contact the author of reference.

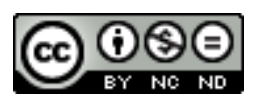

This work is licensed under a Attribution-NonCommercial-NoDerivatives 4.0 International (CC BY-NC-ND 4.0). 


\title{
Motivations for the practice of adventure and nature physical activities on young people
}

\author{
NUNO SILVA ${ }^{1,2} \square$, MARCO BATISTA ${ }^{1,2,3}$, ANTÓNIO FAUSTINO ${ }^{1,2}$, AFONSO LERCAS $^{1}$, JOÃO \\ PETRICA ${ }^{1,2}$ \\ ${ }^{1}$ Polytechnic Institute of Castelo Branco, Portugal \\ ${ }^{2}$ Sport, Health and Exercise Research Unit (SHERU), Portugal \\ ${ }^{3}$ Research in Education and Community Intervention, RECl- Instituto Piaget, Portugal
}

\begin{abstract}
Despite the lack of prominence given to adventure and nature physical activities (ANPA) that involve direct contact with nature, there has been a growing interest in the integration of this type of activities in the schools' curriculum due to its' positive effects in students' physical, social and psychological development. In this context, the goal of this study is to examine what are the antecedents of public schools' students' choice to participate in this type of activities. To achieve this goal, we conducted a study ( $n=183$, 3rd cycle of the Portuguese basic education) in which we compared the motivation of public school's students that offer and don't offer ANPAs in the engagement or disengagement in physical activities, using the Questionnaire of Motivation in Sport Activities (QMSA) and the Survey of Motivation for Absence of Sport Activities (SMASP). Our results suggest that students that practice ANPAs are more motivated to engage on the activities to develop their physical fitness and skill development. In relation to student's who don't practice, we notice that even to there are many that can do ANPAs in school don't practice mainly because the alleged lack of time. Keywords: Adventure and nature physical activities; Self-determination: Motivation; Physical education.
\end{abstract}

Corresponding author. Instituto Politécnico de Castelo Branco, Av. Pedro Alvares Cabral 12, 6000-084 Castelo Branco, Portugal.

E-mail: ruthij@unex.es

Supplementary Issue: Spring Conferences of Sports Science. International Seminar of Physical Education, Leisure and Health, 17-19 June 2019. Castelo Branco, Portugal.

JOURNAL OF HUMAN SPORT \& EXERCISE ISSN 1988-5202

(c) Faculty of Education. University of Alicante.

doi:10.14198/jhse.2019.14.Proc4.82 


\section{INTRODUCTION}

In general, sport has been considered a very important component for a healthier lifestyle (Melo \& Campos, 2015; Pereira, 2013), where we can include ANPA in which it's possible to enjoy the multiple benefits and practice in a more natural context (Serrano \& Petrica, 2011). So, we decided to investigate what are the motivations of young people for the practice or not, of these activities considering if the school offers in their curriculum this kind of activities.

\section{MATERIAL AND METHODS}

\section{Participants}

Were evaluated 183 students from two schools with and without the educational offer of ANPA. Students have ages between 12 and 19 years old $(13,95 \pm 1,17)$, which $86(47 \%)$ are females and $97(53 \%)$ are males.

\section{Measures}

We used Questionnaire of Motivation for Physical Activities (QMPA; Serpa \& Frias, 1991) traduced and adapted from Participation Motivation Questionnaire (PMQ; Gill et al, 1983) and the Survey of Motivations for Absence of Sport (SMAS; Pereira \& Vasconcelos-Raposo, 1998).

\section{Procedures}

Before any student could participate on this study we talked to the school's directors and asked for permission to the student's guardians. After that we made a guide and explain to the Physical Education teacher's how students had to fill the questionnaires.

\section{Analysis}

With the data collected and inserted in SPSS (version 21.0) we started by the descriptive values of our variables, made the normality test (Kolmogorov-Smirnov) and then used the independent T-test for the parametric variables (emotions and status) and for the rest of the variables, non-parametric we used U-MannWhitney.

\section{RESULTS}

We hypothesised that students that practice ANPAs in school have better motivational values for the practice, than students who don't have access in school. In table 2 we show the variables of both instruments with their descriptive values and the level of significance considering if the school offers ANPAs or not.

Table 1. Descriptive values and level of significance of our variables considering Scholar Offer of ANPA

\begin{tabular}{ccccccc}
\hline Variables & $\begin{array}{c}\text { Mean } \pm \text { Stand. } \\
\text { Deviation }\end{array}$ & $\begin{array}{c}\text { Scholar } \\
\text { Offer }\end{array}$ & $\mathbf{N}$ & Mean & $\begin{array}{c}\text { Stand. } \\
\text { Deviation }\end{array}$ & Sig. \\
\hline \multicolumn{2}{c}{ Questionnaire of Motivation for Physical Activities (QMPA) } \\
\hline Specific Affiliation & $3.86 \pm 0.82$ & Yes & 50 & 3.74 & 0.71 & 0.28 \\
& & No & 110 & 3.60 & 0.82 & \\
General Affiliation & $3.64 \pm 0.79$ & Yes & 50 & 3.86 & 0.81 & 0.98 \\
Completion & & No & 112 & 3.86 & 0.83 & \\
Skill Development & $3.02 \pm 0.93$ & Yes & 50 & 3.25 & 0.84 & $0.02^{*}$ \\
& $3.78 \pm 0.77$ & No & 111 & 2.91 & 0.95 & 0.92 \\
\hline
\end{tabular}




\begin{tabular}{|c|c|c|c|c|c|c|}
\hline \multirow{3}{*}{ Emotions } & & No & 112 & 3.78 & 0.82 & \multirow{3}{*}{0.28} \\
\hline & & Yes & 50 & 3.61 & 0.87 & \\
\hline & $3.0 \angle \pm 0.80$ & No & 109 & 3.47 & 0.86 & \\
\hline \multirow[t]{2}{*}{ Status } & $3.02 \pm 0.85$ & Yes & 50 & 3.14 & 0.79 & \multirow[t]{2}{*}{0.22} \\
\hline & & No & 109 & 2.96 & 0.87 & \\
\hline Physical Fitness & $4.13 \pm 0.75$ & $\begin{array}{l}\text { res } \\
\text { No }\end{array}$ & $\begin{array}{c}50 \\
112\end{array}$ & $\begin{array}{l}4.09 \\
4.15\end{array}$ & 0.76 & 0.5 \\
\hline \multirow{2}{*}{ Pleasure } & & Yes & 50 & 3.58 & 0.69 & \multirow{2}{*}{0.36} \\
\hline & $3.47 \pm 0.85$ & No & 112 & 3.42 & 0.91 & \\
\hline \multicolumn{7}{|c|}{ Survey of Motivations for Absence of Sport (SMAS) } \\
\hline Sports & & Yes & 142 & 2.05 & 0.77 & \multirow{2}{*}{0.49} \\
\hline Aversion/Dissatisfaction & $2.06 \pm 0.16$ & No & 15 & 2.19 & 0.58 & \\
\hline \multirow{2}{*}{ Aesthetics/Incompetence } & $227+079$ & Yes & 141 & 2.23 & 0.81 & \multirow{2}{*}{0.15} \\
\hline & $2.27 \pm 0.19$ & No & 16 & 2.54 & 0.63 & \\
\hline \multirow{2}{*}{ Lack of Support/Conditions } & $2.35 \pm 0.79$ & Yes & 147 & 2.34 & 0.78 & \multirow{2}{*}{0.47} \\
\hline & & No & 14 & 2.5 & 0.90 & \\
\hline \multirow{2}{*}{$\begin{array}{l}\text { Disinterest for Physical } \\
\text { Effort }\end{array}$} & $2.24 \pm 0.84$ & Yes & 147 & 2.22 & 0.86 & \multirow{2}{*}{0.28} \\
\hline & & No & 16 & 2.46 & 0.64 & \\
\hline Lack of Time & $2.57 \pm 0.97$ & Yes & 146 & 2.51 & $\begin{array}{l}0.95 \\
102\end{array}$ & $0.04^{*}$ \\
\hline
\end{tabular}

\section{DISCUSSION}

The results show that students with scholar offer have higher mean values on most of the motivational variables for the practice, than students who don't have ANPAs school offer, on which we find statistical significance on "competition". For the absence of practice, students with no offer show higher mean values on all the variables, and we only found statistical differences on the "lack of time". These results are opposites to studies like Costa (2005) and more recently Melo e Gomes (2017), however in relation to the most valued motivations for the practice (physical fitness, skills development and pleasure), the results are shared with some studies (Veigas et al., 2009; Sousa, 2004; Cid, 2002). The lack of time it's the most valued motivational variable for the absence of practice, which is also referred by a recent publication of the World Health Organization (2018) and other authors (Melo \& Gomes, 2017; Correia, 2014; Fernandes et al., 2005).

\section{CONCLUSIONS}

In relation to the motivation to practice ANPAs we see that there aren't many students practicing AFANs on school, maybe not mainly because of the offer but also for the lack of time.

\section{REFERENCES}

Cid, L. F. (2002). Alteração dos motivos para a prática desportiva das crianças e jovens. Lecturas: Educación Física y Deportes, 8(55), 1-2.

Correia, T. M. (2014). Dissertação de Mestrado em Ensino da Educação Física nos Ensinos Básico e Secundário. Maia: Instituto Universitário da Maia. https://doi.org/10.22355/exaequo.2015.31.02

Costa, P. (2005). Motivação para a prática de actividade física de aventura na natureza. Coimbra: Faculdade de Ciências da Universidade de Coimbra. https://doi.org/10.24873/..rpemd.2017.05.012 
Fernandes, H., Lázaro, J., \& Vasconcelos-Raposo, J. (2005). Razões para a não prática desportiva em adultos: Estudo comparativo entre a realidade rural e urbana. Motricidade, 1(2), 106-114.

Melo, R., \& Campos, F. (2015). Hábitos Desportivos e Perspetivas de Participação em Atividades Físicas Desportivas dos Estudantes do Politécnico de Coimbra. Exedra: Revista Ciêntifica, 94-108.

Melo, R., \& Gomes, R. (2017). Nature sports participation: Understanding demand, practice profile, motivations and constraints. European Journal of Tourism Research, 16, 108-135.

Pereira, F. C. (2013). Motivação para a prática de atividades físicas de aventura. Rio Claro: Universidade Estadual Paulista "Júlio de Esquita Filho" Instituto de Biociências. https://doi.org/10.18362/bjta.v6.i2.11 


\title{
The Cooperative Games with children: Communitarianism and citizenship
}

\author{
ANTÓNIO CAMILO CUNHA 1 , CHRISTINE VARGAS LIMA² \\ ${ }^{1}$ Universidade do Minho (UMinho), Portugal \\ 2Universidade Estadual do Centro Oeste (UNICENTRO), Brazil. CIEC, Portugal
}

\begin{abstract}
The communication is based on an investigation (of doctorate realized by Christine Vargas Lima - 2019) on the cooperative games. The central problem was the cooperative games and the construction of citizenship. This problem originated in the observation of children plagued by floods in southern Brazil in 2014 and in the verification that the game (s) (cooperative) was decisive in mitigating pain and at the same time was decisive in the relationship, affective, collaborative relationships between children and between children and adults. Faced with this empirical evidence, a cut in the investigation was established, in which a school of the neighbourhood reached by the floods was analysed. Thus, through participant observation, field diary and interviews, it was attempted to know the characteristics, representations, languages about games (cooperative) of thirty five children. One of the results (conclusions - we will present the ones related to children) of this study showed that cooperative games constitute a first manifestation of "living together" and therefore one of the first forms of human organization - communitarianism. Keywords: Games; Cooperation; Childhood; Citizenship; Education; Praxis.
\end{abstract}

Corresponding author. Rua Jesuíno Marcondes, 520 - Santa Cruz, Guarapuava, PR - Brasil.

E-mail: cvargaslima@gmail.com

Supplementary Issue: Spring Conferences of Sports Science. International Seminar of Physical Education, Leisure and Health, 17-19 June 2019. Castelo Branco, Portugal.

JOURNAL OF HUMAN SPORT \& EXERCISE ISSN 1988-5202

(c) Faculty of Education. University of Alicante.

doi:10.14198/jhse.2019.14.Proc4.82 


\section{INTRODUCTION}

This research aims to deepen the studies related to cooperative games and the construction of citizenship (Garairgordobil , 2009; Camilo Cunha, 2014; Solerm 2002; Brown, 1994; Brotto, 2001; Brougère, 2002; Lima, 2018). It originated the observation of children plagued by the floods in southern Brazil in 2014, where it was possible to observe that the Cooperative Games were decisive in alleviating pain and, at the same time, creating affective and collaborative bonds between children and they and the adults. To contribute to this challenge, with a view to better education, we propose to carry out an investigation with cooperative games with school children. Learning focused on dialogue, on relationships between people, on creation and on meaning, are constitutive forms of being a citizen. In this context, we take Cooperative Games as an investigative undertaking, because it is our conviction (based on experience and literature) that cooperative games contribute to the communication, with the citizen relations of children and adults, for the attribution of meanings, not only in school contexts, but also in the social fabric. We believe that through cooperative play, we can glimpse learning, with pleasurable and committed experiences - foundations for a committed and citizen's adult life.

\section{MATERIAL AND METHODS}

To obtain the data, interviews with parents, students and teachers of the Municipal School of Guarapuava, Paraná, Brazil, were carried out. In addition to the interviews, participant observation was used, and this contributed to a better understanding of the field of study. The instruments selected for the collection of research data are: participant observations, field diary and interviews. (Lakatos, 2010; Ludke \& André, 2013; Bardin, 2011; Yin, 2010; Stake, 2011; Graue, 2003).

\section{Participants}

The sample consisted of thirty five children, eight parents, nine teachers and one employee of the Municipal School of Guarapuava, in the state of Paraná, Brazil. The children investigated are students of the third year of elementary school, aged between seven and eleven, are middle class low and reside in Vila Bela, Vila Jardim das Américas, Aeroporto, Batel and Vila São Vicente, the latter with greater number of children in school.

\section{Measures}

Since this is a case study with a qualitative approach, the research interviews were elaborated according to the practical intervention in which one of the methodological stages of the research was studied, as well as validated by two female doctoral professors from the State University of the Central West - UNICENTRO, Parana Brazil.

\section{Procedures}

The instruments selected for the collection of research data are: participant observations, field diary and interviews. They are procedures compatible with the research and are consistent with the qualitative approach, Stake (2011), whose characteristics are of the human understanding and interpretation, representing the personal experiences of the participants in specific situations.

\section{Analysis}

By means of interviews and practical intervention, these prayers are decoded into categories (according to Bardin) and subsequently into subcategories. We also carried out a trilogy of the interviews (parents, students and teachers) based on a theoretical framework where the importance of the connections that must be made 
by these groups was perceived, so that the school can propose an interdisciplinary project that fills the voids and fragilities existing in the school routine.

\section{RESULTS}

Before the data analysis, a practical pedagogical proposal involving the research actors was elaborated.

\section{DISCUSSION}

Children play regardless of the state they are in. But they need to be presented and presented to the world around them. There is a need for an interaction between family, school and society. Public policies must meet the needs of the school so that these children can assume the role of citizenship.

\section{CONCLUSIONS}

After the investigative walk, we arrive at the main conclusions of this study that are presented in sequence through a synthesis of the representations and practices of children, teachers, parents.

- The praxis of cooperative games was constituted as a form of construction of the citizenship of the children. The games allowed the dialogue, the interest, the very close relationship between them. As the group decided and with the autonomy they were given to play, they were building new relationships and moving with sovereignty and wisdom to a desired citizenship. It has been confirmed that children's patterns of behaviour result from the model to which they are exposed. When they are "plastered", static / instrumentalized, for a long time, in the classroom portfolios, they demonstrated this behaviour in the games through more rigid attitudes. Conversely, when children play freely they are creative and spontaneous in their relationships and attitudes.

- We also find that when they are exposed to certain rules decided by the groups, some of them feel threatened to "like" the rules of adults and express aggressive attitudes, because they do not know another way to position themselves on what is new and strange to them. That is why we believe that it is important to foster dialogue among children so that they demonstrate and develop positive values in the group and feel confident in the autonomy they are offered to continue their natural evolution. At the beginning of the pedagogical intervention with games, the children brought from their daily feelings contradictions, sometimes sadness, fear, sometimes joy, laughter, contentment. With the cooperative games, there was the possibility of dialogue and played equally. This communication is perceived in the language and practice of games / bodies, which allowed for the overcoming and communion raising in them the idea of individual (s) and citizens.

- $\quad$ The children liked to play in the community, because they played with their brothers, cousins and classmates at school. Their statements were very significant, highlighting the ideas / practices of cooperation, solidarity and participation. Most kids enjoyed playing collective games, this is another essential feature of cooperative games. Games like pick-up, futpar, stars, little feet and little hands were some games more cited by the children. In them, the children felt more freedom and autonomy in playing, expressing the idea of being respected in their needs and opinions.

\section{REFERENCES}

Brown, G. (1994). Jogos Cooperativos: Teoria e Prática. São Leopoldo, RS: Editora Sinodal. 
Camilo Cunha, A. (2014). Multiculturalismo e Educação da diversidade: Crítica/Crítica. Coleção Intuições ( $1^{\mathrm{a} e}$ edição). Santo Tirso, junho de 2014 IEC-UM.

Freire, B. (2002). O Jogo: entre o riso e o choro. São Paulo: Spicione.

Ludke, M. \& Andre, M. (2013).Pesquisa em Educação: abordagem qualitativa. São Paulo. Editora Pedagógica e Universidade.

Lima, C. (2018). Os Jogos Cooperativos com crianças:Construindo caminhos de cidadania. Tese de Doutoramento em Estudos da Criança. Instituto de Educação da Universidade do Minho. https://doi.org/10.22355/exaequo.2015.32.09 


\title{
Assessent of Portuguese wheelchair basketball team motivation and anxiety levels
}

\author{
JOÃO ROCHA ${ }^{1,2} \triangleleft$, ANA DOMINGUES ${ }^{1}$, INÊS FERNANDES ${ }^{1}$, JOÃO DOMINGUES ${ }^{1}$, SANDRA \\ RIBEIRO1, PAULO SILVEIRA ${ }^{1,2}$, MARCO BATISTA ${ }^{1,2}$, JOÃO SERRANO ${ }^{1,2}$, JOÃO PETRICA ${ }^{1,2}$, RUI \\ PAULO 1,2, PEDRO MENDES ${ }^{1,2}$, SAMUEL HONORIO1,2, JORGE SANTOS ${ }^{1,2}$, ANTÓNIO FAUSTINO ${ }^{1,2}$ \\ ${ }^{1}$ Escola Superior de Educação do Instituto Politécnico de Castelo Branco, Portugal \\ ${ }^{2}$ Sport Health \& Exercise Research Unit, Portugal
}

\begin{abstract}
Adapted sports is an organized activity with the objective of improving physical and psychic conditions, it represents one of the most important factors promoting educational success, inclusion and psychosocial development (Celestino \& Pereira, 2017). The objective of the study focuses on motivational and anxiety aspects of high-competition athletes from the Portuguese wheelchair basketball team (WCB). We sought to observe how this special and elite population reacts psychologically to sports practice adapted to high performance, more precisely the relationship between motivation and the classification of athletes in the WCB according to their type of disability (acquired or congenital) and anxiety with the classification of athletes in the WCB according to their type of disability (acquired or congenital). The sample consisted on 14 athletes of WCB, males gender, and aged between 19 to 40 years. The instruments used were questionnaires: The Behavioural Regulation in Sport Questionnaire; The Basic Psychological Needs in Exercise Scale; Sport Competition Anxiety Test. We collect the information defined by the model of analysis referenced in the literature, having been treated in the SPSS software program. We conclude that sports practice has a significant positive effect on autonomous motivation and in the control of anxiety levels in adapted sports athletes. In the motivation, statistically significant differences were observed among athletes according to their classification. In the anxiety, when relating the variables, the athletes with a classification higher than 3 obtained more positive results. Keywords: Adapted sport; Wheelchair basketball Portuguese National team; Motivation; Anxiety.
\end{abstract}

Corresponding author. Escola Superior de Educação do Instituto Politécnico de Castelo Branco, Portugal.

E-mail: joao.rocha@ipcb.pt

Supplementary Issue: Spring Conferences of Sports Science. International Seminar of Physical Education, Leisure and Health, 17-19 June 2019. Castelo Branco, Portugal.

JOURNAL OF HUMAN SPORT \& EXERCISE ISSN 1988-5202

(c) Faculty of Education. University of Alicante.

doi:10.14198/jhse.2019.14.Proc4.82

S1812 | 2019| Proc4| VOLUME 14

C 2019 University of Alicante 


\section{INTRODUCTION}

Batista (2019) mentions several authors (Leal, Miranda and Carmo, 2013, Vallerand, 2007, Ryan \& Deci, $2000 \mathrm{a}$, among others) who consider that there are three types of motivation, the intrinsic, which refers to the realization of the activity by personal interest and the peaceful feelings that come from this same practice. The extrinsic motivation that is associated with practice by external factors. And the amotivation as lack or absence of intent to perform certain behaviours. In the sense of understanding the levels of motivation and anxiety of the athletes of the national basketball team in wheelchair, we observe the role and explanatory power of the competencies of confrontation with stressful and problematic situations have been taking on an Increasing role in the scientific literature of sports psychology (Dias, 2005). Questionnaires have already been applied in the population with motor disabilities, but not at the level of adapted sports from elite basketball in Portugal. Thus, we want to see if there are statistically significant differences in the levels of motivation and anxiety confronted the classification of the athletes in WCB according with type of disability, where we believe that players with more motor capacities are less anxious.

\section{MATERIAL E METHODS}

The methodology employed in the implementation of this study is quantitative, descriptive, exploratory and transversal, similar to the according with Batista et al. (2018).

\section{Participants}

In this study, 14 athletes from the national team of WCB, aged between 19 and 40 years, $(\dot{X}=28.79)$ with motor impairment, of the acquired or congenital type, participated. The athletes have an average of 10.50 years of practice, 3.07 weekly workouts and consequently 2.29 hours of weekly training.

\section{Instruments}

The questionnaires applied were: The Behavioural Regulation in Sport Questionnaire (Monteiro, Moutão \& Cid, 2018); The Basic Psychological Needs in Exercise Scale (Monteiro, Marinho, Moutão, Couto, Antunes \& Cid, 2016); Sport Competition Anxiety Test (Serpa, Pereira \& Freitas, 1991).

\section{Procedures}

After applying the questionnaires, we proceeded to its analysis through the statistical program SPSS (Statistical Package for the Social Sciences) in its version 20.0.

\section{Analysis}

We used a descriptive statistic that allowed us to reach minimum values, maximum, averages, standard deviation. In order to understand whether there were statistically significant differences in the levels of motivation and anxiety confronted the classification of the athletes in WCB and the type of disability, we resorted to the correlation and comparison test, according to their normal or unnormal distribution. We checked through the Cronbach's alpha tests to assess the level of internal consistency of each dimension of the questionnaire in order to estimate the uniformity of the items and their reliability. After assessing the internal consistency of the questionnaires, we verify the normality of the sample through Shapiro-Wilk, and the parametric and non-parametric tests were used. 


\section{RESULTS}

Regarding motivation, the athletes showed a high autonomous motivation ( $\dot{X}=6.34)$, presenting low values of amotivation ( $\dot{X}=1.64)$ and controlled motivation $(\dot{X}=3.38)$, there was still a non-normal distribution in this variable according to the type of Disability and classification groups $(p<0.05)$, where statistically significant differences between athletes were observed. In the basic psychological needs the autonomy, relation and competence perception, the athletes present high results in the autonomy perception ( $\dot{X}=4.07)$, in the relationship perception $(\dot{X}=4.50)$ and in the competence perception $(\dot{X}=4.04)$. In anxiety, athletes in general indicated $(\dot{X}=16.36)$ points related to anxiety trait. When the variables were related, the athletes with a classification higher than 3 obtained more positive results $(\dot{X}=1.55)$, which measured that athletes with a rating lower than $3(\dot{X}=1.70)$ tended to be more nervous. Regarding the type of disability, we observed the same orientation of data.

\section{DISCUSSION}

The high results that the Portuguese national team of WCB presents in the self-determined motivation for the practice of physical activity will meet the studies of Rolo (2003), Ferreira (2017) and Lercas (2018), where the latter author indicates results of a motivation Autonomous ( $\dot{X}=5.77)$. In the study by Corrêa (2016) We observed that older athletes $(\dot{X}=15.69)$ and with longer practice time tended to have lower anxiety values, and these results were in the same way of our population.

\section{CONCLUSIONS}

The athletes of the Portuguese WCB team show high values at the level of autonomous motivation, where we see that in the basic psychological needs, more specifically in the autonomous, competent and relations perception as moderate correlation with the Autonomous motivation, showing lower values of amotivation and controlled motivation. As for anxiety, athletes with a rating higher than 2.5 points are less likely to be nervous, thus showing that athletes with lower ranking tend to be more nervous, confirming our hypothesis.

\section{REFERENCES}

Batista, M., Lercas, A., Santos, J., Honório, S., Serrano, J. \& Petrica, J. (2018). Practice motivation and life satisfaction of athletes of team sports: Comparative study between adapted and regular sport. Journal of Human Sport \& Exercise, 14(1), p. 20-29. https://doi.org/10.14198/jhse.2019.14.proc1.03

Celestino, T. \& Pereira, A. (2017). Os valores e a ética no desporto adaptado: uma revisão bibliográfica. Revista Científica da FPDD, 3(1), 20-28.

Corrêa, W. (2016). Basquete Sobre Rodas: Análise da Ansiedade Pré-Competitiva. Revista da Associação Brasileira de Atividade Motora Adaptada, 17(2), 31-36.

Dias, C. (2005). Do stress e ansiedade às emoções no desporto: Da importância da sua compreensão à necessidade da sua gestão. Braga: Instituto de Educação e Psicologia da Universidade do Minho. https://doi.org/10.21011/apn.2017.1502

Monteiro, D.; Moutão, J. \& Cid, L. (2018). Validation of the Behavioral Regulation Sport Questionnaire in Portuguese athletes, Revista de Psicologia del Desporte 27(1) 145 - 150. https://doi.org/10.1037/t68847-000

Monteiro, D.; Marinho, D.; Moutão, J.; Couto, N.; Antunes, R. \& Cid, L. (2017). Adaptation and validation of the portuguese version of Basic Psychological Needs Exercise Scale to the sport domain and 
invariance across football ans swimming. Revista Motricidade, 12(4), 51-61. https://doi.org/10.6063/motricidade.9372

Serpa, S. \& Santos, A. (1991). Ansiedade competitiva. Relação treinador atleta - Estudo da influência do treinador em atletas de ténis. Tese de licenciatura. Cruz-Quebrada: Faculdade de Motricidade Humana.

Serpa, S.; Pereira, F. \& Freitas, M. (1991). Sport Competition Anxiety Test. Adaptação à população portuguesa. Cruz Quebrada: Faculdade de Motricidade Humana.

\section{(c) (i) $\ominus$}

This work is licensed under a Attribution-NonCommercial-NoDerivatives 4.0 International (CC BY-NC-ND 4.0). 


\title{
Assessment of physical capacities of the Portuguese wheelchair basketball team
}

\author{
JOÃO ROCHA ${ }^{1,2} \triangleleft$, ANA DOMINGUES ${ }^{1}$, INÊS FERNANDES ${ }^{1}$, JOÃO DOMINGUES ${ }^{1}$, SANDRA \\ RIBEIRO1, PAULO SILVEIRA ${ }^{1,2}$, MARCO BATISTA ${ }^{1,2}$, JOÃO SERRANO ${ }^{1,2}$, JOÃO PETRICA ${ }^{1,2}$, RUI \\ PAULO 1,2, PEDRO MENDES ${ }^{1,2}$, SAMUEL HONORIO1,2, JORGE SANTOS ${ }^{1,2}$, ANTÓNIO FAUSTINO ${ }^{1,2}$ \\ ${ }^{1}$ Escola Superior de Educação do Instituto Politécnico de Castelo Branco, Portugal \\ ${ }^{2}$ Sport Health \& Exercise Research Unit, Portugal
}

\begin{abstract}
Wheelchair basketball (WCB) is an intermittent effort sport, where resistance is considered as the most important physical capacity, associated with repeated intense effort during a long period. The aim of this study is to classify the levels of physical capacities in athletes of the national team of WCB. In this wat, we examined speed, endurance, agility, power of upper limbs and handgrip, according to adapted sports practice. Our sample consisted of 14 athletes from the Portuguese WCB national team at the senior level, aged between 19 and 40 years. Through the application of several physical tests, we collected the information defined by our analysis model, and treated in the SPSS software program. In general, we can conclude that compared with other studies we observed that there are significant differences in the level of Speed, agility tests where Portuguese WCB national team shown higher performance we see the opposite in power and endurance tests. Keywords: Adapted sport; Portuguese wheelchair Basketball National Team; Speed; Agility; Power; Resistance.
\end{abstract}

Corresponding author. Escola Superior de Educação do Instituto Politécnico de Castelo Branco, Portugal.

E-mail: joao.rocha@ipcb.pt

Supplementary Issue: Spring Conferences of Sports Science. International Seminar of Physical Education, Leisure and Health, 17-19 June 2019. Castelo Branco, Portugal.

JOURNAL OF HUMAN SPORT \& EXERCISE ISSN 1988-5202

(c) Faculty of Education. University of Alicante.

doi:10.14198/jhse.2019.14.Proc4.82 


\section{INTRODUCTION}

Wheelchair basketball (WCB) is defined in physical and physiological terms by Yanci et al. (2015), as an intermittent activity, which combines repeated moments of short and intense exercise that include fast running, acceleration, deceleration, dynamic changes of position, maintenance and obtaining new positions in the field. As for the level of intensity, the same authors consider that it is characterized by numerous periods of high or maximum intensity exercise and sprint actions with brief periods of recovery between. They also mention that both aerobic and anaerobic energy systems are activated to meet the energetic demands of the muscles during the game. Thus, physical fitness measurements (Sprint, agility, strength, heart rate, lactate concentrations) are usually included in test batteries to evaluate the performance of WCB players (Vanlandewijck et al., 2004) serving as control, to realize the player's performance. The aim of this study is to assess the level of physical performance of the athletes of the national selection of BCR, and to perceive their performance status in relation to the results of other studies.

\section{MATERIAL AND METHODS}

The methodology employed in the implementation of this study is quantitative, descriptive, exploratory and transversal.

\section{Participants}

In this study, 14 athletes from the national team of WCB, aged between 19 and 40 years, $(\dot{X}=28.79)$ with motor disability, of acquired or congenital type, participated. The athletes have an average of 10.50 years of practice, 3.07 weekly workouts and consequently 2.29 hours of weekly training.

\section{Instruments}

The physical tests applied were: Modified Agility Test (Belasco e Silva, 1998), Medical Ball Throw Test (Johnson and Nelson, 1986), Running Anaerobic Sprint Test (Draper \& Whyte, 1997), Handgrip Test (Yanci et al., 2015), Yo-Yo test Interval resistance (Krustrup et al., 2003).

\section{Procedure}

After the results registration of the tests, we proceeded to its analysis through the statistical program SPSS (Statistical Package for the Social Sciences) in its version 2.0, thus guaranteeing the credibility of data collection.

\section{Analysis}

When the data were collected, we used the categorical variables analysis through descriptive statistics that allowed us to reach minimum values, maximum, means, standard deviation. In order to know the existence of statistically significant differences in the levels of physical capacity, taking into account speed, agility, endurance, upper limbs and handgrip, we used the correlation test, due to its Normal or non-distribution, so as to accept or reject our study hypotheses.

\section{RESULTS}

The athletes revealed in the upper limbs power test with a basketball ball $\dot{X}=10.33$ meters. The $3 \mathrm{~kg}$ medicinal ball Launch Test presented $\dot{X}=5.34$ meters. At the handgrip level, the left-hand values of $\dot{X}=28.38 \mathrm{~kg}$ and values with the right-hand of $\dot{X}=27.62 \mathrm{~kg}$ were obtained. In the speed test $(20 \mathrm{~m})$ without ball, players presented $\dot{X}=5.34$ seconds and with ball $\dot{X}=6.18$ seconds. The agility test obtained $\dot{X}=13.99$ seconds. The 
Yo-Yo test showed $\dot{X}=790$ meters, with a standard deviation value of $440 \mathrm{~m}$, which indicates very distinct values among the athletes.

\section{DISCUSSION}

Comparing the results obtained with the study by Yanci et al., (2015), in the Yo-Yo test, the players of the Portuguese WCB National Team presented lower values $(\dot{X}=790 \pm 440 \mathrm{~m}$.) than those obtained in Yanci et al., (2015) test $(\dot{X}=1014 \pm 396,15 \mathrm{~m})$. In the power test of upper limbs with basketball ball $\dot{X}=10.33$ meters, above $(8.39 \pm 1.77 \mathrm{~m})$ of Yanci et al., (2015) test, but Lower to what this author points out of the results obtained by the players of the Belgian 1st League of WCB of $14.6 \pm 4.2 \mathrm{~m}$. In the throwing test with medicinal ball of $3 \mathrm{~kg}$ present $\dot{X}=5.34$ meters higher than $3.9 \pm 1.1$ of Ferreira et al, (2017). In Handgrip obtained values with the left hand $\dot{X}=28.38 \mathrm{~kg}$, less than $47.6 \pm 11.5$ of Ferreira et al, (2017), with the right-hand obtained values $\dot{X}=27.62 \mathrm{~kg}$ less than $52.5 \pm 10.7$ of Ferreira et al, (2017). In the test of the speed without ball, the players present values $\dot{X}=5.34$ seconds, lower to the study by Yanci et al., (2015) in relation to the test with ball showed values of $\dot{X}=6.18$ seconds, also lower to $6.59 \mathrm{~s}$ of the same authors. At the level of agility, $\dot{X}=$ 13.99 seconds lower than $15.91+1.35$ obtained by Belasco Junior and Silva (1998).

\section{CONCLUSIONS}

When comparing results with other studies of the same type, we found that the speed and agility tests of the players from the Portuguese WCB National Team are better than the comparative studies. When comparing to the strength and endurance tests (Throwing, Handgrip and Endurance), the values are lower. A possible justification for performance that we observed, can be that the elite level in Portugal and their teams with regard to the WCB give a great deal of development of the specific capabilities of the BCR game and less attention to general physical component workout.

\section{REFERENCES}

Belasco, D. \& Oliveira, R. (1997). Consistência dos resultados do teste de corrida em ziguezague de Barrow (modificado) em jogadores de basquetebol em cadeira de rodas. São Paulo: Escola Paulista de Medicina. https://doi.org/10.29381/0103-8559/2019290134-42

Coutts D. (1992). Dynamics of wheelchair basketball. Med Sci Sports Exer. 24, 231-234.

Draper, N. \& Whyte, G. (1997). Here's a new running based test of anaerobic performance for which you need only a stopwatch and a calculator. Peak Performance, 97, 3-5.

Gil-Agudo, A., Del Ama-Espinosa, A., Crespo-Ruiz B. (2010). Wheelchair basketball quantification. Phys Med Rehabil Clin North Am, 21(1), 141-156. https://doi.org/10.1016/j.pmr.2009.07.002

Ferreira, S. (2017). Morphological characteristics, muscle strength, and anaerobic power performance of wheelchair basketball players. Revista Brasileira de Cineantropometria Desempenho Humano, 17(3), 343-353.

Krustrup, P.; Mohr, M.; Amstrup, T.; Rysgaard, T.; Johansen, J.; Steensberg, A.; Pedersen, K. \& Bangsbo, J. (2003). The yo-yo intermittent recovery test: physiological response, reliability, and validity. Medicine Science Sports Exercise, 35(4), 697-705. https://doi.org/10.1249/01.mss.0000058441.94520.32

Lopes, C. (2005). Análise das capacidades de resistência, força e velocidade na periodização de modalidades intermitentes. Dissertação de Mestrado. Brasil: Faculdade de Educação Física da Universidade de Campinas. https://doi.org/10.32385/rpmgf.v29i2.11056 
Vanlandewijck, C., et al., (2004). The relationship between functional potential and field performance in elite female wheelchair basketball players. J Sports Sci, 22, 668-675. https://doi.org/10.1080/02640410310001655750

Yanci, J., et al., (2015). Sprint, agility, strength and endurance capacity in wheelchair basketball players. Biology of Sport,32(1),71-78. https://doi.org/10.5604/20831862.1127285

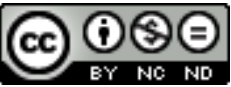

This work is licensed under a Attribution-NonCommercial-NoDerivatives 4.0 International (CC BY-NC-ND 4.0). 


\title{
Sociocultural animation in 1st cycle for educational sucess
}

\author{
BRUNO TRINDADE 1,3 , MARIA JOSÉ CONDE ${ }^{2}$, RICARDO POCINHO $^{3}$, JOÃO SERRANO 4,5 , RUI \\ PAULO 4,5 \\ ${ }^{1}$ Câmara Municipal de Castelo Branco, Portugal \\ 2University of Salamanca, Spain \\ ${ }^{3}$ Escola Superior de Educação e Ciências Sociais, Instituto Politécnico de Leiria, Portugal \\ ${ }^{4}$ Escola Superior de Educação, Instituto Politécnico de Castelo Branco, Portugal \\ ${ }^{5}$ Sport, Health and Exercise Research Unit (SHERU), Portugal
}

\begin{abstract}
Sociocultural animation is relevant in the development of the child, because valuing play, prevents conflicts and favours social relations. At school, this interrelation with the playful makes the action of Sociocultural Animation applied to education helps to build the personality of the child, instructing rules and rules of socialization in a playful way. The present article aims to show the pedagogical perspective of the relationship between the Sociocultural Animation and the education, verifying the perception that the students and teachers reveal about it. the present study tried to demonstrate the value that the Sociocultural Animation has in the integration and success in school, in the perception of the own students and their respective teachers of the Basic Education. In this study, participated 609 students and 36 teachers from the School Grouping, $1^{\circ}$ St Cycle School. The questionnaire "Adaptation of the Program Implementation Assessment Scale" was administered to collect information from students and teachers regarding the importance and impact of socio-cultural animation in the school context. From the descriptive statistical analysis it was possible to verify that students and teachers recognize the importance of non-formal education to promote school success. Keywords: Sociocultural animation; Child education; Non-formal education; Growth.
\end{abstract}

Corresponding author. Câmara Municipal de Castelo Branco, Portugal.

E-mail: pmaga@ipb.pt

Supplementary Issue: Spring Conferences of Sports Science. International Seminar of Physical Education, Leisure and Health, 17-19 June 2019. Castelo Branco, Portugal.

JOURNAL OF HUMAN SPORT \& EXERCISE ISSN 1988-5202

(c) Faculty of Education. University of Alicante.

doi:10.14198/jhse.2019.14.Proc4.82 


\section{INTRODUCTION}

One of the permanent needs with which the child is born is to play. It is through this activity that she develops her skills, her social, affective, cognitive and physical abilities (Macedo et al., 2005). By playing the children express their interests, desires and desires. Play, games and recreations are forms of insertion in everyday life, and in this way the child exposes his thoughts, organizes and disorganizes, builds and rebuilds his world, acquiring social rules and values (Lopes et al., 2010) . The ludic activities promoted by the Animation Technician allow a better knowledge of the individual and the group. The child learns not only to express himself individually but also to listen, agree or disagree with the opinions of colleagues. Playing the child makes learning that makes him a more capable, respectful, tolerant and autonomous adult (Lopes, 2006). If the playful activities that Sociocultural Animation makes possible to be an integral part of the child's education (with the same relevance given to the formal actions provided by the educational system), they would not only benefit the children, but also the society where they will insert themselves as adults in the future next. The ludic component integrates the work of Sociocultural Animation, in that it is seen not only as a "joke", but also as a resource that facilitates the social construction of the child, its development process, helping in the formation of his own knowledge. Integrating Sociocultural Animation, associated with play, in an educational context, linking a model of formal education with a non-formal educational component, allows the child: to develop oral and corporal expression; strengthen social, intellectual and affective skills; reduce violence and aggression; establish healthy social relationships; build your own knowledge (Jardim \& Pereira, 2006).

\section{MATERIAL AND METHODS}

Sociocultural Animation in primary schools should be seen as a priority strategy to promote socialization and prevention of social exclusion as well as other problems that are inherent to it (eg school drop-out). The purpose of this study was to analyse the importance of socio-cultural activities for 1st cycle students, as well as for teachers, and to verify the existing perception about the impact of socio-cultural animation in the school context. More specifically, it was intended to study students' perceptions about the preventive impact that sociocultural animation might have on school problems (e.g. social exclusion, school dropout, indiscipline, bullying).

\section{Participants}

This study had the participation of 609 students, studying in the elementary school of the Nuno Álvares School Grouping, located in Castelo Branco, Portugal. The students were between 6 and 10 years old and were distributed between the 1 st year and the 4 th year of schooling. This study had, also, the participation of 36 teachers from the school group.

\section{Measures}

The collection of information to be used to analyse the study object was performed through the administration of a questionnaire - Adaptation of the Program Implementation Evaluation Scale (Jardim \& Pereira, 2006). The students and the teachers should respond according to a Likert scale, with a rating from 1 (Bad) to 5 (Very good).

\section{Procedures}

All participants agreed to individual participation in this research study, thus respecting the informed consent necessary and inherent in any type of field study. Authorization was sought from the parents. 


\section{Analysis}

The statistical analysis was performed with the SPSS (Statistical Package for the Social Sciences) version 22.0 for Windows, and involved measures of descriptive statistics and inferential statistics.

\section{RESULTS}

The results show that the students consider that the activities of socio-cultural animation are very important in educational education $(76.3 \%)$ and the teachers consider the integration of socio-cultural animation also interesting (58.3\%). Most of the students also mention that these activities promote success in learning, motivating students to school $(71.3 \%)$ and teachers also reinforce the existence of a link between activities and the greater interest in school content $(61.1 \% \%)$. The students also show that you like more of the school when there are activities of sociocultural animation $(74.7 \%)$, and the teachers say that the activities seem to contribute to the students' enjoyment of the school (69.4\%). In this sense, both students (71.2\%) and teachers $(75 \%)$ affirm that it is important to integrate socio-cultural animation into the curriculum, in order to promote school success. Sociocultural animation is seen as a potential educational offer by students $(71.9 \%)$ and by teachers $(77.8 \%)$.

\section{DISCUSSION}

The results show that the students consider that the activities of socio-cultural animation developed allow to promote school success, as well as interest in school contents, motivation to study and learn and the best relationship between students and teachers. Teachers also verify the positive relationship between school and socio-cultural animation, reinforcing that students actually benefit from these activities, being more committed, interested and focused on learning. The results confirm the fundamental role of Sociocultural Animation in the school context. The teachers, whose answers show that they recognize the merit of the animation technicians and do not feel threatened by their presence in the schools or by the students, who, even subject to an extensive curriculum, approve the entry of this area in their school curriculum, because it offers differentiated activities, that the other areas / disciplines in the same curriculum do not allow. The Sociocultural Animation employs fundamental techniques that allow to embrace the comprehensive dimensions that are part of the formation of the young, creating conditions for the realization of learning in a non-formal context and for the internalization of social rules. This Grouping provides concrete help in the complementarity of the educational process of parents / guardians in the development of their children / learners. With this study, we try to demonstrate the value of the role of the Sociocultural Animator in the enrichment of playing as a social activity of childhood. We believe that Play should play an essential role in education and the Sociocultural Animator is the fundamental element for this to happen, creating games, spaces, materials and providing social games.

\section{CONCLUSION}

With this study, the value of the role of the Sociocultural Animator in the enrichment of the kitten as a social activity of childhood. We believe that Playing must be an essential element in education and the Sociocultural Animator is the fundamental for this to happen, playing games, spaces, materials and providing social games. We intend to recognize one of the actions of Sociocultural Animation in the education of children, with an integration of Sociocultural Animation Techniques in the teams schools and non-grouped schools, Animation activities at all levels of education (from pre-school to teaching secondary). This research will undoubtedly serve to strengthen a task of with a broad education, providing new horizons and knowledge. 


\section{REFERENCES}

Jardim, J. \& Pereira, A. (2006). Competências pessoais e sociais: Guia prático para a mudança positiva. Porto: Edições ASA.

Lopes, M. S. (2006). Animação sociocultural em Portugal. Chaves: Editora Intervenção Associação para a Promoção e Divulgação Cultural.

Lopes, M., Galinha, A. \& Loureiro, M. (2010). Animação e bem-estar psicológico. Metodologias de intervenção sociocultural e educativa, Intervenção, Chaves.

Macedo, Lino de; Petty, Ana Lúcia Sicoli; Passos, Norimar Christie. (2005) Os jogos e o lúdico na aprendizagem. Porto Alegre: Artmed. 


\title{
Is running kinematics of university trainned students changed by hipertrophy training? A pilot study
}

\author{
PEDRO CARVALHO ${ }^{1}$, MIGUEL MORAIS $^{1}$, MÁRIO J. COSTA ${ }^{1,2}$ \\ ${ }^{1}$ Polytechnic Institute of Guarda, Portugal \\ ${ }^{2}$ Research Centre in Sports Sciences, Health Sciences and Human Development, CIDESD, Portugal
}

\begin{abstract}
The aim of this study was to investigate if running kinematics are changed by a single session of hypertrophy training. Four male subjects were filmed while running at two different speeds ( $8 \mathrm{~km} / \mathrm{h}$ and $16 \mathrm{~km} / \mathrm{h}$ ) for $1 \mathrm{~min}$ before (pre-test) and after (post-test) a hypertrophy training session. Strength training was composed by squatting with bar, deadlift, and leg press at a $60 \%$ of RM. Three sets of 10 repetitions were performed in each exercise. The Kinovea software was used to determine: (i) knee angle at heal strike (aHS); (ii) knee angle in the midstance (aMS); (iii) knee angle at toe off (aTO); (iv) minimum knee angle on the swing (aSw); (v) trunk incline ( $\mathrm{TI}$ ) and; (vi) time of three cycles (Tcycle). Results were interpreted using descriptive statistics as mean and standard deviation. There was an increase of aHS (2 and $7 \%$ for $8 \mathrm{~km} / \mathrm{h}$ and $16 \mathrm{~km} / \mathrm{h}$, respectively). Changes in aMS and aTO were trivial (0.7-1.5\%). The aSw increased $2.3 \%$ and $6.7 \%$ at $8 \mathrm{k} / \mathrm{h}$ and $16 \mathrm{~km} / \mathrm{h}$, respectively. A greater $\mathrm{Tl}$ and a reduced Tcycle was seen around $5 \%$. Thus, it seems that a strength training session focusing on hypertrophy of the lower limbs will induce important muscular deficitis, that can impair running kinematics. Keywords: Strength; Biomechanics; Training; Running.
\end{abstract}

Corresponding author. Av. Dr. Francisco Sá Carneiro, 6300-559, Guarda, Portugal.

E-mail: mario.costa@ipg.pt

Supplementary Issue: Spring Conferences of Sports Science. International Seminar of Physical Education, Leisure and Health, 17-19 June 2019. Castelo Branco, Portugal.

JOURNAL OF HUMAN SPORT \& EXERCISE ISSN 1988-5202

(c) Faculty of Education. University of Alicante.

doi:10.14198/jhse.2019.14.Proc4.82 


\section{INTRODUCTION}

Normally, the act of running is performed in the gym before or after a strength training session. It is expected that, neuromuscular fatigue induced by weights training may impose changes in running kinematics and hence, increase the susceptibility to injury. However, scientific evidence on that topic remains scarce. This study aimed to investigate if running kinematics can be affected by a single session of hypertrophy training.

\section{MATERIAL AND METHODS}

\section{Participants}

Four male subjects ( $23.5 \pm 1.65$ years, $178 \pm 187 \mathrm{~cm}$ of height and $83 \pm 4.12 \mathrm{~kg}$ of body mass) were recruited considering some inclusion criteria: (i) be physically active; (ii) have at least two years of experience in resistance training sessions; (iii) not show any skeletal-muscular injury in the previous six months.

\section{Measures}

The following measures were taken: (i) knee angle at heal strike (aHS); (ii) knee angle in the midstance (aMS); (iii) knee angle at toe off (aTO); (iv) minimum knee angle on the swing (aSw); (v) trunk incline (TI) and; (vi) time of three cycles (Tcycle).

\section{Procedures}

The subjects were filmed in the sagittal and frontal planes with a pair of cameras (Go Pro model hero5, United States) with a sampling rate of $60 \mathrm{~Hz}$, at a 1,5 meters distance and 1 meter in height. Body markers were applied in the most important anatomical regions such as the lateral malleolus, lateral condyle of the femur, great trochanter and acromion. With the dermatographic pencil the patellar tendon and medial portion of the gastrocnemius were identified. All subjects performed running ergometer (BH fitness, Portugal) at two different speeds $(8 \mathrm{~km} / \mathrm{h}$ and later at $16 \mathrm{~km} / \mathrm{h}$ ) for $1 \mathrm{~min}$. Each subject repeated the same procedure after the strength training protocol.

The muscle hypertrophy training was adapted from Bompa (2000) aiming to exercise the lower limbs. The chosen exercises were: squat with bar, deadlift and leg press. Three sets of 10 repetitions were performed at $60 \%$ of the $1 \mathrm{RM}$, with 1 min of recovery between sets and 2 min between exercises. The 1RM test was conducted as described elsewhere (Heyward, 2010).

\section{Analysis}

Descriptive statistics were used to interpret the results computing mean and standard deviation. Data processing was done using the Kinovea motion analysis software. Only 3 consecutive running cycles were retrieved for further analysis.

\section{RESULTS}

Table 1 shows the kinematic changes from the pre to the post workout at both speeds. There was a trend for an increase in knee joint angle from pre to post training, at both 8 and $16 \mathrm{~km} / \mathrm{h}$. In contrast, the remaining angles seem to decrease, along with the three cycles duration, suggesting an increase in the stride frequency due to fatigue. 
Table 1. Kinematic characteristics at pre and post workout at both running velocities

\begin{tabular}{lcccccc}
\hline & \multicolumn{2}{c}{ Pre-workout } & \multicolumn{2}{c}{ Post-workout } & \multicolumn{2}{c}{$\%$ Variation } \\
& $8 \mathrm{~km} / \mathrm{h}$ & $16 \mathrm{~km} / \mathrm{h}$ & $8 \mathrm{~km} / \mathrm{h}$ & $16 \mathrm{~km} / \mathrm{h}$ & $8 \mathrm{~km} / \mathrm{h}$ & $16 \mathrm{~km} / \mathrm{h}$ \\
\hline aHS $\left(^{\circ}\right)$ & 159.25 & 153.00 & 162.75 & 163.75 & 2.20 & 7.03 \\
aMS $\left(^{\circ}\right)$ & 143.75 & 134.75 & 142.50 & 135.75 & -0.87 & 0.74 \\
aTO $\left(^{\circ}\right)$ & 146.75 & 154.50 & 148.75 & 156.75 & 1.36 & 1.46 \\
aSw $\left(^{\circ}\right)$ & 96.50 & 78.00 & 99.00 & 83.25 & 2.59 & 6.73 \\
TI $\left({ }^{\circ}\right)$ & 88.25 & 84.75 & 83.25 & 81.75 & -5.67 & -3.54 \\
Tcycle $(\mathrm{s})$ & 2.27 & 2,04 & 2.14 & 1.94 & -5.84 & -5.02 \\
\hline
\end{tabular}

\section{DISCUSSION}

It is consensual that hypertrophy training induces muscle and neural fatigue, and may cause changes in normal patterns of movement (Arruda, et al., 2010). The highest change detected from pre to post workout was in the aHS at $16 \mathrm{~km} / \mathrm{h}$, suggesting that the muscular capacity of the lower limbs were reduced, with less condition to absorb the impact. This variation from pre to post-test allow us to understand that, in the heal strike phase, the knee is in greater extension, forcing the anterior cruciate ligament, increasing the incidence of injury (Lima \& Monaco, 2014). An increase in trunk inclination was also noticed. This may indicate a greater requirement under the muscles responsible for the torso extension, inducing an increased load in the lumbar region. However, this seems to be a positive motor behaviour. According with previous reports, this may relieve pressure at the patellofemoral joint (Teng, 2014). No less important was the increase in the stride frequency. An increased frequency directly affects kinetics, kinematics and peak impact during running, becoming a mechanism that influences both recovery and injury risk of athletes (Schubert et al., 2014). However, we believe that its adjustment was due mainly to elevated neuromuscular fatigue, affecting the voluntary activation and motor units, and consequent reduction of contraction velocity.

\section{CONCLUSIONS}

A hypertrophy training session for the lower limbs seems to induce important muscular changes, which impair the act of running. In this sense, exercise instructors should supervise their clients at this stage of the workout, aiming to maintain a fairly motor pattern in order to avoid the occurrence of injury.

\section{REFERENCES}

Arruda, D, Assumpção, C, Urtado, C, Dorta, Rosa, M, Zabaglia, R., \& Souza, T. (2010). Relação entre treinamento de força e redução do peso corporal. Rev. Bras. Exercício. 4. 605-609.

Bompa, T., \& Cornachia, L. (2000). Treino de Força Consciente . Phorte Editora.

Heyward, V. (2010). Avaliação e Prescrição do Exercício. Artmed.

Lima, D. \& Monaco, J. (2014). A relação entre o desequilibrio muscular entre quadricipites e isquiotibiais e a lesão de LCA em atletas. Pindamonhangaba.

Schubert, A. Kempf, J. \& Heiderscheit, B. (2014). Influence of Stride Frequency and Length on Running Mechanics: A systematic Review. American Orthopaedic Society for Sports Medicine. 6. 210-217. https://doi.org/10.1177/1941738113508544

Teng, H. \& Powers, C. (2014). Sagittal Plane Trunk Posture Influences Patellofemoral Joint Stress During Running. journal orthopaedic therapy. 44. 785-792. https://doi.org/10.2519/jospt.2014.5249 


\section{(c) $\underset{\mathrm{EY}}{\mathrm{NO}} \mathrm{\Theta}$}

This work is licensed under a Attribution-NonCommercial-NoDerivatives 4.0 International (CC BY-NC-ND 4.0). 